\title{
SYNTHESE UND UMLAGERUNG VON BICYCLOBUTYL-1-OLEN UND SPIRO[3.4]OCTAN-5-OLEN: EIN NEUER ZUGANG ZU ( \pm )-endo-CAPNELLEN
}

\author{
DISSERTATION \\ Zur Erlangung des Doktorgrades \\ der Mathematisch-Naturwissenschaftlichen Fakultäten \\ der Georg-August-Universität zu Göttingen
}

vorgelegt von

Klaus Mandelt

aus Göttingen

Göttingen 2000 
D7

Referent: Prof. Dr. L. Fitjer

Korreferent: Prof. Dr. A. de Meijere

Tag der mündlichen Prüfung: 30.10.2000 
Die vorliegende Arbeit wurde unter der Leitung von Herrn Prof. Dr. L. Fitjer in der Zeit von Juli 1997 bis September 2000 am Organisch-Chemischen Institut der Georg-AugustUniversität zu Göttingen durchgeführt.

An dieser Stelle möchte ich Herrn Prof. Dr. L. Fitjer für die Anregung zu diesem Thema, sein stetes Interesse am Fortgang der Arbeit, die ausgezeichneten Arbeitsbedingungen und zahlreichen fördernden Diskussionen danken. 
Meiner Familie 


\section{INHALTSVERZEICHNIS}

Seite

EINLEITUNG

ALLGEMEINER TEIL

1 Synthese und Umlagerung substituierter Bicyclobutyl-1-ole

1.1 Synthese von 2-Methyl-, 1,2-Dimethyl- und 1,2,2-

Trimethyl-cyclobutylchlorid 82a,b, 86a,b und 97

1.2 Generierung und Carboxylierung der Grignard-Reagenzien 21a,b,

22a,b und 23: 2-Methyl- (81a,b), 1,2-Dimethyl- (86a,b) und 1,2,2-

Trimethyl-cyclobutancarbon- säure (100)

1.3 Versuche zur Addition der Grignard-Reagenzien 8, 21a,b, 22a,b und 23 an Cyclobutanone: 2,1'-Dimethyl- (104b), 2,2,1'-Trimethyl- (105), 2'-Methyl- (106a,b) und 1',2'-Dimethyl-bicyclobutyl-1-ol (107a,b)

1.4 Stereochemische Zuordnung der Cyclobutancarbonsäuren 81a,b und

98a,b, der Cyclobutylchloride 82a,b und 86a,b sowie der Bicyclobutyl-1-ole 106a,b und 107a,b

1.5 Umlagerung der Bicyclobutyl-1-ole 104b, 105, 106a, 106b, 107a und 107b zu mono- (108), di- (109) und trimethylierten Bicyclo[3.3.0] octenen $(\mathbf{1 1 8}, \mathbf{1 2 0})$

2 Synthese und Umlagerung potentieller Vorläufer von ( \pm )-endo-Capnellen (44) mit Bicyclobutyl-Teilstruktur: 1,4,4-Trimethyl-6-(2'-methyl-cyclobutyl)bicyclo-[3.2.0]heptan-6-ole

2.1 Darstellung der 1,4,4-Trimethyl-6-(2'-methyl-cyclobutyl)-bicyclo[3.2.0]heptan-6- ole $\mathbf{4 6}, \mathbf{4 7}, \mathbf{4 8}$ und 49 
2.2 Säurekatalysierte Umlagerung von 46 und 49: Bicyclo[3.2.0]hept6-yl-Cycloheptenyl-Umlagerung unter Bildung von Dienen

2.3 Umsetzung von $\mathbf{4 6}$ und $\mathbf{4 9}$ mit Thionylchlorid in Pyridin: Bildung von Cyclobutyl-cyclobutenen und Bicyclobutylidenen

3 Synthese und Umlagerung potentieller Vorläufer von ( \pm )-endo-Capnellen (44) mit Spirooctyl-Teilstruktur: (1 $\left.\mathrm{R}^{*}, 5 \mathrm{R}^{*}, 6 \mathrm{R}^{*}, 2^{\prime} \mathrm{R}^{*}, 3^{\prime} \mathrm{R}^{*}\right)-(\mathbf{6 0}),\left(1 \mathrm{R}^{*}, 5 \mathrm{R}^{*}\right.$, $\left.6 \mathrm{R}^{*}, 2^{\prime} \mathrm{S}^{*}, 3^{\prime} \mathrm{R}^{*}\right)-(64)$ und $\left(1 \mathrm{R}^{*}, 5 \mathrm{R}^{*}, 6 \mathrm{R}^{*}, 2^{\prime} \mathrm{R}^{*}, 3^{\prime} \mathrm{S}^{*}\right)-1,4,4,3^{\prime}$-Tetramethylspiro $\left\{\right.$ bicyclo-[3.2.0]heptan-6, $1^{\prime}$-cyclopentan-2'-ol\} (66)

3.1 Synthese von $\left(1 \mathrm{R}^{*}, 5 \mathrm{R}^{*}, 6 \mathrm{R}^{*}\right)-1,4,4$-Trimethylspiro\{bicyclo[3.2.0]heptan-6, $1^{\prime}$-cyclopentan-2'-on $\}$ (57)

3.2 Methylierung und Reduktion von $57 \mathrm{zu}$ den Spirooctanolen 60, 64 und 66

3.3 Röntgenstrukturanalysen von $\mathbf{6 0}$ und $\mathbf{6 6}$

3.4 Säurekatalysierte Umlagerung der Spirooctanole $\mathbf{6 0}$ und $\mathbf{6 6}$ zu den Tetramethyl- octahydro-1H-cyclopenta[a]pentalenen 67-70 und (士)-endo-Capnellen (44)

3.4.1 Produkte und zeitlicher Verlauf der säurekatalysierten Umlagerung von 60

3.4.2 Produkte und zeitlicher Verlauf der säurekatalysierten Umlagerung von 66

3.4.3 Der Mechanismus der Umlagerungen von 60 und 66 
SPEKTRENANHANG

$\begin{array}{ll}\text { IR-Spektren } & 91\end{array}$

$\begin{array}{ll}{ }^{1} \mathrm{H}-\mathrm{NMR}-S p e k t r e n & 102\end{array}$

${ }^{13}$ C-NMR-Spektren $\quad 121$

$\begin{array}{ll}\text { ABBILDUNGSVERZEICHNIS } & 168\end{array}$

LITERATURVERZEICHNIS 177 


\section{EINLEITUNG}

Die säurekatalysierte Umlagerung von Bicyclobutyl-1-olen und Bicyclobutylidenen [2(3)-4-5] ist eine potentiell wertvolle Methode zum Aufbau von Bicyclo[3.3.0]octenen ${ }^{1}$ (Schema 1). Nachteilig ist, dass substituierte Vertreter aufgrund der Bildungsweise der benötigten Edukte [1-2(3)] nur über substituierte Cyclobutanone und / oder substituierte Wittig- bzw. Grignard-Reagenzien zugänglich sind. Substituierte Cyclobutanone sind in großer Zahl verfügbar ${ }^{2}$, auf substituierten Cyclobutanen basierende Grignard- und WittigReagenzien dagegen rar. In der Abteilung wurde deshalb damit begonnen diesem Mangel abzuhelfen.

\section{Schema 1}

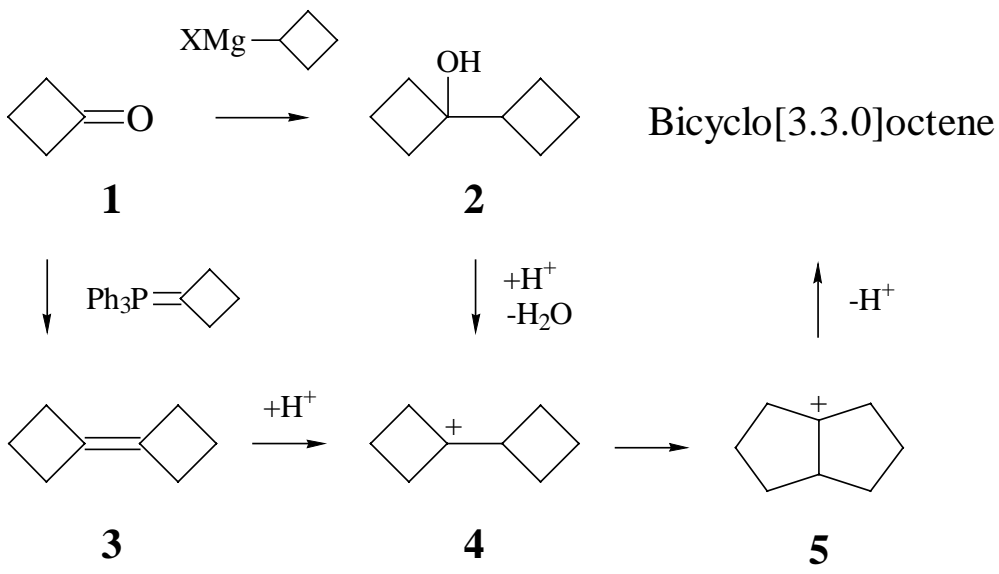

\section{Schema 2}

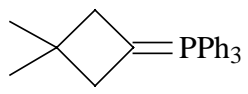

6

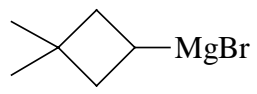

7

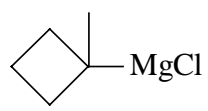

8

Bei den ersten auf substituierten Cyclobutanen basierenden Wittig- bzw. GrignardReagenzien handelte es sich um $\mathbf{6}^{3}, 7^{4}$ und $\mathbf{8}^{5}$ (Schema 2). Sämtliche Reagenzien wurden zu Naturstoffsynthesen genutzt (Schema 3). Das Wittig-Reagenz 6 diente zur Synthese der Bicyclobutylidene 9 und 12 sowie nach Umlagerung zu den Bicyclooctenen 10 bzw. 13 zur Synthese der Sesquiterpene $( \pm)$-Ceratopicanol $(\mathbf{1 1})^{6}$ und $( \pm)$-Hirsuten $(\mathbf{1 4})^{6}$. Die Grignard- 
Reagenzien 7 und 8 dienten zur Darstellung der Cyclobutylcarbinole 15 bzw. 18a,b sowie nach Umlagerung zu 16 bzw. 19a,b zur Synthese der Sesquiterpene ( \pm )-Cerapicol $(\mathbf{1 7})^{4}$ bzw. $( \pm)$-Cuparen $(\mathbf{2 0 a})^{5}$ und $( \pm)$-Herberten $(\mathbf{2 0 b})^{5}$.

\section{Schema 3}

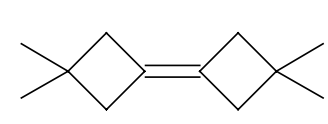

9

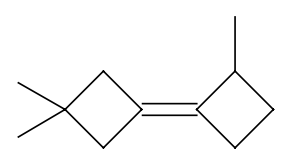

12

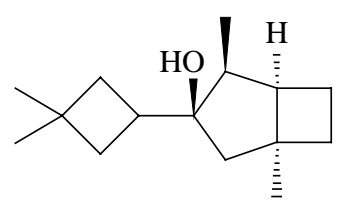

15

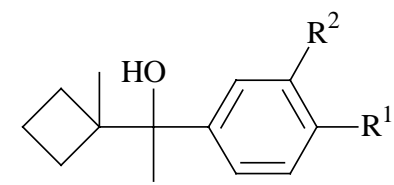

$\mathrm{p}-\mathrm{TsOH}$

$\mathrm{CF}_{3} \mathrm{COOH}$<smiles>CC(=O)OC[C@H]1C[C@@]2(C)CC[C@@H]1[C@H]1CC(C)(C)C[C@H]1C2</smiles>

16

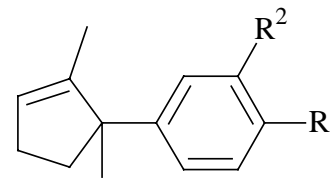

19a

19b
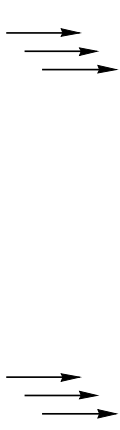

$11( \pm)$-Ceratopicanol

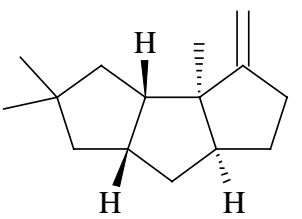

14 ( \pm )-Hirsuten 18a $\mathrm{R}^{1}=\mathrm{CH}_{3}, \mathrm{R}^{2}=\mathrm{H}$ $18 b \mathrm{R}^{1}=\mathrm{H}, \mathrm{R}^{2}=\mathrm{CH}_{3}$
13<smiles>CC1(C)CC2[C@@H]3CC[C@@](C)(C3)[C@@H]2C[C@H]1CO</smiles>

17 (士)-Cerapicol<smiles>[R]c1ccc(C2(C)CCCC2(C)C)cc1[R]</smiles>

20a ( \pm )-Cuparen

20b ( \pm )-Herberten

Es lag auf der Hand, dass mit den aufgeführten Beispielen das Synthesepotential säurekatalysierter Umlagerungen von Bicyclobutylidenen und Bicyclobutyl-1-olen keineswegs ausgeschöpft war. Es war vielmehr zu erwarten, dass sich für die vorhandenen wie für neu darzustellende Reagenzien weitere Anwendungsmöglichkeiten ergeben würden. Im ersten Teil der vorliegenden Arbeit sollten die hierzu notwendigen Beurteilungskriterien erarbeitet werden.

Hierzu war geplant, mit 21a,b, 22a,b und $\mathbf{2 3}$ drei weitere auf substituierten Cyclobutanen basierende Grignard-Reagenzien darzustellen, durch Carboxylierung zu 
charakterisieren und zusammen mit $\mathbf{8}$ bezüglich ihrer Reaktivität gegenüber den Cyclobutanonen 24-26 zu testen (Schema 4). Gebildete Additionsprodukte sollten umgelagert und auf diese Weise die über Bicyclobutyl-Bicyclooctyl-Umlagerungen zugänglichen Substitutionsmuster von Bicyclooctenen eingegrenzt werden. Besonders interessiert waren wir an 27a,b als erhoffte Produkte einer Addition von 22a,b an 26, deren Umlagerung bei günstigem Verlauf zu 28 als potentiellem Vorläufer des Sesquiterpens $\left( \pm\right.$ )-Ptychanolid $(\mathbf{2 9})^{7}$ hätte führen können. Für dieses Sesquiterpen gibt es bis heute keine Synthese ${ }^{8}$.

\section{Schema 4}

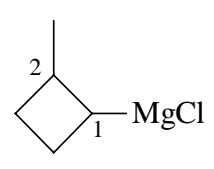

21a $1 R^{*}, 2 S^{*}$

21b $1 R^{*}, 2 R^{*}$

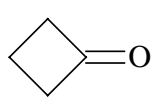

24

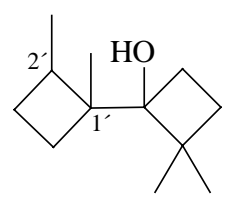

27a $1^{\prime} R^{*}, 2 S^{*}$ 27b $1^{\prime} R^{*}, 2^{\prime} R^{*}$

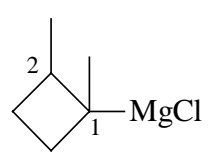

22a $1 R^{*}, 2 S^{*}$

22b $1 R^{*}, 2 R^{*}$

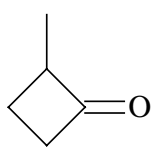

25

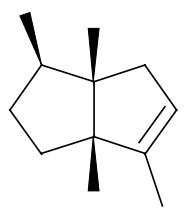

28

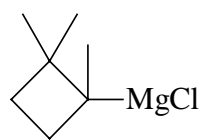

23

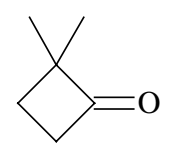

26

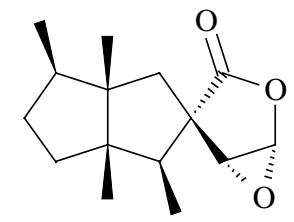

29 ( \pm )-Ptychanolid

Nach Eingrenzung der Möglichkeiten zur Synthese mono- bis tetramethylierter Bicyclooctene durch Synthese und Umlagerung von Bicyclobutyl-1-olen sollten im zweiten Teil der Arbeit gezielt solche Edukte synthetisiert und umgelagert werden, die direkt zu Sesquiterpen-Kohlenwasserstoffen führen konnten. Hierzu kamen insbesondere solche Verbindungen in Frage, die nach einer mit dem Retrosyntheseprogramm CARESY ${ }^{9}$ in Verbindung mit dem Programm HUNTER ${ }^{10}$ durchgeführten Analyse säurekatalysiert zu dem Sesquiterpen $\left( \pm\right.$ )-endo-Capnellen $(\mathbf{4 4})^{11}$ umlagern sollten. Hierdurch sollte gleichzeitig der prognostische Wert von a priori-Analysen kationischer Umlagerungen für Naturstoffsynthesen überprüft werden. 
Nach CARESY ist das Bicyclobutyl-Kation 30 ein direkter Vorläufer von $( \pm)$-endoCapnellen (44) (30-38-42-43-44) (Schema 5). Es schien allerdings keineswegs unwahrscheinlich, dass auch bei Isomeren von 30 (31-33) sowie bei Isomeren von 38 (34-37, 39-41) auf der Stufe zu 42 analoger tricyclopentanoider Kationen über Eliminierungs- und Reprotonierungsreaktionen die für $\mathbf{4 4}$ charakteristische cis-anti-cis-Stereochemie etabliert werden würde. Schwieriger zu beurteilen war die Frage nach der Regiochemie. Gleichwohl sollte versucht werden, Vorläufer von 30-33 sowie von 34-41 in Form von Alkoholen darzustellen und säurekatalysiert umzulagern.

\section{Schema 5}

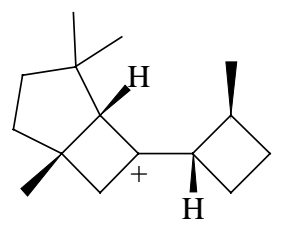

30<smiles>CCCCCC</smiles>

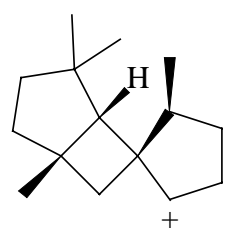

34

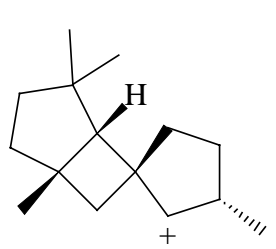

38

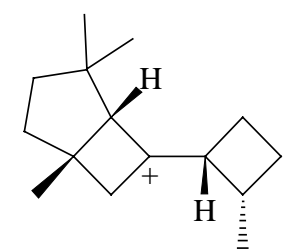

31

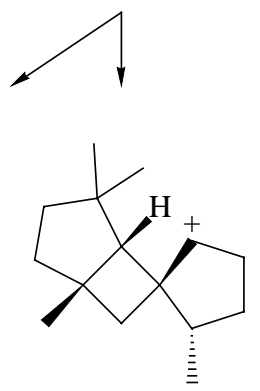

35

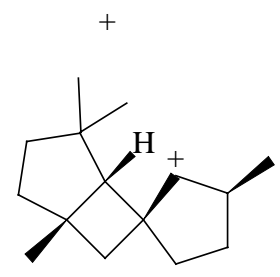

39

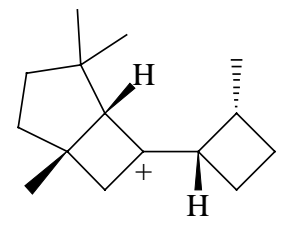

32
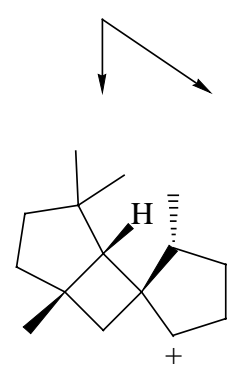

36

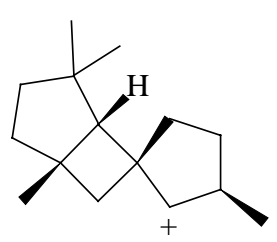

40

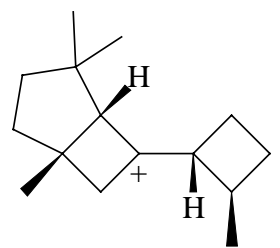

33
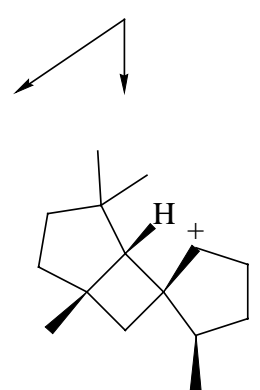

37

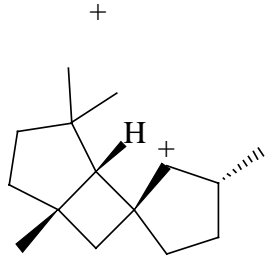

41

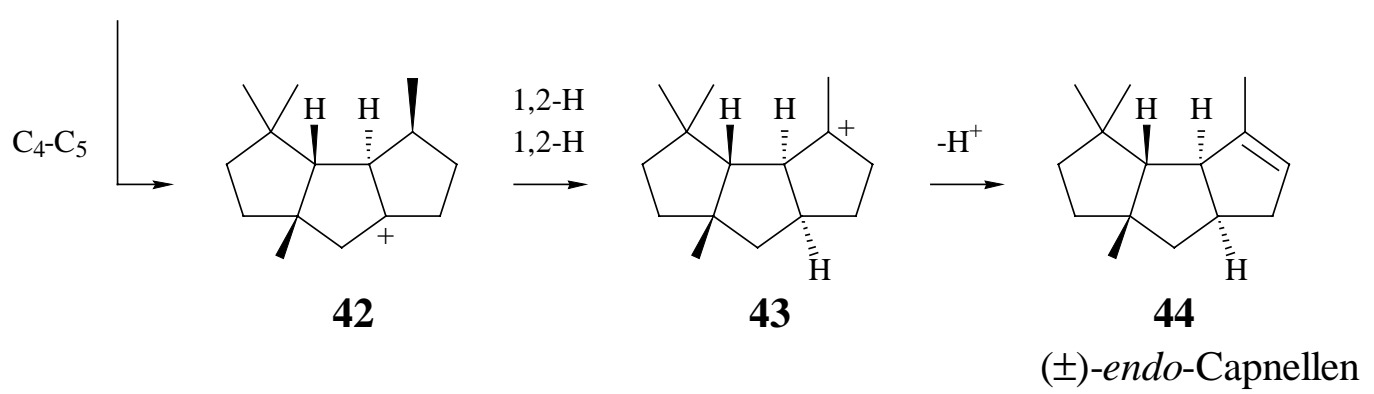


Zur Darstellung von Vorläufern der Bicyclobutyl-Kationen 30-33 sollten die GrignardReagenzien 21a,b an das in einer früheren Arbeit ${ }^{12}$ dargestellte bicyclische Keton $\mathbf{4 5}$ addiert werden. Bei einem konkurrierenden exo/endo-Angriff konnten hierbei mit 46-53 bis zu acht diastereomere Alkohole entstehen, die jedoch unter ionisierenden Bedingungen paarweise zu den erwünschten Bicyclobutyl-Kationen 30-33 kollabieren mussten (Schema 6).

\section{Schema 6}

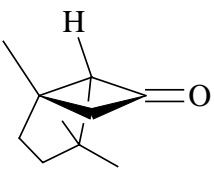

45

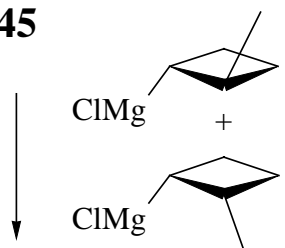

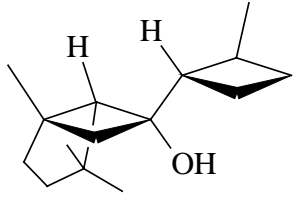

46

$+$

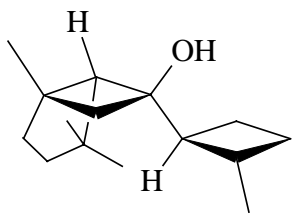

50

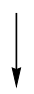

30

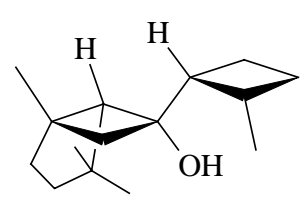

47

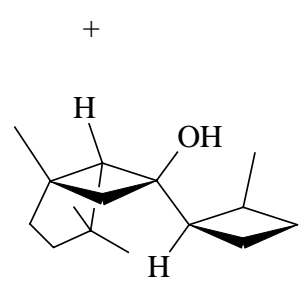

51

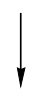

31

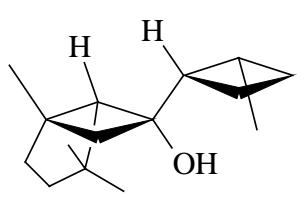

48

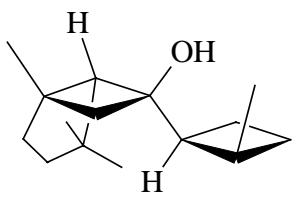

52

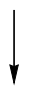

32

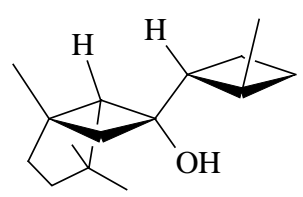

49

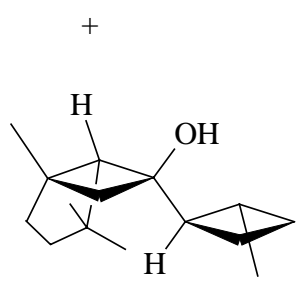

53

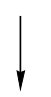

33

Von den Spirooctyl-Kationen 34-41 schienen lediglich Vorläufer von 38-41 zugänglich. $\mathrm{Zu}$ ihrer Darstellung sollte das Bicyclobutyliden 54 epoxidiert und anschließend einer Oxaspirohexan-Cyclopentanon-Umlagerung ${ }^{13}$ unterworfen werden [54-55(56)-57(58)]. Hier rechneten wir aufgrund früherer Erfahrungen ${ }^{14}$ mit ähnlichen Systemen mit einer regioselektiven Ringerweiterung des peripheren Cyclobutanringes unter Bildung der 
Spirooctanone 57 und 58. Die folgenden Schritte einer Monomethylierung und Reduktion konnten bei nicht stereospezifischem Verlauf mit 59-66 wiederum bis zu acht diastereomere Alkohole liefern, die jedoch unter ionisierenden Bedingungen, ähnlich wie 46-53, paarweise die erwünschten Spirooctyl-Kationen 38-41 liefern mussten (Schema 7).

\section{Schema 7}

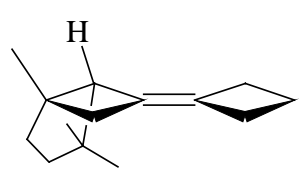

54

$\downarrow$ MCPB

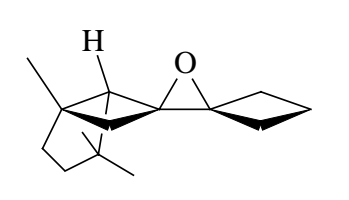

55

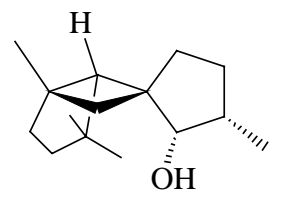

59

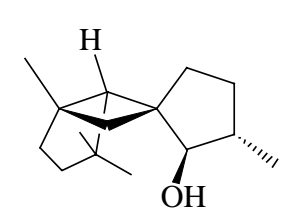

63

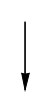

38

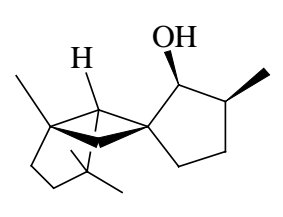

60

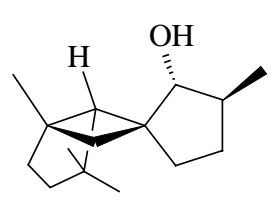

64

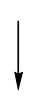

39

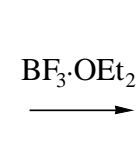

57

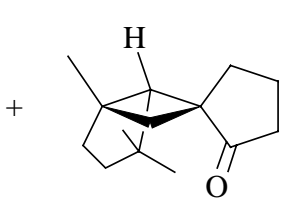

58

1. $\mathrm{LDA} / \mathrm{CH}_{3} \mathrm{I}$

r. 2. $\mathrm{LiAlH}_{4}$

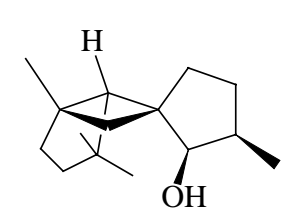

61

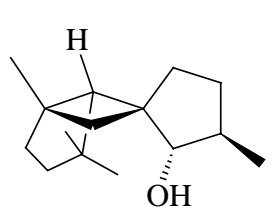

65

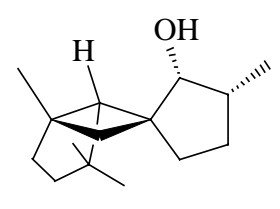

62

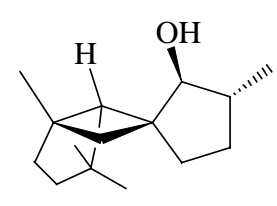

66

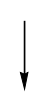

40

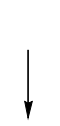

41 
Natürlich hatten wir die Erwartung, dass es bei der Mehrzahl der mit $\mathbf{4 5}$ und $\mathbf{5 4}$ durchzuführenden Reaktionen nicht zu einer statistischen Produktverteilung kommen würde. So rechneten wir bei der Addition von 21a,b an $\mathbf{4 5}$ wie bei der Epoxidierung von 54 mit einer ausgeprägten exo-Selektivität und bei der Umlagerung von 55 mit einer Begünstigung von 57. Bei den Folgereaktionen hielten wir die stereodifferenzierenden Faktoren im Zuge der Monomethylierung für schwach, im Zuge der nachfolgenden Reduktion dagegen für stark. Als Hauptprodukte erwarteten wir daher bei 45 die Bicyclobutyl-1-ole 46-49 und bei 54 die Spirooctanole 60 und 62. In jedem Fall war klar, dass die durchzuführenden Reaktionen von einer sorgfältigen Klärung der Regio- und Stereochemie begleitet sein mussten.

\section{Schema 8}

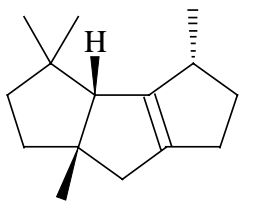

67

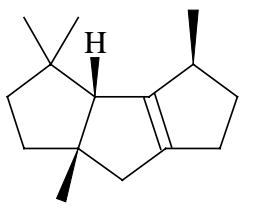

69

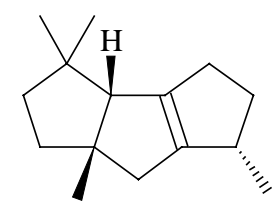

72

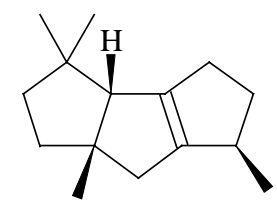

75
$-27.8$

$-29.7$

$-29.6$

$-29.4$

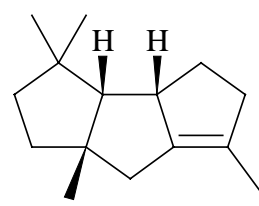

76

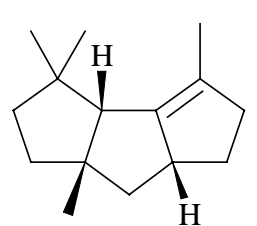

70

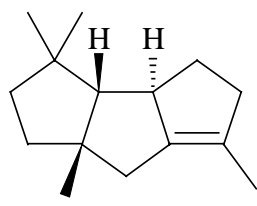

73

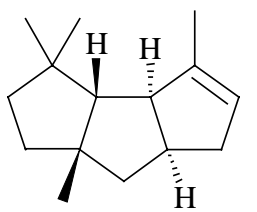

$-28.2$

44

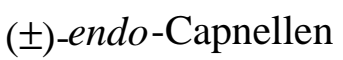

$-23.3$

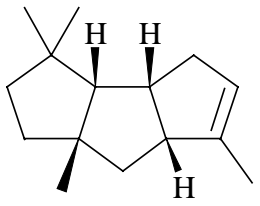

77 
Bezüglich der Struktur der zu erwartenden Umlagerungsprodukte schien bedeutsam, dass von den jeweils sechs isomeren linearen Triquinanen einheitlicher Regiochemie (44, 6771 bzw. 72-77) ein Auftreten der cis-syn-cis-konfigurierten Vertreter 70 und 71 bzw. 76 und 77 nach Rechnungen mit HUNTER $^{10}$ in Verbindung mit MMP2 ${ }^{15}$ zumindest unter äquilibrierenden Bedingungen wenig wahrscheinlich war (Schema 8). Von den verbleibenden jeweils vier Isomeren waren die ${ }^{1} \mathrm{H}$ - und ${ }^{13} \mathrm{C}$ - Daten von $\mathbf{4 4}^{11}$ bekannt. Eine Unterscheidung der restlichen Isomeren schien möglich.

Mit der vorliegenden Arbeit wurde versucht, die Anwendungsbreite der BicyclobutylBicyclooctyl-Umlagerung durch Entwicklung und Einsatz neuer Reagenzien zum Aufbau entsprechender Edukte zu erweitern. Sie beschreibt im ersten Teil die Darstellung neuer, auf substituierten Cyclobutanen basierender Grignard-Reagenzien (21a,b, 22a,b, 23), deren Addition an Cyclobutanone $(\mathbf{2 4}, \mathbf{2 5}, \mathbf{2 6})$ sowie die Umlagerung der gebildeten Produkte, im zweiten Teil die Synthese und Umlagerung von 45 abgeleiteter Bicyclobutyl-1-ole, und im dritten Teil die Synthese und Umlagerung von 54 abgeleiteter Spirooctanole. Die Versuche lieferten variabel substituierte Bicyclooctene, neue Cycloheptadiene und öffneten einen neuen Zugang zu ( \pm )-endo-Capnellen (44). 


\section{ALLGEMEINER TEIL}

\section{Synthese und Umlagerung substituierter Bicyclobutyl-1-ole}

Die Bicyclobutyl-Bicylooctyl-Umlagerung ist eine potentiell wertvolle Methode zum Aufbau von Bicyclo[3.3.0]octenen ${ }^{1}$. Sie besitzt ein hohes thermodynamisches Gefälle und kann unter anderem durch saure Dehydratisierung von Bicyclobutyl-1-olen ausgelöst werden (2-4-78-5). Bicyclobutyl-1-ole wiederum können durch Umsetzung von Cyclobutanonen mit Cyclobutyl-Grignard-Reagenzien dargestellt werden (1-2) ${ }^{4,5}$. Nach Umlagerung befinden sich Substituenten des Cyclobutanons in Ring A und Substituenten des Cyclobutyl-GrignardReagenzes in Ring B (Schema 9).

\section{Schema 9}

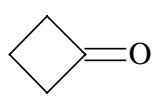

1

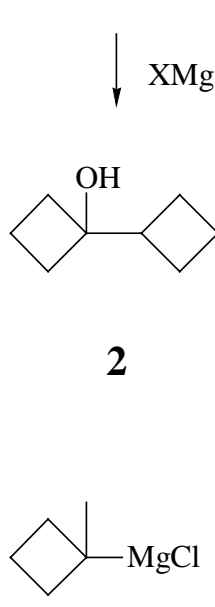

8

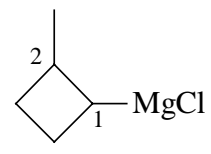

21a $1 R^{*}, 2 S^{*}$ $21 \mathrm{~b} 1 R^{*}, 2 R^{*}$

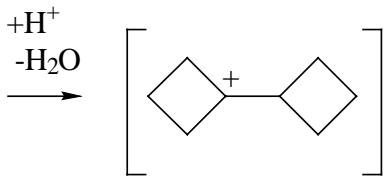

4

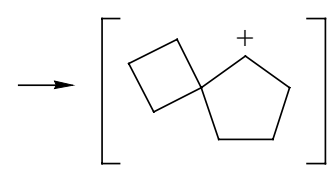

78

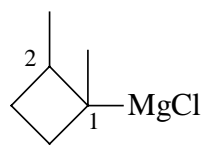

22a $1 R^{*}, 2 S^{*}$ 22b $1 R^{*}, 2 R^{*}$
Bicyclo[3.3.0]octene

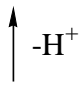

A B

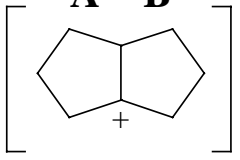

5

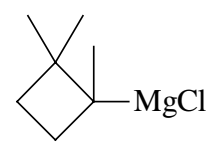

23

Im Gegensatz zu substituierten Cyclobutanonen ${ }^{2}$ sind substituierte CyclobutylGrignard-Reagenzien $\operatorname{rar}^{4,5}$. Um den Anwendungsbereich der Bicyclobutyl-BicyclooctylUmlagerung zu erweitern, sollten daher mit 21a,b, 22a,b und 23 drei bisher unbekannte Vertreter dargestellt und zusammen mit dem bereits früher beschriebenen $\mathbf{8}^{5}$ zur Synthese unterschiedlich substituierter Bicyclobutyl-1-ole eingesetzt werden. Sämtliche Bicyclobutyl- 
1-ole sollten anschließend dehydratisiert und die zu erwartenden Bicyclooctene bezüglich ihrer Struktur charakterisiert werden.

\subsection{Synthese von 2-Methyl-, 1,2-Dimethyl- und 1,2,2-Trimethyl-cyclobutylchlorid 82a,b, 86a,b und 97}

Eine unerwünschte Nebenreaktion bei der Generierung von Grignard-Reagenzien ist die Grignard-Kupplung. Insbesondere sekundäre und tertiäre Bromide und Iodide neigen dazu ${ }^{16}$. Wir haben daher von vornherein versucht, die erwünschten Cyclobutyl-GrignardVerbindungen 21a,b, 22a,b und 23 aus den entsprechenden Chloriden 82a,b, 86a,b und 97 zu generieren. Von diesen Verbindungen waren 82a,, $\mathbf{b}^{17}$ und 86a, $\mathbf{b}^{18}$ bekannt. Allerdings fehlten bei 82a,b eine überzeugende stereochemische Zuordnung und bei $\mathbf{8 6 a}, \mathbf{b}$ präparative Details.

\section{Schema 10}
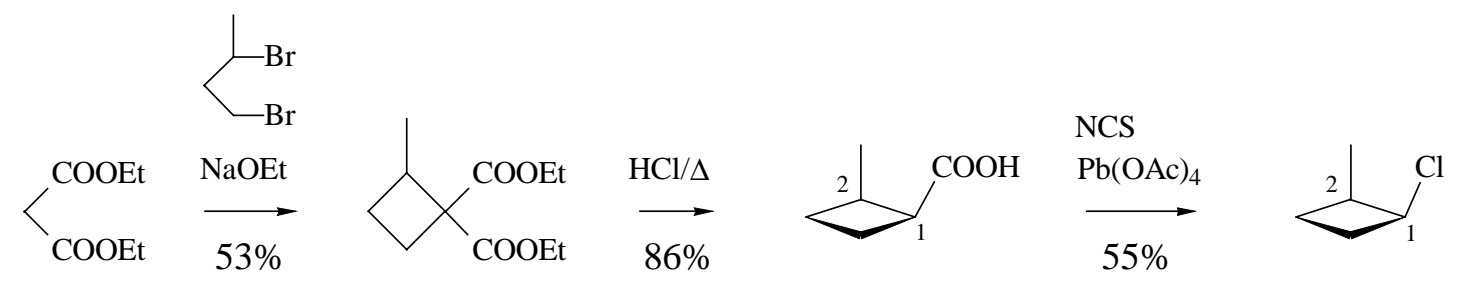

79

80

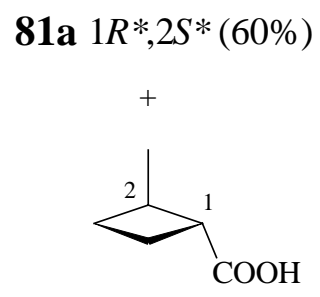

82a $1 R^{*}, 2 S^{*}(33 \%)$

81b $1 R^{*}, 2 R^{*}(40 \%)$

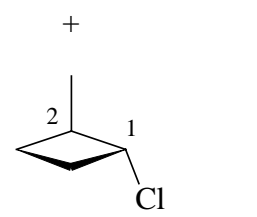

82b $1 R^{*}, 2 R^{*}(67 \%)$

Zur Darstellung der Cyclobutylchloride 82a,b folgten wir zunächst der Vorschrift von Cason und Allen ${ }^{19}$ zur Darstellung von Cyclobutancarbonsäure und unterwarfen das 60:40Gemisch der gebildeten 2-Methyl-cyclobutancarbonsäuren ${ }^{20-23}$ 81a und 81b anschließend nach Sorensen ${ }^{17}$ einem Hunsdiecker-Abbau in der Variante von Grob $^{24}$ (79-80-81a,b-82a,b). Dabei erhielten wir unter teilweiser Umkehr der Stereochemie ein 33:67-Gemisch der 2Methyl-cyclobutylchloride 82a und 82b (Schema 10). Von allen Stereoisomeren wurden 
analytisch reine Proben präparativ gaschromatographisch isoliert. Die stereochemische Zuordnung ist unter 1.4 beschrieben.

Zur Darstellung der Cyclobutylchloride 86a,b haben wir das durch photochemische Cyclisierung von käuflichem 2,3-Dimethyl-butadien-1,3 leicht zugängliche 1,2-Dimethylcyclobuten $^{25} \mathbf{8 5}$ eingesetzt (Schema 11). Behandelte man 85 in Analogie zur Darstellung des 8 zugrunde liegenden Chlorids ${ }^{26}$ bei $0^{\circ} \mathrm{C}$ mit konzentrierter Salzsäure, so bildete sich unter Cyclobutyl-Cyclopropylmethyl-Homoallyl-Umlagerung und zweifacher Addition von Chlorwasserstoff das bekannte 1,3-Dichlor-3-methylpentan ${ }^{27}$ (84) (85-89-88-87-83-84). Die Ringöffnung ließ sich durch Einleiten von trockenem Chlorwasserstoff in eine auf $-78^{\circ} \mathrm{C}$ gekühlte Lösung von 85 in Pentan vermeiden. Auf diese Weise erhielten wir nach fraktionierter Destillation in $77 \%$ Ausbeute ein 40:60-Gemisch der beiden erwünschten Cyclobutylchloride 86a und 86b. Auch hier wurden zur stereochemischen Zuordnung (vgl. 1.4) gaschromatographisch reine Proben abgetrennt. Die ${ }^{1} \mathrm{H}$ - und ${ }^{13} \mathrm{C}-\mathrm{NMR}-\mathrm{Daten}$ stimmten mit Literaturdaten ${ }^{18}$ überein.

\section{Schema 11}

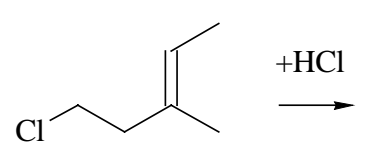

83

$+\mathrm{Cl}^{-}$

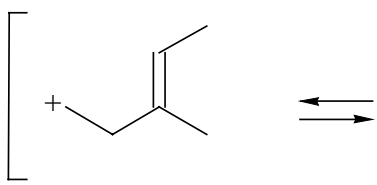

87<smiles>CCC(C)(Cl)CCCl</smiles>

84

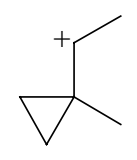

88
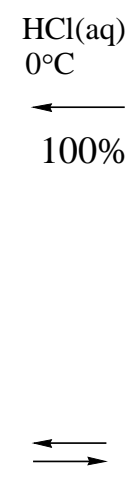

Nach den Erfahrungen mit 85 sollte die Darstellung des bisher unbekannten Cyclobutylchlorids 97 von vornherein durch Einleiten von trockenem Chlorwasserstoff in eine auf $78^{\circ} \mathrm{C}$ gekühlte Lösung eines geeigneten Olefins in Pentan erfolgen. Als Olefin wählten wir 1,1-Dimethyl-2-methylencyclobutan ${ }^{28}$ (91). Zu seiner Synthese wurde das bekannte $\beta$ - 
Hydroxysulfid ${ }^{29} 90$ nach einer Methode von Trost ${ }^{30}$ säurekatalysiert in 2,2-Dimethylcyclobutanon $^{31-34}$ (26) umgelagert und anschließend methyleniert (Schema 12).

\section{Schema 12}

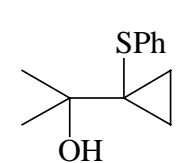

90

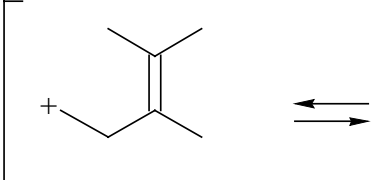

92

$\downarrow+\mathrm{Cl}^{-}$<smiles>CC(C)=C(C)CCCl</smiles>

$\mathbf{9 5}(9 \%)$

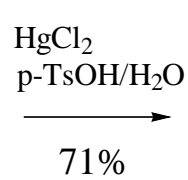

26<smiles>C[C+](C)C1(C)CC1</smiles>

93<smiles>CC(CCCl)C(C)(C)Cl</smiles>

$96(25 \%)$
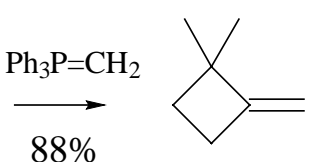

91 $\mid \begin{aligned} & \mathrm{HCl}(\mathrm{g}) /-78^{\circ} \mathrm{C} \\ & +\mathrm{H}^{+}\end{aligned}$

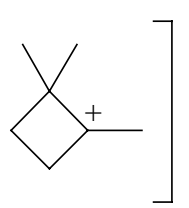

94
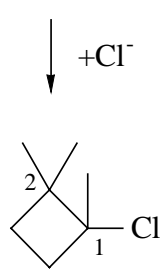

97 (66\%; 44\% isoliert)

Behandelte man 91 unter den angegebenen Bedingungen mit Chlorwasserstoff, so bildete sich ein 66:25:9-Gemisch aus drei Verbindungen. Das Hauptprodukt ließ sich in 44\% isolierter Ausbeute destillativ abtrennen und erwies sich als das erwünschte 1,2,2-Trimethylcyclobutylchlorid (97). Sein ${ }^{1} \mathrm{H}-\mathrm{NMR}-$ Spektrum (Abb. 28) zeigt die erwarteten Singuletts für die Methylprotonen bei $\delta=0.99,1.24$ und 1.61 (je 3H) und die zu erwartenden Dubletts von Dubletts von Dubletts für die Methylenprotonen bei $\delta=1.60(\mathrm{~J}=10.5,10.5,9 \mathrm{~Hz}, 1 \mathrm{H}), 1.73$ $(\mathrm{J}=10.5,10.5,3 \mathrm{~Hz}, 1 \mathrm{H}), 2.08(\mathrm{~J}=12,9,3 \mathrm{~Hz}, 1 \mathrm{H})$ und $2.44(\mathrm{~J}=12,10.5,10.5 \mathrm{~Hz}, 1 \mathrm{H}) . \mathrm{Im}$ ${ }^{13} \mathrm{C}-\mathrm{NMR}-$ Spektrum (Abb. 68) finden sich die zu fordernden sieben Resonanzen für drei primäre, zwei sekundäre und zwei quartäre Kohlenstoffe, darunter die Resonanz des Chlorcarbinyl-Kohlenstoffs bei $\delta=72.99 \mathrm{ppm}$. Aus dem Destillationsrückstand ließen sich präparativ gaschromatographisch reine Proben eines Mono- und eines Dichlorids gewinnen. 
Bei dem Monochlorid handelte es sich nach Ausweis seiner spektroskopischen Daten um das bereits in einer früheren Arbeit ${ }^{35}$ aufgetretene und durch Cyclobutyl-Cyclopropylmethyl-Homoallyl-Umlagerung entstanden zu denkende 5-Chlor-2,3-dimethyl-pent-2-en (95) (94-93-92-95). Bei dem Dichlorid handelte es sich um das ebenfalls bekannte ${ }^{36}$ 1,4-Dichlor3,4-dimethyl-pentan (96). Seine Bildung bedingt eine Addition von Chlorwasserstoff an 95.

\subsection{Generierung und Carboxylierung der Grignard-Reagenzien 21a,b, 22a,b und 23:} 2-Methyl- (81a,b), 1,2-Dimethyl- (86a,b) und 1,2,2-Trimethyl-cyclobutancarbonsäure (100)

Nachdem die Cyclobutylchloride 82a,b, 86a,b und 97 in ausreichender Menge zur Verfügung standen, sollten sie zu den Grignard-Reagenzien 21a,b, 22a,b bzw. 23 umgesetzt und diese wiederum durch Carboxylierung charakterisiert werden. Bei den zu erwartenden Cyclobutancarbonsäuren interessierten sowohl die Ausbeuten als Maß für die Konzentration der Grignard-Reagenzien als auch die stereochemische Zusammensetzung als Indiz für eine eventuelle Stereoselektion.

Zur Generierung der Grignard-Reagenzien versetzte man eine Suspension von mit Iod aktiviertem Magnesium in wasserfreiem Ether (82a,b THF) unter Argon mit einer Lösung des (der) entsprechenden Chlorids (Chloride) $(\mathbf{8 2 a}, \mathbf{b}, \mathbf{8 6 a}, \mathbf{b}, \mathbf{9 7})$ in wasserfreiem Ether $(\mathbf{8 2 a}, \mathbf{b}$ THF) und erhitzte nach Zugabe eines Tropfens Brom das Reaktionsgemisch solange zum Sieden, bis eine begleitende gaschromatographische Kontrolle den vollständigen Verbrauch des (der) Chlorids (Chloride) anzeigte. In die so erhaltenen Grignard-Lösungen wurde anschließend bei $0^{\circ} \mathrm{C}$ trockenes Kohlendioxid eingeleitet. Nach $2 \mathrm{~h}$ wurde sauer aufgearbeitet und nach Extraktion die Gesamtausbeute an Cyclobutancarbonsäure (n) bestimmt. Analytisch reine Proben erhielt man durch präparative Gaschromatographie.

Aus dem 33:67-Gemisch der Cyclobutylchloride 82a und 82b erhielt man so in $85 \%$ isolierter Ausbeute ein 30:70-Gemisch der unter 1.1 beschriebenen Cyclobutancarbonsäuren 81a und 81b (Schema 13). Die erwünschten Grignard-Reagenzien 21a und 21b wurden demnach in hoher Ausbeute gebildet und übertrugen die Stereochemie der Chloride nahezu unverändert auf die Säuren. Wir vermuten, dass dies nicht auf eine konfigurative Stabilität der 
Grignard-Reagenzien zurückzuführen ist. Wahrscheinlicher ist ein Gleichgewicht ${ }^{37}$ zwischen $^{3}$ 21a und 21b und eine stereochemische Diskriminierung im Zuge der Addition. Leider konnte keines der beiden stereoisomeren Chloride rein eingesetzt werden, um diese Frage zu klären.

\section{Schema 13}

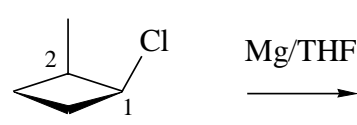

82a $1 R^{*}, 2 S^{*}(33 \%)$

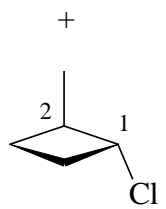

82b $1 R^{*}, 2 R^{*}(67 \%)$

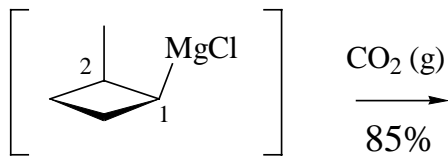

21a $1 R^{*}, 2 S^{*}$

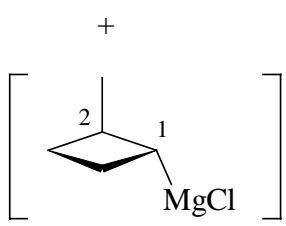

21b $1 R^{*}, 2 R^{*}$

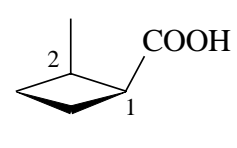

81a $1 R^{*}, 2 S^{*}(30 \%)$

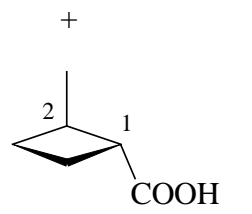

81b $1 R^{*}, 2 R^{*}(70 \%)$

Auch die beiden stereoisomeren Cyclobutylchloride 86a und 86b lieferten nach Generierung der zugehörigen Grignard-Reagenzien durch Carboxylierung ein Gemisch stereoisomerer Säuren (Schema 14). Diesmal war die Ausbeute mit 55\% niedriger, die Stereodifferenzierung zwischen 98a und 98b mit 14:86 dagegen höher. Auch hier vermuten wir ein Gleichgewicht zwischen 22a und 22b und eine Stereodifferenzierung im Zuge der Addition.

\section{Schema 14}
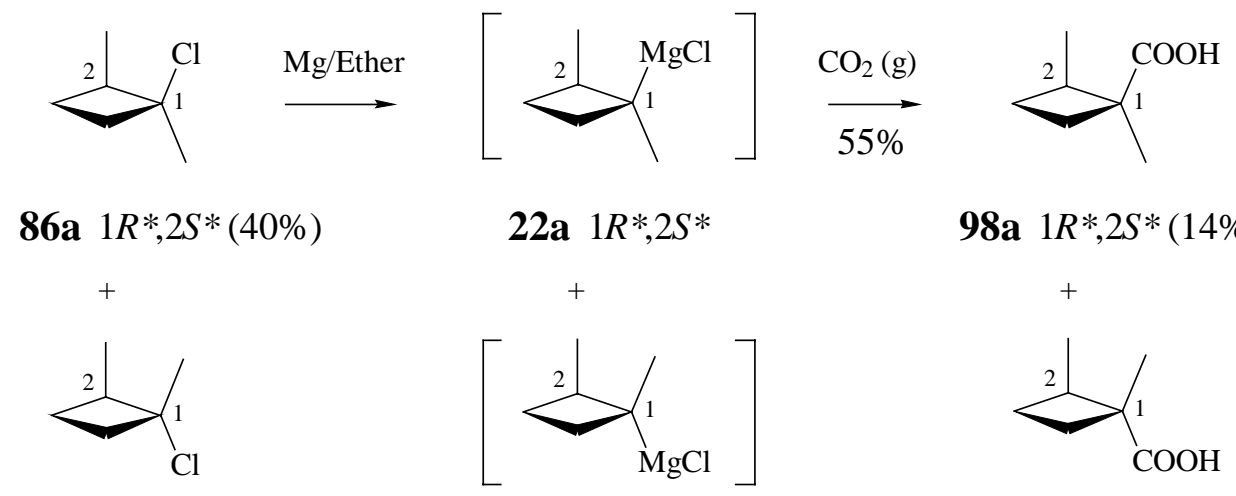

98a $1 R^{*}, 2 S^{*}(14 \%)$

86b $1 R^{*}, 2 R^{*}(60 \%)$

22b $1 R^{*}, 2 R^{*}$

98b $1 R^{*}, 2 R^{*}(86 \%)$ 
Im Gegensatz zu den 2-Methyl-cyclobutancarbonsäuren 81a und 81b waren die 1,2Dimethyl-cyclobutancarbonsäuren 98a und 98b unbekannt. Ihre Strukturen ergaben sich jedoch zweifelsfrei aus ihren spektroskopischen Daten. Im ${ }^{1}$ H-NMR-Spektrum (Abb. 29, 30) zeigten beide Verbindungen u.a. jeweils ein Singulett und ein Dublett für die Protonen der Methylgruppen [98a: 1.38 (s, 3H), $1.06(\mathrm{~d}, 3 \mathrm{H})$; 98b: 1.27 (s, 3H), $0.97(\mathrm{~d}, 3 \mathrm{H})$ ], und im ${ }^{13} \mathrm{C}$ NMR-Spektrum (Abb. 69, 70) die zu fordernden sieben Resonanzen zutreffender Multiplizität einschließlich der Resonanz für das Carbonylkohlenstoffatom (98a: 182.66; 98b: 184.49). Zur stereochemischen Zuordnung vgl. 1.4.

Das verbliebene Chlorid 97 lieferte nach Umsetzung mit Magnesium in Ether und Carboxylierung in $80 \%$ Ausbeute ein 1:1-Gemisch einer kristallinen, gesättigten und einer flüssigen, ungesättigten Monocarbonsäure (Schema 15). Bei der gesättigten Säure handelte es sich nach Ausweis ihrer spektroskopischen Daten um die erwartete 1,2,2-Trimethyl-cyclobutancarbonsäure 100. So zeigte ihr ${ }^{1} \mathrm{H}-\mathrm{NMR}-$ Spektrum (Abb. 31) u.a. drei Singuletts für die Protonen der Methylgruppen $\left(\delta=1.03,1.12,1.33\right.$, je $3 \mathrm{H}$ ), und ihr ${ }^{13} \mathrm{C}$-NMR-Spektrum (Abb. 71) drei Resonanzen für Methylgruppen $(\delta=19.90,23.85,25.53)$, zwei Resonanzen für Methylengruppen $(\delta=25.40,30.07)$ und drei Resonanzen für quartäre Kohlenstoffatome $[\delta=$ 40.51, 48.58, $182.83(\mathrm{C}=\mathrm{O})]$.

\section{Schema 15}

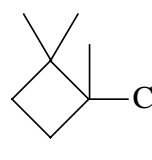

97
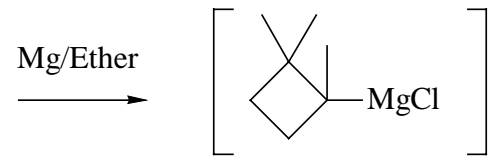

99<smiles>CC(C)=C(C)CCCl</smiles>

101
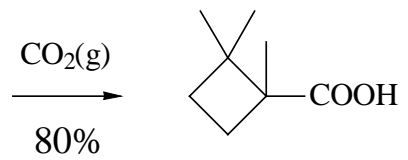

$100(50 \%)$

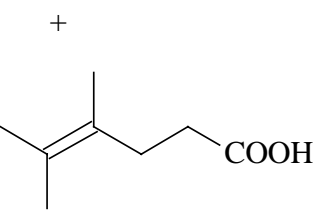

$102(50 \%)$ 
Bei der ungesättigten Säure handelte es sich nach Ausweis ihrer spektroskopischen Daten um die bekannte ${ }^{39,40}$ 4,5-Dimethyl-hex-4-ensäure (102). Ihre Bildung kann nur über eine anionische Ringöffnung auf der Stufe von 99 erklärt werden. Umlagerungen tertiärer zu primären Grignard-Verbindungen sind bekannt ${ }^{41}$.

\subsection{Versuche zur Addition der Grignard-Reagenzien 8, 21a,b, 22a,b und 23 an Cyclobutanone: 2,1'-Dimethyl- (104b), 2,2,1'-Trimethyl- (105), 2'-Methyl- $(106 a, b)$ und 1',2’-Dimethyl-bicyclobutyl-1-ol (107a,b)}

Nach der erfolgreichen Generierung und Charakterisierung der neuen, auf substituierten Cyclobutanen basierenden Grignard-Reagenzien 21a,b, 22a,b und 23 sollten diese, zusammen mit dem bereits früher beschriebenen Grignard-Reagenz 8, zur Synthese unterschiedlich substituierter Bicyclobutyl-1-ole genutzt werden. Da wir 8 in einer früheren Arbeit $^{5}$ bereits an einige unsubstituierte Ketone einschließlich Cyclobutanon addiert hatten, sollte zuerst seine Reaktivität gegenüber substituierten Cyclobutanonen untersucht werden. Anschließend sollten 21a,b, 22a,b und 23 an unsubtituiertes Cyclobutanon addiert werden. Ziel war eine Umlagerung der zu erwartenden Bicyclobutyl-1-ole und damit eine Eingrenzung über Bicyclobutyl-Bicyclooctyl-Umlagerungen zugänglicher Substitutions-muster von Bicyclooctenen.

Bei den Versuchen zur Addition der Grignard-Reagenzien 8, 21a,b, 22a,b und 23 an die

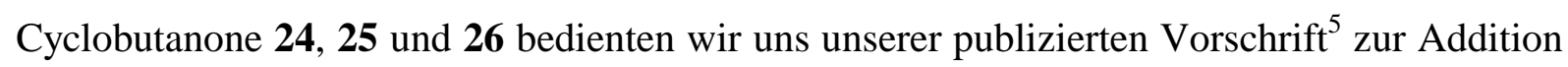
von $\mathbf{8}$ an Ketone. Hierbei handelt es sich um eine für leicht enolisierbare Ketone empfohlene Methode $^{42}$, bei der die Enolisierung durch Einwirkung von wasserfreiem Certrichlorid weitgehend unterdrückt wird. Zur experimentellen Realisierung wurden die Cyclobutanone mit zwei Moläquivalenten Certrichlorid in Tetrahydrofuran komplexiert und anschließend bei $-78^{\circ} \mathrm{C}$ mit dem entsprechenden Grignard-Reagenz umgesetzt. Nach 15 min bei $-78^{\circ} \mathrm{C}$ und $4 \mathrm{~h}$ bei Raumtemperatur lieferten Aufarbeitung und Chromatographie die zugehörigen Bicyclobutyl-1-ole. 
Die Addition von aus 103 generiertem 8 an 2-Methyl-cyclobutanon ${ }^{6}$ (25) lieferte in 59\% Ausbeute ein einziges Produkt. Dass es sich hierbei um eines von zwei denkbaren 2,1'Dimethyl-bicyclobutyl-1-olen 104 handelte, machte bereits das ${ }^{1} \mathrm{H}-\mathrm{NMR}-\mathrm{Spektrum}$ (Abb. 42) mit Resonanzen für insgesamt 18 Protonen deutlich. Darunter befanden sich das zu fordernde Singulett $(\delta=1.07,3 \mathrm{H})$ und Dublett $(\delta=1.00, \mathrm{~J}=7 \mathrm{~Hz}, 3 \mathrm{H})$ für die Protonen der Methylgruppen. Auch das ${ }^{13} \mathrm{C}-\mathrm{NMR}-$ Spektrum (Abb. 82) zeigte die nach Anzahl, Lage und Multiplizität zu fordernden zehn Signale, darunter eine charakteristische Resonanz für das die Hydroxylgruppe tragende Kohlenstoffatom $(\delta=80.99)$. Bezüglich der Stereochemie nehmen wir an, dass durch selektiven Angriff von 8 auf die der Methylgruppe von 25 abgewandten Seite 104b entsteht (Schema 16).

\section{Schema 16}

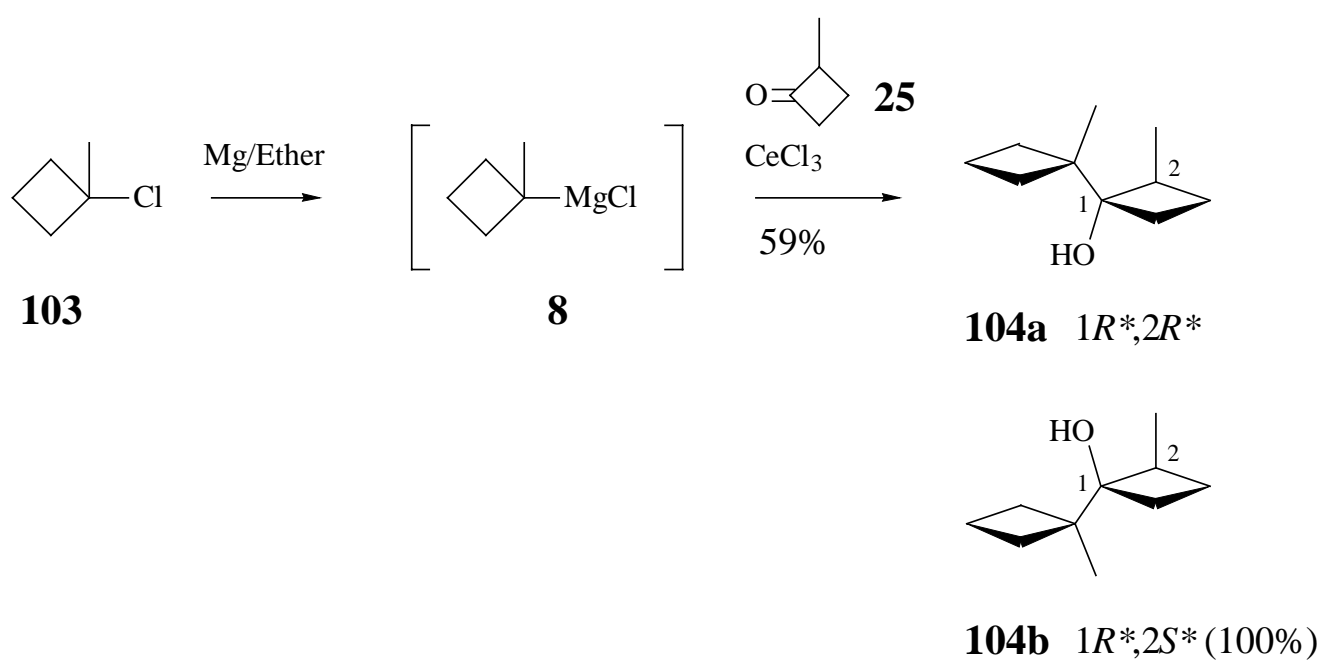

Bei der Addition von 8 an 2,2-Dimethyl-cyclobutanon (26) bildete sich das erwartete 2,2,1'-Trimethyl-bicyclobutyl-1-ol (105) in einer Ausbeute von nurmehr 27\% (Schema 17). Signifikant für die Struktur waren im ${ }^{1}$ H-NMR-Spektrum (Abb. 43) drei Singuletts für die Methylgruppen $(\delta=0.92,1.02,1.19$, je $3 \mathrm{H})$, und im ${ }^{13} \mathrm{C}-\mathrm{NMR}-\mathrm{Spektrum}$ (Abb. 83) die Resonanz für das die Hydroxylgruppe tragende Kohlenstoffatom $(\delta=82.96)$. Bedenkt man, dass 8 an unsubstituiertes Cyclobutanon (24) in $87 \%$ Ausbeute addiert, so wird der starke Einfluss sterischer Faktoren bei der Addition an 2-Methyl-cyclobutanon (25) und 2,2Dimethyl-cyclobutanon (26) deutlich. 


\section{Schema 17}

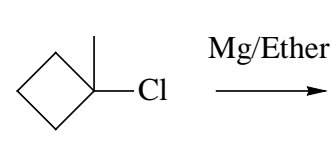

103

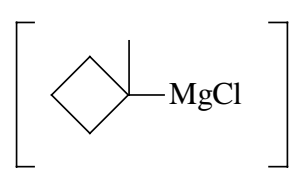

8

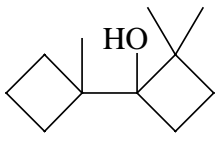

105

Die Umsetzung von 21a,b mit Cyclobutanon (24) lieferte in 76\% Ausbeute ein 45:55Gemisch der 2'-Methyl-bicyclobutyl-1-ole 106a und 106b (Schema 18). Beide Diastereomere zeigten im ${ }^{1}$ H-NMR-Spektrum (Abb. 44, 45) u.a. ein Dublett für eine gekoppelte Methylgruppe [106a: 1.07, $\mathrm{J}=7 \mathrm{~Hz}, 3 \mathrm{H}) ; \mathbf{1 0 6 b}: 1.07, \mathrm{~J}=7 \mathrm{~Hz}, 3 \mathrm{H})$ ] und im ${ }^{13} \mathrm{C}-\mathrm{NMR}$-Spektrum (Abb. 84, 85) die nach Anzahl und Lage zu erwartenden neun Resonanzen für ein primäres, fünf sekundäre, zwei tertiäre und ein quartäres Kohlenstoffatom. Die stereochemischen Zuordnung erfolgte wie in den übrigen Fällen über den $\gamma$-gauche-Effekt und ist unter 1.4 erläutert.

\section{Schema 18}

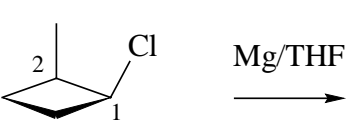

82a $1 R^{*}, 2 S^{*}(33 \%)$

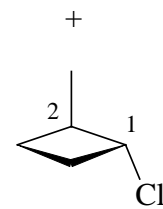

82b $1 R^{*}, 2 R^{*}(67 \%)$

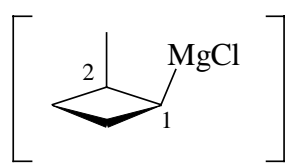

21a $1 R^{*}, 2 S^{*}$

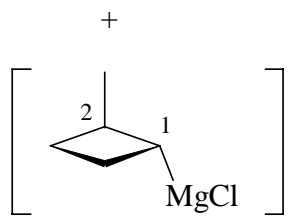

21b $1 R^{*}, 2 R^{*}$
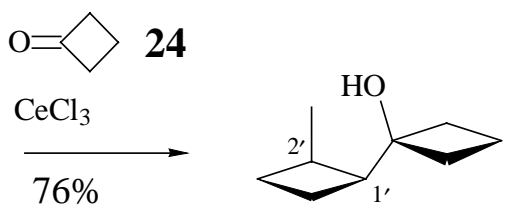

106a $1^{\prime} R^{*}, 2 S^{*}(45 \%)$

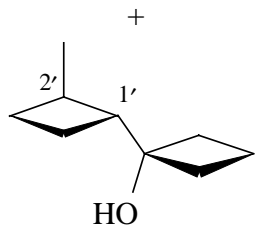

106b $1^{\prime} R^{*}, 2^{\prime} R^{*}(55 \%)$

Die bei der Addition von 21a,b an Cyclobutanon (24) beobachtete Ausbeute wurde mit 22a,b nicht mehr erreicht. Hier bildeten sich nur noch 9\% eines 33:67-Gemisches der 1',2'Dimethyl-bicyclobutyl-1-ole 107a und 107b (Schema 19). Der Versuch einer Addition von 23 an 24 schlug sogar gänzlich fehl. Dies zeigt, dass die Addition von Cyclobutyl-GrignardVerbindungen an Cyclobutanone nicht nur durch $\alpha$-Substituenten im Keton, sondern auch durch $\alpha$-Substituenten im Grignard-Reagenz verhindert werden kann. 


\section{Schema 19}

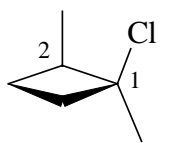

$\mathrm{Mg} /$ Ether

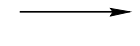

86a $1 R^{*}, 2 S^{*}(40 \%)$

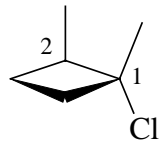

86b $1 R^{*}, 2 R^{*}(60 \%)$

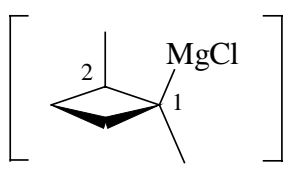

22a $1 R^{*}, 2 S^{*}$

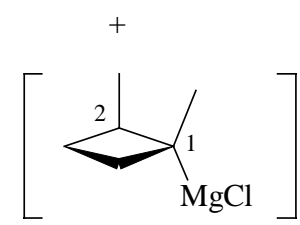

22b $1 R^{*}, 2 R^{*}$
24

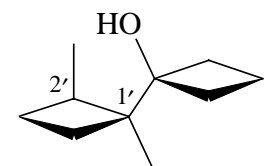

107a $1^{\prime} R^{*}, 2 S^{*}(33 \%)$

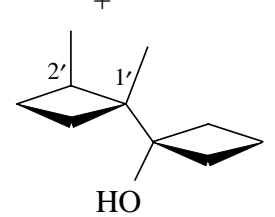

107b $1^{\prime} R^{*}, 2^{\prime} R^{*}(67 \%)$

Insgesamt ist festzustellen, dass von den getesteten Cyclobutyl-Grignard-Reagenzien 8, 21a,b, 22a,b und 23 nur 8 und 21a,b in brauchbaren Ausbeuten an Cyclobutanone zu addieren sind.

\subsection{Stereochemische Zuordnung der Cyclobutancarbonsäuren 81a,b und 98a,b, der Cyclobutylchloride 82a,b und 86a,b sowie der Bicyclobutyl-1-ole 106a,b und 107a,b}

Obwohl die 2-Methyl-cyclobutancarbonsäuren 81a,b bereits mehrmals synthetisiert worden $\operatorname{sind}^{20-23}$, herrscht bezüglich ihrer Stereochemie erhebliche Verwirrung. Wir erhielten diese Säuren analog einer Vorschrift ${ }^{19}$ für unsubstituierte Cyclobutancarbonsäure als 60:40Gemisch des cis- $\left(1 R^{*}, 2 S^{*}\right)(\mathbf{8 1 a})$ und des trans-Isomers $\left(1 R^{*}, 2 R^{*}\right)(\mathbf{8 1 b})(\operatorname{vgl} .1 .1)$. Da sich die in der Literatur angegebenen ${ }^{1} \mathrm{H}-\mathrm{NMR}-\mathrm{Daten}^{22,23}$ widersprachen und die ${ }^{13} \mathrm{C}-\mathrm{NMR}$ Daten $^{23}$ mit unseren unvereinbar waren, haben wir den bekannten ${ }^{8,43} \gamma$-Effekt, d.h. den Hochfeldshift der ${ }^{13} \mathrm{C}$-Resonanzen 1,2-cis-orientierter Substituenten zur stereochemischen Zuordnung herangezogen. Dieser Effekt war bereits früher zur stereochemischen Zuordnung der 1,2-Dimethyl-cyclobutylchloride 86a,b verwendet worden $^{8}$. Er diente uns nicht nur zur stereochemischen Zuordnung der Cyclobutancarbonsäuren 81a,b, sondern auch der Cyclobutylchloride 82a,b sowie der Bicyclobutyl-1-ole 106a,b und 107a,b (Schema 20). 


\section{Schema 20}

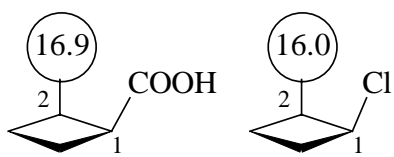

81a $1 R^{*}, 2 S^{*}$

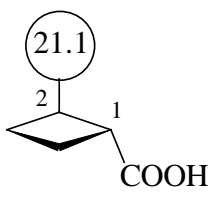

81b $1 R^{*}, 2 R^{*}$

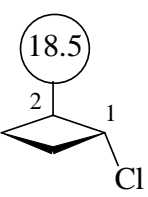

82b $1 R^{*}, 2 R^{*}$
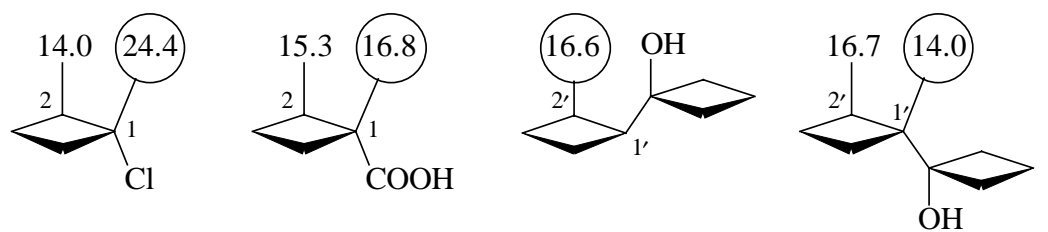

$98 \mathrm{~b} 1 R^{*}, 2 R^{*}$

106a $1^{\prime} R^{*}, 2^{\prime} S^{*}$

107b $1^{\prime} R^{*}, 2^{\prime} R^{*}$
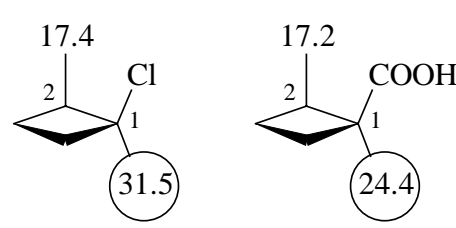

98a $1 R^{*}, 2 S^{*}$
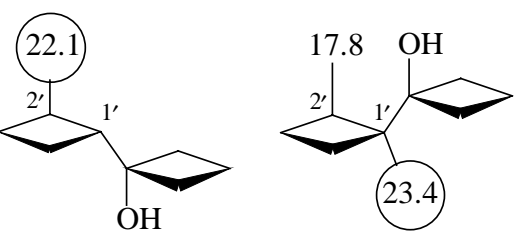

106b $1^{\prime} R^{*}, 2^{\prime} R^{*} \quad 107 \mathbf{a} 1^{\prime} R^{*}, 2^{\prime} S^{*}$

Zur Auswertung der ${ }^{13} \mathrm{C}$-NMR-Spektren wurden die Methylgruppen zunächst über ${ }^{13} \mathrm{C}$ ${ }^{1} \mathrm{H}$-Korrelationsspektren als an tertiäre oder quartäre Kohlenstoffatome gebunden identifiziert. Anschließend wurden die ${ }^{13} \mathrm{C}$-chemischen Verschiebungen derjenigen Methylgruppen, die reine 1,2-cis- oder 1,2-trans-Beziehungen zu anderen Atomen $(\mathrm{Cl})$ oder Atomgruppen $\left(\mathrm{CH}_{3}\right.$, $\mathrm{COOH}, \mathrm{c}-\mathrm{C}_{4} \mathrm{H}_{6} \mathrm{OH}$ ) aufwiesen, als stereochemische Indikatoren (eingekreist) verwendet. In allen Fällen und unabhängig von einem eventuell vorhandenen geminalen Substituenten war die Differenz der chemischen Verschiebungen zwischen den Stereoisomeren groß genug (2.5 - 7.6 ppm) um eine eindeutige Zuordnung zu treffen. Im Fall der 2-Methyl-cyclobutylchloride 82a,b stimmte die Zuordnung mit einer früheren, auf ${ }^{1} \mathrm{H}-\mathrm{NMR}-\mathrm{Daten}$ basierenden Zuordnung überein ${ }^{8}$.

\subsection{Umlagerung der Bicyclobutyl-1-ole 104b, 105, 106a, 106b, 107a und 107b zu mono- (108), di- (109) und trimethylierten Bicyclo[3.3.0]octenen $(118,120)$}

Die durch Addition der Grignard-Reagenzien 8, 21a,b und 22a,b an Cyclobutanone erhaltenen Bicyclobutyl-1-ole 104b, 105, 106a, 106b, 107a und 107b sollten abschließend einer durch Säure ausgelösten Bicyclobutyl-Bicyclooctyl-Umlagerung ${ }^{1}$ unterworfen werden. Als Produkte erwarteten wir mono- bis trimethylierte Bicyclooctene. 
Zur experimentellen Realisierung wurden die Alkohole mit jeweils einem Moläquivalent einer 0.074 M Lösung von wasserfreier p-Toluolsulfonsäure in Benzol versetzt und $3 \mathrm{~h}$ bei $70^{\circ} \mathrm{C}$ gehalten. Dabei bildete sich in allen Fällen jeweils ein einziges Produkt, das gaschromatographisch isoliert und spektroskopisch identifiziert wurde. Das trimethylierte Bicyclobutyl-1-ol 105 behandelten wir auch mit Thionylchlorid in Pyridin. Unter diesen Bedingungen ist eine Reprotonierung gebildeter Olefine unmöglich, so dass hier eher von Kationen im Frühstadium der Umlagerung abgeleitete Produkte zu erwarten waren.

Die beiden diastereomeren 2'-Methyl-bicyclobutyl-1-ole 106a und 106b lieferten mit pToluolsulfonsäure in jeweils quantitativer Ausbeute das Bicycloocten 108 ${ }^{44-48}$ (Schema 21), die beiden diastereomeren 1',2'-Dimethyl-bicyclobutyl-1-ole 107a und 107b sowie das 2,1'Dimethyl-bicyclobutyl-1-ol 104b in jeweils quantitativer Ausbeute das Bicycloocten 109 $9^{49-52}$ (Schema 22). Beide Produkte wurden anhand ihrer bekannten ${ }^{1} \mathrm{H}$ - und / oder ${ }^{13} \mathrm{C}-\mathrm{NMR}-\mathrm{Daten}$ identifiziert.

\section{Schema 21}

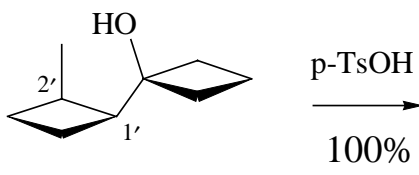

106a $1^{\prime} R^{*}, 2 S^{*}$<smiles>CC1=CCC2CCC[C@H]12</smiles>

108

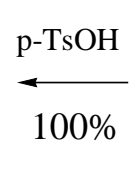

$106 \mathbf{b} 1^{\prime} R^{*}, 2^{\prime} R^{*}$

\section{Schema 22}

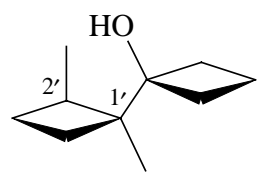

$\underset{100 \%}{\stackrel{\mathrm{p}-\mathrm{TsOH}}{\longrightarrow}}$

107a $1^{\prime} R^{*}, 2^{\prime} S^{*}$

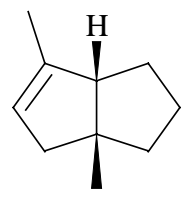

109
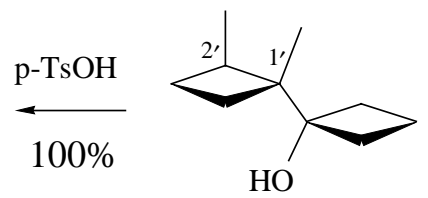

107b $1^{\prime} R^{*}, 2^{\prime} R^{*}$

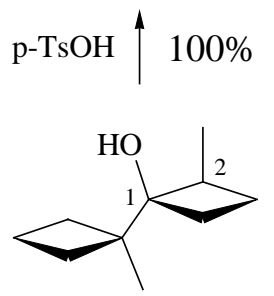

104b $1 R^{*}, 2 S^{*}$ 
Bezüglich der Produktbildung liegt auf der Hand, dass sie in allen Fällen thermodynamisch kontrolliert erfolgt sein muss. Über die tatsächlich beschrittenen Wege kann allerdings erst dann Auskunft gegeben werden, wenn die zu durchlaufenden Kationen samt zugehöriger Neutralkörper analysiert sind.

Eine derartige Analyse wurde für das von 2,2,1'-Trimethyl-bicyclobutyl-1-ol (105) abgeleitete Primärkation 110 durchgeführt. Dabei beschränkten wir uns auf tertiäre Kationen und davon abgeleitete Neutralkörper und analysierten potentielle Umlagerungswege mit unseren Programmen CARESY ${ }^{9}$ und HUNTER ${ }^{10}$ in Verbindung mit MMP2 ${ }^{15}$ (Schema 23).

\section{Schema 23}

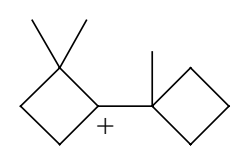

$110(+188.0)$<smiles>C[Te]</smiles><smiles>CC1(C)CCCC12CCCC2(C)C</smiles>

$113(+174.1)$

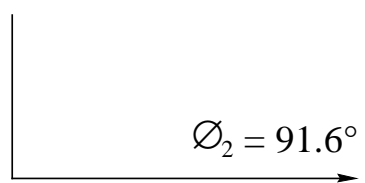

$\varnothing_{1}=14.6^{\circ}$

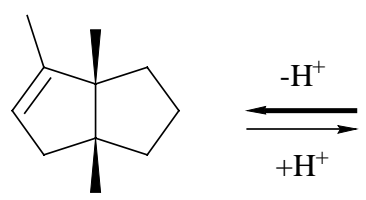

$118(-19.7)$

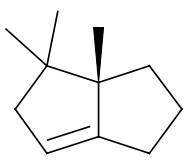

$111(-11.5)$ $-\mathrm{H}^{+} \mid \uparrow+\mathrm{H}^{+}$

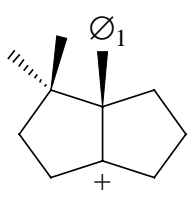

$114(+145.7)$

$$
\uparrow \varnothing_{1}=0.9^{\circ}
$$<smiles></smiles>
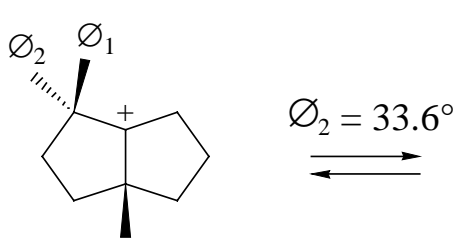

$116(+141.2)$

$$
\Uparrow \varnothing_{1}=21.2^{\circ}
$$

$115(-2.5)$

$-\mathrm{H}^{+} \uparrow \mid+\mathrm{H}^{+}$

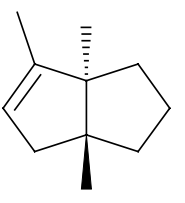

$112(-11.4)$

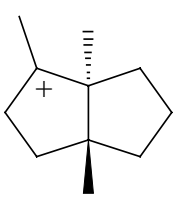

$117(+159.5)$

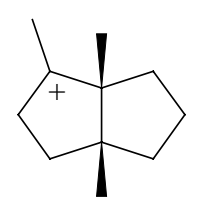

$119(+143.1)$

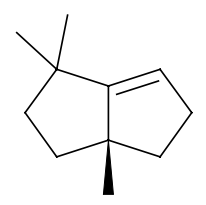

$120(-14.6)$ 
Nach den für Kationen und abgeleitete Olefine berechneten Standardbildungsenthalpien $(\mathrm{kcal} / \mathrm{mol})$ sowie den Diederwinkeln $(\varnothing)$ zwischen leerem Orbital und zu verschiebender Bindung war zu erwarten, dass 105 nach Protonierung und Abspaltung der Hydroxylgruppe zu 110 über 113, 114, 116 und 119 in 118 als thermodynamisch stabilstem Bicycloocten umlagern würde. Die bei Einwirkung von Thionylchlorid in Pyridin zu erwartenden Produkte waren weniger klar. Hierbei konnte es sich um die von 114 abgeleiteten Olefine 111 und / oder 112, und / oder um das von 116 abgeleitete Olefin 120 handeln. Ein Auftreten des trans-verknüpften Bicyclooctens 115 (116-117-115) schlossen wir aufgrund eines ungünstigen Diederwinkels in 116 sowie ungünstiger Bildungsenthalpien von 117 und 115 aus.

\section{Schema 24}

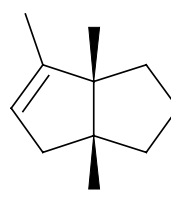

118

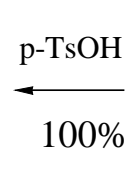

$100 \%$
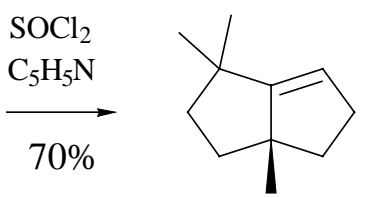

120

Wie die Umlagerungsversuche zeigten, lieferte 105 mit p-Toluolsulfonsäure in Benzol in quantitativer Ausbeute das Bicycloocten 118 und mit Thionylchlorid in Pyridin in 70\% Ausbeute das Bicycloocten 120 (Schema 24). Das Bicycloocten 118 gab sich im ${ }^{1} \mathrm{H}-\mathrm{NMR}$ Spektrum (Abb. 52) bereits dadurch zu erkennen, dass sich unter den Resonanzen für insgesamt 18 Protonen neben einem breiten Singulett für ein vinylisches Proton $(\delta=5.13,1 \mathrm{H})$ auch ein breites Singulett für eine vinylisch gebundene Methylgruppe befand $(\delta=1.56,3 \mathrm{H})$. Ein solches Strukturelement findet sich, außer bei 115, als thermodynamisch ungünstigstem Bicycloocten überhaupt, nur bei 118. Die Struktur von 120 war weniger leicht zu klären. Hier ergaben sich eindeutige Unterscheidungsmerkmale zu 111 und 112 erst durch ${ }^{1} \mathrm{~J}_{\mathrm{CH}^{-}}$(HMQC) (Abb. 103) ${ }^{2,3} \mathrm{~J}_{\mathrm{HH}^{-}}(\mathrm{COSY})$ (Abb. 104) und ${ }^{2,3} \mathrm{~J}_{\mathrm{CH}^{-}}$Korrelationsspektren (HMBC) (Abb. 105).

Insgesamt haben die Versuche zur Synthese und Umlagerung von Bicyclobutyl-1-olen gezeigt, dass das größte Problem in einer mit dem Substitutionsgrad steigenden sterischen Hinderung bei der Synthese der Edukte liegt. So erwiesen sich von den in 
Additionsreaktionen an Cyclobutanone getesteten Cyclobutyl-Grignard-Reagenzien lediglich die monomethylierten Reagenzien 8 und 21a,b, nicht jedoch die di- bzw. trimethylierten Reagenzien 22a,b und 23 als brauchbar. Letztere reagierten selbst mit unsubstituiertem Cyclobutanon nicht (23) oder nur schleppend (22a,b). Dagegen verliefen die Umlagerungen der synthetisierten Bicyclobutyl-1-ole 104b, 105, 106a,b und 107a,b sehr effektiv und lieferten unter thermodynamischer Kontrolle jeweils ein einziges Produkt. Insbesondere die Umlagerungen 106a,b-108 und 104b-109 scheinen auch unter präparativen Gesichtspunkten brauchbar.

\section{Synthese und Umlagerung potentieller Vorläufer von ( \pm )-endo-Capnellen (44) mit Bicyclobutyl-Teilstruktur: 1,4,4-Trimethyl-6-(2’-methyl-cyclobutyl)-bicyclo- [3.2.0]heptan-6-ole}

Nachdem die Cyclobutyl-Grignard-Reagenzien 8, 21a,b, 22a,b und 23 bezüglich ihrer Eignung zum Aufbau methylierter Bicyclobutyl-1-ole getestet waren (vgl. 1.3), sollten im zweiten Teil der vorliegenden Arbeit gezielt solche Vertreter synthetisiert und umgelagert werden, die nach einer mit unserem Retrosyntheseprogramm CARESY ${ }^{9}$ in Verbindung mit dem Programm HUNTER ${ }^{10}$ durchgeführten Analyse direkt zu dem Sesquiterpen $( \pm$ )-endoCapnellen $^{11}$ (44) führen konnten. Hierbei handelte es sich um die Bicyclobutyl-1-ole 46 und 50, die nach Ionisierung über eine regio- und stereospezifische Bicyclobutyl-BicyclooctylUmlagerung mit anschließender doppelter 1,2-Hydridverschiebung direkt das cis-anti-ciskonfigurierte 44 liefern sollten [46(50)-30-38-42-43-44]. Es schien allerdings keineswegs ausgeschlossen, dass auch bei Isomeren von 46 (47-49) und 50 (51-53) nach Ionisierung und Bicyclobutyl-Bicyclooctyl-Umlagerung auf der Stufe zu 42 analoger tricyclopentanoider Kationen über Eliminierungs- und Reprotonierungsreaktionen die für $\mathbf{4 4}$ charakteristische cisanti-cis-Konfiguration etabliert werden würde. Auch 47-49 und / oder 51-53 waren daher als potentielle Edukte einer Umlagerung zu 44 willkommen (Schema 25). 
Schema 25

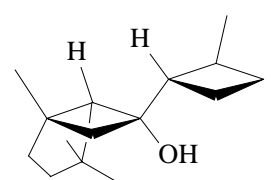

46

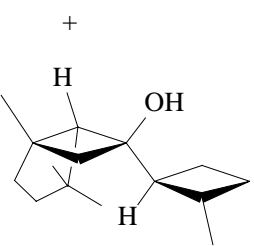

50

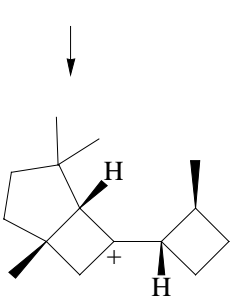

30
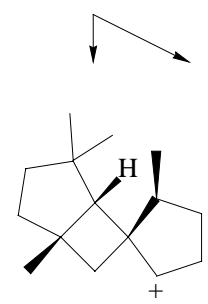

34

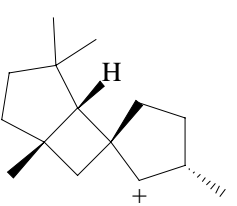

38

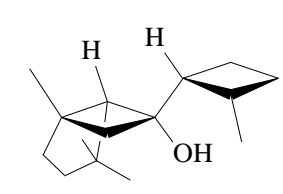

47

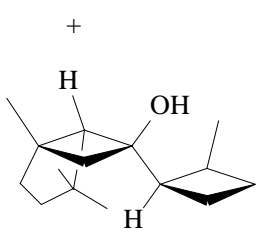

51

$\downarrow$

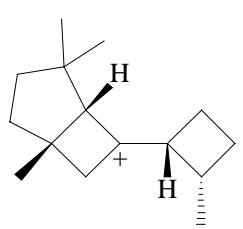

31<smiles>C1CC1</smiles>

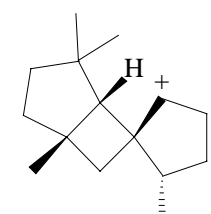

35

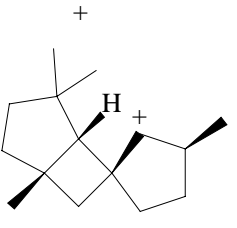

39

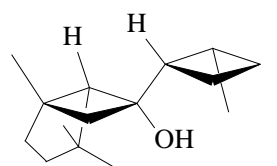

48

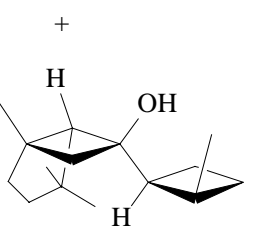

52

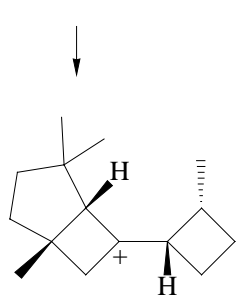

32
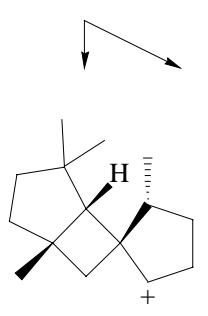

36

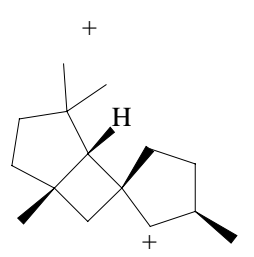

40

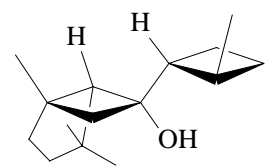

49

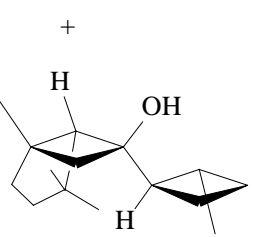

53

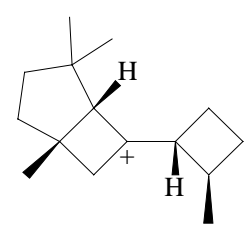

33

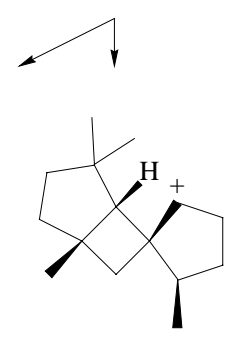

37

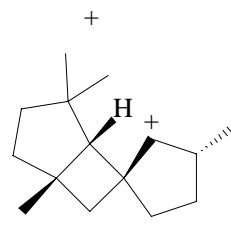

41

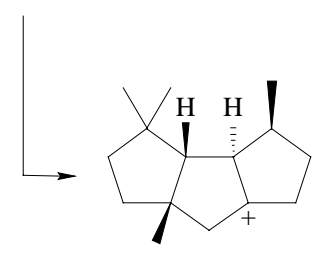

42

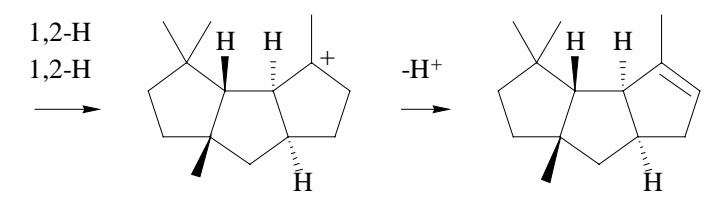

43
44

(士)-endo-Capnellen 


\subsection{Darstellung der 1,4,4-Trimethyl-6-(2'-methyl-cyclobutyl)-bicyclo[3.2.0]heptan-6- ole $46,47,48$ und 49}

Zur Darstellung der angestrebten 1,4,4-Trimethyl-6-(2'-methyl-cyclobutyl)-bicyclo[3.2.0]heptan-6-ole bot sich an, das in einer früheren Arbeit ${ }^{12}$ dargestellte bicyclische Keton 45 nach Komplexierung mit Certrichlorid mit den unter 1.2 beschriebenen diastereomeren Grignard-Reagenzien 21a,b umzusetzen. Ein konkurrierender exo / endo-Angriff vorausgesetzt, konnten dabei mit 46-53 bis zu acht diastereomere Alkohole entstehen.

Zur experimentellen Realisierung verfuhren wir wie für die Darstellung der Bicyclobutyl-1-ole 106a,b beschrieben (vgl. 1.3) und erhielten nach Aufarbeitung ein Gemisch aus vier Alkoholen. Chromatographie an Kieselgel in Pentan/Ether (99:1) lieferte zwei der vier Alkohole in Ausbeuten von 11 bzw. 16\% rein, die beiden anderen in einer Ausbeute von 36\% als 45:55-Gemisch. Alle Alkohole zeigten in ihren ${ }^{1} \mathrm{H}-\mathrm{NMR}-(\mathrm{Abb} .48,49,50)$ und ${ }^{13} \mathrm{C}$ NMR-Spektren (Abb 88, 89, 90) die für 1,4,4-Trimethyl-6-(2’-methyl-cyclobutyl)-bicyclo[3.2.0]heptan-6-ole zu erwartenden Resonanzen. Da lediglich vier von acht denkbaren Alkoholen gebildet worden waren, gingen wir davon aus, dass es sich hierbei um die aus einem exo-Angriff von 21a,b auf 45 resultierenden Diastereomeren 46-49 handelte (Schema 26).

\section{Schema 26}

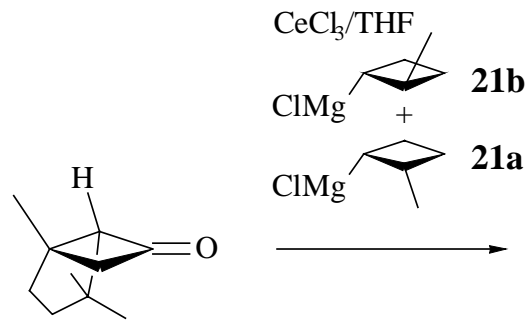

45

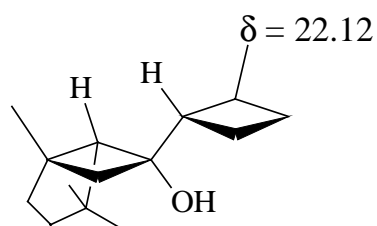

$461 S^{*}, 5 S^{*}, 6 S^{*}, 1^{\prime} S^{*}, 2^{\prime} S^{*}$

(45 von $36 \%, R_{f}=0.22$ )

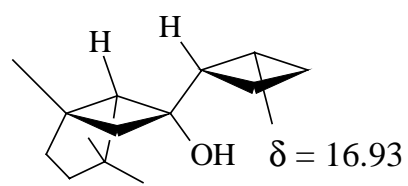

$471 S^{*}, 5 S^{*}, 6 S^{*}, 1^{\prime} S^{*}, 2^{\prime} R^{*}$ $\left(11 \%, R_{\mathrm{f}}=0.33\right)$

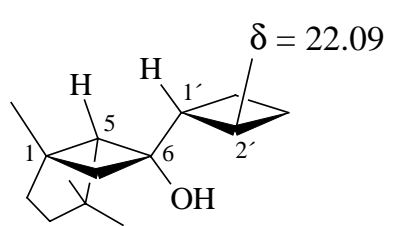

$491 S^{*}, 5 S^{*}, 6 S^{*}, 1^{\prime} R^{*}, 2^{\prime} R^{*}$ $\left(55\right.$ von $\left.36 \%, R_{\mathrm{f}}=0.22\right)$

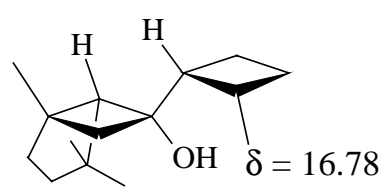

$481 S^{*}, 5 S^{*}, 6 S^{*}, 1^{\prime} R^{*}, 2^{\prime} S^{*}$ $\left(16 \%, R_{f}=0.28\right)$ 
Zur stereochemischen Zuordnung verwendeten wir, wie schon bei den Bicyclobutyl-1olen 106a,b und 107a,b (vgl. 1.4), den $\gamma$-gauche-Effekt ${ }^{43}$, d.h. die Hochfeldverschiebung der ${ }^{13} \mathrm{C}$-Resonanzen der aus den Grignard-Reagenzien stammenden Methylgruppen durch cisständige Substituenten ${ }^{43}$. Hierzu wurden die fraglichen Methylgruppen zunächst über ${ }^{1} \mathrm{~J}_{\mathrm{CH}^{-}}$ Korrelationsspektren identifiziert und anschließend als stereochemische Indikatoren benutzt. Dabei zeigte sich, dass die beiden rein isolierten Alkohole $\left[\delta\left(\mathrm{CH}_{3}\right)=16.93(47)\right.$ bzw. 16.78 (48)] im Cyclobutylrest cis-, die beiden Alkohole des 45:55-Gemisches $\left[\delta\left(\mathrm{CH}_{3}\right)=22.12\right.$ (46) bzw. 22.09 (49)] dagegen trans-konfiguriert sind. Ob die Zuordnung innerhalb der Paare $\mathbf{4 7 / 4 8}$ und $\mathbf{4 6 / 4 9}$ richtig ist, wissen wir nicht. Sie ist willkürlich und könnte vertauscht sein.

\subsection{Säurekatalysierte Umlagerung von 46 und 49: Bicyclo[3.2.0]hept-6-yl- Cycloheptenyl-Umlagerung unter Bildung von Dienen}

Nach der erfolgreichen Synthese der 1,4,4-Trimethyl-6-(2'-methyl-cyclobutyl)-bicyclo[3.2.0]heptan-6-ole 46-49 sollte geprüft werden, ob sie säurekatalysiert zu ( \pm )-endoCapnellen $^{11}$ (44) umlagern. Da 46 als direkter Vorläufer des Kations 30 anzusehen war, und dieses von CARESY als aussichtsreichter Kandidat einer Umlagerung zu $\mathbf{4 4}$ ausgewiesen war, verwendeten wir für die Umlagerungsversuche das 45:55-Gemisch von 46 und 49.

Zur säurekatalysierten Umlagerung wurde das Gemisch aus 46 und 49 mit Nafion NR $50^{53}$ in siedendem Ether behandelt und die Reaktion gaschromatographisch überwacht. Nach $23 \mathrm{~h}$ waren die Edukte verbraucht und vier Hauptprodukte in Anteilen von 9\%, 34\%, 20\% und $12 \%$ entstanden. Präparative Gaschromatographie lieferte alle Produkte rein.

Nach ihren hochaufgelösten Massen besaßen alle Verbindungen die Summenformel $\mathrm{C}_{15} \mathrm{H}_{24}$ und nach in ihren ${ }^{13} \mathrm{C}$-NMR-Spektren handelte es sich bei allen um Diene. Damit war klar, dass es sich nicht um tricyclische, sondern um bicyclische Systeme handelte. Die naheliegendste Hypothese war, dass die von 46 bzw. 49 abgeleiteten Primärkationen 30 und 33 nicht die erwünschte Cyclobutylmethyl-Cyclopentyl-Umlagerung ${ }^{1}$, sondern eine Cyclobutyl-Homoallyl-Umlagerung ${ }^{54}$ eingegangen waren und dass es sich bei den Produkten um von 121 abgeleitete Diene handelte (Schema 27). Diese Hypothese wurde durch eingehende Strukturuntersuchungen bestätigt. Diese umfassten die Auswertung der ${ }^{1} \mathrm{H}-$ (Abb. 
54, 55, 56, 57) und ${ }^{13} \mathrm{C}-\mathrm{NMR}-$ Spektren (Abb. 93, 94, 95, 96), die Auswertung von ${ }^{1} \mathrm{~J}_{\mathrm{CH}^{-}}$ (HMQC) (Abb.106, 108, 111, 113) und ${ }^{2,3} \mathrm{~J}_{\mathrm{HH}^{-}}$Korrelationsspektren (COSY) (Abb. 107, 109, $112,114)$ sowie bei der in $34 \%$ Ausbeute entstandenen Komponente auch die Auswertung eines ${ }^{2,3} \mathrm{~J}_{\mathrm{CH}}$-Korrelationsspektrums (HMBC) (Abb.110).

\section{Schema 27}

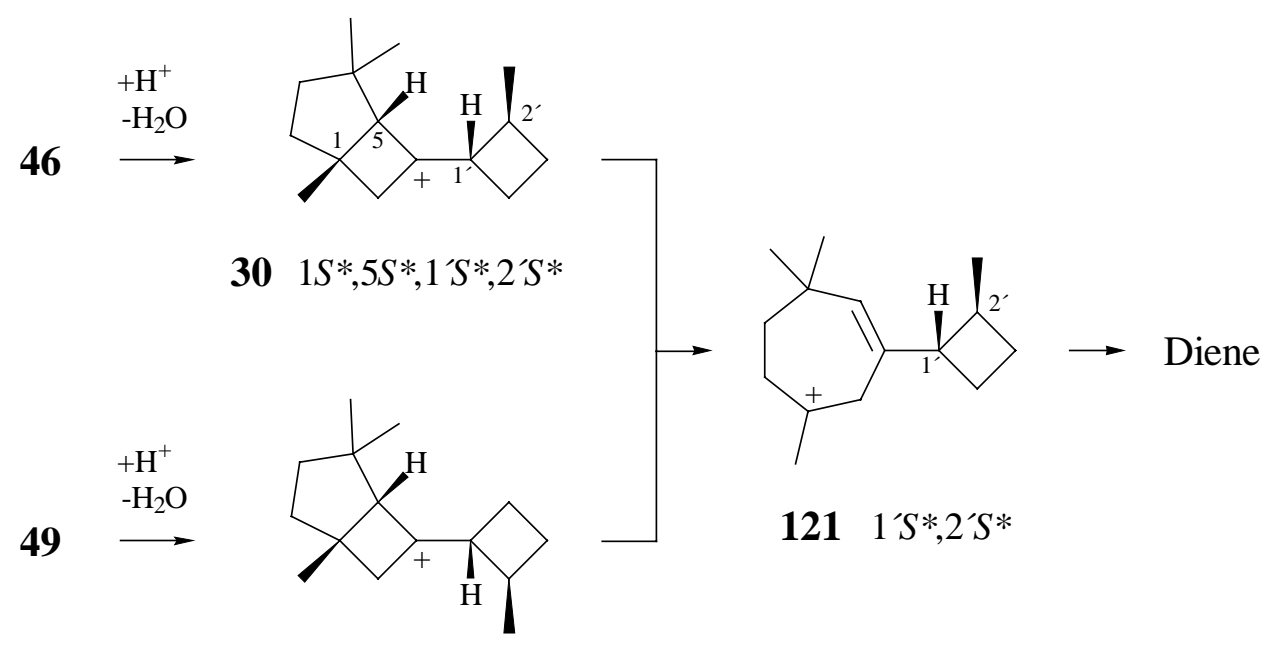

$331 S^{*}, 5 S^{*}, 1^{\prime} R^{*}, 2^{\prime} R^{*}$

Zur Auswertung wurden die ${ }^{13} \mathrm{C}$-Resonanzen von hohem zu tiefem Feld durchnumeriert und jeder Resonanz die laut ${ }^{1} \mathrm{~J}_{\mathrm{CH}}$-Korrelation zugehörigen Protonen zugeordnet. Die resultierenden $\mathrm{CH}_{3^{-}}, \mathrm{CH}_{2^{-}}$und $\mathrm{CH}-\mathrm{Gruppen}$ wurden anschließend über ${ }^{2,3} \mathrm{~J}_{\mathrm{HH}^{-}}$Korrelationen (bei der in $34 \%$ Ausbeute gebildeten Komponente auch über eine ${ }^{2,3} \mathbf{J}_{\mathrm{CH}}-$ Korrelation) $\mathrm{zu}$ größeren Fragmenten (fett gezeichnet) zusammengefügt und mit den verbliebenen Einzelfragmenten zu den angegebenen Gesamtstrukturen ergänzt. Hierbei gingen wir davon aus, dass die Abfolge der Kohlenstoffatome im ursprünglichen Fünfring durch die Umlagerung nicht verändert wird. Es resultierten zwei (2-Methyl-cyclobutyl)-cycloheptadiene $(\mathbf{1 2 2}, \mathbf{1 2 3})$ und zwei (2-Methyl-cyclobutyliden)-cycloheptene $(\mathbf{1 2 4}, \mathbf{1 2 5})$ (Schema 28). 


\section{Schema 28}
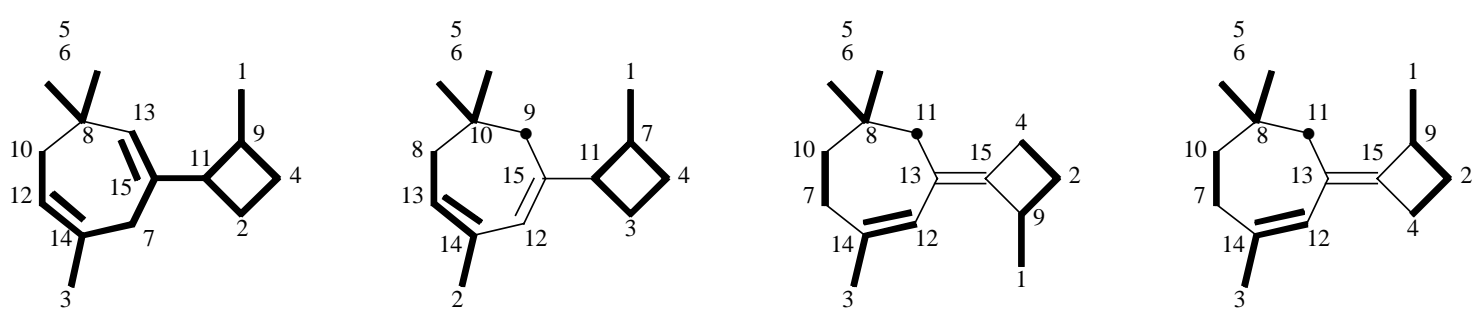

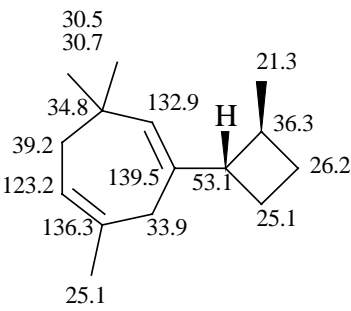

$122(9 \%)$

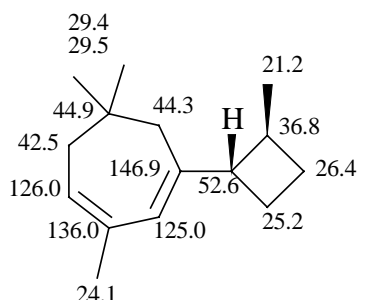

$123(34 \%)$

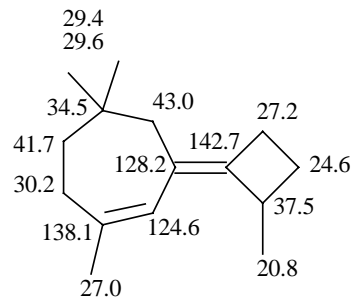

$124(20 \%)$

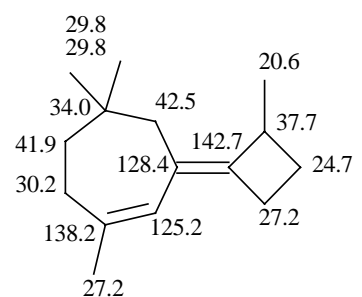

$125(12 \%)$

126

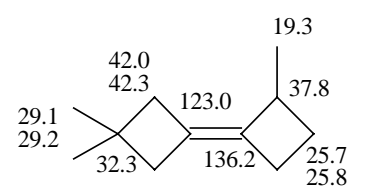

Aufgrund der Lage der ${ }^{13} \mathrm{C}$-Resonanzen der Methylgruppen in den Cyclobutanringen von $122(\delta=21.3)$ und $123(\delta=21.2)$ ist sicher, dass die Methylgruppen und die Cycloheptadienylreste in beiden Fällen trans-ständig sind (vgl. hierzu auch die Zuordnungen in 1.4 und 2.1). Willkürlich ist dagegen die stereochemische Zuordnung in den (2-Methylcyclobutyliden)-cycloheptenen $\mathbf{1 2 4}$ und $\mathbf{1 2 5}$ als cis bzw. trans. Hier sind die Unterschiede in den ${ }^{13} \mathrm{C}$-chemischen Verschiebungen so gering $(\Delta \delta \leq 0.6 \mathrm{ppm})$, dass keine Argumente für eine stereochemische Differenzierung resultieren. Wie ein Vergleich der ${ }^{13} \mathrm{C}-\mathrm{NMR}$-Daten von 124 und 125 mit denen des Bicyclobutylidens 126 zeigt $^{6}$, sind nur die Globalstrukturen sicher. Die stereochemische Zuordnung könnte vertauscht sein.

Bezüglich der Produktbildung ist festzustellen, dass mit einer Umlagerung der Bicyclobutyl-1-ole 46 und 49 zu den Dienen 122-125 nicht unbedingt zu rechnen war. So hatte das in einer früheren Arbeit ${ }^{12}$ untersuchte Bicyclobutyliden 54 nach Protonierung zu 127 durch zweifache Cyclobutylmethyl-Cyclopentyl-Umlagerung glatt das erwünschte 
tricyclische Olefin 128, und nicht etwa durch Cyclobutyl-Homoallyl-Umlagerung von 129 abgeleitete bicyclische Diene geliefert (Schema 29). Gleichwohl ist die bei $\mathbf{4 6}$ und 49 beobachtete Öffnung eines Bicyclo[3.2.0]hept-6-yl-Kations zu einem Cycloheptenyl-Kation nicht ohne Beispiel. Insbesondere für Systeme mit endo-ständiger Abgangsgruppe sind mehrere Fälle bekannt ${ }^{55,56}$.

\section{Schema 29}

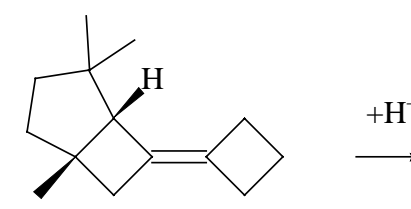

54

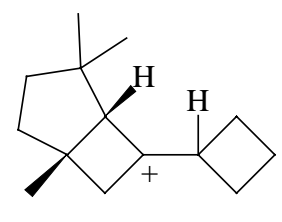

127

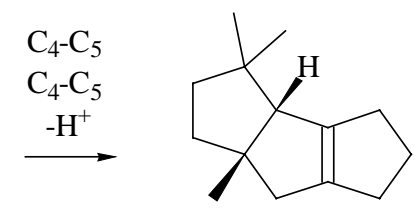

128

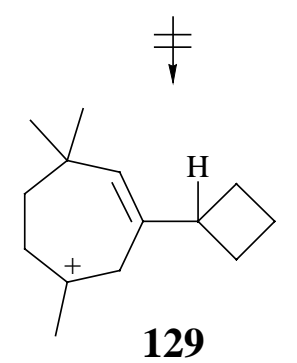

\subsection{Umsetzung von 46 und 49 mit Thionylchlorid in Pyridin: Bildung von Cyclo- butyl-cyclobutenen und Bicyclobutylidenen}

In Anbetracht der unterschiedlichen Reaktionsweisen der Bicyclobutyl-1-ole 46 und 49 sowie des Bicyclobutylidens 54 (vgl. 2.2) schien es sinnvoll zu versuchen, 46 und 49 durch Umsetzung mit Thionylchlorid in Pyridin in Cyclobutyl-cyclobutene und / oder Bicyclobutylidene zu überführen, um diese dann säurekatalysiert umzulagern. Bei einer zu 54 analogen Reaktionsweise hätten die erwünschten tricyclischen Olefine, darunter auch $( \pm)$ endo-Capnellen (44), doch noch entstehen können.

Zur experimentellen Realisierung wurde eine Lösung von 46 und 49 in Pyridin mit zwei Moläquivalenten Thionylchlorid $3 \mathrm{~h}$ bei $0^{\circ} \mathrm{C}$ gehalten. Laut gaschromatographischer Analyse enthielt das Reaktionsgemisch nach dieser Zeit vier Hauptkomponenten in Anteilen von 37, 29, 10 und 7\%. Leider erwies sich der 37\%-Anteil als Gemisch mehrerer Olefine, das nicht aufgetrennt und auch nicht als solches analysiert werden konnte. Von den verbliebenen 
Komponenten erwies sich die in 7\% gebildete Komponente als das bereits bekannte 122 (vgl. 2.2). Die in $29 \%$ bzw.10\% gebildeten Komponenten waren neu (Schema 30).

\section{Schema 30}

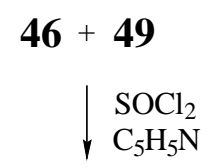

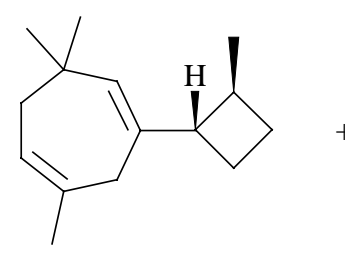

$122(7 \%)$

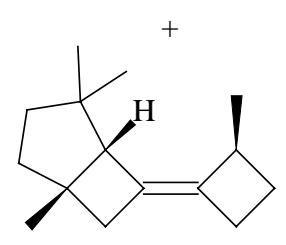

$132(10 \%)$

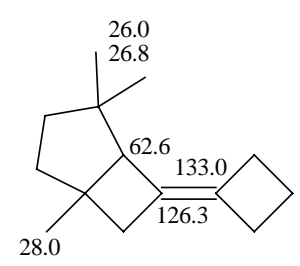

54

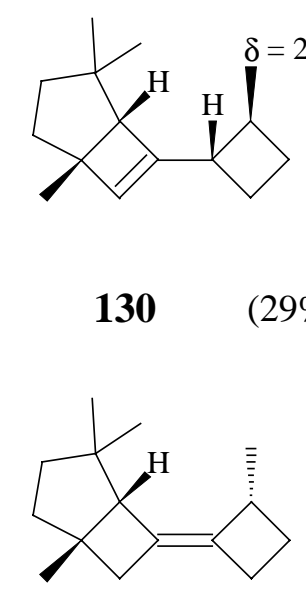

133

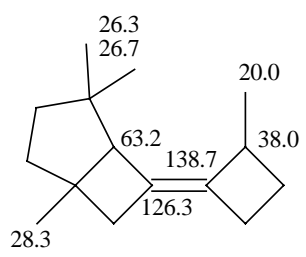

$132(133,134,135)$

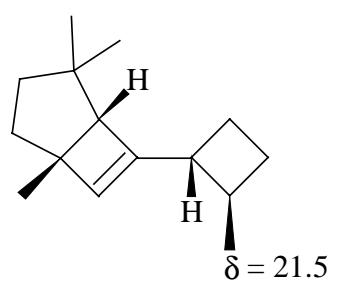

131

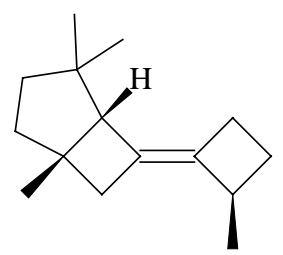

134

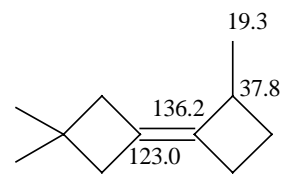

126

Bei der in 29\% Ausbeute gebildeten Komponente handelte es sich um ein 1:1-Gemisch zweier Monoolefine mit einer Summenformel $\mathrm{C}_{15} \mathrm{H}_{24}$ (MS). Dies bedeutete, dass es sich um tricyclische Systeme handeln musste. Das ${ }^{1} \mathrm{H}-\mathrm{NMR}$-Spektrum (Abb. 58) zeigte Resonanzen für jeweils 24 Protonen, darunter Singuletts für jeweils drei ungekoppelte Methylgruppen $[\delta=$ 0.88, 0.89, 1.02, 1.03 (je 3H), $1.33(6 \mathrm{H})$ ], Dubletts für jeweils eine gekoppelte Methylgruppe $(\delta=1.04,1.08, \mathrm{~J}=6.8 \mathrm{~Hz}$, je $3 \mathrm{H})$, sowie ein breites Singulett für insgesamt zwei olefinische Protonen $(\delta=5.66)$. Das ${ }^{13} \mathrm{C}-\mathrm{NMR}-$ Spektrum (Abb. 97) zeigte einen doppelten Satz von Resonanzen identischer Multiplizitäten und nahezu identischer chemischer Verschiebungen, so dass die Vermutung eines Vorliegens von Diastereomeren nahelag. Insbesondere die ${ }^{13} \mathrm{C}$ - 
chemischen Verschiebungen der gekoppelten Methylgruppen ( $\delta=21.4$ und 21.5) machten deutlich, dass die trans-Verknüpfung der Substituenten im Cyclobutanring der Edukte erhalten geblieben war (vgl. auch die Zuordnungen unter 1.4, 2.1 und 2.2). Wir glauben daher, dass es sich bei den fraglichen Verbindungen um die Cyclobutyl-cyclobutene 130 und 131 handelt, ohne aufgrund der spektroskopischen Daten zwischen den beiden Diastereomeren differenzieren zu können. Analoge Eliminierungen sind bei der Umsetzung von Bicyclobutyl-1-olen mit Thionylchlorid in Pyridin bereits mehrfach beobachtet worden $^{57,58}$.

Auch bei der in $10 \%$ Ausbeute gebildeten Komponente handelte es sich um ein Monoolefin mit einer Summenformel $\mathrm{C}_{15} \mathrm{H}_{24}$ (MS) und damit um ein tricyclisches System. Dieses Olefin war tetrasubstituiert und wurde über sein ${ }^{1} \mathrm{H}$ - (Abb. 59) und ${ }^{13} \mathrm{C}-\mathrm{NMR}$ Spektrum (Abb. 98) sowie über ein ${ }^{1} J_{\mathrm{CH}}$ (HMQC) (Abb. 115) und ${ }^{2,3} \mathrm{~J}_{\mathrm{HH}^{-}}$ Korrelationsspektrum (COSY) (Abb. 116) als eines von vier diastereomeren Bicyclobutylidenen 132-135 identifiziert. Die Übereinstimmung der ${ }^{13} \mathrm{C}$-Resonanzen primärer, tertiärer und quartärer Kohlenstoffatome mit den entsprechenden Resonanzen im Bicycloheptyliden- bzw. 2-Methyl-cyclobutyliden-Teil von $\mathbf{5 4}^{12}$ und $\mathbf{1 2 6}^{6}$ kann als zusätzlicher Nachweis gelten. Um welches Diastereomer es sich tatsächlich handelt, wissen wir nicht.

Insgesamt ist festzustellen, dass $\mathbf{4 6}$ und $\mathbf{4 9}$ bei der Umsetzung mit Thionylchlorid in Pyridin in nur untergeordnetem Maße Ringöffnung zu 122 erleiden. Hauptprodukte sind die Cyclobutyl-cyclobutene 130 und 131 sowie das Bicyclobutyliden 132 (133,134,135). Allerdings war ihr Anteil mit insgesamt 39\% zu gering, um sie im Gemisch mit 7\% 122 und $37 \%$ unaufgeklärter Produkte in Versuche einer säurekatalysierten Umlagerung zu ( \pm )-endoCapnellen (44) einzusetzen. Wir haben daher nach anderen Wegen gesucht, die von CARESY prognostizierten Umlagerungen zu realisieren. 
3 Synthese und Umlagerung potentieller Vorläufer von ( \pm )-endo-Capnellen (44) mit Spirooctyl-Teilstruktur: $\quad\left(1 R^{*}, 5 R^{*}, 6 R^{*}, 2^{\prime} R^{*}, 3^{\prime} R^{*}\right)-\quad(60), \quad\left(1 R^{*}, 5 R^{*}, 6 R^{*}\right.$, $\left.2^{\prime} S^{*}, 3^{\prime} R^{*}\right)-(64)$ und $\left(1 R^{*}, 5 R^{*}, 6 R^{*}, 2^{\prime} R^{*}, 3^{\prime} S^{*}\right)-1,4,4,3^{\prime}$-Tetramethylspiro\{bicyclo[3.2.0]heptan-6, $1^{\prime}$-cyclopentan-2'-ol\} (66)

Nachdem die Versuche zur Synthese von ( \pm )-endo-Capnellen (44) über eine Bicyclobutyl-Bicyclooctyl-Umlagerung von 46 und 49 nicht zum erwünschten Ergebnis geführt hatten (vgl. 2.2), sollte versucht werden, die im Erstschritt der Umlagerung erwarteten, aber nicht gebildeten Spirooctyl-Kationen 38 und 41 und / oder ihre Diastereomere 39 und 40 direkt zu erzeugen. Hierzu mußten die zugehörigen Alkohole dargestellt werden (Schema 31).

\section{Schema 31}

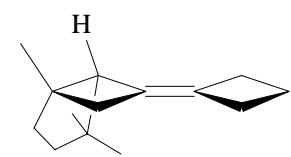

54 MCPB

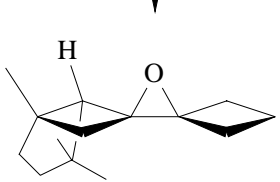

55

$+$

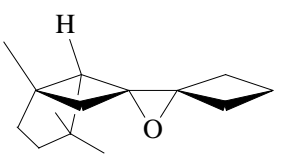

56

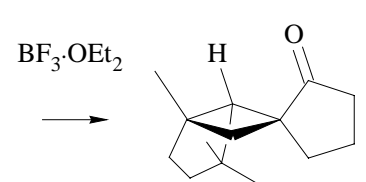

57

$+$

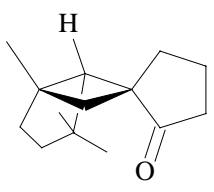

58

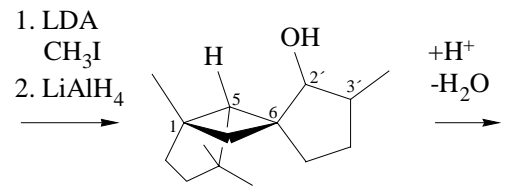

$60\left(1 R^{*}, 5 R^{*}, 6 R^{*}, 2^{\prime} R^{*}, 3^{\prime} R^{*}\right)$ $62\left(1 R^{*}, 5 R^{*}, 6 R^{*}, 2^{\prime} S^{*}, 3^{\prime} S^{*}\right)$ $64\left(1 R^{*}, 5 R^{*}, 6 R^{*}, 2^{\prime} S^{*}, 3^{\prime} R^{*}\right)$ $66\left(1 R^{*}, 5 R^{*}, 6 R^{*}, 2^{\prime} R^{*}, 3^{\prime} S^{*}\right)$

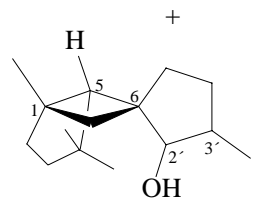

$59\left(1 R^{*}, 5 R^{*}, 6 S^{*}, 2^{\prime} R^{*}, 3^{\prime} R^{*}\right)$ $61\left(1 R^{*}, 5 R^{*}, 6 S^{*}, 2^{\prime} S^{*}, 3^{\prime} S^{*}\right)$ $63\left(1 R^{*}, 5 R^{*}, 6 S^{*}, 2^{\prime} S^{*}, 3^{\prime} R^{*}\right)$ $65\left(1 R^{*}, 5 R^{*}, 6 S^{*}, 2^{\prime} R^{*}, 3^{\prime} S^{*}\right)$

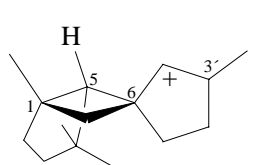

$39\left(1 R^{*}, 5 R^{*}, 6 S^{*}, 3^{\prime} S^{*}\right)$ $41\left(1 R^{*}, 5 R^{*}, 6 S^{*}, 3^{\prime} R^{*}\right)$

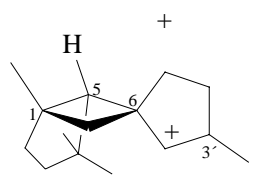

$\mathbf{3 8}\left(1 R^{*}, 5 R^{*}, 6 R^{*}, 3^{\prime} S^{*}\right)$ $40\left(1 R^{*}, 5 R^{*}, 6 R^{*}, 3^{\prime} R^{*}\right)$

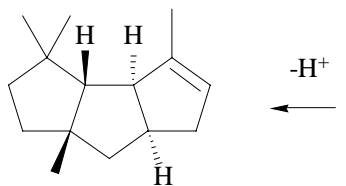

44 ( \pm )-endo-Capnellen

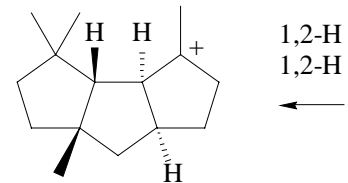

43

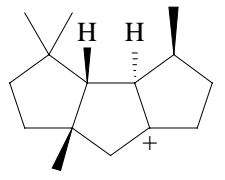

42 
Wie bereits bemerkt, sollte hierzu das Bicyclobutyliden 54 epoxidiert und anschließend einer Oxaspirohexan-Cyclopentanon-Umlagerung ${ }^{13}$ unterworfen werden [54-55(56)-57(58)]. Hierbei rechneten wir aufgrund früherer Erfahrungen ${ }^{14}$ mit ähnlichen Systemen mit einer Ringerweiterung des peripheren Cyclobutanringes und damit einer Bildung der Spirooctanone 57 und 58. Die Folgeschritte einer Methylierung und Reduktion konnten bei mangelnder Selektivität mit 59-66 bis zu acht diastereomere Alkohole liefern, die jedoch unter ionisierenden Bedingungen paarweise in die erwünschten Spirooctyl-Kationen 38-41 übergehen mussten. Wir hatten allerdings die Hoffnung, dass zumindest die Reduktion der monomethylierten Ketone stereoselektiv ablaufen würde ${ }^{59}$.

Von den Spirooctyl-Kationen 38-41 war 38 durch CARESY als potentieller Vorläufer von ( \pm )-endo-Capnellen (44) ausgewiesen (38-42-43-44) ${ }^{9}$. Allerdings konnte auch jedes andere Diastereomer nach selektiver 1,2-Verschiebung der höher substituierten Cyclobutanbindung über De- und Reprotonierungsreaktionen in das cis-anti-cis-verknüpfte ( \pm )-endo-Capnellen (44) übergehen. Wie wir nachfolgend zeigen werden, traten derartige Umlagerungen tatsächlich ein.

\subsection{Synthese von $\left(1 R^{*}, 5 R^{*}, 6 R^{*}\right)-1,4,4-$ Trimethylspiro\{bicyclo[3.2.0]heptan-6,1'- cyclopentan-2'-on\} (57)}

Zur Synthese der für Methylierungsversuche benötigten Spirooctanone $\mathbf{5 7}$ und $\mathbf{5 8}$ wurde das Bicyclobutyliden 54 zunächst epoxidiert. Diese Reaktion war bei Verwendung von 1.3 Moläquivalenten m-Chlorperbenzoesäure in Dichlormethan innerhalb von $15 \mathrm{~min}$ bei $0^{\circ} \mathrm{C}$ komplett und lieferte nach Chromatographie in 83 bzw. 5\% isolierter Ausbeute die beiden diastereomeren Epoxide 55 und 56 als farblose Öle. Beide Verbindungen zeigten im ${ }^{1} \mathrm{H}$ NMR-Spektrum (Abb. 33, 34) Resonanzen für insgesamt 22 Protonen, darunter drei Singuletts für die Methylgruppen $[\delta=0.89,1.12,1.35$ (55); 0.84, 1.00, $1.26(\mathbf{5 6})]$. Auch die ${ }^{13}$ C-NMR-Spektren (Abb. 73, 74) entsprachen der Erwartung. Sie enthielten jeweils 14 Resonanzen zutreffender Lage und Multiplizität, darunter charakteristische Resonanzen für die periphere Methylengruppe des spiroanellierten Cyclobutans $[\delta=12.94(\mathbf{5 5}) ; 11.93(\mathbf{5 6})]$ sowie für die Kohlenstoffatome des Oxirans $[\delta=66.20,68.56(\mathbf{5 5}) ; 63.81,65.06(\mathbf{5 6})]$. Bezüglich der Stereochemie nehmen wir an, dass es sich bei der Majoritätskomponente um 
das aus einem exo-Angriff der Persäure auf 54 resultierende Epoxid 55 handelt. Dieses wurde für die nachfolgende Umlagerung rein eingesetzt (Schema 32).

\section{Schema 32}

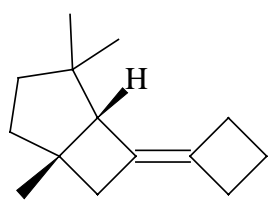

54

$87 \%$ МСРB

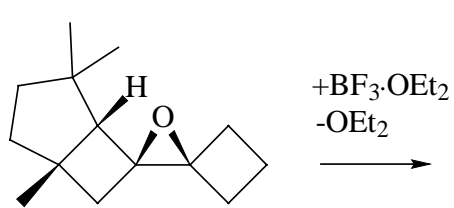

$55(94 \%)$

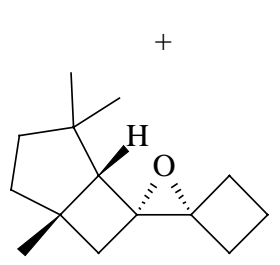

$56(6 \%)$

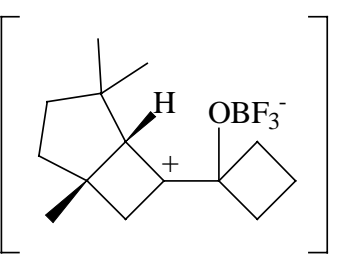

136

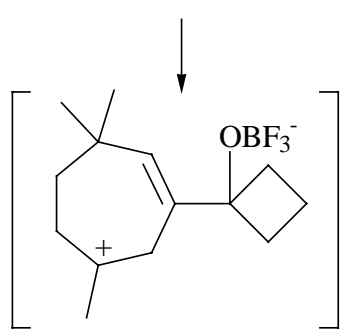

137

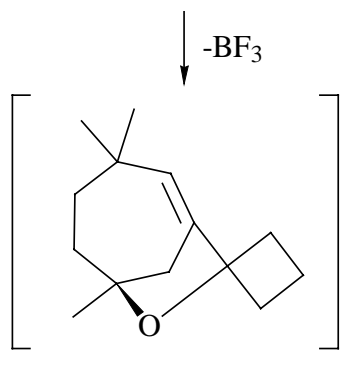

139

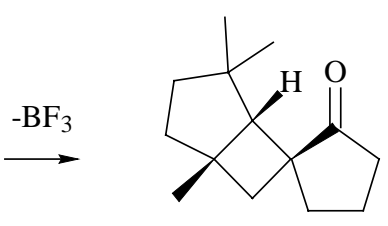

$57(51 \%)$

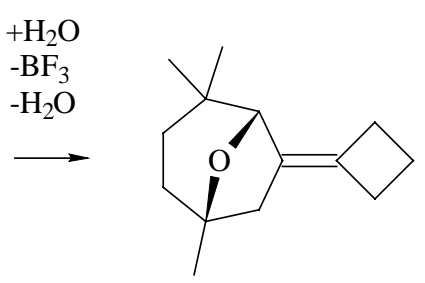

$138(8 \%)$

$\uparrow-\mathrm{BF}_{3}$

$+\mathrm{BF}_{3}$

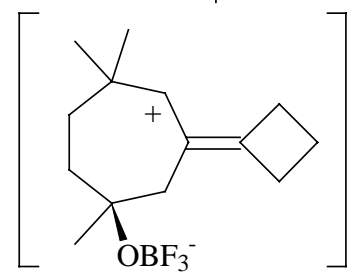

140

Zur Umlagerung versetzte man eine auf $0^{\circ} \mathrm{C}$ gekühlte Lösung von $\mathbf{5 5}$ in trockenem Dichlormethan mit 0.025 Moläquivalenten Bortrifluorid-Etherat. Nach 15 min lieferten Aufarbeitung und Chromatographie in 51 bzw. 8\% isolierter Ausbeute ein Keton und einen Ether mit einer Molmasse von jeweils 206. Arbeitete man bei Raumtemperatur und 
verwendete nicht getrocknetes Dichlormethan und 0.050 Moläquivalente BortrifluoridEtherat, so lag die isolierte Ausbeute des Ketons bei $41 \%$ und die des Ethers bei $45 \%$.

Das ${ }^{1} \mathrm{H}-\mathrm{NMR}-$ Spektrum des Ethers (Abb. 36) zeigte Resonanzen für insgesamt 22 Protonen, darunter drei Singuletts für an gesättigte Kohlenstoffatome gebundene Methylgruppen $(\delta=0.75,1.05,1.30)$ sowie ein breites Singulett für das Proton einer Methingruppe in Nachbarstellung zu Sauerstoff $\left(\delta=3.87\right.$ ). Das ${ }^{13}$ C-NMR-Spektrum (Abb. 76) enthielt die zu erwartenden 14 Resonanzen, darunter Resonanzen für einen Ether $[\delta=$ $\left.79.90\left(\mathrm{C}_{\text {quart }}\right), 85.35\left(\mathrm{C}_{\text {tert }}\right)\right]$ und ein tetrasubstituiertes Olefin $\left[\delta=131.03\left(\mathrm{C}_{\text {quart }}\right), 131.14\right.$ $\left.\left(\mathrm{C}_{\text {quart }}\right)\right]$ mit der Partialstruktur eines Cyclobutylidens $\left[\delta=17.39\left(\mathrm{C}_{\mathrm{sek}}\right)\right]$. Gleichwohl mussten zur endgültigen Klärung der Struktur ${ }^{1} \mathrm{~J}_{\mathrm{CH}^{-}}$(HMQC) (Abb. 117), ${ }^{2,3} \mathrm{~J}_{\mathrm{HH}^{-}}$(COSY) (Abb. 118) und ${ }^{2,3} \mathrm{~J}_{\mathrm{CH}}$-Korrelationsspektren (HMBC) (Abb. 119) herangezogen werden. Hieraus folgte zweifelsfrei, dass es sich bei dem fraglichen Ether um 138 handelte. Seine Bildung erklären wir durch eine Cyclobutyl-Homoallyl-Umlagerung von 136 zu 137 mit nachfolgender Transposition des Sauerstoffs über 139 als Zwischenprodukt (136-137-139-140-138). Alternativ könnte 137 auch zu einem Diol hydratisiert und anschließend unter Allylumkehr dehydratisiert worden sein (Schema 32).

Bei dem als Hauptprodukt isolierten Keton konnte es sich nach Ausweis seiner spektroskopischen Daten um das Spirooctanon 57 oder 58 handeln. So zeigte sein IRSpektrum (Abb. 9) eine für Fünfringketone charakteristische $\mathrm{C}=\mathrm{O}-$ Valenzschwingung ${ }^{60}$ bei $1732 \mathrm{~cm}^{-1}$, und sein ${ }^{13} \mathrm{C}-\mathrm{NMR}-S p e k t r u m$ (Abb.75) unter den 14 Resonanzen zutreffender Multiplizität eine Resonanz für ein Carbonyl-Kohlenstoffatom $\left[\delta=221.05\left(\mathrm{C}_{\text {quart }}\right)\right]$. Mechanistisch war eine Oxaspirohexan-Cyclopentanon-Umlagerung unter Inversion der Konfiguration am Endpunkt der Umlagerung und damit eine Bildung von 57 am wahrscheinlichsten ${ }^{61}$. Gleichwohl war ein Entscheid auf der Basis der spektroskopischen Daten nicht zu treffen. Er ergab sich erst durch Kristallstrukturanalysen auf der Stufe der Alkohole (vgl. 3.3). Danach handelte es sich bei dem durch Oxaspiropentan-CyclopentanonUmlagerung von $\mathbf{5 5}$ gebildeten Keton tatsächlich um 57. 


\subsection{Methylierung und Reduktion von 57 zu den Spirooctanolen 60, 64 und 66}

Nachdem das Spirooctanon 57 in ausreichender Menge zur Verfügung stand, haben wir es in Methylierungsversuche eingesetzt. Dabei erwies sich als vorteilhaft, zur Generierung des Lithiumenolats lediglich ein Moläquivalent Lithiumdiisopropylamid (LDA) zu verwenden. Unter diesen Bedingungen war der Umsatz zwar unvollständig, dafür war der Anteil an dimethyliertem Produkt gering. Ein präparativer Ansatz mit je einem Moläquivalent LDA und 57 sowie fünf Moläquivalenten Methyliodid lieferte innerhalb $2 \mathrm{~h}$ bei $-78^{\circ} \mathrm{C}$ nach Aufarbeitung und Chromatographie 79\% eines 54:46-Gemisches der monomethylierten Ketone 141 und 142, 3\% dimethyliertes Keton 143 sowie 13\% unumgesetztes 57 (Schema 33).

\section{Schema 33}

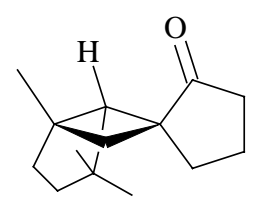

57

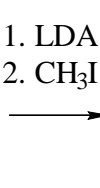

$\mathrm{CH}_{3} \mathrm{I}$

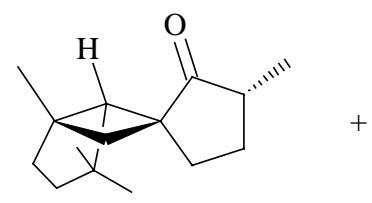

141

$(79 \%, 54: 46)$

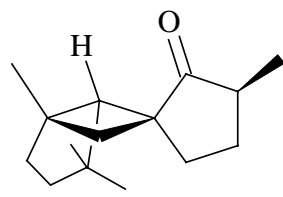

142

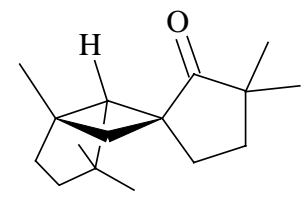

$143(3 \%)$

$$
\downarrow \mathrm{LiAlH}_{4}
$$

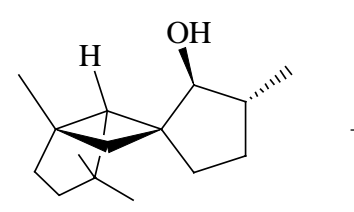

$66(49 \%)$

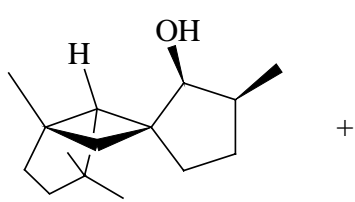

$60(29 \%)$

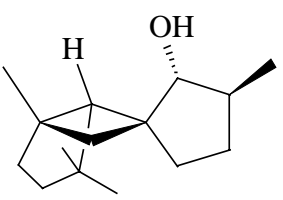

$64(10 \%)$

Dass es sich bei dem in 79\% Ausbeute isolierten 54:46-Gemisch um monomethylierte Produkte handelte, machte bereits das ${ }^{1} \mathrm{H}-\mathrm{NMR}-$ Spektrum (Abb. 37) deutlich. Es enthält neben je drei Singuletts für ungekoppelte Methylgruppen $[\delta=0.83,1.04,1.23$ (141); 0.82, 1.06, $1.24(\mathbf{1 4 2})]$ je ein Dublett für eine gekoppelte Methylgruppe $[\delta=1.13, \mathrm{~J}=7.0 \mathrm{~Hz}(\mathbf{1 4 1})$ : 1.03, J = 7.5 Hz (142)]. Das ${ }^{1} \mathrm{H}-\mathrm{NMR}-$ Spektrum des dimethylierten 143 (Abb. 38) war eindeutig. Es zeigte fünf Singuletts für fünf ungekopppelte Methylgruppen $(\delta=0.83,0.93$, 1.06, 1.09, 1.23). Natürlich war die Stereochemie von 141 und 142 aus den angegebenen ${ }^{1} \mathrm{H}$ - 
NMR-Daten nicht abzulesen. Sie ergab sich erst aus einer spektroskopischen und kristallographischen Untersuchung der von 141 und 142 abgeleiteten Alkohole.

Zu deren Darstellung wurde das 54:46-Gemisch der beiden monomethylierten Ketone mit zwei Moläquivalenten Lithiumaluminiumhydrid in Ether solange unter Rückfluss erhitzt, bis eine dünnschichtchromatographische Kontrolle vollständigen Umsatz anzeigte (40 min). Aufarbeitung und Chromatographie lieferte drei Alkohole in isolierten Ausbeuten von 49, 29 und 10\%. Bei den Majoritätsalkoholen handelte es sich um farblose Feststoffe [Schmp. 78$\left.80^{\circ} \mathrm{C}(\mathbf{6 6}), 94-95^{\circ} \mathrm{C}(\mathbf{6 0})\right]$, der Minoritätsalkohol war ein Öl.

\section{Schema 34}

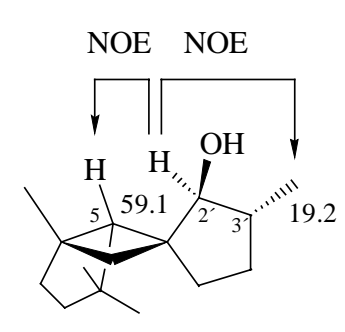

66

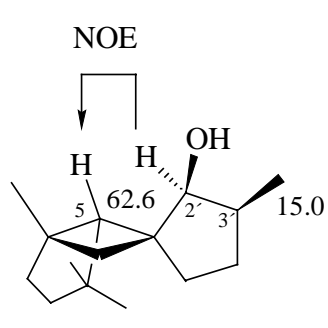

60

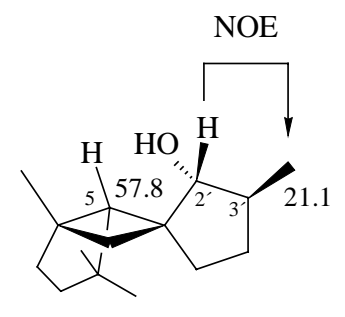

64

Zur Festlegung der Stereochemie haben wir von allen Alkoholen neben einem ${ }^{1} \mathrm{H}$ - und ${ }^{13} \mathrm{C}$-NMR-Spektrum auch ein ${ }^{1} \mathrm{~J}_{\mathrm{CH}}$-Korrelationsspektrum (HMQC) und ein NOESY-Spektrum aufgenommen. Alle Alkohole zeigten im ${ }^{1} \mathrm{H}-\mathrm{NMR}-$ Spektrum (Abb. 39, 40, 41) neben drei Singuletts für ungekoppelte Methylgruppen $[\delta=0.80,1.05,1.21(\mathbf{6 6}), 0.78,1.07,1.22(\mathbf{6 0})$, $0.83,1.05,1.23(64)]$ und einem Dublett für eine gekoppelte Methylgruppe $[\delta=1.03, \mathrm{~J}=6.0$ $\mathrm{Hz}(\mathbf{6 6}) ; 1.00, \mathrm{~J}=7.0 \mathrm{~Hz}(\mathbf{6 0}) ; 1.07 \mathrm{~J}=7.5 \mathrm{~Hz}(\mathbf{6 4})]$ ein Dublett für das Proton $\mathrm{H}-2^{\prime}[\delta=3.17$, $\mathrm{J}=7.9 \mathrm{~Hz}(\mathbf{6 6}) ; 3.50, \mathrm{~J}=4.1 \mathrm{~Hz}(\mathbf{6 0}), 3.43 \mathrm{~J}=7.5 \mathrm{~Hz}(\mathbf{6 4})])$. Da die Kopplung von H-2` zu H-3' trotz charakteristischer Unterschiede aufgrund der konformativen Beweglichkeit des Fünfringes als stereochemischer Indikator ausfiel, haben wir hierzu die ${ }^{13} \mathrm{C}$-chemischen Verschiebungen der Methylgruppen an C-3’ herangezogen $[\delta=15.0(\mathbf{6 0}), 21.1(\mathbf{6 4}), 19.2$ (66)] und unter Berücksichtigung des $\gamma$-Effektes, d.h. einer durch cis-ständige Substituenten bewirkten Hochfeldverschiebung interpretiert. Danach waren die Hydroxyl- und Methylgruppen in 60 cis-, in 64 und 66 dagegen trans-konfiguriert. Leider waren die durch $\gamma$ Effekte bewirkten Unterschiede in den ${ }^{13} \mathrm{C}$-chemischen Verschiebungen der Brückenkopf- 
atome C-5 weniger ausgeprägt $[\delta=62.6(\mathbf{6 0}), 57.8(\mathbf{6 4}), 59.1$ (66)]. Sie hätten sonst zur Festlegung der gesamten Stereochemie dienen können. Glücklicherweise waren die NOESYSpektren ergiebiger. Sie zeigten bei 66 (Abb. 122) eine starke Kopplung zwischen H-2` und H-5 sowie der Methylgruppe an C-3', bei 60 (Abb. 120) eine starke Kopplung zwischen H-2' und H-5, nicht jedoch zwischen H-2' und der Methylgruppe an C-3', sowie bei 64 (Abb. 121) eine Kopplung zwischen H-2' und der Methylgruppe an C-3', nicht jedoch zu H-5 (Schema 34). Die aus den ${ }^{13} \mathrm{C}$-chemischen Verschiebungen der Methylgruppen abgeleitete relative Konfiguration der Hydroxyl- und Methylgruppen war damit bestätigt und die Gesamtkonfiguration jedes der drei Alkohole festgelegt.

\subsection{Röntgenstrukturanalysen von 60 und 66}

Um letzte Zweifel an der Struktur und Stereochemie der Spirooctanole 60, 64 und 66 zu beseitigen und damit die Stereochemie der zu ihrer Darstellung eingesetzten Ketone festlegen zu können, wurden die als Feststoffe angefallenen Majoritätsalkohole aus Pentan (60) bzw. einem 9:1-Gemisch aus Pentan und Ether (66) kristallisiert und anschließend einer Röntgenstrukturanalyse unterworfen. Dabei erwies sich die aus spektroskopischen Daten abgeleitete Struktur und Stereochemie beider Alkohole als korrekt. Daraus folgt, dass es sich bei dem zur Methylierung eingesetzten Keton um $\mathbf{5 7}$ und bei den dabei erhaltenen Produkten aufgrund der Produktverteilung bei der nachfolgenden Reduktion um 141 (Majorität) bzw. 142 (Minorität) handelt.

Mechanistisch war eine Synchronumlagerung des Oxaspiropentans 55 unter Bildung von $57 \mathrm{zu}$ erwarten $^{61}$. Gleiches gilt für die mangelnde Stereoselektivität bei dessen Methylierung zu 141 und 142. Die bei der anschließenden Reduktion beobachtete Produktverteilung allerdings überrascht. Hier hätten wir erwartet, dass der Einfluss der Methylgruppe gegenüber jedem anderen Einfluss überwiegt und damit aus beiden Edukten bezüglich der Hydroxyl- und Methylgruppe cis-konfigurierte Produkte entstehen ${ }^{59}$. Dies ist jedoch bei 141 nicht der Fall. Der Grund dafür ist unklar. 


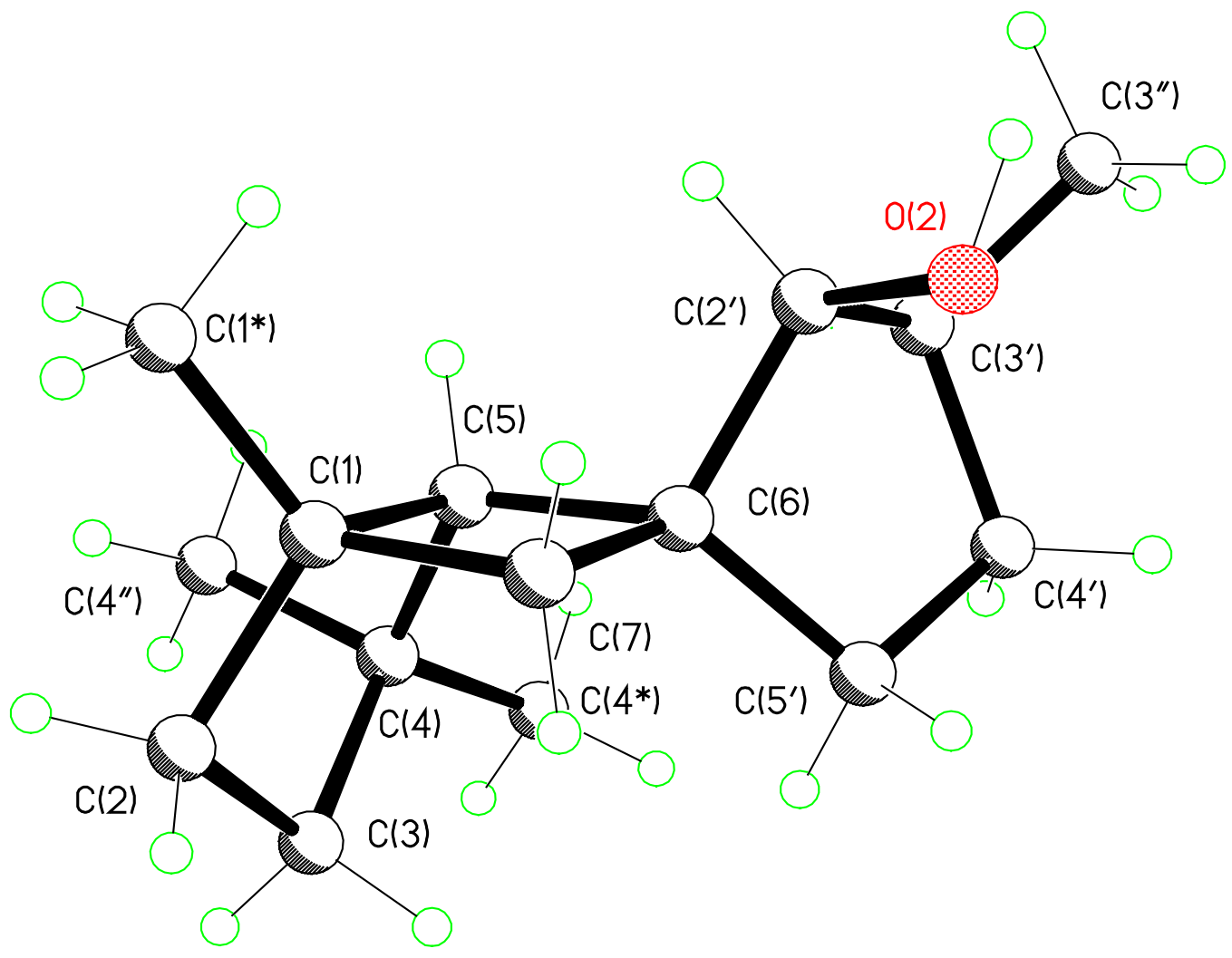

Abb.1 Kristallstruktur von 60.

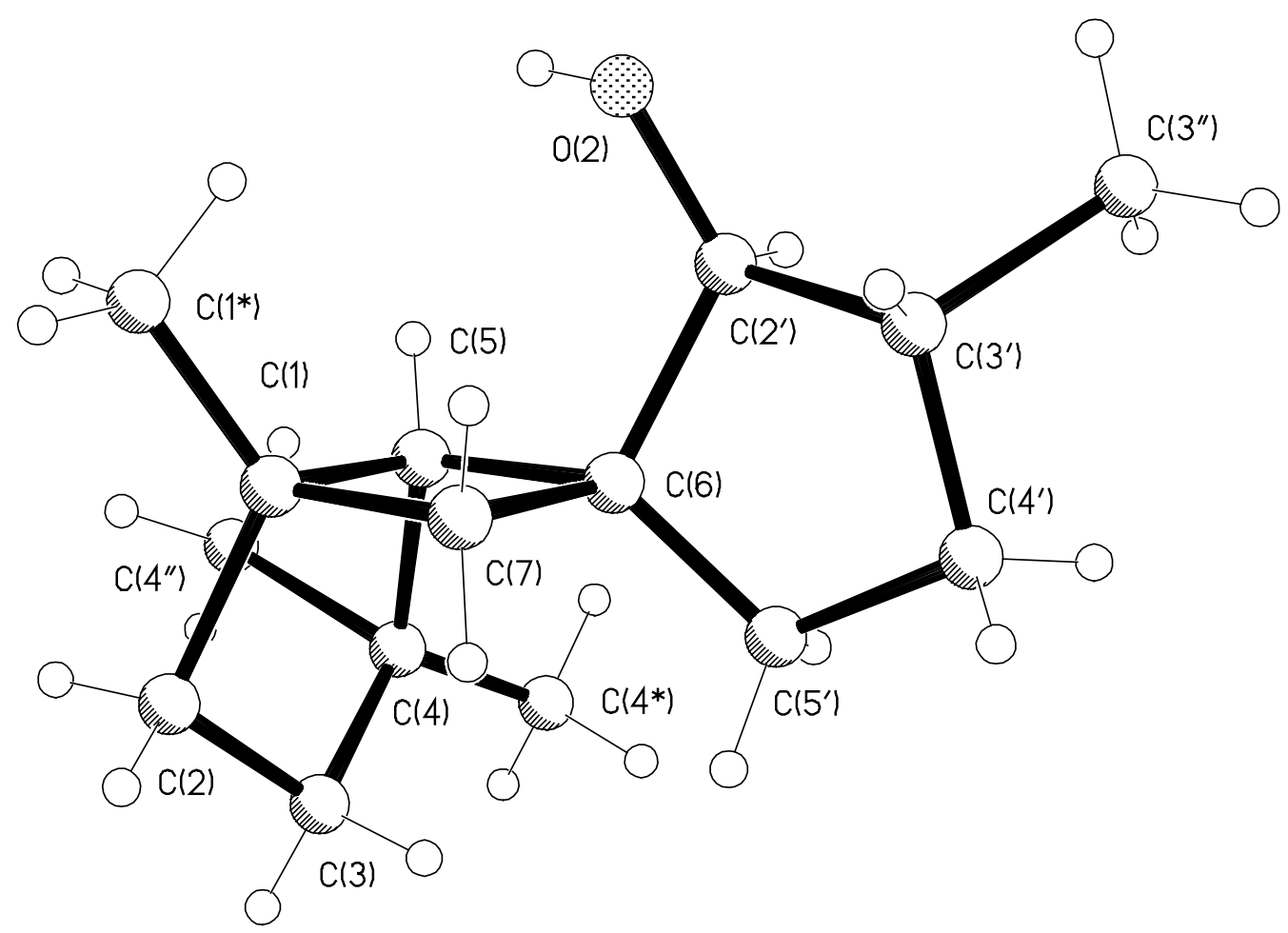

Abb.2 Kristallstruktur von 66. 


\subsection{Säurekatalysierte Umlagerung der Spirooctanole 60 und 66 zu den Tetramethyl- octahydro-1H-cyclopenta[a]pentalenen 67-70 und ( \pm )-endo-Capnellen (44)}

Nachdem die Synthese von Spirooctanolen gelungen war und mit 60 und 66 zwei Vertreter mit kristallographisch gesicherter Struktur zur Verfügung standen, sollte geprüft werden, in welche Produkte sie bei Einwirkung von Säure übergehen. Dabei sollten beide Alkohole getrennt eingesetzt und der Fortgang der Umlagerungen zeitaufgelöst gaschromatographisch dokumentiert werden. Wesentlich für den Erfolg der angestrebten Synthese von $( \pm)$-endo-Capnellen $(\mathbf{4 4})^{11}$ war, ob im Erstschritt der Umlagerung eine hierzu notwendige selektive 1,2-Verschiebung der höher substituierten Cyclobutanbindung eintreten würde. Da 1,2-Verschiebungen in der Regel unter Inversion der Konfiguration am Endpunkt der Umlagerung ablaufen, schien die Stellung der Hydroxylgruppe in $\mathbf{6 0}$ wie $\mathbf{6 6}$ für den gewünschten Verlauf günstig.

\section{Schema 35}

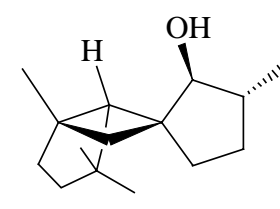

66

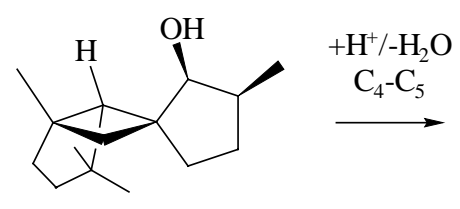

60
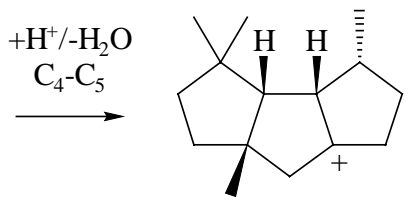

144

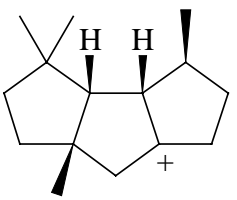

145

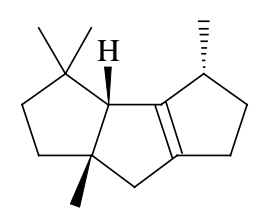

$67(-27.8)$

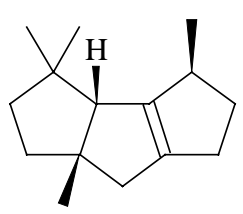

$69(-29.7)$

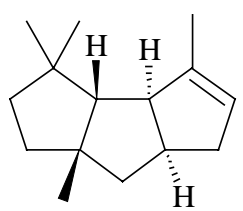

$44(-28.2)$

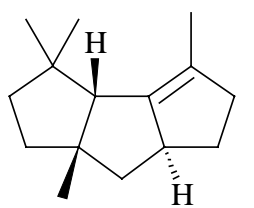

$68(-29.2)$

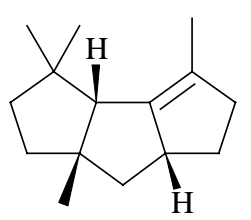

$70(-26.2)$

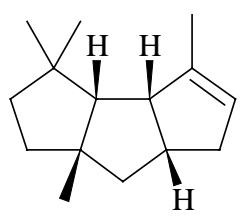

$71(-24.5)$

$( \pm)$-endo-Capnellen

Eine 1,2-Verschiebung der höher substituierten Cyclobutanbindung vorausgesetzt, war unter äquilibrierenden Bedingungen nach Kraftfeldrechnungen mit HUNTER ${ }^{10}$ in Verbindung 
mit MMP2 ${ }^{15}$ mit einer Bildung von maximal sechs Olefinen zu rechnen (Schema 35). Von diesen schienen 67, 68, 69 und ( \pm )-endo-Capnellen (44) aufgrund der berechneten Standardbildungsenthalpien (in $\mathrm{kcal} / \mathrm{mol}$ ) gegenüber $\mathbf{7 0}$ und $\mathbf{7 1}$ begünstigt.

Zur Realisierung der Umlagerungen wurden die Alkohole zu jeweils einem Moläquivalent einer $0.074 \mathrm{M}$ Lösung von wasserfreier p-Toluolsulfonsäure in Benzol gegeben und $45 \mathrm{~h}$ bei $25^{\circ} \mathrm{C}$ bzw. $6 \mathrm{~h}$ bei $70^{\circ} \mathrm{C}$ gerührt. Dabei wurde der Fortgang der Umlagerungen gaschromatographisch überwacht. Analytisch reine Proben der gebildeten Produkte isolierte man präparativ gaschromatographisch. Die Strukturaufklärung erfolgte durch Auswertung der ${ }^{1} \mathrm{H}$ - und ${ }^{13} \mathrm{C}$-NMR-Spektren in Verbindung mit ${ }^{1} \mathrm{~J}_{\mathrm{CH}^{-}}$(HMQC) und ${ }^{2,3} \mathrm{~J}_{\mathrm{HH}}$-Korrelationsspektren (COSY). Die ${ }^{1} \mathrm{H}$ - und ${ }^{13} \mathrm{C}$-NMR-Daten von ( \pm )-endo-Capnellen (44) waren bekannt ${ }^{11}$.

\subsubsection{Produkte und zeitlicher Verlauf der säurekatalysierten Umlagerung von 60}

Lagerte man 60 bei $25^{\circ} \mathrm{C}$ um, so bildete sich anfänglich ein einziges Produkt. Dieses Produkt erreichte nach $45 \mathrm{~h}$ bei $97 \%$ Umsatz einen Anteil von $95 \%$ und war auch bei $70^{\circ} \mathrm{C}$ anfänglich dominant. Allerdings bildeten sich bei dieser Temperatur im weiteren Verlauf zwei neue Produkte, darunter das erwünschte ( \pm )-endo-Capnellen (44), das anhand seiner bekannten spektroskopischen Daten ${ }^{11}$ leicht identifiziert werden konnte. Nach 6 h bei $70^{\circ} \mathrm{C}$ war das Gleichgewicht erreicht. Zu diesem Zeitpunkt enthielt das Reaktionsgemisch $42 \%( \pm)$ endo-Capnellen (44) sowie 32\% des zuerst und 26\% des später mit $\mathbf{4 4}$ gebildeten Produktes. Weiteres Erhitzen änderte die Zusammensetzung nicht mehr. Um sicherzustellen, dass es sich um ein echtes Gleichgewicht handelte, wurden die beiden unbekannten Produkte abgetrennt und als Gemisch äquilibriert. Dabei bildeten sich bei $70^{\circ} \mathrm{C}$ ebenso wie aus reinem $6042 \% 44$. Damit war klar, dass $\mathbf{6 0}$ unter selektiver 1,2-Verschiebung der höher substituierten Cyclobutanbindung umgelagert hatte und dass die neuen Produkte die aus dem spiroanellierten Cyclopentanring von 60 stammende Methylgruppe in derselben Position wie in $\mathbf{4 4}$ gebunden enthielten.

Wie bereits bemerkt, wurden zur Strukturaufklärung die ${ }^{1} \mathrm{H}-(\mathrm{Abb} .61,62)$ und ${ }^{13} \mathrm{C}$ NMR-Spektren (Abb. 100, 101) in Verbindung mit ${ }^{1} \mathrm{~J}_{\mathrm{CH}^{-}}$(HMQC) (Abb. 123, 125) und ${ }^{2,3} \mathrm{~J}_{\mathrm{HH}^{-}}$ 
Korrelationsspektren (COSY) (Abb. 124, 126) herangezogen. Bei dem zuerst gebildeten Produkt handelte es sich laut ${ }^{13} \mathrm{C}-\mathrm{NMR}$-Spektrum um ein tetrasubstituiertes Olefin $[\delta=$ $\left.143.30\left(\mathrm{C}_{\text {quart }}\right), 150.14\left(\mathrm{C}_{\text {quart }}\right)\right]$, das laut ${ }^{1} \mathrm{H}-\mathrm{NMR}-$ Spektrum drei Methylgruppen an gesättigten quartären $(\delta=0.82,1.01,1.14$, s, je $3 \mathrm{H})$, und eine an einem gesättigten tertiären Kohlenstoffatom gebunden enthielt $(\delta=0.95, \mathrm{~d}, \mathrm{~J}=6.8 \mathrm{~Hz}, 3 \mathrm{H})$. Auch bei dem später gebildeten Produkt handelte es sich um ein tetrasubstituiertes Olefin $\left[\delta=127.90\left(\mathrm{C}_{\text {quart }}\right)\right.$, $\left.147.60\left(\mathrm{C}_{\text {quart }}\right)\right]$, das drei Methylgruppen an gesättigten quartären $(\delta=0.79,1.07,1.15$, s, je $3 \mathrm{H})$, die vierte jedoch an ein vinylisches Kohlenstoffatom gebunden enthielt $(\delta=1.59$, br s, 3H). Damit war klar, dass es sich bei dem zuerst gebildeten Produkt um 67 oder 69, und bei dem später gebildeten Produkt um 68 oder 70 handeln musste. Dieses Ergebnis wurde durch die Auswertung der Korrelationsspektren gestützt. Sie ergab die in Schema 36 fett gezeichneten Fragmente.

\section{Schema 36}
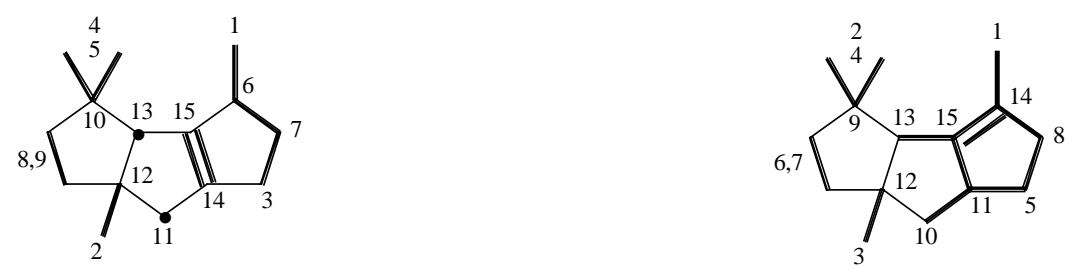

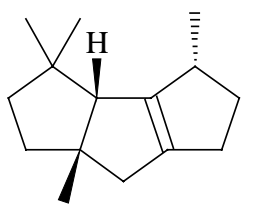

$67(-27.8)$

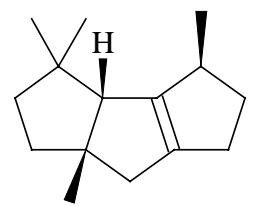

$69(-29.7)$

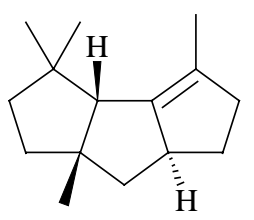

$68(-29.2)$

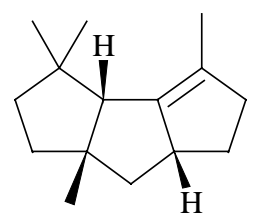

$\mathbf{7 0}(-26.2)$

Der Entscheid zugunsten von 69 als Primärprodukt und 68 als Folgeprodukt ergab sich aus der Überlegung, dass (a) die Stereochemie der Methylgruppe von 60 im Erstschritt der Umlagerung erhalten bleibt, (b) das Primärprodukt thermodynamisch mit den später gebildeten Produkten konkurrieren kann, und (c) im weiteren Verlauf der Umlagerung das jeweils stabilere von zwei denkbaren Stereoisomeren gebildet werden sollte. Einzelheiten zum Verlauf der Umlagerung von 60 finden sich in den Tabellen 1 und 2. Der Mechanismus der Umlagerung wird zusammen mit dem von 66 unter 3.4 .3 diskutiert. 
Tab.1: Produkte und zeitlicher Verlauf der säurekatalysierten Umlagerung von $\mathbf{6 0}$ bei $25^{\circ} \mathrm{C}$

\begin{tabular}{|c|c|c|c|c|c|c|}
\hline \multicolumn{2}{|c|}{$\begin{array}{l}1 \mathrm{Moläquivalent} \\
0.074 \mathrm{M} \mathrm{p}-\mathrm{TsOH} \\
\text { in Benzol, } 25^{\circ} \mathrm{C}\end{array}$} & \multirow[t]{2}{*}{69} & \multirow[t]{2}{*}{67} & \multirow[t]{2}{*}{70} & \multirow[t]{2}{*}{68} & \multirow[t]{2}{*}{44} \\
\hline Zeit & Umsatz & & & & & \\
\hline $20 \mathrm{~min}$ & $9 \%$ & $100 \%$ & - & - & - & - \\
\hline $60 \mathrm{~min}$ & $18 \%$ & $100 \%$ & - & - & - & - \\
\hline $3 \mathrm{~h}$ & $40 \%$ & $100 \%$ & - & - & - & - \\
\hline $4 \mathrm{~h}$ & $61 \%$ & $98 \%$ & - & - & - & - \\
\hline $20 \mathrm{~h}$ & $94 \%$ & $96 \%$ & - & - & $1 \%$ & $1 \%$ \\
\hline $45 \mathrm{~h}$ & $97 \%$ & $95 \%$ & - & - & $2 \%$ & $2 \%$ \\
\hline
\end{tabular}

Tab.2:Produkte und zeitlicher Verlauf der säurekatalysierten Umlagerung von 60 bei $70^{\circ} \mathrm{C}$

\begin{tabular}{|c|c|c|c|c|c|c|}
\hline \multicolumn{2}{|c|}{$\begin{array}{l}1 \mathrm{Moläquivalent} \\
0.074 \mathrm{M} \mathrm{p}-\mathrm{TsOH} \\
\text { in Benzol, } 70^{\circ} \mathrm{C}\end{array}$} & \multirow[t]{2}{*}{69} & \multirow[t]{2}{*}{67} & \multirow[t]{2}{*}{70} & \multirow[t]{2}{*}{68} & \multirow[t]{2}{*}{44} \\
\hline Zeit & Umsatz & & & & & \\
\hline $10 \mathrm{~min}$ & $100 \%$ & $95 \%$ & - & - & $1 \%$ & $4 \%$ \\
\hline $20 \mathrm{~min}$ & $100 \%$ & $91 \%$ & - & - & $3 \%$ & $5 \%$ \\
\hline $40 \mathrm{~min}$ & $100 \%$ & $88 \%$ & - & - & $5 \%$ & $7 \%$ \\
\hline $60 \mathrm{~min}$ & $100 \%$ & $81 \%$ & - & - & $8 \%$ & $11 \%$ \\
\hline $2 \mathrm{~h}$ & $100 \%$ & $62 \%$ & - & - & $15 \%$ & $23 \%$ \\
\hline $4 \mathrm{~h}$ & $100 \%$ & $36 \%$ & - & - & $24 \%$ & $39 \%$ \\
\hline $6 \mathrm{~h}$ & $100 \%$ & $32 \%$ & - & - & $26 \%$ & $42 \%$ \\
\hline
\end{tabular}




\subsubsection{Produkte und zeitlicher Verlauf der säurekatalysierten Umlagerung von 66}

Lagerte man 66 bei $25^{\circ} \mathrm{C}$ um, so betrug der Umsatz nach 45 h erst $78 \%$. Zu diesem Zeitpunkt enthielt das Gemisch 8\% 69, 9\% ( \pm )-endo-Capnellen (44), 46\% eines 65:35Gemisches aus einem unbekannten Olefin und 68, sowie $24 \%$ eines weiteren unbekannten Olefins. Arbeitete man bei $70^{\circ} \mathrm{C}$, so enthielt das Gemisch nach $6 \mathrm{~h} 25 \% \mathbf{6 8}, 33 \% \mathbf{6 9}$ und $42 \%$ ( \pm )-endo-Capnellen (44), und damit dieselben Komponenten in derselben Zusammensetzung wie bei der Umlagerung von 60 (Tab.2). Alle bekannten Umlagerungsprodukte wurden durch Vergleich ihrer spektroskopischen Daten mit denen authentischer Proben identifiziert.

Das nach $45 \mathrm{~h}$ bei $25^{\circ} \mathrm{C}$ in $24 \%$ Ausbeute isolierte unbekannte Olefin war nach Ausweis seines ${ }^{13} \mathrm{C}$-NMR-Spektrums (Abb. 102) tetrasubstituiert $\left[\delta=126.33\left(\mathrm{C}_{\text {quart }}\right), 146.14\left(\mathrm{C}_{\text {quart }}\right)\right]$ und enthielt laut ${ }^{1} \mathrm{H}-\mathrm{NMR}-$ Spektrum (Abb. 63) wie $\mathbf{6 8}$ neben drei an gesättigte Kohlenstoffatome gebundene Methylgruppen $(\delta=0.85,1.11,1.16, \mathrm{~s}$, je $3 \mathrm{H})$ eine vinylisch gebundene Methylgruppe $\left(\delta=1.67, \mathrm{~m}_{\mathrm{c}}, 3 \mathrm{H}\right)$. Die naheliegende Vermutung, es könnte sich bei dem fraglichen Olefin um ein Stereoisomer von $\mathbf{6 8}$ handeln, wurde durch Auswertung der ${ }^{1} \mathrm{~J}_{\mathrm{CH}^{-}}$(HMQC) (Abb. 127), ${ }^{2,3} \mathrm{~J}_{\mathrm{HH}^{-}}$(COSY) (Abb. 128) und ${ }^{2,3} \mathrm{~J}_{\mathrm{CH}^{-}}$Korrelationsspektren (HMBC) (Abb. 129) bestätigt. Dabei ergab sich ein mit 68 identisches Gerüst und damit die Stereochemie von 70 zwangsläufig (Schema 37).

\section{Schema 37}

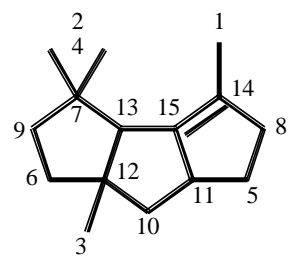

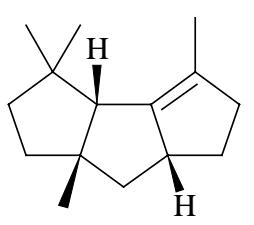

$70(-26.2)$

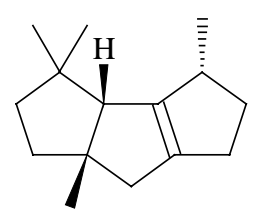

$67(-27.8)$ 
Das im Gemisch mit 68 angefallene unbekannte Olefin war nach Ausweis seines ${ }^{13} \mathrm{C}$ NMR-Spektrums (Abb. 99) ebenfalls tetrasubstituiert $\left[\delta=121.98\left(\mathrm{C}_{\text {quart }}\right), 143.10\left(\mathrm{C}_{\text {quart }}\right)\right]$ und enthielt laut ${ }^{1} \mathrm{H}$-NMR-Spektrum (Abb. 60) wie 69 drei ungekoppelte $(\delta=0.77,1.05,1.07$, je $3 \mathrm{H})$ und eine gekoppelte Methylgruppe $(\delta=1.07, \mathrm{~d}, \mathrm{~J}=6.8 \mathrm{~Hz}, 3 \mathrm{H})$. In diesem Fall konnten keine Korrelationsspektren zur Strukturaufklärung herangezogen werden. Berücksichtigt man, dass es sich bei dem fraglichen Olefin um ein Primärprodukt handelt, und bedenkt man, dass auch im Erstschritt der Umlagerung von 66 die Stereochemie der Methylgruppe erhalten bleibt, so erscheint ein Auftreten von 67 als Stereoisomer von 69 im Frühstadium der Umlagerung zwingend.

Auch bei 66 wurde der Verlauf der Umlagerung gaschromatographisch verfolgt. Einen Überblick geben die Tabellen 3 und 4.

Tab.3: Produkte und zeitlicher Verlauf der säurekatalysierten Umlagerung von 66 bei $25^{\circ} \mathrm{C}$

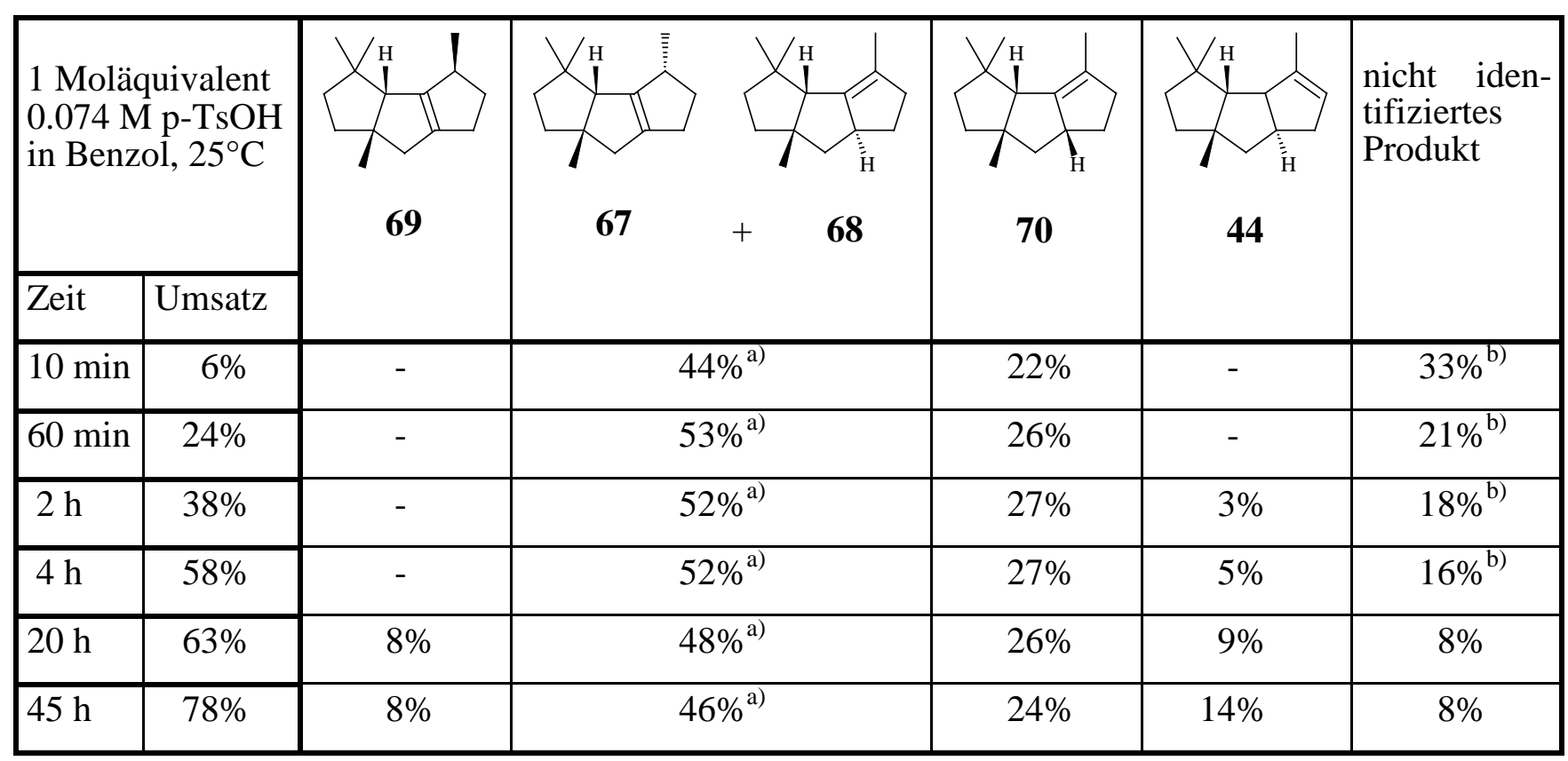

(a) Gemisch aus 67 und 68, das nach $45 \mathrm{~h}$ aus $65 \% \mathbf{6 7}$ und 35\% 68 bestand. (b) Gemisch eines nicht identifizierten Produktes mit 69, dessen Komponenten anfänglich wegen mangelnder Trennschärfe nicht quantifiziert werden konnten. 
Tab.4: Produkte und zeitlicher Verlauf der säurekatalysierten Umlagerung von 66 bei $70^{\circ} \mathrm{C}$

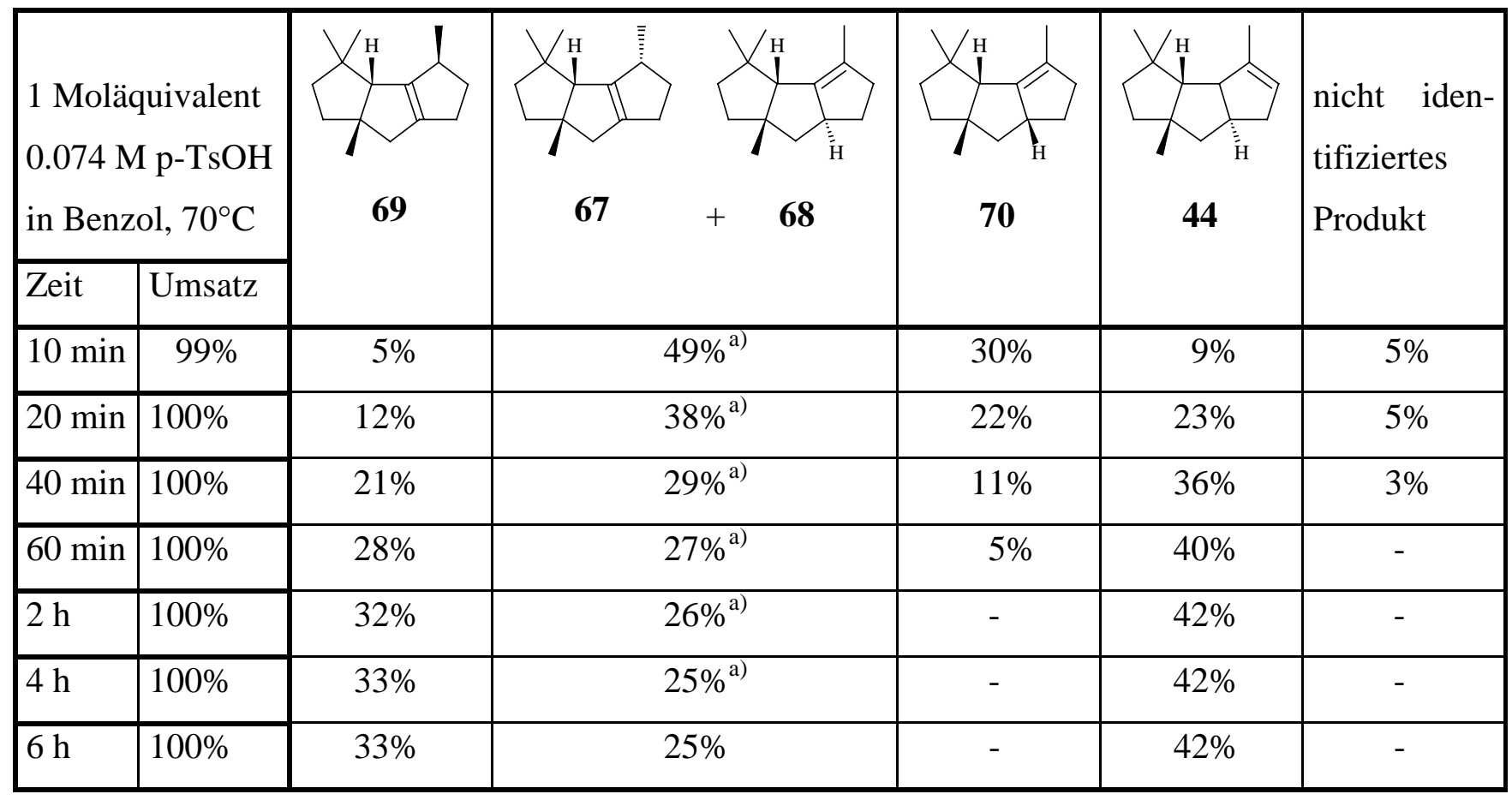

(a) Vermutlich ein Gemisch (vgl. hierzu die Ergebnisse der Umlagerung bei $25^{\circ}$ in Tab.3). Nach $6 \mathrm{~h}$ reines 68.

\subsubsection{Der Mechanismus der Umlagerungen von 60 und 66}

Wichtigstes Ergebnis der säurekatalysierten Umlagerungen von 60 und 66 ist, dass in beiden Fällen im Erstschritt der Umlagerung eine selektive 1,2-Verschiebung der höher substituierten Cyclobutanbindung beobachtet wird (69-145 bzw. 66-144). Ob dies auf einer Synchronumlagerung beruht oder ob die Regiochemie erst nach Ionisierung auf der Stufe der freien Kationen 39 bzw. 41 entschieden wird, wissen wir nicht. Diese Frage ließe sich durch Umlagerung von 64 klären. Treten dabei Regioisomere von 67-71 auf, so handelt es sich um eine Synchronumlagerung. Ist das Gegenteil der Fall, werden freie Kationen durchlaufen. 


\section{Schema 38}

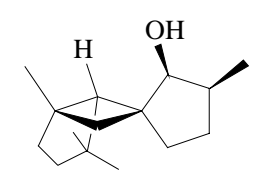

60

$\downarrow+\mathrm{H}^{+} /-\mathrm{H}_{2} \mathrm{O}$

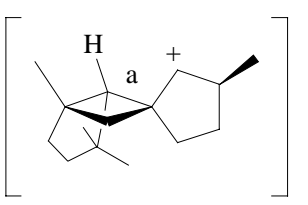

39

$\downarrow 1,2-\mathrm{a}$

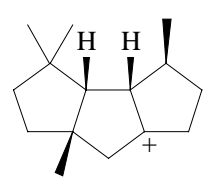

1,2-H

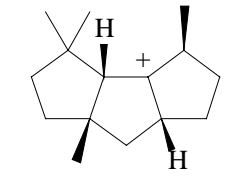

147

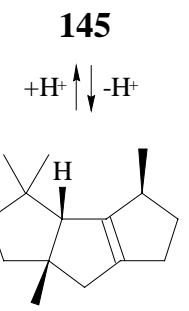

$69(-29.7)(33 \%)$

$\stackrel{+\mathrm{H}^{+}}{+\mathrm{H}^{+}}$

$\underset{-\mathrm{H}^{+}}{\stackrel{+\mathrm{H}^{+}}{\rightleftarrows}}$

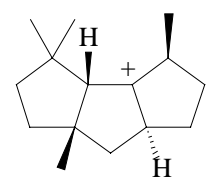

149

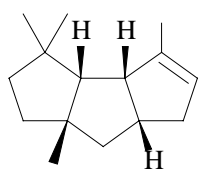

$71(-24.5)$

$+\mathrm{H}^{+} \downarrow \uparrow-\mathrm{H}^{+}$

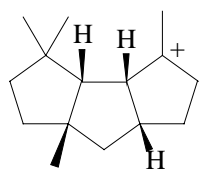

146

$-\mathrm{H}^{+} \downarrow \uparrow+\mathrm{H}^{+}$
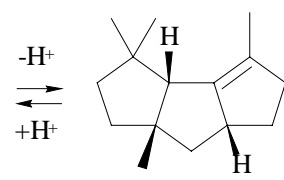

$70(-26.2)$
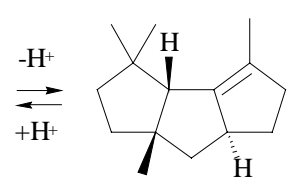

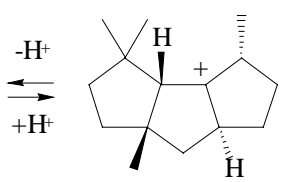

150

$68(-29.2)(25 \%)$

$$
-\mathrm{H}^{+} \uparrow \boldsymbol{r}^{\wedge} \mathrm{H}^{+}
$$

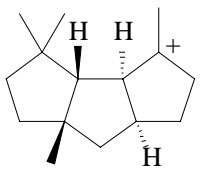

43

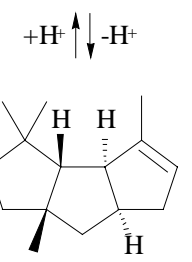

$44(-28.2)(42 \%)$

$( \pm)$-endo-Capnellen

148

(t)-endo-Capnellen

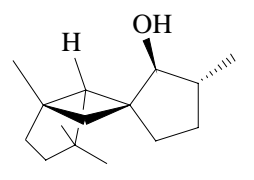

66

$+\mathrm{H}^{+} /-\mathrm{H}_{2} \mathrm{O}$

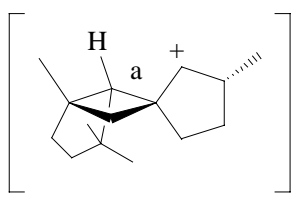

41

1,2-a

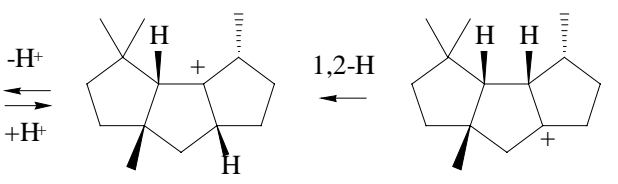

$\underbrace{+\mathrm{H}^{+}}_{-\mathrm{H}^{+}}+\mathrm{H}^{+} \uparrow \downarrow-\mathrm{H}^{+}$

$\stackrel{+\mathrm{H}^{+}}{-\mathrm{H}^{+}}$

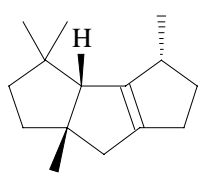

$67(-27.8)$

Unabhängig vom Mechanismus der Ringerweiterung leiten sich von den dabei gebildeten Kationen 145 und 144 mit 69 bzw. 67 die ersten Produkte ab. 69 kann unter kinetischer Kontrolle sogar als alleiniges Produkt erhalten werden, während 67 von Anfang 
an mit 70 vergesellschaftet ist. In der dann folgenden Phase der Äquilibrierung isomerisieren das aus 60 gebildete 69 sowie die aus 66 gebildeten 67 und 70 zu identischen Gemischen aus $33 \%$ 69, $25 \% 68$ und 42\% ( \pm )-endo-Capnellen (44). Dies geschieht über Protonierungs- und Deprotonierungsreaktionen (Schema 38).

Interessant ist, dass nach mit MMP2 berechneten Standardbildungsenthalpien (in $\mathrm{kcal} / \mathrm{mol}) 69$ (-29.7) mit 68 (-29.2) und ( \pm )-endo-Capnellen (44) (-28.2) konkurrieren kann, 67 (-27.8) und 70 (-26.2) dagegen nicht. Folgerichtig bleibt ein Teil von 69 im Verlauf der Äquilibrierung erhalten, während 67 und 70 verschwinden, und 71 (-24.5) gar nicht erst auftritt. Dies zeigt, dass Kraftfeldrechnungen insbesondere für thermodynamisch kontrollierte Reaktionen einen hohen prognostischen Wert besitzen. 


\section{ZUSAMMENFASSUNG}

Mit der vorliegenden Arbeit sollte das Synthesepotential von Bicyclobutyl-Bicyclooctyl-Umlagerungen zum Aufbau unterschiedlich substituierter di- und tricyclopentanoider Systeme ausgelotet werden. Hierzu sollten neue Cyclobutyl-Grignard-Reagenzien dargestellt, an Cyclobutanone addiert und die gebildeten Bicyclobutyl-1-ole säurekatalysiert umgelagert werden. Alternativ sollten die im Erstschritt gebildeten Spirooctyl-Kationen direkt aus entsprechenden Vorläufern generiert werden. Eines der Syntheseziele war ( \pm )-endo-Capnellen (44).

Die außer 8 erstmals dargestellten Grignard-Reagenzien, 21a,b, 22a,b und 23 erwiesen sich als sterisch anspruchsvoll. So reagierte 8 mit 25 und 26, 21a,b und 22a,b nur noch mit 24, und 23 reagierte nicht. Die erhaltenen mono- $(\mathbf{1 0 6 a}, \mathbf{b})$, di- $(\mathbf{1 0 4 b}, 107 \mathbf{a}$ b) und trimethylierten Bicyclobutyl-1-ole (105) lieferten säurekatalysiert in jeweils quantitativer Ausbeute die zugehörigen mono- (108), di- (109) bzw. trimethylierten Bicyclooctene (118).

\section{Schema 39}

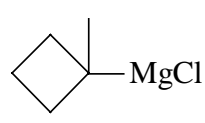

8

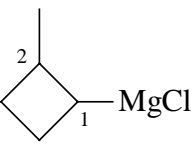

$21 \mathbf{a}\left(1 R *, 2 S^{*}\right)$

$21 \mathbf{b}\left(1 R^{*}, 2 R *\right)$

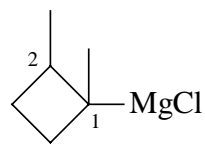

22a $\left(1 R^{*}, 2 S^{*}\right)$

22b $\left(1 R^{*}, 2 R *\right)$

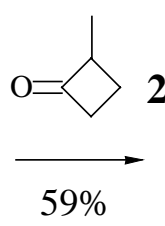

25

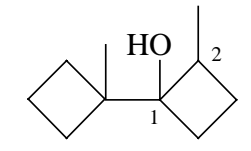

$\underset{100 \%}{\stackrel{\mathrm{H}^{+}}{\longrightarrow}}$

$104 \mathbf{b}\left(1 R^{*}, 2 S^{*}\right)$
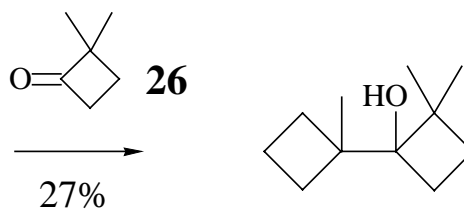

$\underset{100 \%}{\stackrel{\mathrm{H}^{+}}{\longrightarrow}}$

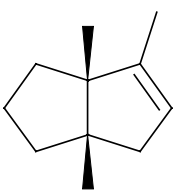

118

105
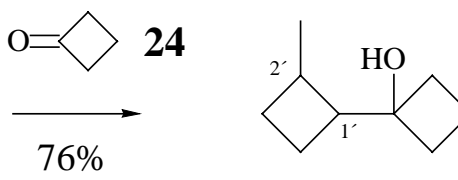

$\underset{100 \%}{\stackrel{\mathrm{H}^{+}}{\longrightarrow}}$

$106 \mathbf{a}\left(1^{\prime} R^{*}, 2 S^{*}\right)$

$106 b\left(1^{\prime} R^{*}, 2^{\prime} R^{*}\right)$
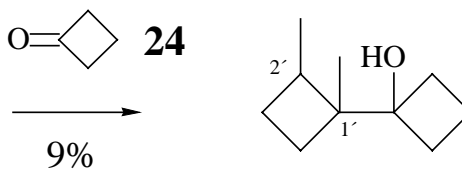

$\underset{100 \%}{\stackrel{\mathrm{H}^{+}}{\longrightarrow}}$

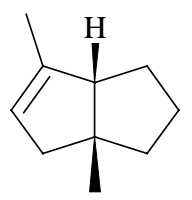

109

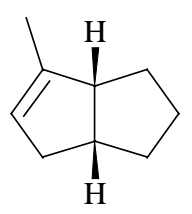

108 
Die mit dem Ziel einer Umlagerung $\mathrm{zu}( \pm)$-endo-Capnellen (44) synthetisierten Bicyclobutyl-1-ole 46 und 49 gingen bei Einwirkung von Säure nicht die erwartete Bicyclobutyl-Bicyclooctyl-, sondern eine Bicycloheptyl-Cycloheptenyl-Umlagerung ein und lieferten ein Gemisch der Cyclobutyl-cycloheptadiene bzw. Cyclobutyliden-cycloheptene 122-125. Mit Thionylchlorid in Pyridin bildeten sich die Cyclobutyl-cyclobutene bzw. Bicyclobutylidene 130-132. In diesem Fall waren die Ausbeuten für eine nachgeschaltete säurekatalysierte Umlagerung zu gering.

\section{Schema 40}

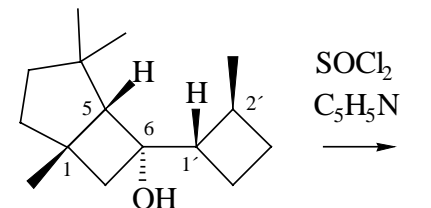

$46\left(1 S^{*}, 5 S^{*}, 6 S^{*}, 1^{\prime} S^{*}, 2^{\prime} S^{*}\right)$

$49\left(1 S^{*}, 5 S^{*}, 6 S^{*}, 1^{\prime} R^{*}, 2^{\prime} R^{*}\right)$ $\downarrow \mathrm{H}^{+}$

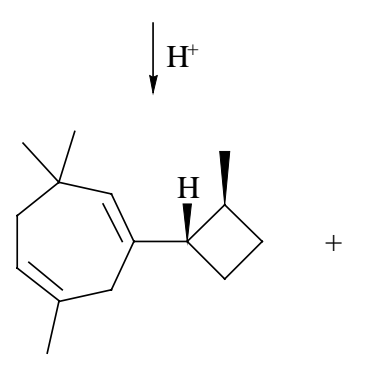

$122(9 \%)$

$122(9 \%)$

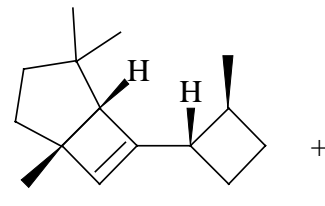

$130(15 \%)$

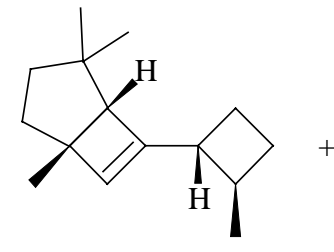

$131(15 \%)$

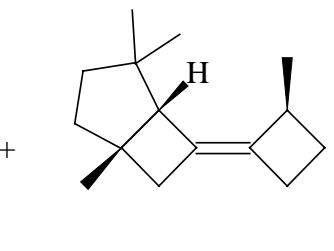

$132(10 \%)$

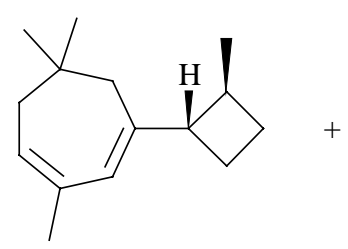

$123(34 \%)$

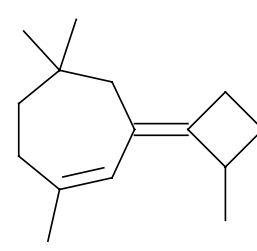

$124(20 \%)$

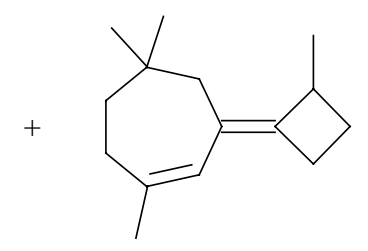

$125(12 \%)$

Die Versuche zur Synthese von ( \pm )-endo-Capnellen (44) durch Umlagerung von Spirooctanolen waren erfolgreich. Ausgehend von dem Bicyclobutyliden 54 gelang die Synthese der benötigten Edukte über eine Sequenz aus Epoxidierung, OxaspiropentanCyclopentanon-Umlagerung, Monomethylierung und Reduktion. Die Struktur und Stereochemie der dabei als Hauptprodukte gebildeten Spirooctanole 60 und $\mathbf{6 6}$ wurde kristallographisch gesichert. Unter der Einwirkung von Säure lieferten beide Alkohole identische Gemische der linearen Triquinane 44, 68 und 69, mit dem gewünschten ( \pm )-endoCapnellen (44) als Hauptprodukt. 


\section{Schema 41}

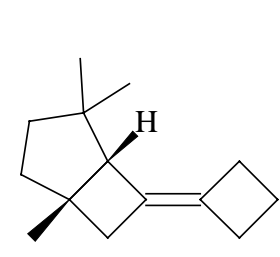

54
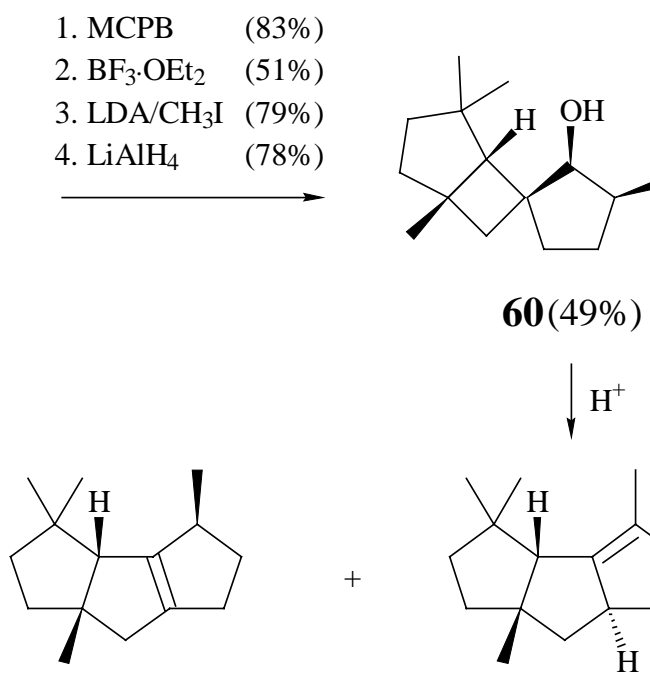

$69(33 \%)$
60(49\%)

$\checkmark \mathrm{H}^{+}$

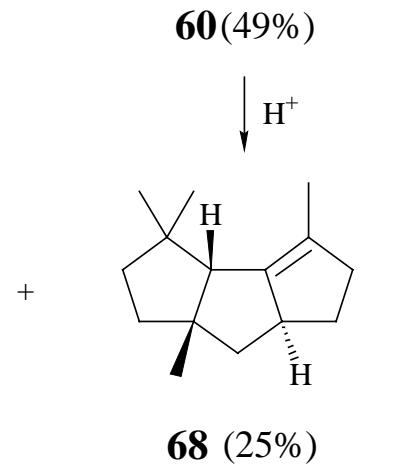

$(25 \%)$

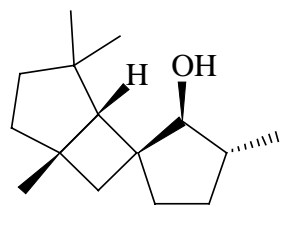

66 $(29 \%)$

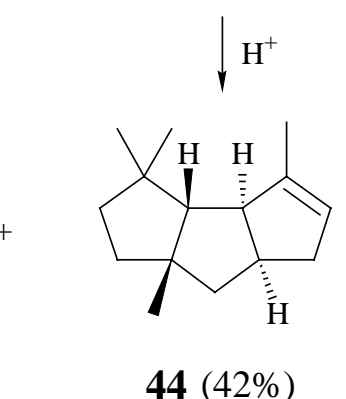

$( \pm)$-endo-Capnellen

Insgesamt hat die vorliegende Arbeit gezeigt, dass Bicyclobutyl-Bicyclooctyl- und Spirooctyl-Bicyclooctyl-Umlagerungen bei geeigneter Wahl der Edukte zu einem gezielten Aufbau bi- und tricyclischer Systeme genutzt werden können. Begleitende Kraftfeldrechnungen können hierzu Leitlinien liefern. 


\section{EXPERIMENTELLER TEIL}

\section{Allgemeine Angaben}

IR-Spektren wurden mit einem Spektrometer Modell 2000 FT-IR der Firma PerkinElmer aufgenommen. ${ }^{1} \mathrm{H}$ - und ${ }^{13} \mathrm{C}-\mathrm{NMR}-$ Spektren wurden mit einem Gerät XL 200, VXR 300 oder VXR 500 der Firma Varian bzw. einem Gerät AMX 300 der Firma Bruker registriert. Chemische Verschiebungen sind als Werte in ppm angegeben und beziehen sich auf Tetramethylsilan $(\delta=0.00)$ als internen Standard. Bei Verwendung anderer Standards wurden folgende chemische Verschiebungen zugrunde gelegt: $\delta_{\mathrm{H}}\left(\mathrm{CHCl}_{3}\right)=7.24, \delta_{\mathrm{C}}\left(\mathrm{CDCl}_{3}\right)=77.00$, $\delta_{\mathrm{H}}\left(\mathrm{C}_{6} \mathrm{D}_{5} \mathrm{H}\right)=7.15$ und $\delta_{\mathrm{C}}\left(\mathrm{C}_{6} \mathrm{D}_{6}\right)=128.00 \mathrm{ppm}$. Multiplizitäten wurden mit den in der Literatur $^{63}$ angegebenen Methoden bestimmt. Massenspektren wurden mit einem Spektrometer Modell MAT 311 A oder MAT 731 der Firma Varian, bzw. einem Spektrometer Modell MAT 95 der Firma Finnigan aufgenommen.

Dünnschichtchromatographische Untersuchungen erfolgten an DC-Plastikfolien Polygram SIL G/UV254 (Schichtdicke $0.25 \mathrm{~mm}$ ) der Firma Macherey \& Nagel. Farblose Substanzen wurden durch Besprühen mit 4 proz. ethanolischer Molybdatophosphorsäure (Merck) und anschließendem Erwärmen sichtbar gemacht. Mit Silbernitrat dotierte DCPlastikfolien wurden durch Eintauchen $(5 \mathrm{~min})$ in eine 10 proz. Lösung von Silbernitrat in Methanol/Wasser (2:1) hergestellt und $1 \mathrm{~h}$ bei $110^{\circ} \mathrm{C}$ getrocknet. Für säulenchromatographische Trennungen stand ein Fraktionssammler Modell LINEAR II der Firma Serva Technik, sowie ein Modell FC 220 der Firma Gilson zur Verfügung. Mit Silbernitrat dotiertes Kieselgel wurde durch Aufschlämmen einer entsprechenden Menge Kieselgels (0.04 - 0.063 mm) in einer Lösung von Silbernitrat in Acetonitril und Abziehen des Lösungsmittels im Rotationsverdampfer hergestellt. Das Material wurde bei $40^{\circ} \mathrm{C} / 20$ Torr bis zur Gewichtskonstanz getrocknet. Angaben über die zur Trennung verwendeten Materialien sowie über die Säulenabmessungen finden sich in den jeweiligen Versuchsbeschreibungen.

Für gaschromatographische Arbeiten standen ein Modell IGC 121 MLR der Firma Delsi Instruments und ein Modell GC 6000 Vega Series 2 der Firma Carlo Erba mit Leitfähigkeitsdetektoren zur Verfügung. Als Trägergas diente Wasserstoff. Der 
Wasserstoffstrom betrug jeweils 40-80 ml/min. Angaben über Art und Abmessungen der Säulen, Trägermaterial, Art der stationären Phasen, Belegung in Prozent und Säulentemperatur finden sich in den jeweiligen Versuchsbeschreibungen. Integrationen wurde mit einem Gerät D 2000 der Firma Merck - Hitachi oder einem Gerät SP 4270 der Firma Carlo Erba durchgeführt. Kapillargaschromatographische Trennungen erfolgten auf einem Modell GC 6000 Vega Series 2 der Firma Carlo Erba mit Flammenionisationsdetektor FID 40 und Split/Splitless-Injektor. Als Trägergas verwendeten wir Wasserstoff ( $2 \mathrm{ml} / \mathrm{min}$ ). Die Injektor- und Detektortemperatur betrug $250^{\circ} \mathrm{C}$.

Schmelzpunkte wurden auf einem Mikroheiztisch der Firma Reichert bestimmt und sind nicht korrigiert.

Die Elementaranalysen wurden von Herrn Hambloch von der Mikroanalytischen Abteilung des Instituts für Organische Chemie der Universität Göttingen durchgeführt.

\section{Arbeitsvorschriften}

$\left(1 R^{*}, 2 S^{*}\right)$-2-Methyl-cyclobutancarbonsäure

(81a) und

$\left(1 R^{*}, 2 R^{*}\right)-2-$ Methylcyclobutancarbonsäure (81b): Für die Darstellung von 81a und 81b wurde die Arbeitsvorschrift für die Synthese von Cyclobutancarbonsäuren von Casen und Allen ${ }^{19}$ verwendet und zuerst hergestellter 2-Methyl-cyclobutancarbonsäurediethylester (80) (201 g, Ausbeute 53\%, Sdp. $95-98^{\circ} \mathrm{C} / 8$ Torr, Lit. ${ }^{20}$ Sdp. $85-87^{\circ} \mathrm{C} / 2.8$ Torr) nach saurer Hydrolyse decarboxyliert. Dies lieferte ein 60:40 Gemisch von 81a und 81b (92 g, Ausbeute 86\%, Sdp. $98^{\circ} \mathrm{C} / 10$ Torr, Lit. $^{21} \mathrm{Sdp} .105^{\circ} \mathrm{C} / 16$ Torr). Proben der reinen Stereoisomere wurden durch präparative gaschromatographische Trennung erhalten [3 m x 1/4" Allglassystem, 15\% FFAP auf Chromosorb W AW/DMCS 60/80 mesh, $160^{\circ} \mathrm{C}$; Retentionszeiten (min): 4.90 (81a) und 5.70 (81b)]. 


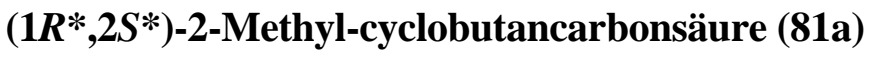

\section{$\mathrm{C}_{6} \mathrm{H}_{10} \mathrm{O}_{2}$}

${ }^{1} \mathbf{H}-\mathbf{N M R}$ (Abb. 26) (200 MHz, $\mathrm{CDCl}_{3}, \mathrm{CHCl}_{3}$ int.): $\delta=1.12 \mathrm{ppm}(\mathrm{d}, \mathrm{J}=7 \mathrm{~Hz}, 3 \mathrm{H}), 1.65\left(\mathrm{~m}_{\mathrm{c}}\right.$, $1 \mathrm{H}), 1.95-2.21(\mathrm{~m}, 2 \mathrm{H}), 2.27-2.40(\mathrm{~m}, 1 \mathrm{H}), 2.80\left(\mathrm{~m}_{\mathrm{c}}, 1 \mathrm{H}\right), 3.25\left(\mathrm{~m}_{\mathrm{c}}, 1 \mathrm{H}\right), 9-10($ br s , $1 \mathrm{H}$, $\mathrm{OH})$.

${ }^{13} \mathrm{C}-\mathrm{NMR}$ (Abb. 64) $\left(50 \mathrm{MHz}, \mathrm{CDCl}_{3}, \mathrm{CDCl}_{3}\right.$ int.): $\delta=16.91 \mathrm{ppm}\left(\mathrm{C}_{\text {prim }}\right), 19.91,26.22\left(\mathrm{C}_{\text {sek }}\right)$, 32.77, $41.69\left(\mathrm{C}_{\text {tert }}\right), 180.48\left(\mathrm{C}_{\text {quart }}\right)$.

$\left(1 R^{*}, 2 R^{*}\right)$-2-Methyl-cylobutancarbonsäure (81b)

\section{$\mathrm{C}_{6} \mathrm{H}_{10} \mathrm{O}_{2}$}

${ }^{1} \mathbf{H}-\mathbf{N M R}$ (Abb. 27) (300 MHz, $\mathrm{CDCl}_{3}, \mathrm{CHCl}_{3}$ int.): $\delta=1.14 \mathrm{ppm}(\mathrm{d}, \mathrm{J}=7 \mathrm{~Hz}, 3 \mathrm{H}), 1.56$ (dddd, $\mathrm{J}=9.5,9.5,9.5,9.0 \mathrm{~Hz}, 1 \mathrm{H}), 1.96-2.20(\mathrm{~m}, 3 \mathrm{H}), 2.53-2.69(\mathrm{~m}, 1 \mathrm{H}), 2.69$ (ddd, $\mathrm{J}=9.5,9.5,9.0$ $\mathrm{Hz}, 1 \mathrm{H}), 10$ - 11 (br s, 1H, OH).

${ }^{13}$ C-NMR (Abb. 65) (50 MHz, $\mathrm{CDCl}_{3}, \mathrm{CDCl}_{3}$ int.): $\delta=21.04 \mathrm{ppm}\left(\mathrm{C}_{\mathrm{sek}}\right), 21.09\left(\mathrm{C}_{\text {prim }}\right), 26.31$ $\left(\mathrm{C}_{\text {sek }}\right), 35.22,45.48\left(\mathrm{C}_{\text {tert }}\right), 181.35\left(\mathrm{C}_{\text {quart }}\right)$.

\section{$\left(1 R^{*}, 2 S^{*}\right)$-2-Methyl-cyclobutylchlorid $(82 \mathrm{a})$ und $\left(1 R^{*}, 2 R^{*}\right)$-2-Methyl-cyclobutylchlorid} (82b): Ein 60:40 Gemisch aus 81a und 81b (28.6 g, 0.25 mol) und N-Chlorsuccinimid (184 g, $1.38 \mathrm{~mol})$ wurden in einem 5:1 (v/v) Gemisch (200 ml) aus Dimethylformamid und Eisessig gelöst. Die Lösung wurde entgast und wasserfreies Bleitetraacetat (107 g, 0.24 mol) hinzugegeben, worauf man nochmals entgaste. Durch vorsichtiges Erwärmen auf $50^{\circ} \mathrm{C}$ trat die exotherme Kohlendioxidentwicklung ein. Unter gelegentlichem Kühlen auf $65^{\circ} \mathrm{C}$, war die Reaktion nach $1.5 \mathrm{~h}$ beendet. Die Reaktionsmischung wurde anschließend mit Pentan (5 x 100 ml) extrahiert, die vereinigten organischen Phasen mit 20\%iger Perchlorsäure ( 2 x $250 \mathrm{ml})$, 10\%iger Natriumcarbonatlösung $(2 \times 250 \mathrm{ml})$ und Wasser $(100 \mathrm{ml})$ gewaschen. Nach dem 
Trocknen über Magnesiumsulfat wurde fraktioniert. Man erhielt so $10.3 \mathrm{~g}$ (40\%) eines 33:67 Gemisches aus 82a und 82b als farblose Flüssigkeit mit einem Siedepunkt von $85-93^{\circ} \mathrm{C}, 760$ Torr. Die ${ }^{1} \mathrm{H}$ - und ${ }^{13} \mathrm{C}-\mathrm{NMR}-D a t e n$ entsprachen denen in der Literatur ${ }^{17}$ gegebenen Daten.

\section{$\left(1 R^{*}, 2 S^{*}\right)-1,2$-Dimethyl-cyclobutylchlorid (86a) und $\left(1 R^{*}, 2 R^{*}\right)-1,2$-Dimethyl-cyclobutyl-} chlorid (86b): In eine auf $-78^{\circ} \mathrm{C}$ gekühlte Lösung von 1,2-Dimethyl-cyclobut-1-en $(\mathbf{8 5})^{25}(5.88$ g, $71.6 \mathrm{mmol})$ in abs. Pentan $(2 \mathrm{ml})$ wurde langsam unter Rühren trockener Chlorwasserstoff eingeleitet. Nach 1.5 h zeigte gaschromatographische Kontrolle [3 m x 1/4" Allglassystem, 15\% OV 101 auf Chromosorb W AW/DMCS 60/80 mesh; Temperaturprogramm: 7 min bei $50^{\circ} \mathrm{C}$, $20^{\circ} / \mathrm{min}$ auf $140^{\circ} \mathrm{C}$; Retentionszeiten ( $\left.\mathrm{min}\right)$ : $2.69(\mathbf{8 5}), 8.21$ (86b), 8.53 (86a)] vollständige Addition an. Die Lösung wurde mit Pentan $(20 \mathrm{ml})$ und Wasser $(20 \mathrm{ml})$ aufgenommen. Die Phasen wurden getrennt und die organische mit gesättigter Natriumhydrogencarbonatlösung $(2 \mathrm{x}$ $25 \mathrm{ml})$ gewaschen. Die wässrige Phase wurde mit Pentan (3 x $20 \mathrm{ml})$ extrahiert und anschließend wurden die vereinigten organischen Phasen mit gesättigter Natriumhydrogencarbonatlösung ( $3 \mathrm{x}$ $35 \mathrm{ml}$ ) gewaschen. Nach dem Trocknen über Magnesiumsulfat wurde die Lösung fraktioniert. Man erhielt so $5.91 \mathrm{~g}(77 \%)$ eines 40:60 Gemisches von 86a und 86b als farblose Flüssigkeit (Sdp.: $73-76^{\circ} \mathrm{C}, 140$ Torr). Die spektroskopischen Daten stimmten mit denen in der Literatur ${ }^{18}$ überein.

2,2-Dimethyl-cyclobutanon (26): $\mathrm{Zu}$ einer Lösung von (1'-Phenylthiocyclopropyl-1'yl)propan-2-ol ${ }^{29}$ (90) $(24.80 \mathrm{~g}, 120 \mathrm{mmol})$ in Tetralin $(40 \mathrm{ml})$ wurden Quecksilberdichlorid (19.01 g, $0.07 \mathrm{~mol})$, p-Toluolsulfonsäuremonohydrat (2.51 g, $0.01 \mathrm{~mol}$ ) und Wasser (2.48 g, 0.14 mol) gegeben und $2 \mathrm{~h}$ bei $70^{\circ} \mathrm{C}$ gerührt. Anschließend wurde auf $140^{\circ} \mathrm{C}$ geheizt und abdestilliert. Dabei wurde weiteres Wasser $(3.5 \mathrm{ml})$ zugegeben. Dann wurden die Phasen des Destillates getrennt, die wässrige Phase mit Natriumchlorid gesättigt und mit Dichlormethan $(2 \times 10 \mathrm{ml})$ extrahiert. Nach Trocknen über Magnesiumsulfat wurde über eine $10 \mathrm{~cm}$ Vigreuxkolonne fraktioniert. Man erhielt so $8.53 \mathrm{~g}(72 \%) 26$ (Sdp.: $106-110^{\circ} \mathrm{C}$, Lit. ${ }^{33} 113.5-114^{\circ} \mathrm{C}, 760$ Torr) als farblose Flüssigkeit. Die ${ }^{1} \mathrm{H}^{32}$ - und ${ }^{13} \mathrm{C}-\mathrm{NMR}-\mathrm{Daten}^{34}$ stimmten mit denen in der Literatur überein. 
1,1-Dimethyl-2-methylencyclobutan (91): $\mathrm{Zu}$ einer Suspension von Triphenylmethylenphosphoniumbromid (37.31 g, $144 \mathrm{mmol})$ in wasserfreiem Xylol $(100 \mathrm{ml})$ wurde portionsweise Natriumhydrid $(2.51 \mathrm{~g}, 104 \mathrm{mmol})$ gegeben und $3.5 \mathrm{~h}$ bei $90^{\circ} \mathrm{C}$ bis zur intensiven Gelbfärbung gerührt. Dann ließ man auf $45^{\circ} \mathrm{C}$ abkühlen und gab 2,2-Dimethyl-cyclobutanon (26) (8.5 g, 87 mmol) langsam hinzu. Nach weiteren $3 \mathrm{~h}$ Rühren bei $60^{\circ} \mathrm{C}$ destillierte man direkt über eine 30 cm Vigreuxkolonne und erhielt so $6.66 \mathrm{~g}(88 \%) 91$ (Sdp.: $83-90^{\circ} \mathrm{C}, 760$ Torr). Die ${ }^{1} \mathrm{H}-\mathrm{NMR}-$ Daten stimmten mit denen in der Literatur ${ }^{28}$ überein.

${ }^{13}$ C-NMR (Abb. 66) (50 MHz, $\mathrm{CDCl}_{3}, \mathrm{CDCl}_{3}$ int.): $\delta=126.62 \mathrm{ppm}\left(\mathrm{C}_{\mathrm{sek}}\right), 27.44\left(\mathrm{C}_{\text {prim }}\right), 31.97$ $\left(\mathrm{C}_{\text {sek }}\right), 44.20$ ( $\left.\mathrm{C}_{\text {quart }}\right), 101.58\left(\mathrm{C}_{\text {sek }}\right), 160.24\left(\mathrm{C}_{\text {quart }}\right)$.

1,2,2-Trimethyl-cyclobutylchlorid (97), 5-Chlor-2,3-dimethyl-pent-2-en (95) und 1,4Dichlor-3,4-dimethyl-pentan (96): In eine auf $-78^{\circ} \mathrm{C}$ gekühlte Lösung von $6.66 \mathrm{~g}$ (69 mmol) 2,2-Dimethyl-1-methylencyclobutan (91) in Pentan $(2 \mathrm{ml})$ wurde trockener Chlorwasserstoff eingeleitet. Nach 3 h zeigte gaschromatographische Kontrolle [3 m x 1/4" Allglassystem, 15\% OV 101 auf Chromosorb W AW/DMCS 60/80 mesh; Temperaturprogramm: 5 min bei $100^{\circ} \mathrm{C}$, $20 \%$ min auf $190^{\circ} \mathrm{C}$; Retentionszeiten (min): 1.22 (91), 3.38 (97) (66\%), $5.76(95)(9 \%), 9.17(96)$ (25\%)] vollständige Addition an. Die Lösung wurde mit Pentan (20 ml) aufgenommen, mit Wasser $(2 \times 20 \mathrm{ml})$, sowie mit gesättigter Natriumhydrogencarbonatlösung (3 x $20 \mathrm{ml})$ gewaschen. Nach dem Trocknen über Magnesiumsulfat, wurde die Lösung über eine $20 \mathrm{~cm}$ Vigreuxkolonne fraktioniert. Man erhielt so $4.03 \mathrm{~g}$ (44\%) (Sdp.: 95 - 105 ${ }^{\circ} \mathrm{C} / 180$ Torr) des Chlorids (97) als klare Flüssigkeit, welche beim Kühlen zu einem glasigem Feststoff erstarrte (Schmp.: $27-30^{\circ} \mathrm{C}$ ). Präparative gaschromatographische Analyse des Rückstandes lieferte 95 und 96 als klare Flüssigkeiten. Das Monochlorid 95 war aus einer früheren Arbeit ${ }^{35}$ bekannt und die ${ }^{1}$ H-NMR-Daten von 96 entsprachen den Literaturdaten ${ }^{36}$.

\section{1,4-Dichlor-3,4-dimethyl-pentan (96)}

${ }^{13}$ C-NMR (Abb. 67) $\left(50 \mathrm{MHz}, \mathrm{CDCl}_{3}, \mathrm{CDCl}_{3}\right.$ int.): $\delta=14.50$ ppm, 30.04, $30.86\left(\mathrm{C}_{\text {prim }}\right), 35.11$ $\left(\mathrm{C}_{\text {sek }}\right), 42.68\left(\mathrm{C}_{\text {tert }}\right), 43.63\left(\mathrm{C}_{\text {sek }}\right), 74.48\left(\mathrm{C}_{\text {quart }}\right)$. 


\section{1,2,2-Trimethyl-cyclobutylchlorid (97)}

\section{$\mathrm{C}_{7} \mathrm{H}_{13} \mathrm{Cl}$}

Ber. C $63.39 \quad$ H 9.88

Gef. C $63.15 \quad$ H 9.84

IR (Abb. 3) (KBr): $820 \mathrm{~cm}^{-1}$ (C-Cl), $2907\left(\mathrm{CH}_{3}\right)$.

${ }^{\mathbf{1}} \mathbf{H}$-NMR (Abb. 28) (300 MHz, $\mathrm{CDCl}_{3}, \mathrm{CHCl}_{3}$ int.): $\delta=0.99 \mathrm{ppm}(\mathrm{s}, 3 \mathrm{H}), 1.24$ (s, 3H), 1.60 $(\mathrm{ddd}, \mathrm{J}=10.5,10.5,9 \mathrm{~Hz}, 1 \mathrm{H}), 1.61(\mathrm{~s}, 3 \mathrm{H}), 1.73(\mathrm{ddd}, \mathrm{J}=10.5,10.5,3 \mathrm{~Hz}, 1 \mathrm{H}), 2.08$ (ddd, J = $12,9,3 \mathrm{~Hz}, 1 \mathrm{H}), 2.44(\mathrm{ddd}, \mathrm{J}=12,10.5,10.5 \mathrm{~Hz}, 1 \mathrm{H})$.

${ }^{13}$ C-NMR (Abb. 68) (75 MHz, $\mathrm{CDCl}_{3}, \mathrm{CDCl}_{3}$ int.): $\delta=23.36$ ppm, 26.90, $27.38\left(\mathrm{C}_{\text {prim }}\right), 30.45$, $36.25\left(\mathrm{C}_{\mathrm{sek}}\right), 44.72,72.99\left(\mathrm{C}_{\text {quart }}\right)$.

MS $(70 \mathrm{eV}): \mathrm{m} / \mathrm{z}(\%)=132\left(\mathrm{M}^{+}, 2\right), 97(5), 56(100)$.

Generierung und Carboxylierung der Grignardreagenzien 21a,b, 22a,b und 23: 2-Methyl(81a,b), 1,2-Dimethyl- (86a,b) und 1,2,2-Trimethyl-cyclobutancarbonsäure (100): Man überschichtete mit Jod aktivierte Magnesiumspäne (304 mg, $12.5 \mathrm{mmol}$ ) mit wasserfreiem Ether (ca. $1.5 \mathrm{ml}$ ) (82a,b: THF) und tropfte anschließend unter Argon und Rühren eine Lösung des (der) entsprechenden Chlorids (Chloride) (82a,b, 86a,b, 97) (10 mmol) in wasserfreiem Ether (82a,b:THF) (0.5 ml) und einen Tropfen Brom hinzu. Nach dem Erwärmen der Lösung bis zum Einsetzten der exothermen Reaktion (Entfärbung), gab man vorsichtig weitere $10 \mathrm{ml}$ Ether (82a,b: THF) hinzu. Nach beendeter Zugabe hielt man noch weitere $2 \mathrm{~h}$ unter Rückfluss, bis laut gaschromatographischer Analyse eines Probehydrolysates [3 m x 1/4" Allglassystem, 15\% OV 101 auf Chromosorb W AW/DMCS 60/80 mesh; Retentionszeiten (min): 2.01 (82a), und 2.51 (82b) bei $90^{\circ} \mathrm{C}, 2.69(\mathbf{8 6 b})$ und $2.85(\mathbf{8 6 a})$ bei $90^{\circ} \mathrm{C}, 3.38(\mathbf{9 7})$ bei $\left.100^{\circ} \mathrm{C}\right]$ das (die) Chlorid (Chloride) verbraucht waren. Die Lösung wurde auf $0^{\circ} \mathrm{C}$ gekühlt und trockenes Kohlendioxid eingeleitet. Nach $2 \mathrm{~h}$ wurde die Reaktionsmischung mit $0.5 \mathrm{~N}$ Salzsäure (20 ml) hydrolysiert und 
die Phasen getrennt. Die wässrige Phase wurde mit konz. Salzsäure auf pH 1 gebracht und mit Ether $(7 \times 20 \mathrm{ml})$ extrahiert. Die vereinigten organischen Phasen wurden anschließend über Magnesiumsulfat getrocknet und die Lösungsmittel über eine $20 \mathrm{~cm}$ Vigreuxkolonne abdestilliert. Endgültiges Konzentrieren am Rotationsverdampfer $\left(20^{\circ} \mathrm{C} / 14\right.$ Torr $)$ lieferte die Cyclobutancarbonsäuren. Analytische Proben der Isomere wurden durch präparative Gaschromatographie erhalten [3 m x 1/4" Allglassystem, 15\% FFAP auf Chromosorb W AW/DMCS 60/80 mesh].

$\left(1 R^{*}, 2 S^{*}\right)$-2-Methyl-cyclobutancarbonsäure (81a) und $\left(1 R^{*}, 2 R^{*}\right)$-2-Methyl-cyclobutancarbonsäure (81b): $970 \mathrm{mg}(85 \%)$ eines 30:70 Gemisches von 81a und 81b. $\left[160^{\circ} \mathrm{C}\right.$; Retentionszeiten (min): 4.90 (81a), 5.70 (81b)]. Die ${ }^{1} \mathrm{H}-\mathrm{NMR}-$ und ${ }^{13} \mathrm{C}-\mathrm{NMR}-\mathrm{Daten}$ stimmten mit denen authentischer Proben überein.

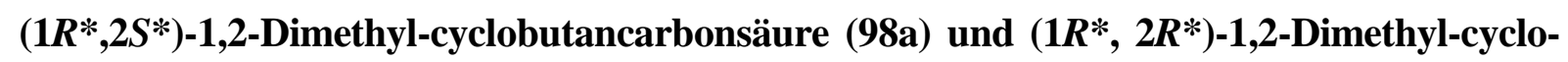
butancarbonsäure (98b): $705 \mathrm{mg}(55 \%)$ eines $14: 86$ Gemisches von $98 \mathrm{a}$ und $\mathbf{9 8 b}$. $\left[175^{\circ} \mathrm{C}\right.$; Retentionszeiten (min): 3.08 (98a), 3.61 (98b)].

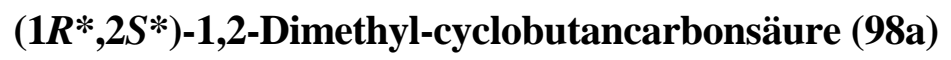

$\mathrm{C}_{7} \mathrm{H}_{12} \mathrm{O}_{2}$

Ber. C $65.60 \quad$ H 9.44

Gef. C $65.25 \quad$ H 9.70

IR (Abb. 4) (KBr): $3525-2500 \mathrm{~cm}^{-1}(\mathrm{COO}-\mathrm{H}), 1685(\mathrm{C}=\mathrm{O})$.

${ }^{1} \mathbf{H}-\mathbf{N M R}$ (Abb. 29) (300 MHz, $\mathrm{CDCl}_{3}, \mathrm{CHCl}_{3}$ int.): $\delta=1.06 \mathrm{ppm}(\mathrm{d}, \mathrm{J}=7 \mathrm{~Hz}, 3 \mathrm{H}), 1.38$ (s, $3 \mathrm{H}), 1.50-1.72(\mathrm{~m}, 2 \mathrm{H}), 2.00-2.15(\mathrm{~m}, 1 \mathrm{H}), 2.20-2.37(\mathrm{~m}, 1 \mathrm{H}), 2.45-2.58(\mathrm{~m}, 1 \mathrm{H}), \mathrm{OH}$ nicht detektiert. 
${ }^{13}$ C-NMR (Abb. 69) $\left(75 \mathrm{MHz}, \mathrm{CDCl}_{3}, \mathrm{CDCl}_{3}\right.$ int.): $\delta=17.15 \mathrm{ppm}\left(\mathrm{C}_{\text {prim }}\right), 23.66\left(\mathrm{C}_{\text {sek }}\right), 24.39$ $\left(\mathrm{C}_{\text {prim }}\right), 27.36\left(\mathrm{C}_{\text {sek }}\right), 40.68\left(\mathrm{C}_{\text {tert }}\right), 47.55,182.66\left(\mathrm{C}_{\text {quart }}\right)$.

MS $(70 \mathrm{eV}): \mathrm{m} / \mathrm{z}(\%)=128\left(\mathrm{M}^{+}, 8\right), 97(59), 87(100)$.

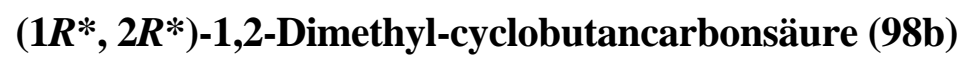

\section{$\mathrm{C}_{7} \mathrm{H}_{12} \mathrm{O}_{2}$}

Ber. C $65.60 \quad$ H 9.44

Gef. C $65.56 \quad$ H 9.62

IR (Abb. 5) (KBr): 3550 - $2200 \mathrm{~cm}^{-1}(\mathrm{COO}-\mathrm{H}), 1685$ ( C=O).

${ }^{\mathbf{1}} \mathbf{H}$-NMR (Abb. 30) (200 MHz, $\mathrm{CDCl}_{3}, \mathrm{CHCl}_{3}$ int.): $\delta=0.97 \mathrm{ppm}(\mathrm{d}, \mathrm{J}=7 \mathrm{~Hz}, 3 \mathrm{H}$ ), 1.27 (s, $3 \mathrm{H}), 1.45-1.70(\mathrm{~m}, 2 \mathrm{H}), 1.85-2.05(\mathrm{~m}, 1 \mathrm{H}), 2.24-2.45(\mathrm{~m}, 1 \mathrm{H}), 2.72\left(\mathrm{~m}_{\mathrm{c}}, 1 \mathrm{H}\right), 10.7-11.7$ (br $\mathrm{s}, 1 \mathrm{H}, \mathrm{OH})$.

${ }^{13}$ C-NMR (Abb. 70) $\left(50 \mathrm{MHz}, \mathrm{CDCl}_{3}, \mathrm{CDCl}_{3}\right.$ int.): $\delta=15.28$ ppm, $16.83\left(\mathrm{C}_{\text {prim }}\right), 23.92,28.63$ $\left(\mathrm{C}_{\text {sek }}\right), 35.86\left(\mathrm{C}_{\text {tert }}\right), 45.39\left(\mathrm{C}_{\text {quart }}\right), 184.49\left(\mathrm{C}_{\text {quart }}\right)$.

MS $(70 \mathrm{eV}): \mathrm{m} / \mathrm{z}(\%)=128\left(\mathrm{M}^{+}, 7\right), 87(100)$.

1,2,2-Trimethyl-cyclobutancarbonsäure (100) und 4,5-Dimethyl-hex-4-en-säure (102): 1.14 g (80\%) eines 51:49 Gemisches von 100 [farbloser Feststoff (Schmp.: $\left.115-117^{\circ} \mathrm{C}\right)$ ] und $\mathbf{1 0 2}^{39,40}$. [Temperaturprogramm: $6 \mathrm{~min}$ bei $180^{\circ} \mathrm{C}, 20^{\circ} \mathrm{C} / \mathrm{min}$ auf $220^{\circ} \mathrm{C}$; Retentionszeiten (min): $4.68(\mathbf{1 0 0})$ und $8.85(\mathbf{1 0 2})]$. 


\section{1,2,2-Trimethyl-cyclobutancarbonsäure (100)}

\section{$\mathrm{C}_{8} \mathrm{H}_{14} \mathrm{O}_{2}$}

Ber. C $67.35 \quad$ H 9.92

Gef. C $67.35 \quad$ H 9.77

IR (Abb. 6) (KBr): 3550 - $2375 \mathrm{~cm}^{-1}(\mathrm{COO}-\mathrm{H}), 1697(\mathrm{C}=\mathrm{O})$.

${ }^{1} \mathbf{H}-\mathbf{N M R}$ (Abb. 31) (200 MHz , $\mathrm{CDCl}_{3}, \mathrm{CHCl}_{3}$ int.): $\delta=1.03$ ppm (s, 3H), 1.12 (s, 3H), 1.33 (s, 3H), 1.44 - 1.54 (m, 2H), 1.75 (ddd, J = 10, 10, $9 \mathrm{~Hz}, 1 \mathrm{H}), 2.50$ (ddd, J = 10, 10, $9 \mathrm{~Hz}, 1 \mathrm{H}), \mathrm{OH}$ nicht detektiert.

${ }^{13}$ C-NMR (Abb. 71) $\left(50 \mathrm{MHz}, \mathrm{CDCl}_{3}, \mathrm{CDCl}_{3}\right.$ int.): $\delta=19.90$ ppm, $23.85\left(\mathrm{C}_{\text {prim }}\right), 25.40\left(\mathrm{C}_{\mathrm{sek}}\right)$, $25.53\left(\mathrm{C}_{\text {prim }}\right), 30.07\left(\mathrm{C}_{\text {sek }}\right), 40.51,48.58,182.83\left(\mathrm{C}_{\text {quart }}\right)$.

MS $(70 \mathrm{eV}): \mathrm{m} / \mathrm{z}(\%)=142\left(\mathrm{M}^{+}, 4\right), 56(100)$.

4,5-Dimethylhex-4-en-säure (102)

\section{$\mathrm{C}_{8} \mathrm{H}_{14} \mathrm{O}_{2}$}

${ }^{\mathbf{1}} \mathbf{H}$-NMR (Abb. 32) $\left(300 \mathrm{MHz}, \mathrm{CDCl}_{3}, \mathrm{CHCl}_{3}\right.$ int.): $\delta=1.63 \mathrm{ppm}(\mathrm{s}, 6 \mathrm{H}), 1.65$ (s, 3H), $2.36(\mathrm{~s}$, $4 \mathrm{H}), \mathrm{OH}$ nicht detektiert.

${ }^{13}$ C-NMR (Abb. 72) (50 MHz, $\mathrm{CDCl}_{3}, \mathrm{CDCl}_{3}$ int.): $\delta=17.98$ ppm, 20.08, $20.63\left(\mathrm{C}_{\text {prim }}\right), 29.60$, $32.87\left(\mathrm{C}_{\mathrm{sek}}\right), 125.34,125.94,180.14\left(\mathrm{C}_{\text {quart }}\right)$.

MS $(70 \mathrm{eV}): \mathrm{m} / \mathrm{z}(\%)=142\left(\mathrm{M}^{+}, 98\right), 83(100)$ 


\section{2,2-Spiro-cyclobutyl-3,3-spiro-[(1 $\left.R^{*}, 5 R^{*}, 6 R^{*}\right)$-1,4,4-trimethyl-bicyclo[3.2.0]heptan-6,6-} yl]-oxiran (55) und 2,2-Spiro-cyclobutyl-3,3-spiro-[(1 $\left.R^{*}, 5 R^{*}, 6 S^{*}\right)$-1,4,4-trimethylbicyclo[3.2.0]heptan-6,6-yl]-oxiran (56): Eine Lösung des Olefins 54 (1.70 g, 8.9 mmol) in Dichlormethan $(30 \mathrm{ml})$ wurde auf $0^{\circ} \mathrm{C}$ gekühlt und m-Chlorperbenzoesäure $(2.74 \mathrm{~g}, 70$ $75 \%$ ig, $11.3 \mathrm{mmol}$ ) portionsweise hinzugegeben und weitere $15 \mathrm{~min}$ bei dieser Temperatur gerührt bis dünnschichtchromatographische Kontrolle [Laufmittel Pentan/Ether 95:5; $\mathrm{R}_{\mathrm{f}}$ Werte: 0.95 (54), 0.57 (56), 0.43 (55)] vollständigen Umsatz anzeigte. Daraufhin wurde $1 \mathrm{~N}$ Natronlauge $(20 \mathrm{ml})$ hinzugegeben und die Phasen getrennt. Die wässrige Phase wurde anschließend mit Dichlormethan (3 x $30 \mathrm{ml}$ ) extrahiert und die vereinigten organischen Phasen über Magnesiumsulfat getrocknet. Nach dem Entfernen der Lösungsmittel am Rotationsverdampfer $\left(20^{\circ} \mathrm{C} / 14\right.$ Torr $)$ lieferte abschließende Chromatographie an Kieselgel (0.05 - $0.20 \mathrm{~mm}$ ) in Pentan/Ether 95:5 (Säule 3.5 x $50 \mathrm{~cm}$ ) $0.093 \mathrm{~g}(5 \%)$ (56) und $1.501 \mathrm{~g}$ $(82 \%)(\mathbf{5 5})$ als farblose Flüssigkeiten.

\section{2,2-Spiro-cyclobutyl-3,3-spiro-[( $\left.R^{*}, 5 R^{*}, 6 R^{*}\right)-1,4,4-$ trimethyl-bicyclo[3.2.0]heptan-6,6- yl]-oxiran (55)}

\section{$\mathrm{C}_{14} \mathrm{H}_{22} \mathrm{O}$}

Ber. C $81.50 \quad$ H 10.75

Gef. C 81.59 H 11.01

IR (Abb. 7) (KBr): $2951 \mathrm{~cm}^{-1}(\mathrm{CH}), 802$ (C-O-C).

${ }^{1} \mathbf{H}-\mathbf{N M R}$ (Abb. 33) (300 MHz, $\mathrm{CDCl}_{3}, \mathrm{CHCl}_{3}$ int.): $\delta=0.89 \mathrm{ppm}(\mathrm{s}, 3 \mathrm{H}), 1.12$ (s, 3H), 1.35 (s, 3H), $1.46-1.59(\mathrm{~m}, 4 \mathrm{H}), 1.60-1.91(\mathrm{~m}, 3 \mathrm{H}), 1.96(\mathrm{~d}, \mathrm{~J}=6 \mathrm{~Hz}, 1 \mathrm{H}), 2.01-2.13(\mathrm{~m}, 1 \mathrm{H})$, $2.15-2.27(\mathrm{~m}, 2 \mathrm{H}), 2.33-2.54(\mathrm{~m}, 2 \mathrm{H})$.

${ }^{13}$ C-NMR (Abb. 73) (50 MHz, $\mathrm{CDCl}_{3}, \mathrm{CDCl}_{3}$ int.): $\delta=12.94 \mathrm{ppm}\left(\mathrm{C}_{\mathrm{sek}}\right), 26.54,27.16,29.54$ $\left(\mathrm{C}_{\text {prim }}\right), 30.38,31.80,37.71,40.21,41.41\left(\mathrm{C}_{\text {sek }}\right), 41.76,42.65\left(\mathrm{C}_{\text {quart }}\right), 64.82\left(\mathrm{C}_{\text {tert }}\right), 66.20$, $68.56\left(\mathrm{C}_{\text {quart }}\right)$. 
MS $(70 \mathrm{eV}): \mathrm{m} / \mathrm{z}(\%)=206\left(1, \mathrm{M}^{+}\right), 121(100), 109(30)$

2,2-Spiro-cyclobutyl-3,3-spiro-[(1R*,5R*,6 $\left.S^{*}\right)$-1,4,4-trimethyl-bicyclo[3.2.0]heptan-6,6yl]-oxiran (56)

\section{$\mathrm{C}_{14} \mathrm{H}_{22} \mathrm{O}$}

Ber. C $81.50 \quad$ H 10.75

Gef. C $81.48 \quad$ H 10.45

IR (Abb. 8) (KBr): $2943 \mathrm{~cm}^{-1}$ (CH), 806 (C-O-C).

${ }^{\mathbf{1}} \mathbf{H}-\mathbf{N M R}$ (Abb. 34) (300 MHz, $\mathrm{CDCl}_{3}, \mathrm{CHCl}_{3}$ int.): $\delta=0.84 \mathrm{ppm}(\mathrm{s}, 3 \mathrm{H}), 1.00$ (s, 3H), 1.26 (s, 3H), $1.46-1.72(\mathrm{~m}, 5 \mathrm{H}), 1.76-1.90(\mathrm{~m}, 2 \mathrm{H}), 1.92-2.03(\mathrm{~m}, 2 \mathrm{H}), 2.04-2.17(\mathrm{~m}, 2 \mathrm{H})$, $2.24-2.38(\mathrm{~m}, 2 \mathrm{H})$.

${ }^{13}$ C-NMR (Abb. 74) $\left(50 \mathrm{MHz}, \mathrm{CDCl}_{3}, \mathrm{CDCl}_{3}\right.$ int. $): \delta=11.93$ ppm $\left(\mathrm{C}_{\text {sek }}\right), 24.51,26.78\left(\mathrm{C}_{\text {prim }}\right)$, 28.47, $28.73\left(\mathrm{C}_{\text {sek }}\right), 29.43\left(\mathrm{C}_{\text {prim }}\right), 38.92,39.11\left(\mathrm{C}_{\text {sek }}\right), 39.56\left(\mathrm{C}_{\text {quart }}\right), 40.30\left(\mathrm{C}_{\text {sek }}\right), 43.00$ $\left(\mathrm{C}_{\text {quart }}\right), 59.55\left(\mathrm{C}_{\text {tert }}\right), 63.81,65.06\left(\mathrm{C}_{\text {quart }}\right)$.

MS $(70 \mathrm{eV}): \mathrm{m} / \mathrm{z}(\%)=206\left(1, \mathrm{M}^{+}\right), 121(100), 109(90)$

$\left(1 R^{*}, 5 R^{*}, 6 R^{*}\right)-1,4,4-T$ rimethylspiro $\left\{\right.$ bicyclo[3.2.0]heptan-6, $1^{\prime}$-cyclopentan-2'-on $\}$ und 6-Cyclobutyliden-1,4,4-trimethyl-8-oxa-bicyclo[3.2.1]octan (138): Eine Lösung von $55(1.41 \mathrm{~g}, 6.8 \mathrm{mmol})$ in wasserfreiem Dichlormethan $(100 \mathrm{ml})$ wurde auf $0^{\circ} \mathrm{C}$ gekühlt und mit Bortrifluoridetheratlösung $(40 \mu \mathrm{l}, \sim 48 \% \mathrm{ig}, \sim 0.17 \mathrm{mmol})$ versetzt. Nach $15 \mathrm{~min}$ zeigte dünnschichtchromatographische Kontrolle [Laufmittel Pentan/Ether 9:1; $\mathrm{R}_{\mathrm{f}}-$ Werte: 0.63 (138), 0.60 (55), 0.46 (57)] vollständigen Umsatz an. Daraufhin wurde 1 N Natronlauge (50 ml) hinzugegeben und die Phasen getrennt. Die wässrige Phase wurde anschließend mit 
Dichlormethan (3 x $40 \mathrm{ml}$ ) extrahiert und die vereinigten organischen Phasen über Magnesiumsulfat getrocknet. Nach dem Entfernen der Lösungsmittel am Rotationsverdampfer $\left(20^{\circ} \mathrm{C} / 14\right.$ Torr) lieferte abschließende Chromatographie an Kieselgel (0.05 - $0.20 \mathrm{~mm}$ ) in Pentan/Ether 9:1 (Säule 3.5 x $45 \mathrm{~cm}$ ) $719 \mathrm{mg}(51 \%)$ (57) und $110 \mathrm{mg}$ (8\%) (138) als farblose Flüssigkeiten, sowie eine nicht identifizierte Verbindung (490 mg, $35 \%)$.

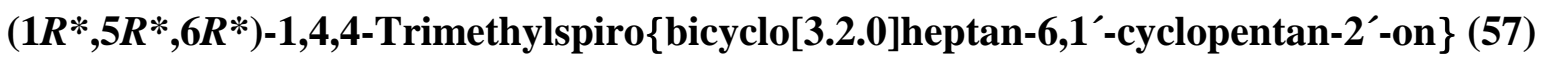

\section{$\mathrm{C}_{14} \mathrm{H}_{22} \mathrm{O}$}

Ber. C $81.50 \quad \mathrm{H} 10.75$

Gef. C $81.30 \quad$ H 10.91

IR (Abb. 9) (KBr): $2941 \mathrm{~cm}^{-1}$ und $2864(\mathrm{CH}), 1732(\mathrm{C}=\mathrm{O})$.

${ }^{1} \mathbf{H}-\mathbf{N M R}$ (Abb. 35) (300 MHz, $\mathrm{CDCl}_{3}, \mathrm{CHCl}_{3}$ int.): $\delta=0.84 \mathrm{ppm}(\mathrm{s}, 3 \mathrm{H}), 1.03$ (s, 3H), 1.23 (s, 3H), 1.38 - $1.70(\mathrm{~m}, 5 \mathrm{H}), 1.72-2.03(\mathrm{~m}, 6 \mathrm{H}), 2.19$ (br s, 1H), $2.29\left(\mathrm{~m}_{\mathrm{c}}, 1 \mathrm{H}\right)$.

${ }^{13}$ C-NMR (Abb. 75) (75 MHz, $\mathrm{CDCl}_{3}, \mathrm{CDCl}_{3}$ int.): $\delta=18.45 \mathrm{ppm}\left(\mathrm{C}_{\text {sek }}\right), 24.99,26.83,30.00$ $\left(\mathrm{C}_{\text {prim }}\right), 34.71,35.24,39.61,40.38\left(\mathrm{C}_{\text {sek }}\right), 40.56\left(\mathrm{C}_{\text {quart }}\right), 42.91\left(\mathrm{C}_{\text {sek }}\right), 43.10,48.81\left(\mathrm{C}_{\text {quart }}\right)$, $55.27\left(\mathrm{C}_{\text {tert }}\right), 221.05\left(\mathrm{C}_{\text {quart }}\right)$.

MS $(70 \mathrm{eV}): \mathrm{m} / \mathrm{z}(\%)=206\left(6, \mathrm{M}^{+}\right), 109(100), 95(70)$.

6-Cyclobutyliden-1,4,4-trimethyl-8-oxa-bicyclo[3.2.1]octan (138)

\section{$\mathrm{C}_{14} \mathrm{H}_{22} \mathrm{O}$}

Ber. C $81.50 \quad \mathrm{H} 10.75$

Gef. C 81.47 H 10.86 
IR (Abb. 10) (KBr): $2927 \mathrm{~cm}^{-1}(\mathrm{CH}), 1032$ und 1000 (C-O-C).

${ }^{1} \mathbf{H}-\mathbf{N M R}$ (Abb. 36) (300 MHz, $\mathrm{CDCl}_{3}, \mathrm{CHCl}_{3}$ int.): $\delta=0.75 \mathrm{ppm}$ (s, 3H), 1.05 (s, 3H), 1.14 $1.34(\mathrm{~m}, 2 \mathrm{H}), 1.30$ (s, 3H), 1.41 (ddd, J = 6, 12, $12 \mathrm{~Hz}, 1 \mathrm{H}), 1.69$ (ddd, J = 6, 12, $12 \mathrm{~Hz}, 1 \mathrm{H})$, 1.87 - 2.03 (m, 2H), $2.08\left(\mathrm{~m}_{\mathrm{c}}, 2 \mathrm{H}\right), 2.43$ - $2.73(\mathrm{~m}, 4 \mathrm{H}), 3.87$ (br s, 1H).

${ }^{13} \mathbf{C}$-NMR (Abb. 76) (50 MHz, $\mathrm{CDCl}_{3}, \mathrm{CDCl}_{3}$ int.): $\delta=17.39 \mathrm{ppm}\left(\mathrm{C}_{\mathrm{sek}}\right), 24.18,26.37,26.86$ $\left(\mathrm{C}_{\text {prim }}\right), 30.30,31.01,31.77,34.00\left(\mathrm{C}_{\text {sek }}\right), 34.66\left(\mathrm{C}_{\text {quart }}\right), 38.55\left(\mathrm{C}_{\text {sek }}\right), 79.90\left(\mathrm{C}_{\text {quart }}\right), 85.35$ $\left(\mathrm{C}_{\text {tert }}\right), 131.03,131.14\left(\mathrm{C}_{\text {quart }}\right)$.

MS $(70 \mathrm{eV}): \mathrm{m} / \mathrm{z}(\%)=206\left(86, \mathrm{M}^{+}\right), 137(100)$

$\left(1 R^{*}, 5 R^{*}, 6 R^{*}, 3 S^{*}\right)-1,4,4,3^{\prime}-T^{\prime}$ Tetramethylspiro\{bicyclo[3.2.0]heptan-6,1'-cyclopentan-2'on \} (141), $\left(1 R^{*}, 5 R^{*}, 6 R^{*}, 3 R^{*}\right)-1,4,4,3^{\prime}$-Tetramethylspiro\{bicyclo[3.2.0]heptan-6,1'cyclopentan-2'-on $\} \quad(142)$ und $\left(1 R^{*}, 5 R^{*}, 6 R^{*}\right)-1,4,4,3^{\prime}, 3^{\prime}$-Pentamethylspiro\{bicyclo[3.2.0]heptan-6,1'-cyclopentan-2'-on\} (143): Zu einer Lösung von Diisopropylamin (505 $\mathrm{mg}, 705 \mu \mathrm{l}, 5 \mathrm{mmol})$ in wasserfreiem Tetrahydrofuran $(10 \mathrm{ml})$ wurden bei $5-10^{\circ} \mathrm{C}$ nacheinander n-Butyllithium (3.13 ml einer 1.6 M Lösung in n-Hexan, $5 \mathrm{mmol}, 5$ - $10 \mathrm{~min}$ Zugabedauer) und 57 (1.03 g, $5 \mathrm{mmol})$ getropft und anschließend $1 \mathrm{~h}$ bei Raumtemperatur gerührt. Daraufhin wurde auf $-78^{\circ} \mathrm{C}$ gekühlt und schnell Iodmethan $(3.55 \mathrm{~g}, 25 \mathrm{mmol}, 1.56$ ml) hinzugegeben. Nachdem gaschromatographische Kontrolle keine weitere Veränderung des Reaktionsgemisches nach $2 \mathrm{~h}$ anzeigte [3 $\mathrm{m} \times 1 / 4 "$ Allglassystem, 15\% FFAP auf Chromosorb W AW/DMCS 60/80 mesh, $180^{\circ} \mathrm{C}$, Retentionszeiten (min): 6.21 (143), 7.84 (141, 142), 8.73 (57)], ließ man die Reaktionsmischung auf Raumtemperatur kommen und hydrolysierte mit gesättigter Ammoniumchloridlösung (10 ml). Anschließend trennte man die Phasen, extrahierte die wässrige Phase mit Pentan (3 x $7 \mathrm{ml}$ ), wusch die vereinigten organischen Phasen mit gesättigter Natriumchloridlösung, trocknete über Magnesiumsulfat und entfernte die Lösungsmittel am Rotationsverdampfer $\left(20^{\circ} \mathrm{C} / 14\right.$ Torr $)$. Der so erhaltene Rückstand wurde an Kieselgel (0.05 - $0.20 \mathrm{~mm})$ in Pentan/Ether 98:2 [Säule 3.5 x 60 cm, DCKontrolle, $\mathrm{R}_{\mathrm{f}}$ - Werte: $\left.0.11(\mathbf{5 7}), 0.17(\mathbf{1 4 3}), 0.22(\mathbf{1 4 1}, \mathbf{1 4 2})\right]$ chromatographiert. Man erhielt 
so $875 \mathrm{mg}(79 \%)(\mathbf{1 4 1}, \mathbf{1 4 2})$ als ein laut kapillargaschromatographischer Analyse [30 m x $0.32 \mathrm{~mm}, 0.25 \mu \mathrm{m}$ DB-FFAP, Temperaturprogramm: $100^{\circ} \mathrm{C}, 10^{\circ} \mathrm{C} / \mathrm{min}$ auf $220^{\circ} \mathrm{C}$, Retentionszeiten (min): 4.72 (46\%), 4.83 (54\%), ] 54:46 Gemisch der beiden Stereoisomeren. Außerdem wurden $35 \mathrm{mg}$ (3\%) (143), sowie $137 \mathrm{mg}$ (13\%) (57) als farblose Flüssigkeiten isoliert.

\section{1 und 142}

\section{$\mathrm{C}_{15} \mathrm{H}_{24} \mathrm{O}$}

Ber. C 81.76 H 10.98

Gef. C 81.84 H 10.62

IR (Abb. 11) (KBr): 2949 und $2866 \mathrm{~cm}^{-1}(\mathrm{CH}), 1730(\mathrm{C}=\mathrm{O})$.

${ }^{1} \mathbf{H}-\mathbf{N M R}$ (Abb. 37) $\left(300 \mathrm{MHz}, \mathrm{CDCl}_{3}, \mathrm{CHCl}_{3}\right.$ int.): $\delta=0.82 \mathrm{ppm}$ (s, 1.4H), 0.83 (s, 1.6H), $1.03(\mathrm{~d}, \mathrm{~J}=7.5 \mathrm{~Hz}, 1.4 \mathrm{H}), 1.04$ (s, 1.6H), 1.06 (s, 1.4H), 1.13 (d, $7 \mathrm{~Hz}, 1.6 \mathrm{H}), 1.23$ (s, 1.6H), $1.24(\mathrm{~s}, 1.4 \mathrm{H}), 1.38$ - $1.63(\mathrm{~m}, 4 \mathrm{H}), 1.67-2.07$ (m, 6H), 2.09 (br s, 0.4H), 2.12 - $2.33(\mathrm{~m}, 1 \mathrm{H})$, 2.20 (br s, 0.6H).

${ }^{13}$ C-NMR (Abb. 77) $\left(50 \mathrm{MHz}, \mathrm{CDCl}_{3}, \mathrm{CDCl}_{3}\right.$ int.): $\delta=16.14 \mathrm{ppm}, 16.45,24.58,25.15$, 26.78, $26.87\left(\mathrm{C}_{\text {prim }}\right), 26.95,27.75\left(\mathrm{C}_{\text {sek }}\right), 29.97,30.06\left(\mathrm{C}_{\text {prim }}\right), 31.86,33.40\left(\mathrm{C}_{\text {sek }}\right), 39.44\left(\mathrm{C}_{\text {tert }}\right)$, 39.60, 39.68, 40.21, $40.50\left(\mathrm{C}_{\text {sek }}\right), 40.59,40.65\left(\mathrm{C}_{\text {quart }}\right), 42.50\left(\mathrm{C}_{\text {tert }}\right), 42.85\left(\mathrm{C}_{\text {sek }}\right), 43.19$ (Koinzidenz zweier Signale, $\left.\left(\mathrm{C}_{\text {quart }}\right)\right), 43.79\left(\mathrm{C}_{\text {sek }}\right), 48.65,48.87\left(\mathrm{C}_{\text {quart }}\right), 55.70,56.45\left(\mathrm{C}_{\text {tert }}\right)$, $222.98,223.61\left(\mathrm{C}_{\text {quart }}\right)$.

MS $(70 \mathrm{eV}): \mathrm{m} / \mathrm{z}(\%)=220\left(8, \mathrm{M}^{+}\right), 109(100)$ 
$\left(1 R^{*}, 5 R^{*}, 6 R^{*}\right)-1,4,4,3^{\prime}, 3^{\prime}$-Pentamethylspiro $\{$ bicyclo[3.2.0]heptan-6,1'-cyclopentan-2'on\} (143)

\section{$\mathrm{C}_{16} \mathrm{H}_{26} \mathrm{O}$}

Ber. C 81.99 H 11.18

Gef. C 82.06 H 10.90

IR (Abb. 12) (KBr): 2942 und $2865 \mathrm{~cm}^{-1}(\mathrm{CH}), 1730(\mathrm{C}=\mathrm{O})$.

${ }^{1} \mathbf{H}-\mathbf{N M R}$ (Abb. 38) (300 MHz, $\mathrm{CDCl}_{3}, \mathrm{CHCl}_{3}$ int.): $\delta=0.83$ (s, 3H), 0.93 (s, 3H), 1.06 (s, 3H), 1.09 (s, 3H), $1.23(\mathrm{~s}, 3 \mathrm{H}), 1.39-1.62(\mathrm{~m}, 4 \mathrm{H}), 1.66\left(\mathrm{~m}_{\mathrm{c}}, 2 \mathrm{H}\right), 1.74-1.92(\mathrm{~m}, 3 \mathrm{H}), 2.08$ (ddd, J = 12, 12, $6 \mathrm{~Hz}, 1 \mathrm{H}), 2.18$ (br s, 1H).

${ }^{13}$ C-NMR (Abb. 78) (75 MHz, $\mathrm{CDCl}_{3}, \mathrm{CDCl}_{3}$ int.): $\delta=24.49$ ppm, 24.97, 26.56, 26.90, 30.01 $\left(\mathrm{C}_{\text {prim }}\right), 31.44,34.51,39.71,40.51\left(\mathrm{C}_{\text {sek }}\right), 40.71,43.26,43.51\left(\mathrm{C}_{\text {quart }}\right), 44.07\left(\mathrm{C}_{\text {sek }}\right), 48.99$ $\left(\mathrm{C}_{\text {quart }}\right), 56.15\left(\mathrm{C}_{\text {tert }}\right), 224.83\left(\mathrm{C}_{\text {quart }}\right)$.

MS $(70 \mathrm{eV}): \mathrm{m} / \mathrm{z}(\%)=234\left(7, \mathrm{M}^{+}\right), 109(100)$.

$\left(1 R^{*}, 5 R^{*}, 6 R^{*}, 2^{\prime} R^{*}, 3^{\prime} R^{*}\right)-1,4,4,3^{\prime}-$ Tetramethylspiro\{bicyclo[3.2.0]heptan-6,1'-cyclopentan-2'-ol\} $(60), \quad\left(1 R^{*}, 5 R^{*}, 6 R^{*}, 2^{\prime} S^{*}, 3^{\prime} R^{*}\right)-1,4,4,3^{\prime}$-Tetramethylspiro\{bicyclo[3.2.0]heptan-6,1'-cyclopentan-2'-ol $\}(64)$ und $\left(1 R^{*}, 5 R^{*}, 6 R^{*}, 2^{\prime} R^{*}, 3^{\prime} S^{*}\right)-1,4,4,3^{\prime}$-Tetramethylspiro\{bicyclo[3.2.0]heptan-6,1'-cyclopentan-2'-ol\} (66): $\mathrm{Zu}$ einer Suspension von Lithiumaluminiumhydrid $(258 \mathrm{mg}, 6.8 \mathrm{mmol})$ in wasserfreiem Ether $(14 \mathrm{ml})$ gab man ein 54:46 Gemisch von 141:142 (750 mg, $3.4 \mathrm{mmol})$ so zu, dass die Reaktionsmischung am Sieden gehalten wurde. Nach beendeter Zugabe erhitzte man 40 min unter Rückfluss [DCKontrolle, Pentan/Ether 9:1, $\mathrm{R}_{\mathrm{f}}$ - Werte: 0.76 (141, 142), 0.25 (66), 0.20 (60), $\left.0.16(\mathbf{6 4})\right]$ bis der Umsatz vollständig war, kühlte auf $0^{\circ} \mathrm{C}$ und hydrolysierte vorsichtig nacheinander mit Wasser $(244 \mu \mathrm{l})$, Natronlauge (15\%ig, $244 \mu \mathrm{l})$ und Wasser $(733 \mu \mathrm{l})$. Der ausgefallene Feststoff wurde abfiltriert und der Rückstand mit Ether $(3 \times 5 \mathrm{ml})$ gewaschen. Die vereinigten 
organischen Phasen über Magnesiumsulfat getrocknet und die Lösungsmittel am Rotationsverdampfer $\left(20^{\circ} \mathrm{C} / 14\right.$ Torr $)$ entfernt. Das so erhaltene Rohprodukt wurde an Kieselgel (0.04 - $0.063 \mathrm{~mm})$ in Pentan/Ether 9:1 chromatographiert (Säule 3.5 x $60 \mathrm{~cm}$ ). Man erhielt so $369 \mathrm{mg}(49 \%) 66$ als farblose Kristalle (Schmp.: $78-80^{\circ} \mathrm{C}$ ), $24 \mathrm{mg}$ (3\%) einer Mischfraktion aus 66 und 60 (47:53), 217 mg (29\%) 60 als farblose Kristalle (Schmp.: 94 $\left.95^{\circ} \mathrm{C}\right)$, sowie $75 \mathrm{mg}(10 \%) 64$ als farbloses Öl.

$\left(1 R^{*}, 5 R^{*}, 6 R^{*}, 2^{\prime} R^{*}, 3^{\prime} R^{*}\right)-1,4,4,3^{\prime}-T^{\prime}$ tramethylspiro\{bicyclo[3.2.0]heptan-6,1'-cyclopentan-2'-ol\} (60)

\section{$\mathrm{C}_{15} \mathrm{H}_{26} \mathrm{O}$}

Ber. C 81.02 H 11.78

Gef. C 80.62 H 11.66

IR (Abb. 13) (KBr): $3635-3020 \mathrm{~cm}^{-1}\left(\mathrm{OH}_{\mathrm{ass}}\right), 2939$ und $2866(\mathrm{CH})$.

${ }^{\mathbf{1}} \mathbf{H}$-NMR (Abb. 39) (300 MHz, $\mathrm{CDCl}_{3}, \mathrm{CHCl}_{3}$ int.): $\delta=0.78$ (s, 3H), 1.00 (d, J = $7 \mathrm{~Hz}, 3 \mathrm{H}$ ), 1.07 (s, 3H), 1.22 (s, 3H), 1.26 (br s, 1H), 1.38 - 1.56 (m, 6H), 1.64 - 1.77 (m, 2H), 1.84 - 2.11 $(\mathrm{m}, 4 \mathrm{H}), 3.50(\mathrm{~d}, \mathrm{~J}=4.1 \mathrm{~Hz}, 1 \mathrm{H})$.

${ }^{13}$ C-NMR (Abb. 79) (75 MHz, $\mathrm{CDCl}_{3}, \mathrm{CDCl}_{3}$ int.): $\delta=14.98$ ppm, 22.91, $28.84\left(\mathrm{C}_{\text {prim }}\right), 29.47$ $\left(\mathrm{C}_{\text {sek }}\right), 30.66\left(\mathrm{C}_{\text {prim }}\right), 31.02\left(\mathrm{C}_{\text {sek }}\right), 35.07\left(\mathrm{C}_{\text {tert }}\right), 37.74,39.57,39.73\left(\mathrm{C}_{\text {sek }}\right), 40.19,42.96,47.63$ $\left(\mathrm{C}_{\text {quart }}\right), 62.56,84.80\left(\mathrm{C}_{\text {tert }}\right)$.

MS $(70 \mathrm{eV}): \mathrm{m} / \mathrm{z}(\%)=222\left(3, \mathrm{M}^{+}\right), 95(100)$ 
$\left(1 R^{*}, 5 R^{*}, 6 R^{*}, 2^{\prime} S^{*}, 3^{\prime} R^{*}\right)-1,4,4,3^{\prime}-$ Tetramethylspiro\{bicyclo[3.2.0]heptan-6,1'-cyclopentan-2'-ol\} (64)

\section{$\mathrm{C}_{15} \mathrm{H}_{26} \mathrm{O}$}

Ber. C 81.02 H 11.78

Gef. C 80.65 H 11.60

IR (Abb. 14) (KBr): $3599 \mathrm{~cm}^{-1}(\mathrm{OH}), 3590$ - $3020\left(\mathrm{OH}_{\text {ass. }}\right), 2943$ und $2864(\mathrm{CH})$.

${ }^{1} \mathbf{H}-\mathbf{N M R}$ (Abb. 40) (300 MHz, $\mathrm{CDCl}_{3}, \mathrm{CHCl}_{3}$ int.): $\delta=0.83 \mathrm{ppm}(\mathrm{s}, 3 \mathrm{H}), 0.87-1.02(\mathrm{~m}, 2 \mathrm{H})$, $1.05(\mathrm{~s}, 3 \mathrm{H}), 1.07$ (d, J = 7.5 Hz, 3H), 1.23 (s, 3H), 1.40 - $1.50(\mathrm{~m}, 3 \mathrm{H}), 1.51$ - 1.61 (m, 3H), $1.72-1.94(\mathrm{~m}, 5 \mathrm{H}), 3.43(\mathrm{~d}, \mathrm{~J}=1.5 \mathrm{~Hz}, 1 \mathrm{H})$.

${ }^{13}$ C-NMR (Abb. 80) (50 MHz, $\mathrm{CDCl}_{3}, \mathrm{CDCl}_{3}$ int.): $\delta=21.09$ ppm, 24.80, 29.02, 30.29 $\left(\mathrm{C}_{\text {prim }}\right), 30.48,33.44,39.60,40.30\left(\mathrm{C}_{\text {sek }}\right), 40.52\left(\mathrm{C}_{\text {quart }}\right), 41.19\left(\mathrm{C}_{\text {tert }}\right), 43.27\left(\mathrm{C}_{\text {quart }}\right), 43.52$ $\left(\mathrm{C}_{\text {sek }}\right), 47.23\left(\mathrm{C}_{\text {quart }}\right), 57.79,88.23\left(\mathrm{C}_{\text {tert }}\right)$.

MS $(70 \mathrm{eV}): \mathrm{m} / \mathrm{z}(\%)=222\left(2, \mathrm{M}^{+}\right), 95(100)$

$\left(1 R^{*}, 5 R^{*}, 6 R^{*}, 2^{\prime} R^{*}, 3^{\prime} S^{*}\right)-1,4,4,3^{\prime}-$ Tetramethyl-spiro $\{$ bicyclo[3.2.0]heptan-6,1'-cyclopentan-2'-ol\} (66)

\section{$\mathrm{C}_{15} \mathrm{H}_{26} \mathrm{O}$}

Ber. C 81.02 H 11.78

Gef. C 80.86 H 11.77

IR (Abb. 15) (KBr): 3630 - $3020 \mathrm{~cm}^{-1}\left(\mathrm{OH}_{\mathrm{ass}}\right), 2930(\mathrm{CH})$. 
${ }^{\mathbf{1}} \mathbf{H}$-NMR (Abb. 41) (300 MHz, $\mathrm{CDCl}_{3}, \mathrm{CHCl}_{3}$ int.): $\delta=0.80 \mathrm{ppm}(\mathrm{s}, 3 \mathrm{H}), 0.96-1.12$ (m, 2H), 1.03 (d, J = 6 Hz, 3H), 1.05 (s, 3H), 1.21 (s, 3H), 1.30 (dd, J = 1, $12 \mathrm{~Hz}, 1 \mathrm{H}), 1.40$ - 1.63 (m, 7H), 1.65 - 1.94 (m, 2H), 1.73 (br s, 1H), 3.17 (d, J = 7.9 Hz, 1H).

${ }^{13}$ C-NMR (Abb. 81) $\left(75 \mathrm{MHz}, \mathrm{CDCl}_{3}, \mathrm{CDCl}_{3}\right.$ int. $): \delta=19.25$ ppm, 24.91, $27.20\left(\mathrm{C}_{\text {prim }}\right), 27.56$ $\left(\mathrm{C}_{\text {sek }}\right), 30.07\left(\mathrm{C}_{\text {prim }}\right), 30.81\left(\mathrm{C}_{\text {sek }}\right), 36.21\left(\mathrm{C}_{\text {tert }}\right), 38.72,39.90\left(\mathrm{C}_{\text {sek }}\right), 40.11\left(\mathrm{C}_{\text {quart }}\right), 40.46\left(\mathrm{C}_{\text {sek }}\right)$, $43.47,46.03\left(\mathrm{C}_{\text {quart }}\right), 59.15,86.96\left(\mathrm{C}_{\text {tert }}\right)$.

MS $(70 \mathrm{eV}): \mathrm{m} / \mathrm{z}(\%)=222\left(3, \mathrm{M}^{+}\right), 95(100)$

Allgemeine Darstellung der Bicyclobutyl-1-ole 104b, 105, 106a,b, 107a,b und der 1,4,4Trimethyl-6-(2’-methyl-cyclobutyl)-bicyclo[3.2.0]heptan-6-ole 46, 47, 48 und 49: Eine Suspension von fein gemahlenem trockenen Certrichlorid (4.93 g, $20 \mathrm{mmol})$ in abs. Tetrahydrofuran $(85 \mathrm{ml})$ wurde unter Argonatmosphäre über Nacht gerührt. Nach Zugabe des entsprechenden Ketons (10 mmol) wurde weitere $2 \mathrm{~h}$ gerührt, dann auf $-78^{\circ} \mathrm{C}$ gekühlt und das entsprechende Grignardreagenz $(15 \mathrm{mmol})$ hinzugegeben. Nach $15 \mathrm{~min}$ bei $-78^{\circ} \mathrm{C}$ und $4 \mathrm{~h}$ bei Raumtemperatur wurde mit $2 \mathrm{~N} \mathrm{HCl}(50 \mathrm{ml})$ hydrolysiert. Die wässrige Phase wurde dann mit Ether (4 x $40 \mathrm{ml}$ ) extrahiert. Anschließend wurden die vereinigten organischen Phasen mit gesättigter Natriumhydrogencarbonatlösung $(60 \mathrm{ml})$ und gesättigter Natriumchloridlösung (60 ml) gewaschen. Nach dem Trocknen über Magnesiumsulfat wurden die Lösungsmittel am Rotationsverdampfer entfernt (Badtemperatur $20^{\circ} \mathrm{C} / 14$ Torr). In allen Fällen lieferte abschließende Chromatographie an Kieselgel (0.05 - 0.20 mm bzw. 0.04 - 0.063 mm (46-49) in Pentan/Ether 3:1 (104b), 5:1 (105, 106a,b, 107a,b) oder 99:1 (46-49) die reinen Alkohole als klare Flüssigkeiten.

$\left(1 R^{*}, 2 S^{*}\right)-2,1^{\prime}$-Dimethyl-bicyclobutyl-1-ol (104b): $841 \mathrm{mg}(59 \%), \mathrm{R}_{\mathrm{f}}=0.24$.

\section{$\mathrm{C}_{10} \mathrm{H}_{18} \mathrm{O}$}

Ber. C $77.87 \quad \mathrm{H} 11.76$

Gef. C $78.15 \quad$ H 11.81 
IR (Abb. 16) (KBr): $3595 \mathrm{~cm}^{-1}(\mathrm{OH}), 3570$ - $3100\left(\mathrm{OH}_{\text {ass. }}\right)$.

${ }^{\mathbf{1}} \mathbf{H}$-NMR (Abb. 42) (300 MHz, $\mathrm{CDCl}_{3}, \mathrm{CHCl}_{3}$ int.): $\delta=1.00 \mathrm{ppm}(\mathrm{d}, \mathrm{J}=7 \mathrm{~Hz}, 3 \mathrm{H}), 1.07$ (s, $3 \mathrm{H}), 1.42$ (br s, 1H, OH), $1.45-1.57(\mathrm{~m}, 3 \mathrm{H}), 1.60-2.20(\mathrm{~m}, 7 \mathrm{H}), 2.40\left(\mathrm{~m}_{\mathrm{c}}, 1 \mathrm{H}\right)$.

${ }^{13}$ C-NMR (Abb. 82) $\left(75 \mathrm{MHz}, \mathrm{CDCl}_{3}, \mathrm{CDCl}_{3}\right.$ int.): $\delta=14.09$ ppm $\left(\mathrm{C}_{\text {sek }}\right), 15.37,21.36\left(\mathrm{C}_{\text {prim }}\right)$, $22.40,28.27,28.43,28.47\left(\mathrm{C}_{\text {sek }}\right), 33.57\left(\mathrm{C}_{\text {tert }}\right), 43.76,80.99\left(\mathrm{C}_{\text {quart }}\right)$.

MS $(70 \mathrm{eV}): \mathrm{m} / \mathrm{z}(\%)=154\left(\mathrm{M}^{+}, 60\right), 137(100)$.

2,2,1'-Trimethyl-bicyclobutyl-1-ol (105): $460 \mathrm{mg}(27 \%), \mathrm{R}_{\mathrm{f}}=0.32$.

\section{$\mathrm{C}_{11} \mathrm{H}_{20} \mathrm{O}$}

Ber. C 78.51 H 11.98

Gef. C 78.77 H 12.01

IR (Abb. 17) (KBr): $3610 \mathrm{~cm}^{-1}(\mathrm{OH}), 3580-3250\left(\mathrm{OH}_{\text {ass. }}\right)$.

${ }^{\mathbf{1}} \mathbf{H}$-NMR (Abb. 43) $\left(300 \mathrm{MHz}, \mathrm{CDCl}_{3}, \mathrm{CHCl}_{3}\right.$ int.): $\delta=0.98$ ppm (s, 3H), 1.02 (s, 3H), 1.19 (s, $3 \mathrm{H}), 1.40(\mathrm{~s}, 1 \mathrm{H}, \mathrm{OH}), 1.30-1.48(\mathrm{~m}, 2 \mathrm{H}), 1.53-2.04(\mathrm{~m}, 6 \mathrm{H}), 2.30\left(\mathrm{~m}_{\mathrm{c}}, 1 \mathrm{H}\right), 2.40\left(\mathrm{~m}_{\mathrm{c}}, 1 \mathrm{H}\right)$.

${ }^{13}$ C-NMR (Abb. 83) (50 MHz, $\mathrm{CDCl}_{3}, \mathrm{CDCl}_{3}$ int.): $\delta=15.32 \mathrm{ppm}\left(\mathrm{C}_{\mathrm{sek}}\right), 20.82,24.47,24.99$ $\left(\mathrm{C}_{\text {prim }}\right), 27.37,29.35,30.00,31.52\left(\mathrm{C}_{\text {sek }}\right), 43.00,44.24,82.96\left(\mathrm{C}_{\text {quart }}\right)$.

MS $(70 \mathrm{eV}): \mathrm{m} / \mathrm{z}(\%)=168\left(\mathrm{M}^{+}, 16\right), 151(100)$

$\left(1^{\prime} R^{*}, 2^{\prime} S^{*}\right)-2^{\prime}$-Methyl-bicyclobutyl-1-ol (106a) und $\left(1^{\prime} R^{*}, 2^{\prime} R^{*}\right)-2^{\prime}$-Methyl-bicyclobutyl-1-ol (106b): $237 \mathrm{mg}(34 \%)$ (106a), $297 \mathrm{mg}$ (42\%) (106b), $\mathrm{R}_{\mathrm{f}}=0.23$ (106a), 0.14 (106b). 
$\left(1^{\prime} R^{*}, 2^{\prime} S^{*}\right)-2^{\prime}-$ Methyl-bicyclobutyl-1-ol (106a)

\section{$\mathrm{C}_{9} \mathrm{H}_{16} \mathrm{O}$}

Ber. C 77.09 H 11.50

Gef. C 77.05 H 11.29

IR (Abb. 18) (KBr): $3593 \mathrm{~cm}^{-1}(\mathrm{OH}), 3595-3020\left(\mathrm{OH}_{\text {ass. }}\right)$.

${ }^{\mathbf{1}} \mathbf{H}-\mathbf{N M R}$ (Abb. 44) (300 MHz, $\mathrm{CDCl}_{3}, \mathrm{CHCl}_{3}$ int.): $\delta=1.07 \mathrm{ppm}(\mathrm{d}, \mathrm{J}=7 \mathrm{~Hz}, 3 \mathrm{H}), 1.40$ - 1.60 (m, 2H), 1.72 (br s, 1H, OH), 1.70 - $1.85(\mathrm{~m}, 1 \mathrm{H}), 1.85$ - $2.06(\mathrm{~m}, 6 \mathrm{H}), 2.06$ - $2.18(\mathrm{~m}, 1 \mathrm{H}), 2.46$ $-2.52(\mathrm{~m}, 2 \mathrm{H})$.

${ }^{13}$ C-NMR (Abb. 84) (50 MHz, $\mathrm{CDCl}_{3}, \mathrm{CDCl}_{3}$ int.): $\delta=12.79 \mathrm{ppm}\left(\mathrm{C}_{\text {sek }}\right), 16.74\left(\mathrm{C}_{\text {prim }}\right), 19.84$, $26.22\left(\mathrm{C}_{\text {sek }}\right), 32.07\left(\mathrm{C}_{\text {tert }}\right), 35.25,35.46\left(\mathrm{C}_{\text {sek }}\right), 43.97\left(\mathrm{C}_{\text {tert }}\right)$; Koinzidenz eines Signal mit $\mathrm{CDCl}_{3}$.

${ }^{13}$ C-NMR (50 MHz, $\mathrm{C}_{6} \mathrm{D}_{6}, \mathrm{C}_{6} \mathrm{D}_{6}$ int.): $\delta=12.85 \mathrm{ppm}\left(\mathrm{C}_{\mathrm{sek}}\right), 17.00\left(\mathrm{C}_{\text {prim }}\right), 20.04,26.80\left(\mathrm{C}_{\text {sek }}\right)$, $32.63\left(\mathrm{C}_{\text {tert }}\right), 36.03,36.41\left(\mathrm{C}_{\text {sek }}\right), 44.20\left(\mathrm{C}_{\text {tert }}\right), 76.37\left(\mathrm{C}_{\text {quart }}\right)$.

MS $(70 \mathrm{eV}): \mathrm{m} / \mathrm{z}(\%)=140\left(93,\left[\mathrm{M}+\mathrm{NH}_{4}-\mathrm{H}_{2} \mathrm{O}\right]^{+}\right), 123(100)$.

$\left(1^{\prime} R^{*}, 2^{\prime} R^{*}\right)-2^{\prime}$-Methyl-bicyclobutyl-1-ol (106b)

\section{$\mathrm{C}_{9} \mathrm{H}_{16} \mathrm{O}$}

Ber. C 77.09 H 11.50

Gef. C 77.15 H 11.33

IR (Abb. 19) (KBr): $3595 \mathrm{~cm}^{-1}(\mathrm{OH}), 3595-3020\left(\mathrm{OH}_{\text {ass. }}\right)$. 
${ }^{1} \mathbf{H}-\mathbf{N M R}$ (Abb. 45) (300 MHz, $\mathrm{CDCl}_{3}, \mathrm{CHCl}_{3}$ int.): $\delta=1.07 \mathrm{ppm}(\mathrm{d}, \mathrm{J}=7 \mathrm{~Hz}, 3 \mathrm{H}), 1.30$ $1.55(\mathrm{~m}, 2 \mathrm{H}), 1.60(\mathrm{~s}, 1 \mathrm{H}, \mathrm{OH}), 1.55-2.16(\mathrm{~m}, 9 \mathrm{H}), 2.16-2.30(\mathrm{~m}, 1 \mathrm{H})$.

${ }^{13}$ C-NMR (Abb. 85) (50 MHz, $\mathrm{CDCl}_{3}, \mathrm{CDCl}_{3}$ int.): $\delta=11.96$ ppm, $18.69\left(\mathrm{C}_{\mathrm{sek}}\right), 22.06\left(\mathrm{C}_{\text {prim }}\right)$, $25.74\left(\mathrm{C}_{\text {sek }}\right), 31.47\left(\mathrm{C}_{\text {tert }}\right), 33.82,34.08\left(\mathrm{C}_{\text {sek }}\right), 50.72\left(\mathrm{C}_{\text {tert }}\right), 75.87\left(\mathrm{C}_{\text {quart }}\right)$.

MS $(70 \mathrm{eV}): \mathrm{m} / \mathrm{z}(\%)=140\left(100,\left[\mathrm{M}+\mathrm{NH}_{4}-\mathrm{H}_{2} \mathrm{O}\right]^{+}\right)$

$\left(1^{\prime} R^{*}, 2^{\prime} S^{*}\right)-1^{\prime}, 2^{\prime}$-Dimethyl-bicyclobutyl-1-ol (107a) und $\left(1^{\prime} R^{*}, 2^{\prime} R^{*}\right)-1^{\prime}, 2^{\prime}$-Dimethylbicyclobutyl-1-ol (107b): $50 \mathrm{mg}(3 \%)$ (107a), $90 \mathrm{mg}(6 \%)(\mathbf{1 0 7 b}), \mathrm{R}_{\mathrm{f}}=0.29$ (107a), 0.22 (107b).

$\left(1^{\prime} R^{*}, 2^{\prime} S^{*}\right)-1^{\prime}, 2^{\prime}$-Dimethyl-bicyclobutyl-1-ol (107a)

\section{$\mathrm{C}_{10} \mathrm{H}_{18} \mathrm{O}$}

Ber. 154.1358

Gef. 154.1357 HRMS

IR (Abb. 20) (KBr): $3599 \mathrm{~cm}^{-1}(\mathrm{OH}), 3590$ - $3020\left(\mathrm{OH}_{\mathrm{ass}}\right), 2956(\mathrm{CH})$.

${ }^{1} \mathbf{H}-\mathbf{N M R}$ (Abb. 46) (300 MHz, $\mathrm{CDCl}_{3}, \mathrm{CHCl}_{3}$ int.): $\delta=1.03 \mathrm{ppm}(\mathrm{s}, 3 \mathrm{H}), 1.04$ (d, J = $7 \mathrm{~Hz}$, $3 \mathrm{H}), 1.30-1.45(\mathrm{~m}, 1 \mathrm{H}), 1.50-1.85(\mathrm{~m}, 5 \mathrm{H}), 1.90-2.20(\mathrm{~m}, 5 \mathrm{H}), 2.55-2.67(\mathrm{~m}, 1 \mathrm{H})$.

${ }^{13}$ C-NMR (Abb. 86) $\left(75 \mathrm{MHz}, \mathrm{CDCl}_{3}, \mathrm{CDCl}_{3}\right.$ int.): $\delta=14.78 \mathrm{ppm}\left(\mathrm{C}_{\text {sek }}\right), 17.77,23.26\left(\mathrm{C}_{\text {prim }}\right)$, $23.62,27.92,32.29,33.52\left(\mathrm{C}_{\mathrm{sek}}\right), 39.35\left(\mathrm{C}_{\text {tert }}\right), 45.30,81.58\left(\mathrm{C}_{\text {quart }}\right)$.

MS (70 eV): m/z (\%) = $154\left(2, \mathrm{M}^{+}\right), 84(100)$.

$\left(1^{\prime} R^{*}, 2^{\prime} R^{*}\right)-1^{\prime}, 2^{\prime}$-Dimethyl-bicyclobutyl-1-ol (107b)

$\mathrm{C}_{10} \mathrm{H}_{18} \mathrm{O}$ 
Ber. 154.1358

Gef. 154.1357 HRMS

IR (Abb. 21) (KBr): $3598 \mathrm{~cm}^{-1}(\mathrm{OH}), 3590$ - $3020\left(\mathrm{OH}_{\mathrm{ass}}\right), 2961(\mathrm{CH})$.

${ }^{1} \mathbf{H}-\mathbf{N M R}$ (Abb. 47) $\left(300 \mathrm{MHz}, \mathrm{CDCl}_{3}, \mathrm{CHCl}_{3}\right.$ int.): $\delta=0.92(\mathrm{~d}, \mathrm{~J}=7 \mathrm{~Hz}, 3 \mathrm{H}), 1.00(\mathrm{~s}, 3 \mathrm{H})$, $1.30-1.56(\mathrm{~m}, 3 \mathrm{H}), 1.46(\mathrm{~s}, 1 \mathrm{H}, \mathrm{OH}), 1.76-1.94(\mathrm{~m}, 5 \mathrm{H}), 2.16-2.42(\mathrm{~m}, 3 \mathrm{H})$.

${ }^{13}$ C-NMR (Abb. 87) $\left(75 \mathrm{MHz}, \mathrm{CDCl}_{3}, \mathrm{CDCl}_{3}\right.$ int.): $\delta=12.37 \mathrm{ppm}\left(\mathrm{C}_{\mathrm{sek}}\right), 14.01,16.67\left(\mathrm{C}_{\text {prim }}\right)$, $23.64,26.28,31.56,31.58\left(\mathrm{C}_{\text {sek }}\right), 31.76\left(\mathrm{C}_{\text {tert }}\right), 45.14,80.85\left(\mathrm{C}_{\text {quart }}\right)$.

MS $(70 \mathrm{eV}): \mathrm{m} / \mathrm{z}(\%)=154\left(1, \mathrm{M}^{+}\right), 84(100)$

$\left(1 S^{*}, 5 S^{*}, 6 S^{*}, 1^{\prime} S^{*}, 2^{\prime} S^{*}\right)-1,4,4-T r i m e t h y l-6-\left(2^{\prime}-\right.$ methyl-cyclobutyl)-bicyclo[3.2.0]heptan-6ol (46), $\left(1 S^{*}, 5 S^{*}, 6 S^{*}, 1^{\prime} S^{*}, 2^{\prime} R^{*}\right)-1,4,4$-Trimethyl-6-(2'-methyl-cyclobutyl)-bicyclo[3.2.0]heptan-6-ol $(47),\left(1 S^{*}, 5 S^{*}, 6 S^{*}, 1^{\prime} R^{*}, 2^{\prime} S^{*}\right)-1,4,4-T r i m e t h y l-6-\left(2^{\prime}\right.$-methyl-cyclobutyl)-bicyclo[3.2.0]heptan-6-ol (48) und $\left(1 S^{*}, 5 S^{*}, 6 S^{*}, 1^{\prime} R^{*}, 2^{\prime} R^{*}\right)-1,4,4-T r i m e t h y l-6-\left(2^{\prime}\right.$-methylcyclobutyl)-bicyclo[3.2.0]heptan-6-ol (49): $165 \mathrm{mg}$ (11\%) (47), $246 \mathrm{mg}$ (16\%) (48) und 560 $\operatorname{mg}(36 \%)$ eines Gemisches aus 46/49; $\mathrm{R}_{\mathrm{f}}=0.33(\mathbf{4 7}), 0.28(\mathbf{4 8}), 0.22$ (46/49).

47

$\mathrm{C}_{15} \mathrm{H}_{26} \mathrm{O}$

Ber. C 81.02 H 11.78

Gef. C $80.85 \quad$ H 11.75

IR (Abb. 23) (KBr): $3620 \mathrm{~cm}^{-1}(\mathrm{OH}), 3580$ - $3020\left(\mathrm{OH}_{\mathrm{ass}}\right), 2944(\mathrm{CH})$.

${ }^{1} \mathbf{H}-\mathbf{N M R}$ (Abb. 49) $\left(300 \mathrm{MHz}, \mathrm{CDCl}_{3}, \mathrm{CHCl}_{3}\right.$ int.): $\delta=0.77 \mathrm{ppm}(\mathrm{s}, 3 \mathrm{H}), 1.07$ (d, J = $7 \mathrm{~Hz}$, $3 \mathrm{H}), 1.15$ (s, 3H), 1.20 (s, 3H), 1.34 - 1.62 (m, 5H), 1.38 (br s, 1H, OH), 1.70 - 2.11 (m, 6H), $2.41\left(\mathrm{~m}_{\mathrm{c}}, 1 \mathrm{H}\right), 2.56\left(\mathrm{~m}_{\mathrm{c}}, 1 \mathrm{H}\right)$. 
${ }^{13}$ C-NMR (Abb. 89) (75 MHz, $\mathrm{CDCl}_{3}, \mathrm{CDCl}_{3}$ int.): $\delta=16.93$ ppm $\left(\mathrm{C}_{\text {prim }}\right), 18.79\left(\mathrm{C}_{\text {sek }}\right), 24.52$ $\left(\mathrm{C}_{\text {prim }}\right), 26.37\left(\mathrm{C}_{\text {sek }}\right), 28.05,30.39\left(\mathrm{C}_{\text {prim }}\right), 31.89\left(\mathrm{C}_{\text {tert }}\right), 38.20\left(\mathrm{C}_{\text {quart }}\right), 40.09,40.28\left(\mathrm{C}_{\text {sek }}\right)$, $43.10\left(\mathrm{C}_{\text {quart }}\right), 46.51\left(\mathrm{C}_{\text {sek }}\right), 48.49\left(\mathrm{C}_{\text {tert }}\right), 64.05\left(\mathrm{C}_{\text {tert }}\right), 73.91\left(\mathrm{C}_{\text {quart }}\right)$.

MS $(70 \mathrm{eV}): \mathrm{m} / \mathrm{z}(\%)=222\left(\mathrm{M}^{+}, 3\right), 95(100)$.

48

\section{$\mathrm{C}_{15} \mathrm{H}_{26} \mathrm{O}$}

Ber. C 81.02 H 11.78

Gef. C 81.10 H 11.86

IR (Abb. 24) (KBr): $3623 \mathrm{~cm}^{-1}(\mathrm{OH}), 3600$ - $3050\left(\mathrm{OH}_{\mathrm{ass}}\right), 2943(\mathrm{CH})$.

${ }^{\mathbf{1}} \mathbf{H}$-NMR (Abb. 50) (300 MHz, $\mathrm{CDCl}_{3}, \mathrm{CHCl}_{3}$ int.): $\delta=0.79 \mathrm{ppm}$ (s, 3H), 1.10 (d, J = $7 \mathrm{~Hz}$, $3 \mathrm{H}), 1.16(\mathrm{~s}, 3 \mathrm{H}), 1.23(\mathrm{~s}, 3 \mathrm{H}), 1.36-1.67(\mathrm{~m}, 7 \mathrm{H}), 1.84-2.15(\mathrm{~m}, 5 \mathrm{H}), 2.34-2.49\left(\mathrm{~m}_{\mathrm{c}}, 1 \mathrm{H}\right)$, $2.49-2.61\left(\mathrm{~m}_{\mathrm{c}}, 1 \mathrm{H}\right)$.

${ }^{13}$ C-NMR (Abb. 90) (75 MHz, $\mathrm{CDCl}_{3}, \mathrm{CDCl}_{3}$ int.): $\delta=16.78 \mathrm{ppm}\left(\mathrm{C}_{\text {prim }}\right), 19.80\left(\mathrm{C}_{\text {sek }}\right), 24.40$ $\left(\mathrm{C}_{\text {prim }}\right), 25.74\left(\mathrm{C}_{\text {sek }}\right), 27.53,30.41\left(\mathrm{C}_{\text {prim }}\right), 31.89\left(\mathrm{C}_{\text {tert }}\right), 38.35\left(\mathrm{C}_{\text {quart }}\right), 40.17,40.40\left(\mathrm{C}_{\text {sek }}\right)$, $43.50\left(\mathrm{C}_{\text {quart }}\right), 46.27\left(\mathrm{C}_{\text {sek }}\right), 47.84,62.09\left(\mathrm{C}_{\text {tert }}\right), 74.10\left(\mathrm{C}_{\text {quart }}\right)$.

MS $(70 \mathrm{eV}): \mathrm{m} / \mathrm{z}(\%)=222\left(\mathrm{M}^{+}, 2\right), 95(100)$ 


\section{$\mathrm{C}_{15} \mathrm{H}_{26} \mathrm{O}$}

Ber. C 81.02 H 11.78

Gef. C 80.91 H 11.66

IR (Abb. 22) (KBr): $3607 \mathrm{~cm}^{-1}(\mathrm{OH}), 3595$ - $3020\left(\mathrm{OH}_{\mathrm{ass} .}\right), 2945(\mathrm{CH})$.

${ }^{1} \mathbf{H}-\mathbf{N M R}(A b b .48)\left(300 \mathrm{MHz}, \mathrm{CDCl}_{3}, \mathrm{CHCl}_{3}\right.$ int.): $\delta=0.80 \mathrm{ppm}(\mathrm{s}, 1.4 \mathrm{H}), 0.81(\mathrm{~s}, 1.6 \mathrm{H})$, $1.08(\mathrm{~d}, \mathrm{~J}=6.5 \mathrm{~Hz}, 1.4 \mathrm{H}), 1.10(\mathrm{~d}, 6.5 \mathrm{~Hz}, 1.6 \mathrm{H}), 1.18(\mathrm{~s}, 4.4 \mathrm{H}), 1.20(\mathrm{~s}, 1.6 \mathrm{H}), 1.29$ - 1.49 $(\mathrm{m}, 3 \mathrm{H}), 1.50-1.70(\mathrm{~m}, 5 \mathrm{H}), 1.71-2.31(\mathrm{~m}, 6 \mathrm{H})$.

${ }^{13}$ C-NMR (Abb. 88) (75 MHz, $\mathrm{CDCl}_{3}, \mathrm{CDCl}_{3}$ int.): $\delta=18.15$ ppm, $18.23\left(\mathrm{C}_{\mathrm{sek}}\right), 22.09,22.12$, 24.25, $24.30\left(\mathrm{C}_{\text {prim }}\right), 25.33,25.46\left(\mathrm{C}_{\text {sek }}\right), 27.76,27.80\left(\mathrm{C}_{\text {prim }}\right), 30.22$ Koinzidenz zweier Signale $\left(\mathrm{C}_{\text {prim }}\right), 30.62,31.02\left(\mathrm{C}_{\text {tert }}\right), 37.84,37.87\left(\mathrm{C}_{\text {quart }}\right), 40.08,40.10,40.50,40.53\left(\mathrm{C}_{\text {sek }}\right)$, 43.10, $43.18\left(\mathrm{C}_{\text {quart }}\right), 44.98,45.08\left(\mathrm{C}_{\text {sek }}\right), 54.74$ Koinzidenz zweier Signale, 61.87, 62.20 $\left(\mathrm{C}_{\text {tert }}\right), 73.07,73.18\left(\mathrm{C}_{\text {quart }}\right)$.

MS $(70 \mathrm{eV}): \mathrm{m} / \mathrm{z}(\%)=222\left(\mathrm{M}^{+}, 3\right), 95(100)$

$\left(3 \mathrm{a} R^{*}, 6 \mathrm{a} S^{*}\right)-6-M e t h y l-1,2,3,3 \mathrm{a}, 4,6 \mathrm{a}-h e x a h y d r o-p e n t a l e n \quad(108), \quad\left(3 \mathrm{a} R^{*}, 6 \mathrm{a} S^{*}\right)-3 \mathrm{a}, 6-\mathrm{Di}-$ methyl-1,2,3,3a,4,6a-hexahydro-pentalen (109) und $\left(3 \mathrm{a} R^{*}, 6 \mathrm{a} S^{*}\right)-3 \mathrm{a}, 6,6 \mathrm{a}-$ Trimethyl1,2,3,3a,4,6a-hexahydro-pentalen (118): Man erhitzte das entsprechende Bicyclobutyl-1-ol (1.0 mmol) (104b, 105, 106a,b und 107a,b) in einer $0.074 \mathrm{M}$ Lösung von wasserfreier pToluolsulfonsäure $(13.5 \mathrm{ml}, 1.0 \mathrm{mmol})$ in Benzol auf $70{ }^{\circ} \mathrm{C}$. Nach $3 \mathrm{~h}$ verdünnte man mit Pentan $(5 \mathrm{ml})$, wusch mit gesättigter Natriumhydrogencarbonatlösung $(3 \times 5 \mathrm{ml})$, extrahierte die vereinigten wässrigen Phasen mit Pentan $(5 \mathrm{ml})$, trocknete die vereinigten organischen Phasen über Molekularsieb $3 \AA$ und engte die Lösung an einer $20 \mathrm{~cm}$ Vigreuxkolonne (Badtemperatur $100{ }^{\circ} \mathrm{C}$ ) ein. Gaschromatographische Analyse [3 m x 1/4" Allglassystem, 15\% OV 101 auf Chromosorb W AW/DMCS 60/80 mesh, Retentionszeiten (min): 3.09 (109), 
$5.22(\mathbf{1 1 8})$ bei $120^{\circ} \mathrm{C}, 2.81(\mathbf{1 0 8})$ bei $130^{\circ} \mathrm{C}$ ] der Rückstände zeigte für alle Proben das Entstehen nur eines Produktes an (108 aus 106a,b; 109 aus 104b und 107a,b; 118 aus 105.). Analytische Proben der klaren Flüssigkeiten wurden durch präparative Gaschromatographie erhalten. Die ${ }^{1} \mathrm{H}-\mathrm{NMR}{ }^{46,48}$ und ${ }^{13} \mathrm{C}-\mathrm{NMR}-\mathrm{Daten}{ }^{44,48}$ von (108) und ${ }^{13} \mathrm{C}-\mathrm{NMR}-\mathrm{Daten}{ }^{44,51}$ von 109 entsprachen den Literaturdaten.

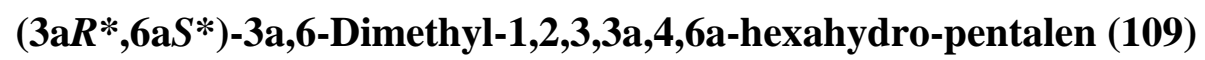

${ }^{1} \mathbf{H}-\mathbf{N M R}$ (Abb. 51) (300 MHz, $\mathrm{CDCl}_{3}, \mathrm{CHCl}_{3}$ int.): $\delta=1.12 \mathrm{ppm}(\mathrm{s}, 3 \mathrm{H}), 1.32-1.72$ (m, $6 \mathrm{H}), 1.62$ (br s, 3H), $2.15\left(\mathrm{~m}_{\mathrm{c}}, 2 \mathrm{H}\right), 2.34\left(\mathrm{~m}_{\mathrm{c}}, 1 \mathrm{H}\right), 5.10$ (br s, 1H).

$\left(3 \mathrm{a} R^{*}, 6 \mathrm{a} S^{*}\right)-3 \mathrm{a}, 6,6 \mathrm{a}-$ Trimethyl-1,2,3,3a,4,6a-hexahydro-pentalen (118)

\section{$\mathrm{C}_{11} \mathrm{H}_{18}$}

Ber. C $87.92 \quad$ H 12.08

Gef. C 88.10 H 12.00

IR (Abb. 25) (KBr): $3050 \mathrm{~cm}^{-1}(\mathrm{C}=\mathrm{C})$.

${ }^{1} \mathbf{H}-\mathbf{N M R}$ (Abb. 52) (300 MHz, $\mathrm{CDCl}_{3}, \mathrm{CHCl}_{3}$ int.): $\delta=0.91 \mathrm{ppm}(\mathrm{s}, 3 \mathrm{H}), 0.98$ (s, 3H), 1.15 $1.50(\mathrm{~m}, 4 \mathrm{H}), 1.50$ - 1.75 (m, 2H), 1.56 (br s, 3H), $2.11\left(\mathrm{~m}_{\mathrm{c}}, 2 \mathrm{H}\right), 5.13$ (br s, 1H).

${ }^{13}$ C-NMR (Abb. 91) (75 MHz, $\mathrm{CDCl}_{3}, \mathrm{CDCl}_{3}$ int.): $\delta=13.03$ ppm, $20.86\left(\mathrm{C}_{\text {prim }}\right), 23.68\left(\mathrm{C}_{\text {sek }}\right)$, $24.80\left(\mathrm{C}_{\text {prim }}\right), 37.96,44.05,47.38\left(\mathrm{C}_{\text {sek }}\right), 49.57,58.16\left(\mathrm{C}_{\text {quart }}\right), 121.81\left(\mathrm{C}_{\text {tert }}\right), 145.44\left(\mathrm{C}_{\text {quart }}\right)$.

MS $(70 \mathrm{eV}): \mathrm{m} / \mathrm{z}(\%)=168\left(100,\left[\mathrm{M}+\mathrm{NH}_{4}\right]^{+}\right)$. 
1,1,3a-Trimethyl-1,2,3,3a,4,5-hexahydro-pentalen (120): $\mathrm{Zu}$ einer Lösung des Bicyclobutyl-1-ols 105 (1.0 mmol) in Pyridin $(3.0 \mathrm{ml})$ wurde bei $0^{\circ} \mathrm{C}$ unter Rühren eine Lösung von Thionylchlorid $(236 \mathrm{mg}, 2.0 \mathrm{mmol})$ in Pyridin $(1 \mathrm{ml})$ gegeben. Nach $3 \mathrm{~h}$ bei $0^{\circ} \mathrm{C}$ zeigte eine gaschromatographische Analyse [3 m x 1/4" Allglassystem, 15\% OV 101 auf Chromosorb W AW/DMCS 60/80 mesh, $120^{\circ} \mathrm{C}$, Retentionzeit (min): 6.19 (120) (70\%)] vollständigen Umsatz an. Man versetzte mit Wasser ( $5 \mathrm{ml}$ ), extrahierte mit Pentan (3 x $5 \mathrm{ml})$, wusch die vereinigten organischen Phasen mit gesättigter Ammoniumchloridlösung (4 x 5 $\mathrm{ml}$ ), trocknete über Molekularsieb $3 \AA$, engte die Lösung an einer $20 \mathrm{~cm}$ Vigreuxkolonne (Badtemperatur $100^{\circ} \mathrm{C}$ ) ein. Präparative Gaschromatographie lieferte reines $\mathbf{1 2 0}$ als farblose Flüssigkeit.

\section{1,1,3a-Trimethyl-1,2,3,3a,4,5-hexahydro-pentalen (120)}

\section{$\mathrm{C}_{11} \mathrm{H}_{18}$}

Ber. 150.1409

Gef. 150.1408 HRMS

${ }^{\mathbf{1}} \mathbf{H}-\mathbf{N M R}$ (Abb. 53) (200 MHz, $\mathrm{CDCl}_{3}, \mathrm{CHCl}_{3}$ int.): $\delta=1.05 \mathrm{ppm}(\mathrm{s}, 3 \mathrm{H}), 1.08$ (s, 3H), 1.13 (s, 3H), 1.38 (ddd, J = 7, 12, $12 \mathrm{~Hz}, 1 \mathrm{H}), 1.52$ - 1.81 (m, 4H), 1.99 (ddd, J = 7, 12, $12 \mathrm{~Hz}, 1 \mathrm{H}$ ), $2.34\left(\mathrm{~m}_{\mathrm{c}}, 1 \mathrm{H}\right), 2.67\left(\mathrm{~m}_{\mathrm{c}}, 1 \mathrm{H}\right), 5.12($ br s, 1H).

${ }^{13}$ C-NMR (Abb. 92) (50 MHz, $\mathrm{CDCl}_{3}, \mathrm{CDCl}_{3}$ int.): $\delta=22.85$ ppm, 30.47, $30.60\left(\mathrm{C}_{\text {prim }}\right), 35.41$ $\left(\mathrm{C}_{\text {sek }}\right), 35.84\left(\mathrm{C}_{\text {quart }}\right), 37.60,42.13,44.33\left(\mathrm{C}_{\text {sek }}\right), 56.43\left(\mathrm{C}_{\text {quart }}\right), 115.14\left(\mathrm{C}_{\text {tert }}\right), 167.66\left(\mathrm{C}_{\text {quart }}\right)$.

MS $(70 \mathrm{eV}): \mathrm{m} / \mathrm{z}(\%)=150\left(29, \mathrm{M}^{+}\right), 135(100)$. 
2,6,6-Trimethyl-4-(2-methylcyclobutyl)-cyclohepta-1,4-dien (122), 3,6,6-Trimethyl-1-(2methyl-cyclobutyl)-cyclohepta-1,3-dien (123), 1,5,5-Trimethyl-3-(2-methyl-cyclobutyliden)-cyclohept-1-en (124) und 1,5,5-Trimethyl-3-(2-methyl-cyclobutyliden)cyclohept-1-en (125): Zu einer Lösung des Alkoholgemisches 46/49 (100 mg, 0.45 mmol) in Ether ( $7 \mathrm{ml}$ ) wurde Nafion NR $50^{53}$ (2.5 g, Aldrich, Perlkörner, 10 - 35 mesh) gegeben und 23 h unter Rückfluss gerührt bis eine gaschromatographische Analyse [3 m x 1/4" Allglassystem, 15\% FFAP auf Chromosorb W AW/DMCS 60/80 mesh, Temperaturprogramm: 8 min bei $150^{\circ} \mathrm{C}, 20^{\circ} \mathrm{C} / \mathrm{min}$ auf $220^{\circ} \mathrm{C}$, Retentionszeiten (min): 4.30 (9\%) (122) 5.73 (34\%) (123), 8.22 (20\%) (124), 9.40 (12\%) (125), 10.79 (46/49)] vollständigen Umsatz anzeigte. Danach wurde mit Pentan $(10 \mathrm{ml})$ aufgenommen und abdekantiert. Die organische Phase wurde mit gesättigter Natriumhydrogencarbonatlösung (4 x $3 \mathrm{ml}$ ) gewaschen und über Molekularsieb 3 $\AA$ getrocknet, anschließend engte man die Lösung an einer $20 \mathrm{~cm}$ Vigreuxkolonne (Badtemperatur $60^{\circ} \mathrm{C}$ ) ein. Analytische Proben wurden durch präparative Gaschromatographie erhalten.

\section{2,6,6-Trimethyl-4-(2-methylcyclobutyl)-cyclohepta-1,4-dien (122)}

$\mathrm{C}_{15} \mathrm{H}_{24}$

Ber. 204.1878

Gef. 204.1878 HRMS

${ }^{1} \mathbf{H}-\mathbf{N M R}$ (Abb. 54) (300 MHz, $\mathrm{C}_{6} \mathrm{D}_{6}, \mathrm{C}_{6} \mathrm{D}_{5} \mathrm{H}$ int.): $\delta=1.05 \mathrm{ppm}(\mathrm{d}, \mathrm{J}=6.5 \mathrm{~Hz}, 3 \mathrm{H}), 1.05$ (s, $6 \mathrm{H}), 1.24-1.41(\mathrm{~m}, 1 \mathrm{H}), 1.59-1.75(\mathrm{~m}, 2 \mathrm{H}), 1.73(\mathrm{~d}, \mathrm{~J}=0.7 \mathrm{~Hz}, 3 \mathrm{H}), 1.84\left(\mathrm{~m}_{\mathrm{c}}, 1 \mathrm{H}\right), 2.00$ $2.31(\mathrm{~m}, 4 \mathrm{H}), 2.61\left(\mathrm{AB}, \Delta \mathrm{v}_{\mathrm{AB}}=24.5 \mathrm{~Hz}, \mathrm{~J}_{\mathrm{AB}}=17 \mathrm{~Hz}, 2 \mathrm{H}\right), 5.18(\mathrm{~d}, \mathrm{~J}=0.8 \mathrm{~Hz}, 1 \mathrm{H}), 5.55\left(\mathrm{~m}_{\mathrm{c}}\right.$, $1 \mathrm{H})$.

${ }^{13}$ C-NMR (Abb. 93) (75 MHz, $\mathrm{C}_{6} \mathrm{D}_{6}, \mathrm{C}_{6} \mathrm{D}_{6}$ int.): $\delta=21.27 \mathrm{ppm}\left(\mathrm{C}_{\text {prim }}\right), 24.41\left(\mathrm{C}_{\text {sek }}\right), 25.07$ $\left(\mathrm{C}_{\text {prim }}\right), 26.15\left(\mathrm{C}_{\text {sek }}\right), 30.53,30.67\left(\mathrm{C}_{\text {prim }}\right), 33.90\left(\mathrm{C}_{\text {sek }}\right), 34.78\left(\mathrm{C}_{\text {quart }}\right), 36.28\left(\mathrm{C}_{\text {tert }}\right), 39.20\left(\mathrm{C}_{\text {sek }}\right)$, $53.11\left(\mathrm{C}_{\text {tert }}\right), 123.23,132.92\left(\mathrm{C}_{\text {tert }}\right), 136.26,145.56$ ( $\left.\mathrm{C}_{\text {quart }}\right)$. 
MS $(70 \mathrm{eV}): \mathrm{m} / \mathrm{z}(\%)=204\left(12, \mathrm{M}^{+}\right), 119(100)$.

\section{3,6,6-Trimethyl-1-(2-methyl-cyclobutyl)-cyclohepta-1,3-dien (123)}

\section{$\mathrm{C}_{15} \mathrm{H}_{24}$}

Ber. 204.1878

Gef. 204.1878 HRMS

${ }^{1}$ H-NMR (Abb. 55) (500 MHz, $\mathrm{C}_{6} \mathrm{D}_{6}, \mathrm{C}_{6} \mathrm{D}_{5} \mathrm{H}$ int.): $\delta=0.96 \mathrm{ppm}(\mathrm{s}, 3 \mathrm{H}), 0.97$ (s, 3H), 1.06 (d, $\mathrm{J}=7 \mathrm{~Hz}, 3 \mathrm{H}), 1.35\left(\mathrm{~m}_{\mathrm{c}}, 1 \mathrm{H}\right), 1.68-1.77(\mathrm{~m}, 2 \mathrm{H}), 1.80$ (br s, 3H), 1.81 - 1.89 (m, 5H), 1.94 $\left(\mathrm{m}_{\mathrm{c}}, 1 \mathrm{H}\right), 2.45\left(\mathrm{~m}_{\mathrm{c}}, 1 \mathrm{H}\right), 5.70(\mathrm{~s}, 1 \mathrm{H}), 5.72\left(\mathrm{~m}_{\mathrm{c}}, 1 \mathrm{H}\right)$.

${ }^{13} \mathrm{C}-\mathrm{NMR}$ (Abb. 94) (75 MHz, $\mathrm{C}_{6} \mathrm{D}_{6}, \mathrm{C}_{6} \mathrm{D}_{6}$ int.): $\delta=21.19,24.07\left(\mathrm{C}_{\text {prim }}\right), 25.17,26.38\left(\mathrm{C}_{\text {sek }}\right)$, 29.40, $29.47\left(\mathrm{C}_{\text {prim }}\right), 36.78\left(\mathrm{C}_{\text {tert }}\right), 42.45,44.25\left(\mathrm{C}_{\text {sek }}\right), 44.92\left(\mathrm{C}_{\text {quart }}\right), 52.55,125.01,125.95$ $\left(\mathrm{C}_{\text {tert }}\right), 136.01,146.92\left(\mathrm{C}_{\text {quart }}\right)$.

MS $(70 \mathrm{eV}): \mathrm{m} / \mathrm{z}(\%)=204\left(40, \mathrm{M}^{+}\right), 119(100)$.

\section{1,5,5-Trimethyl-3-(2-methyl-cyclobutyliden)-cyclohept-1-en (124)}

\section{$\mathrm{C}_{15} \mathrm{H}_{24}$}

Ber. 204.1878

Gef. 204.1878 HRMS

${ }^{1} \mathbf{H}$-NMR (Abb. 56) $\left(500 \mathrm{MHz}, \mathrm{C}_{6} \mathrm{D}_{6}, \mathrm{C}_{6} \mathrm{D}_{5} \mathrm{H}\right.$ int.): $\delta=0.96 \mathrm{ppm}(\mathrm{s}, 6 \mathrm{H}), 1.25$ (d, J = $7 \mathrm{~Hz}$, $3 \mathrm{H}), 1.36$ - 1.49 (m, 2H), 1.56 - 1.63 (m, 1H), 1.72 (br s, 3H), 1.88 - 1.97 (m, 1H), 2.00 - 2.17 (m, 4H), $2.50-2.59(\mathrm{~m}, 1 \mathrm{H}), 2.64-2.74(\mathrm{~m}, 1 \mathrm{H}), 3.03-3.10(\mathrm{~m}, 1 \mathrm{H}), 6.07$ (br s, 1H). 
${ }^{13}$ C-NMR (Abb. 95) (75 MHz, $\mathrm{C}_{6} \mathrm{D}_{6}, \mathrm{C}_{6} \mathrm{D}_{6}$ int.): $\delta=20.84 \mathrm{ppm}\left(\mathrm{C}_{\text {prim }}\right), 24.64\left(\mathrm{C}_{\text {sek }}\right), 27.01$ $\left(\mathrm{C}_{\text {prim }}\right), 27.17\left(\mathrm{C}_{\mathrm{sek}}\right), 29.37,29.59\left(\mathrm{C}_{\text {prim }}\right), 30.24\left(\mathrm{C}_{\text {sek }}\right), 34.47\left(\mathrm{C}_{\text {quart }}\right), 37.48\left(\mathrm{C}_{\text {tert }}\right), 41.74$, $43.04\left(\mathrm{C}_{\text {sek }}\right), 124.56\left(\mathrm{C}_{\text {tert }}\right), 128.23,138.14,142.68\left(\mathrm{C}_{\text {quart }}\right)$.

MS $(70 \mathrm{eV}): \mathrm{m} / \mathrm{z}(\%)=204\left(48, \mathrm{M}^{+}\right), 119(98), 162(100)$

\section{1,5,5-Trimethyl-3-(2-methyl-cyclobutyliden)-cyclohept-1-en (125)}

\section{$\mathrm{C}_{15} \mathrm{H}_{24}$}

Ber. 204.1878

Gef. 204.1878 HRMS

${ }^{1} \mathbf{H}-\mathbf{N M R}$ (Abb. 57) (500 MHz, $\mathrm{C}_{6} \mathrm{D}_{6}, \mathrm{C}_{6} \mathrm{D}_{5} \mathrm{H}$ int.): $\delta=0.97 \mathrm{ppm}(\mathrm{s}, 3 \mathrm{H}), 0.99$ (s, 3H), 1.22 (d, $\mathrm{J}=7 \mathrm{~Hz}, 3 \mathrm{H}), 1.39\left(\mathrm{~m}_{\mathrm{c}}, 1 \mathrm{H}\right), 1.48\left(\mathrm{~m}_{\mathrm{c}}, 1 \mathrm{H}\right), 1.54$ - $1.63(\mathrm{~m}, 1 \mathrm{H}), 1.74$ ( br s, 3H), 1.89 - 2.15 (m, 4H), 2.16 (br s, 1H), $2.59\left(\mathrm{~m}_{\mathrm{c}}, 1 \mathrm{H}\right), 2.71-2.80(\mathrm{~m}, 1 \mathrm{H}), 2.97$ - 3.07 (m, 1H), 5.97 (br s, $1 \mathrm{H})$.

${ }^{13}$ C-NMR (Abb. 96) (125 MHz, $\mathrm{C}_{6} \mathrm{D}_{6}, \mathrm{C}_{6} \mathrm{D}_{6}$ int.): $\delta=20.60 \mathrm{ppm}\left(\mathrm{C}_{\text {prim }}\right), 24.74\left(\mathrm{C}_{\text {sek }}\right), 27.16$ $\left(\mathrm{C}_{\text {prim }}\right), 27.18\left(\mathrm{C}_{\text {sek }}\right), 29.76,29.79\left(\mathrm{C}_{\text {prim }}\right), 30.24\left(\mathrm{C}_{\text {sek }}\right), 34.04\left(\mathrm{C}_{\text {quart }}\right), 37.72\left(\mathrm{C}_{\text {tert }}\right), 41.90$, $42.49\left(\mathrm{C}_{\text {sek }}\right), 125.18\left(\mathrm{C}_{\text {tert }}\right), 128.40,138.09,142.72\left(\mathrm{C}_{\text {quart }}\right)$.

MS $(70 \mathrm{eV}): \mathrm{m} / \mathrm{z}(\%)=204\left(20, \mathrm{M}^{+}\right), 119(100)$

2,6,6-Trimethyl-4-(2-methylcyclobutyl)-cyclohepta-1,4-dien (122), 1,4,4-Trimethyl-6-(2methyl-cyclobutyl)-bicyclo[3.2.0]hept-6-en (130 und 131), 1,4,4-Trimethyl-6-(2-methylcyclobutyliden)-bicyclo[3.2.0]heptan (132/133/134/135): $\mathrm{Zu}$ einer Lösung des Alkoholgemisches $46 / 49$ (1.0 mmol) in Pyridin $(3.0 \mathrm{ml})$ wurde bei $0^{\circ} \mathrm{C}$ unter Rühren eine Lösung von Thionylchlorid $(236 \mathrm{mg}, 2.0 \mathrm{mmol})$ in Pyridin $(1 \mathrm{ml})$ gegeben. Nach $3 \mathrm{~h}$ bei $0^{\circ} \mathrm{C}$ zeigte eine gaschromatographische Analyse [3 m x 1/4" Allglassystem, 15\% FFAP auf 
Chromosorb W AW/DMCS 60/80 mesh, Temperaturprogramm: $8 \mathrm{~min}$ bei $150^{\circ} \mathrm{C}, 20^{\circ} \mathrm{C} / \mathrm{min}$ auf $220^{\circ} \mathrm{C}$, Retentionszeiten (min): 2.50 (29\%), 50:50 (130:131), 3.26 (10\%) (132/133/134/135), $4.83(7 \%)(122)]$ vollständigen Umsatz an. Man versetzte mit Wasser (5 ml), extrahierte mit Pentan (3 x $5 \mathrm{ml}$ ), wusch die vereinigten organischen Phasen mit gesättigter Ammoniumchloridlösung (4 x $5 \mathrm{ml}$ ), trocknete über Molekularsieb $3 \AA$, engte die Lösung an einer $20 \mathrm{~cm}$ Vigreuxkolonne (Badtemperatur $100^{\circ} \mathrm{C}$ ) ein. Analytische Proben der farblosen Flüssigkeiten wurden durch präparative Gaschromatographie erhalten. Die spektoskopischen Daten von 122 stimmten mit denen authentischen Materials überein.

\section{1,4,4-Trimethyl-6-(2-methyl-cyclobutyl)-bicyclo[3.2.0]hept-6-en (130 und 131)}

\section{$\mathrm{C}_{15} \mathrm{H}_{24}$}

Ber. 204.1878

Gef. 204.1878 HRMS

${ }^{1} \mathbf{H}$-NMR (Abb. 58) $\left(300 \mathrm{MHz}, \mathrm{C}_{6} \mathrm{D}_{6}, \mathrm{C}_{6} \mathrm{D}_{5} \mathrm{H}\right.$ int.): $\delta=0.88 \mathrm{ppm}(\mathrm{s}, 1.5 \mathrm{H}), 0.89$ (s, 1.5H), $1.02(\mathrm{~s}, 1.5 \mathrm{H}), 1.03(\mathrm{~s}, 1.5 \mathrm{H}), 1.04(\mathrm{~d}, \mathrm{~J}=6.8 \mathrm{~Hz}, 1.5 \mathrm{H}), 1.08$ (d, J = 6.4 Hz, 1.5H), 1.33 (s, $3 \mathrm{H}), 1.29-1.54(\mathrm{~m}, 4 \mathrm{H}), 1.57-1.74(\mathrm{~m}, 0.5 \mathrm{H}), 1.75-2.04(\mathrm{~m}, 4 \mathrm{H}), 2.12$ (br s, 0.5H), 2.15 (br s, $0.5 \mathrm{H}), 2.24-2.50(\mathrm{~m}, 1.5 \mathrm{H}), 5.66$ (br s, $1 \mathrm{H})$.

${ }^{13}$ C-NMR (Abb. 97) (75 MHz, $\mathrm{C}_{6} \mathrm{D}_{6}, \mathrm{C}_{6} \mathrm{D}_{6}$ int.): $\delta=21.41 \mathrm{ppm}, 21.50\left(\mathrm{C}_{\text {prim }}\right), 23.90\left(\mathrm{C}_{\mathrm{sek}}\right)$, 24.21, $24.27\left(\mathrm{C}_{\text {prim }}\right), 25.76\left(\mathrm{C}_{\text {sek }}\right), 25.94\left(\mathrm{C}_{\text {prim }}\right), 26.20\left(\mathrm{C}_{\text {sek }}\right), 26.53\left(\mathrm{C}_{\text {prim }}\right), 26.71\left(\mathrm{C}_{\text {sek }}\right), 27.62$, $27.68\left(\mathrm{C}_{\text {prim }}\right), 32.26,32.32\left(\mathrm{C}_{\text {sek }}\right), 36.28,38.00\left(\mathrm{C}_{\text {tert }}\right), 38.40$ (2 $\left.\mathrm{C}_{\text {quart }}\right), 39.27,39.30\left(\mathrm{C}_{\text {sek }}\right)$, $43.88,44.13\left(\mathrm{C}_{\text {tert }}\right), 50.94,50.98\left(\mathrm{C}_{\text {quart }}\right), 63.67,64.25,131.14,131.28\left(\mathrm{C}_{\text {tert }}\right), 152.38,152.50$ $\left(\mathrm{C}_{\text {quart }}\right)$.

MS $(70 \mathrm{eV}): \mathrm{m} / \mathrm{z}(\%)=204\left(20, \mathrm{M}^{+}\right), 119(100)$. 


\section{1,4,4-Trimethyl-6-(2-methyl-cyclobutyliden)-bicyclo[3.2.0]heptan (132/133/134/135)}

\section{$\mathrm{C}_{15} \mathrm{H}_{24}$}

Ber. 204.1878

Gef. 204.1878 HRMS

${ }^{1} \mathbf{H}-\mathbf{N M R}$ (Abb. 59) (300 MHz, $\mathrm{C}_{6} \mathrm{D}_{6}, \mathrm{C}_{6} \mathrm{D}_{5} \mathrm{H}$ int.): $\delta=0.89 \mathrm{ppm}(\mathrm{s}, 3 \mathrm{H}), 1.11$ (s, 3H), 1.15 (d, $\mathrm{J}=6.7 \mathrm{~Hz}, 3 \mathrm{H}), 1.25(\mathrm{~s}, 3 \mathrm{H}), 1.35-1.71(\mathrm{~m}, 4 \mathrm{H}), 1.86(\mathrm{ddd}, \mathrm{J}=7,12,12 \mathrm{~Hz}, 1 \mathrm{H}), 1.98$ - 2.11 $(\mathrm{m}, 1 \mathrm{H}), 2.25-2.38(\mathrm{~m}, 3 \mathrm{H}), 2.44-2.71(\mathrm{~m}, 2 \mathrm{H}), 2.87-3.03(\mathrm{~m}, 1 \mathrm{H})$.

${ }^{13}$ C-NMR (Abb.98) $\left(75 \mathrm{MHz}, \mathrm{C}_{6} \mathrm{D}_{6}, \mathrm{C}_{6} \mathrm{D}_{6}\right.$ int.): $\delta=19.95 \mathrm{ppm}\left(\mathrm{C}_{\text {prim }}\right), 25.65\left(\mathrm{C}_{\mathrm{sek}}\right), 26.30$, $26.74\left(\mathrm{C}_{\text {prim }}\right), 27.15\left(\mathrm{C}_{\text {sek }}\right), 28.29\left(\mathrm{C}_{\text {prim }}\right), 37.98\left(\mathrm{C}_{\text {tert }}\right), 39.03,39.16,40.32\left(\mathrm{C}_{\text {sek }}\right), 43.73,43.85$ $\left(\mathrm{C}_{\text {quart }}\right) 63.02\left(\mathrm{C}_{\text {tert }}\right), 126.29,138.73\left(\mathrm{C}_{\text {quart }}\right)$.

MS $(70 \mathrm{eV}): \mathrm{m} / \mathrm{z}(\%)=204\left(20, \mathrm{M}^{+}\right), 119(100)$.

(3aR*,3bR*,6aR*,7aR*)-3,3,4,7a-Tetramethyl-2,3,3a,3b,6,6a,7,7a-octahydro-1H-cyclopenta[a]pentalen $\quad(44), \quad\left(3 \mathrm{aR} *, 4 \mathrm{R}^{*}, 7 \mathrm{aR} *\right)-3,3,4,7 \mathrm{a}-$ Tetramethyl-2,3,3a,4,5,6,7,7a-

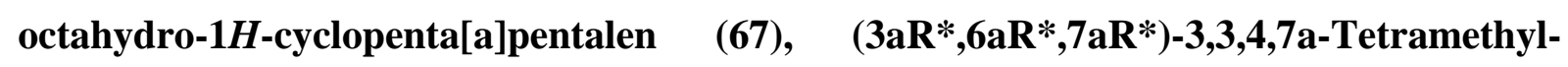
2,3,3a,5,6,6a,7,7a-octahydro-1H-cyclopenta[a]pentalen $(68),\left(3 a R^{*}, 4 R^{*}, 7 a R^{*}\right)-3,3,4,7 a-$ Tetramethyl-2,3,3a,4,5,6,7,7a-octahydro-1H-cyclopenta[a]pentalen (69) und $\left(3 \mathrm{aR}^{*}, 6 \mathrm{aS} *, 7 \mathrm{aR} *\right)-3,3,4,7 \mathrm{a}-$ Tetramethyl-2,3,3a,5,6,6a,7,7a-octahydro-1H-cyclopenta[a]pentalen (70): Man gab Alkohol 60, bzw. 66 (10 mg, $0.045 \mathrm{mmol})$ zu einer 0.074 M Lösung von wasserfreier p-Toluolsulfonsäure $(0.61 \mathrm{ml}, 0.045 \mathrm{mmol})$ in Benzol bei $25^{\circ} \mathrm{C} \mathrm{bzw} .70^{\circ} \mathrm{C}$ und untersuchte den Verlauf der Umlagerung sowie die Zusammensetzung der Reaktionsmischung durch Entnahme von Probehydrolysaten gaschromatographisch $[3 \mathrm{~m} \mathrm{x}$ 1/4" Allglassystem, 15\% FFAP auf Chromosorb W AW/DMCS 60/80 mesh, Temperaturprogramm: $8 \mathrm{~min}$ bei $150^{\circ} \mathrm{C}, 20^{\circ} \mathrm{C} / \mathrm{min}$ auf $220^{\circ} \mathrm{C}$, Retentionszeiten (min): 3.10 (69), 4.04 (67, 68), 5.29 (44), 5.64 (70), 15.44 (66), 15.97 (60)]. Aufgrund der so erhaltenen Ergebnisse [s. S. 44 Tab.1 und Tab.2 (60) und S. $46 / 47$ Tab.3 und Tab.4 (66)] wurden entsprechende präparative Ansätze zur Identifikation der Produkte durchgeführt: 


\section{(3aR*,4R*,7aR*)-3,3,4,7a-Tetramethyl-2,3,3a,4,5,6,7,7a-octahydro-1H-cyclopenta[a]-}

pentalen (69): Man gab den Alkohol $60(50 \mathrm{mg}, 0.22 \mathrm{mmol})$ zu einer $0.074 \mathrm{M}$ Lösung von wasserfreier p-Toluolsulfonsäure $(3.04 \mathrm{ml}, 0.22 \mathrm{mmol})$ in Benzol bei $25^{\circ} \mathrm{C}$ und untersuchte den Verlauf der Umlagerung [s. S. 44 Tab.1] sowie die Zusammensetzung der Reaktionsmischung durch Entnahme von Probehydrolysaten gaschromatographisch $[3 \mathrm{~m} \mathrm{x}$ 1/4" Allglassystem, 15\% FFAP auf Chromosorb W AW/DMCS 60/80 mesh, Temperaturprogramm: $8 \mathrm{~min}$ bei $150^{\circ} \mathrm{C}, 20^{\circ} \mathrm{C} / \mathrm{min}$ auf $220^{\circ} \mathrm{C}$, Retentionszeiten (min): 3.10 (69) (95\%), $4.04(68)(2 \%), 5.29(44)(2 \%), 15.97(60)]$. Nach 45 h verdünnte man mit Pentan (5 $\mathrm{ml}$ ), wusch mit gesättigter Natriumhydrogencarbonatlösung $(3 \times 5 \mathrm{ml})$, extrahierte die vereinigten wässrigen Phasen mit Pentan $(5 \mathrm{ml})$, trocknete die vereinigten organischen Phasen über Molekularsieb $3 \AA$ und engte die Lösung an einer $20 \mathrm{~cm}$ Vigreuxkolonne (Badtemperatur $100{ }^{\circ} \mathrm{C}$ ) ein. Präparative Gaschromatographie lieferte 69 als klare Flüssigkeit.

$\left(3 \mathrm{aR}^{*}, 4 \mathrm{R}^{*}, 7 \mathrm{aR} *\right)-3,3,4,7 \mathrm{a}-$ Tetramethyl-2,3,3a,4,5,6,7,7a-octahydro-1H-cyclopenta[a]pentalen (69)

\section{$\mathrm{C}_{15} \mathrm{H}_{24}$}

Ber. 204.1878

Gef. 204.1878 HRMS

${ }^{1} \mathbf{H}-\mathbf{N M R}$ (Abb. 62) (300 MHz, $\mathrm{CDCl}_{3}, \mathrm{CHCl}_{3}$ int.): $\delta=0.82 \mathrm{ppm}(\mathrm{s}, 3 \mathrm{H}), 0.95$ (d, J = $6.8 \mathrm{~Hz}$, $3 \mathrm{H}), 1.01(\mathrm{~s}, 3 \mathrm{H}), 1.14(\mathrm{~s}, 3 \mathrm{H}), 1.33-1.71(\mathrm{~m}, 5 \mathrm{H}), 1.90\left(\mathrm{~m}_{\mathrm{c}}, 1 \mathrm{H}\right), 1.97\left(\mathrm{~m}_{\mathrm{c}}, 1 \mathrm{H}\right), 2.04-2.14$ $(\mathrm{m}, 3 \mathrm{H}), 2.31-2.44(\mathrm{~m}, 1 \mathrm{H}), 2.63\left(\mathrm{~m}_{\mathrm{c}}, 1 \mathrm{H}\right)$.

${ }^{13}$ C-NMR (Abb. 101) (125 MHz, $\mathrm{CDCl}_{3}, \mathrm{CDCl}_{3}$ int.): $\delta=19.77$ ppm, $25.28\left(\mathrm{C}_{\text {prim }}\right), 28.57$ $\left(\mathrm{C}_{\text {sek }}\right), 30.09,30.76\left(\mathrm{C}_{\text {prim }}\right), 36.76\left(\mathrm{C}_{\text {tert }}\right), 37.24,39.71,41.94\left(\mathrm{C}_{\text {sek }}\right), 42.56\left(\mathrm{C}_{\text {quart }}\right), 46.60\left(\mathrm{C}_{\text {sek }}\right)$, $57.07\left(\mathrm{C}_{\text {quart }}\right), 64.35\left(\mathrm{C}_{\text {tert }}\right), 143.30,150.14\left(\mathrm{C}_{\text {quart }}\right)$.

MS $(70 \mathrm{eV}): \mathrm{m} / \mathrm{z}(\%)=204\left(40, \mathrm{M}^{+}\right), 135(100)$. 
$\left(3 \mathrm{aR}^{*}, 3 \mathrm{bR} *, 6 \mathrm{aR} *, 7 \mathrm{aR} *\right)-3,3,4,7 \mathrm{a}-T e t r a m e t h y l-2,3,3 \mathrm{a}, 3 \mathrm{~b}, 6,6 \mathrm{a}, 7,7 \mathrm{a}-\mathrm{octahydro}-1 H$-cyclopenta[a]pentalen $\quad(44), \quad\left(3 \mathrm{aR}^{*}, 6 \mathrm{aR}^{*}, 7 \mathrm{aR} *\right)-3,3,4,7 \mathrm{a}-$ Tetramethyl-2,3,3a,5,6,6a,7,7aoctahydro-1H-cyclopenta[a]pentalen $(68)$ und $\left(3 \mathrm{aR} *, 4 \mathrm{R}^{*}, 7 \mathrm{aR} *\right)-3,3,4,7 \mathrm{a}-\mathrm{Tetramethyl-}$ 2,3,3a,4,5,6,7,7a-octahydro-1H-cyclopenta[a]pentalen (69): Man gab den Alkohol 60 (50 $\mathrm{mg}, 0.22 \mathrm{mmol}$ ) zu einer 0.074 M Lösung von wasserfreier p-Toluolsulfonsäure (3.04 ml, $0.22 \mathrm{mmol}$ ) in Benzol bei $70^{\circ} \mathrm{C}$ und untersuchte die Zusammensetzung der Reaktionsmischung durch Entnahme von Probehydrolysaten gaschromatographisch $[3 \mathrm{~m} \mathrm{x}$ 1/4" Allglassystem, 15\% FFAP auf Chromosorb W AW/DMCS 60/80 mesh, Temperaturprogramm: $8 \mathrm{~min}$ bei $150^{\circ} \mathrm{C}, 20^{\circ} \mathrm{C} / \mathrm{min}$ auf $220^{\circ} \mathrm{C}$, Retentionszeiten (min): 3.10 (69) (32\%), 4.04 (68) (26\%), 5.29 (44) (42\%)]. Nach 6 h verdünnte man mit Pentan (5 ml), wusch mit gesättigter Natriumhydrogencarbonatlösung (3 x $5 \mathrm{ml}$ ), extrahierte die vereinigten wässrigen Phasen mit Pentan $(5 \mathrm{ml})$, trocknete die vereinigten organischen Phasen über Molekularsieb $3 \AA$ And engte die Lösung an einer $20 \mathrm{~cm}$ Vigreuxkolonne (Badtemperatur 100 $\left.{ }^{\circ} \mathrm{C}\right)$ ein. Präparative Gaschromatographie lieferte analytische Proben der klaren Flüssigkeiten. Die ${ }^{1}$ H-NMR-Daten und ${ }^{13} \mathrm{C}-\mathrm{NMR}-D a t e n$ von 44 stimmten mit den in der Literatur ${ }^{11}$ angegebenen Daten, die von $\mathbf{6 9}$ mit denen authentischen Materials, überein.

$(3 \mathrm{aR} *, 6 \mathrm{aR} *, 7 \mathrm{aR} *)-3,3,4,7 \mathrm{a}-$ Tetramethyl-2,3,3a,5,6,6a,7,7a-octahydro-1H-cyclopenta[a]pentalen (68)

$\mathrm{C}_{15} \mathrm{H}_{24}$

Ber. 204.1878

Gef. 204.1878 HRMS

${ }^{1} \mathbf{H}-\mathbf{N M R}$ (Abb. 61) $\left(300 \mathrm{MHz}, \mathrm{CDCl}_{3}, \mathrm{CHCl}_{3}\right.$ int.): $\delta=0.79 \mathrm{ppm}(\mathrm{s}, 3 \mathrm{H}), 0.87(\mathrm{dd}, \mathrm{J}=12.1$, $12.0 \mathrm{~Hz}, 1 \mathrm{H}), 1.07$ (s, 3H), $1.15(\mathrm{~s}, 3 \mathrm{H}), 1.28\left(\mathrm{~m}_{\mathrm{c}}, 1 \mathrm{H}\right), 1.38-1.64(\mathrm{~m}, 4 \mathrm{H}), 1.59$ (br s, 3H), $1.76(\mathrm{dd}, \mathrm{J}=7.5,12.1 \mathrm{~Hz}, 1 \mathrm{H}), 2.01$ (dddd, J = 1.1, 7.5, 7.5, $12.1 \mathrm{~Hz}, 1 \mathrm{H}), 1.99$ (br s, 1H), $2.26\left(\mathrm{~m}_{\mathrm{c}}, 1 \mathrm{H}\right), 2.61\left(\mathrm{~m}_{\mathrm{c}}, 1 \mathrm{H}\right), 2.93\left(\mathrm{~m}_{\mathrm{c}}, 1 \mathrm{H}\right)$.

${ }^{13}$ C-NMR (Abb. 100) (75 MHz, $\mathrm{CDCl}_{3}, \mathrm{CDCl}_{3}$ int.): $\delta=14.90 \mathrm{ppm}, 25.85,30.65,31.16$ $\left(\mathrm{C}_{\text {prim }}\right), 31.80,40.21,41.93,42.05\left(\mathrm{C}_{\text {sek }}\right), 43.56\left(\mathrm{C}_{\text {quart }}\right), 49.32\left(\mathrm{C}_{\text {sek }}\right), 52.02\left(\mathrm{C}_{\text {tert }}\right), 56.68$ $\left(\mathrm{C}_{\text {quart }}\right), 59.31\left(\mathrm{C}_{\text {tert }}\right), 127.90,147.60\left(\mathrm{C}_{\text {quart }}\right)$. 
MS $(70 \mathrm{eV}): \mathrm{m} / \mathrm{z}(\%)=204\left(24, \mathrm{M}^{+}\right), 133(100)$.

$\left(3 \mathrm{aR} *, 4 \mathrm{R}^{*}, 7 \mathrm{aR} *\right)-3,3,4,7 \mathrm{a}-$ Tetramethyl-2,3,3a,4,5,6,7,7a-octahydro-1H-cyclopenta[a]pentalen (67), (3aR*,6aR*,7aR*)-3,3,4,7a-Tetramethyl-2,3,3a,5,6,6a,7,7a-octahydro-1Hcyclopenta[a]pentalen $\quad(68)$ und $\quad\left(3 \mathrm{aR}^{*}, 6 \mathrm{aS} *, 7 \mathrm{aR} *\right)-3,3,4,7 \mathrm{a}-$ Tetramethyl2,3,3a,5,6,6a,7,7a-octahydro-1H-cyclopenta[a]pentalen (70): Man gab den Alkohol 66 (50 mg, $0.22 \mathrm{mmol}$ ) zu einer $0.074 \mathrm{M}$ Lösung von wasserfreier p-Toluolsulfonsäure $(3.04 \mathrm{ml}$, $0.22 \mathrm{mmol})$ in Benzol bei $70^{\circ} \mathrm{C}$. Nach 10 min verdünnte man mit Pentan $(5 \mathrm{ml})$, wusch mit gesättigter Natriumhydrogencarbonatlösung (3 x $5 \mathrm{ml})$, extrahierte die vereinigten wässrigen Phasen mit Pentan (5 ml), trocknete die vereinigten organischen Phasen über Molekularsieb 3 $\AA$ und engte die Lösung an einer $20 \mathrm{~cm}$ Vigreuxkolonne (Badtemperatur $100{ }^{\circ} \mathrm{C}$ ) ein. Präparative Gaschromatographie [3 m x 1/4" Allglassystem, 15\% FFAP auf Chromosorb W AW/DMCS $60 / 80$ mesh, Temperaturprogramm: $8 \mathrm{~min}$ bei $150^{\circ} \mathrm{C}, 20^{\circ} \mathrm{C} / \mathrm{min}$ auf $220^{\circ} \mathrm{C}$, Retentionszeiten (min): 3.10 (69) (5\%), 4.04 (67, 68) (49\%), 5.29 (44) (9\%), 5.64 (70) (30\%), 15.44 (66) (1\%)] lieferte analytische Proben der klaren Flüssigkeiten. Die spektroskopischen Daten von 44, 68 und 69 stimmten mit denen authentischen Materials überein.

$\left(3 \mathrm{aR} *, 4 \mathrm{R}^{*}, 7 \mathrm{aR} *\right)-3,3,4,7 \mathrm{a}-$ Tetramethyl-2,3,3a,4,5,6,7,7a-octahydro-1H-cyclopenta[a]pentalen $(67)$

$\mathrm{C}_{15} \mathrm{H}_{24}$

${ }^{\mathbf{1}} \mathbf{H}-\mathbf{N M R}$ (Abb. 60) (300 MHz, $\mathrm{CDCl}_{3}, \mathrm{CHCl}_{3}$ int.): $\delta=0.77 \mathrm{ppm}$ (s, 3H), 1.05 (s, 3H), 1.07 $(\mathrm{d}, \mathrm{J}=6.8 \mathrm{~Hz}, 3 \mathrm{H}), 1.07$ (s, 3H).

${ }^{13}$ C-NMR (Abb. 99) (75 MHz, $\mathrm{CDCl}_{3}, \mathrm{CDCl}_{3}$ int.): $\delta=21.51 \mathrm{ppm}, 24.41\left(\mathrm{C}_{\text {prim }}\right), 29.12,29.75$, $31.14\left(\mathrm{C}_{\text {prim }}\right), 37.67\left(\mathrm{C}_{\text {sek }}\right), 38.23\left(\mathrm{C}_{\text {tert }}\right), 38.67\left(\mathrm{C}_{\text {sek }}\right), 41.78\left(\mathrm{C}_{\text {quart }}\right), 44.08,46.66\left(\mathrm{C}_{\text {sek }}\right), 58.31$ $\left(\mathrm{C}_{\text {quart }}\right), 68.01\left(\mathrm{C}_{\text {tert }}\right), 145.97,148.24\left(\mathrm{C}_{\text {quart }}\right)$. 
$\left(3 \mathrm{aR}^{*}, 6 \mathrm{aS} *, 7 \mathrm{aR} *\right)-3,3,4,7 \mathrm{a}-$ Tetramethyl-2,3,3a,5,6,6a,7,7a-octahydro-1H-cyclopenta[a]pentalen (70)

\section{$\mathrm{C}_{15} \mathrm{H}_{24}$}

Ber. 204.1878

Gef. 204.1878 HRMS

${ }^{\mathbf{1}} \mathbf{H}$-NMR (Abb. 63) (300 MHz, $\mathrm{CDCl}_{3}, \mathrm{CHCl}_{3}$ int.): $\delta=0.85 \mathrm{ppm}(\mathrm{s}, 3 \mathrm{H}), 1.04$ (dd, J = 12, 12 $\mathrm{Hz}, 1 \mathrm{H}), 1.11(\mathrm{~s}, 3 \mathrm{H}), 1.16(\mathrm{~s}, 3 \mathrm{H}), 1.31\left(\mathrm{~m}_{\mathrm{c}}, 1 \mathrm{H}\right), 1.41-1.46(\mathrm{~m}, 1 \mathrm{H}), 1.53-1.69(\mathrm{~m}, 4 \mathrm{H})$, $1.65-1.68\left(\mathrm{~m}_{\mathrm{c}}, 3 \mathrm{H}, \mathrm{CH}_{3}\right), 1.87$ (ddd, 6, 6, $\left.11.5 \mathrm{~Hz}, 1 \mathrm{H}\right), 1.99\left(\mathrm{~m}_{\mathrm{c}}, 1 \mathrm{H}\right), 2.31\left(\mathrm{~m}_{\mathrm{c}}, 1 \mathrm{H}\right), 2.56$ $\left(\mathrm{m}_{\mathrm{c}}, 1 \mathrm{H}\right), 3.08\left(\mathrm{~m}_{\mathrm{c}}, 1 \mathrm{H}\right)$.

${ }^{13}$ C-NMR (Abb. 102) (125 MHz, $\mathrm{CDCl}_{3}, \mathrm{CDCl}_{3}$ int.): $\delta=16.94 \mathrm{ppm}, 24.65,29.88,31.22$ $\left(\mathrm{C}_{\text {prim }}\right), 31.14,39.14\left(\mathrm{C}_{\text {sek }}\right), 42.21\left(\mathrm{C}_{\text {quart }}\right), 43.43,44.64,46.80\left(\mathrm{C}_{\text {sek }}\right), 55.70\left(\mathrm{C}_{\text {tert }}\right), 59.33$ $\left(\mathrm{C}_{\text {quart }}\right), 60.53\left(\mathrm{C}_{\text {tert }}\right), 126.33,146.14\left(\mathrm{C}_{\text {quart }}\right)$.

MS $(70 \mathrm{eV}): \mathrm{m} / \mathrm{z}(\%)=204\left(50, \mathrm{M}^{+}\right), 135(100)$.

\section{(3aR*,3bR*,6aR*,7aR*)-3,3,4,7a-Tetramethyl-2,3,3a,3b,6,6a,7,7a-octahydro-1H-cyclo-} penta[a]pentalen $[( \pm)$-endo-Capnellen (44)]: $\mathrm{Zu}$ einer Lösung von wasserfreier pToluolsulfonsäure $(23.1 \mathrm{ml}, 0.074 \mathrm{M}, 1.71 \mathrm{mmol})$ in Benzol wurde bei $70^{\circ} \mathrm{C}$ ein aus der Reduktion von 141 und 142 erhaltenes Alkoholgemisch (381 mg, $1.71 \mathrm{mmol}$ ) gegeben. Nach $5.5 \mathrm{~h}$ verdünnte man mit Pentan $(10 \mathrm{ml})$, wusch mit gesättigter Natriumhydrogencarbonatlösung ( $3 \times 10 \mathrm{ml}$ ), extrahierte die vereinigten wässrigen Phasen mit Pentan $(10 \mathrm{ml})$, trocknete die vereinigten organischen Phasen über Molekularsieb $3 \AA$ und engte die Lösung an einer $20 \mathrm{~cm}$ Vigreuxkolonne (Badtemperatur $110{ }^{\circ} \mathrm{C}$ ) ein. Danach entfernte man die restlichen Lösungsmittel am Rotationsverdampfer ( $20^{\circ} \mathrm{C} / 14$ Torr). Chromatographie des so erhaltenen Rückstandes an mit 10\% Silbernitrat dotiertem Kieselgel (0.04 - $0.063 \mathrm{~mm}$ ) in Pentan [Säule 2 x $50 \mathrm{~cm}$, DC-Kontrolle, $\mathrm{R}_{\mathrm{f}}$ - Werte: 0.83 (69), 0.80 (68), 0.50 (44)] lieferte $48 \mathrm{mg}(14 \%)$ 69, $85 \mathrm{mg}$ (24\%) einer Mischfraktion von 69 (56) und 68 
(44), 44 mg (13\%) 68, sowie 129 mg (37\%) (44). Die spektoskopischen Daten stimmten mir denen von authentischem Material, bzw. den Literaturdaten ${ }^{11}$ überein.

\section{Kristallstrukturdaten}

Röntgenstrukturanalyse von $\left(1 R^{*}, 5 R^{*}, 6 R^{*}, 2^{\prime} R^{*}, 3^{\prime} R^{*}\right)-1,4,4,3^{\prime}$-Tetramethylspiro\{bicyclo[3.2.0]heptan-6,1'-cyclo-pentan-2'-ol\} (60), und $\left(1 R^{*}, 5 R^{*}, 6 R^{*}, 2^{\prime} R^{*}, 3^{\prime} S^{*}\right)$ 1,4,4,3'-Tetramethylspiro\{bicyclo[3.2.0]heptan-6,1'-cyclopentan-2'-ol $\}$ (66): Farblose Blöcke von 60 und 66 (Molekülformel $\mathrm{C}_{15} \mathrm{H}_{26} \mathrm{O}, \mathrm{M}=222.37$ ) wurden aus Pentan (60), bzw. Pentan/Ether 9:1 (66) erhalten und an einem Stoe-Vierkreisdiffraktometer (Mo-K $\alpha$-Strahlung, $\left.2 \theta=3.51-25.01^{\circ}\right)$ bei $\mathrm{T}=200(2) \mathrm{K}$ vermessen. Für 66 ergaben sich folgende Kristalldaten: Raumgruppe $\mathrm{P} \overline{1}, \mathrm{a}=12.019 \AA, \mathrm{b}=12.080 \AA, \mathrm{c}=20.751 \AA, \alpha=87.0^{\circ}, \beta=86.80^{\circ}, \gamma=72.57^{\circ}$ $\mathrm{Z}=8$. Die vier unabhängigen Moleküle konnten mit direkten Methoden bestimmt werden. Die Qualität der Messdaten reichte für eine Verfeinerung jedoch nicht aus. Nur 3536 der 7139 unabhängigen Reflexe sind signifikant $\left(\mathrm{F}_{0}>4 \sigma\left(\mathrm{F}_{0}\right)\right)$. Für $\mathbf{6 0}$ sind die Kristalldaten in Tabelle 5 und die Atomkoordinaten in Tabelle 6 aufgeführt. 
Tabelle 5: Kristalldaten und Strukturverfeinerung von 60.

Wellenlänge:

Kristallsystem:

Raumgruppe:

Zelldimensionen:

Zellvolumen:

$\mathrm{Z}:$

Dichte (berechnet):

Absorptionskoeffizient:

$\mathrm{F}(000)$ :

Kristallgröße:

Index Grenzen:

GesammelteReflexe

Unabhängige Reflexe

Strukturverfeinerung:

Daten / restraints / Parameter:

Goodness-of-fit an $\mathrm{F}^{2}$

Endgültige R-Werte [I > $2 \sigma(\mathrm{I})]$

R-Werte (sämtliche Daten)

Größtes Maximum und Minimum
$0.71073 \AA$

Tetragonal

I4(1)/a

$\mathrm{a}=26.691(4) \AA$

$\alpha=90^{\circ}$

$\mathrm{b}=26.691(4) \AA$

$\beta=90^{\circ}$

$\mathrm{c}=7.836(2) \AA$

$5582.4(16) \AA^{3}$

$\gamma=90^{\circ}$

16

$1.201 \mathrm{Mg} / \mathrm{m}^{3}$

$0.072 \mathrm{~mm}^{-1}$

2176

$0.60 \times 0.60 \times 0.20 \mathrm{~mm}^{3}$

$-31 \leq \mathrm{h} \leq 31,-31 \leq \mathrm{k} \leq 31,-4 \leq 1 \leq 9$

4365

2455 [R(int) $=0.0274]$

Full-matrix least-squares on $\mathrm{F}^{2}$

2448 / 0 / 149

1.058

$\mathrm{R} 1=0.0590, \mathrm{wR} 2=0.1418$

$\mathrm{R} 1=0.0885, \mathrm{wR} 2=0.1755$

0.406 und $-0.300 \mathrm{e} . \AA^{-3}$ 
Tabelle 6: Atomkoordinaten ( x 10 ${ }^{4}$ ) und äquivalente isotrope Auslenkungsparameter $\left(\AA^{2} \quad \mathrm{x} \quad 10^{3}\right)$ für $\left(1 R^{*}, 5 R^{*}, 6 R^{*}, 2^{\prime} R^{*}, 3^{\prime} R^{*}\right)-1,4,4,3^{\prime}$-Tetramethylspiro\{bicyclo[3.2.0]heptan-6,1'-cyclo-pentan-2'-ol\} (60). U(eq) wird berechnet als ein Drittel der Spur des orthogonalen $\mathrm{U}_{\mathrm{ij}}$-Tensors.

\begin{tabular}{lccrc}
\hline & $\mathbf{x}$ & $\mathbf{y}$ & $\mathbf{z}$ & $\mathbf{U}(\mathbf{e q})$ \\
\hline $\mathrm{C}(1)$ & $6063(1)$ & $5611(1)$ & $-602(3)$ & $40(1)$ \\
$\mathrm{C}\left(1^{*}\right)$ & $6502(1)$ & $5845(1)$ & $-1533(4)$ & $67(1)$ \\
$\mathrm{O}(2)$ & $5391(1)$ & $6872(1)$ & $1181(2)$ & $43(1)$ \\
$\mathrm{C}\left(2^{\prime}\right)$ & $5722(1)$ & $6515(1)$ & $1963(3)$ & $33(1)$ \\
$\mathrm{C}(2)$ & $5975(1)$ & $5057(1)$ & $-1041(4)$ & $53(1)$ \\
$\mathrm{C}\left(3^{\prime}\right)$ & $5639(1)$ & $6465(1)$ & $3876(3)$ & $42(1)$ \\
$\mathrm{C}(3)$ & $5767(1)$ & $4825(1)$ & $581(4)$ & $46(1)$ \\
$\mathrm{C}\left(3^{\prime \prime}\right)$ & $5637(1)$ & $6958(1)$ & $4855(4)$ & $61(1)$ \\
$\mathrm{C}\left(4^{*}\right)$ & $5818(1)$ & $5001(1)$ & $3785(4)$ & $56(1)$ \\
$\mathrm{C}(4)$ & $6046(1)$ & $5093(1)$ & $2031(3)$ & $39(1)$ \\
$\mathrm{C}\left(4^{\prime \prime}\right)$ & $6591(1)$ & $4906(1)$ & $2090(4)$ & $56(1)$ \\
$\mathrm{C}\left(4^{\prime}\right)$ & $5151(1)$ & $6162(1)$ & $3995(4)$ & $51(1)$ \\
$\mathrm{C}\left(5^{\prime}\right)$ & $5102(1)$ & $5889(1)$ & $2284(3)$ & $39(1)$ \\
$\mathrm{C}(5)$ & $6060(1)$ & $5640(1)$ & $1398(3)$ & $32(1)$ \\
$\mathrm{C}(6)$ & $5587(1)$ & $5993(1)$ & $1303(3)$ & $32(1)$ \\
$\mathrm{C}(7)$ & $5580(1)$ & $5929(1)$ & $-646(3)$ & $41(1)$ \\
\hline
\end{tabular}


SPEKTRENANHANG

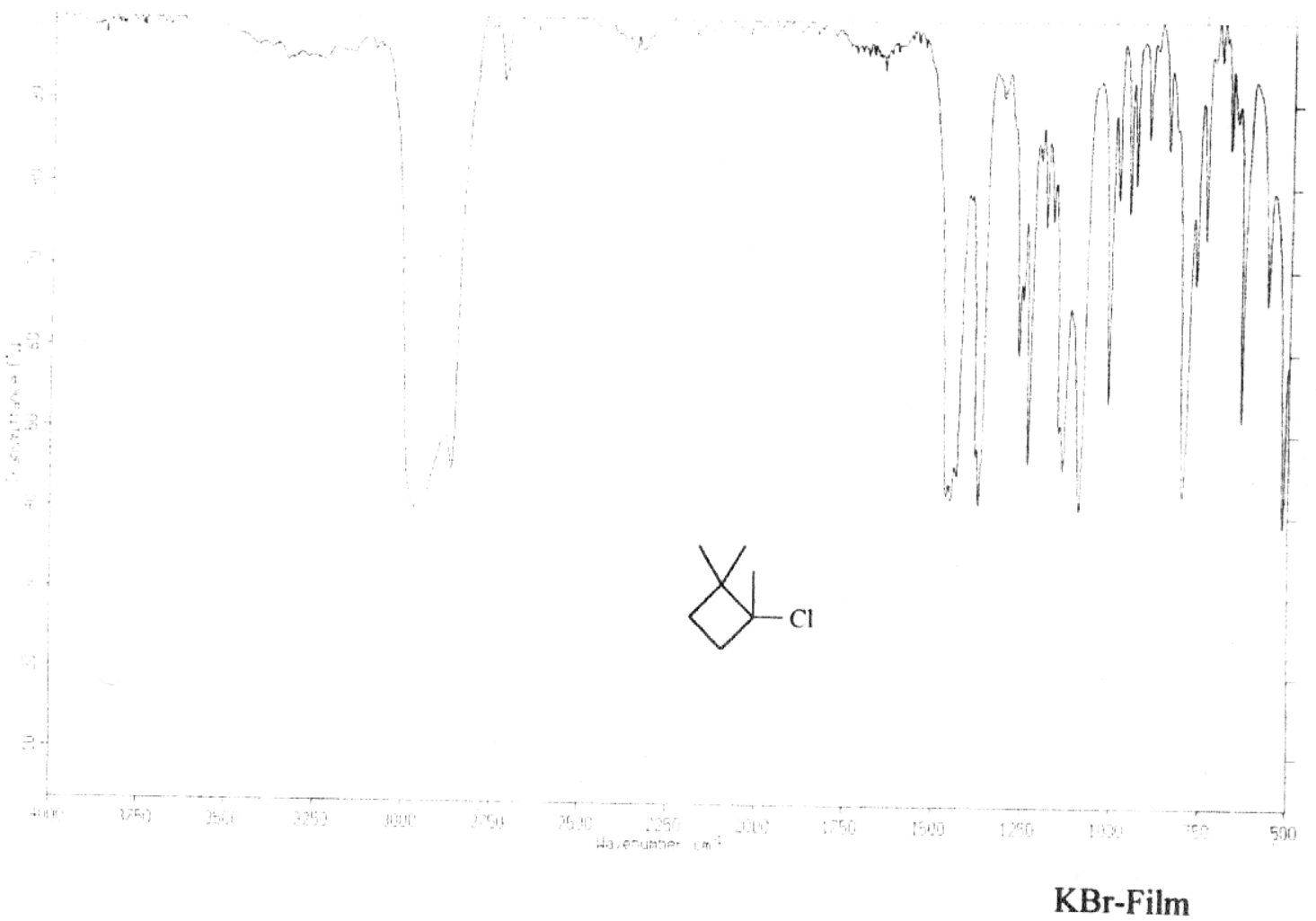

Abb. 3: IR-Spekrum von 1,2,2-Trimethyl-cyclobutylchlorid (97)

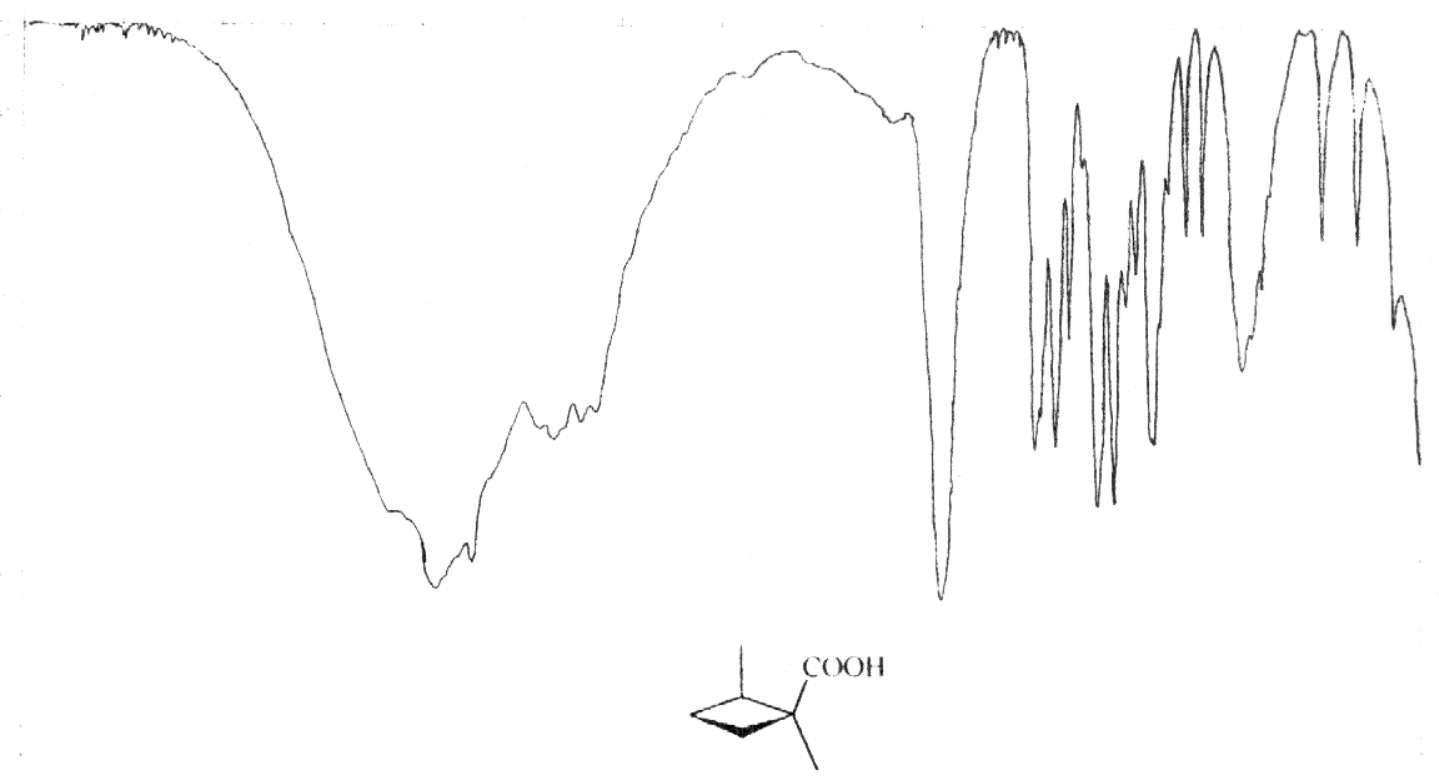

Abb. 4: IR-Spektrum von $\left(1 R^{*}, 2 S^{*}\right)$-1,2-Dimethyl-cyclobutancarbonsäure (98a) 


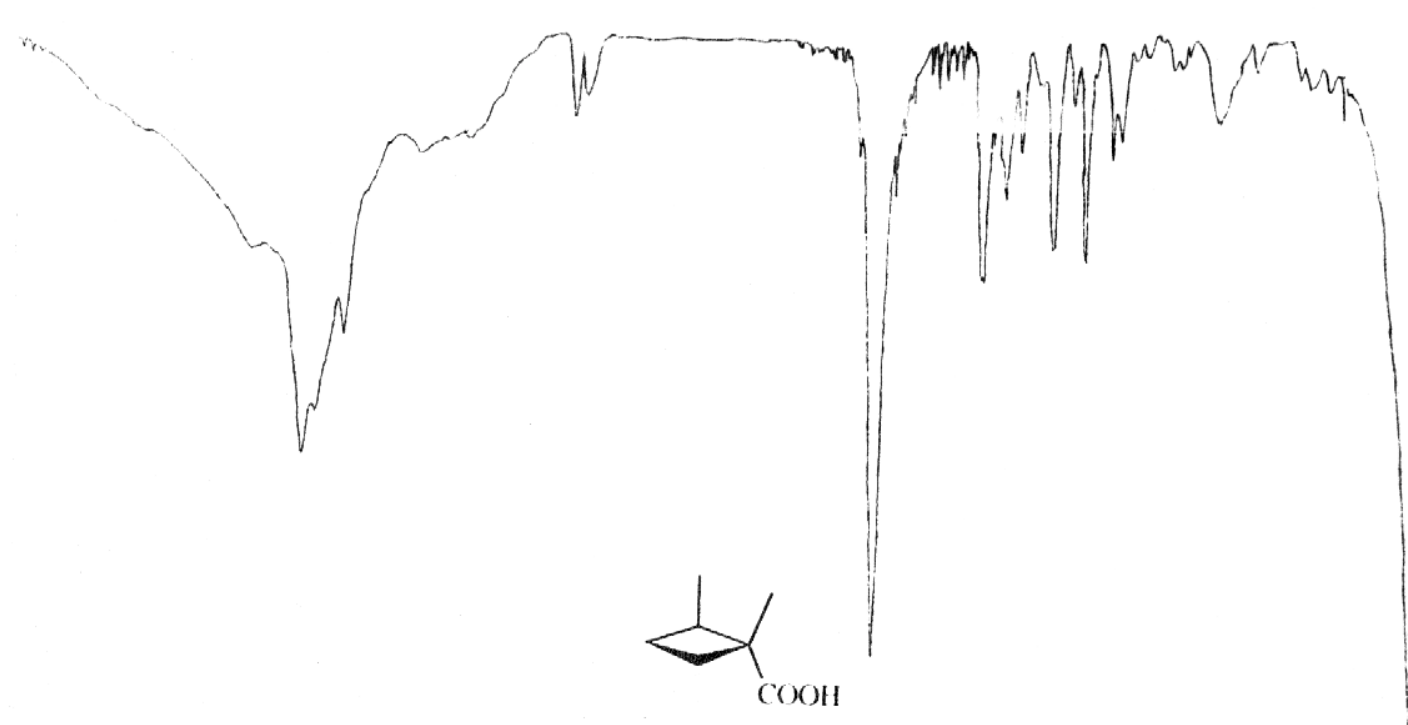

KBr-Film

Abb. 5: $\quad$ IR-Spektrum von $\left(1 R^{*}, 2 R^{*}\right)$-1,2-Dimethyl-cyclobutancarbonsäure (98b)

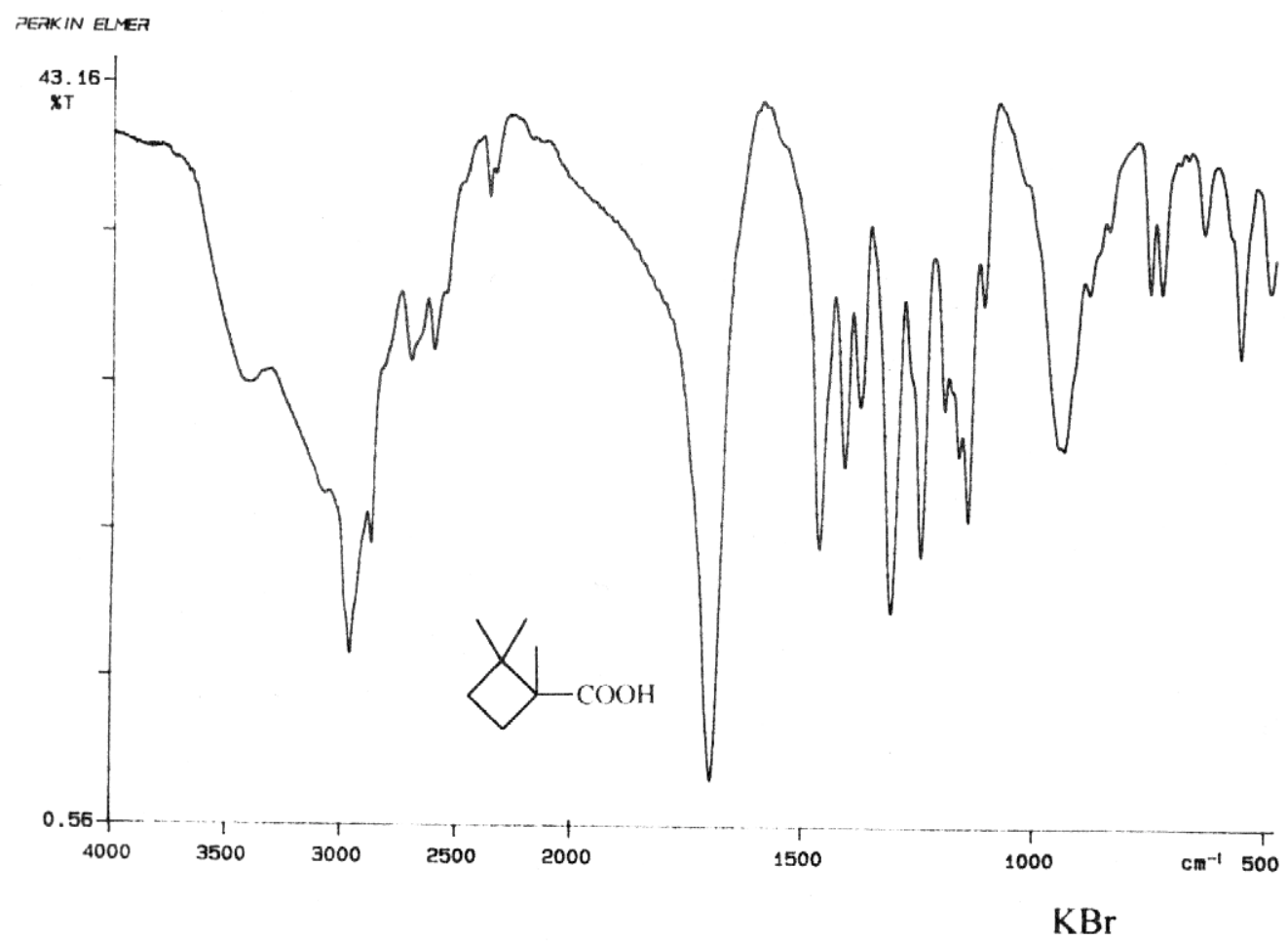

Abb. 6: $\quad$ IR-Spektrum von 1,2,2-Trimethyl-cyclobutancarbonsäure (100) 


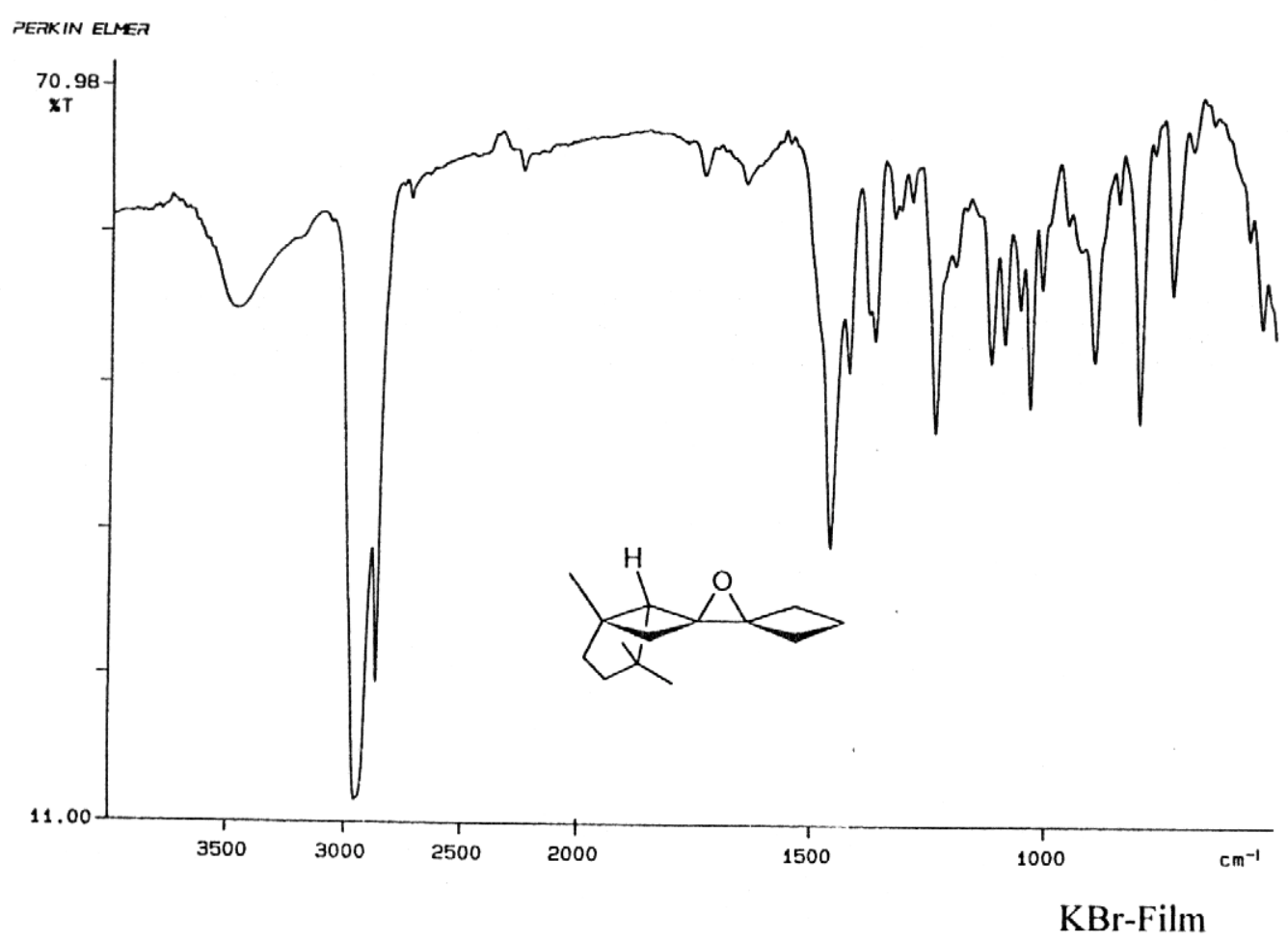

Abb. 7: IR-Spektrum von 2,2-Spiro-cyclobutyl-3,3-spiro-[(1 $\quad$ I $\left., 5 R^{*}, 6 R^{*}\right)$-1,4,4-trimethylbicyclo[3.2.0]heptan-6,6-yl]-oxiran (55)

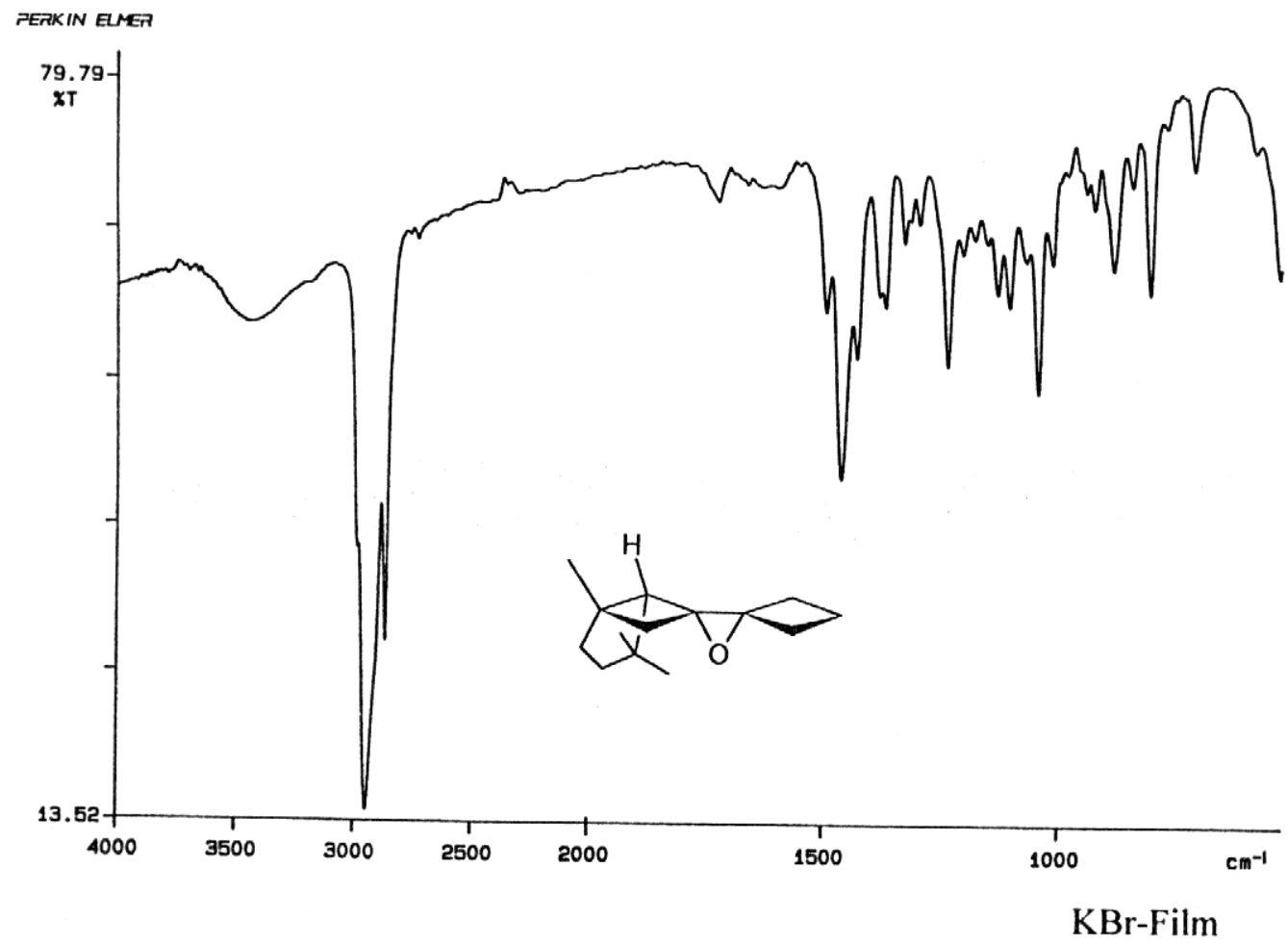

Abb. 8: $\quad$ IR-Spektrum von 2,2-Spiro-cyclobutyl-3,3-spiro-[( $\left.1 R^{*}, 5 R^{*}, 6 S^{*}\right)$-1,4,4-trimethylbicyclo[3.2.0]heptan-6,6-yl]-oxiran (56) 


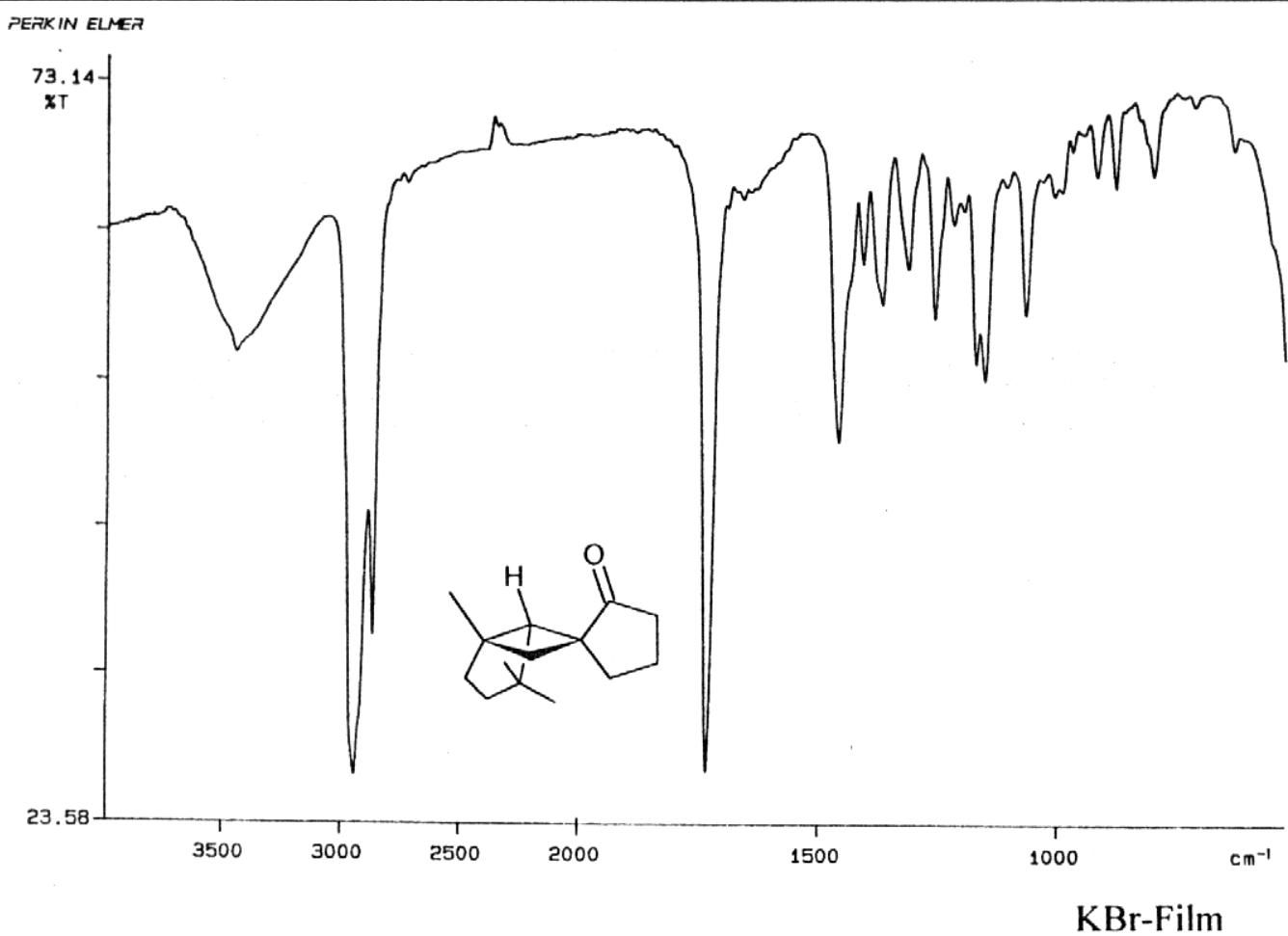

Abb. 9: IR-Spektrum von $\left(1 R^{*}, 5 R^{*}, 6 R^{*}\right)-1,4,4$-Trimethylspiro\{bicyclo[3.2.0]heptan$6,1^{\prime}$-cyclopentan-2'-on\} (57)

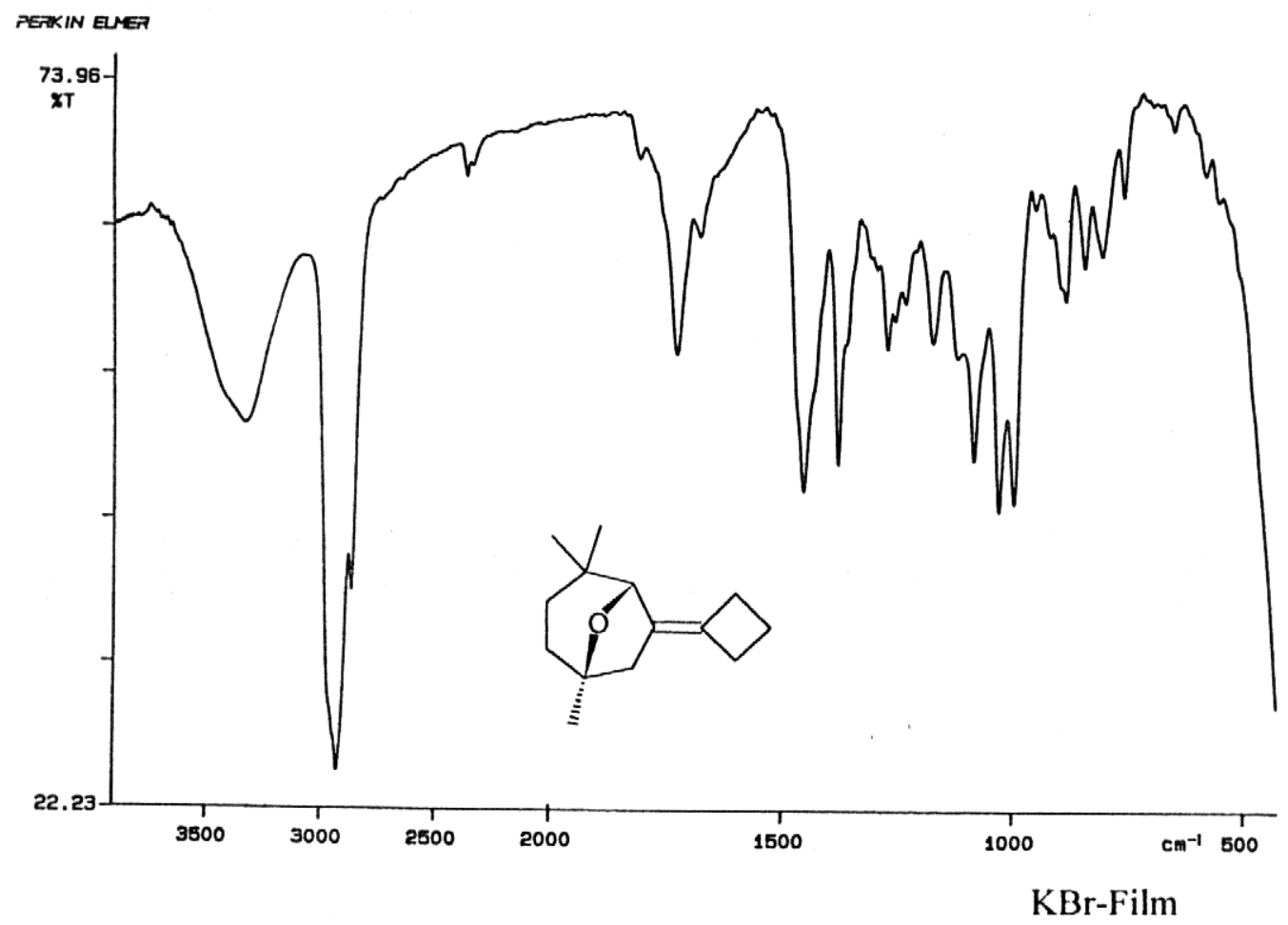

Abb. 10: IR-Spektrum von 6-Cyclobutyliden-1,4,4-trimethyl-8-oxa-bicyclo[3.2.1]octan (138) 


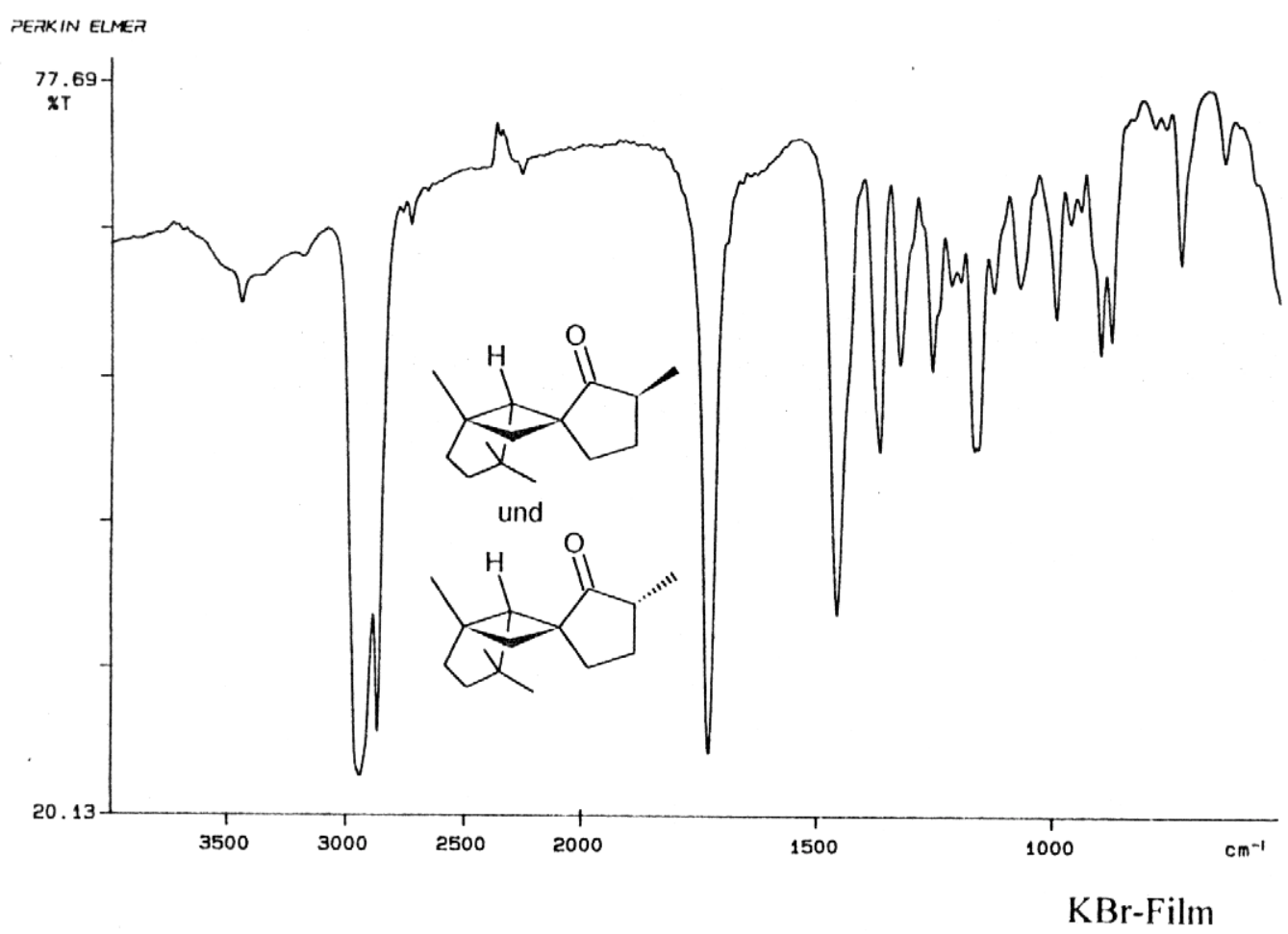

Abb. 11: IR-Spektrum von $\left(1 R^{*}, 5 R^{*}, 6 R^{*}, 3 S^{*}\right)-1,4,4,3^{\prime}$-Tetramethylspiro $\{$ bicyclo[3.2.0]heptan-6, $1^{\prime}$-cyclopentan-2'-on $\}$ (141) und $\left(1 R^{*}, 5 R^{*}, 6 R^{*}, 3 R^{*}\right)-1,4,4,3^{\prime}-$ Tetramethylspiro\{bicyclo[3.2.0]heptan-6,1'-cyclopentan-2'-on $\}$ (142)

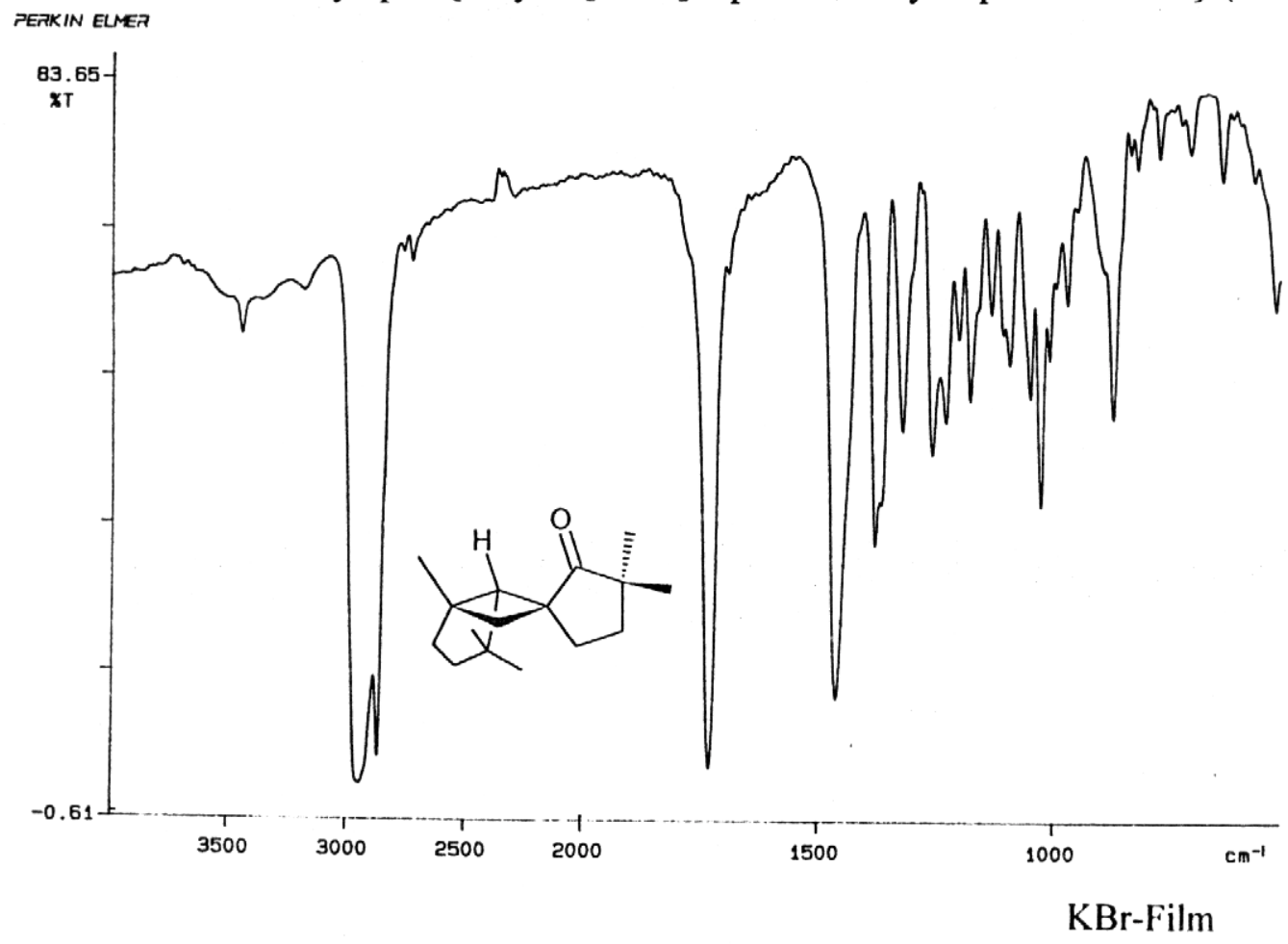

Abb. 12: IR-Spektrum von $\left(1 R^{*}, 5 R^{*}, 6 R^{*}\right)-1,4,4,3^{\prime}, 3^{\prime}$-Pentamethylspiro bicyclo[3.2.0]heptan-6, $1^{\prime}$-cyclopentan-2'-on\} (143) 


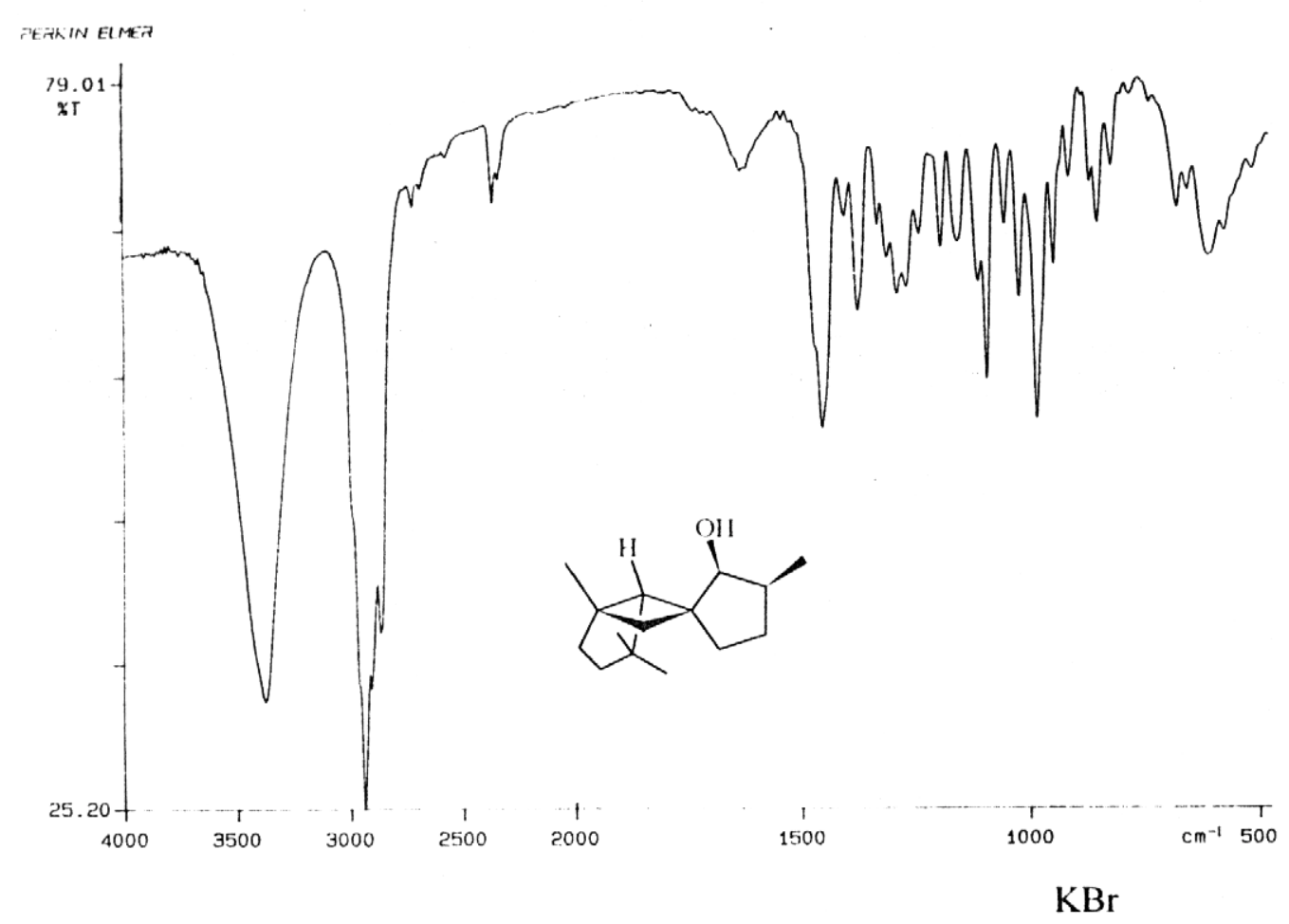

Abb. 13: IR-Spektrum von $\left(1 R^{*}, 5 R^{*}, 6 R^{*}, 2^{\prime} R^{*}, 3^{\prime} R^{*}\right)-1,4,4,3^{\prime}-$ Tetramethylspiro \{bicyclo[3.2.0]heptan-6,1'-cyclo-pentan-2'-ol\} (60)

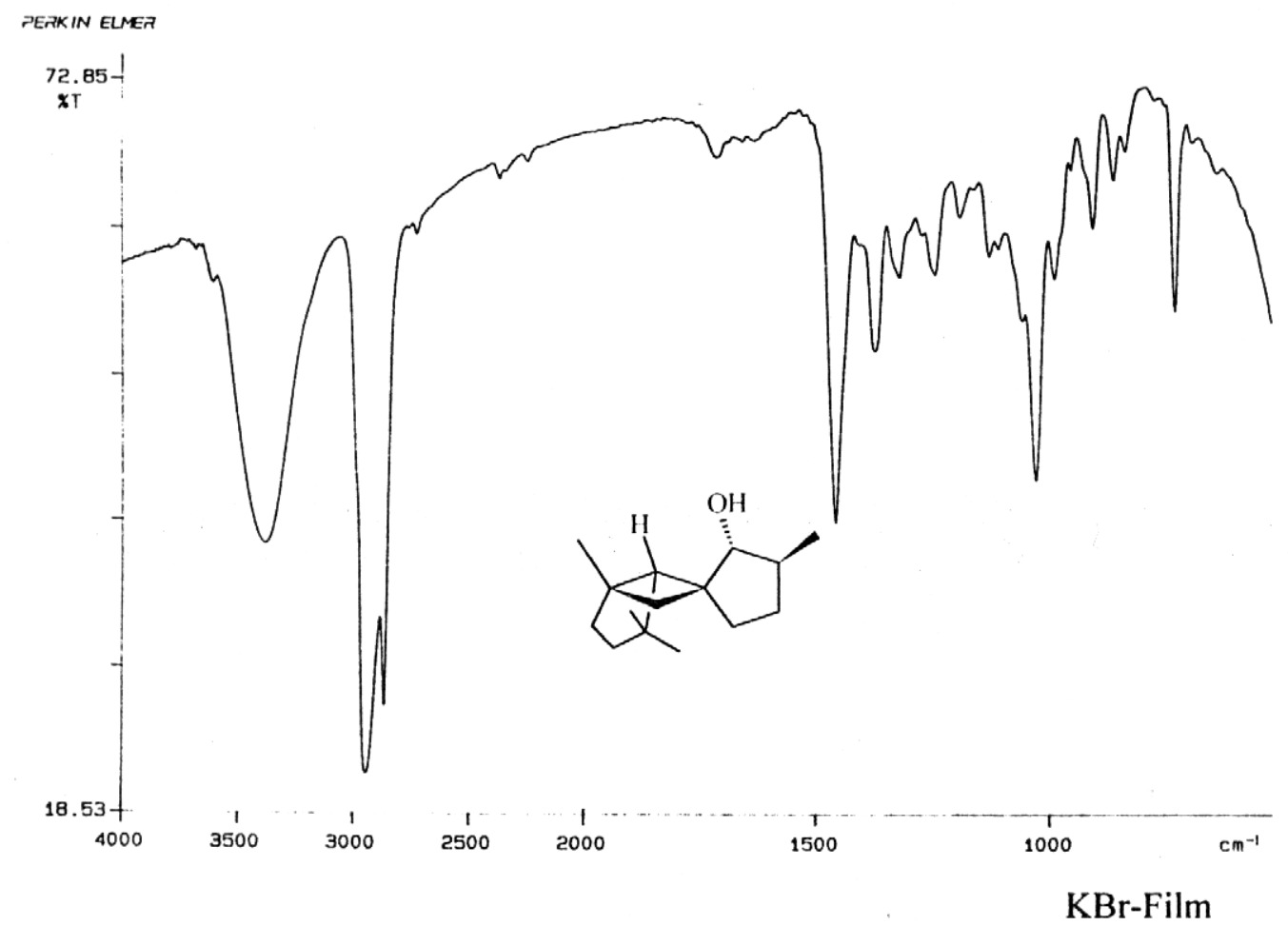

Abb. 14: IR-Spektrum von $\left(1 R^{*}, 5 R^{*}, 6 R^{*}, 2^{\prime} S^{*}, 3^{\prime} R^{*}\right)-1,4,4,3^{\prime}$-Tetramethylspiro bicyclo[3.2.0]heptan-6,1'-cyclopentan-2'-ol\} (64) 


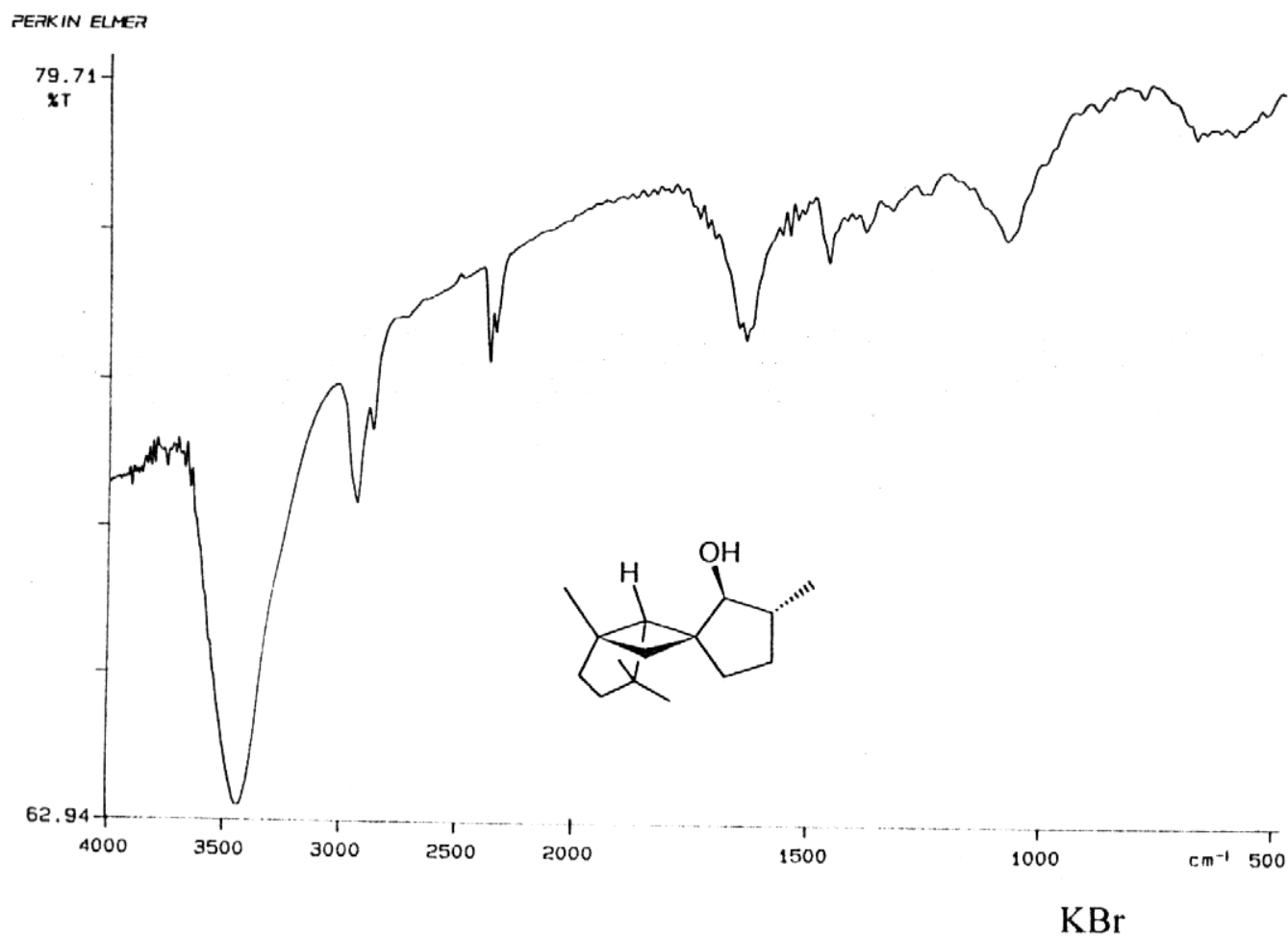

Abb. 15: IR-Spektrum von $\left(1 R^{*}, 5 R^{*}, 6 R^{*}, 2^{\prime} R^{*}, 3^{\prime} S^{*}\right)-1,4,4,3^{\prime}$-Tetramethylspiro bicyclo[3.2.0]heptan-6,1'-cyclopentan-2'-ol\} (66)

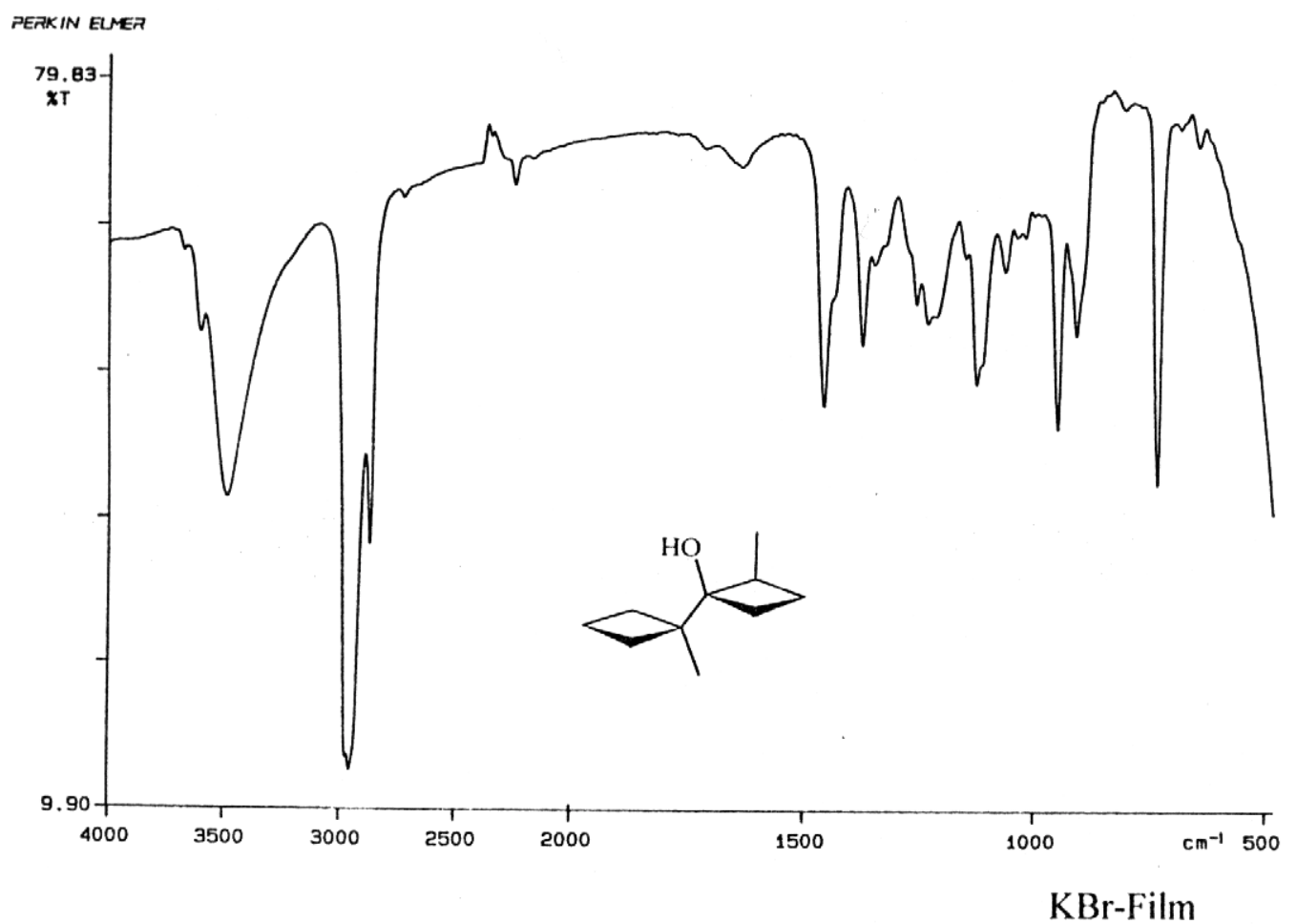

Abb. 16: IR-Spektrum von $\left(1 R^{*}, 2 S^{*}\right)-2,1^{\prime}$-Dimethyl-bicyclobutyl-1-ol (104b) 


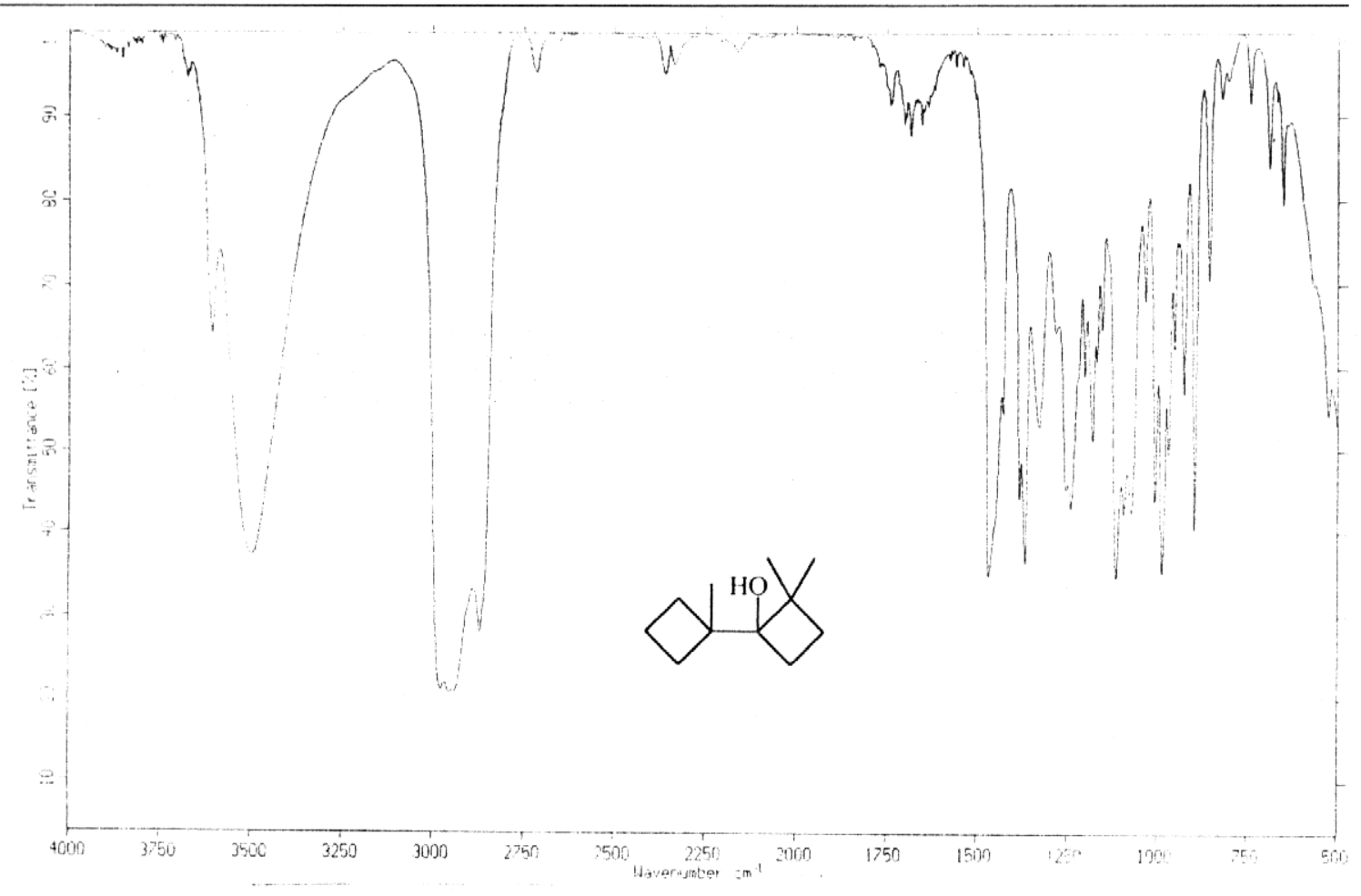

KBr-Film

Abb. 17: IR-Spektrum von 2,2,1'-Trimethyl-bicyclobutyl-1-ol (105)

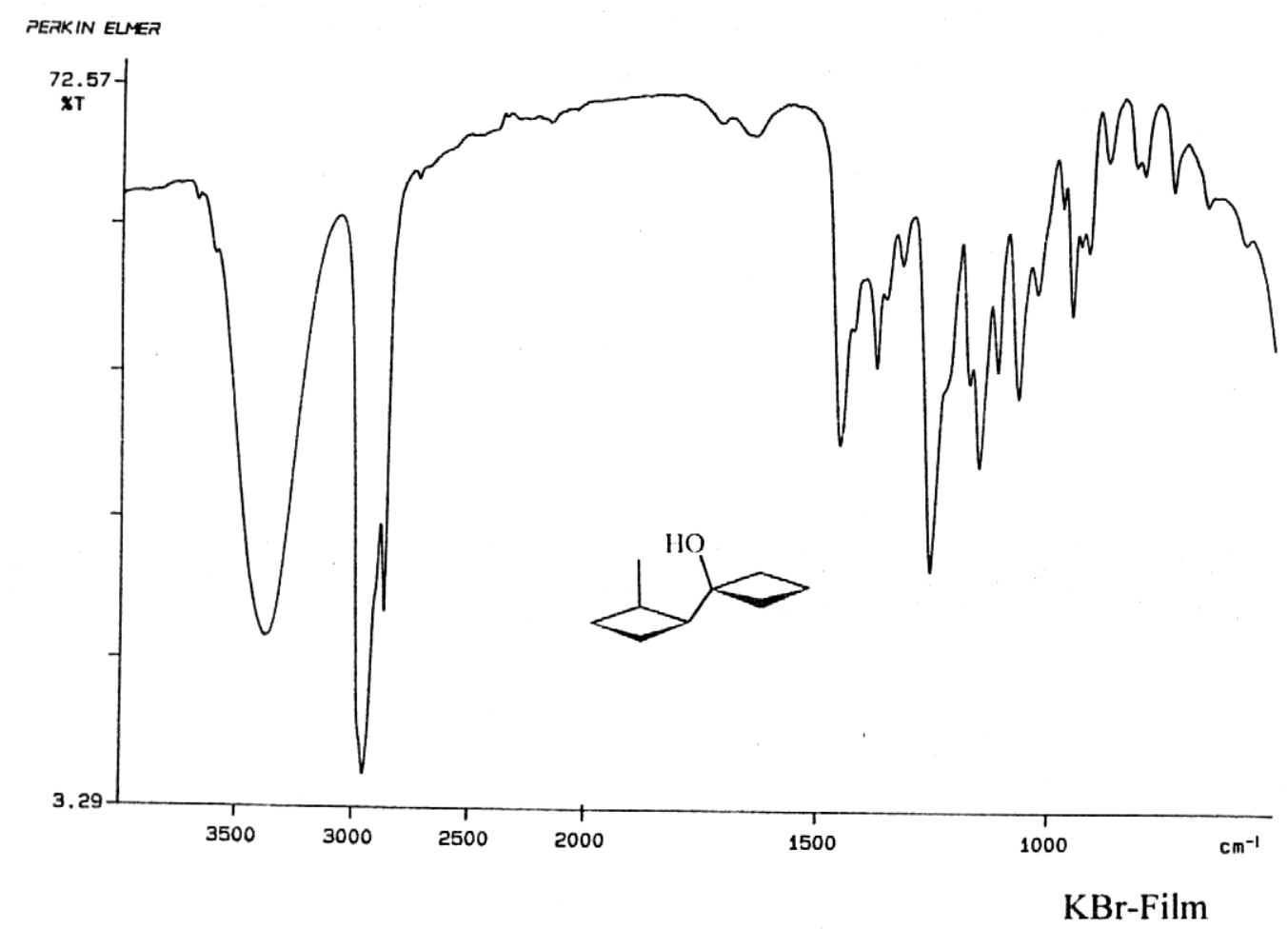

Abb. 18: IR-Spektrum von $\left(1^{\prime} R^{*}, 2^{\prime} S^{*}\right)-2^{\prime}$-Methyl-bicyclobutyl-1-ol (106a) 


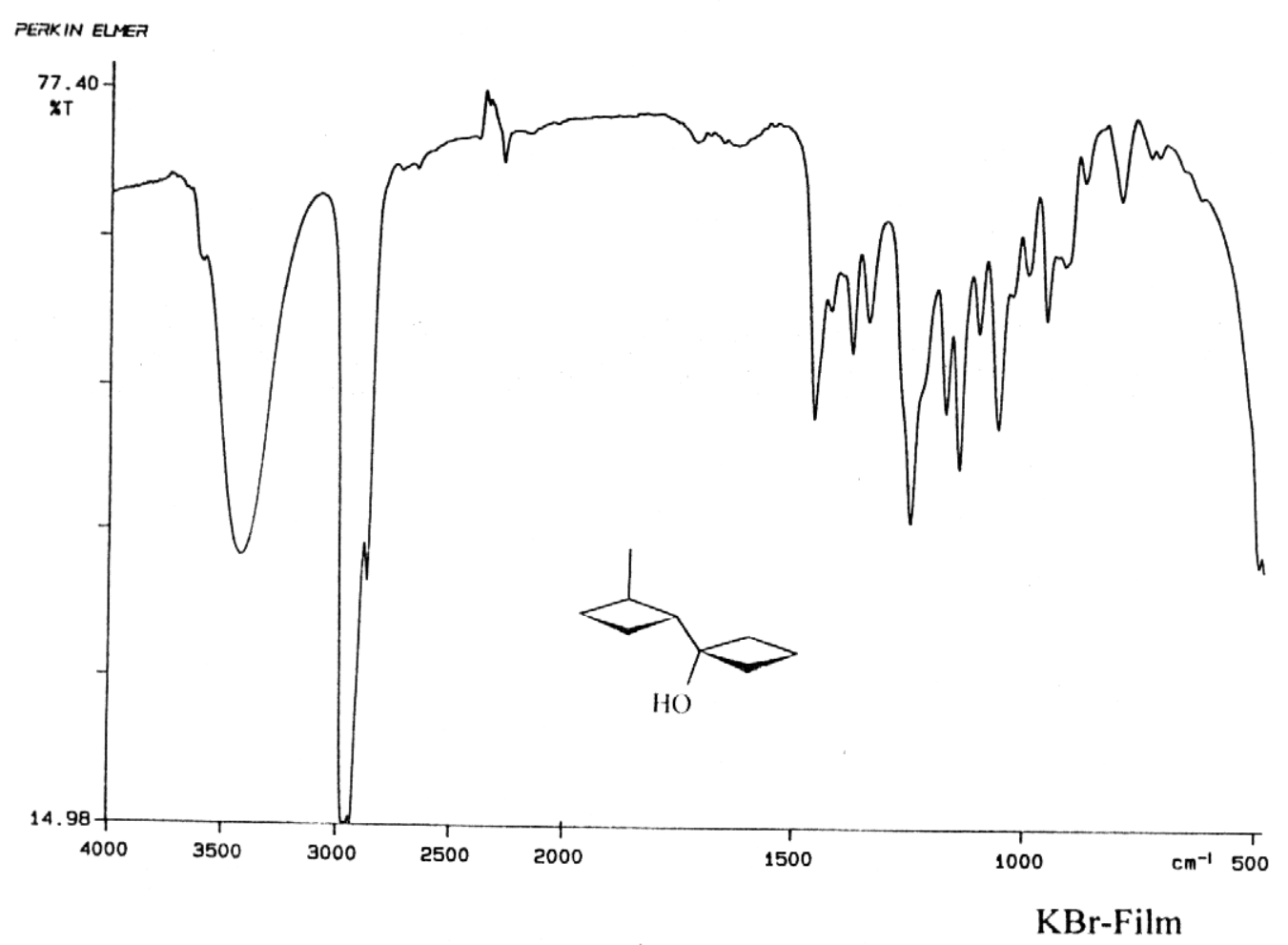

Abb. 19: IR-Spektrum von $\left(1^{\prime} R^{*}, 2^{\prime} R^{*}\right)-2^{\prime}$-Methyl-bicyclobutyl-1-ol (106b)

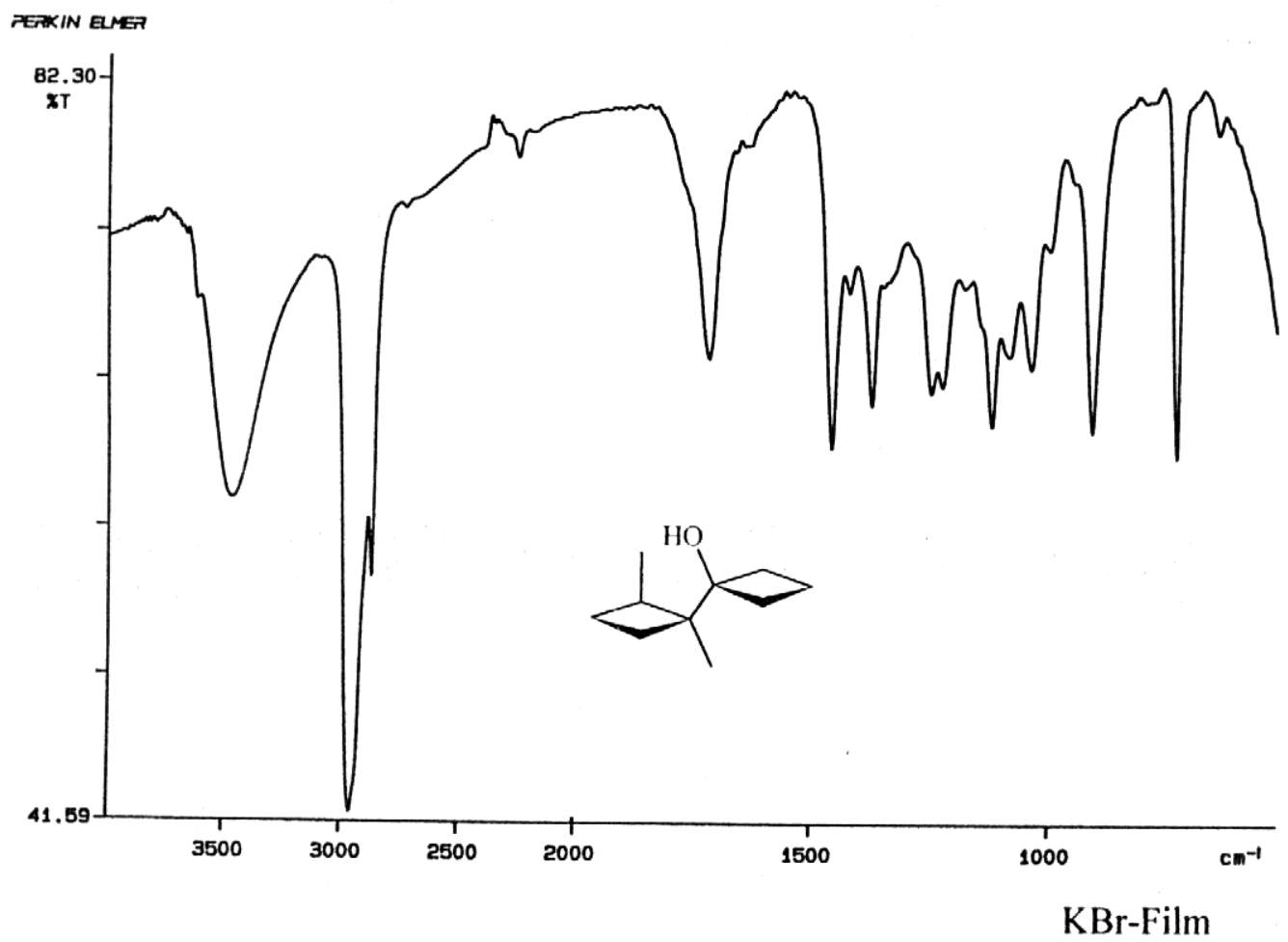

Abb. 20: IR-Spektrum von $\left(1^{\prime} R^{*}, 2^{\prime} S^{*}\right)-1^{\prime}, 2^{\prime}$-Dimethyl-bicyclobutyl-1-ol (107a) 


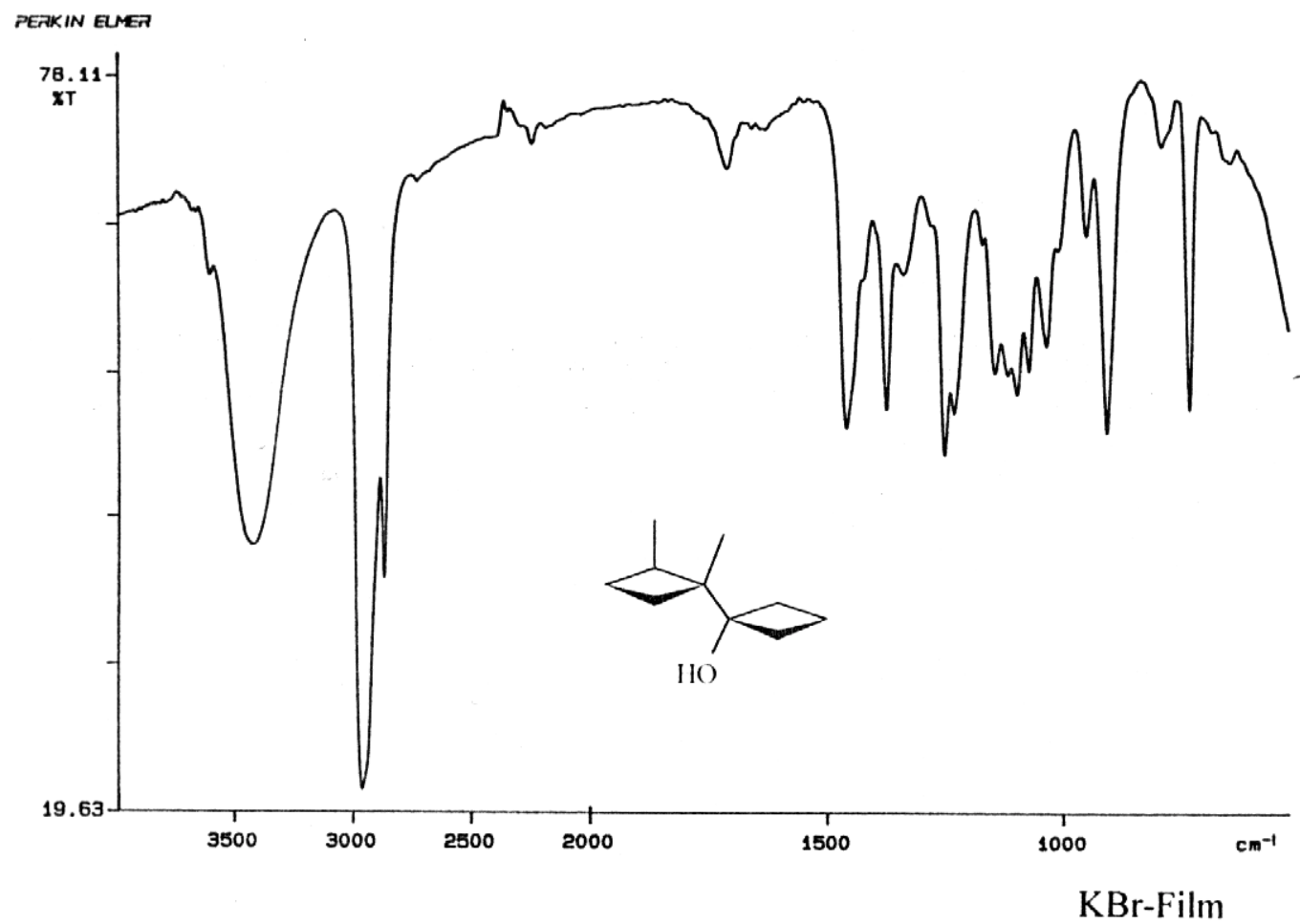

Abb. 21: $\quad$ IR-Spektrum von $\left(1^{\prime} R^{*}, 2^{\prime} R^{*}\right)-1^{\prime}, 2^{\prime}$-Dimethyl-bicyclobutyl-1-ol (107b)

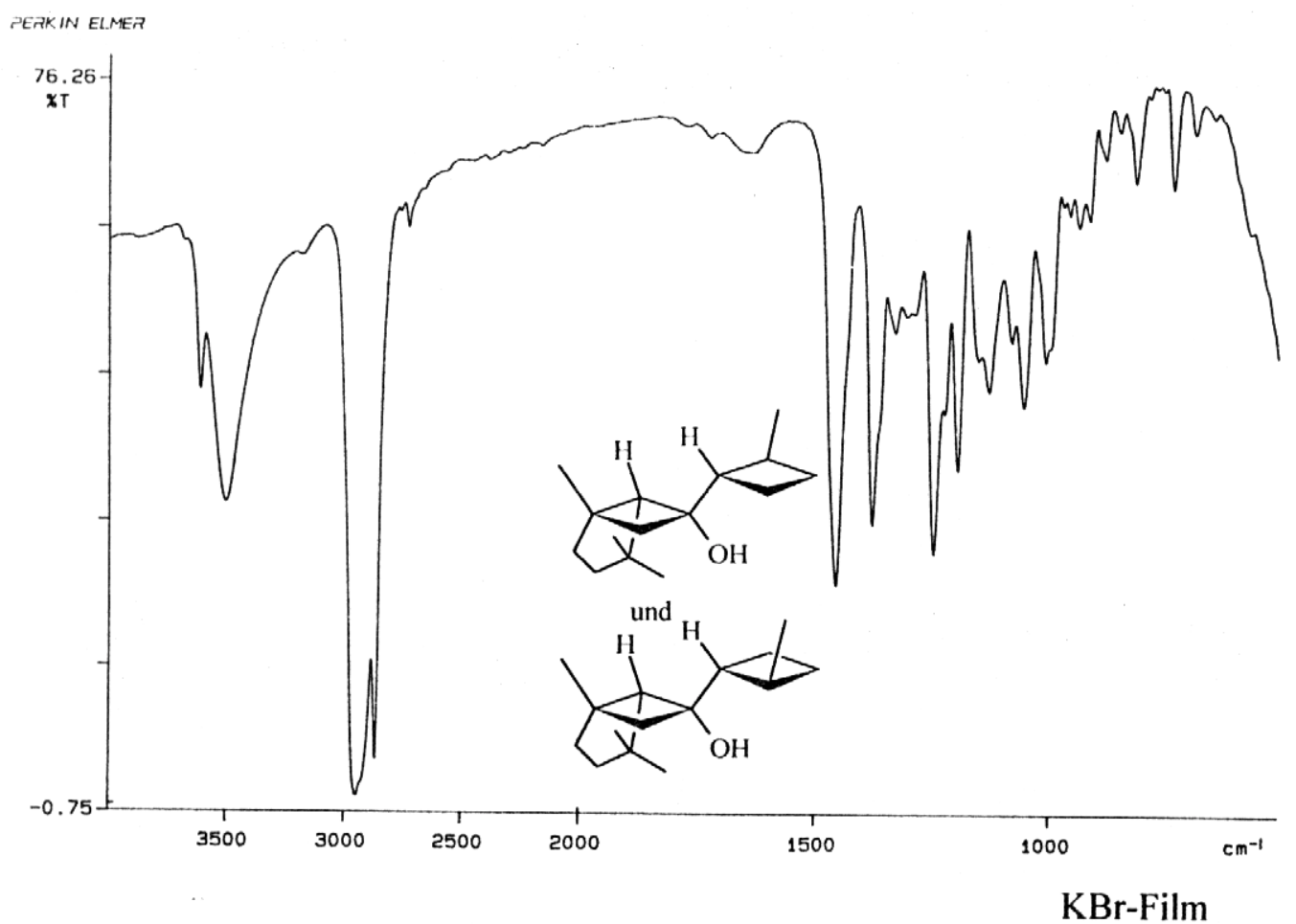

Abb. 22: IR-Spektrum von $\left(1 S^{*}, 5 S^{*}, 6 S^{*}, 1^{\prime} S^{*}, 2^{\prime} S^{*}\right)-1,4,4-$ Trimethyl-6-(2'-methyl-cyclobutyl)-bicyclo[3.2.0]heptan-6-ol (46) und $\left(1 S^{*}, 5 S^{*}, 6 S^{*}, 1^{\prime} R^{*}, 2^{\prime} R^{*}\right)-1,4,4$-Trimethyl-6-(2'-methyl-cyclobutyl)-bicyclo[3.2.0]heptan-6-ol (49) 


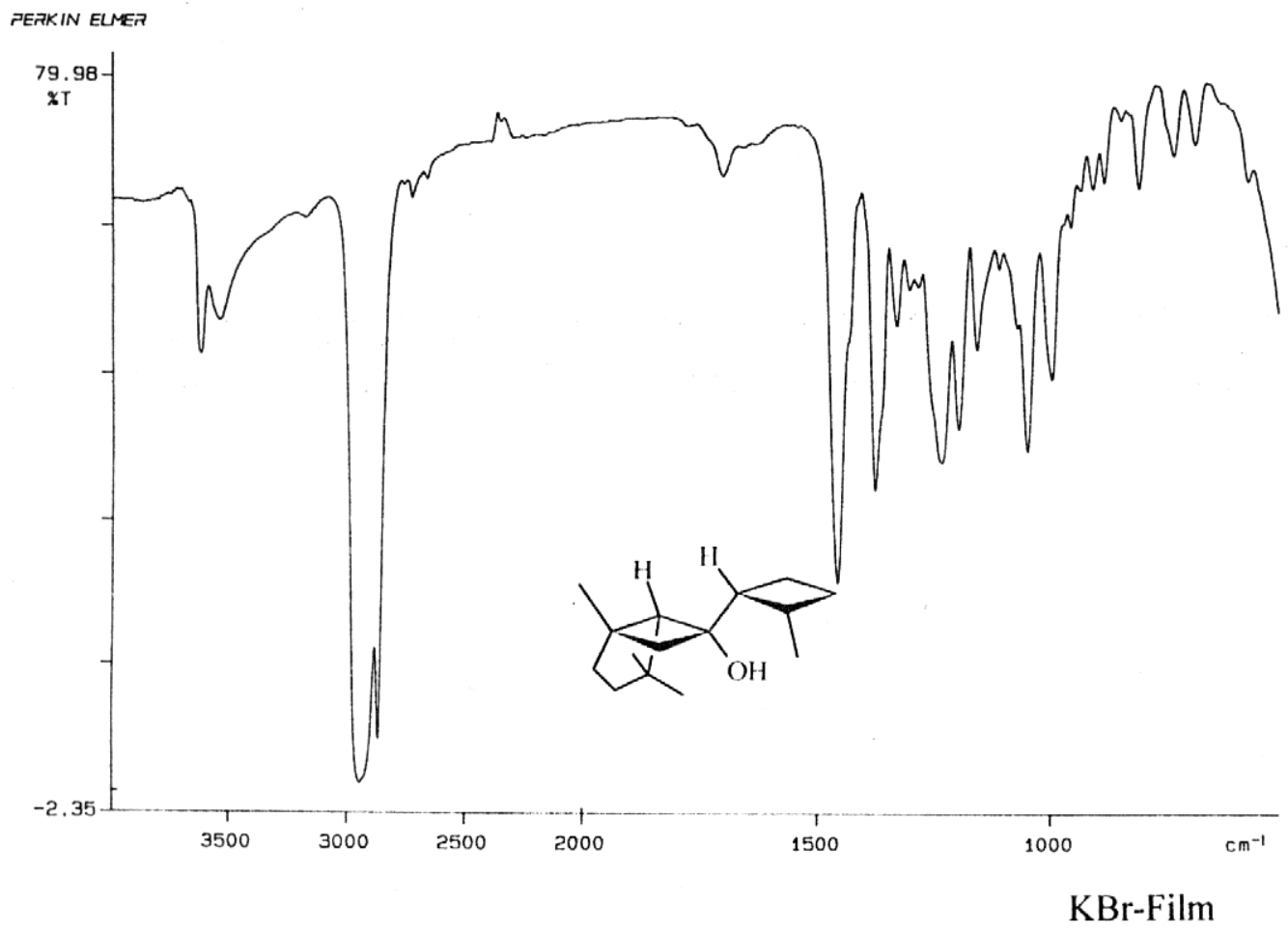

Abb. 23: IR-Spektrum von $\left(1 S^{*}, 5 S^{*}, 6 S^{*}, 1^{\prime} S^{*}, 2^{\prime} R^{*}\right)-1,4,4-$ Trimethyl-6-(2'-methyl-cyclobutyl)-bicyclo[3.2.0]heptan-6-ol (47)

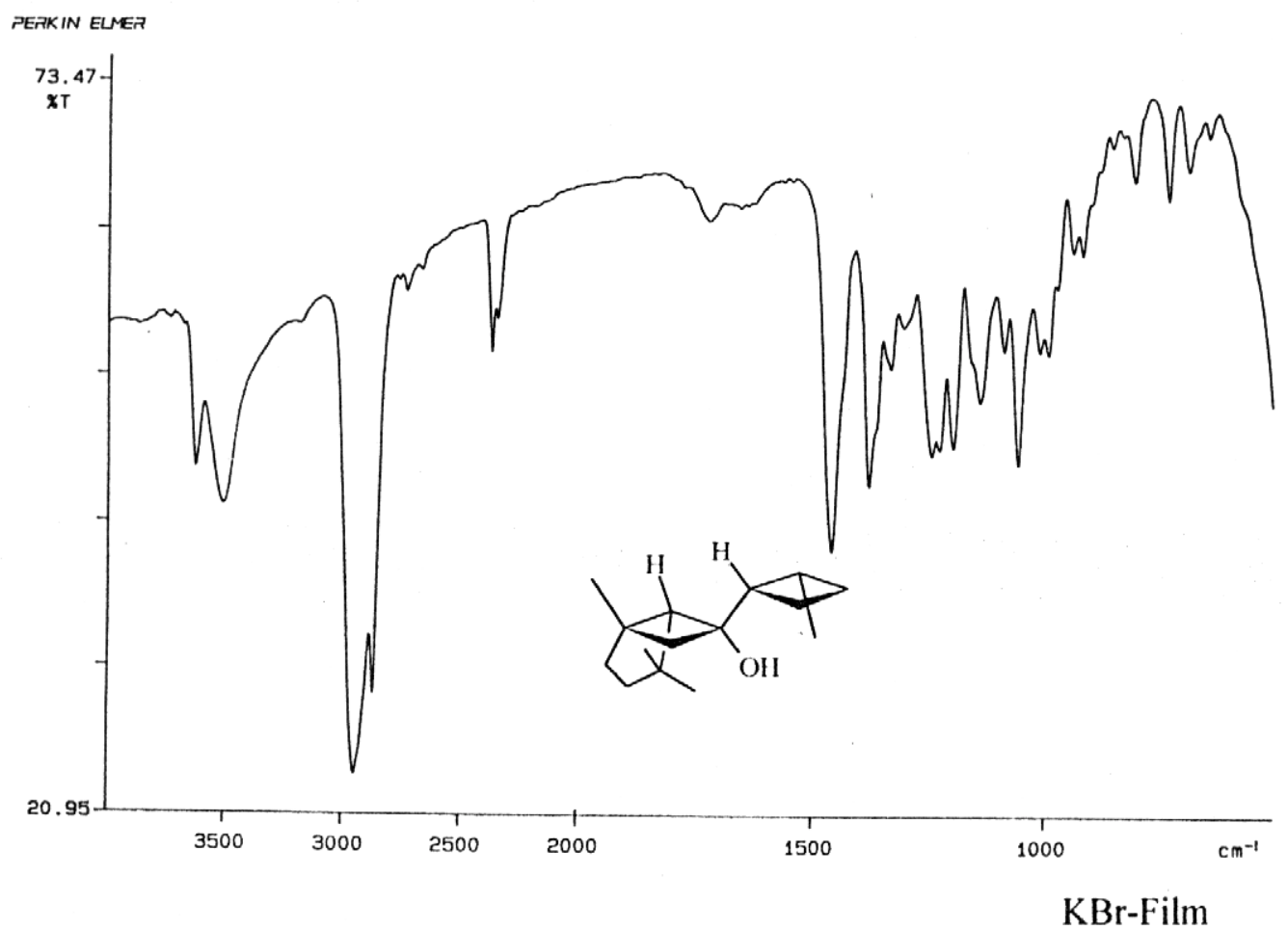

Abb. 24: IR-Spektrum von $\left(1 S^{*}, 5 S^{*}, 6 S^{*}, 1^{\prime} R^{*}, 2^{\prime} S^{*}\right)-1,4,4$-Trimethyl-6-(2'-methyl-cyclobutyl)-bicyclo[3.2.0]heptan-6-ol (48) 


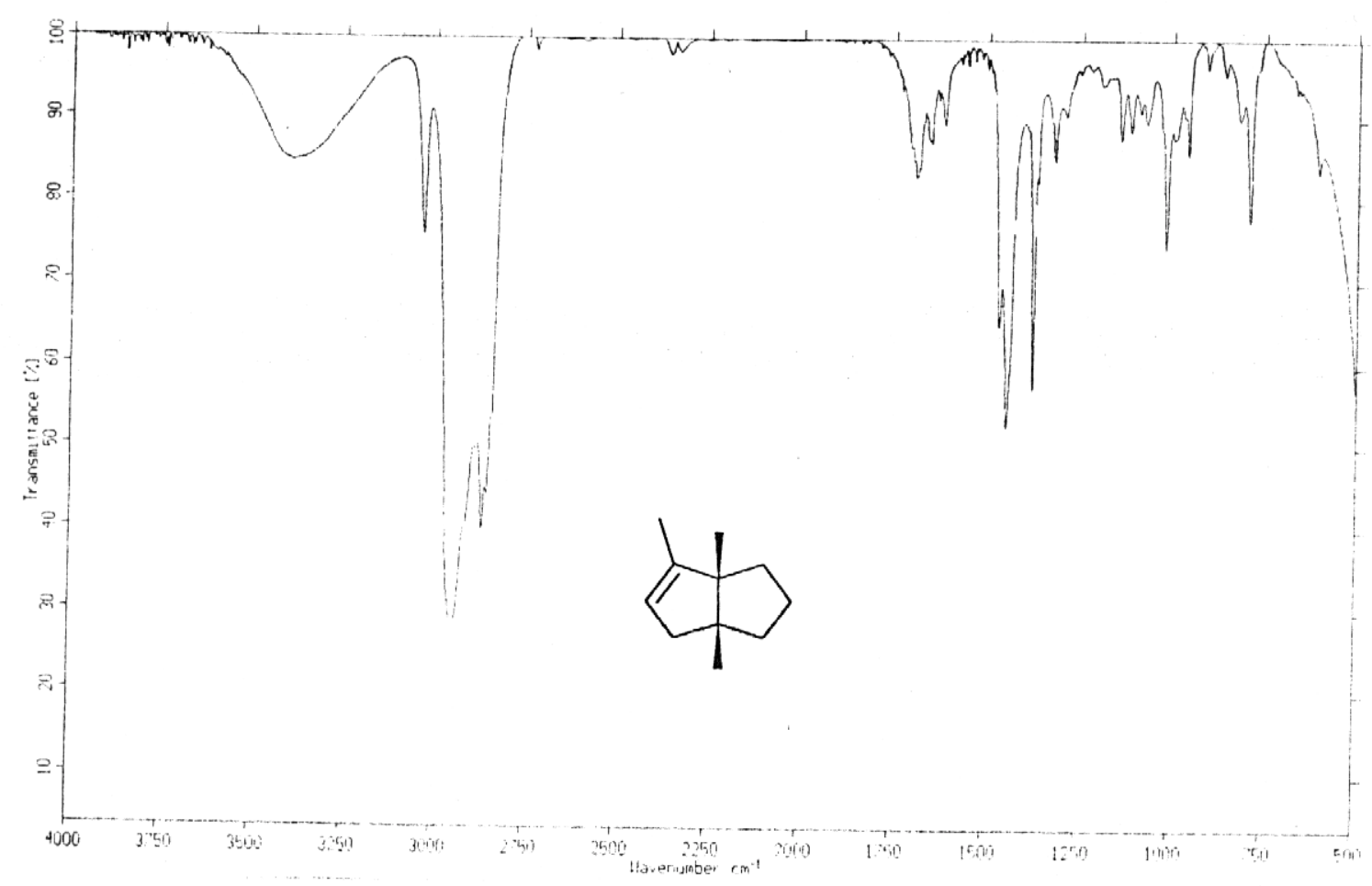

KBr-Film

Abb. 25: IR-Spektrum von $\left(3 \mathrm{a} R^{*}, 6 \mathrm{a} S^{*}\right)-3 \mathrm{a}, 6,6 \mathrm{a}-$ Trimethyl-1,2,3,3a,4,6a-hexahydropentalen (118)

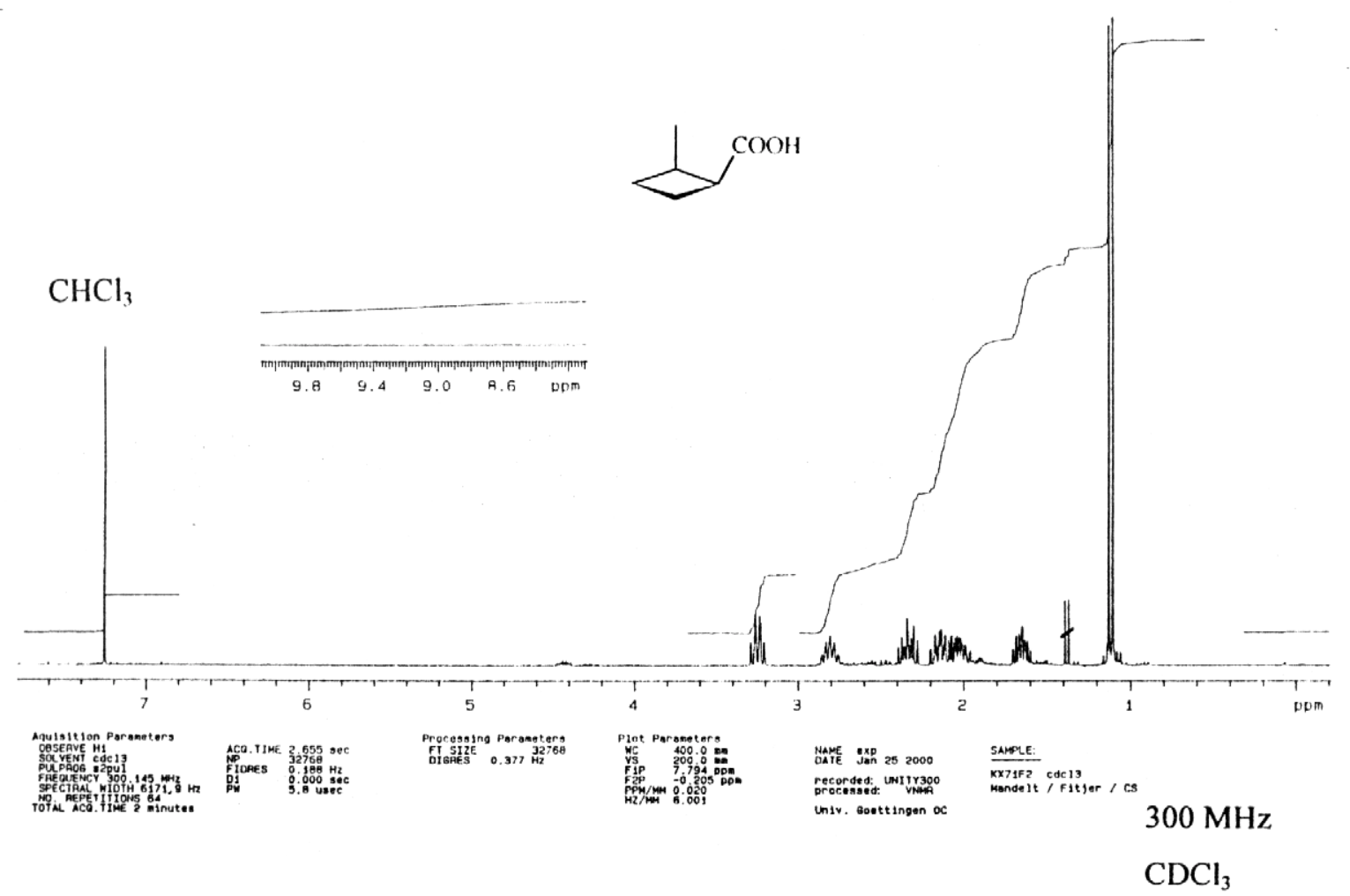

Abb. 26: $\quad{ }^{1}$ H-NMR-Spektrum von $\left(1 R^{*}, 2 S^{*}\right)$-2-Methyl-cyclobutancarbonsäure (81a) 


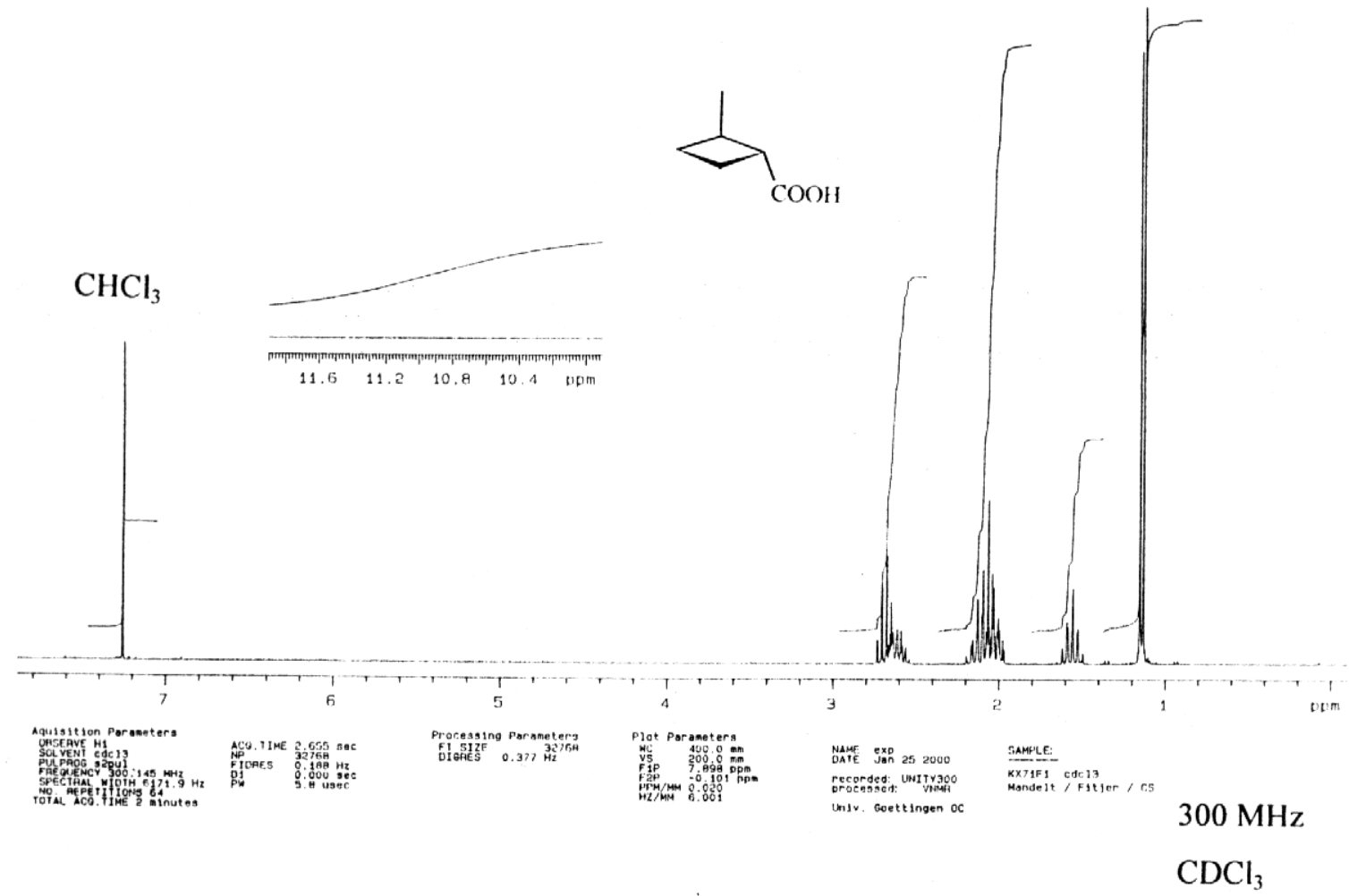

Abb. 27: $\quad{ }^{1}$ H-NMR-Spektrum von $\left(1 R^{*}, 2 R^{*}\right)$-2-Methyl-cylobutancarbonsäure $(\mathbf{8 1 b})$

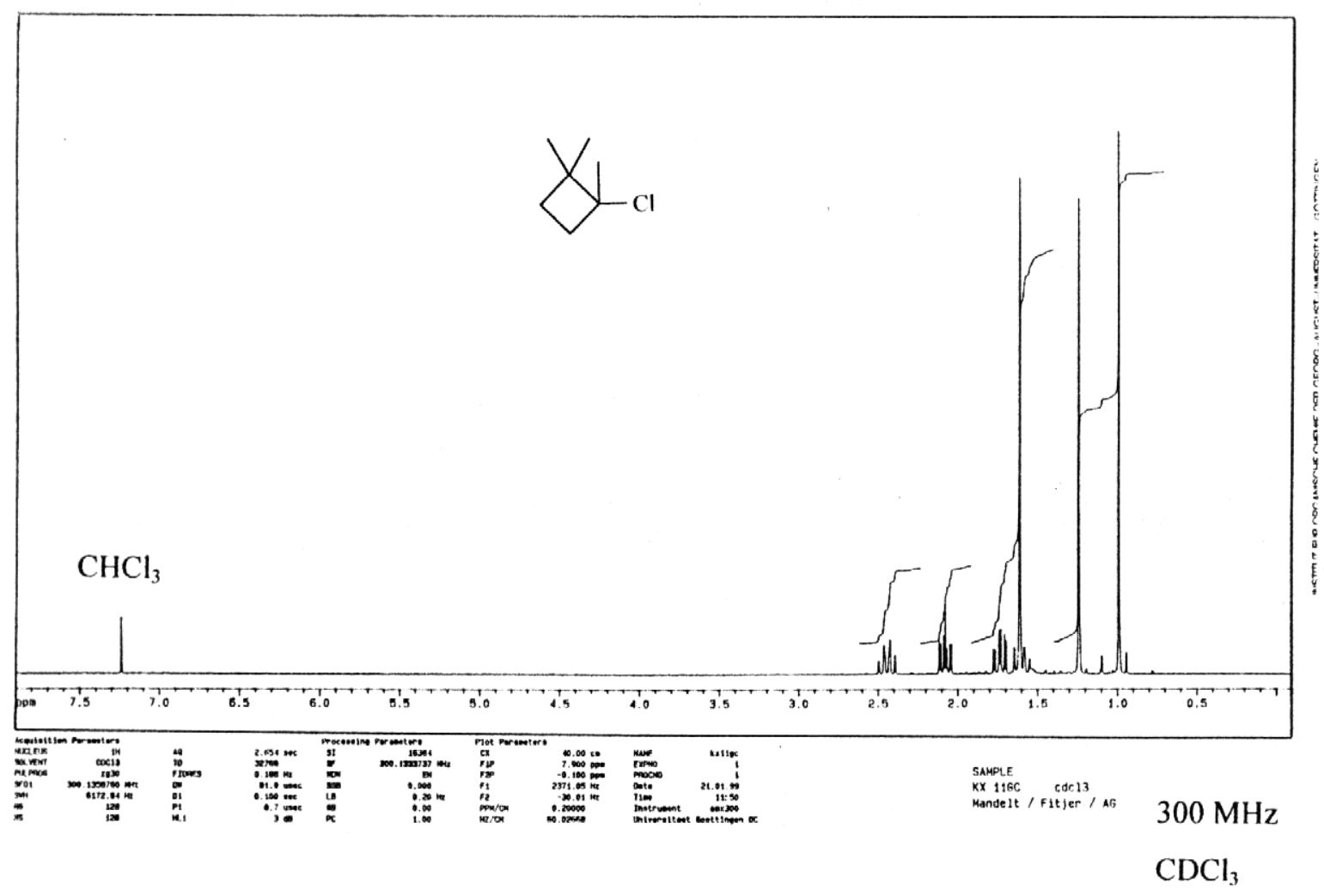

Abb. 28: $\quad{ }^{1}$ H-NMR-Spektrum von 1,2,2-Trimethyl-cyclobutylchlorid (97) 


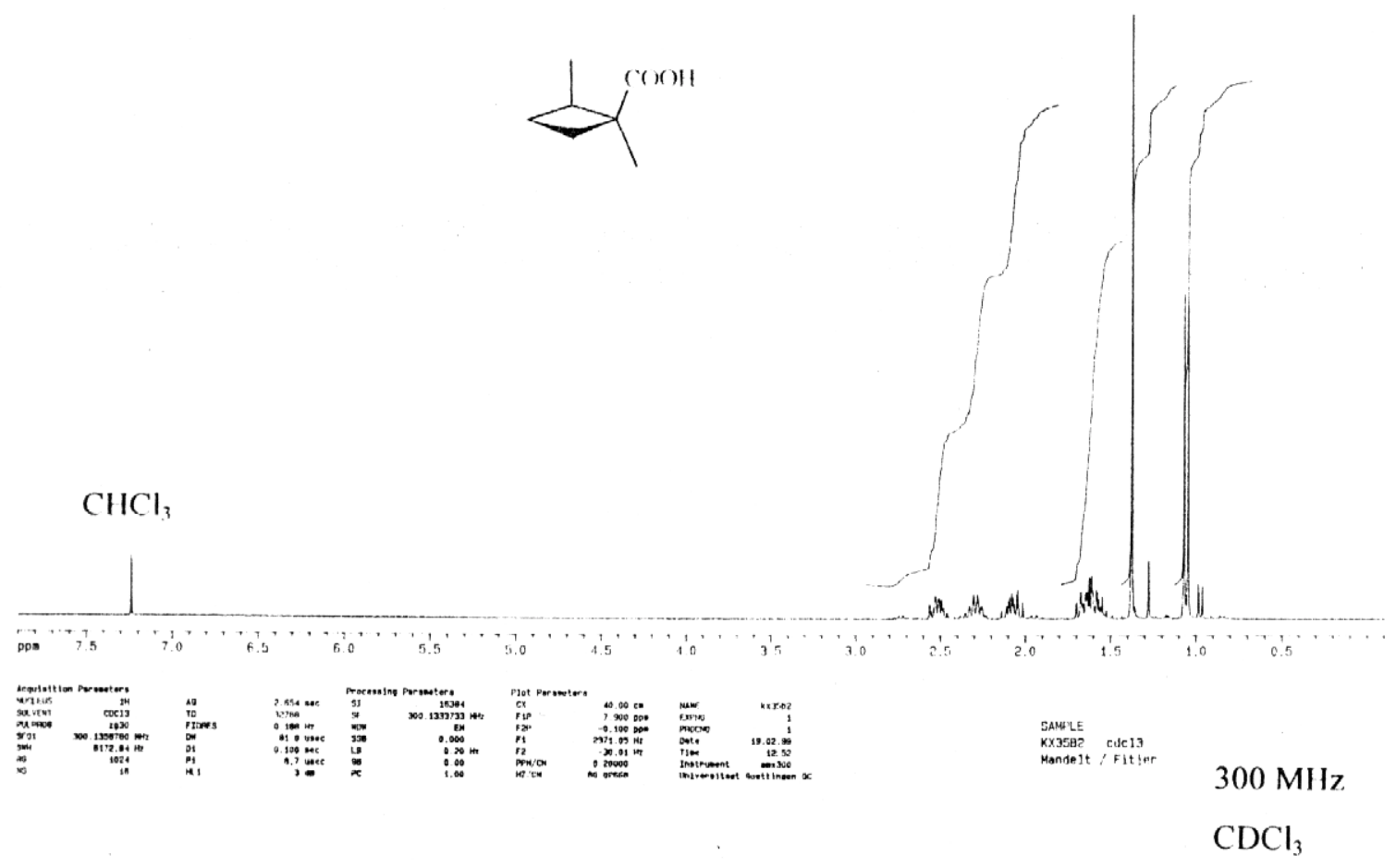

Abb. 29: $\quad{ }^{1}$ H-NMR-Spektrum von $\left(1 R^{*}, 2 S^{*}\right)$-1,2-Dimethyl-cyclobutancarbonsäure (98a)

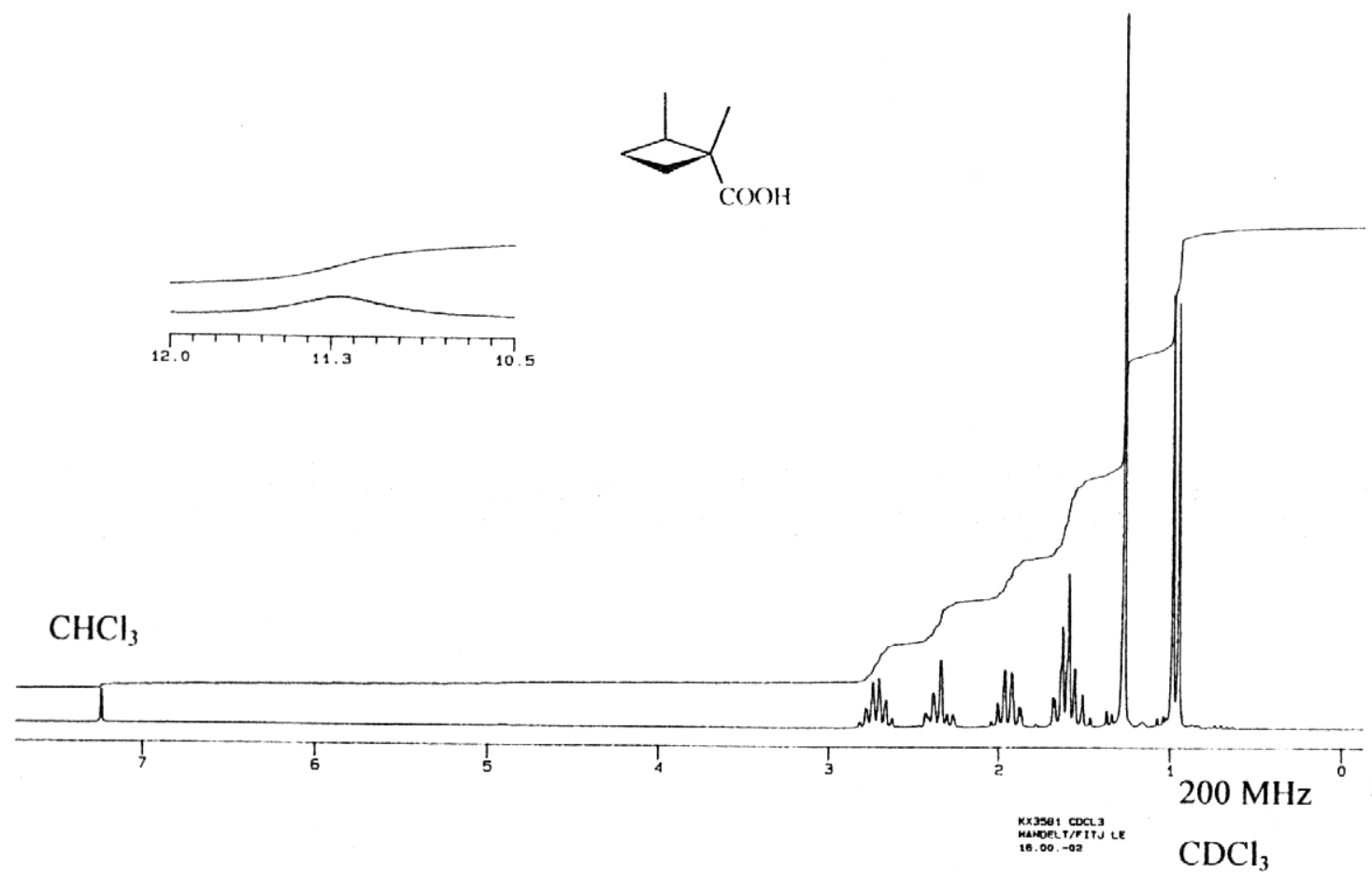

Abb. 30: $\quad{ }^{1} \mathrm{H}-\mathrm{NMR}$-Spektrum von $\left(1 R^{*}, 2 R^{*}\right)$-1,2-Dimethyl-cyclobutancarbonsäure (98b) 


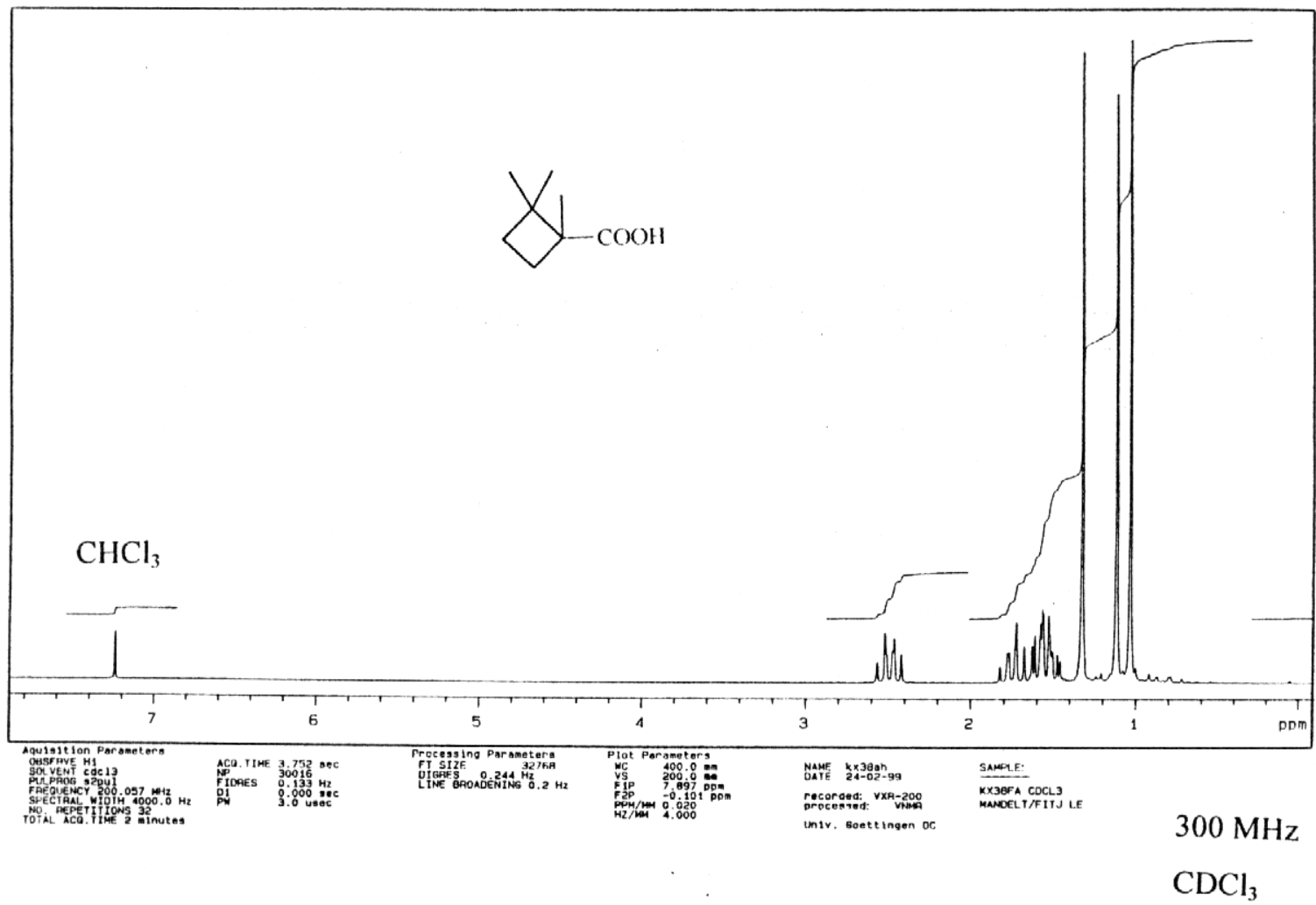

Abb. 31: $\quad{ }^{1}$ H-NMR-Spektrum von 1,2,2-Trimethyl-cyclobutancarbonsäure (100)

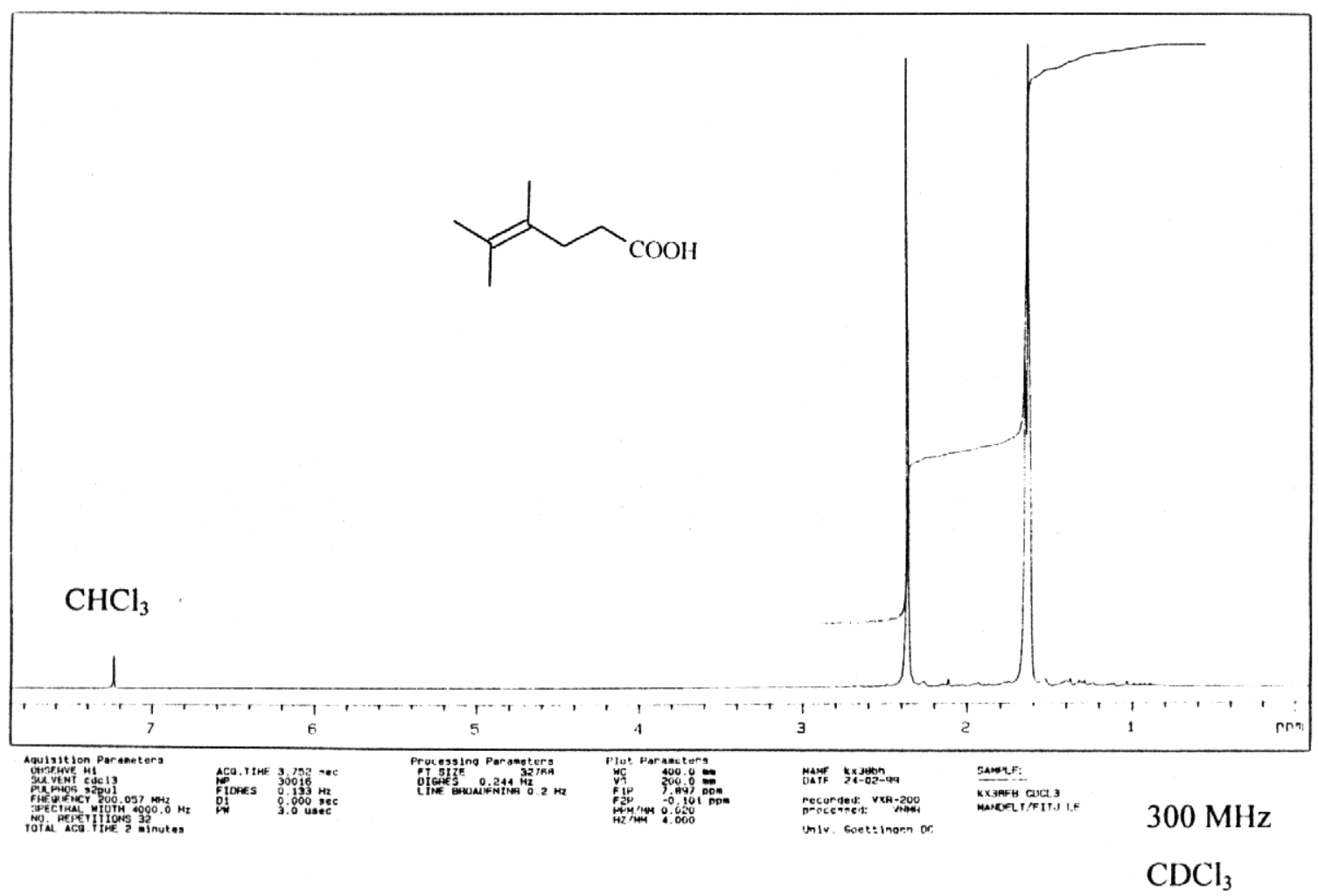

Abb. 32: $\quad{ }^{1}$ H-NMR-Spektrum von 4,5-Dimethyl-hex-4-en-säure (102) 

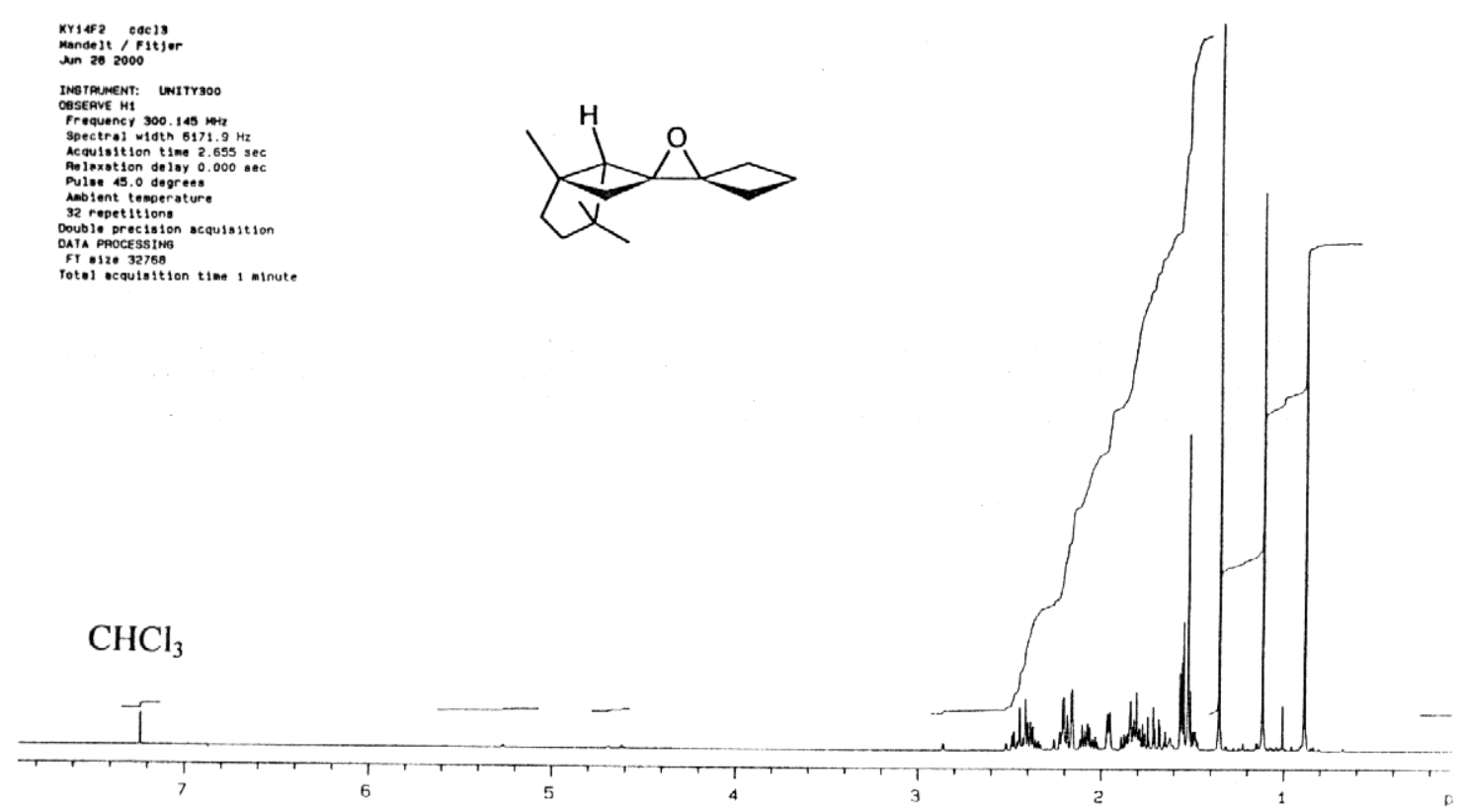

$300 \mathrm{MHz}$

$\mathrm{CDCl}_{3}$

Abb. 33: $\quad$ H-NMR-Spektrum von 2,2-Spiro-cyclobutyl-3,3-spiro[( $\left.1 R^{*}, 5 R^{*}, 6 R^{*}\right)-1,4,4-$ trimethyl-bicyclo[3.2.0]heptan-6,6-yl]-oxiran (55)

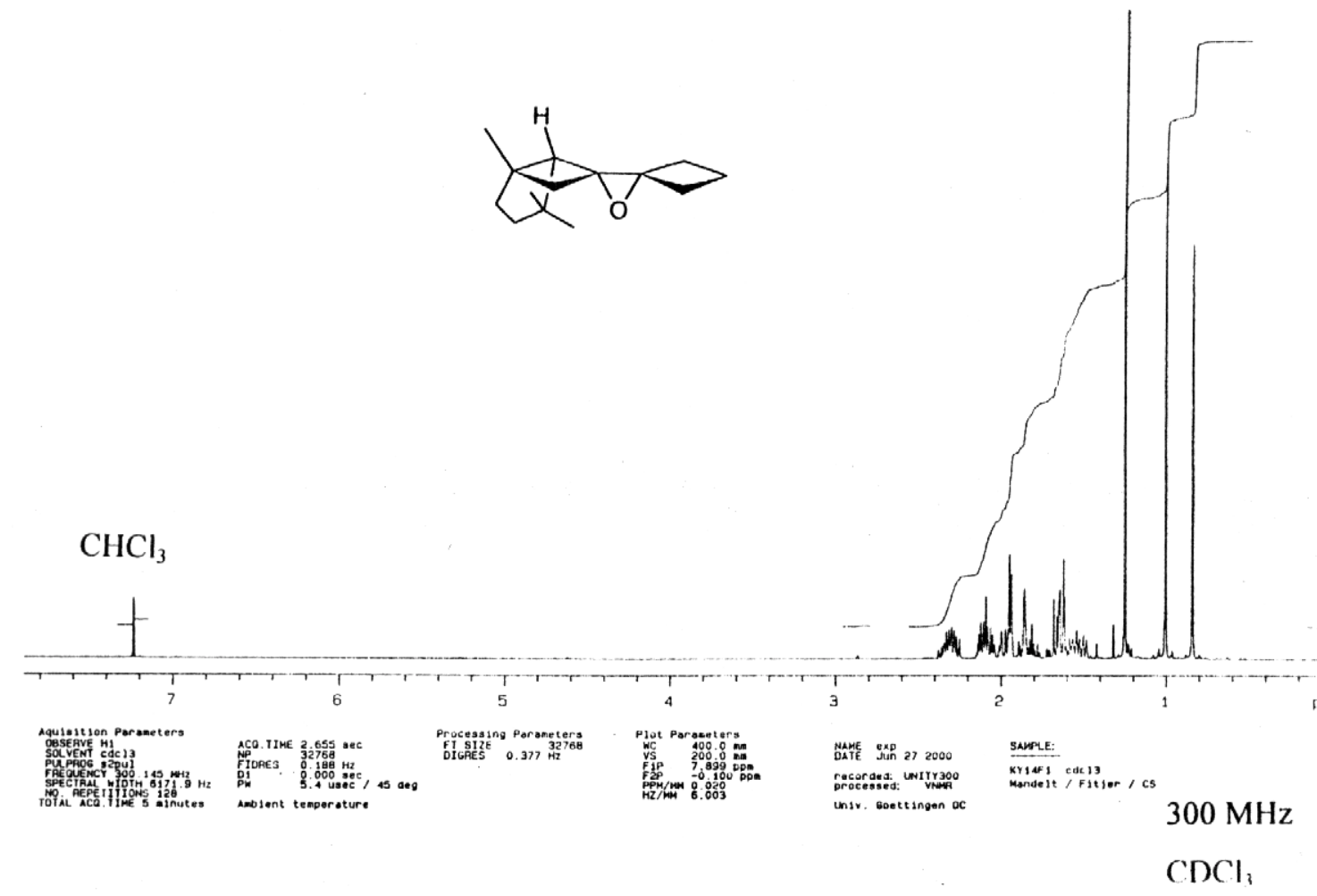

Abb. 34: $\quad{ }^{1} \mathrm{H}$-NMR-Spektrum von 2,2-Spiro-cyclobutyl-3,3-spiro-[(1 $\left.R^{*}, 5 R^{*}, 6 S^{*}\right)-1,4,4-$ trimethyl-bicyclo[3.2.0]heptan-6,6-yl]-oxiran (56) 


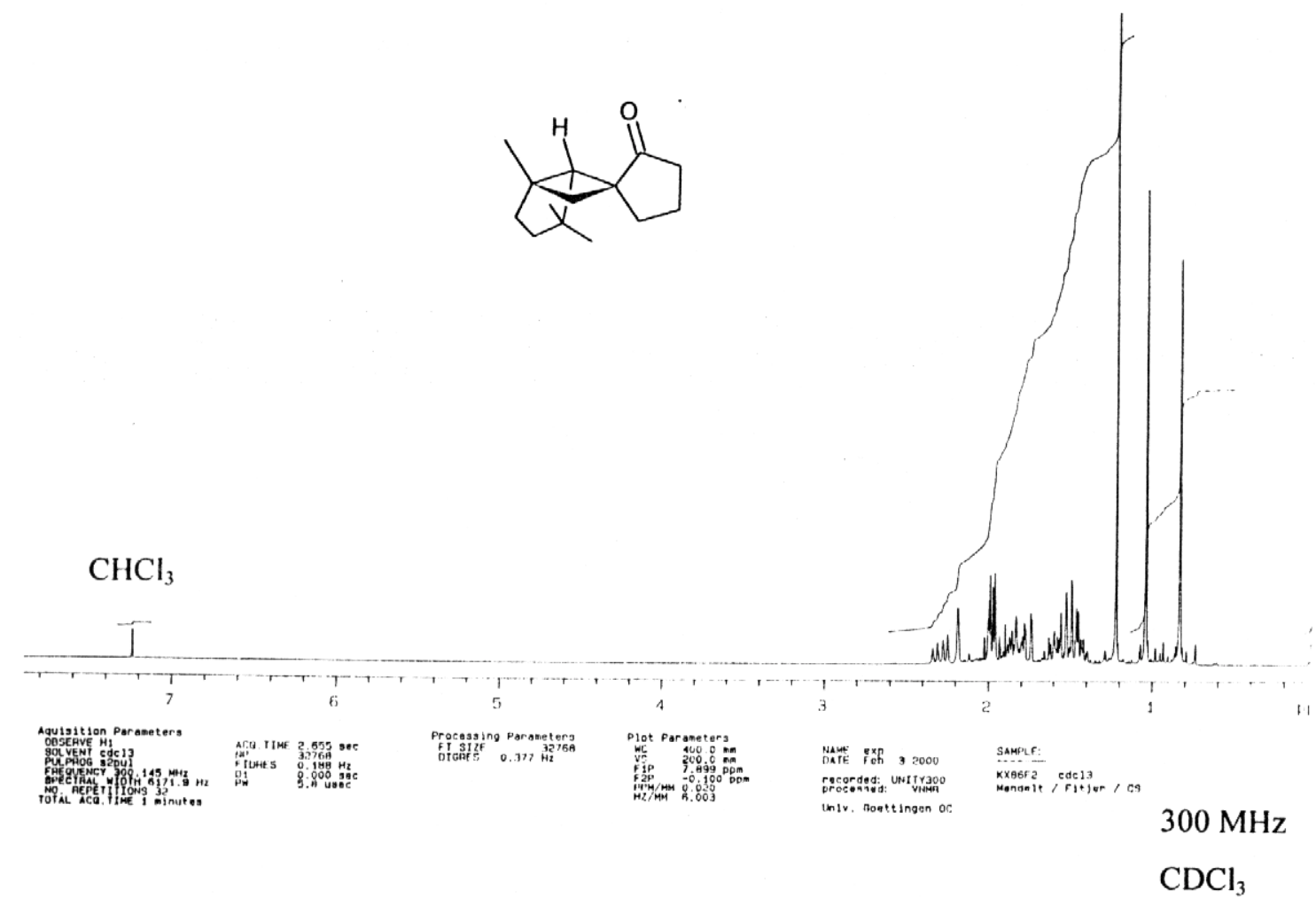

Abb. 35: $\quad{ }^{1} \mathrm{H}-\mathrm{NMR}-\mathrm{Spektrum}$ von $\left(1 R^{*}, 5 R^{*}, 6 R^{*}\right)-1,4,4-$ Trimethylspiro\{bicyclo[3.2.0]heptan-6,1'-cyclopentan-2'-on\} (57)

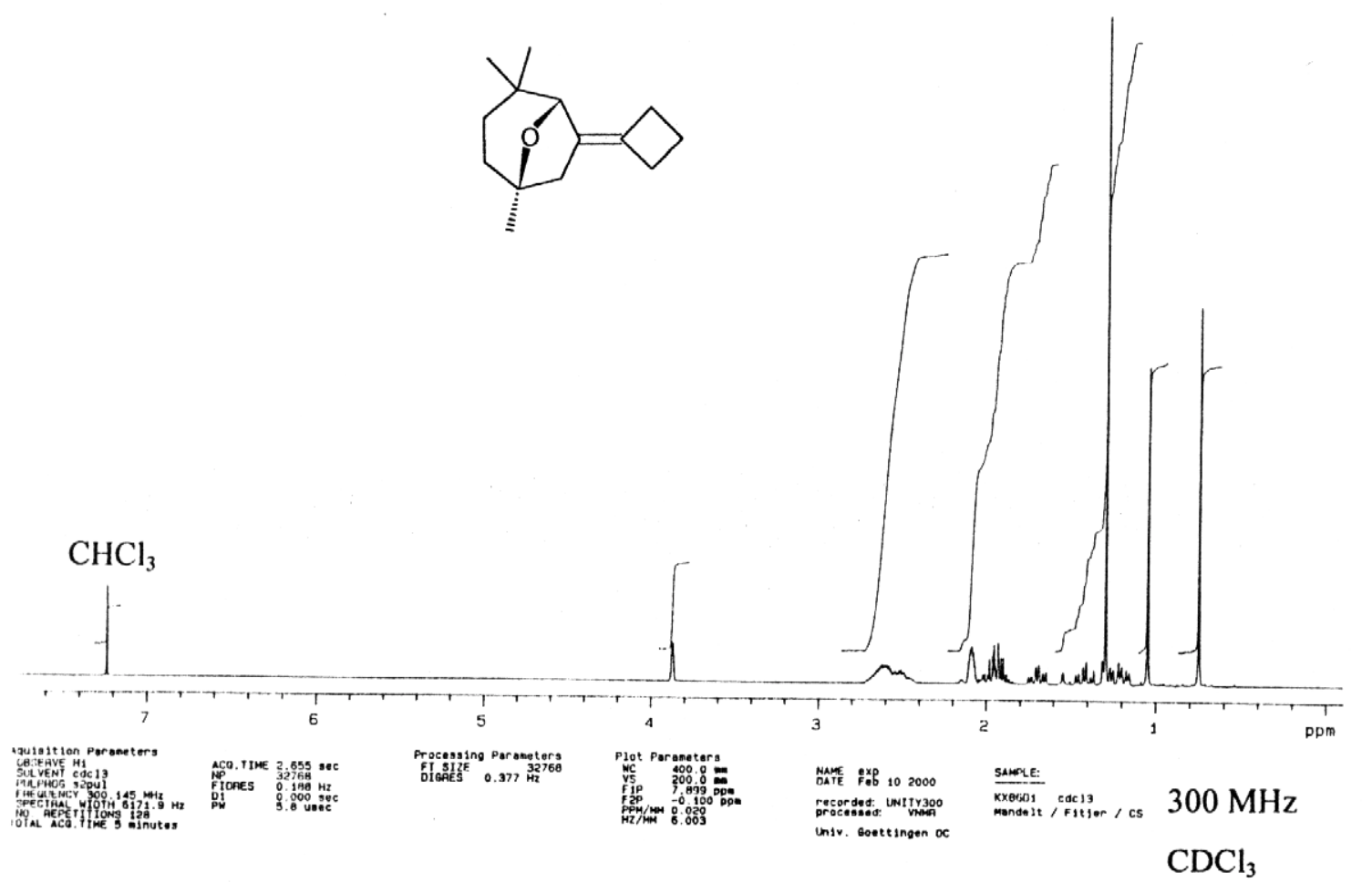

Abb. 36: $\quad$ 'H-NMR-Spektrum von 6-Cyclobutyliden-1,4,4-trimethyl-8-oxa-bicyclo[3.2.1]octan (138) 


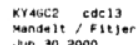
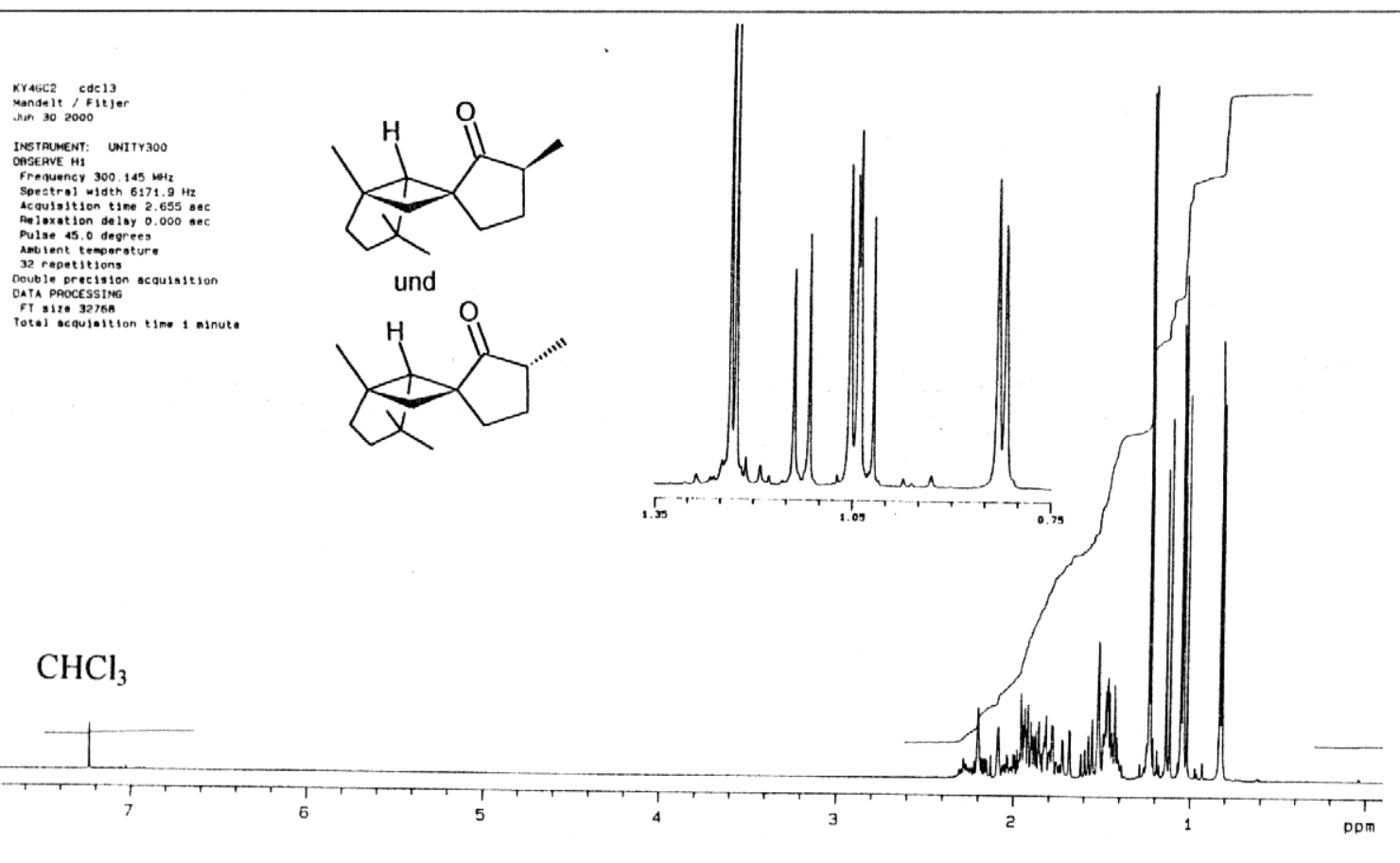

$\mathrm{CHCl}_{3}$

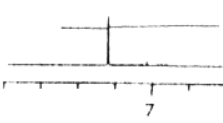

6

5

$300 \mathrm{MHz}$

$\mathrm{CDCl}_{3}$

Abb. 37: $\quad{ }^{1} \mathrm{H}-\mathrm{NMR}-S$ pektrum von $\left(1 R^{*}, 5 R^{*}, 6 R^{*}, 3 S^{*}\right)-1,4,4,3^{\prime}$-Tetramethylspiro \{ bicyclo[3.2.0]heptan-6,1'-cyclopentan-2'-on $\}$ (141) und $\left(1 R^{*}, 5 R^{*}, 6 R^{*}, 3 R^{*}\right)-1,4,4,3^{\prime}-$ Tetramethylspiro\{bicyclo[3.2.0]heptan-6,1'-cyclopentan-2'-on $\}$ (142)

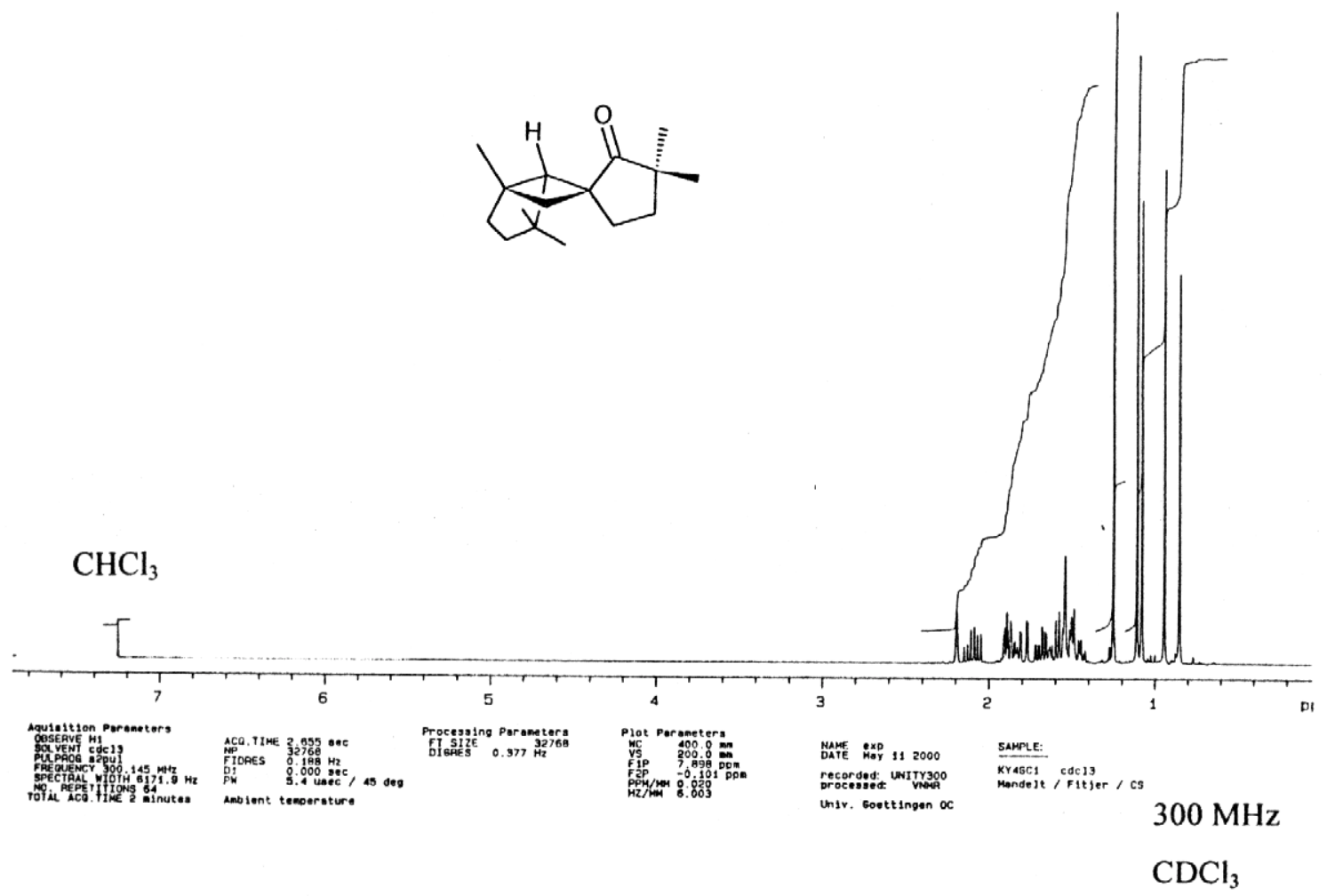

Abb. 38: $\quad{ }^{1} \mathrm{H}-\mathrm{NMR}$-Spektrum von $\left(1 R^{*}, 5 R^{*}, 6 R^{*}\right)-1,4,4,3^{\prime}, 3^{\prime}$-Pentamethylspiro \{bicyclo[3.2.0]heptan-6,1'-cyclopentan-2'-on\} (143) 


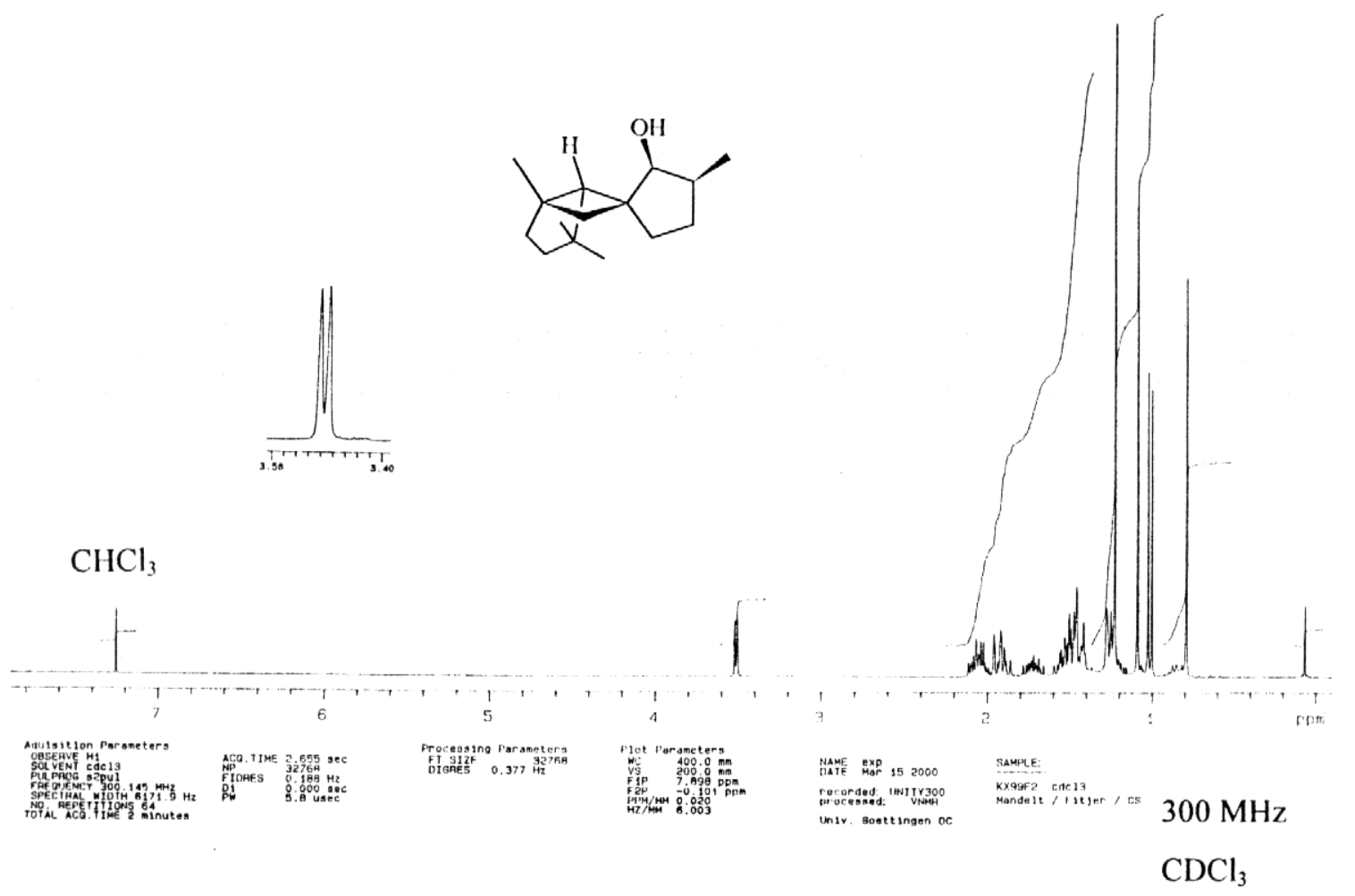

Abb. 39: $\quad{ }^{1} \mathrm{H}-\mathrm{NMR}-\mathrm{Spe}$ trum von $\left(1 R^{*}, 5 R^{*}, 6 R^{*}, 2^{\prime} R^{*}, 3^{\prime} R^{*}\right)-1,4,4,3^{\prime}$-Tetramethylspiro$\{$ bicyclo[3.2.0]heptan-6,1'-cyclopentan-2'-ol $\}(60)$

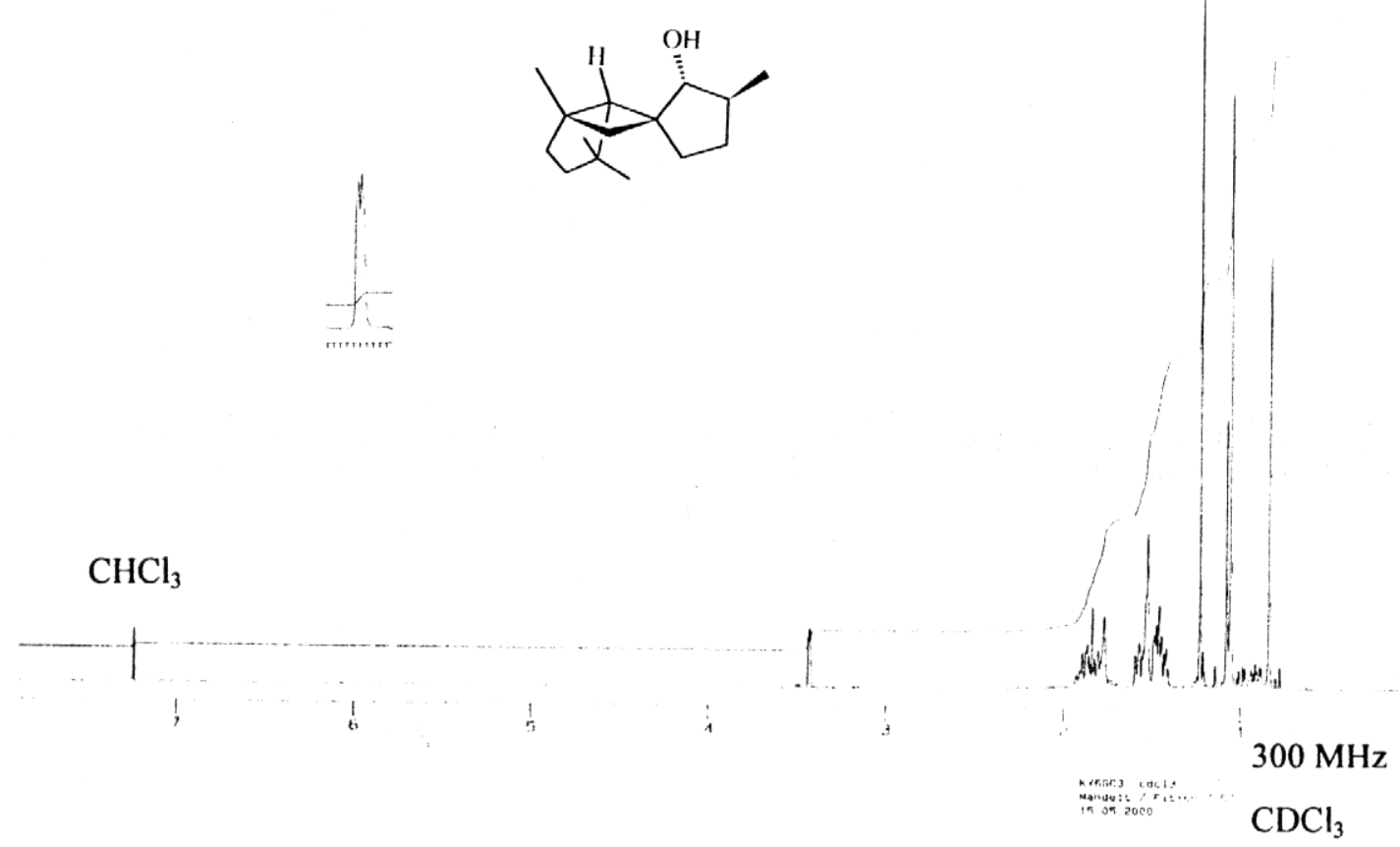

Abb. 40: $\quad{ }^{1} \mathrm{H}$-NMR-Spektrum von $\left(1 R^{*}, 5 R^{*}, 6 R^{*}, 2^{\prime} S^{*}, 3^{\prime} R^{*}\right)-1,4,4,3^{\prime}-$ Tetramethylspiro$\{$ bicyclo[3.2.0]heptan-6,1'-cyclopentan-2'-ol\} (64) 


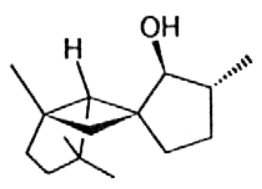

$\mathrm{CHCl}_{3}$
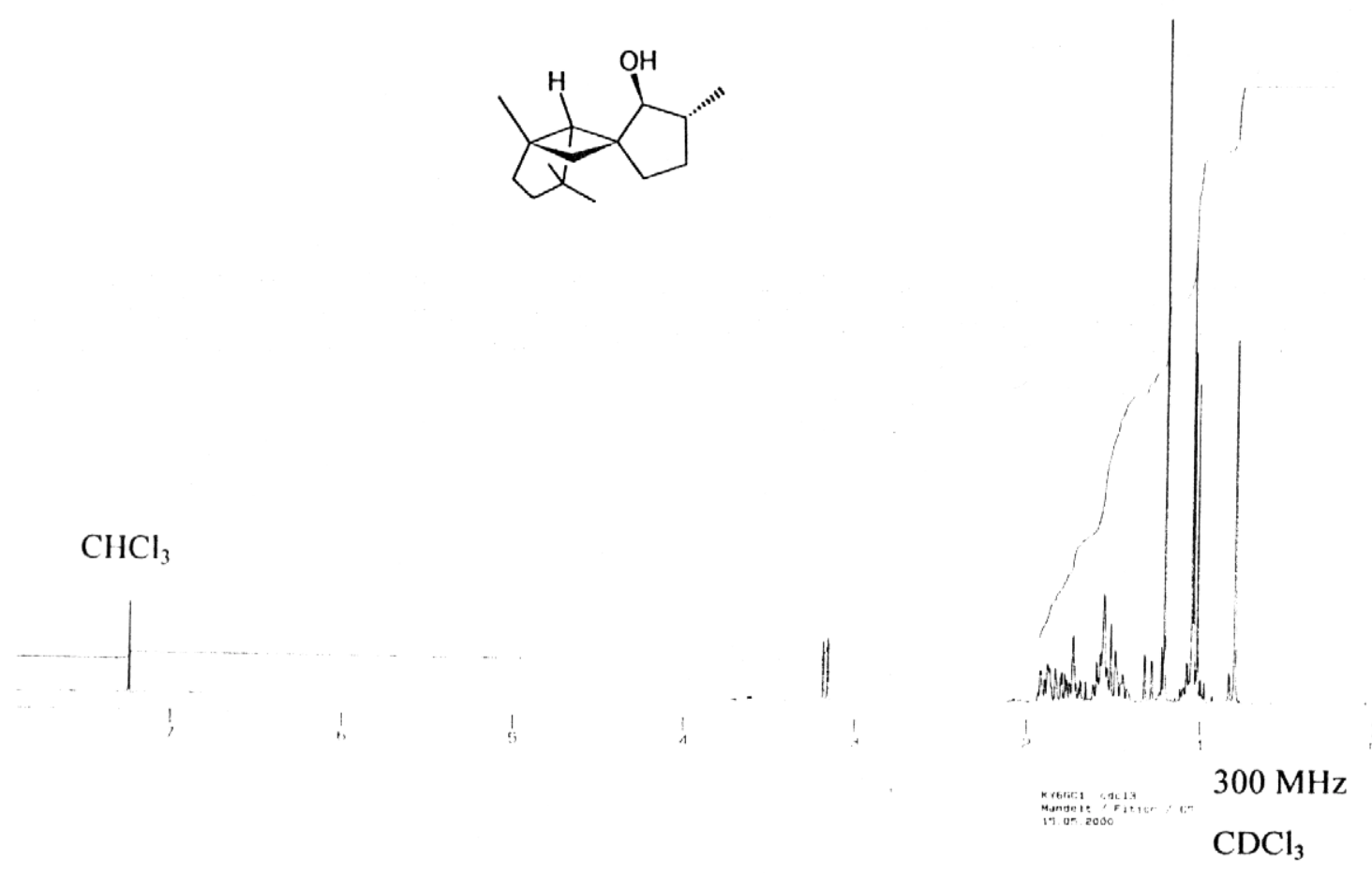

Abb. 41: $\quad{ }^{1} \mathrm{H}-\mathrm{NMR}-\mathrm{Spe}$ trum von $\left(1 R^{*}, 5 R^{*}, 6 R^{*}, 2^{\prime} R^{*}, 3^{\prime} S^{*}\right)-1,4,4,3^{\prime}$-Tetramethylspiro\{bicyclo[3.2.0]heptan-6,1'-cyclopentan-2'-ol $\}(66)$

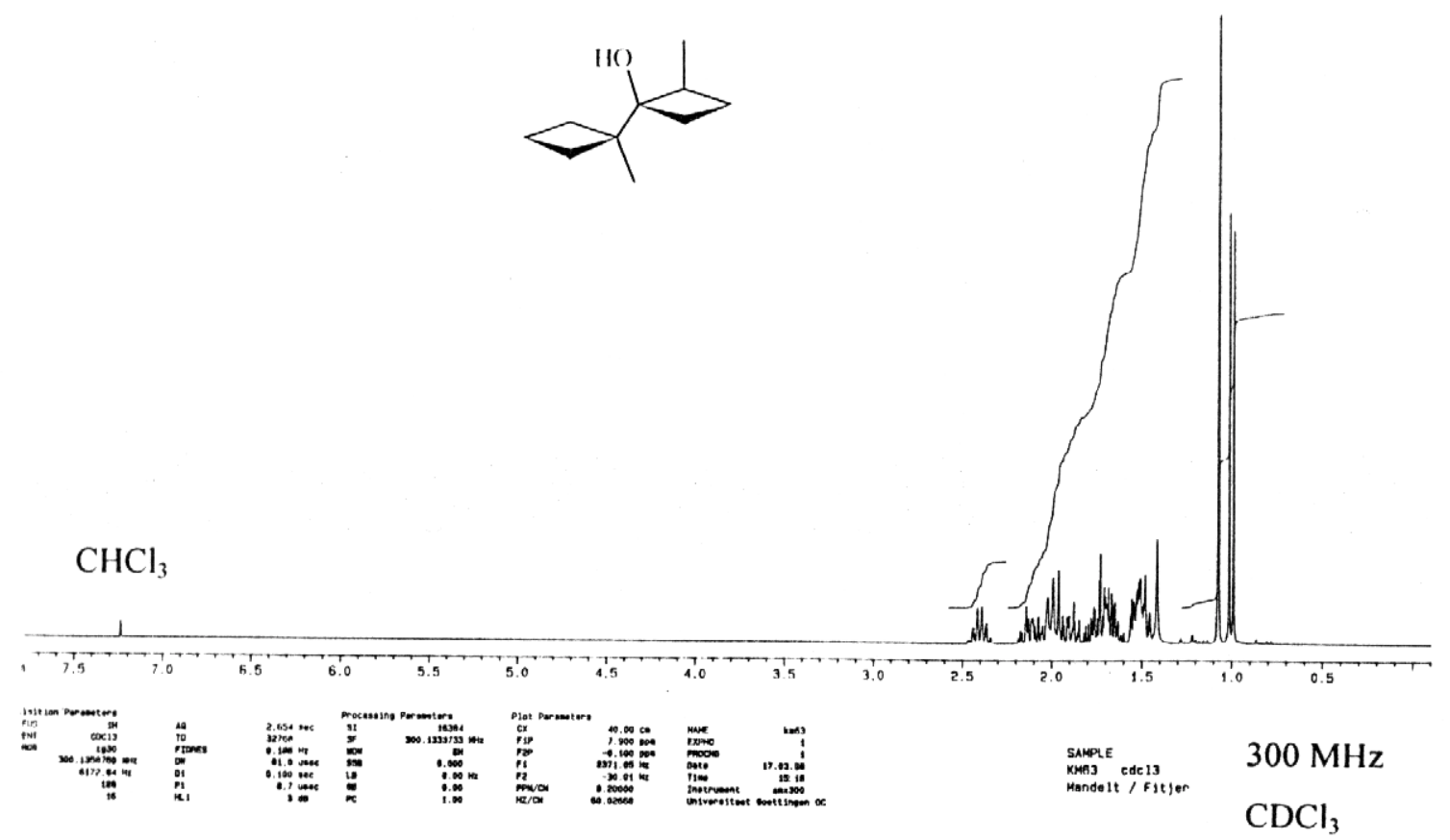

Abb. 42: $\quad{ }^{1}$ H-NMR-Spektrum von $\left(1 R^{*}, 2 S^{*}\right)-2,1^{\prime}$-Dimethyl-bicyclobutyl-1-ol (104b) 


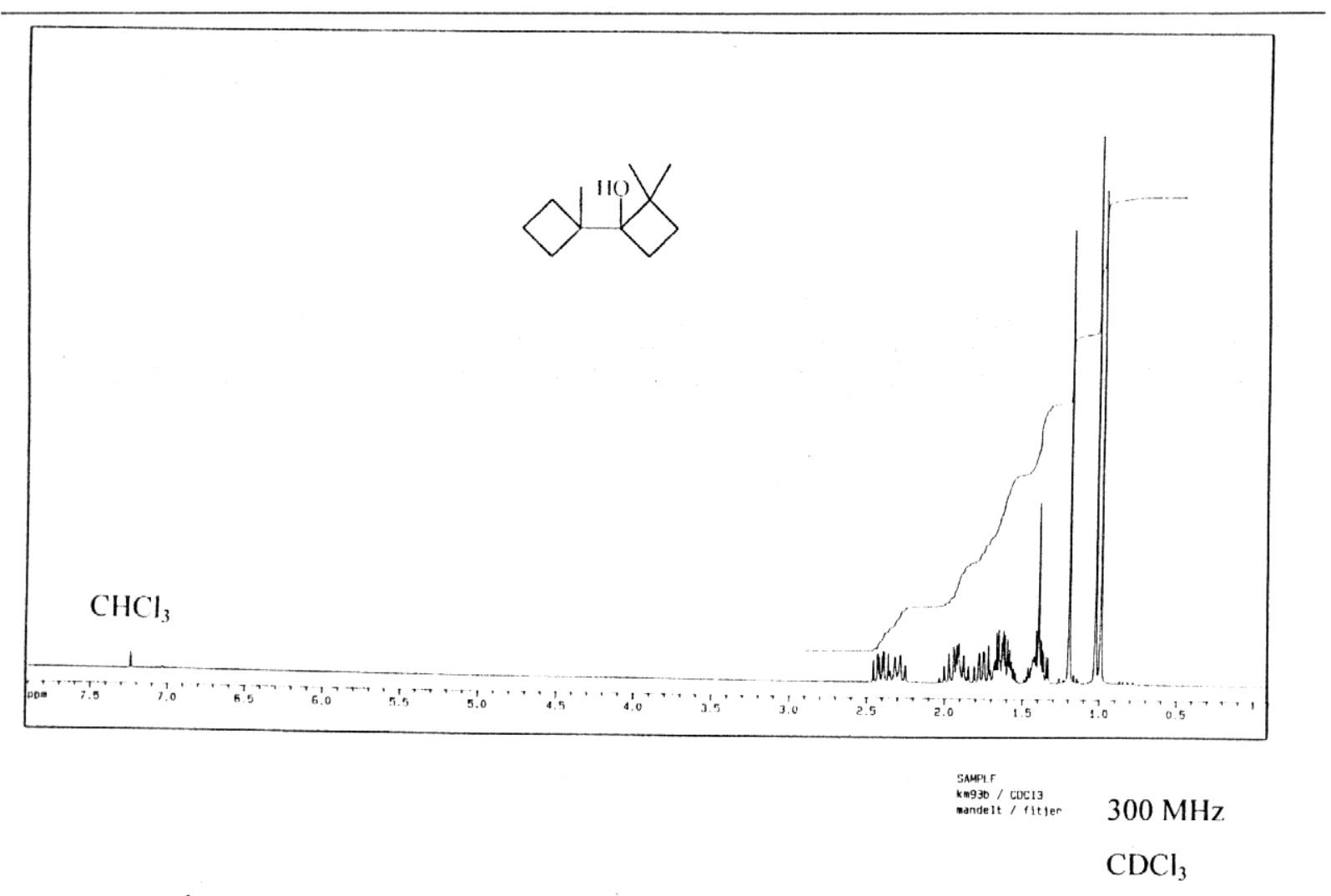

Abb. 43: $\quad{ }^{1}$ H-NMR-Spektrum von 2,2,1'-Trimethyl-bicyclobutyl-1-ol (105)

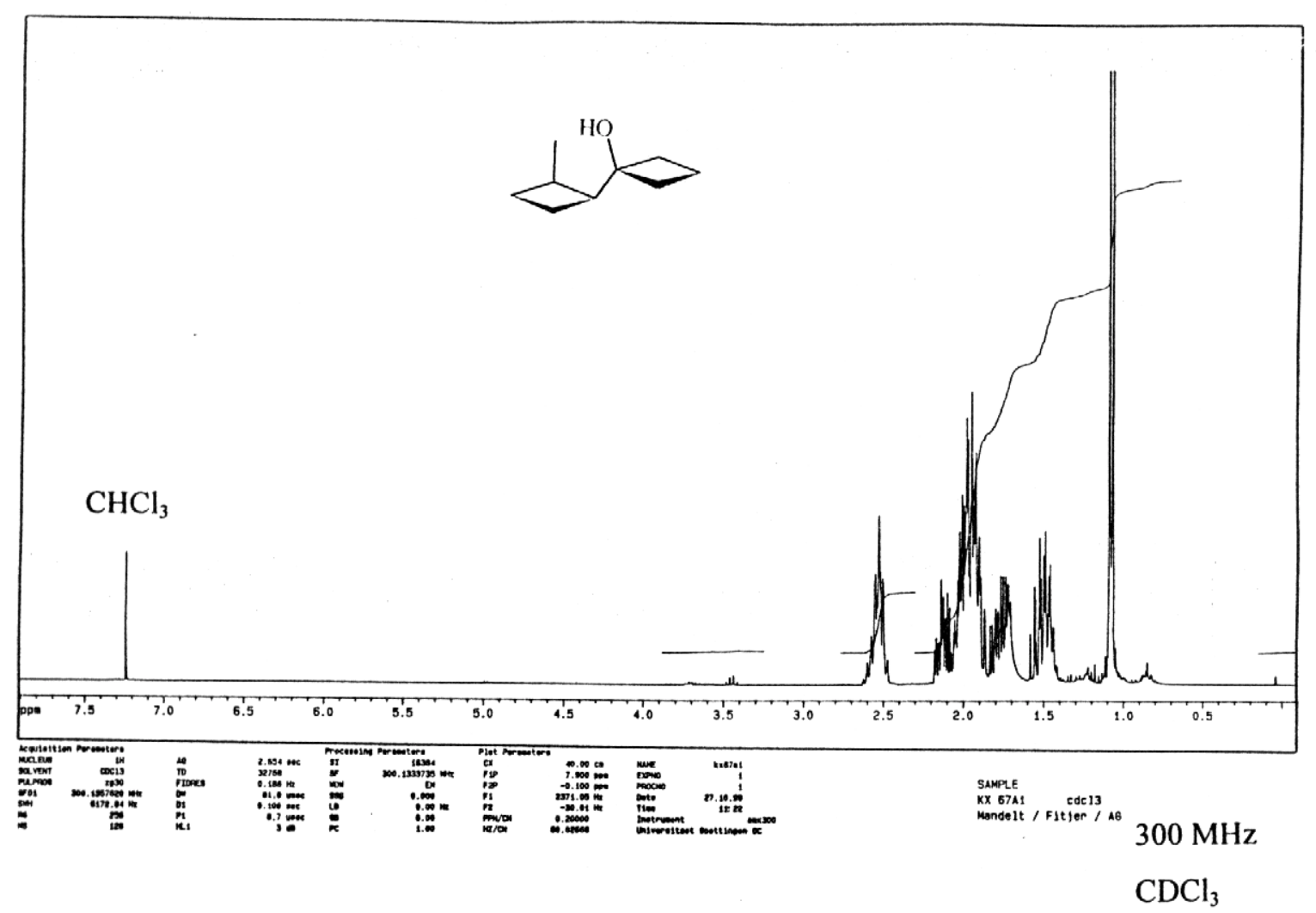

Abb. 44: $\quad{ }^{1}$ H-NMR-Spektrum von $\left(1^{\prime} R^{*}, 2^{\prime} S^{*}\right)-2^{\prime}$-Methyl-bicyclobutyl-1-ol (106a) 


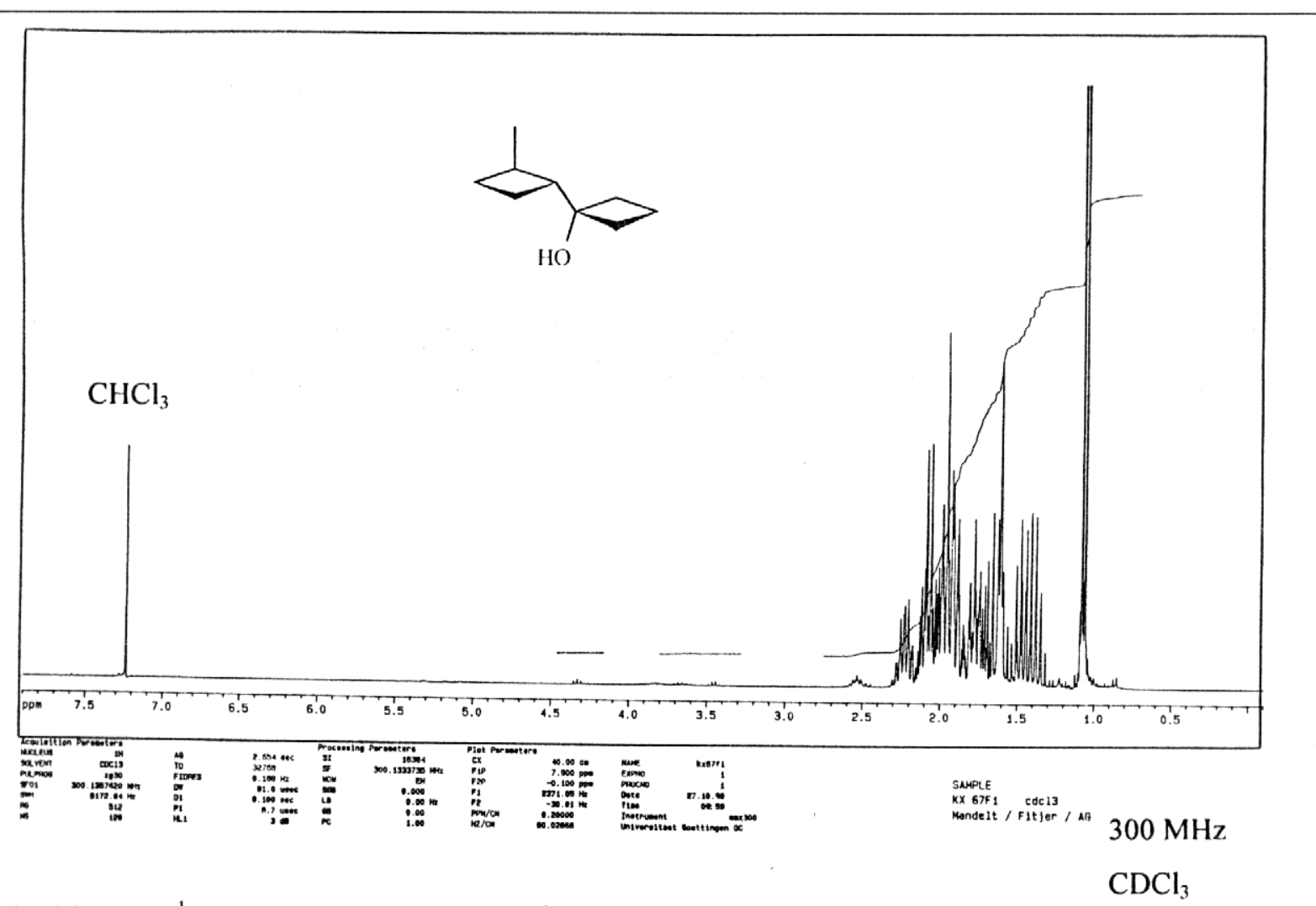

Abb. 45: $\quad{ }^{1} \mathrm{H}-\mathrm{NMR}-\mathrm{Spektrum}$ von $\left(1^{\prime} R^{*}, 2^{\prime} R^{*}\right)-2^{\prime}$-Methyl-bicyclobutyl-1-ol (106b)

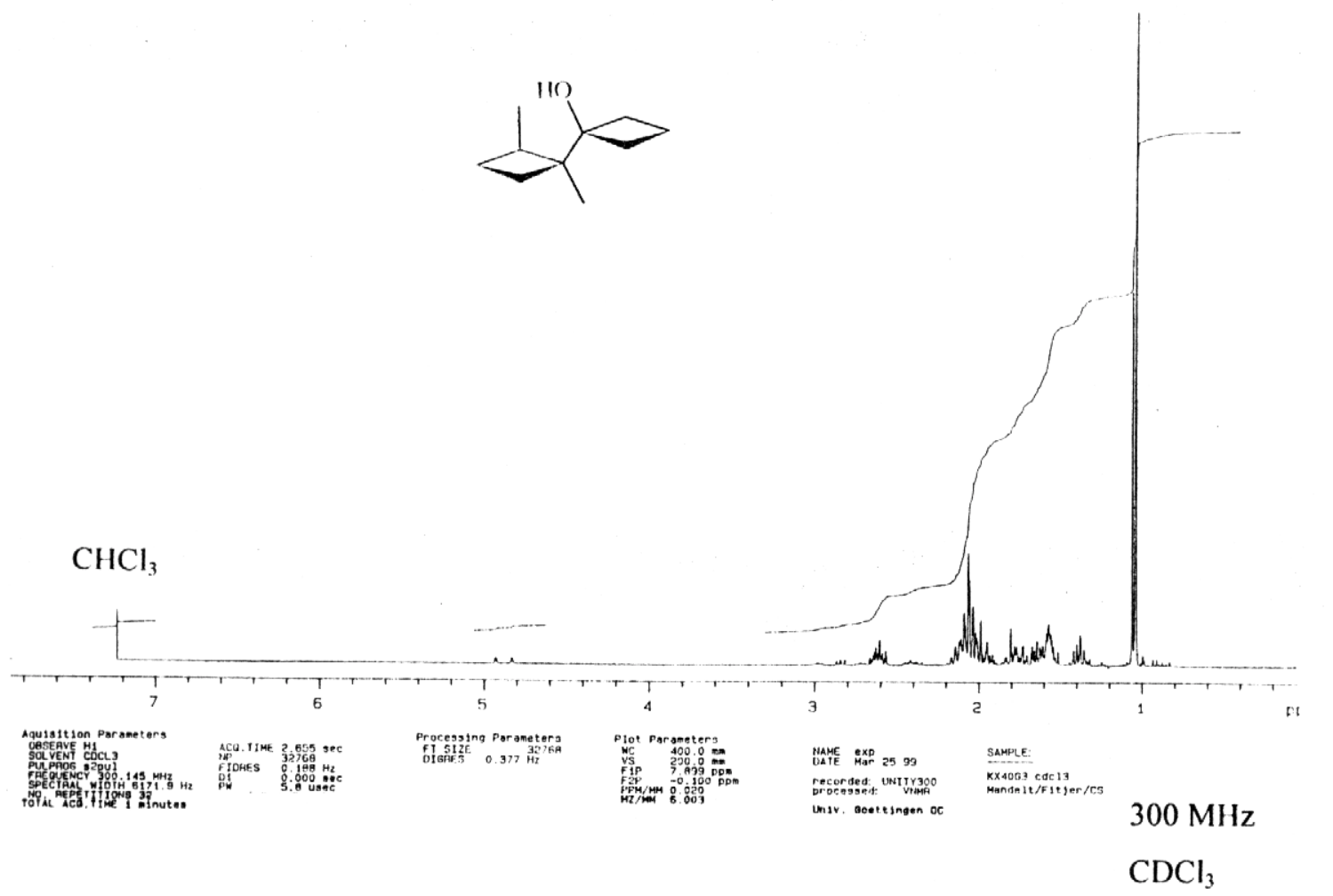

Abb. 46: $\quad{ }^{1} \mathrm{H}-\mathrm{NMR}-\mathrm{Spektrum}$ von $\left(1^{\prime} R^{*}, 2^{\prime} S^{*}\right)-1^{\prime}, 2^{\prime}$-Dimethyl-bicyclobutyl-1-ol (107a) 


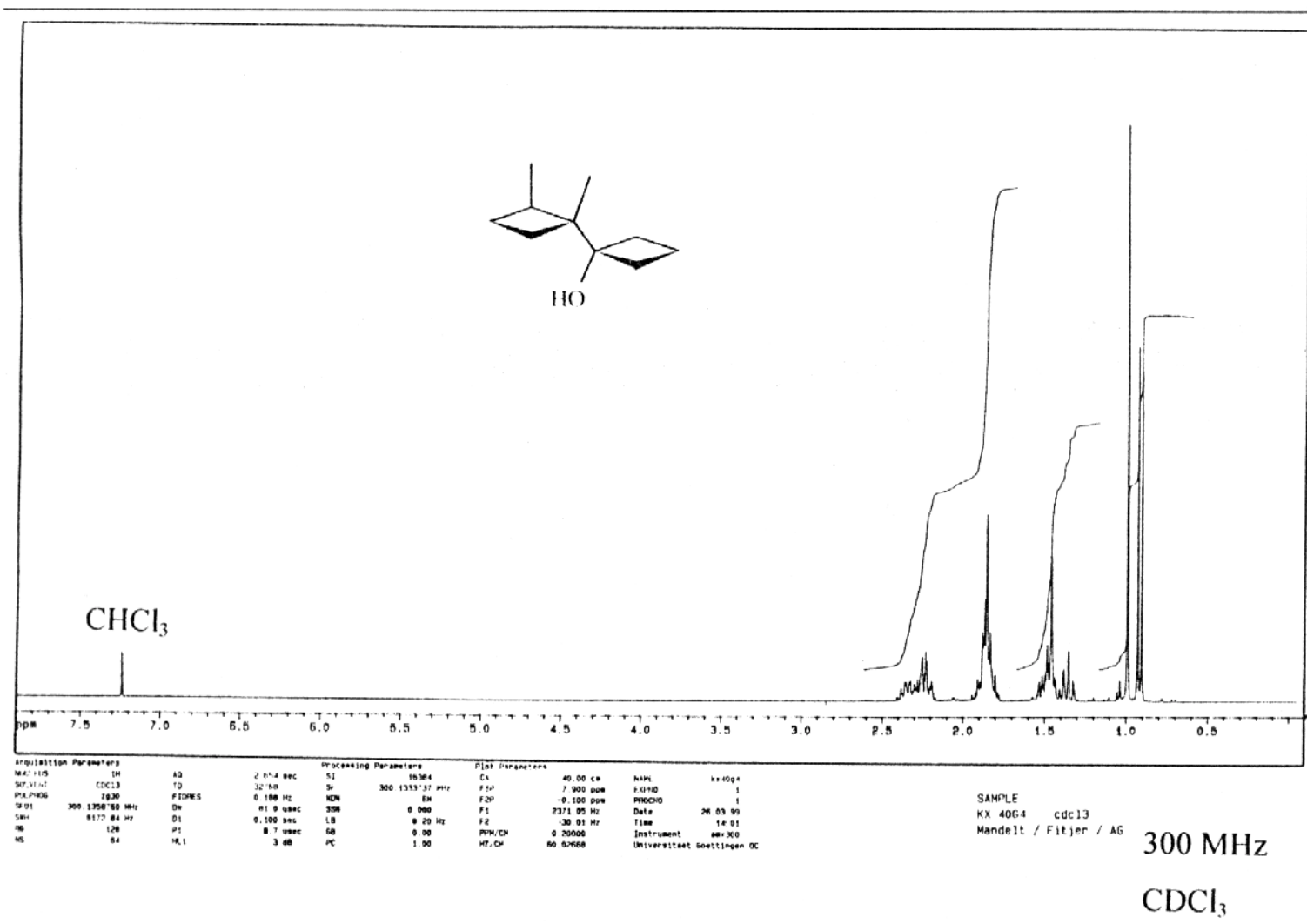

Abb. 47: $\quad{ }^{1} \mathrm{H}-\mathrm{NMR}$-Spektrum von $\left(1^{\prime} R^{*}, 2^{\prime} R^{*}\right)-1^{\prime}, 2^{\prime}$-Dimethyl-bicyclobutyl-1-ol (107b)

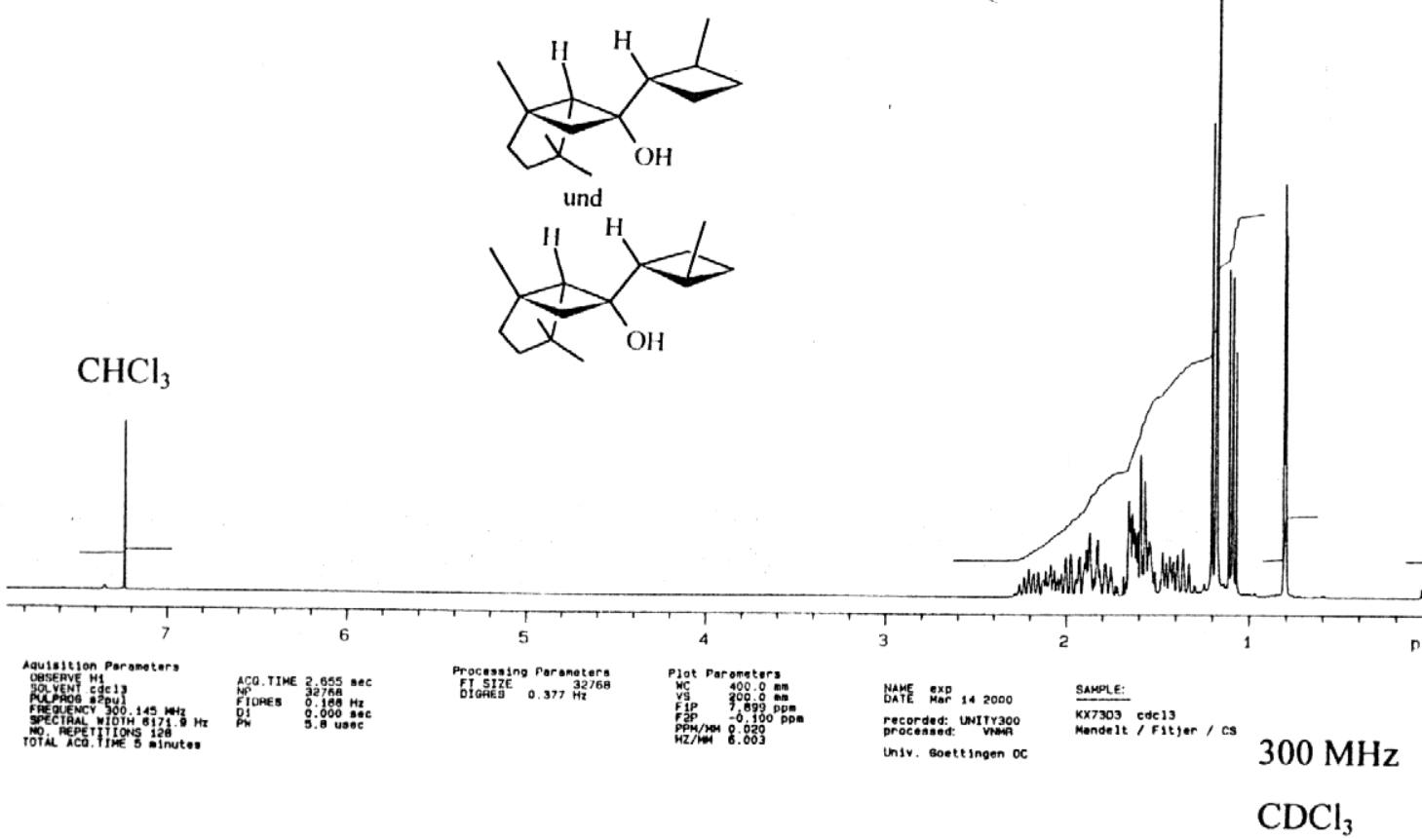

Abb. 48: $\quad{ }^{1}$ H-NMR-Spektrum von $\left(1 S^{*}, 5 S^{*}, 6 S^{*}, 1^{\prime} S^{*}, 2^{\prime} S^{*}\right)$-1,4,4-Trimethyl-6-(2'-methylcyclobutyl)-bicyclo[3.2.0]heptan-6-ol (46) und $\left(1 S^{*}, 5 S^{*}, 6 S^{*}, 1^{\prime} R^{*}, 2^{\prime} R^{*}\right)-1,4,4-$ Trimethyl-6-(2'-methyl-cyclobutyl)-bicyclo[3.2.0]heptan-6-ol (49) 


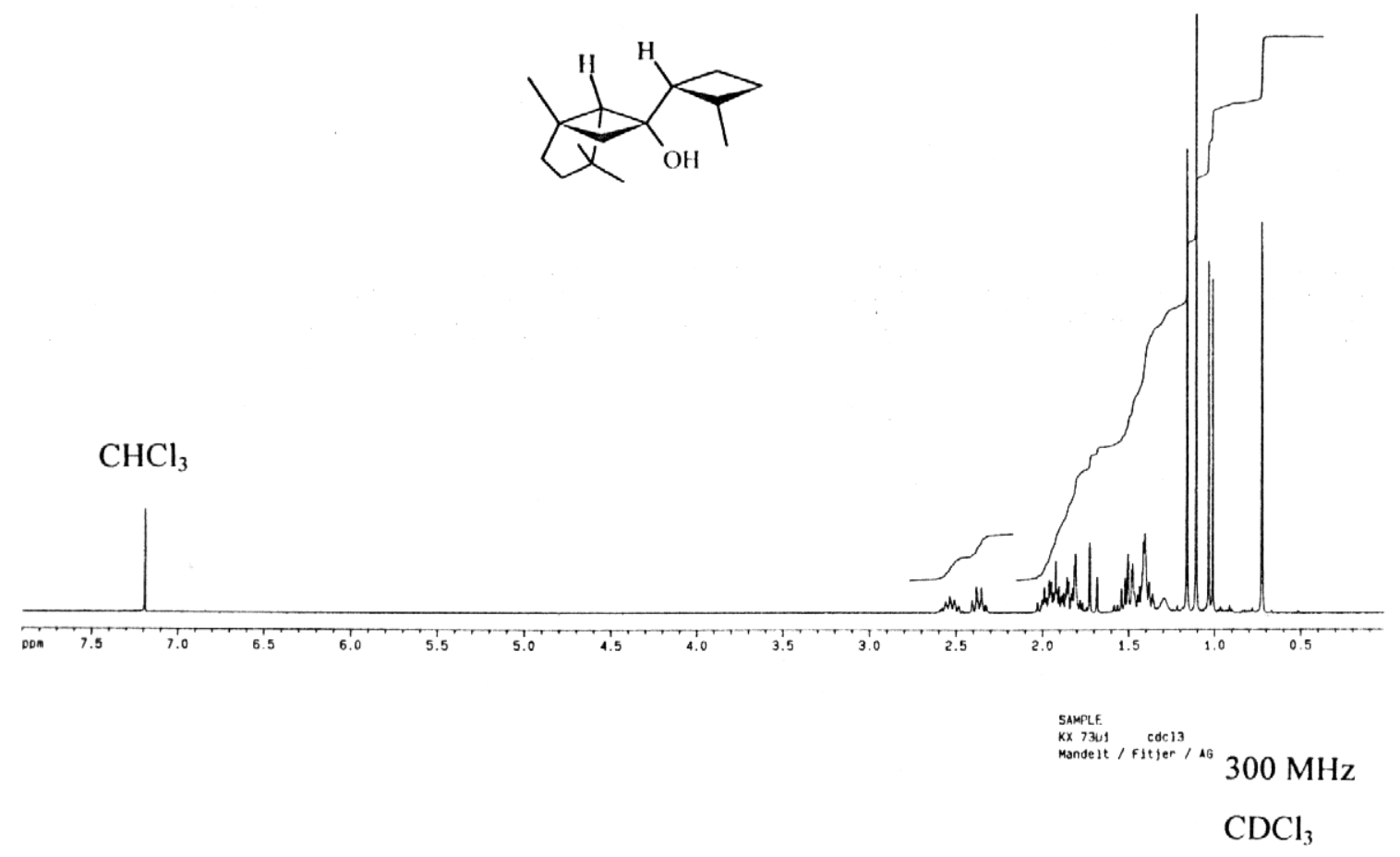

Abb. 49: $\quad{ }^{1}$ H-NMR-Spektrum von $\left(1 S^{*}, 5 S^{*}, 6 S^{*}, 1^{\prime} S^{*}, 2^{\prime} R^{*}\right)-1,4,4$-Trimethyl-6-(2'-methylcyclobutyl)-bicyclo[3.2.0]heptan-6-ol (47)

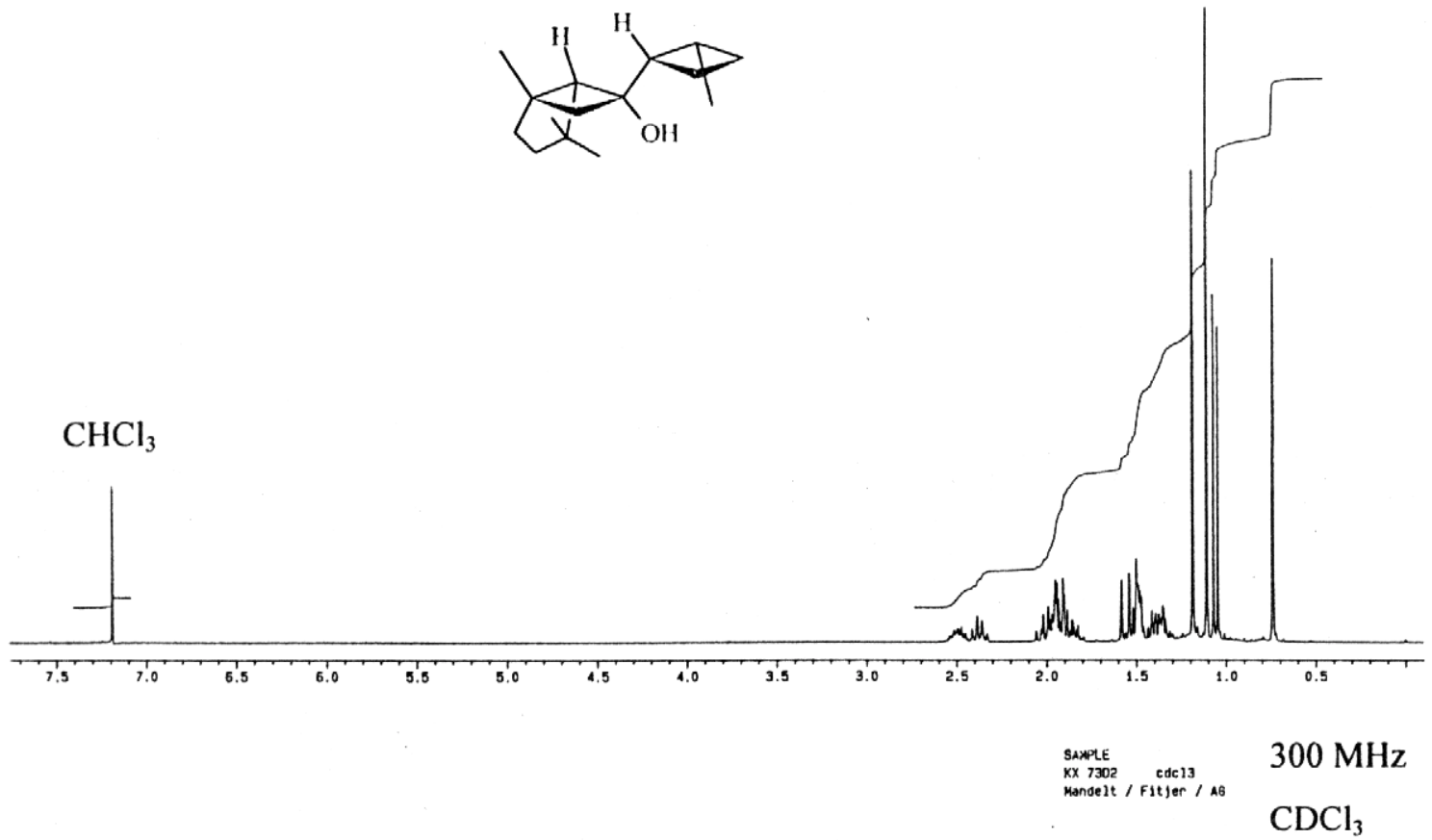

Abb. 50: $\quad{ }^{1}$ H-NMR-Spektrum von $\left(1 S^{*}, 5 S^{*}, 6 S^{*}, 1^{\prime} R^{*}, 2^{\prime} S^{*}\right)-1,4,4-$ Trimethyl-6-(2'-methylcyclobutyl)-bicyclo[3.2.0]heptan-6-ol (48) 


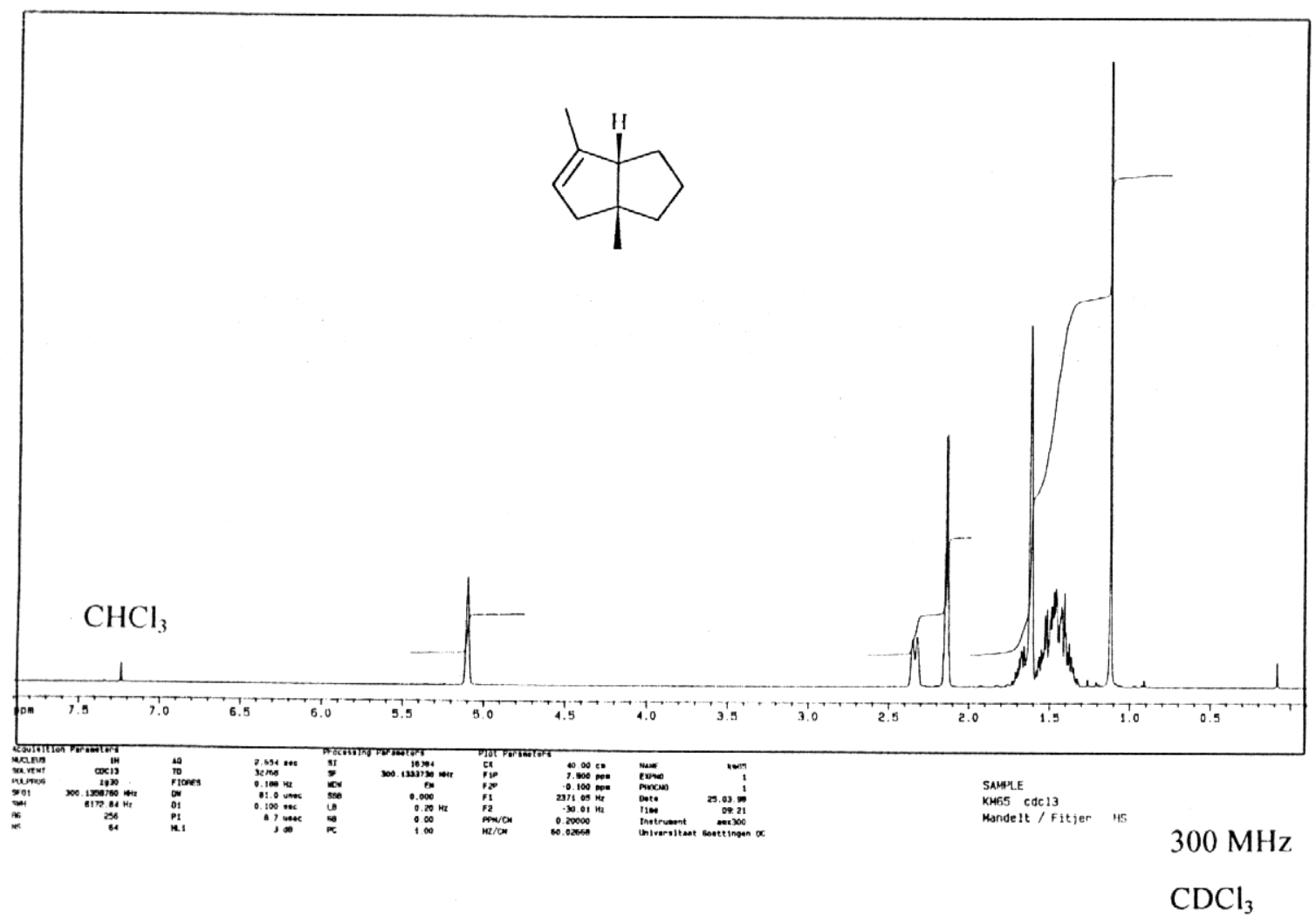

Abb. 51: $\quad{ }^{1} \mathrm{H}-\mathrm{NMR}-$ Spektrum von $\left(3 \mathrm{a} R^{*}, 6 \mathrm{a} S^{*}\right)-3 \mathrm{a}, 6$-Dimethyl-1,2,3,3a,4,6a-hexahydropentalen (109)

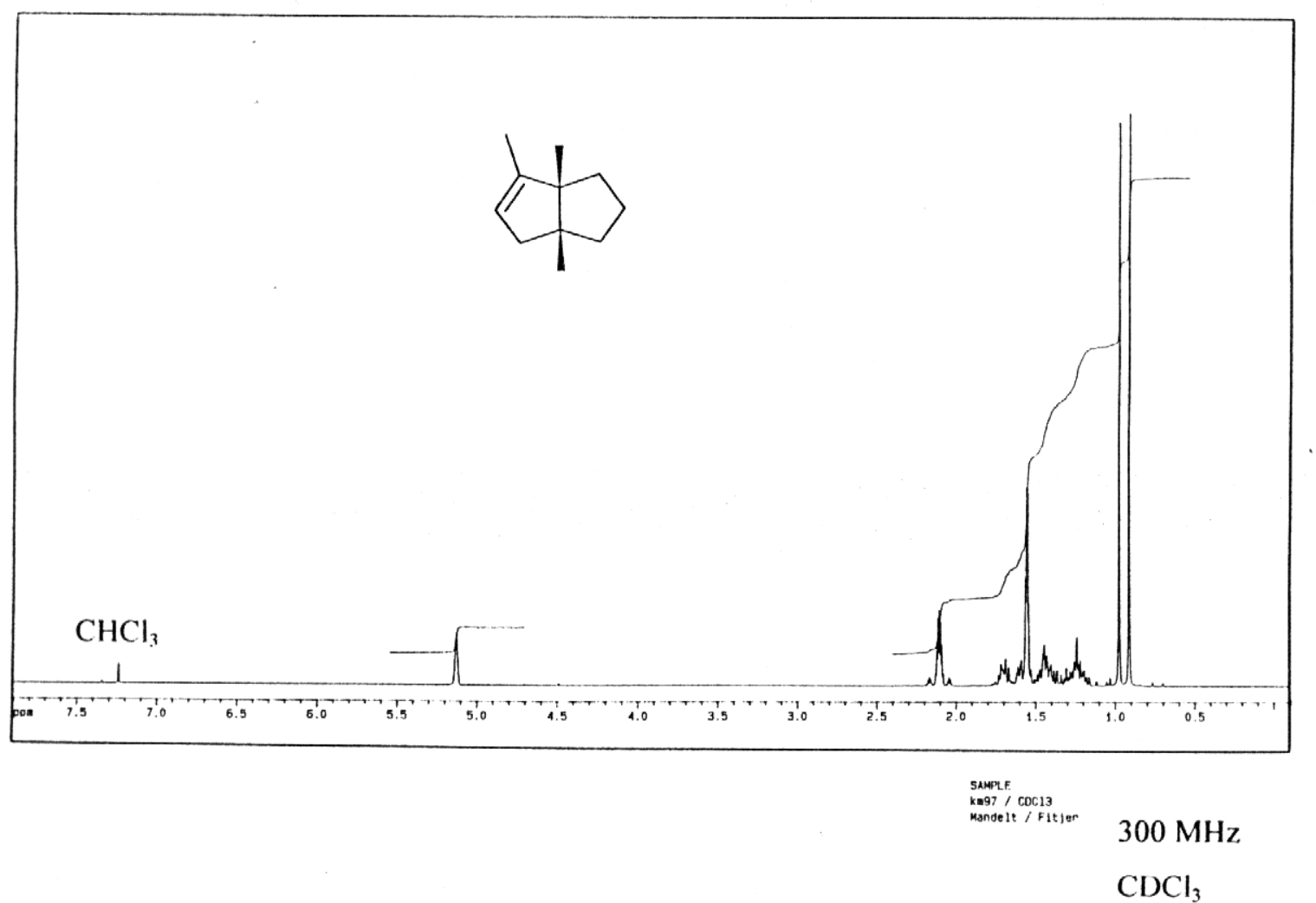

Abb. 52: $\quad$ 'H-NMR-Spektrum von ( $\left.3 \mathrm{a} R^{*}, 6 \mathrm{a} S^{*}\right)-3 \mathrm{a}, 6,6 \mathrm{a}$-Trimethyl-1,2,3,3a,4,6a-hexahydropentalen (118) 

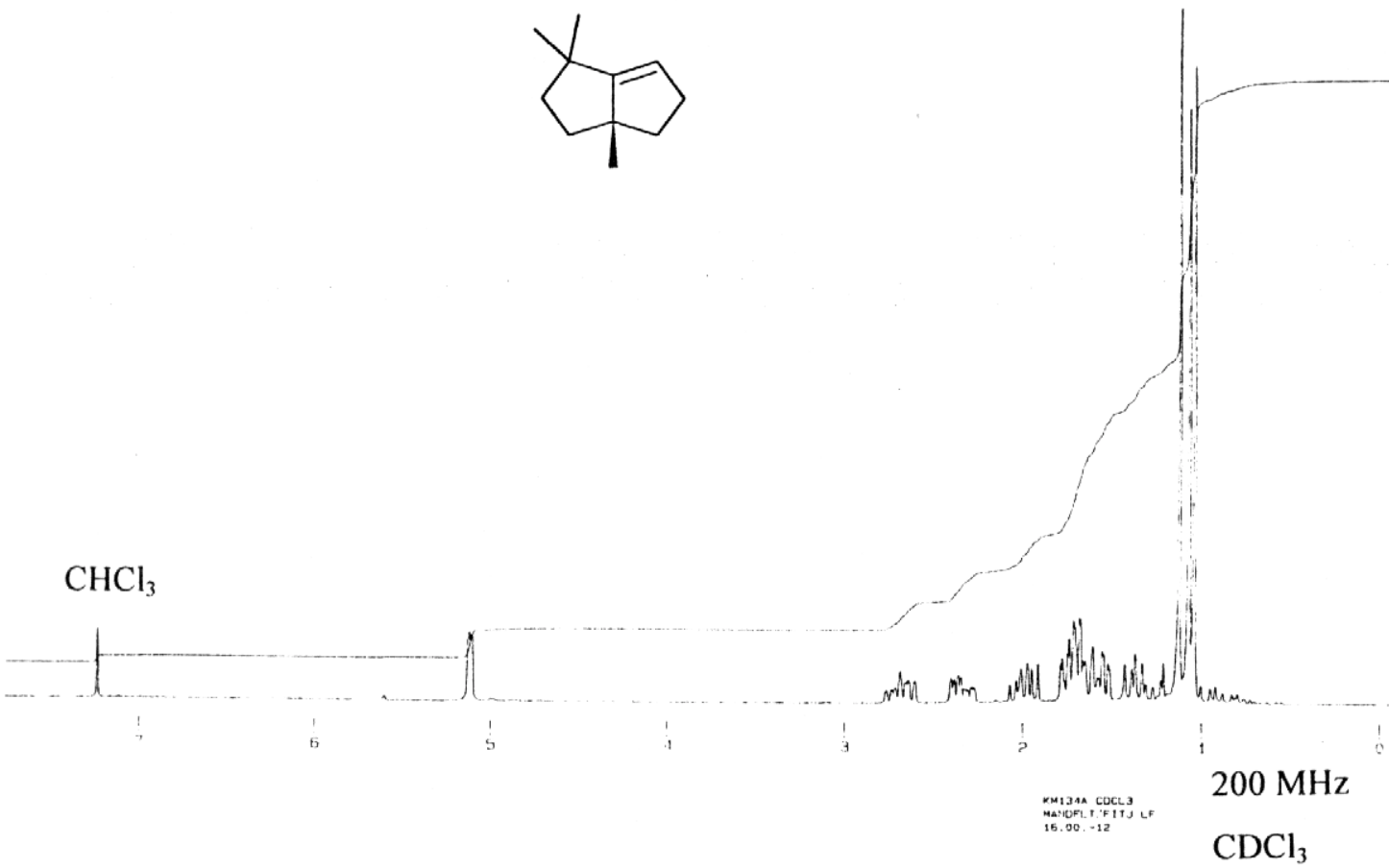

Abb. 53: $\quad{ }^{1}$ H-NMR-Spektrum von 1,1,3a-Trimethyl-1,2,3,3a,4,5-hexahydro-pentalen (120)

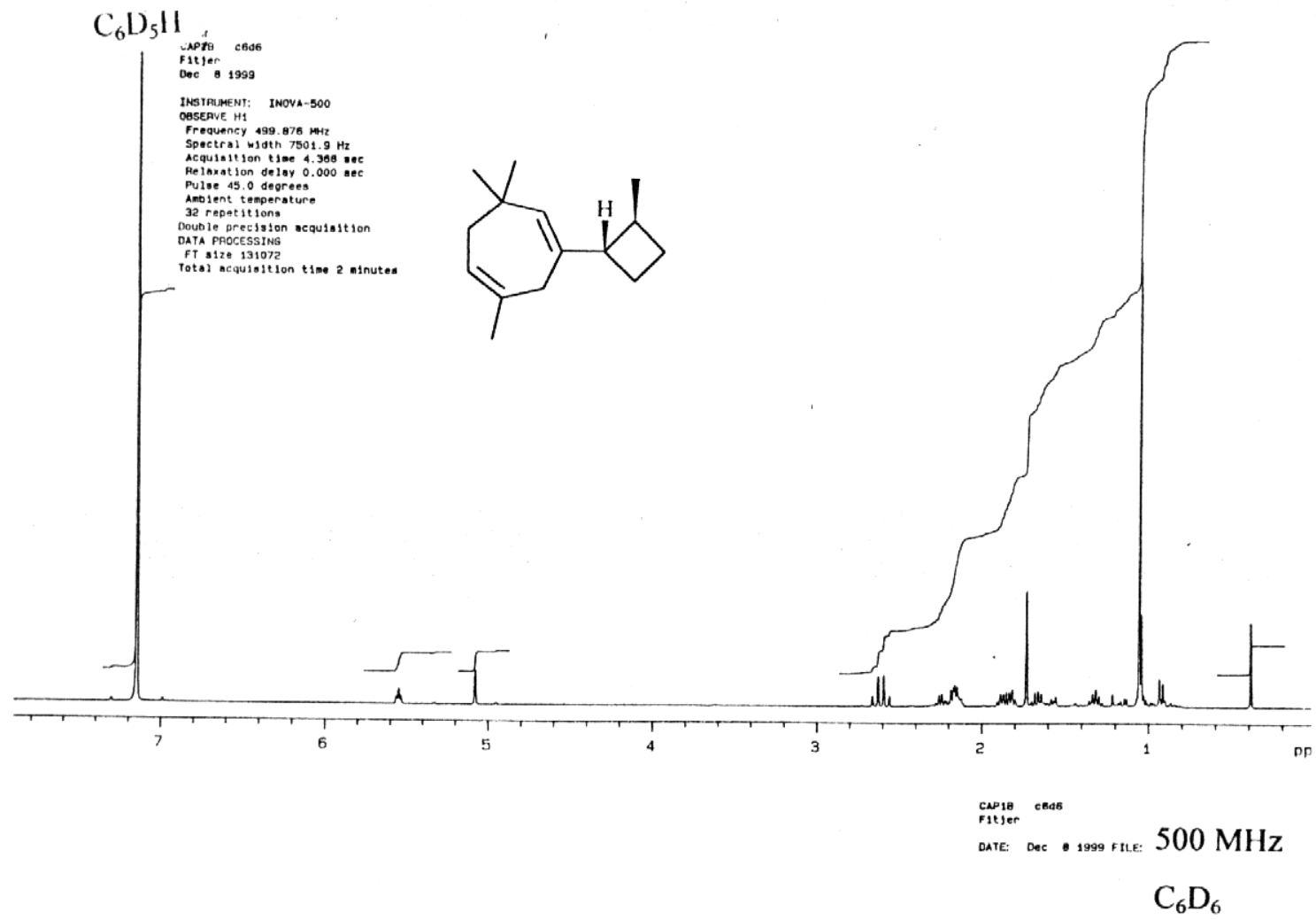

Abb. 54: $\quad$ 'H-NMR-Spektrum von 2,6,6-Trimethyl-4-(2-methylcyclobutyl)-cyclohepta1,4-dien (122) 


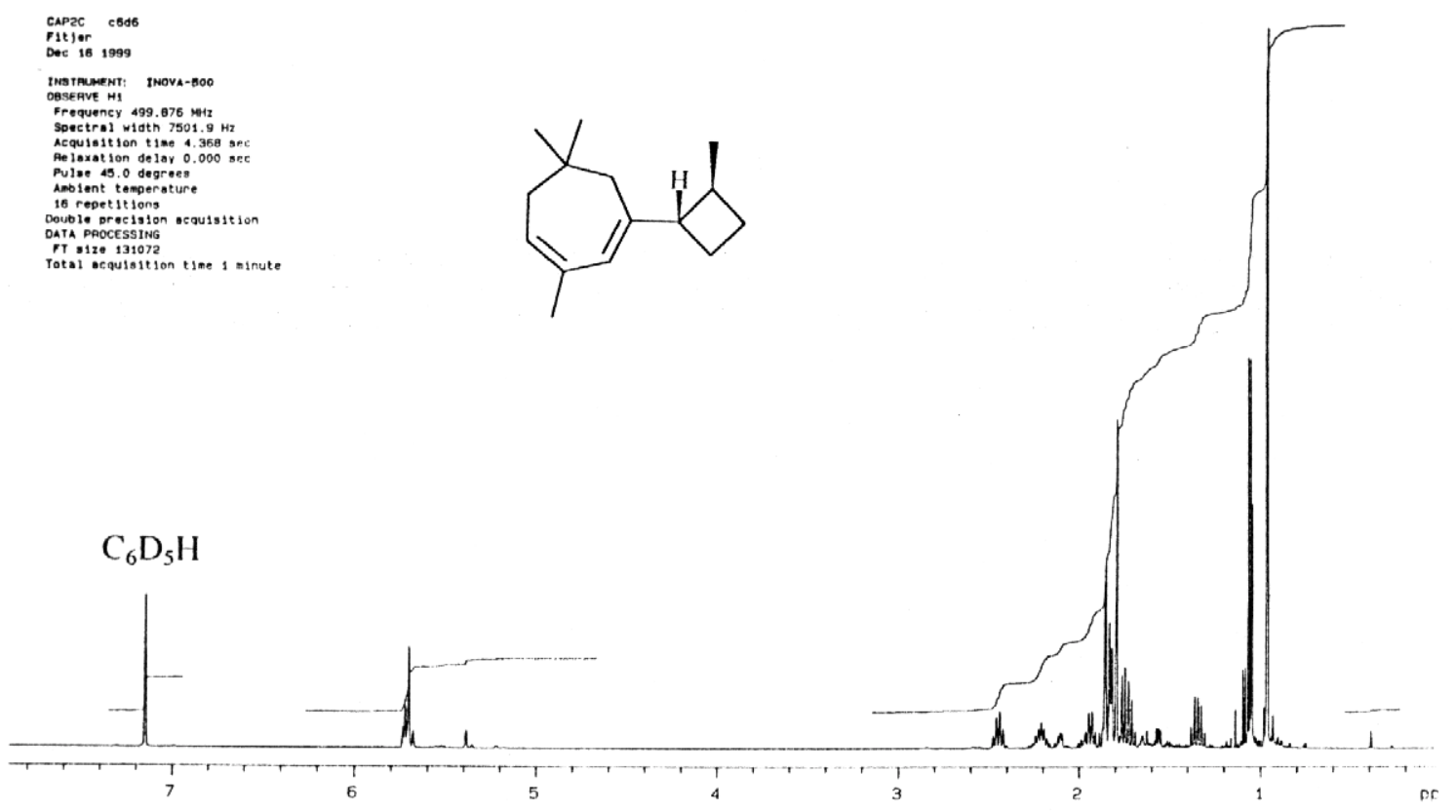

$500 \mathrm{MHz}$

$\mathrm{C}_{6} \mathrm{D}_{6}$

Abb. 55: $\quad{ }^{1}$ H-NMR-Spektrum von 3,6,6-Trimethyl-1-(2-methyl-cyclobutyl)-cyclohepta1,3-dien (123)
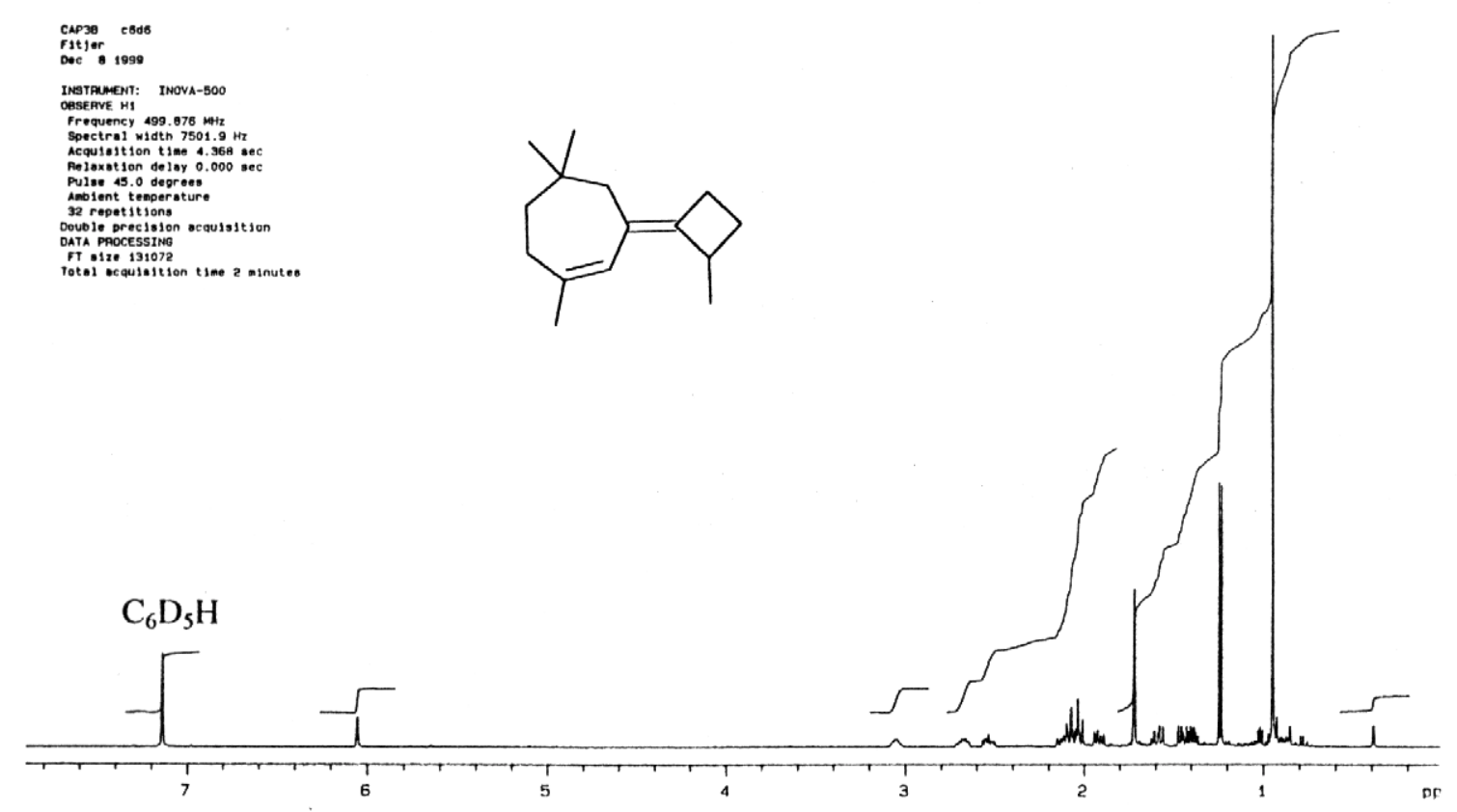

\section{$500 \mathrm{MHz}$}

$\mathrm{C}_{6} \mathrm{D}_{6}$

Abb. 56: $\quad{ }^{1}$ H-NMR-Spektrum von 1,5,5-Trimethyl-3-(2-methyl-cyclobutyliden)-cyclohept-1-en (124) 


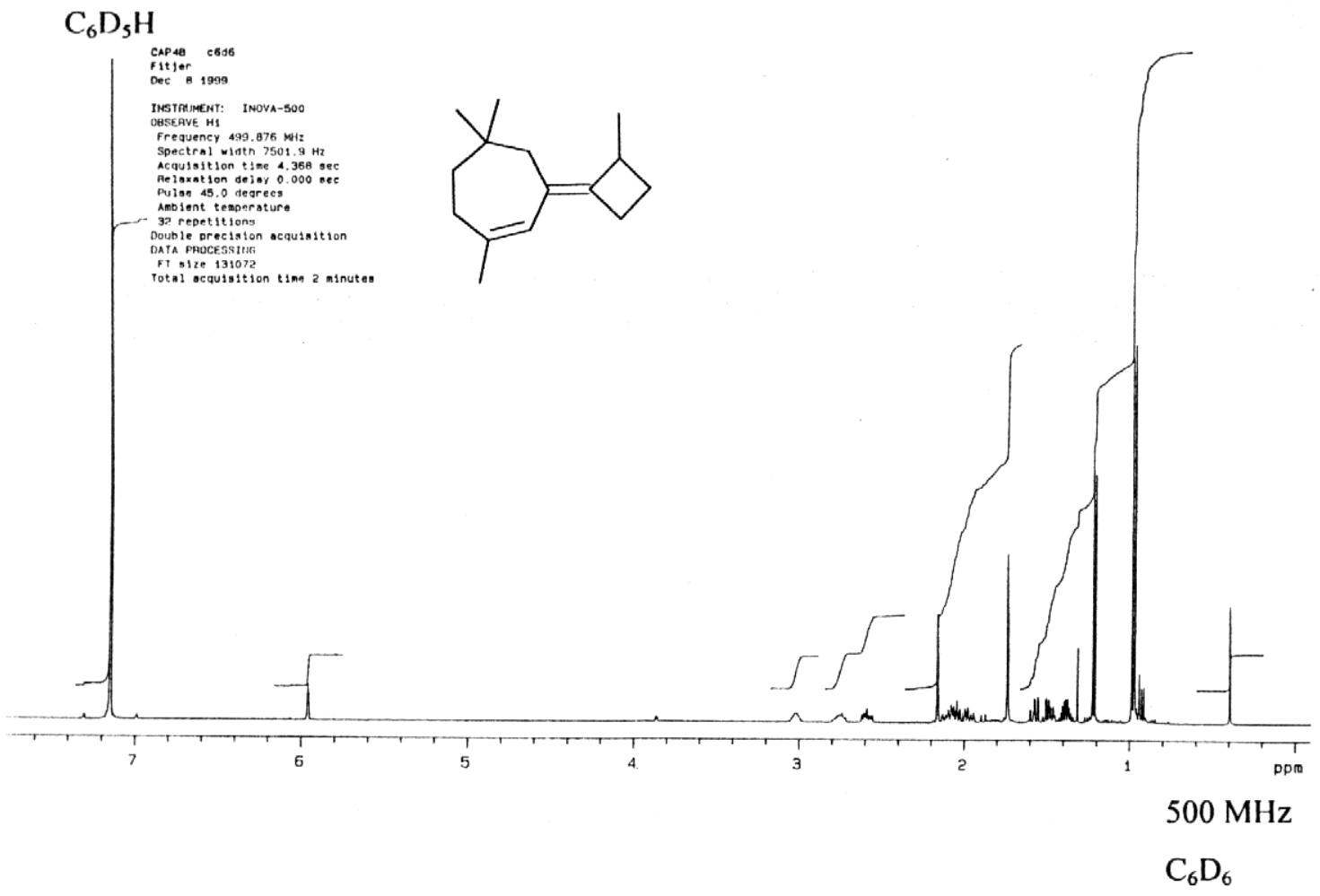

Abb. 57: $\quad{ }^{1}$ H-NMR-Spektrum von 1,5,5-Trimethyl-3-(2-methyl-cyclobutyliden)-cyclohept-1-en (125)
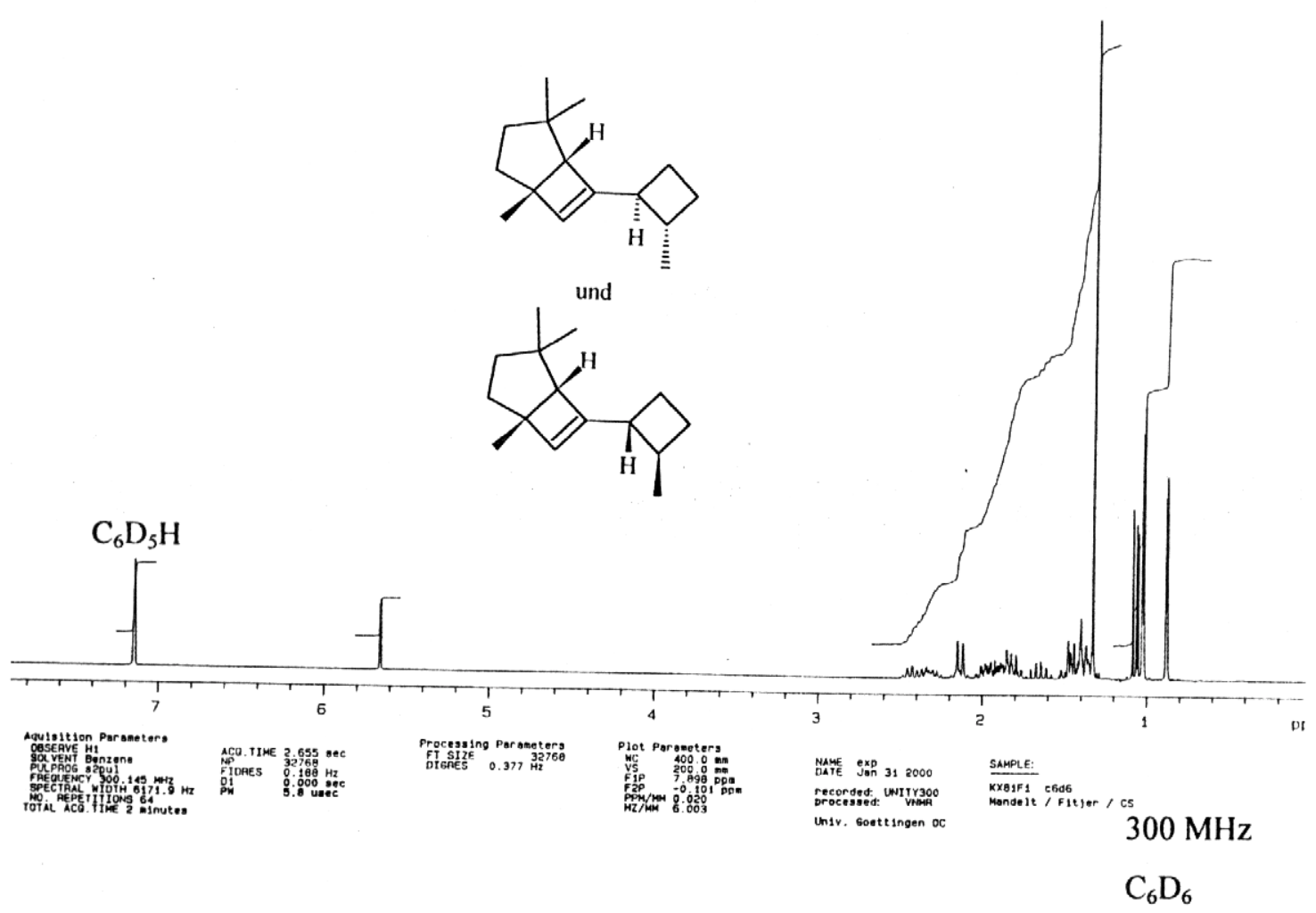

Abb. 58: $\quad{ }^{1}$ H-NMR-Spektrum von 1,4,4-Trimethyl-6-(2-methyl-cyclobutyl)-bicyclo[3.2.0]hept-6-en (130 und 131) 


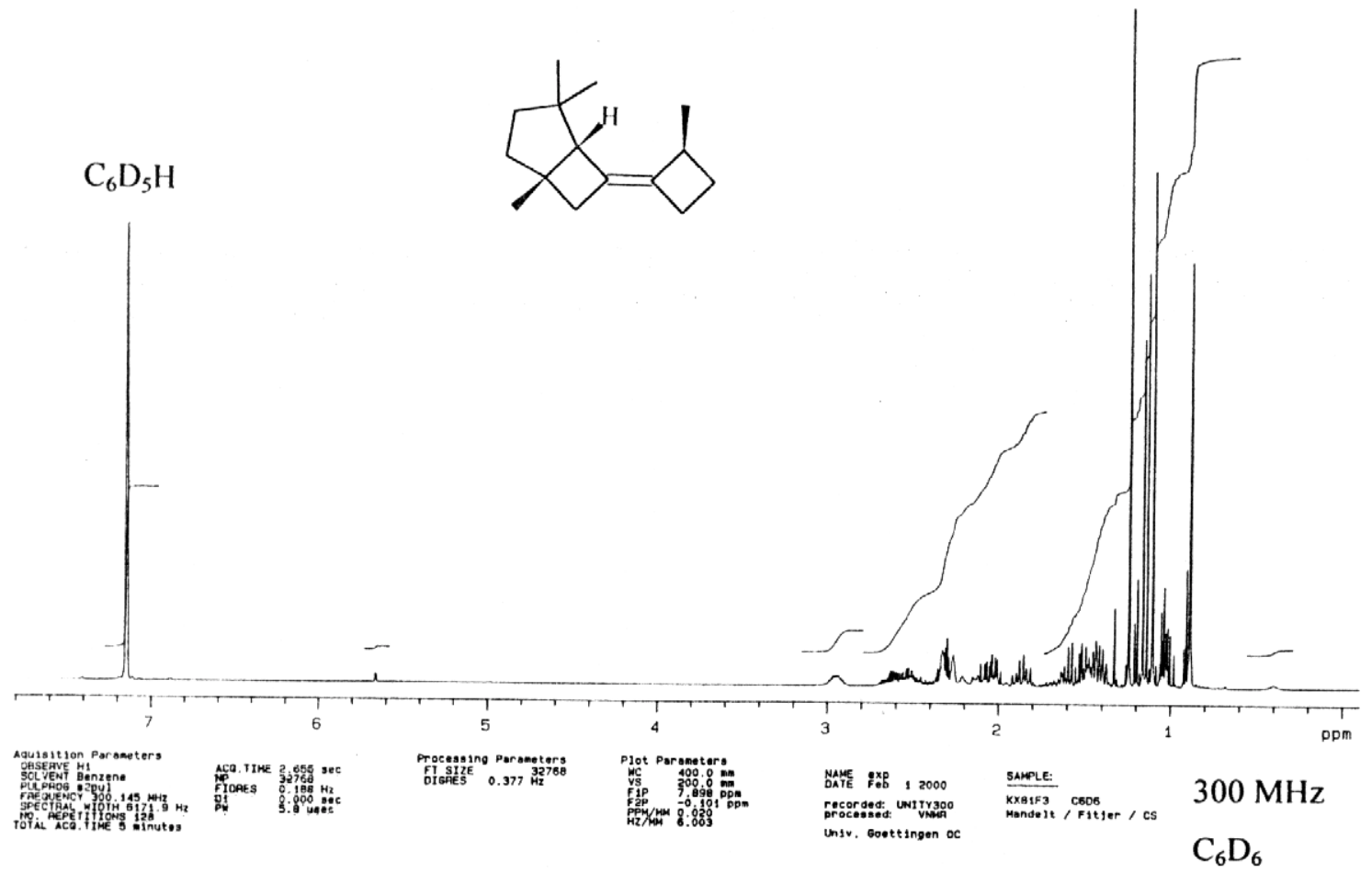

Abb. 59: $\quad{ }^{1}$ H-NMR-Spektrum von 1,4,4-Trimethyl-6-(2-methyl-cyclobutyliden)-bicyclo[3.2.0]heptan (132/133/134/135)

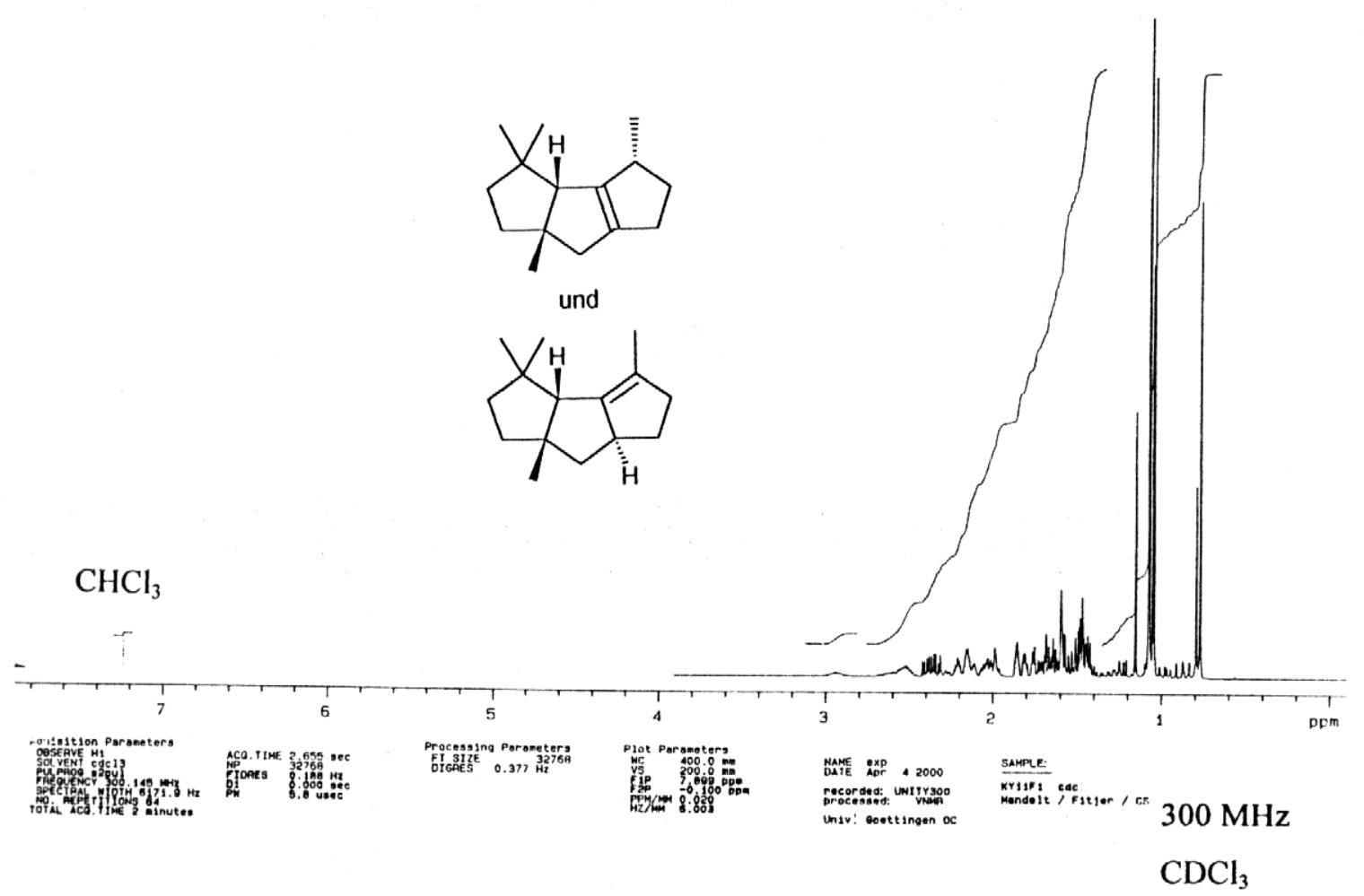

Abb. 60: $\quad{ }^{1} \mathrm{H}-\mathrm{NMR}-$ Spektrum von $\left(3 \mathrm{aR} *, 4 \mathrm{R}^{*}, 7 \mathrm{aR} *\right)-3,3,4,7 \mathrm{a}-$ Tetramethyl-2,3,3a,4,5,6,7,7a-octahydro- $1 H$-cyclopenta[a]pentalen (67) und ( $\left.3 \mathrm{aR}^{*}, 6 \mathrm{aR}^{*}, 7 \mathrm{aR}^{*}\right)-3,3,4,7 \mathrm{a}-$ Tetramethyl-2,3,3a,5,6,6a,7,7a-octahydro-1 $H$-cyclopenta[a]pentalen (68) 

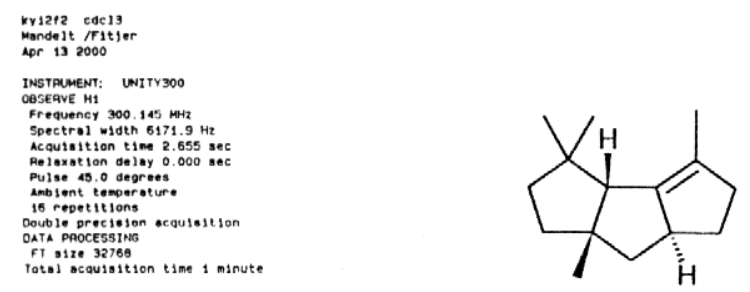

Totol acauisition time 1 minute

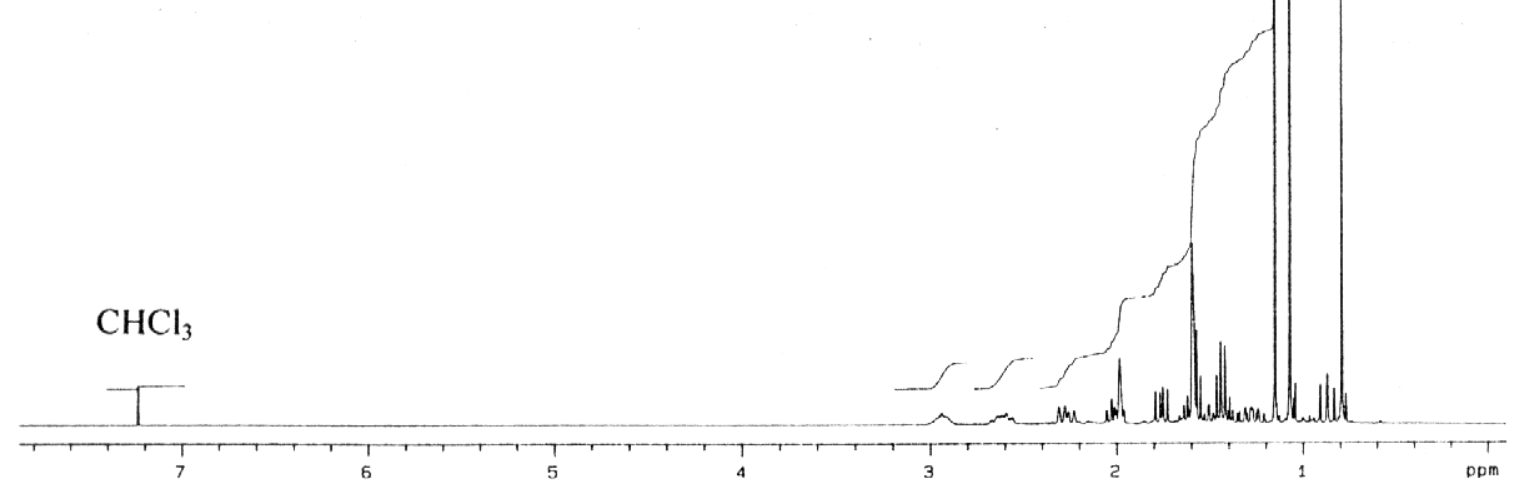

$300 \mathrm{MHz}$

$\mathrm{CDCl}_{3}$

Abb. 61: $\quad{ }^{1} \mathrm{H}-\mathrm{NMR}-$ Spektrum von $\left(3 \mathrm{aR}^{*}, 6 \mathrm{aR} *, 7 \mathrm{aR} *\right)-3,3,4,7 \mathrm{a}-$ Tetramethyl2,3,3a,5,6,6a,7,7a-octahydro- $1 H$-cyclopenta[a]pentalen (68)

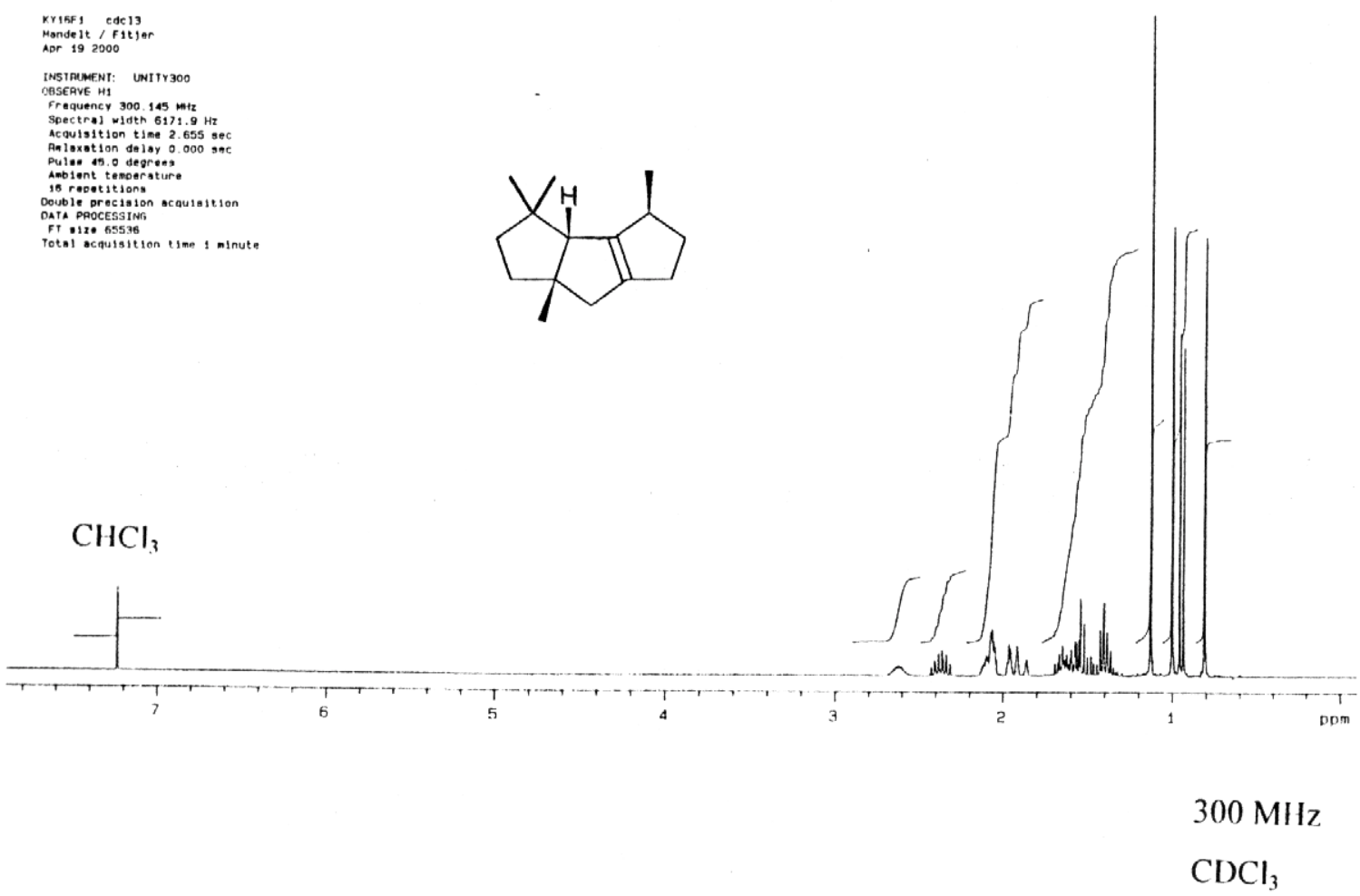

Abb. 62: $\quad{ }^{1} \mathrm{H}-\mathrm{NMR}-$ Spektrum von ( $\left.3 \mathrm{aR}^{*}, 4 \mathrm{R}^{*}, 7 \mathrm{aR}^{*}\right)-3,3,4,7 \mathrm{a}-$ Tetramethyl2,3,3a,4,5,6,7,7a-octahydro- $1 H$-cyclopenta[a]pentalen (69) 


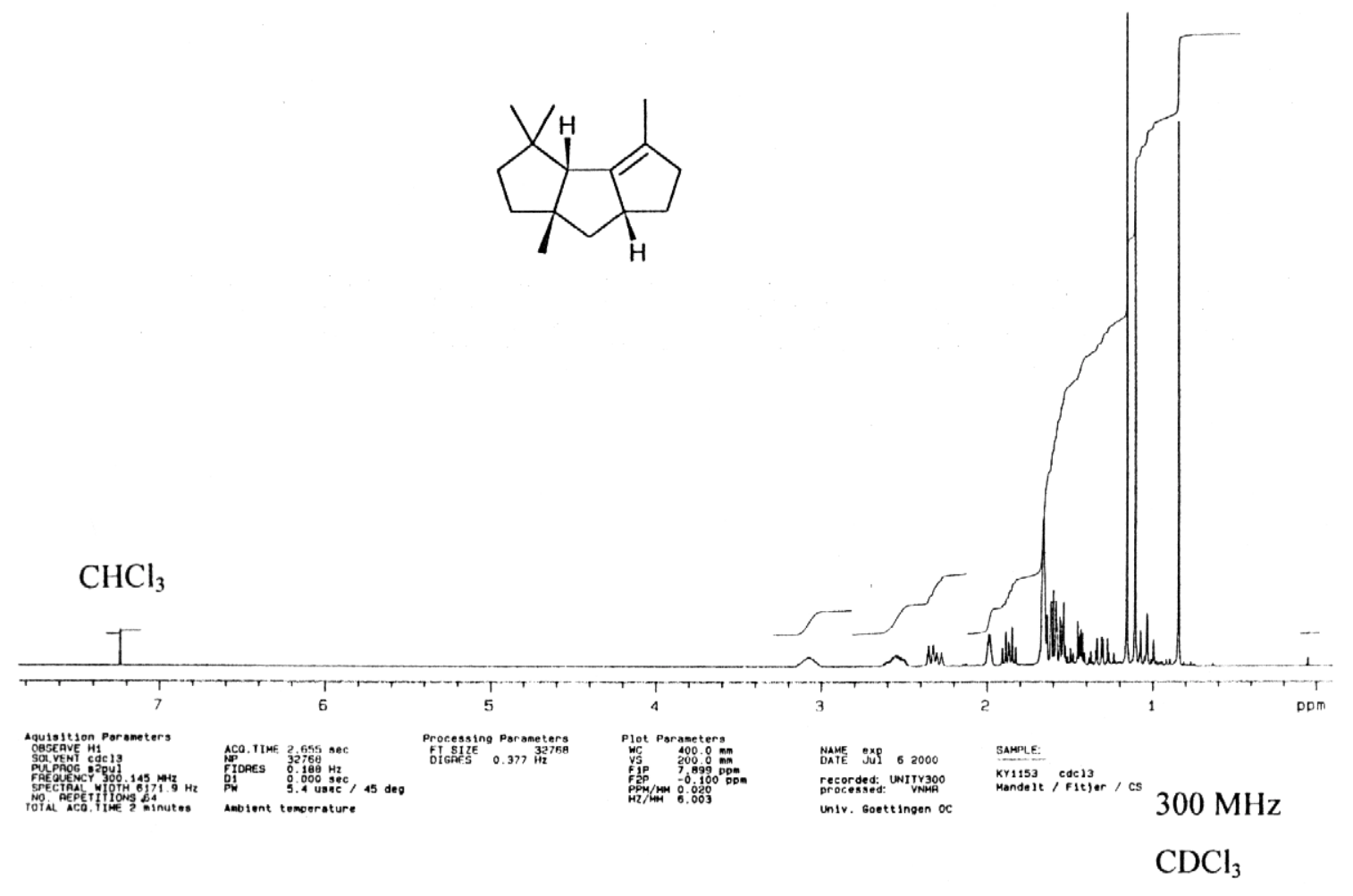

Abb. 63: 'H-NMR-Spektrum von (3aR*,6aS*,7aR*)-3,3,4,7a-Tetramethyl2,3,3a,5,6,6a,7,7a-octahydro- $1 H$-cyclopenta[a]pentalen (70)
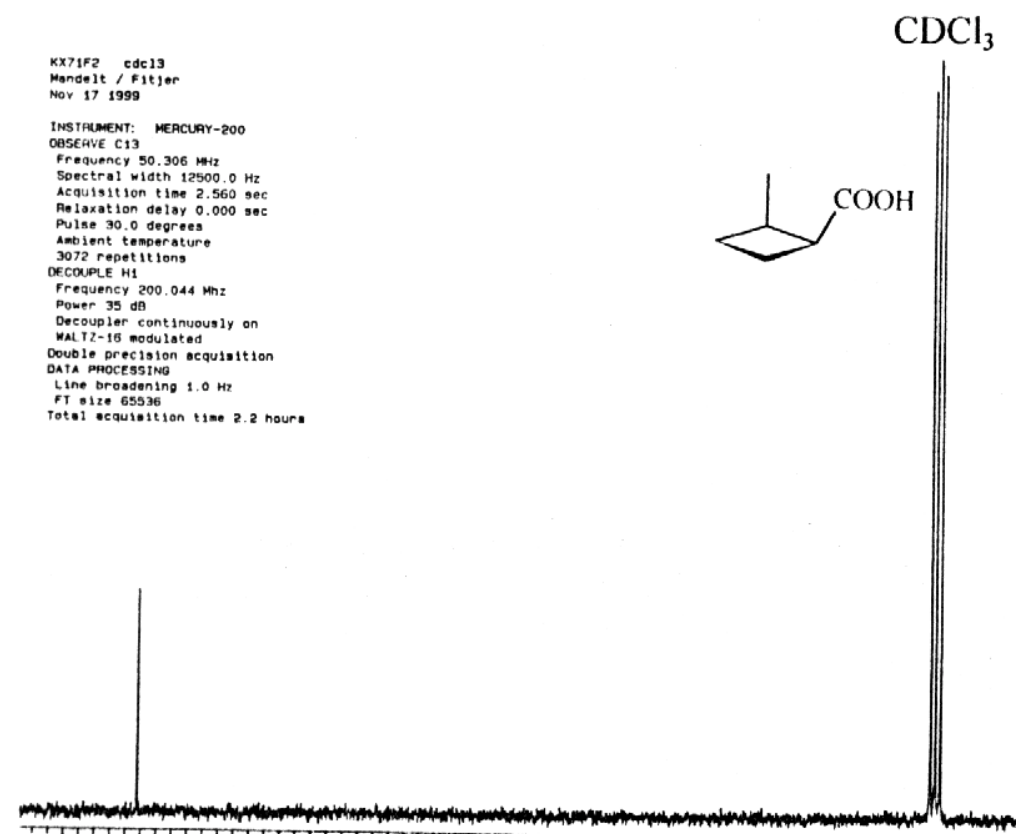

180

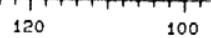

80

60

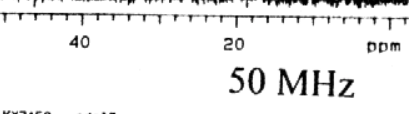

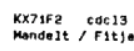

DATE: Nov $171999 \quad \mathrm{CDCl}_{3}$

Abb. 64: $\quad{ }^{13}$ C-NMR-Spektrum von $\left(1 R^{*}, 2 S^{*}\right)$-2-Methyl-cyclobutancarbonsäure (81a) 


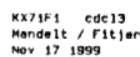

INSTRUMENT: MEACUAY-200
OASEAVE C13

Frequency 50,306 mats

Soectral w1dth $12500.0 \mathrm{~Hz}$
acquigition time $2.560 \mathrm{goc}$

Ae laxation delay 0.000 se

Puse 30 O degrees
Anbient tomoratur.

3072 repetits
DECOUPE H1

erequency 200.044 Mnz

Power 35 al
Decoup ler cant snuousiy

Mal 12-16 modulated

ATA Processina

Cine brosacening $0.5 \mathrm{~Hz}$

fotal acquisittion time 2.2 hours

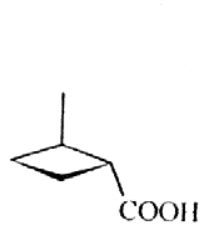

$\mathrm{COOH}$

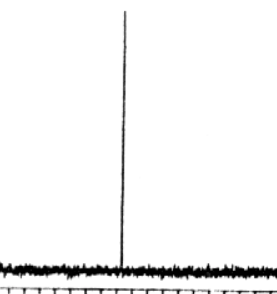

180

160

140

120

100

80

60

20

$50 \mathrm{MHz}$

KX71F 1 cde13
Handelt fitjer

DATE: Nov 171999

$\mathrm{CDCl}_{3}$

Abb. 65: $\quad{ }^{13} \mathrm{C}-\mathrm{NMR}$-Spektrum von $\left(1 R^{*}, 2 R^{*}\right)$-2-Methyl-cylobutancarbonsäure (81b)
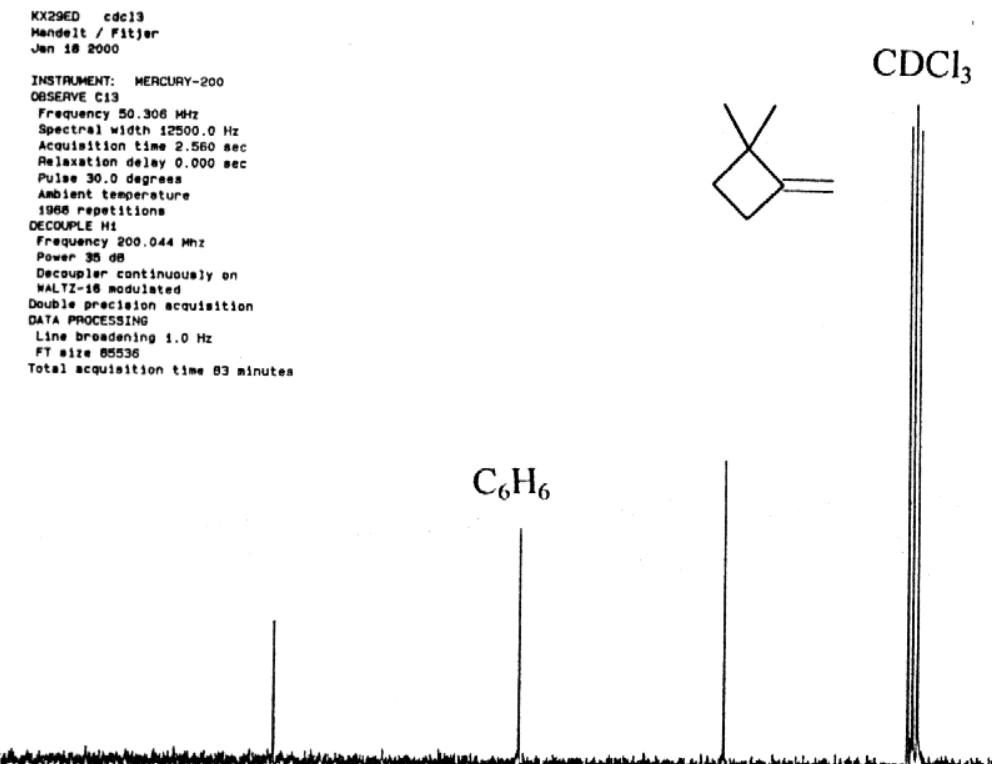

180

160

120

100

80

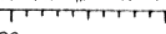

$50 \mathrm{MHz}^{\mathrm{ppm}}$

KXzged cde13
Mandelt / Fitjor

DATE: Jon 182000

$\mathrm{CDCl}_{3}$

Abb. 66: $\quad{ }^{13}$ C-NMR-Spektrum von 1,1-Dimethyl-2-methylencyclobutan (91) 


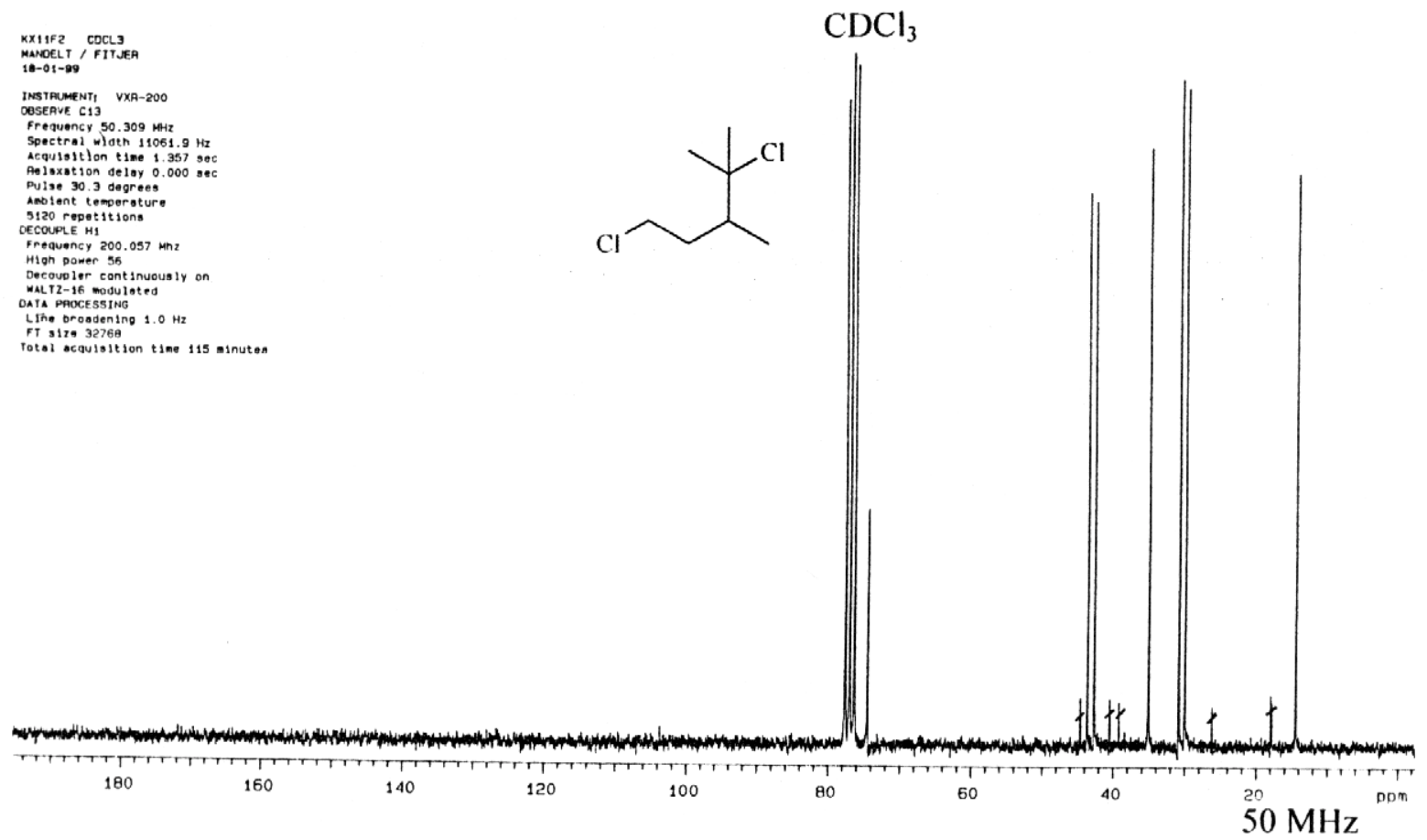

$\mathrm{CDCl}_{3}$

Abb. 67: $\quad{ }^{13}$ C-NMR-Spektrum von 1,4-Dichlor-3,4-dimethyl-pentan (96)
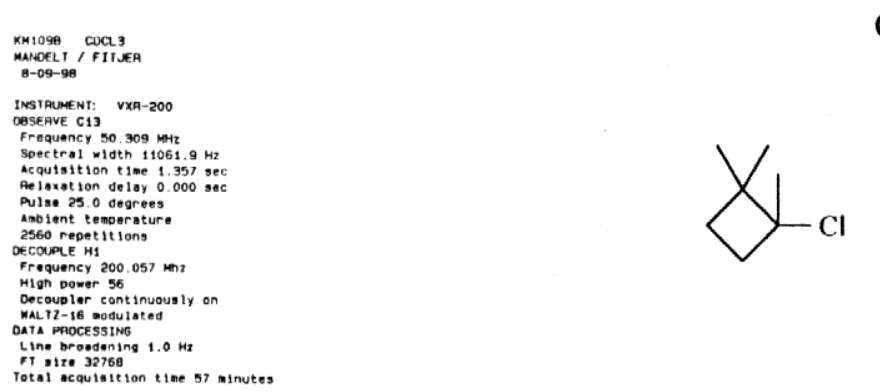

$\mathrm{CDCl}_{3}$

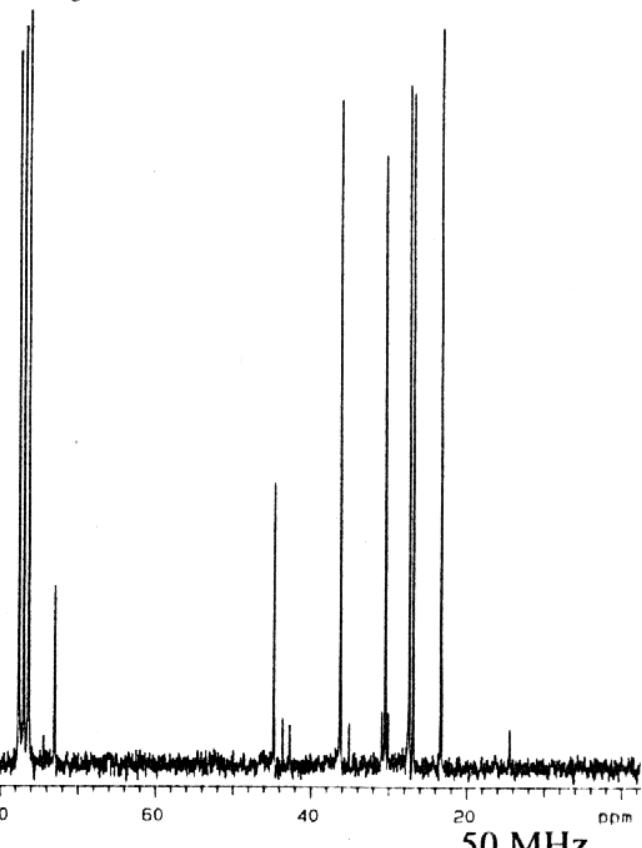

$\mathrm{CDCl}_{3}$

Abb. 68: $\quad{ }^{13}$ C-NMR-Spektrum von 1,2,2-Trimethyl-cyclobutylchlorid (97) 


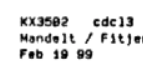

INSTPUMENT: UNITY3OO
OASERVE C13

OQSERVE C13
Frequeney 75.479 MHI

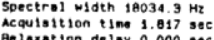

Aaloxation delay 0.000

Ambient temoeratur.

3412 reppetitions
OECOUPLE H1

Frequency 300.143 Mhx

Decoup 1 er continuous $1 y$ on

Double precision ocquistits

Ling brodedening $1.0 \mathrm{~Hz}$

FT G12e s5336
Totai ocquisition time 103 minutes

$\mathrm{CDCl}_{3}$
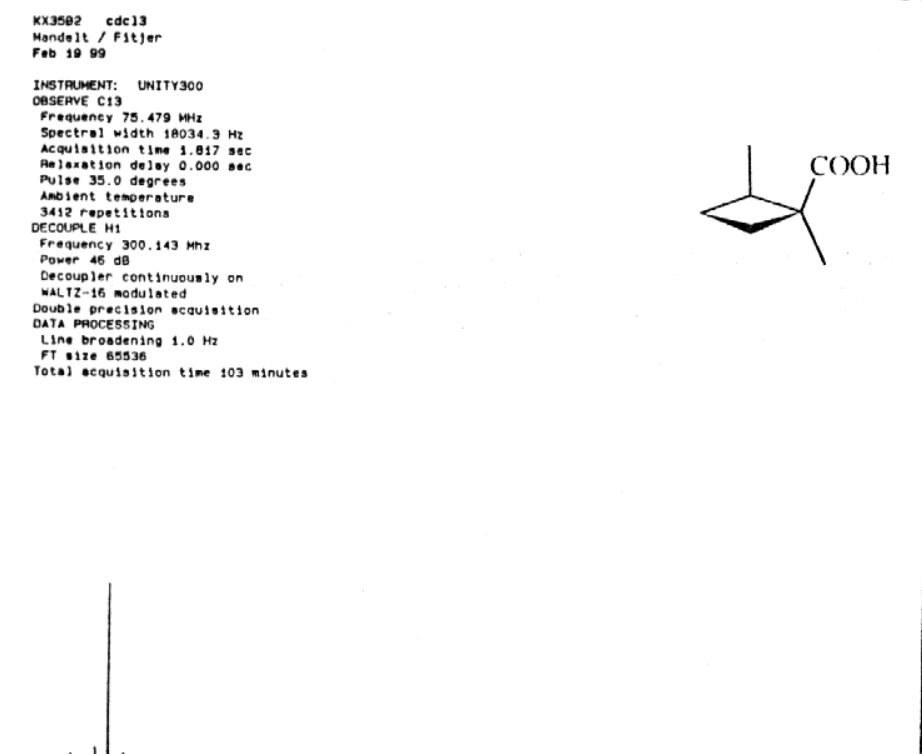

180

160

140

120

100

80

60

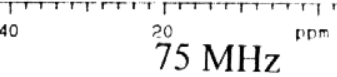

KX35B2
Mande It if Itjer DATE: Fob 1999

$\mathrm{CDCl}_{3}$

Abb. 69: $\quad{ }^{13}$ C-NMR-Spektrum von $\left(1 R^{*}, 2 S^{*}\right)$-1,2-Dimethyl-cyclobutancarbonsäure (98a)

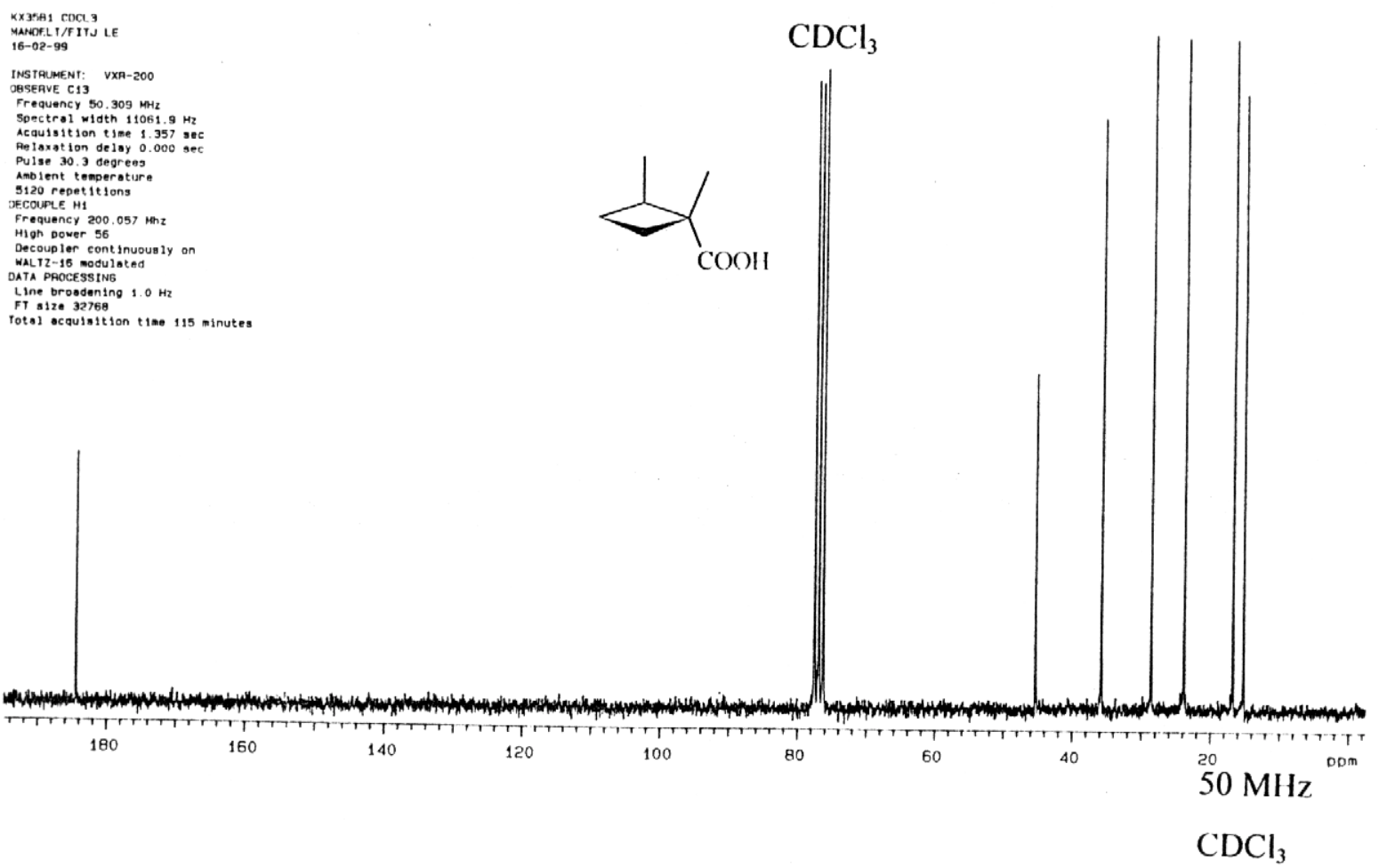

Abb. 70: $\quad{ }^{13}$ C-NMR-Spektrum von $\left(1 R^{*}, 2 R^{*}\right)$-1,2-Dimethyl-cyclobutancarbonsäure (98b) 


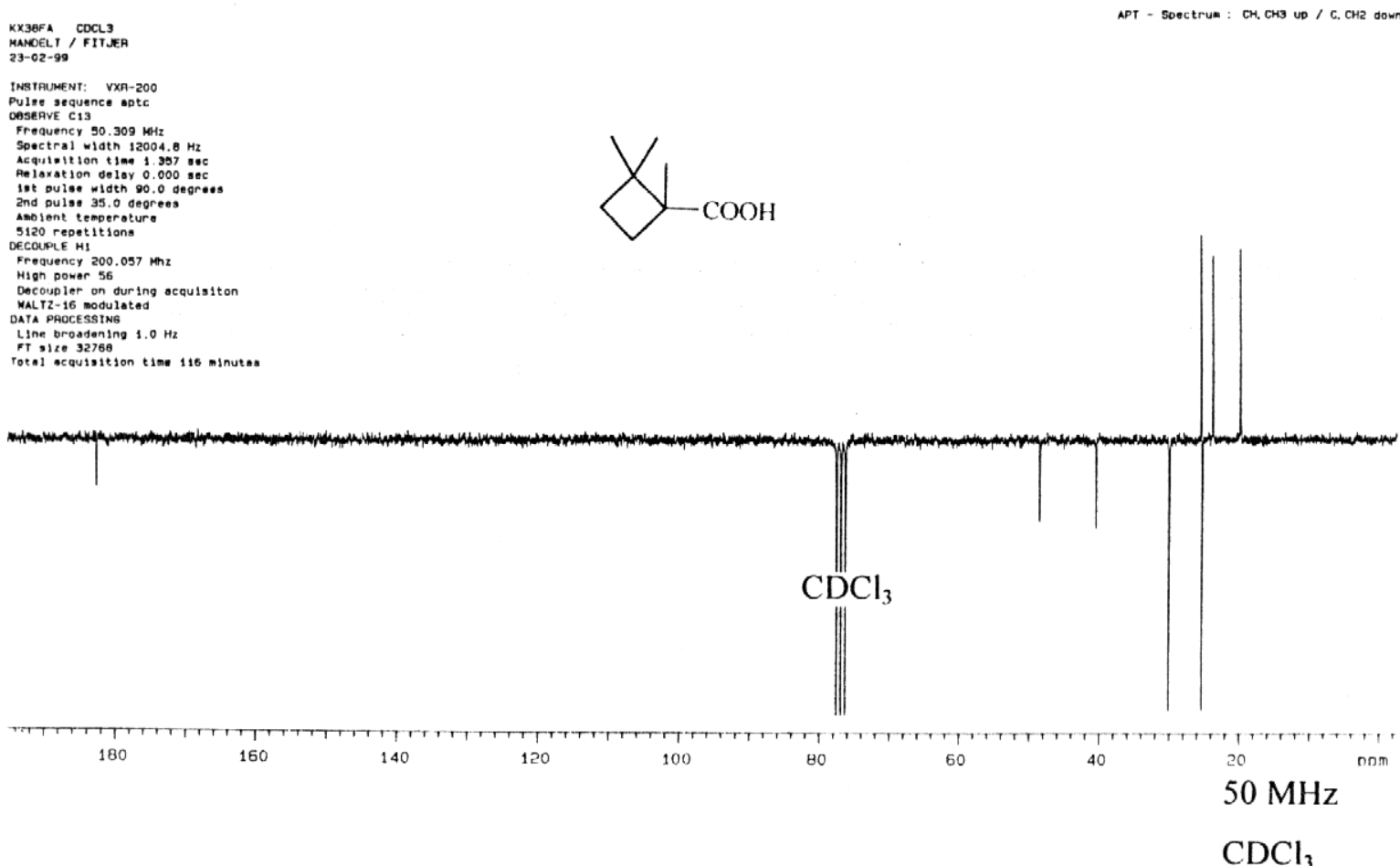

Abb. 71: $\quad{ }^{13}$ C-NMR-Spektrum von 1,2,2-Trimethyl-cyclobutancarbonsäure (100)
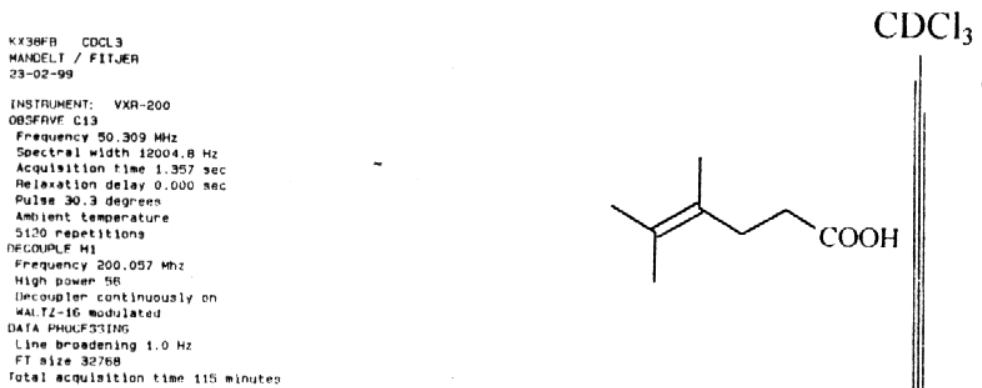

rotal acquisition time 115 minute
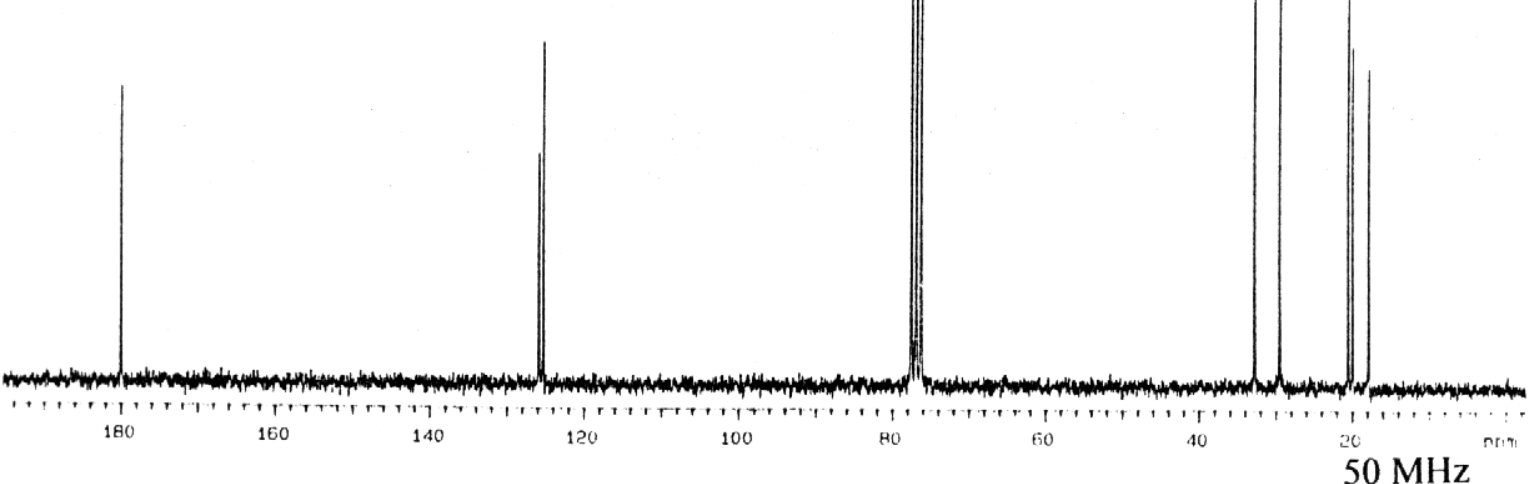

$\mathrm{CDCl}_{3}$

Abb. 72: $\quad{ }^{13}$ C-NMR-Spektrum von 4,5-Dimethylhex-4-en-säure (102) 


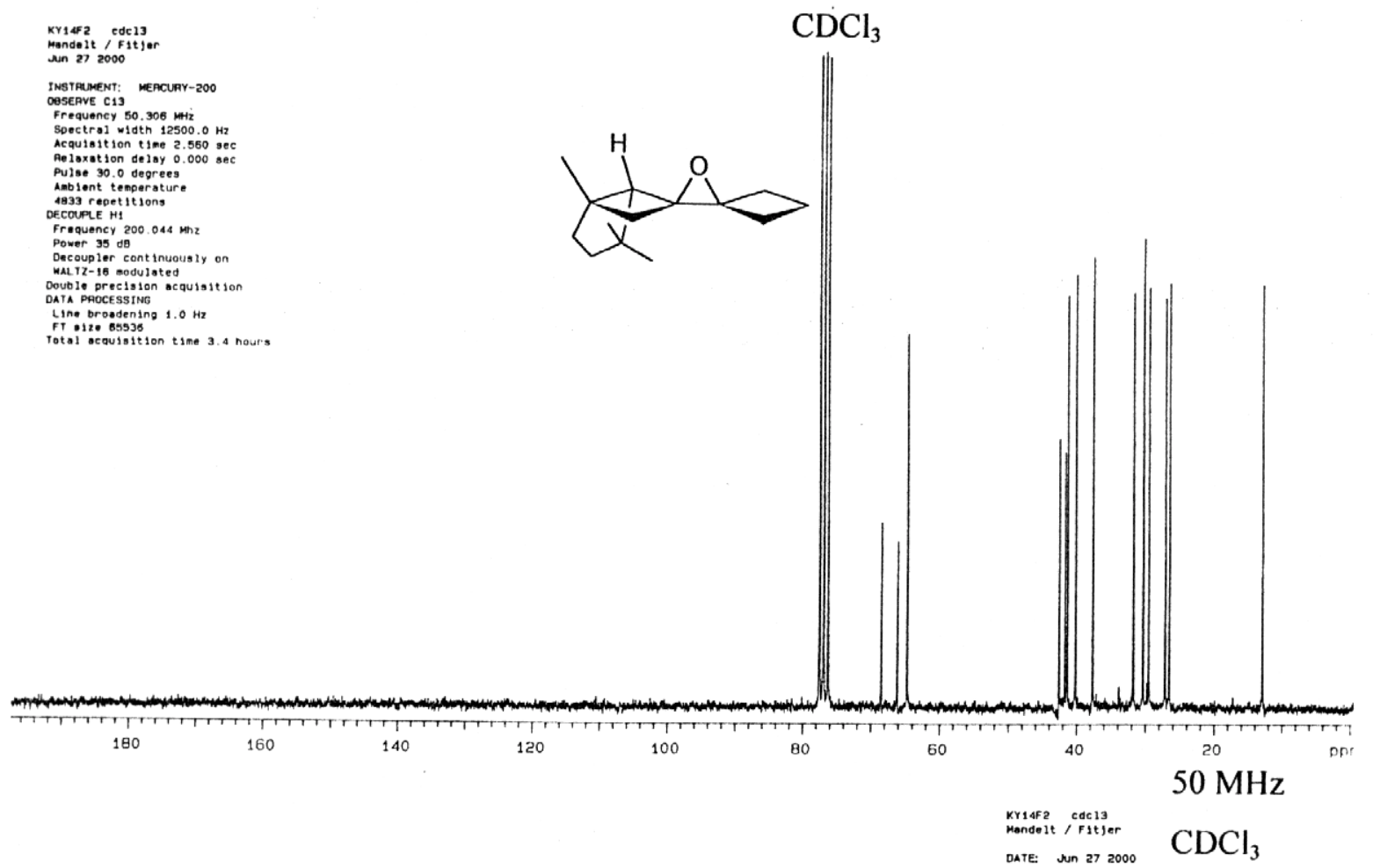

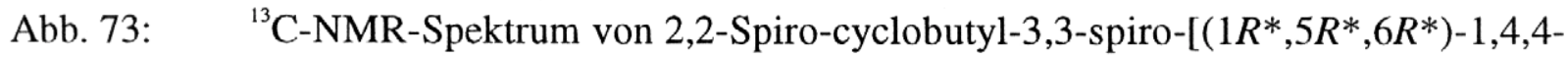
trimethyl-bicyclo[3.2.0]heptan-6,6-yl]-oxiran (55)

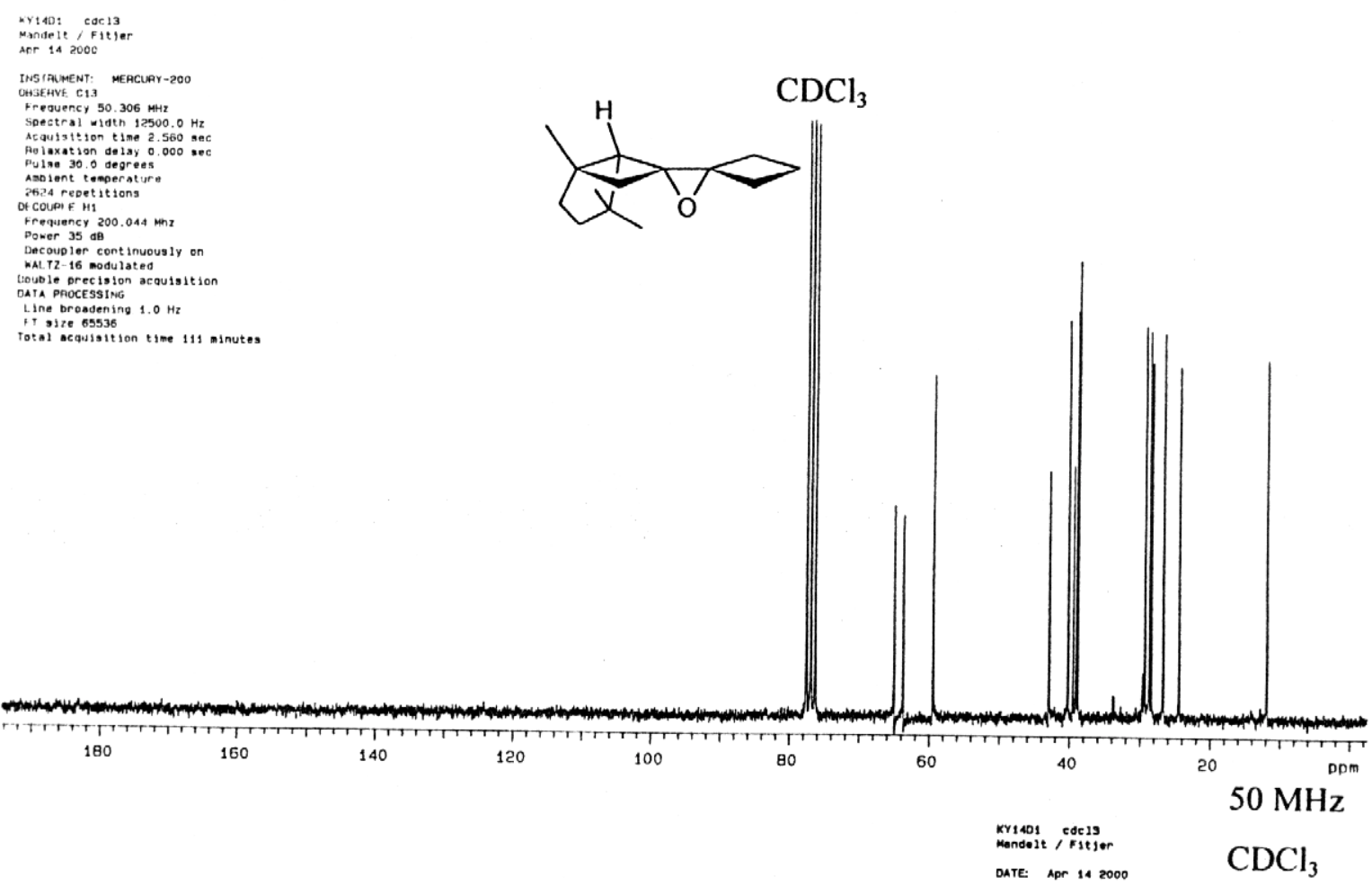

Abb. 74: $\quad{ }^{13} \mathrm{C}-\mathrm{NMR}-\mathrm{Spektrum}$ von 2,2-Spiro-cyclobutyl-3,3-spiro-[(1 $\left.R^{*}, 5 R^{*}, 6 S^{*}\right)-1,4,4-$ trimethyl-bicyclo[3.2.0]heptan-6,6-yl]-oxiran (56) 


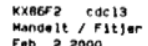

INSTRUMENT: MEACUAY-200

OESEAvE C13
Frequency $50.305 \mathrm{mtz}$

Frequency $50.305 \mathrm{HHz}$
Soectral w1dth $12500.0 \mathrm{~Hz}$

Acquisition time $2.560 \mathrm{gec}$
pe laxation de iay $0.000 \mathrm{gec}$

Puise 30.0 degrees.

Ambient temperatur
5120 repetitions

COUPLE H:

Decoupler cont inuousiy

Doub je precision acquisition

DATA PAOCESSTMG
Lino broosenting $1.0 \mathrm{~Hz}$

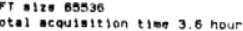

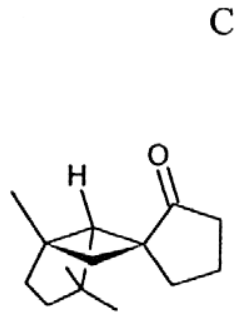

$\mathrm{CDCl}_{3}$
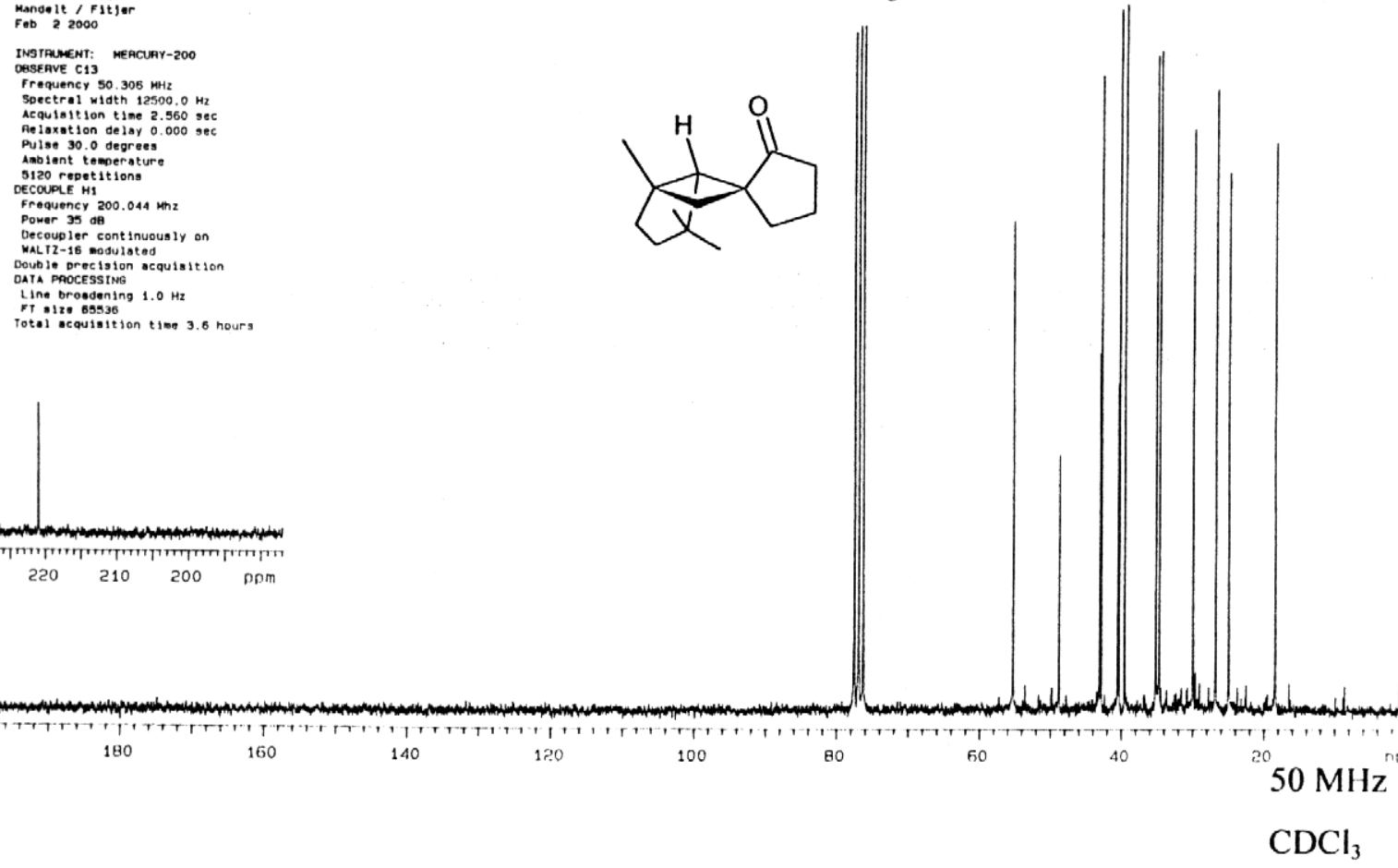

Abb. 75: $\quad{ }^{13} \mathrm{C}-\mathrm{NMR}-\mathrm{Spektrum}$ von $\left(1 R^{*}, 5 R^{*}, 6 R^{*}\right)-1,4,4$-Trimethylspiro \{ bicyclo-

[3.2.0]heptan-6,1'-cyclopentan-2'-on $\}(57)$
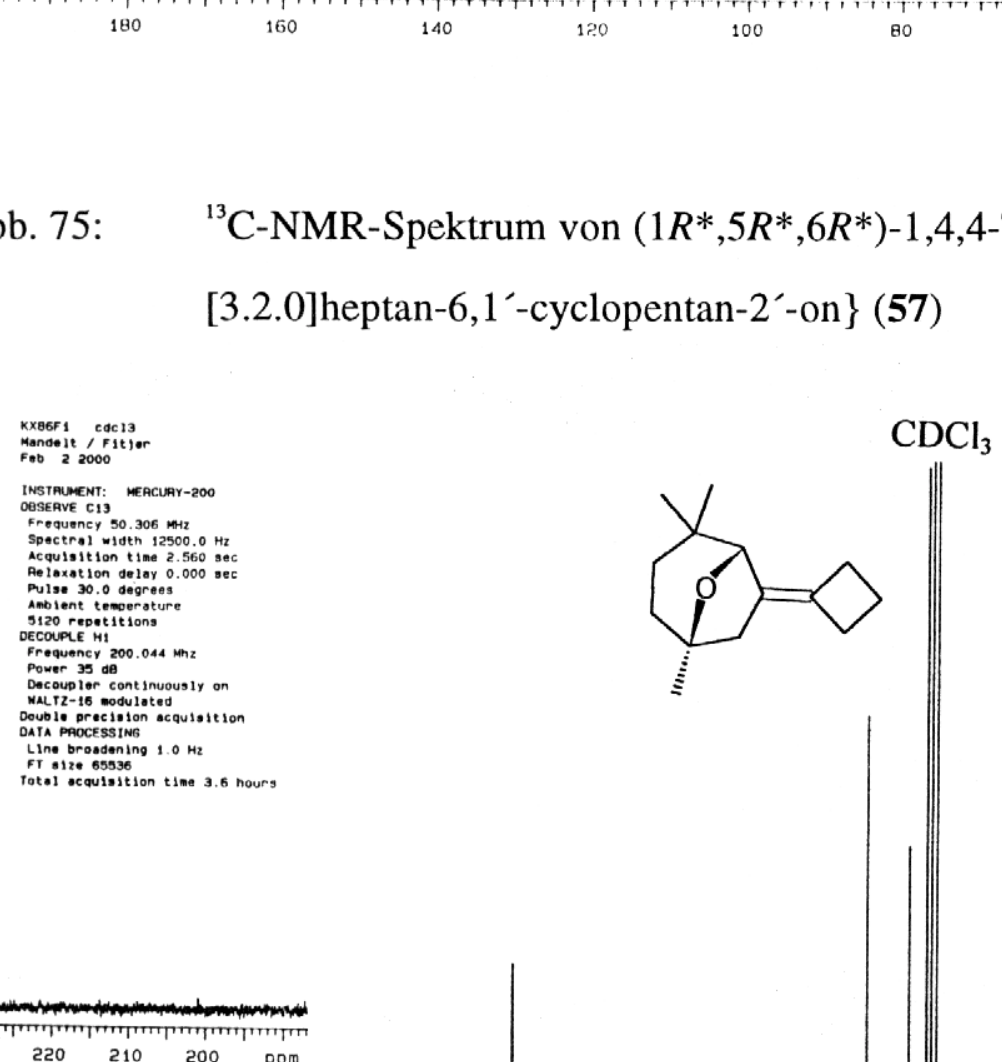

100

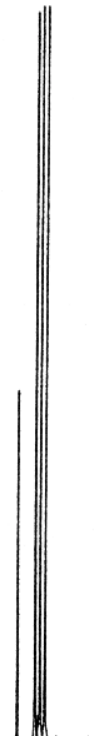

160

140

120

100

80

60

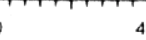

40

$50 \mathrm{MHz}$

$\mathrm{CDCl}_{3}$

Abb. 76: $\quad{ }^{13}$ C-NMR-Spektrum von 6-Cyclobutyliden-1,4,4-trimethyl-8-oxa-bicyclo-

[3.2.1]octan (138) 


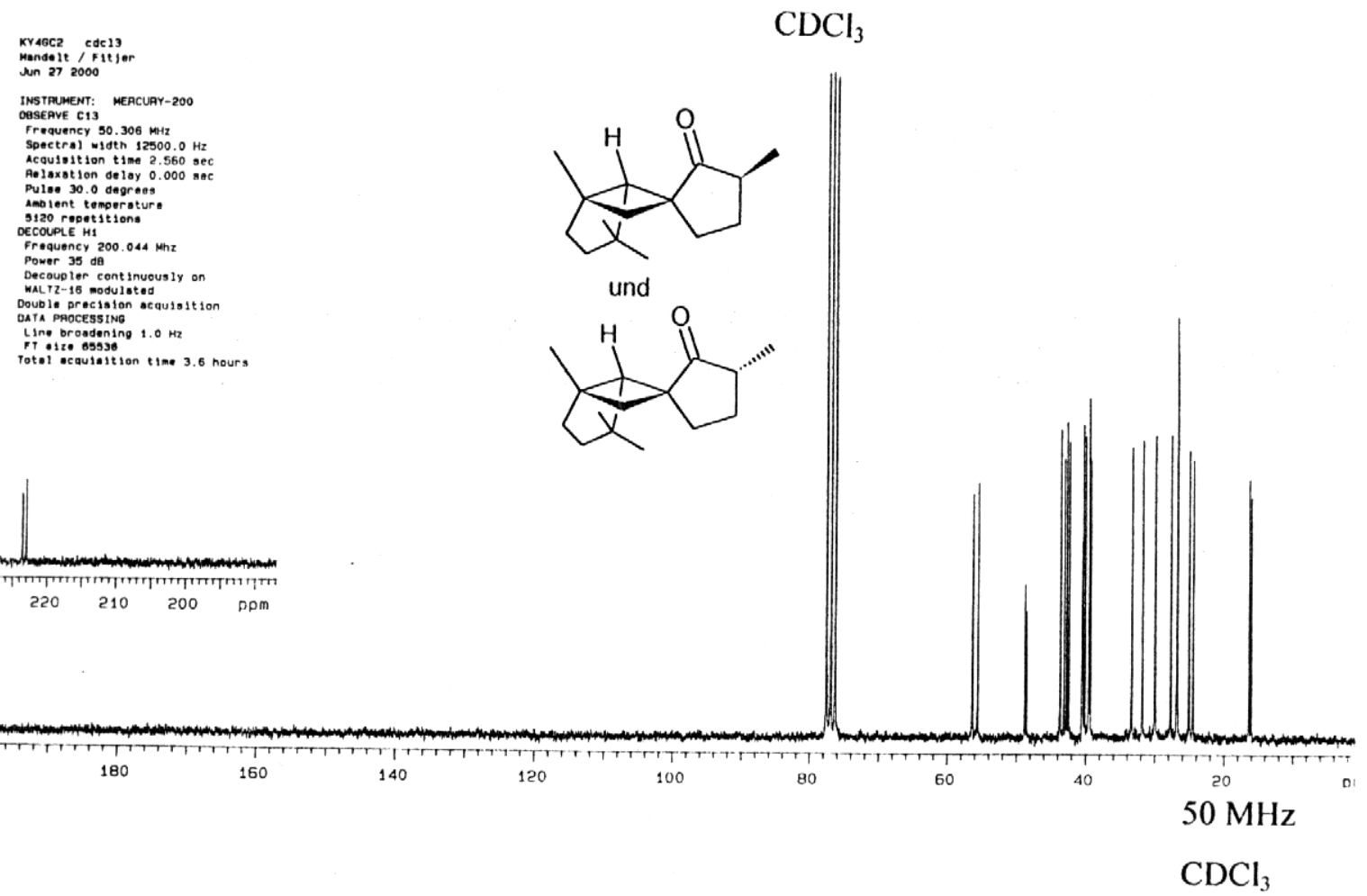

Abb. 77: $\quad{ }^{13} \mathrm{C}-\mathrm{NMR}-\mathrm{Spektrum}$ von $\left(1 R^{*}, 5 R^{*}, 6 R^{*}, 3 S^{*}\right)-1,4,4,3^{\prime}$-Tetramethylspiro- $\{$ bicyclo-

[3.2.0]heptan-6,1'-cyclopentan-2'-on $\}(141)$ und $\left(1 R^{*}, 5 R^{*}, 6 R^{*}, 3 R^{*}\right)-1,4,4,3^{\prime}$ -

Tetramethylspiro\{bicyclo[3.2.0]heptan-6,1'-cyclopentan-2'-on $\}$ (142)

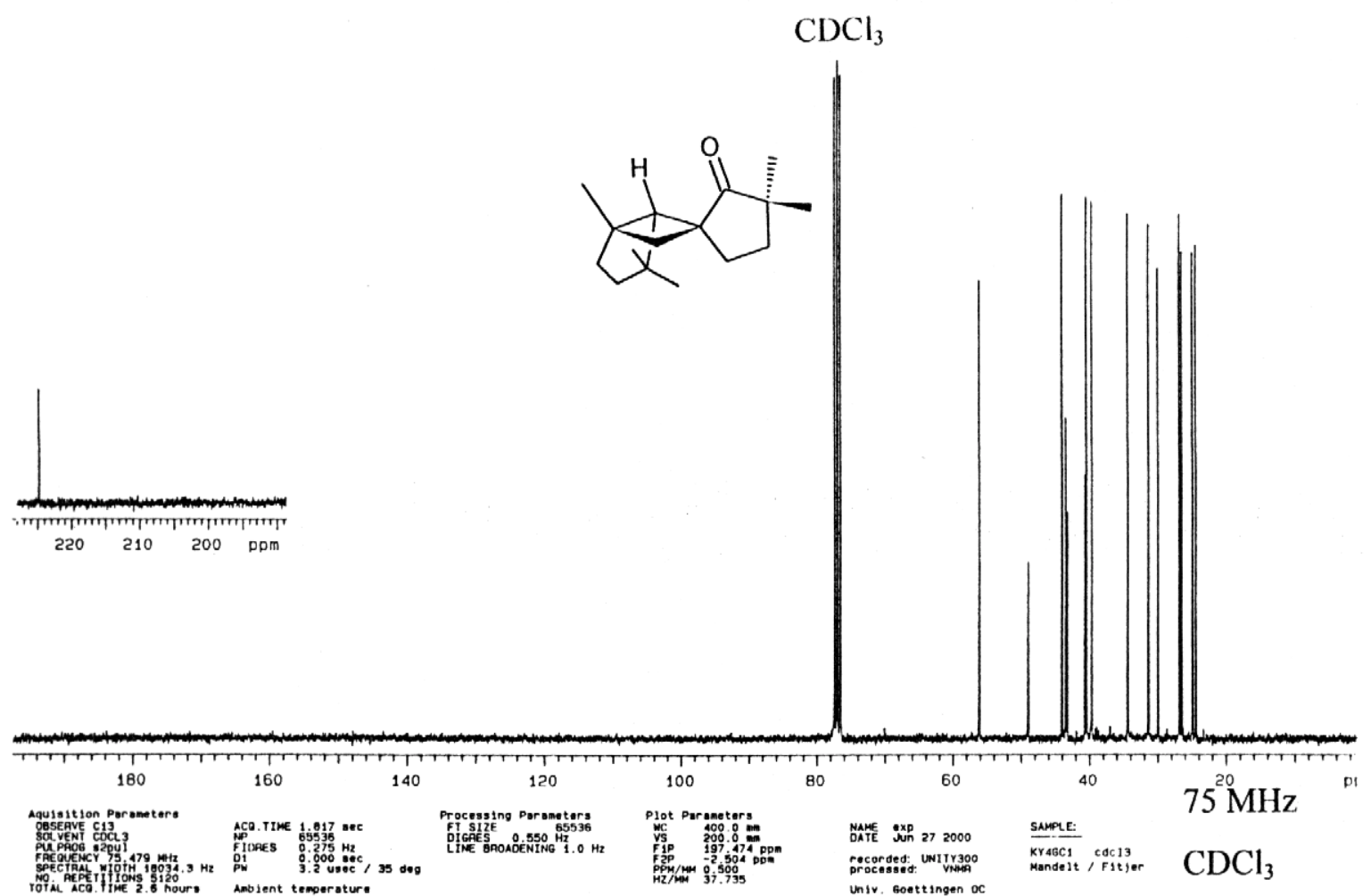

Abb. 78: $\quad{ }^{13} \mathrm{C}-\mathrm{NMR}-$ Spektrum von $\left(1 R^{*}, 5 R^{*}, 6 R^{*}\right)-1,4,4,3^{\prime}, 3^{\prime}$-Pentamethylspiro \{bicyclo-

[3.2.0]heptan-6, $1^{\prime}$-cyclopentan-2'-on $\}$ (143) 

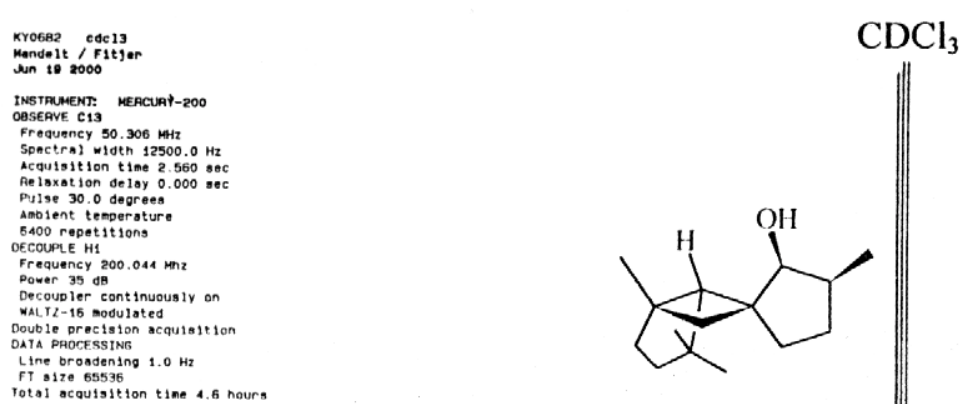

36 a 1.02

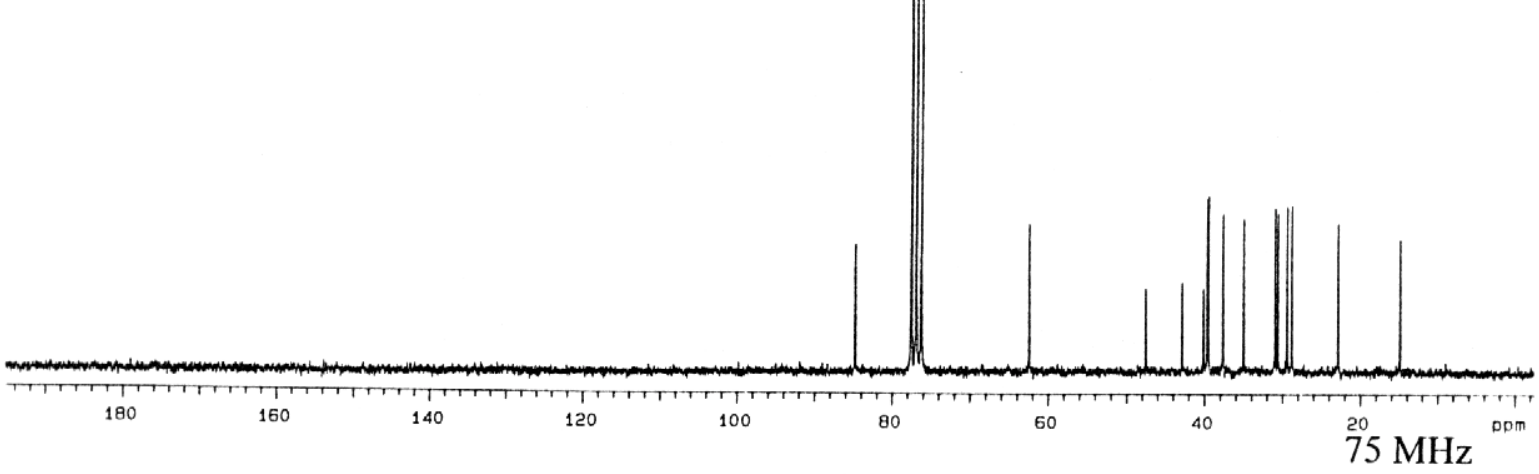

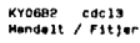

$\mathrm{CDCl}_{3}$

Abb. 79: $\quad{ }^{13} \mathrm{C}-\mathrm{NMR}-\mathrm{Spektrum}$ von $\left(1 R^{*}, 5 R^{*}, 6 R^{*}, 2^{\prime} R^{*}, 3^{\prime} R^{*}\right)-1,4,4,3^{\prime}$-Tetramethylspiro$\{$ bicyclo[3.2.0]heptan-6,1'-cyclopentan-2'-ol $\}(60)$
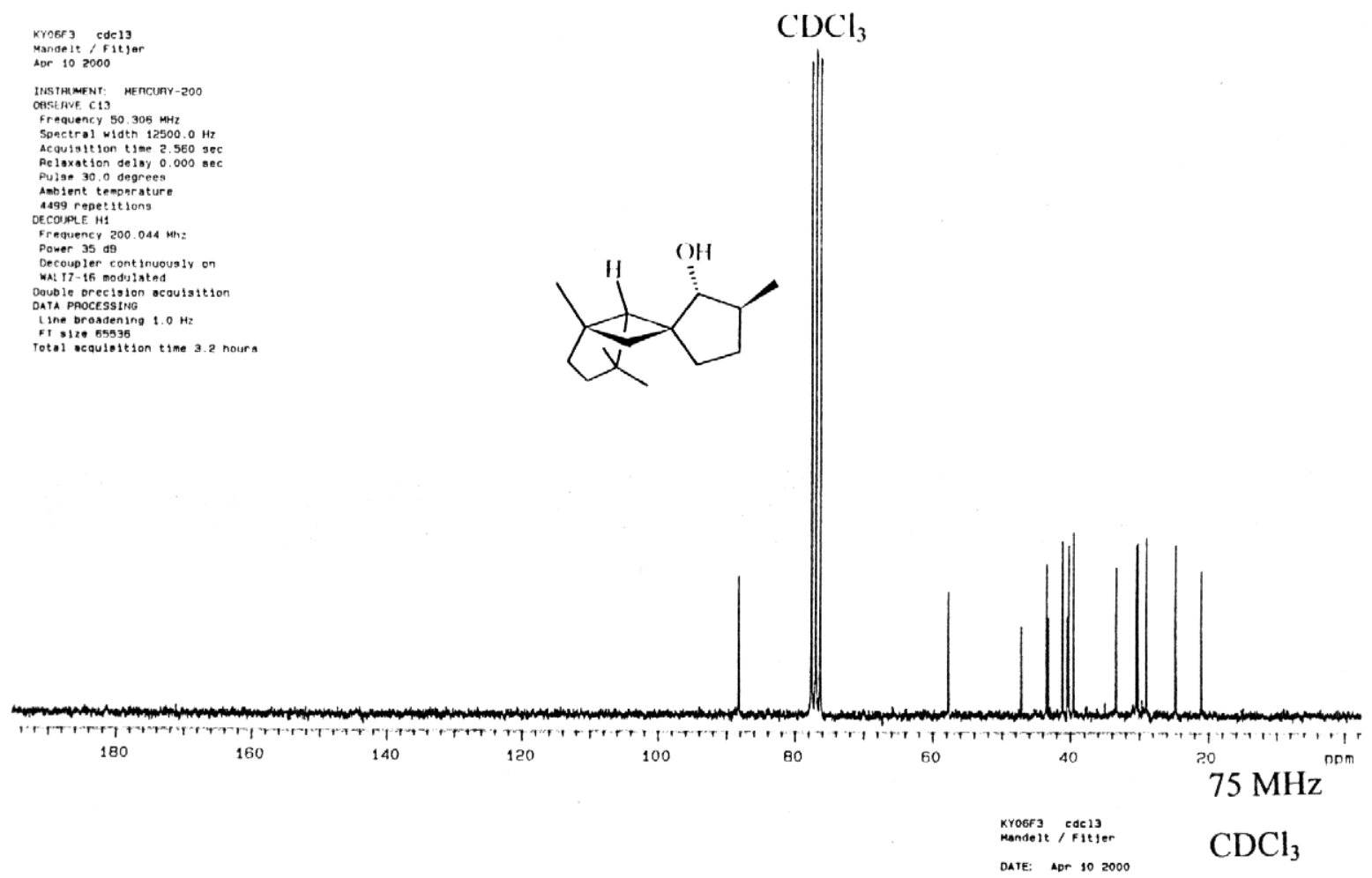

Abb. 80: $\quad{ }^{13}$ C-NMR-Spektrum von $\left(1 R^{*}, 5 R^{*}, 6 R^{*}, 2^{\prime} S^{*}, 3^{\prime} R^{*}\right)-1,4,4,3^{\prime}$-Tetramethylspiro\{bicyclo[3.2.0]heptan-6,1'-cyclopentan-2'-ol\} (64) 


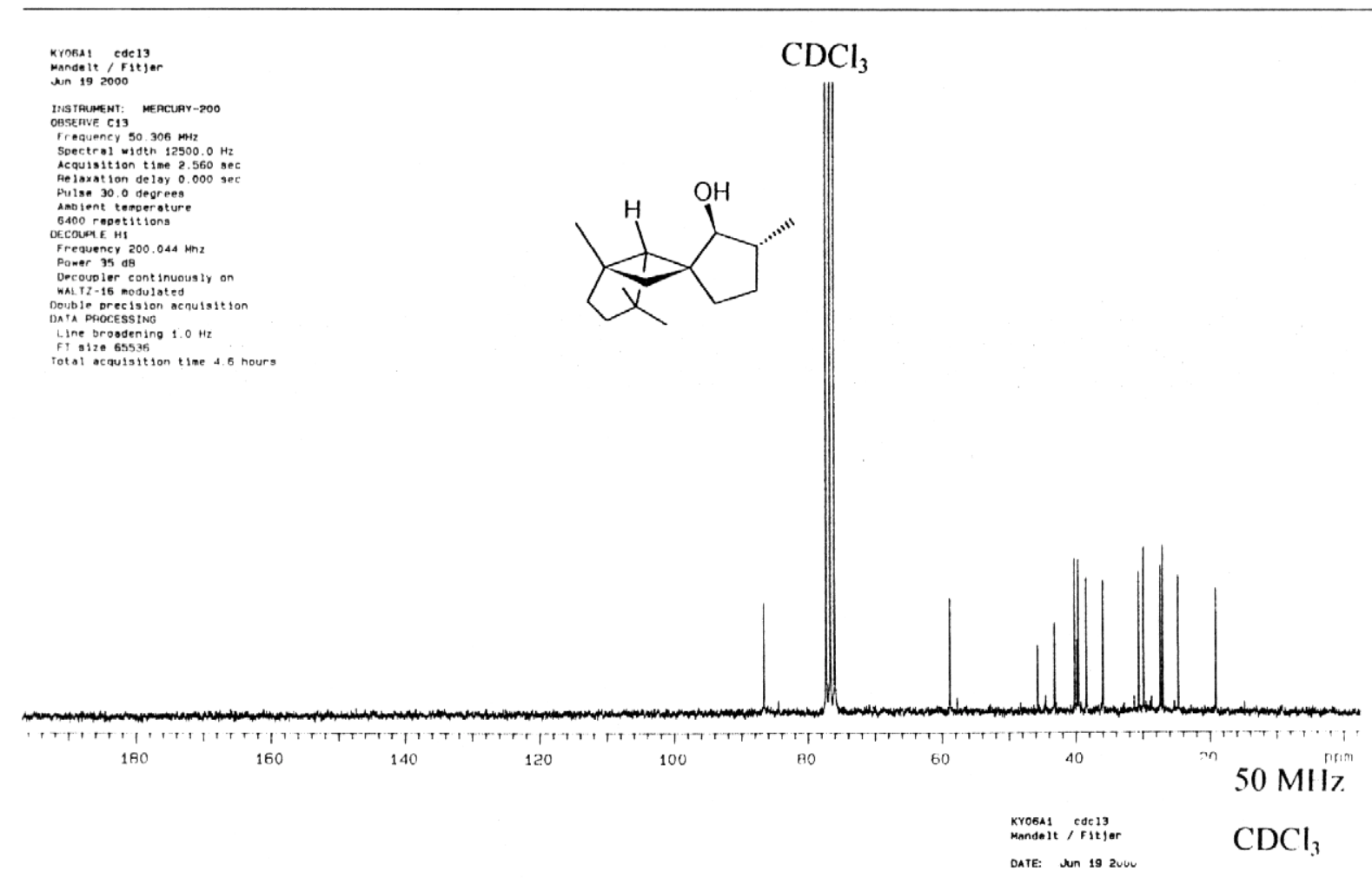

Abb. 81: $\quad{ }^{13} \mathrm{C}-\mathrm{NMR}-\mathrm{Spektrum}$ von $\left(1 R^{*}, 5 R^{*}, 6 R^{*}, 2^{\prime} R^{*}, 3^{\prime} S^{*}\right)-1,4,4,3^{\prime}$-Tetramethylspiro$\{$ bicyclo[3.2.0]heptan-6,1'-cyclopentan-2'-ol $\}(66)$
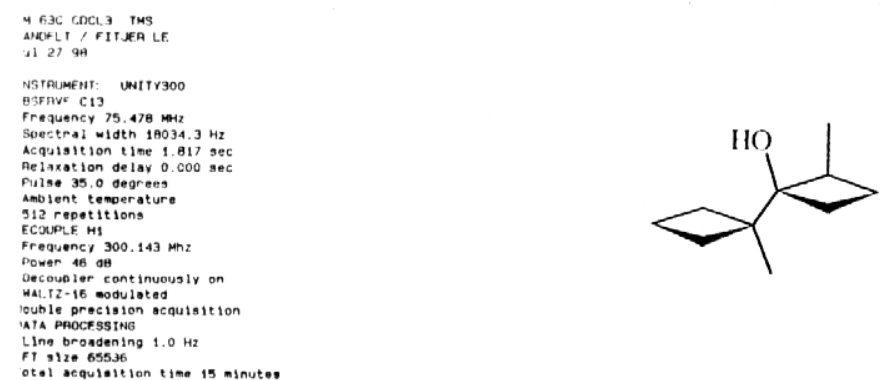

Ling brousdenting $1.0 \mathrm{~Hz}$

otol sequibition tine 15 minute.

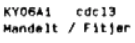
OATE: ven 19 2uv

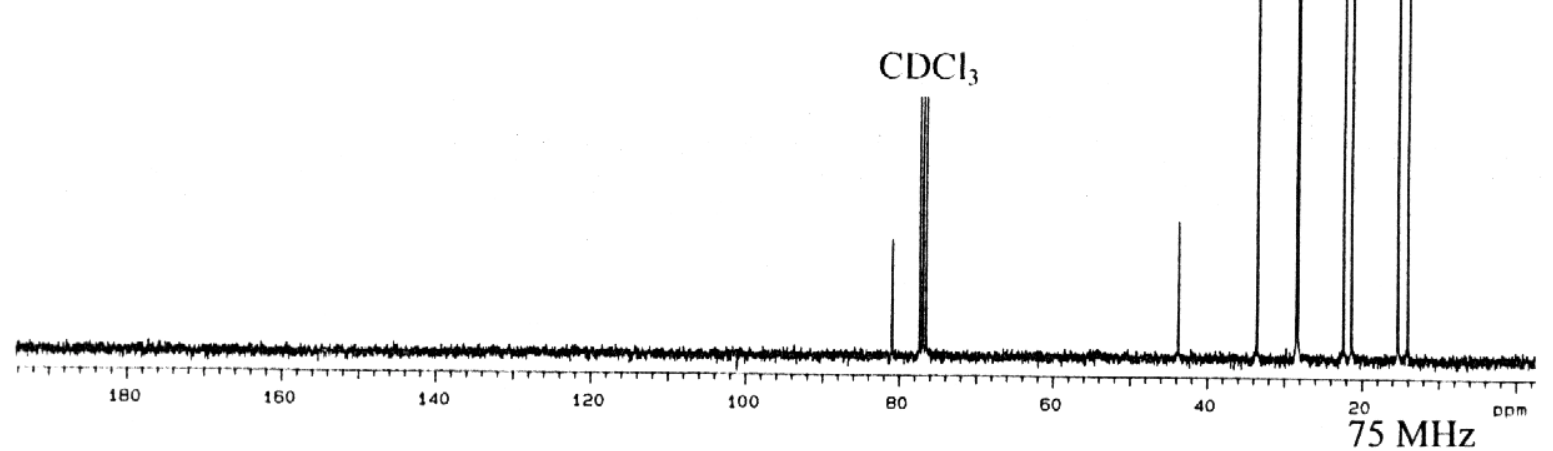

$\mathrm{CDCl}_{3}$

Abb. 82: $\quad{ }^{13}$ C-NMR-Spektrum von $\left(1 R^{*}, 2 S^{*}\right)-2,1^{\prime}$-Dimethyl-bicyclobutyl-1-ol (104b) 


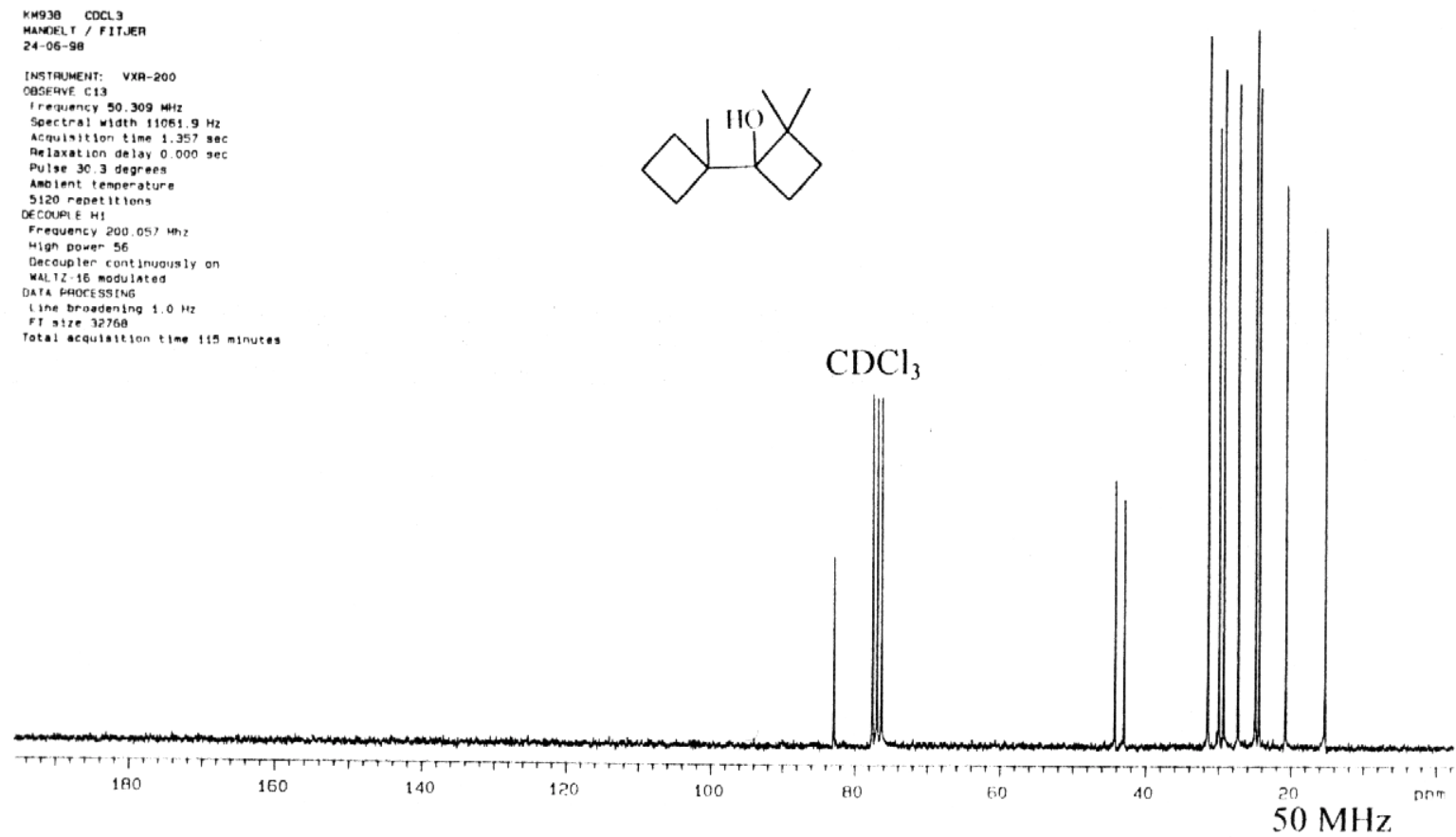

$\mathrm{CDCl}_{3}$

Abb. 83: $\quad{ }^{13}$ C-NMR-Spektrum von 2,2,1'-Trimethyl-bicyclobutyl-1-ol (105)
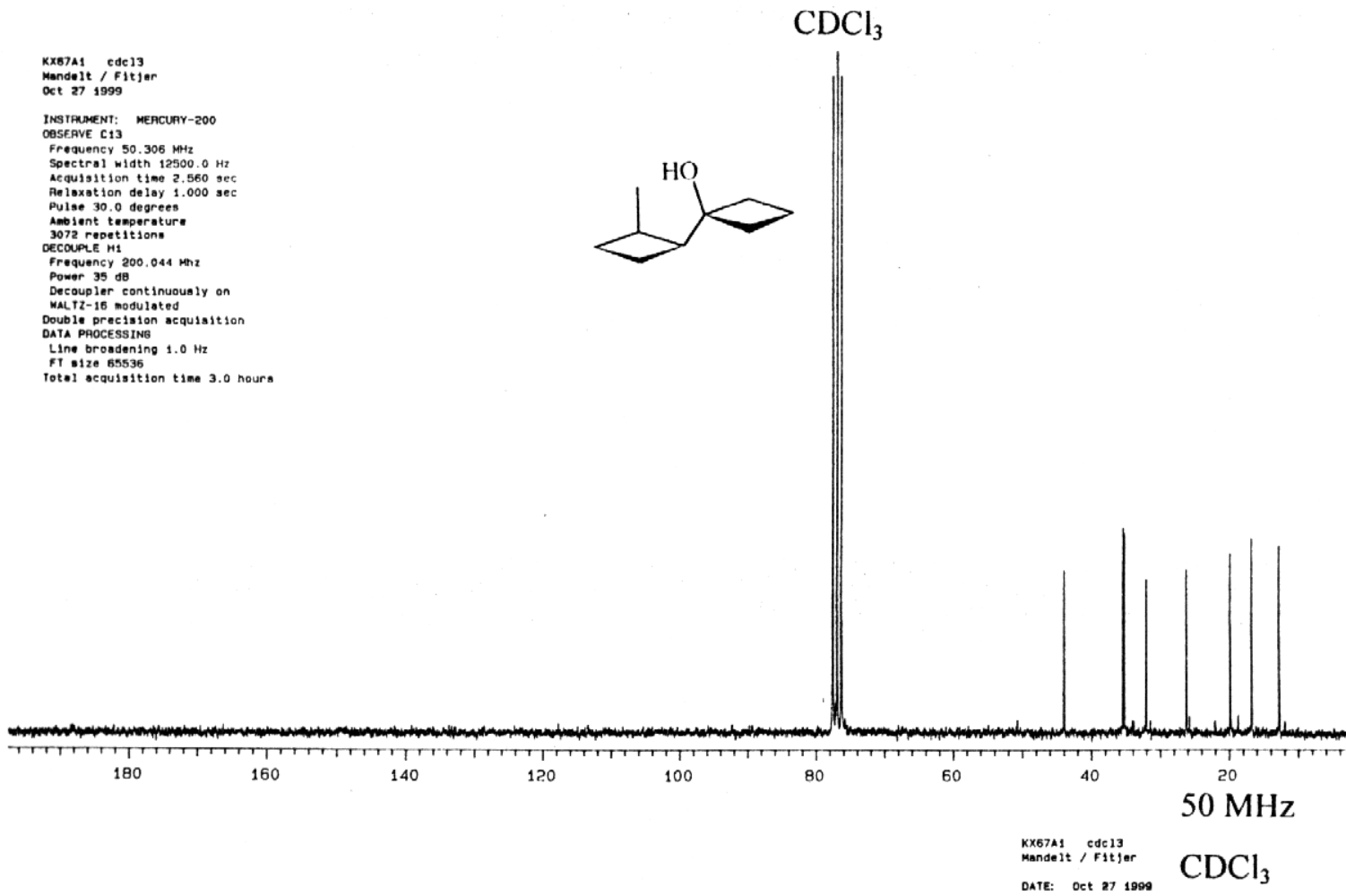

Abb. 84: $\quad{ }^{13}$ C-NMR-Spektrum von $\left(1^{\prime} R^{*}, 2^{\prime} S^{*}\right)-2^{\prime}$-Methyl-bicyclobutyl-1-ol (106a) 


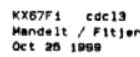

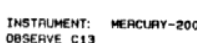

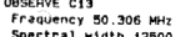

Spectral wideth $12500.0 \mathrm{~Hz}$
Acauts 1 tition time 2.560
sec

Pulse 30.0 dearrees
Ambient Cempersture

2560 repetitions

DECOUPLE HA

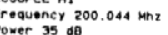

Docounler continuously on

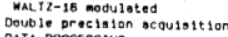

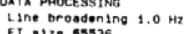

Tots: acquistition time 2.5 hours

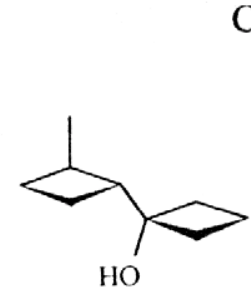

$\mathrm{CDCl}_{3}$

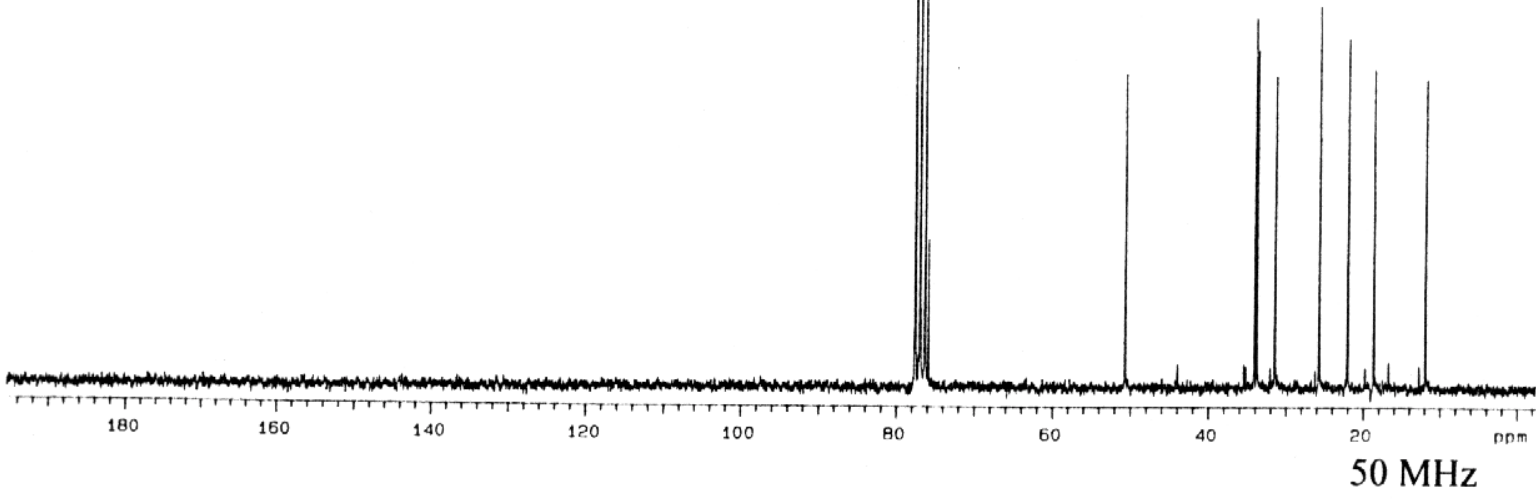

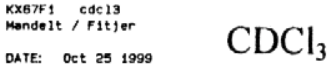

Abb. 85: $\quad{ }^{13} \mathrm{C}-\mathrm{NMR}-$ Spektrum von $\left(1^{\prime} R^{*}, 2^{\prime} R^{*}\right)-2^{\prime}$-Methyl-bicyclobutyl-1-ol (106b)

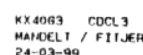

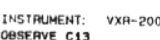

Frequency 50.309 Mot

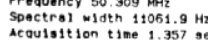

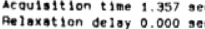

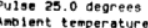

5120 repet 1 ter tons

Erequency 200.057 Hinz

Decoupler continuously on

waLT T2-16 modulated

DAAA Processins

Ft $131203276 \mathrm{~A}$

(1)

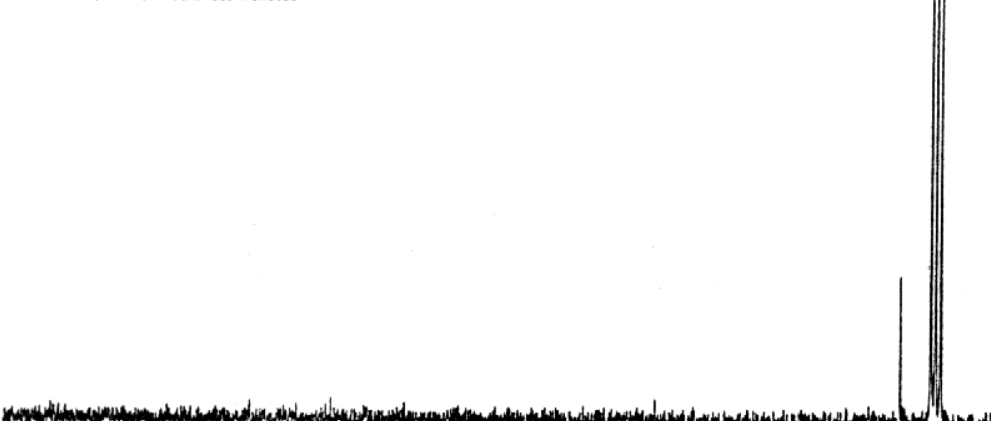

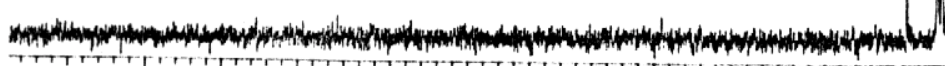

180

160
120

100

80

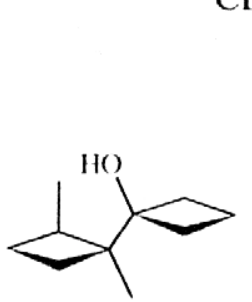

$\mathrm{Cl}_{3}$

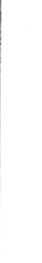

$\mathrm{CDCl}_{3}$

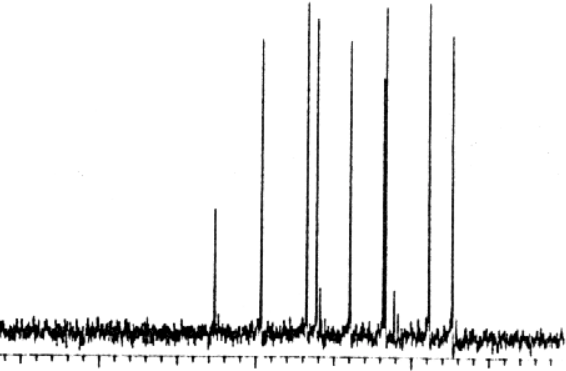

60

40

$50 \mathrm{MHz}$

$\mathrm{CDCl}_{3}$

Abb. 86: $\quad{ }^{13}$ C-NMR-Spektrum von $\left(1^{\prime} R^{*}, 2^{\prime} S^{*}\right)-1^{\prime}, 2^{\prime}$-Dimethyl-bicyclobutyl-1-ol (107a) 


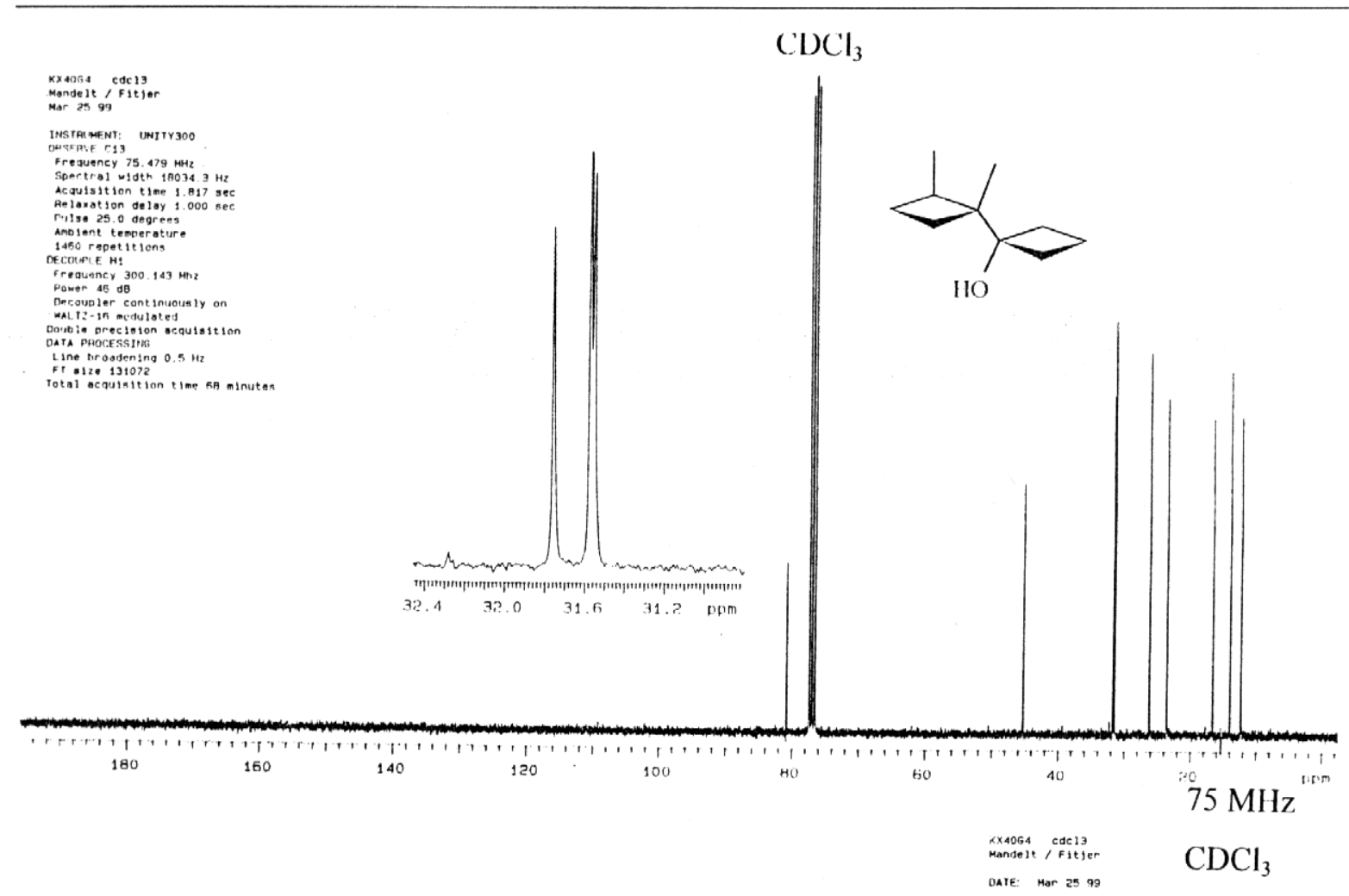

Abb. 87: $\quad{ }^{13} \mathrm{C}-\mathrm{NMR}$-Spektrum von $\left(1^{\prime} R^{*}, 2^{\prime} R^{*}\right)-1^{\prime}, 2^{\prime}$-Dimethyl-bicyclobutyl-1-ol (107b)

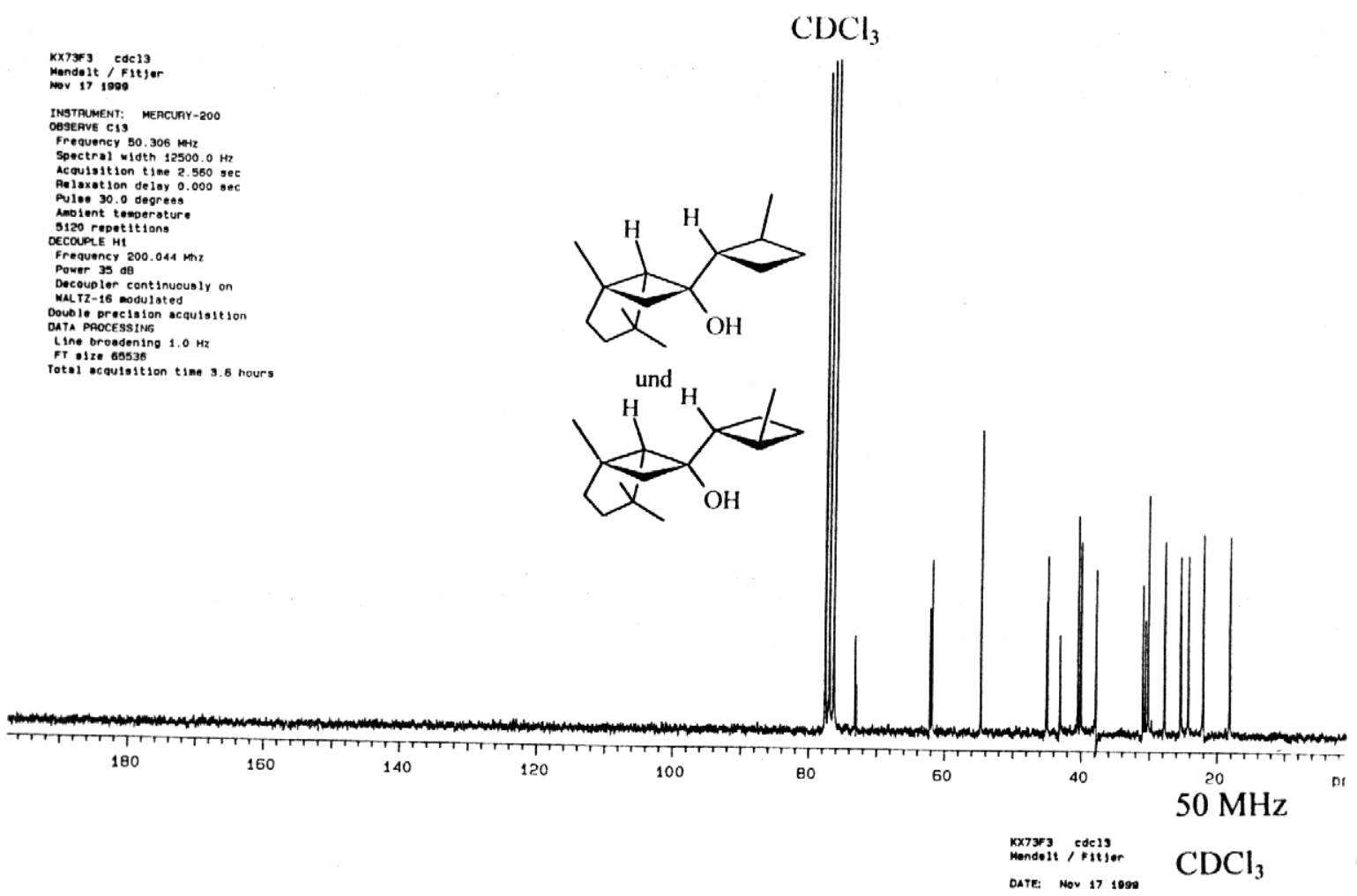

Abb. 88: $\quad{ }^{13} \mathrm{C}-\mathrm{NMR}-\mathrm{Spektrum}$ von $\left(1 S^{*}, 5 S^{*}, 6 S^{*}, 1^{\prime} S^{*}, 2^{\prime} S^{*}\right)$-1,4,4-Trimethyl-6-(2'-methylcyclobutyl)-bicyclo[3.2.0]heptan-6-ol (46) und $\left(1 S^{*}, 5 S^{*}, 6 S^{*}, 1^{\prime} R^{*}, 2^{\prime} R^{*}\right)-1,4,4$ Trimethyl-6-(2'-methyl-cyclobutyl)-bicyclo[3.2.0]heptan-6-ol (49) 

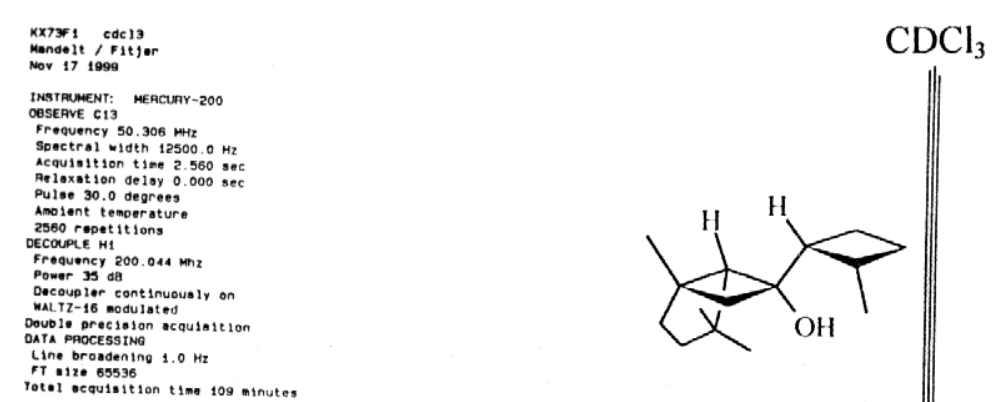

retol ocquisition time 109 minutes
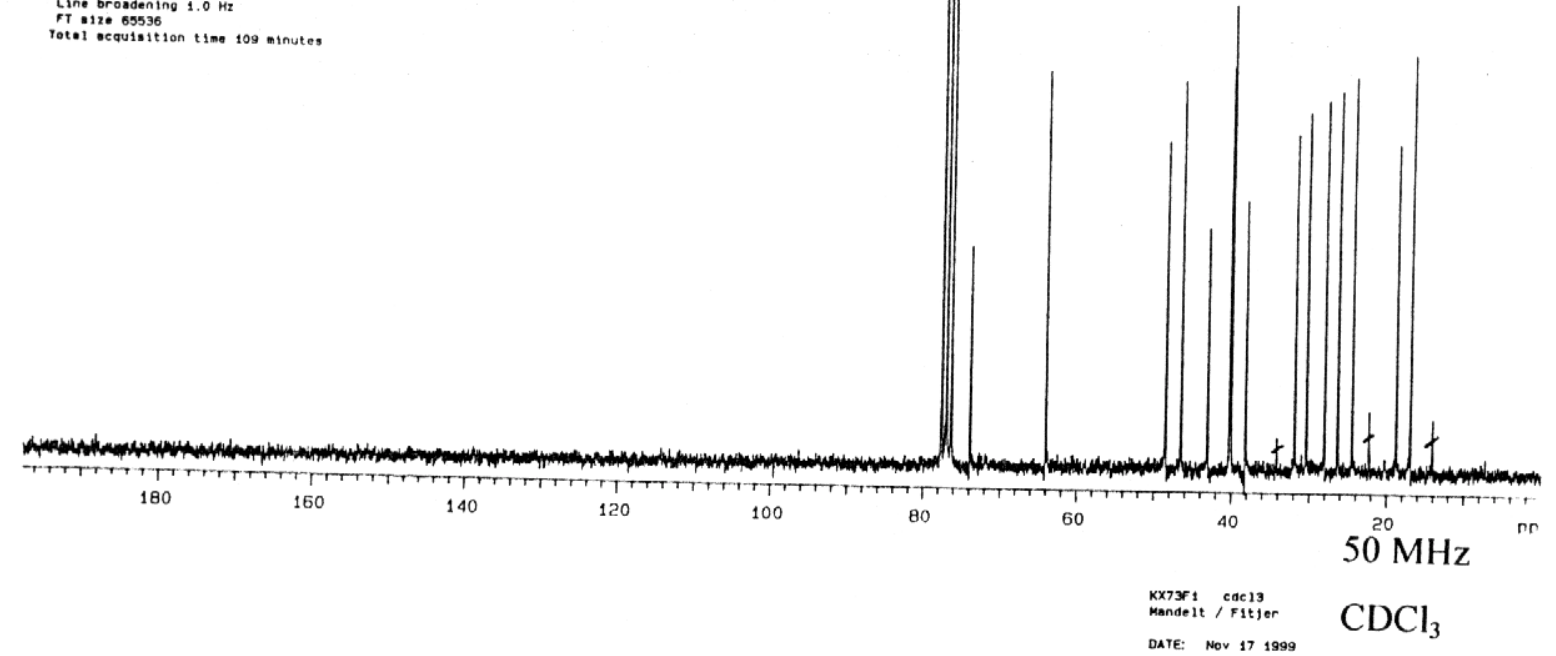

Abb. 89: $\quad{ }^{13}$ C-NMR-Spektrum von $\left(1 S^{*}, 5 S^{*}, 6 S^{*}, 1^{\prime} S^{*}, 2^{\prime} R^{*}\right)-1,4,4-$ Trimethyl-6- $\left(2^{\prime}-\right.$ methyl-cyclobutyl)-bicyclo[3.2.0]heptan-6-ol (47)
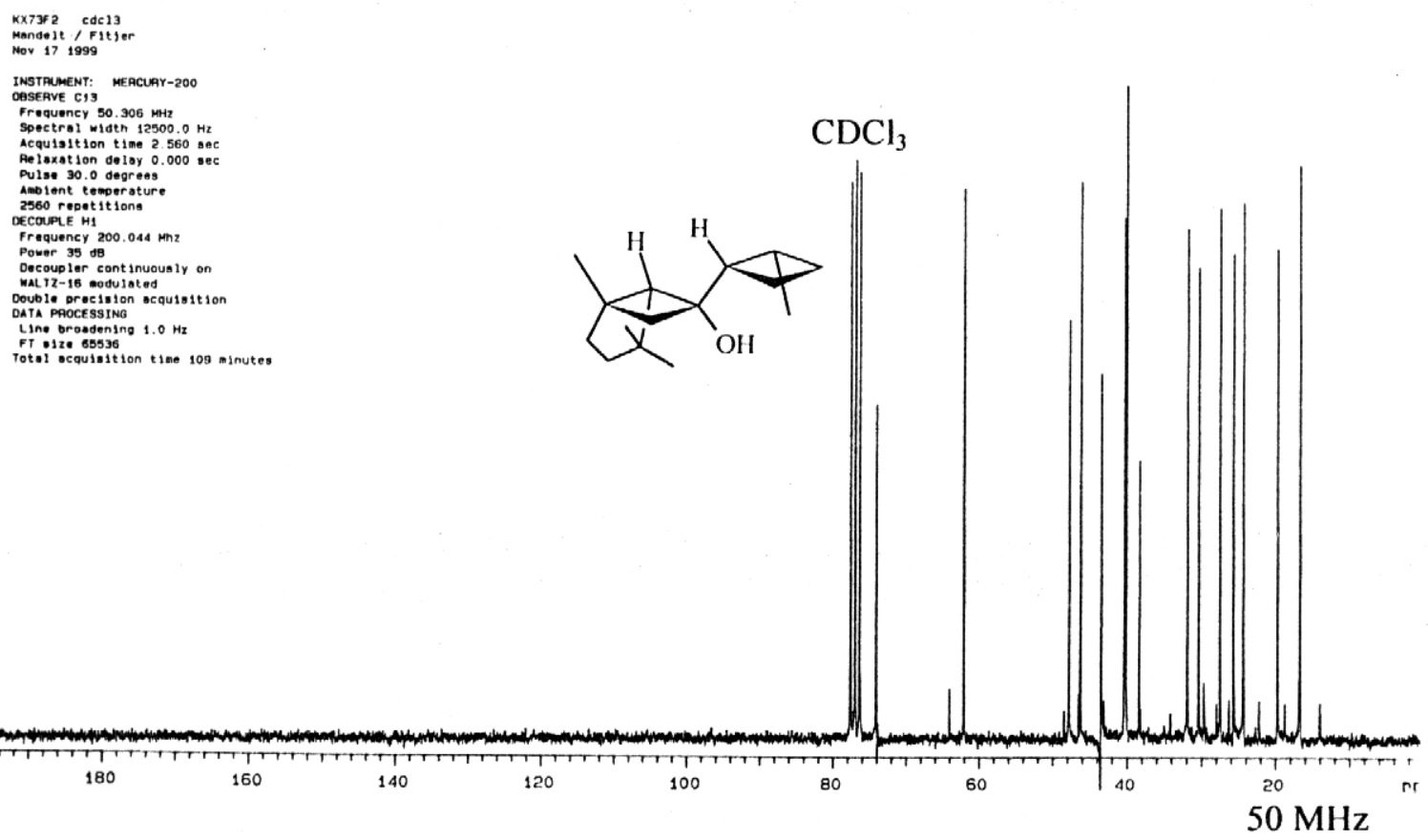

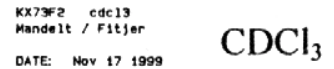

Abb. 90: $\quad{ }^{13} \mathrm{C}-\mathrm{NMR}-\mathrm{Spektrum}$ von $\left(1 S^{*}, 5 S^{*}, 6 S^{*}, 1^{\prime} R^{*}, 2^{\prime} S^{*}\right)-1,4,4-$ Trimethyl-6-(2'-methylcyclobutyl)-bicyclo[3.2.0]heptan-6-ol (48) 


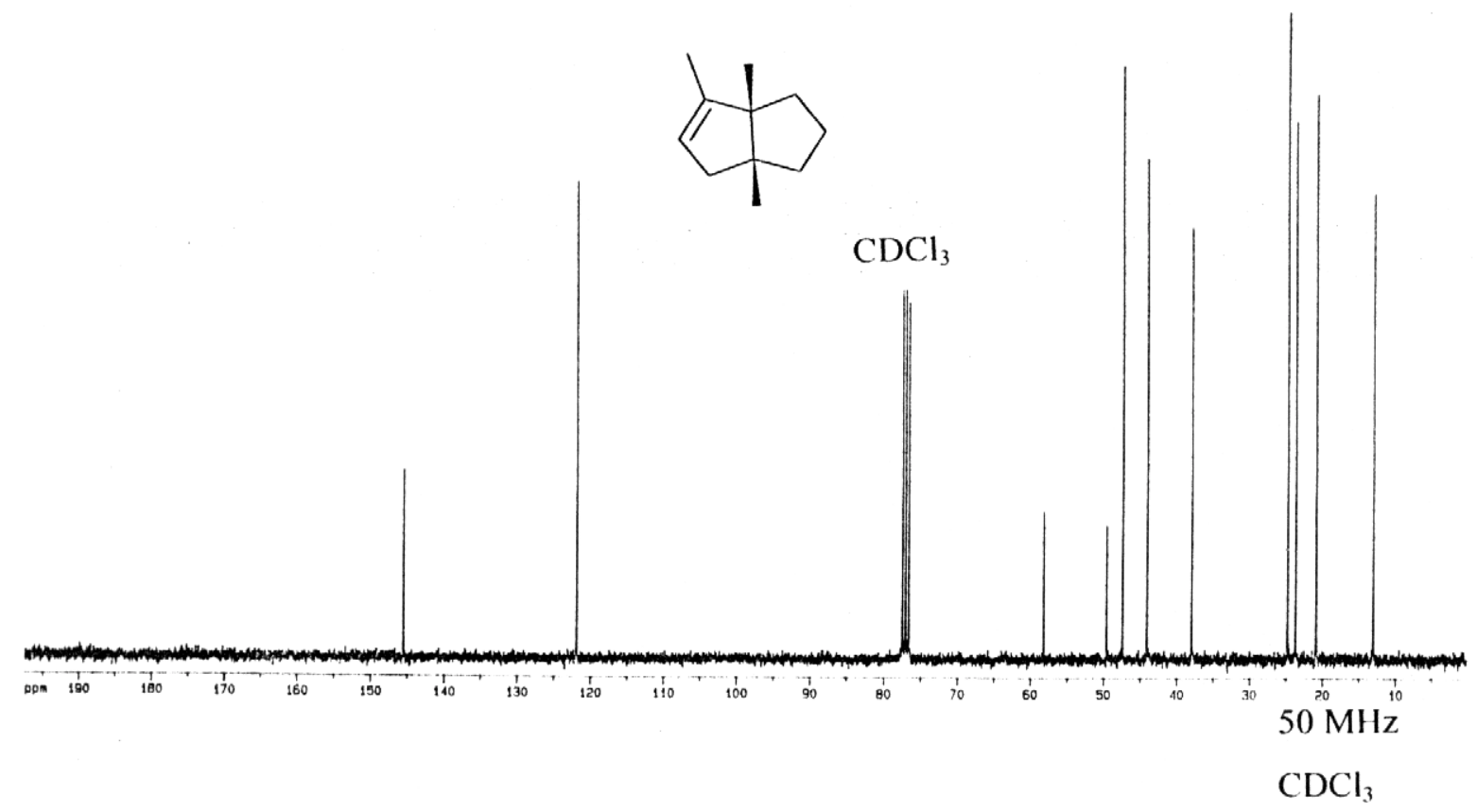

Abb. 91: $\quad{ }^{13}$ C-NMR-Spektrum von (3a $\left.R^{*}, 6 \mathrm{a} S^{*}\right)-3 \mathrm{a}, 6,6 \mathrm{a}-$ Trimethyl-1,2,3,3a,4,6a-hexahydropentalen (118)
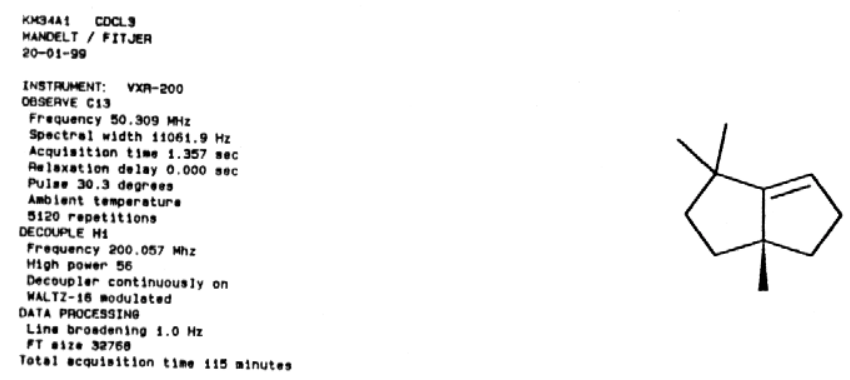

Totol ocquistition timo 115 annutes

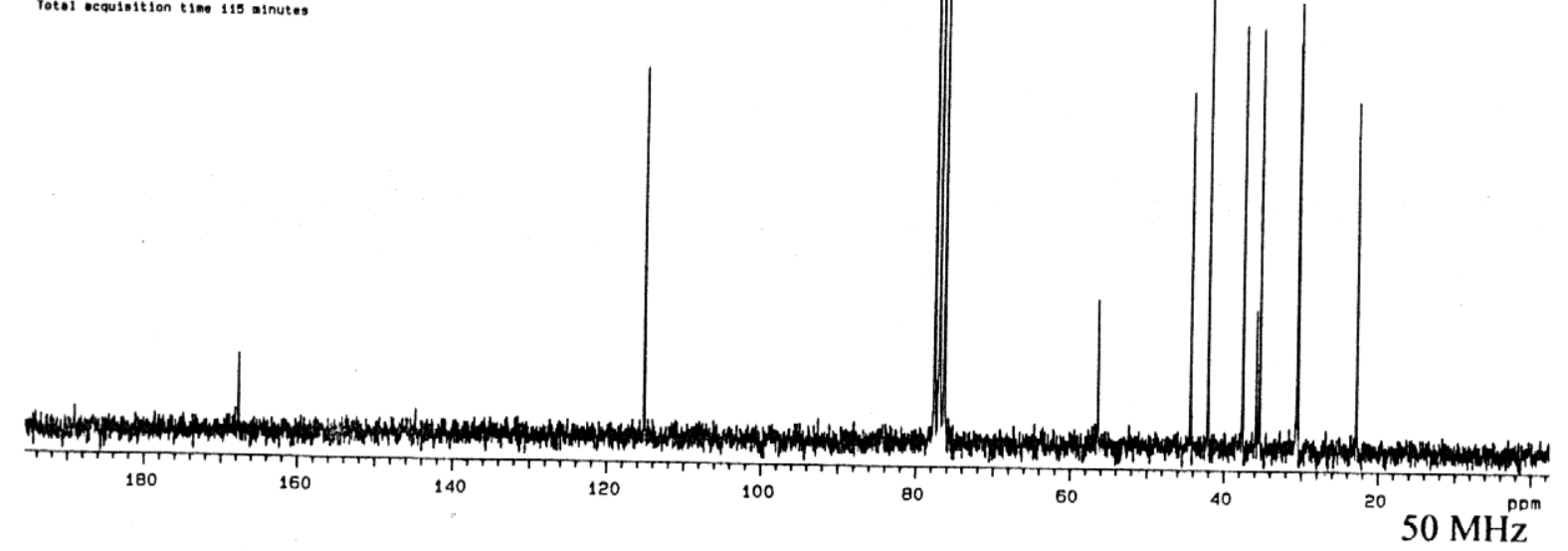

$\mathrm{CDCl}_{3}$

Abb. 92: $\quad{ }^{13}$ C-NMR-Spektrum von 1,1,3a-Trimethyl-1,2,3,3a,4,5-hexahydro-pentalen (120) 


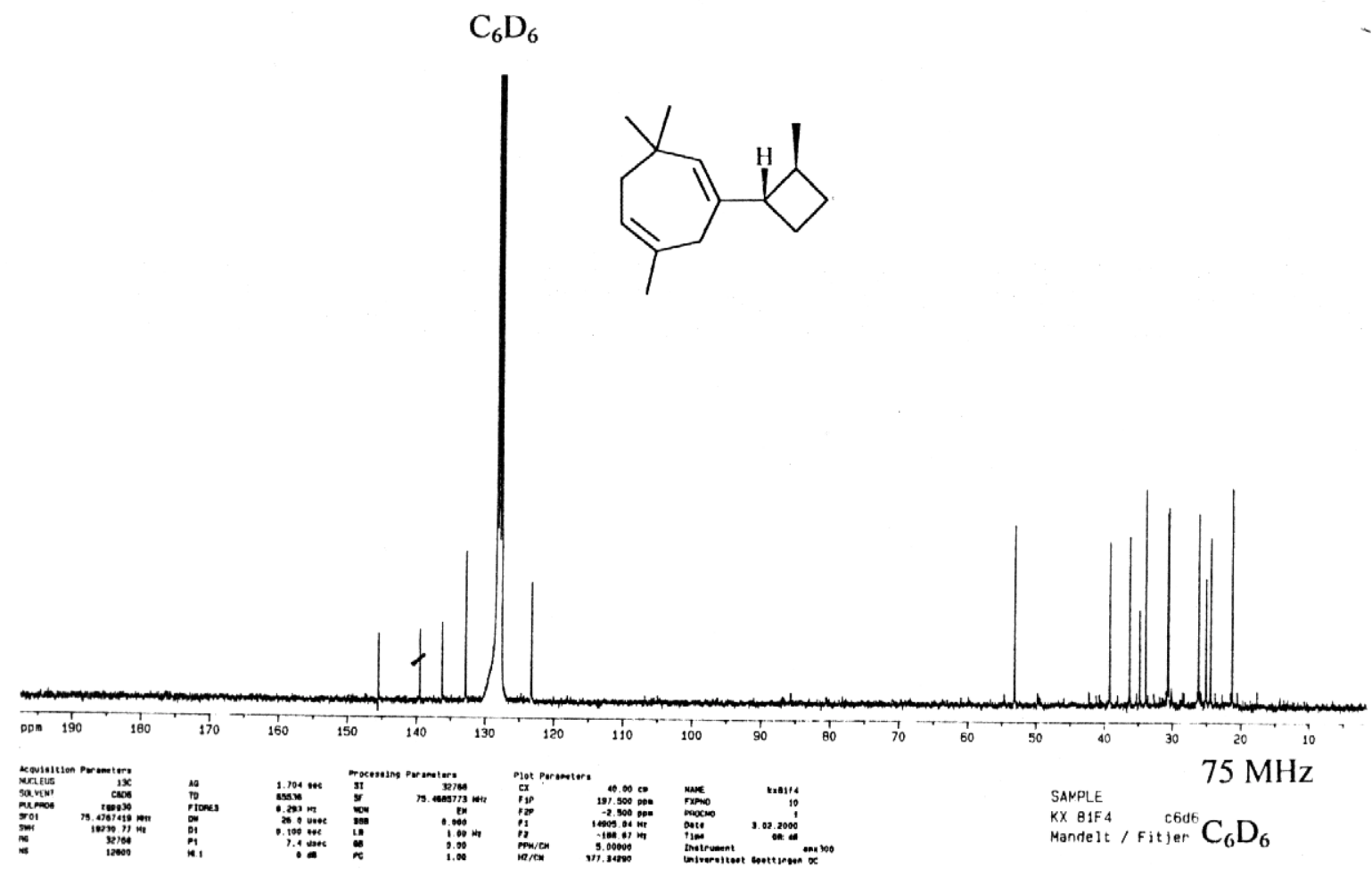

Abb. 93: $\quad{ }^{13}$ C-NMR-Spektrum von 2,6,6-Trimethyl-4-(2-methylcyclobutyl)-cyclohepta-1,4dien (122)

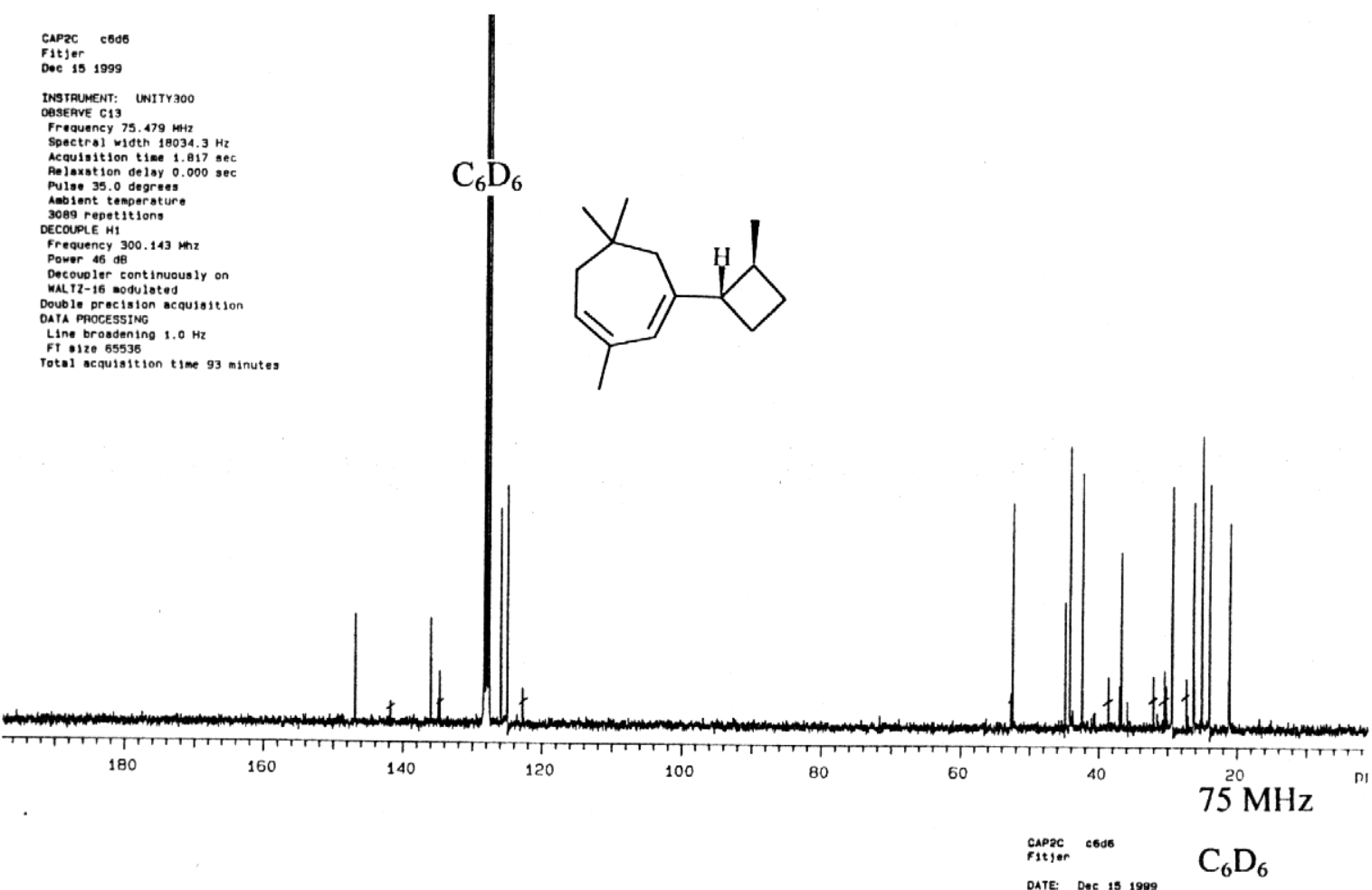

Abb. 94: $\quad{ }^{13}$ C-NMR-Spektrum von 3,6,6-Trimethyl-1-(2-methyl-cyclobutyl)-cyclohepta-1,3dien (123) 


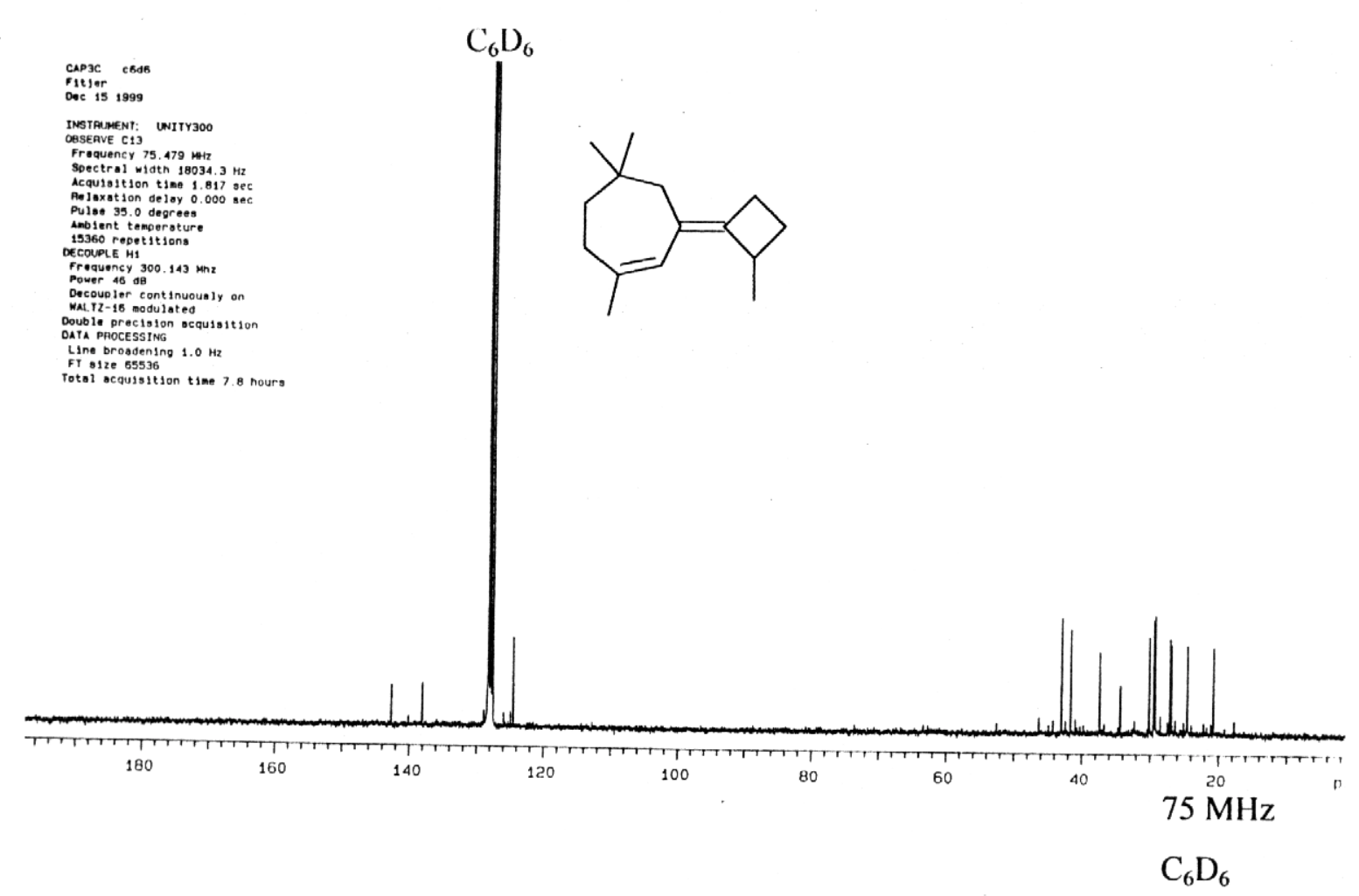

Abb. 95: $\quad{ }^{13}$ C-NMR-Spektrum von 1,5,5-Trimethyl-3-(2-methyl-cyclobutyliden)-cyclohept-1-en (124)

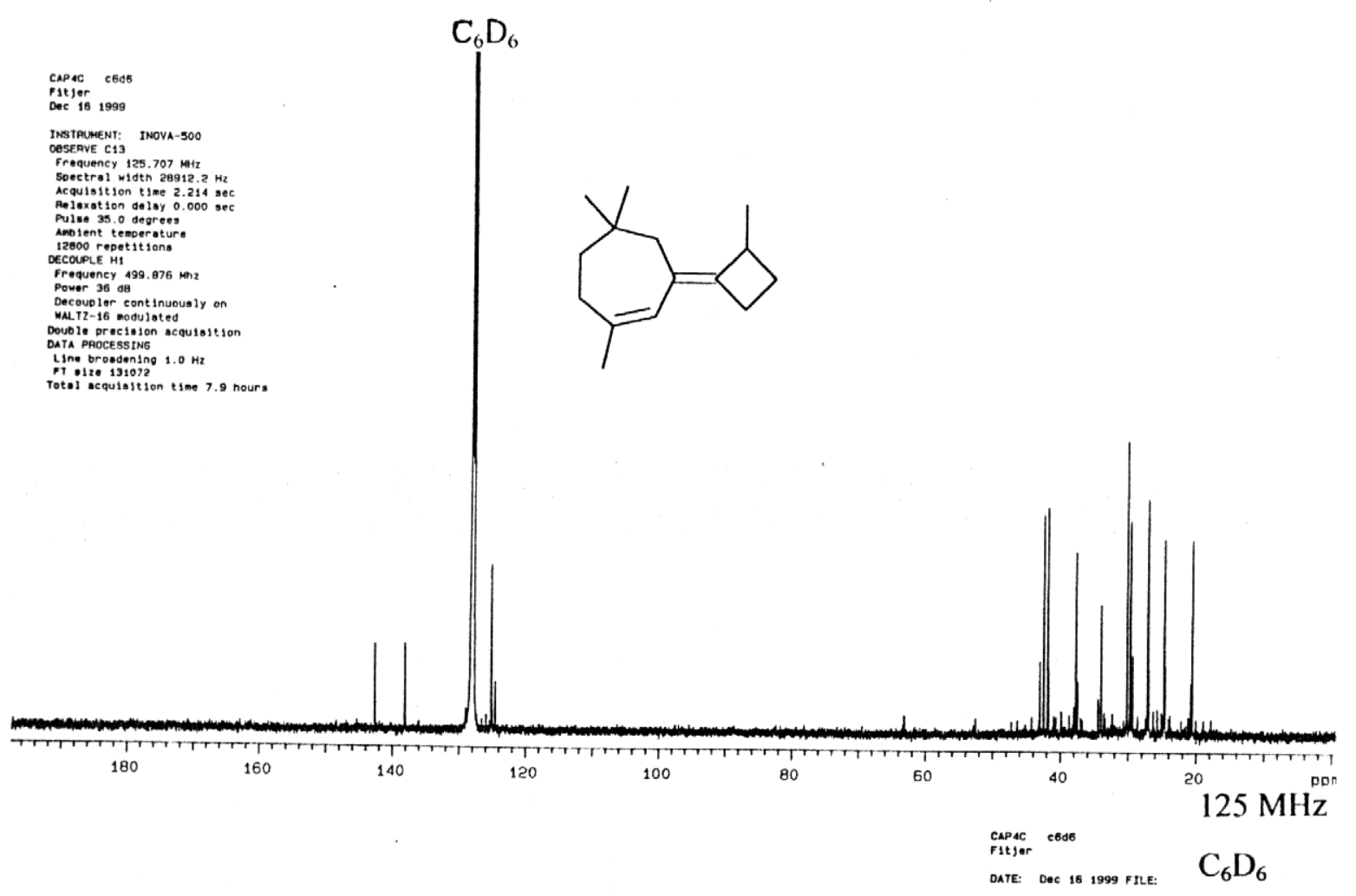

Abb. 96: $\quad{ }^{13}$ C-NMR-Spektrum von 1,5,5-Trimethyl-3-(2-methyl-cyclobutyliden)-cyclohept-1-en (125) 


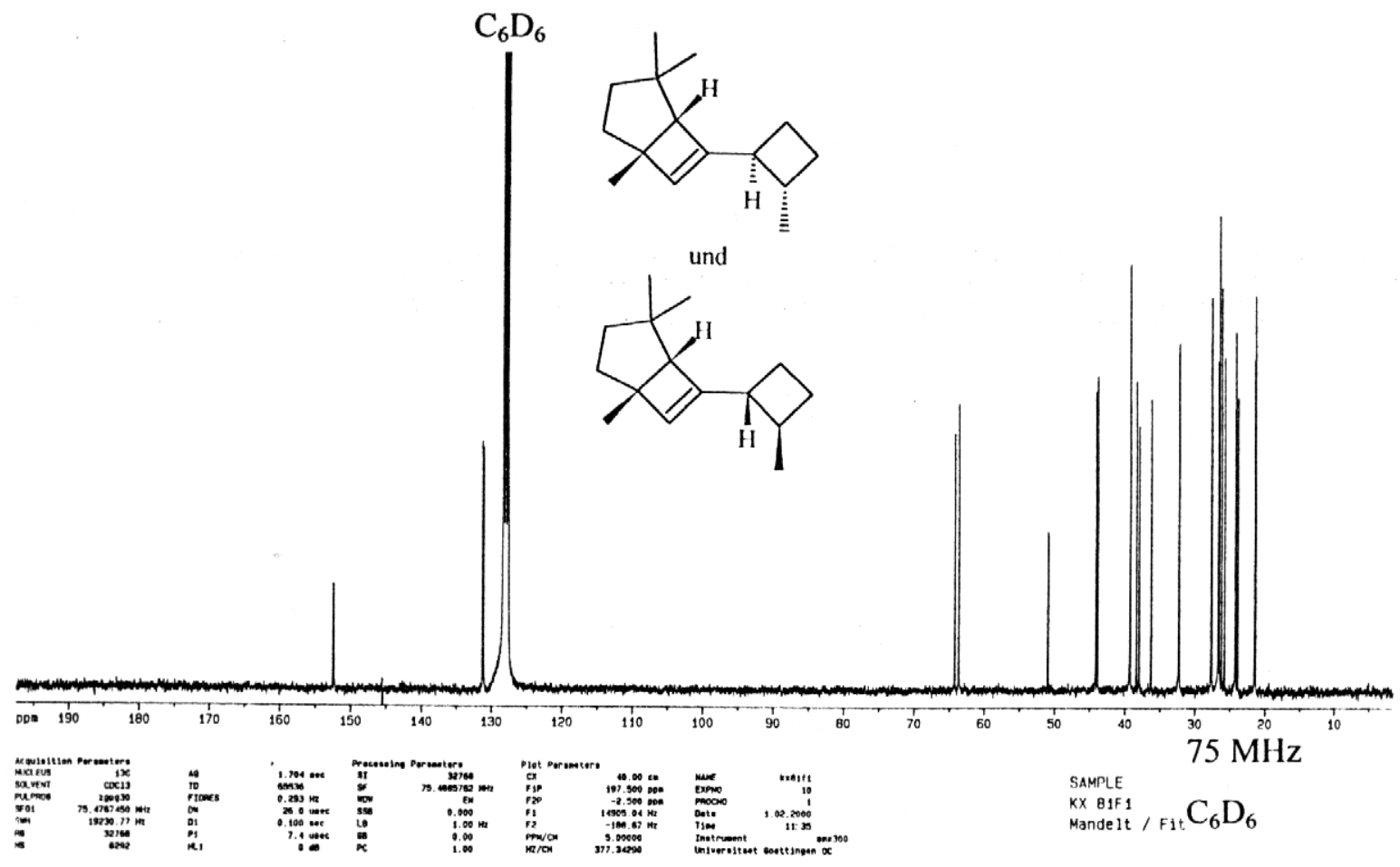

Abb. 97: $\quad{ }^{13}$ C-NMR-Spektrum von 1,4,4-Trimethyl-6-(2-methyl-cyclobutyl)-bicyclo[3.2.0]hept-6-en (130 und 131)
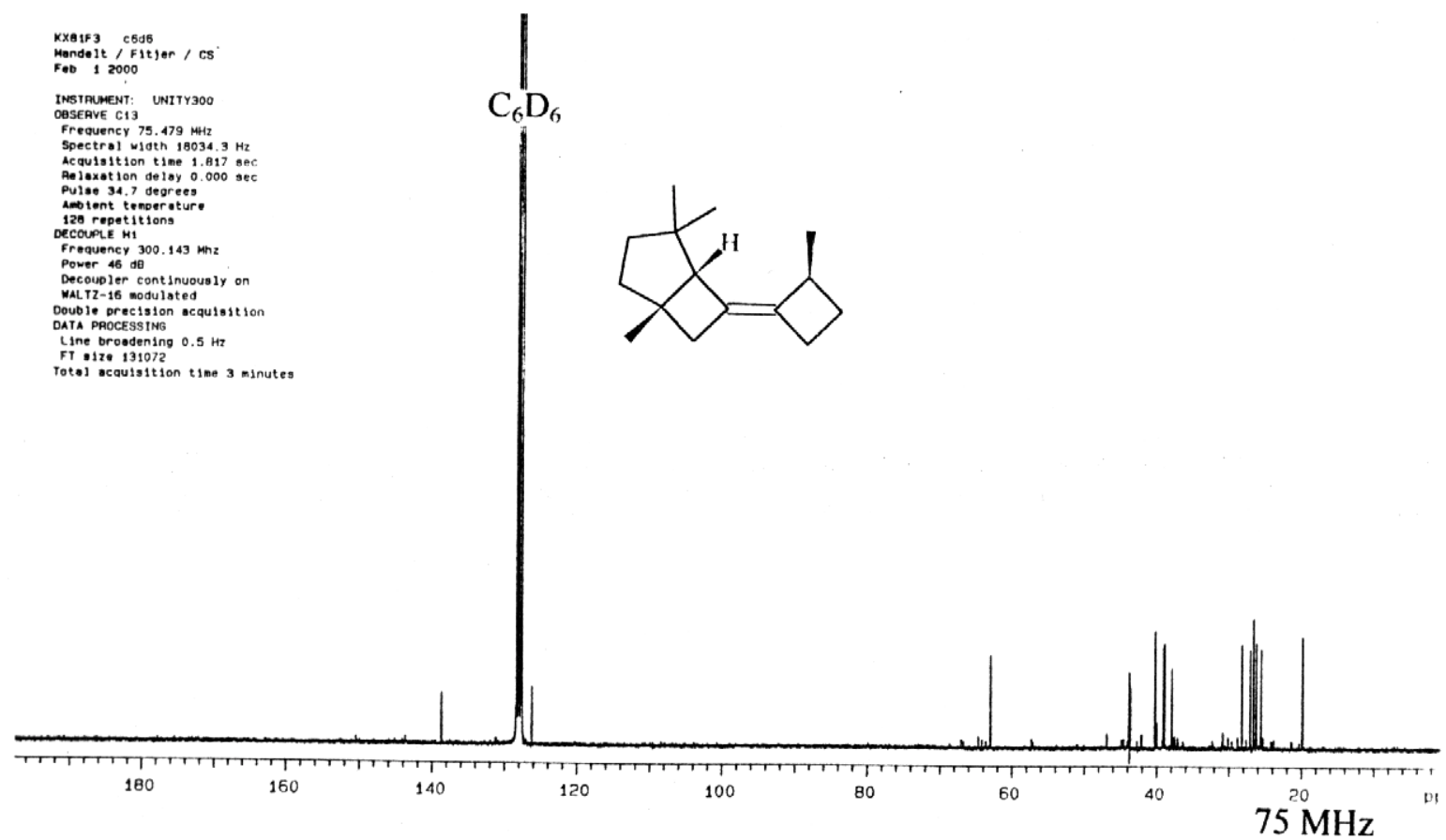

$\mathrm{C}_{6} \mathrm{D}_{6}$

Abb. 98: $\quad{ }^{13}$ C-NMR-Spektrum von 1,4,4-Trimethyl-6-(2-methyl-cyclobutyliden)-bicyclo[3.2.0]heptan (132/133/134/135) 


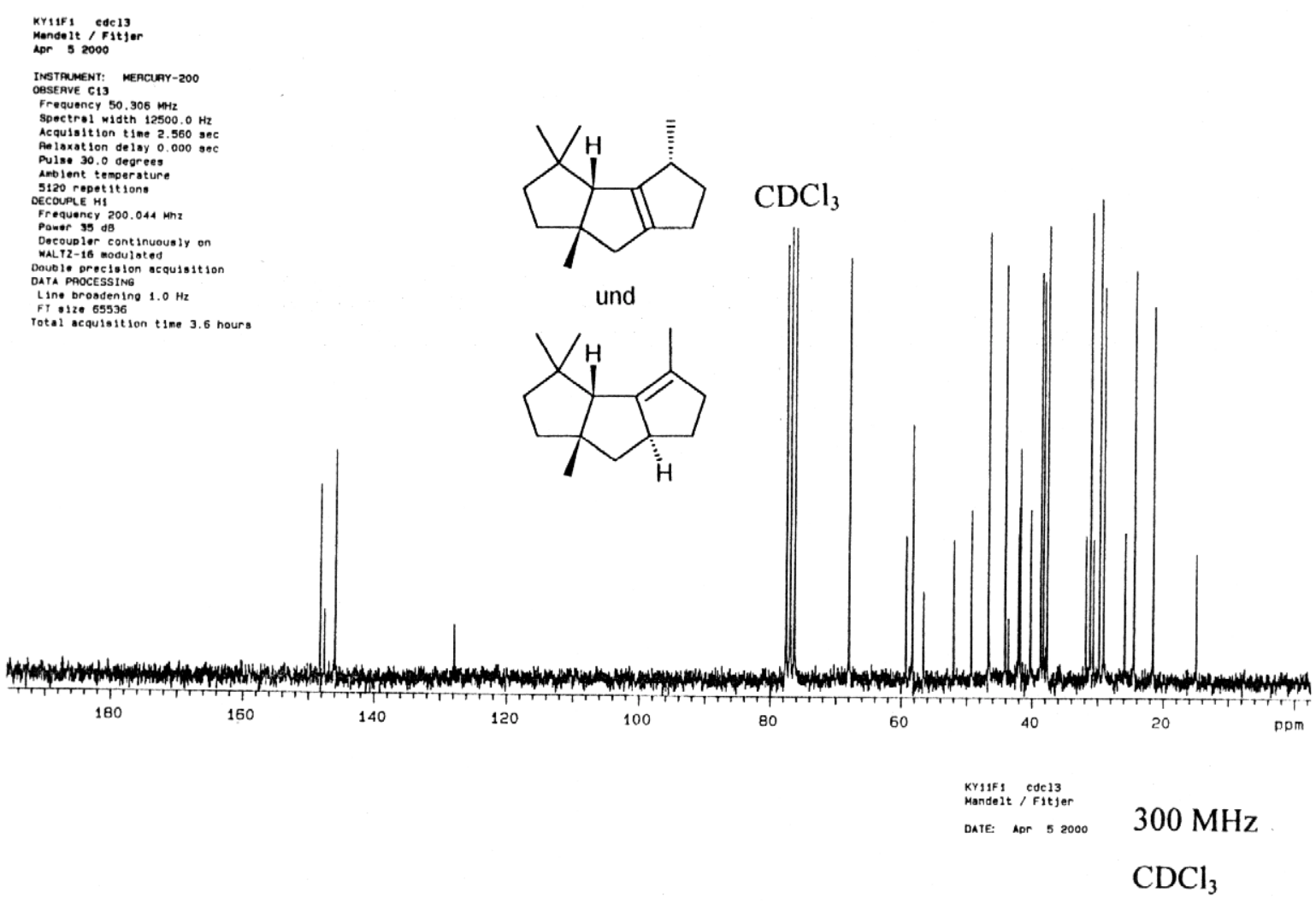

Abb. 99: $\quad{ }^{13}$ C-NMR-Spektrum von (3aR*,4R*,7aR*)-3,3,4,7a-Tetramethyl-2,3,3a,4,5,6,7,7a-octahydro- $1 H$-cyclopenta[a]pentalen $(67)$ und $\left(3 \mathrm{aR} *, 6 \mathrm{aR}^{*}, 7 \mathrm{aR} *\right)-3,3,4,7 \mathrm{a}-$ Tetramethyl-2,3,3a,5,6,6a,7,7a-octahydro-1H-cyclopenta[a]pentalen (68)
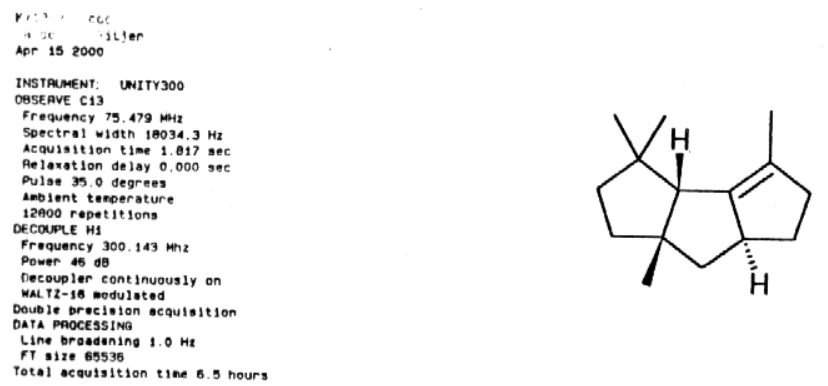

Total ecquilstition tine 8.5 hours

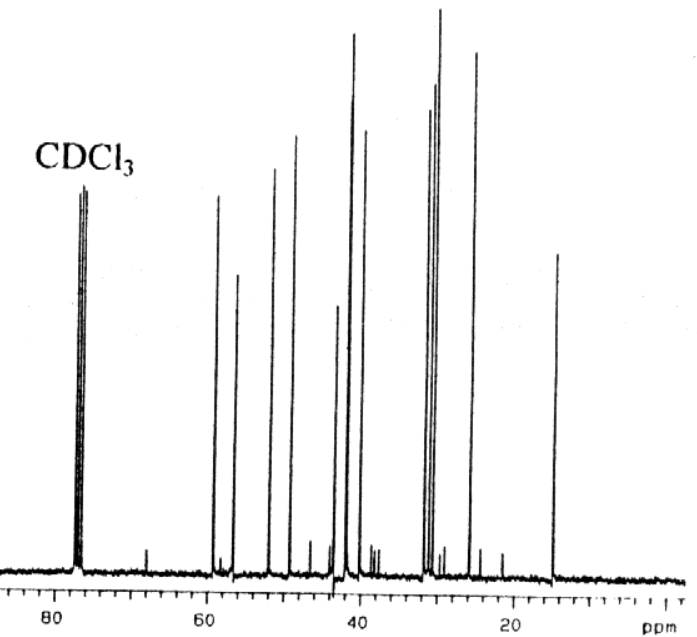

Mandelt If Fitjer
DATE: Apr 152000 $300 \mathrm{MHz}$ $\mathrm{CDCl}_{3}$

Abb. 100: $\quad{ }^{13}$ C-NMR-Spektrum von (3aR*,6aR*,7aR*)-3,3,4,7a-Tetramethyl2,3,3a,5,6,6a,7,7a-octahydro-1H-cyclopenta[a]pentalen (68) 


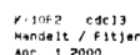

INSI TUMAEN: UNITY300

Frefuency $75,479 \mathrm{MHz}$
Spectral width 19034,3

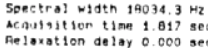

Pulse 35 . o degrees

1513 redetitlons

OECOUPLE H1

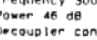

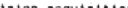

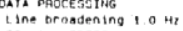

[oes] acquiletition tine 45 minutes
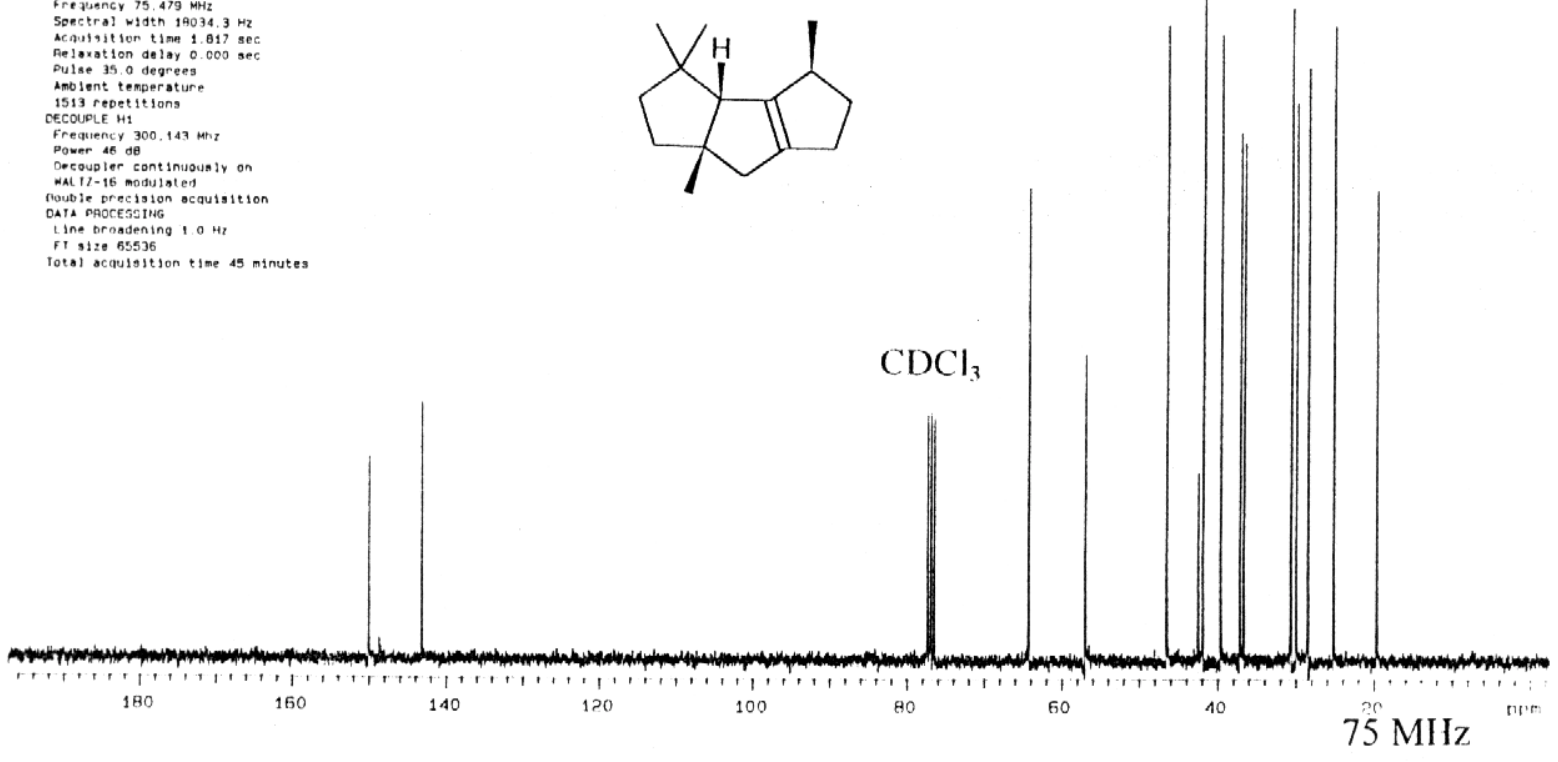

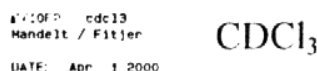

Abb. 101: $\quad{ }^{13} \mathrm{C}-\mathrm{NMR}-$ Spektrum von (3aR*,4R*,7aR*)-3,3,4,7a-Tetramethyl2,3,3a,4,5,6,7,7a-octahydro- $1 H$-cyclopenta[a]pentalen (69)
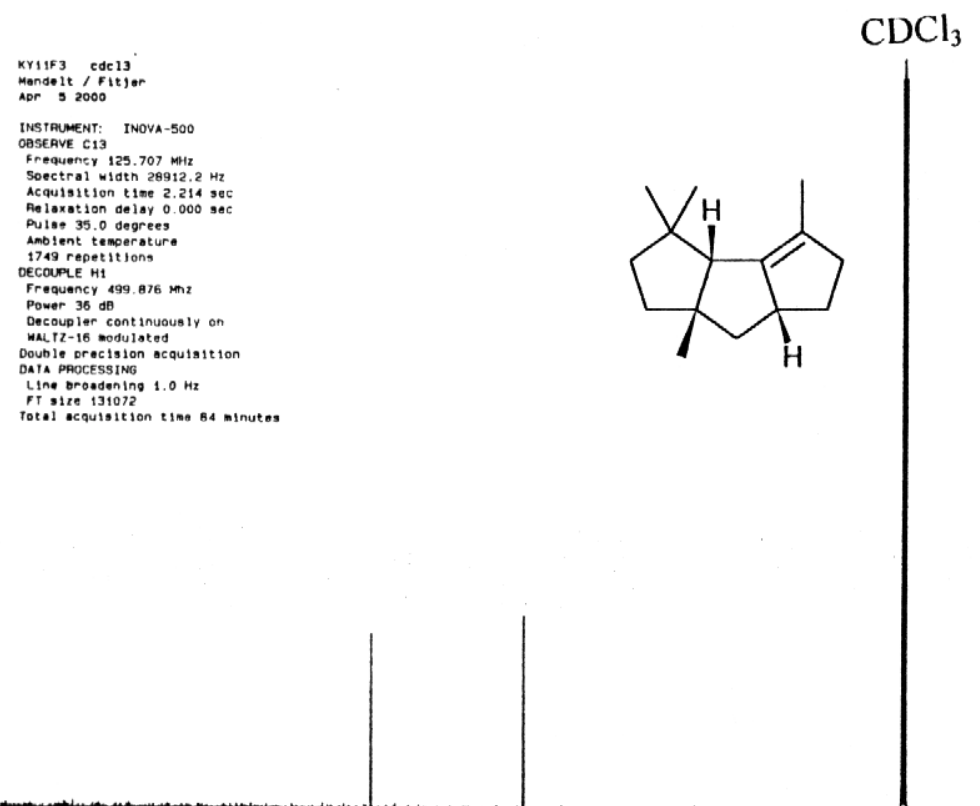

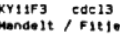

Abb. 102: $\quad{ }^{13} \mathrm{C}-\mathrm{NMR}-$ Spektrum von $(3 \mathrm{aR} *, 6 \mathrm{aS} *, 7 \mathrm{aR} *)-3,3,4,7 \mathrm{a}-$ Tetramethyl- 


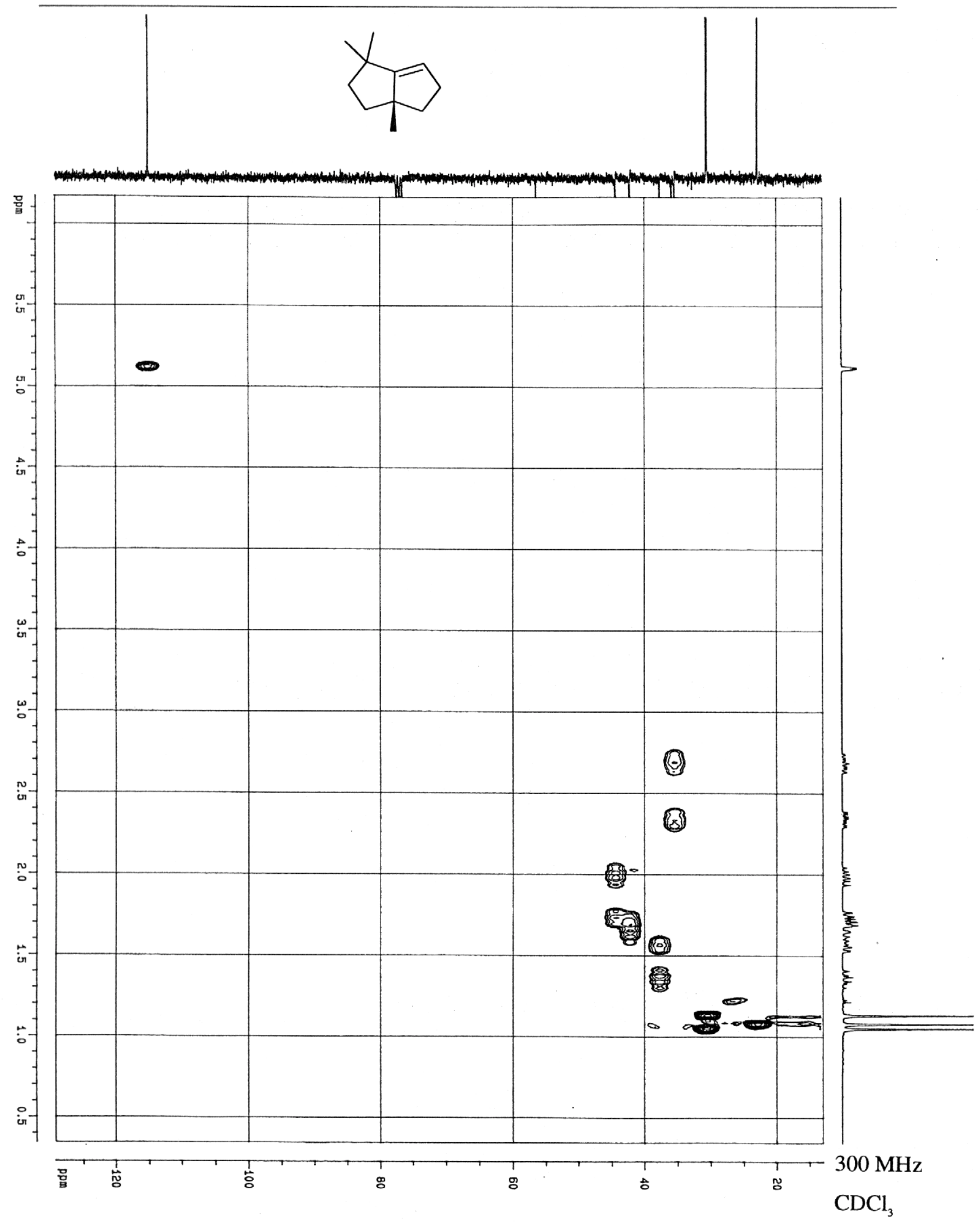

Abb. 103: HMQC-Spektrum von 1,1,3a-Trimethyl-1,2,3,3a,4,5-hexahydro-pentalen (120) 

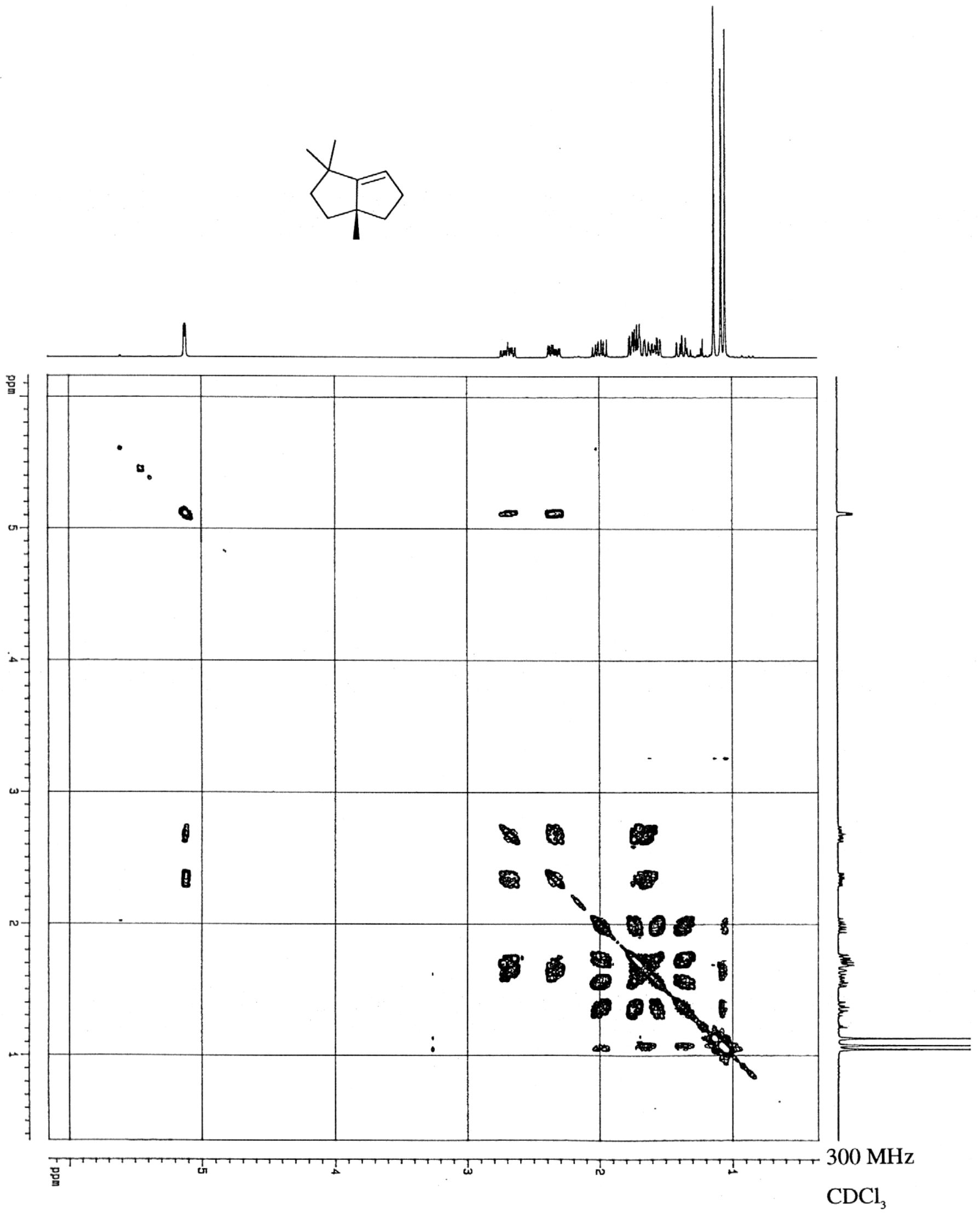

Abb. 104: COSY-Spektrum von 1,1,3a-Trimethyl-1,2,3,3a,4,5-hexahydro-pentalen (120) 


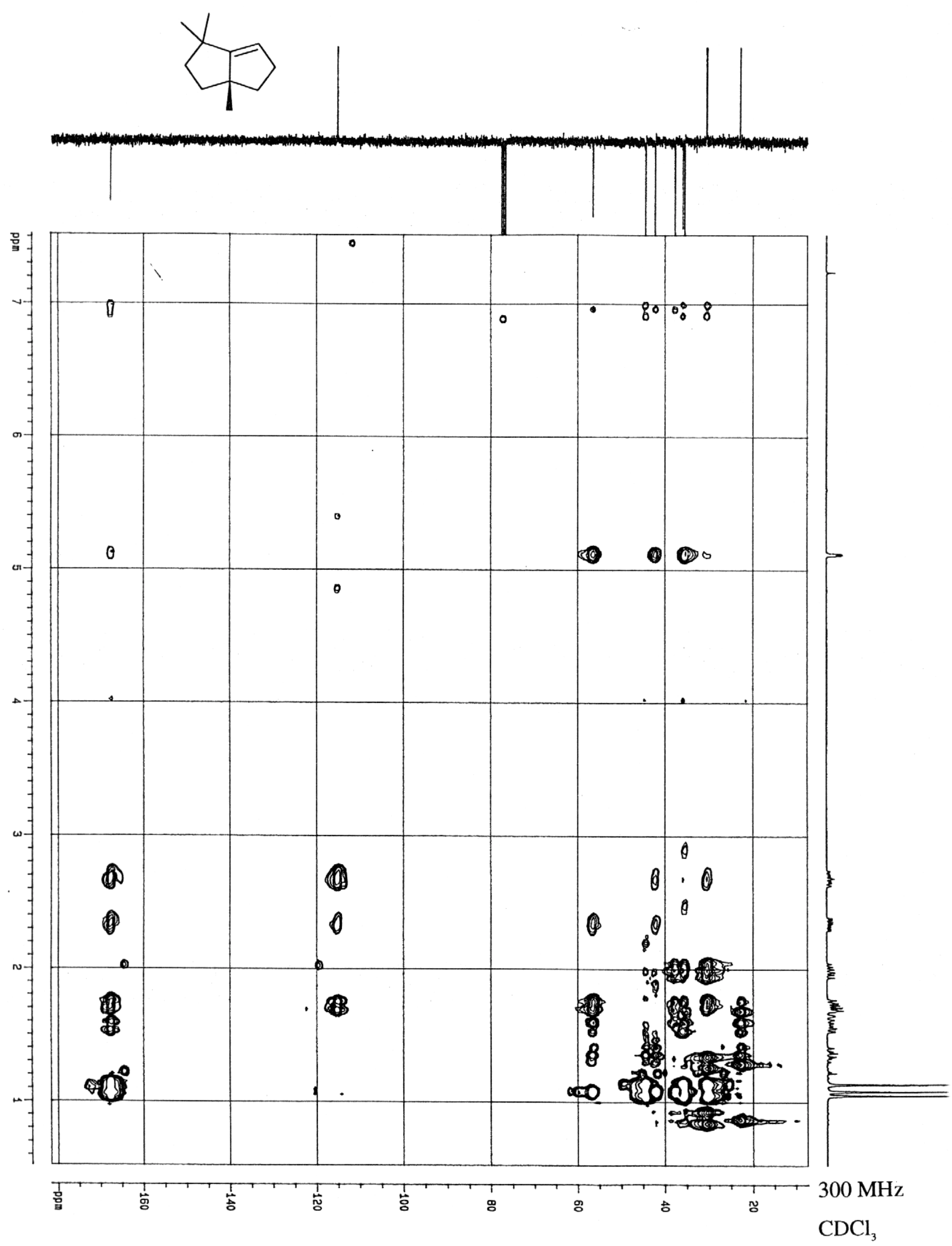

Abb. 105: HMBC-Spektrum von 1,1,3a-Trimethyl-1,2,3,3a,4,5-hexahydro-pentalen (120) 


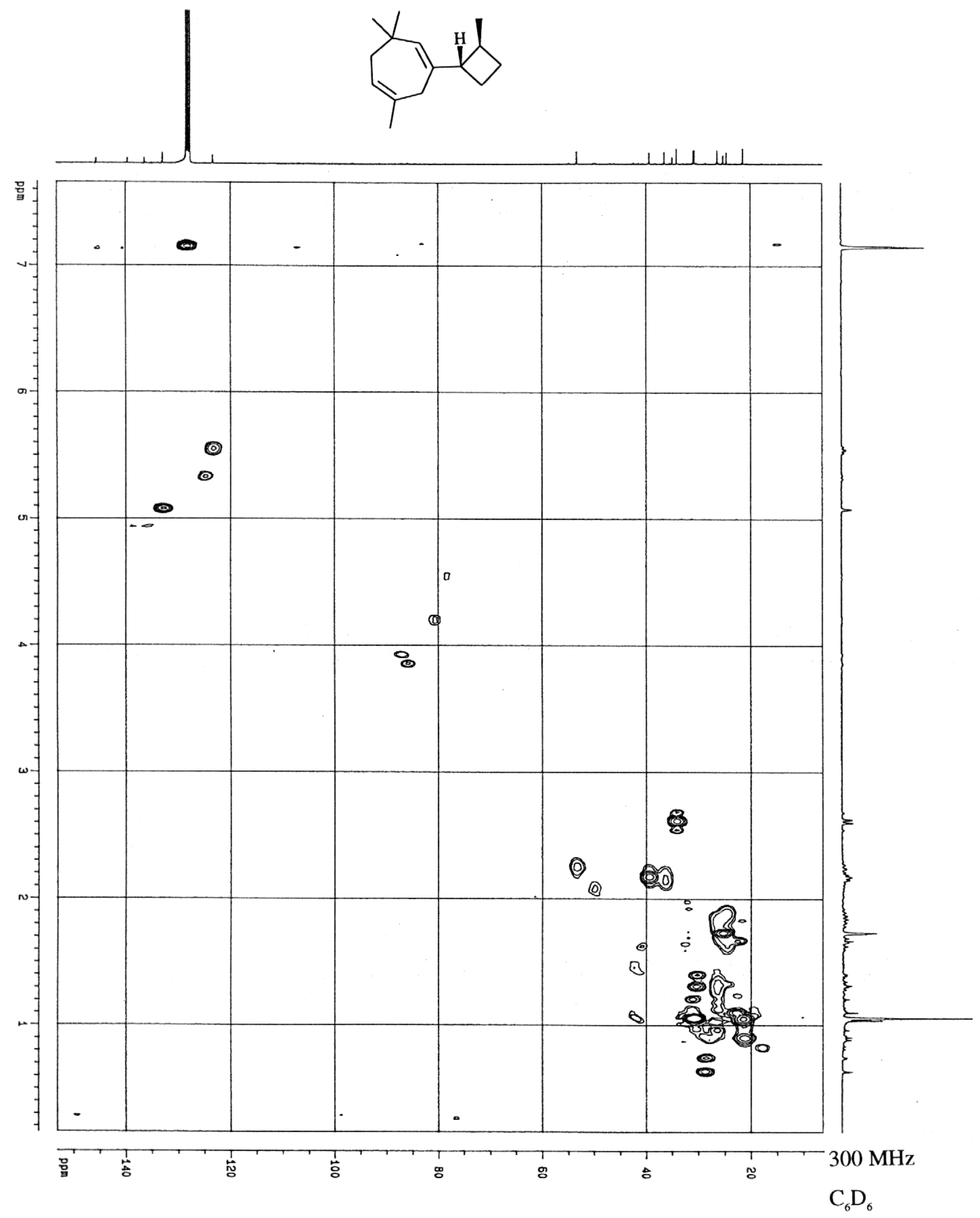

Abb. 106: HMQC-Spektrum von 2,6,6-Trimethyl-4-(2-methylcyclobutyl)-cyclohepta-1,4dien (122) 


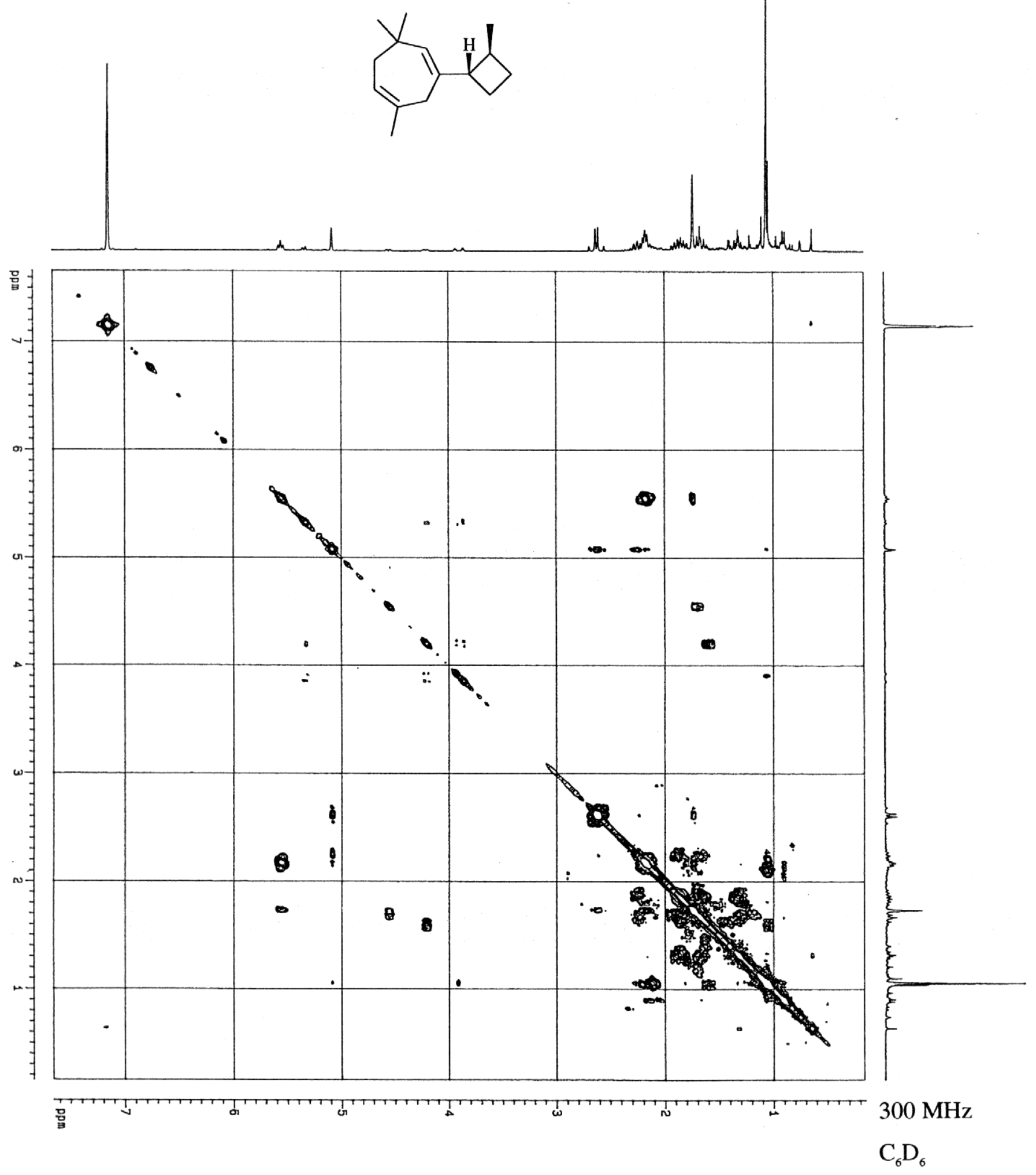

Abb. 107: COSY-Spektrum von 2,6,6-Trimethyl-4-(2-methylcyclobutyl)-cyclohepta-1,4dien (122) 


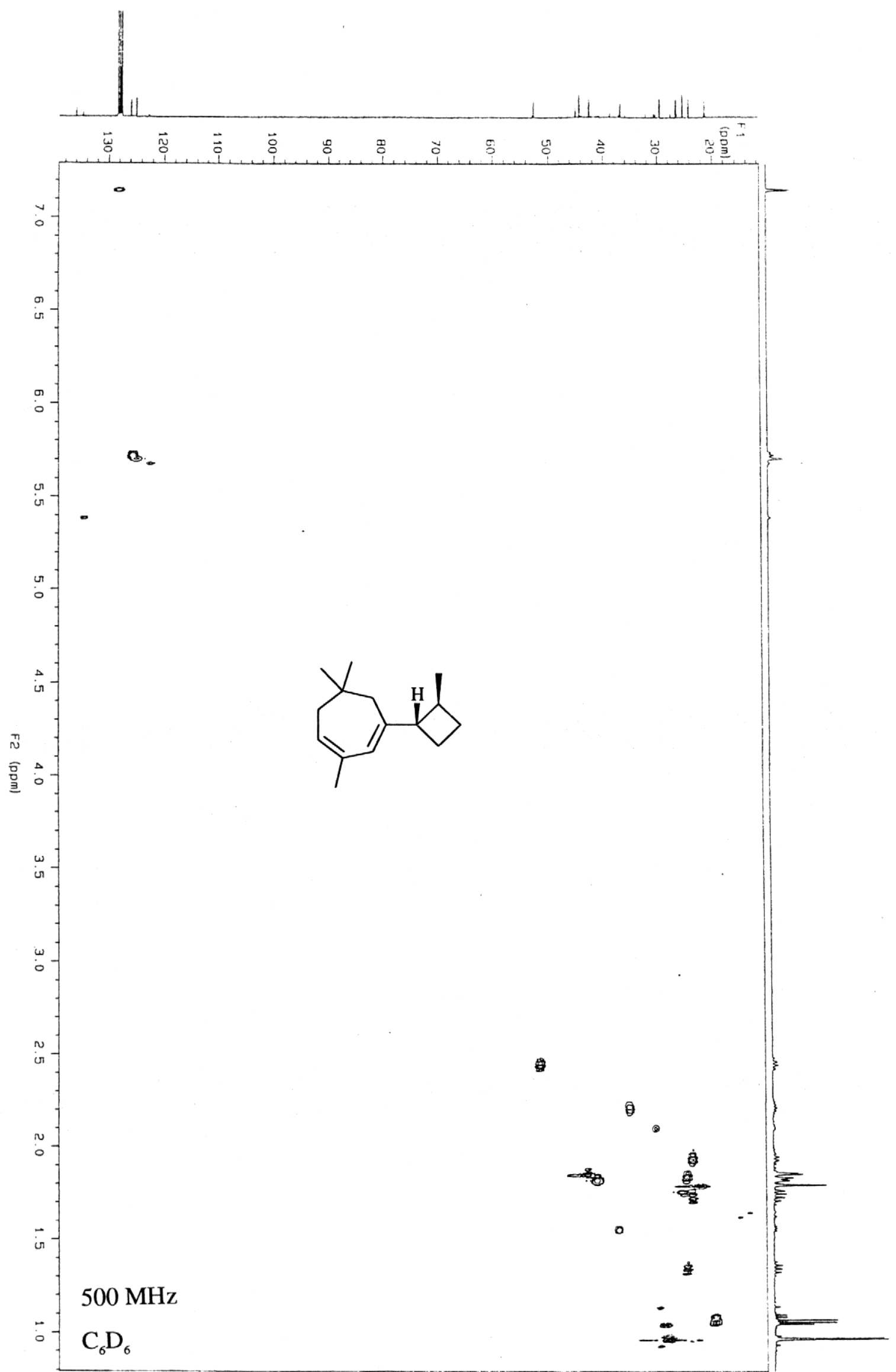

Abb. 108: HMQC-Spektrum von 3,6,6-Trimethyl-1-(2-methyl-cyclobutyl)-cyclohepta-1,3dien (123) 


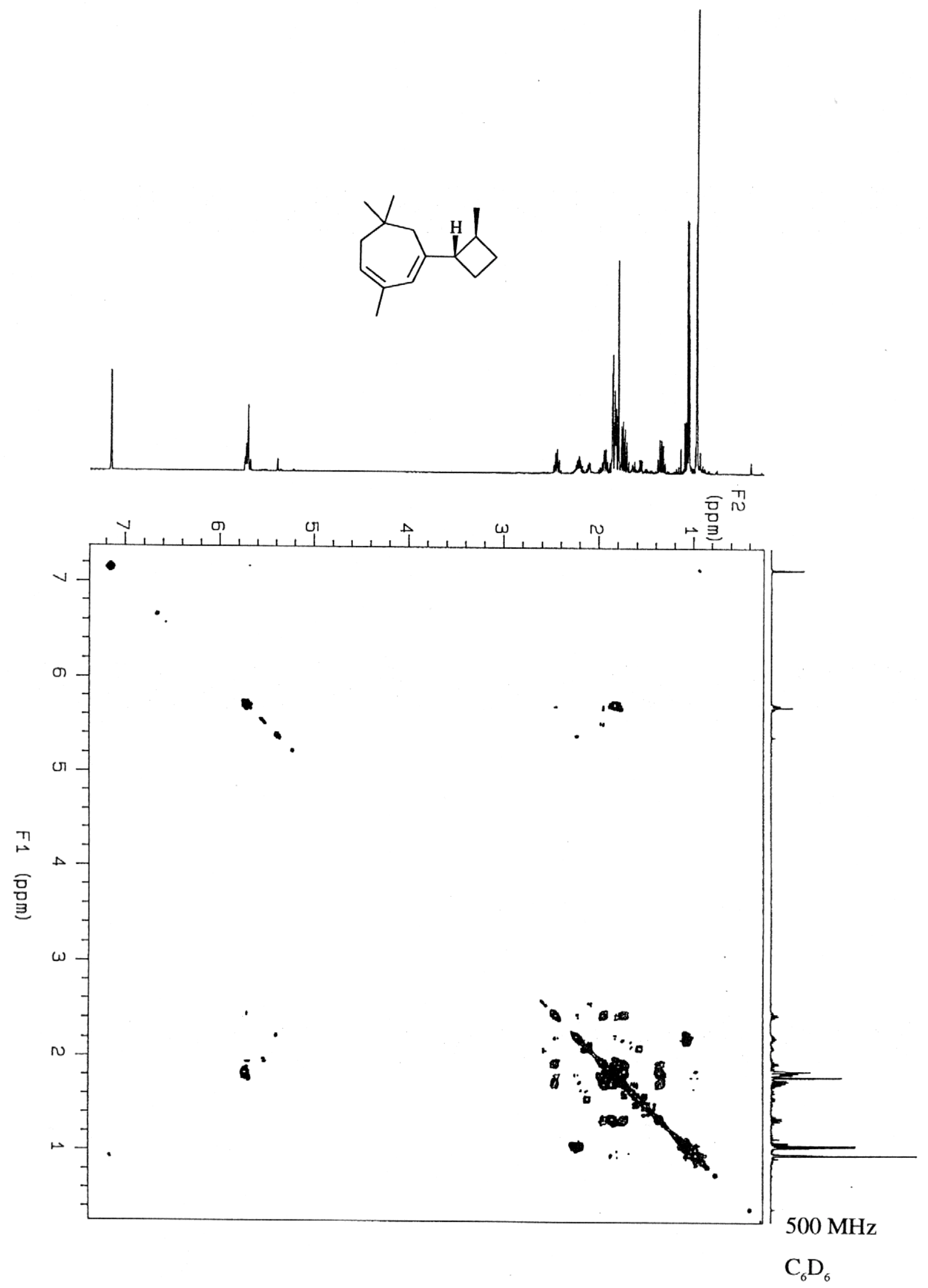

Abb. 109: COSY-Spektrum von 3,6,6-Trimethyl-1-(2-methyl-cyclobutyl)-cyclohepta-1,3dien (123) 


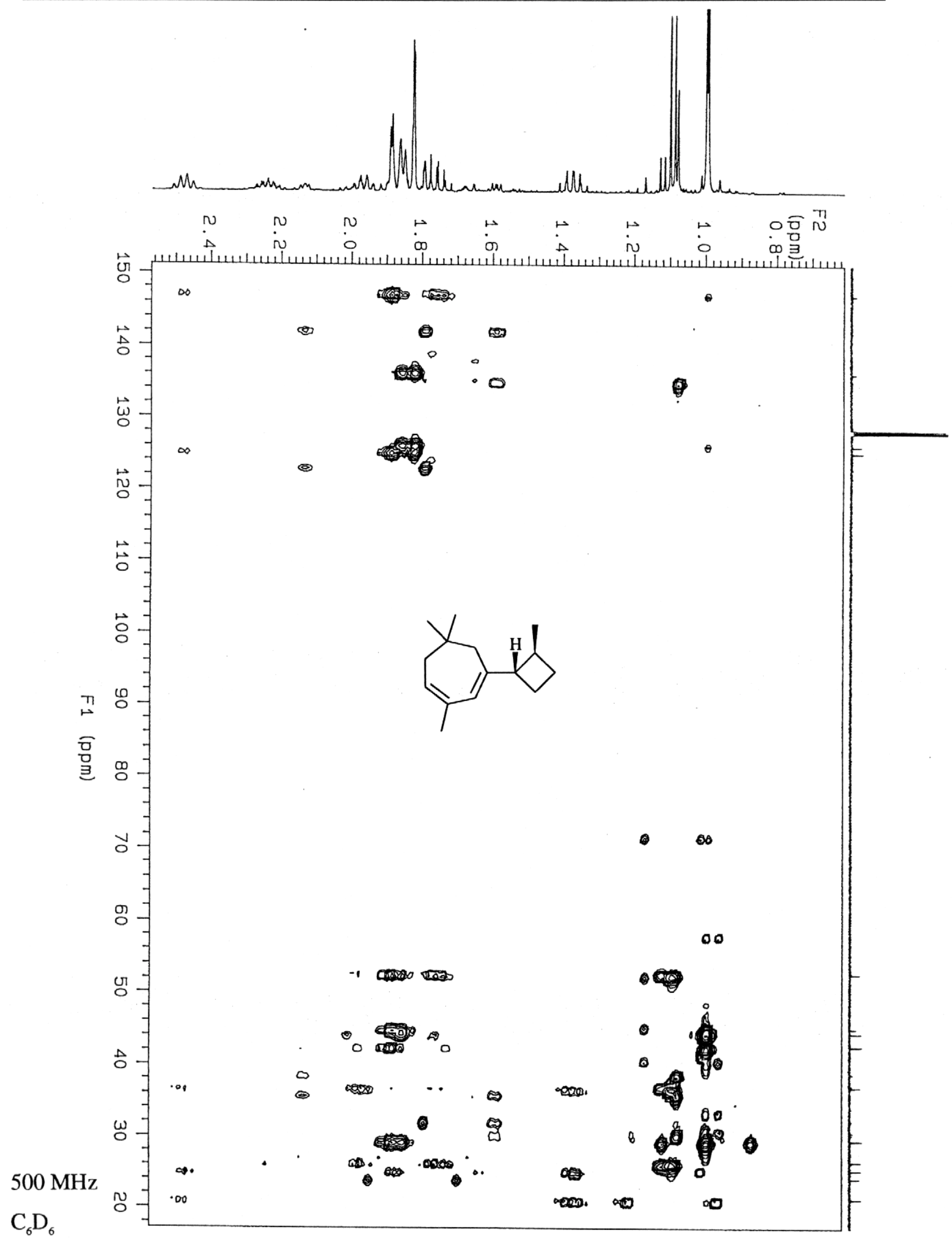

Abb. 110: HMBC-Spektrum von 3,6,6-Trimethyl-1-(2-methyl-cyclobutyl)-cyclohepta-1,3dien (123) 


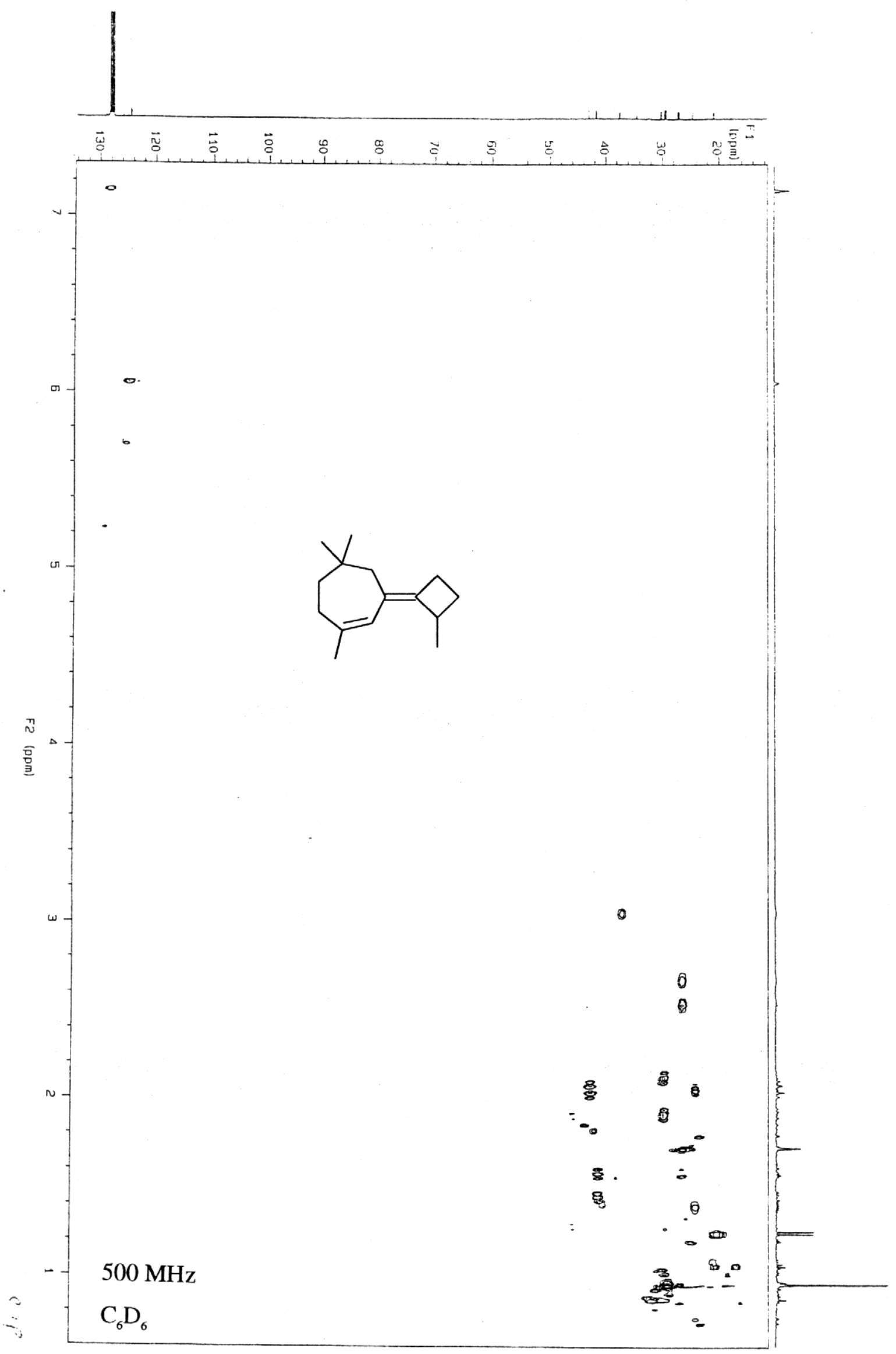

Abb. 111: HMQC-Spektrum von 1,5,5-Trimethyl-3-(2-methyl-cyclobutyliden)-cyclohept-1-en (124) 

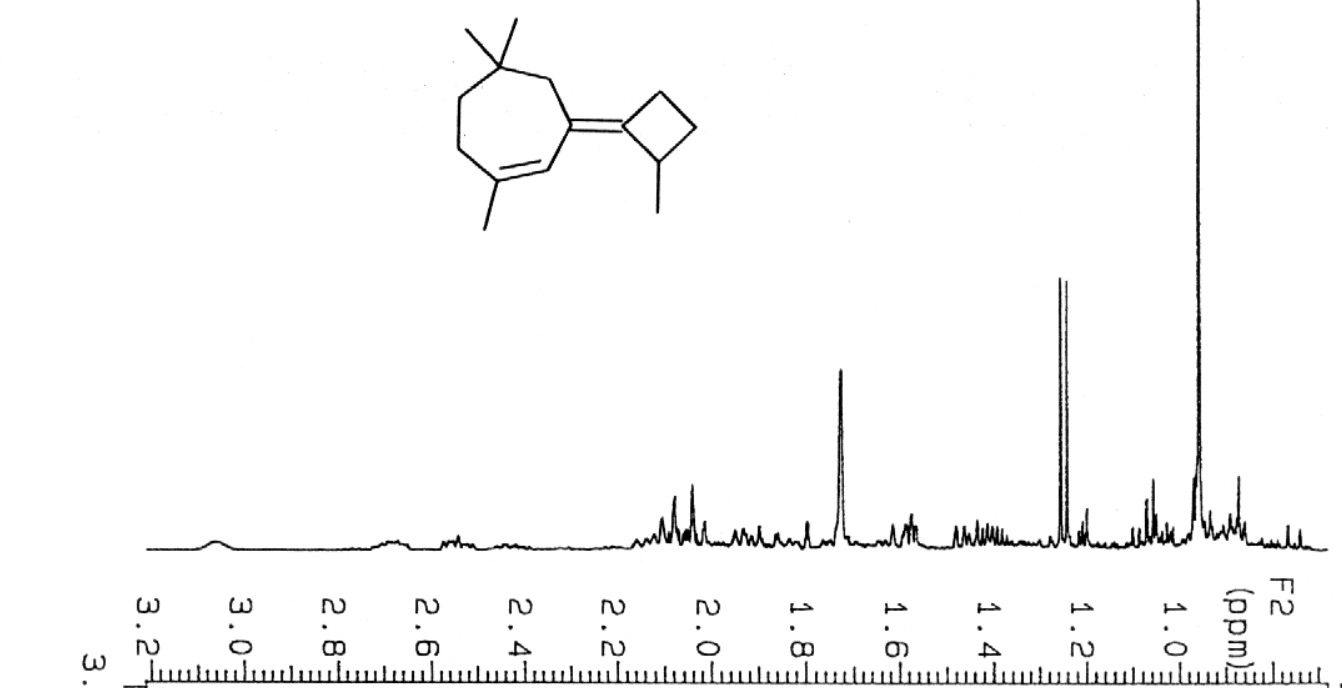

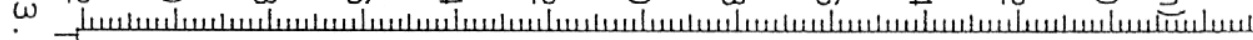

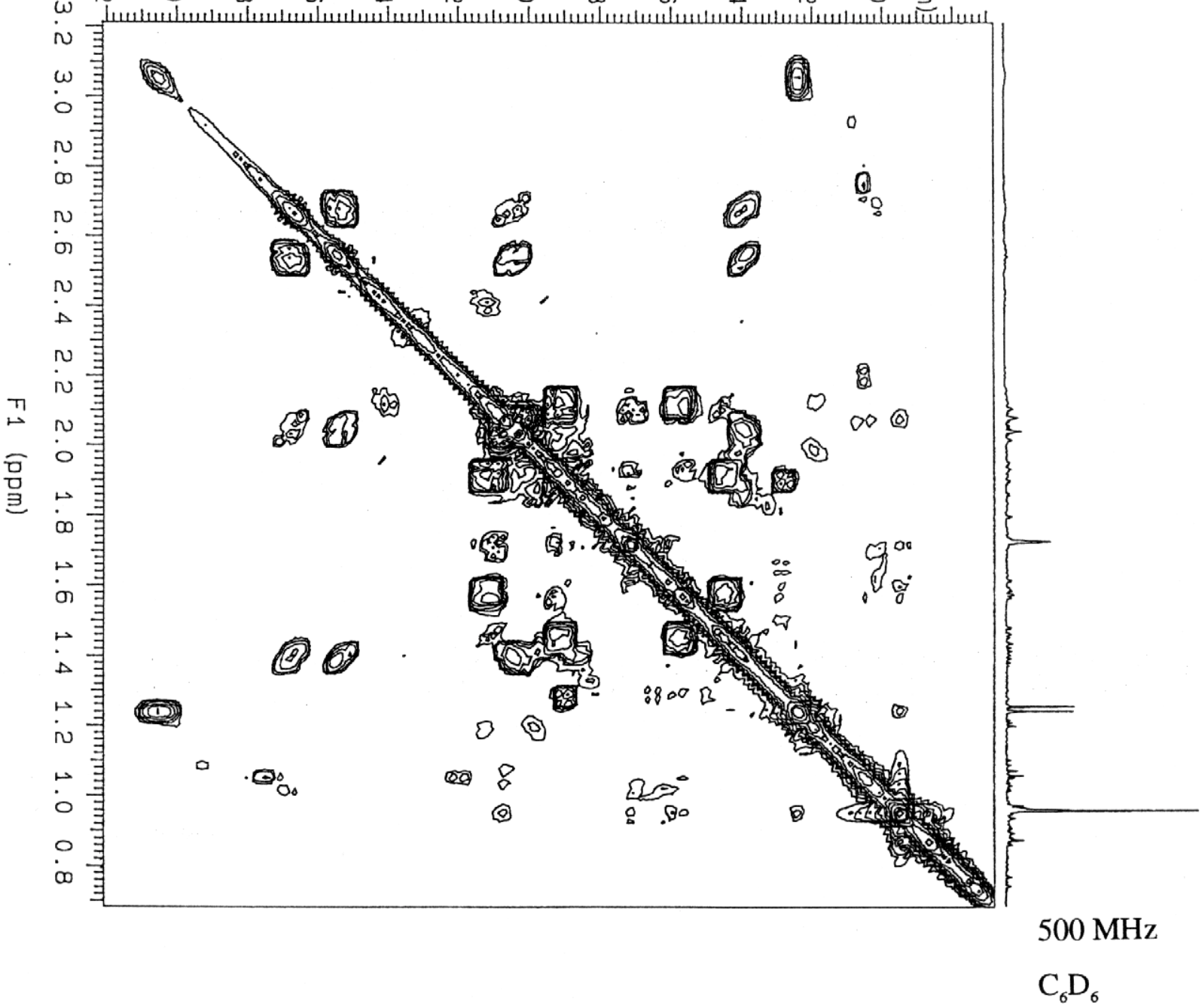

Abb. 112: COSY-Spektrum von 1,5,5-Trimethyl-3-(2-methyl-cyclobutyliden)-cyclohept-1-en (124) 


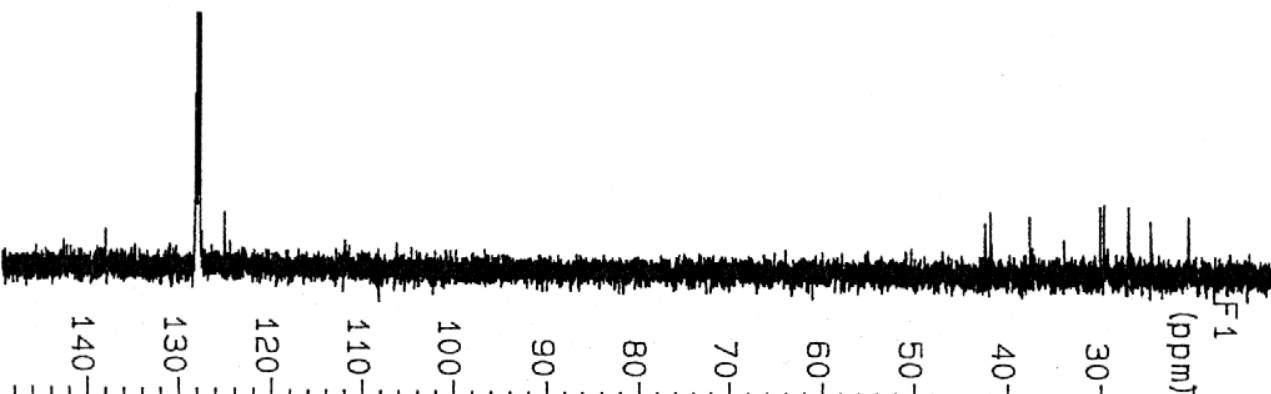

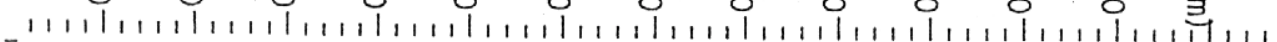

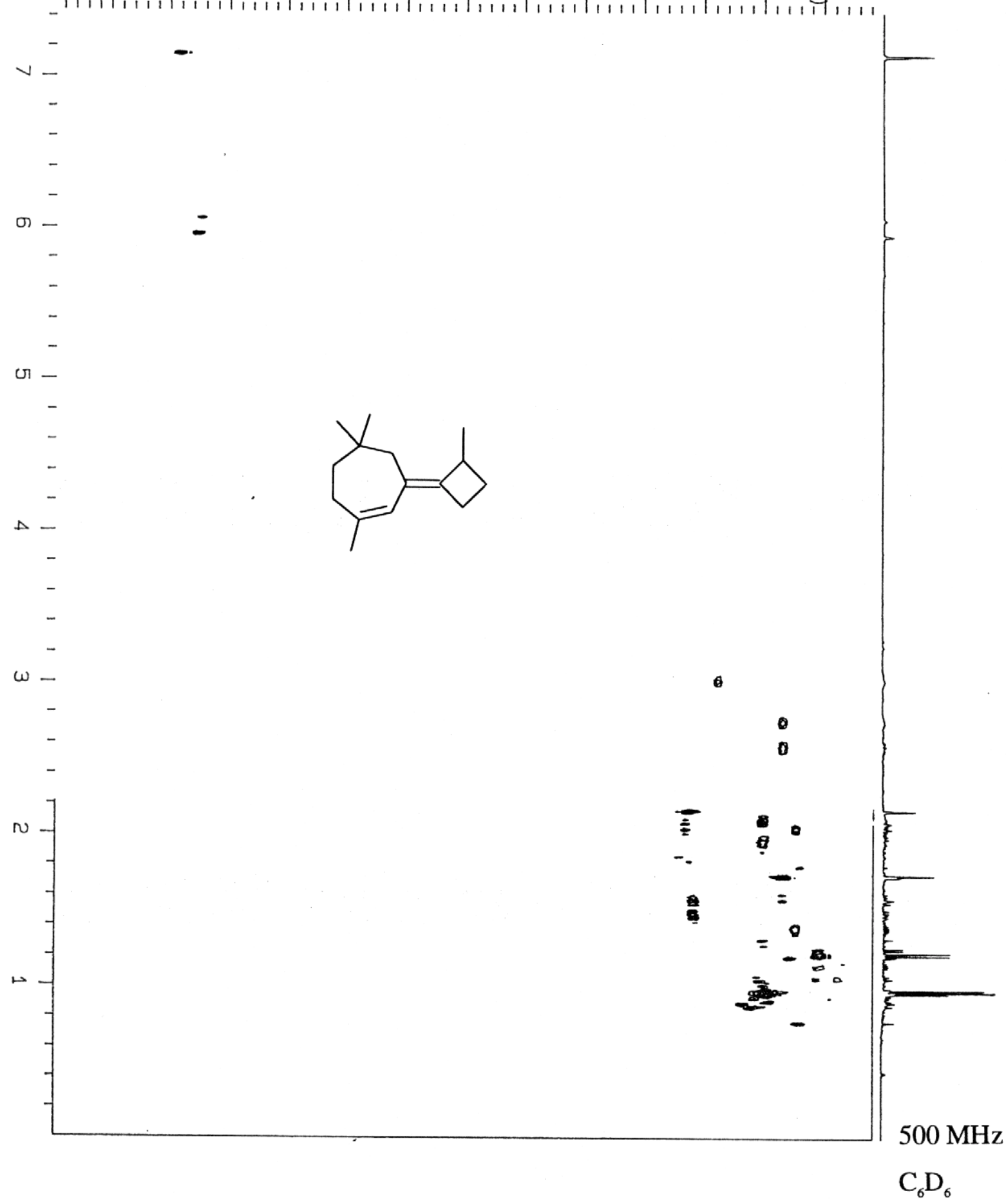

Abb. 113: HMQC-Spektrum von 1,5,5-Trimethyl-3-(2-methyl-cyclobutyliden)-cyclohept-1-en (125) 


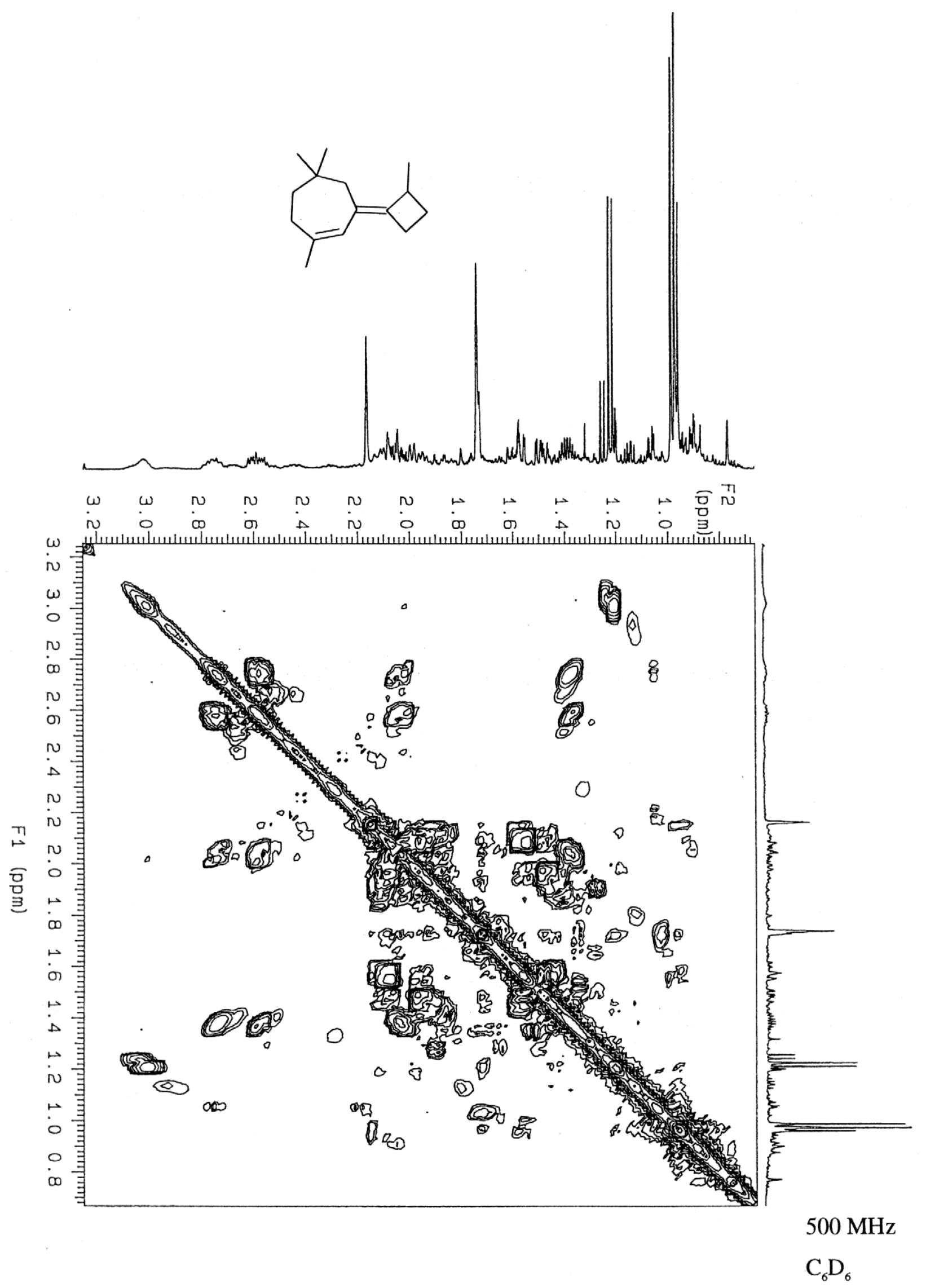

Abb. 114: COSY-Spektrum von 1,5,5-Trimethyl-3-(2-methyl-cyclobutyliden)-cyclohept-1-en (125) 


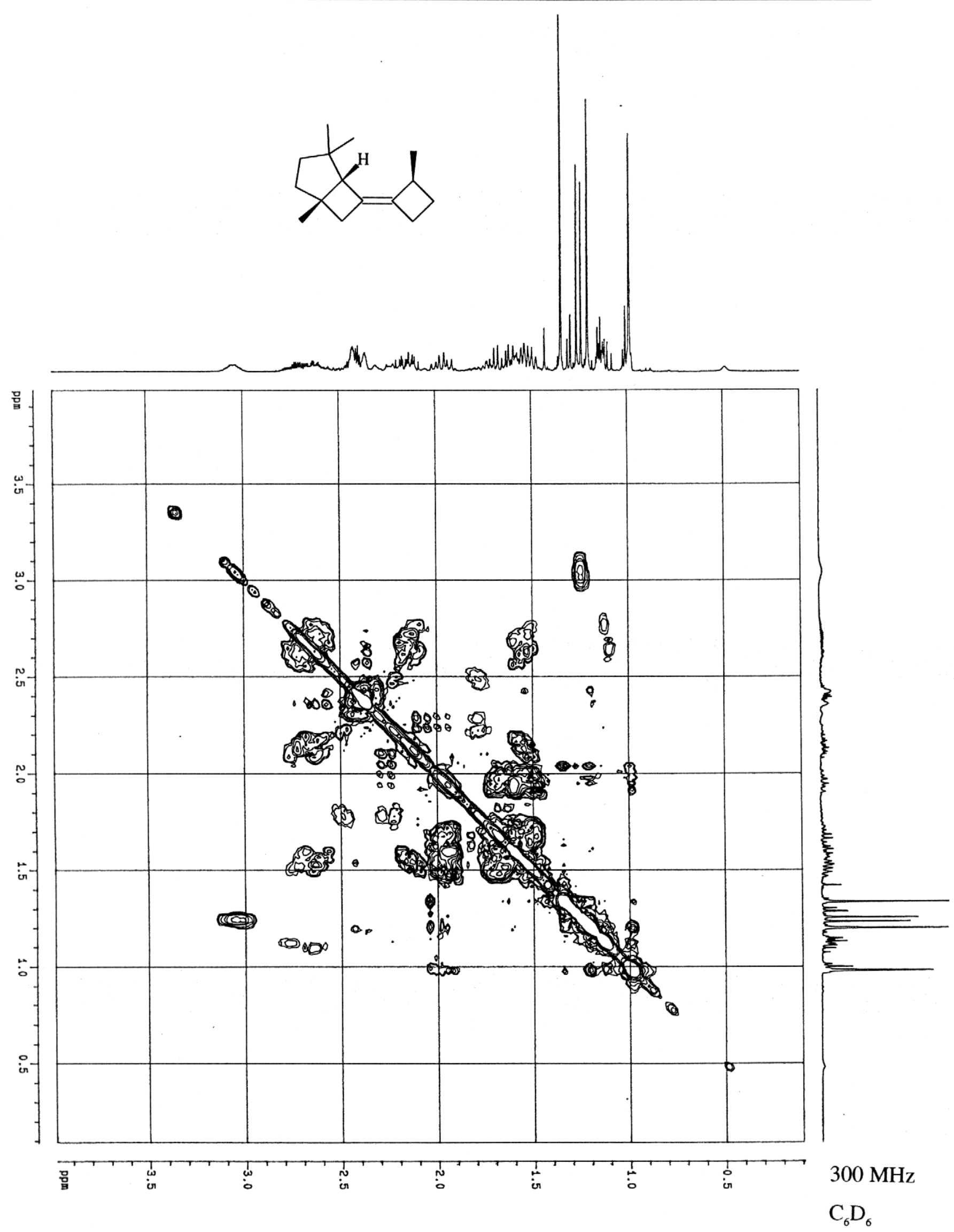

Abb. 115: HMQC-Spektrum von 1,4,4-Trimethyl-6-(2-methyl-cyclobutyliden)-bicyclo[3.2.0]heptan $(\mathbf{1 3 2} / \mathbf{1 3 3} / \mathbf{1 3 4 / 1 3 5 )}$ 

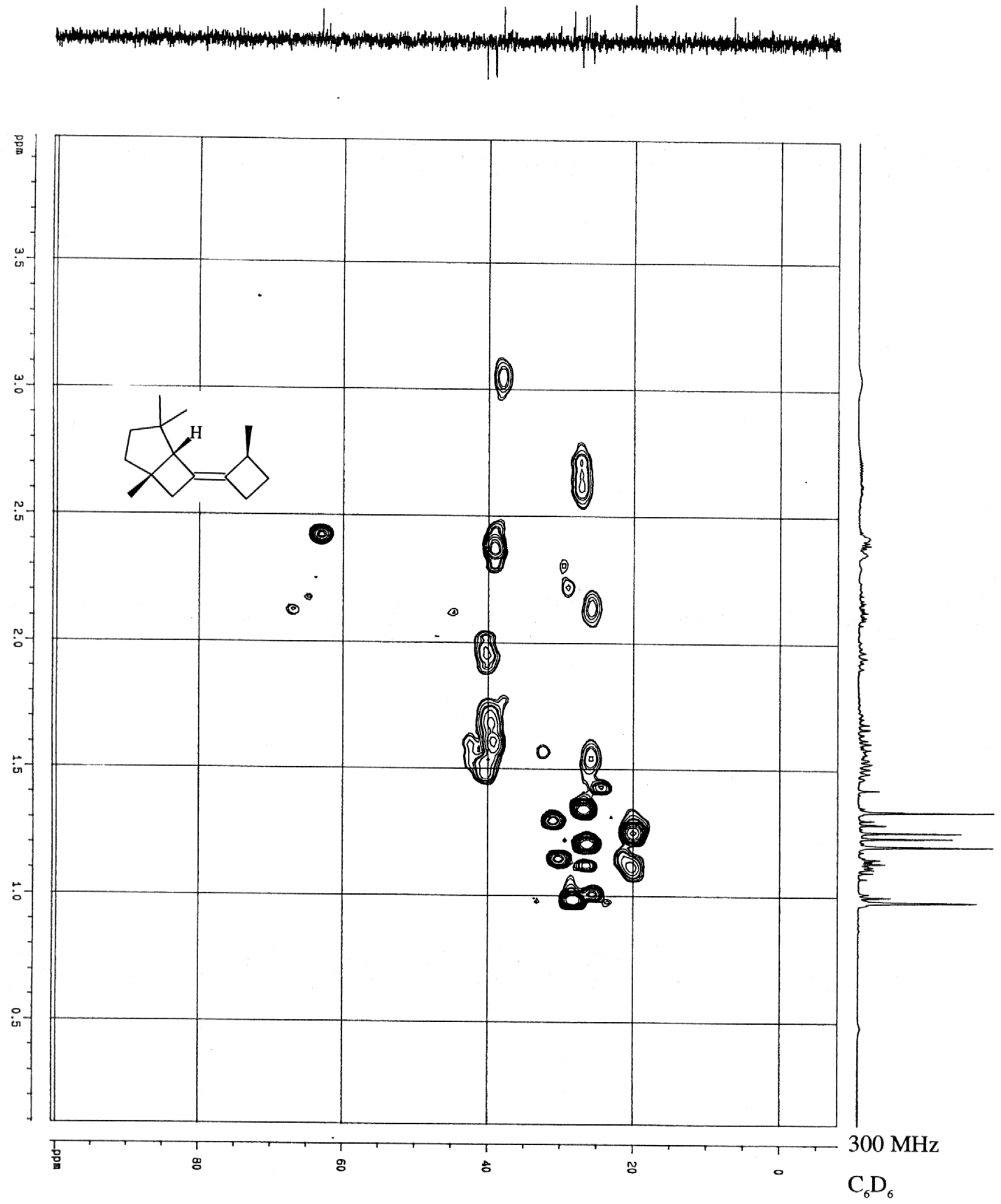

Abb. 116: COSY-Spektrum von 1,4,4-Trimethyl-6-(2-methyl-cyclobutyliden)-bicyclo[3.2.0]heptan (132/133/134/135) 


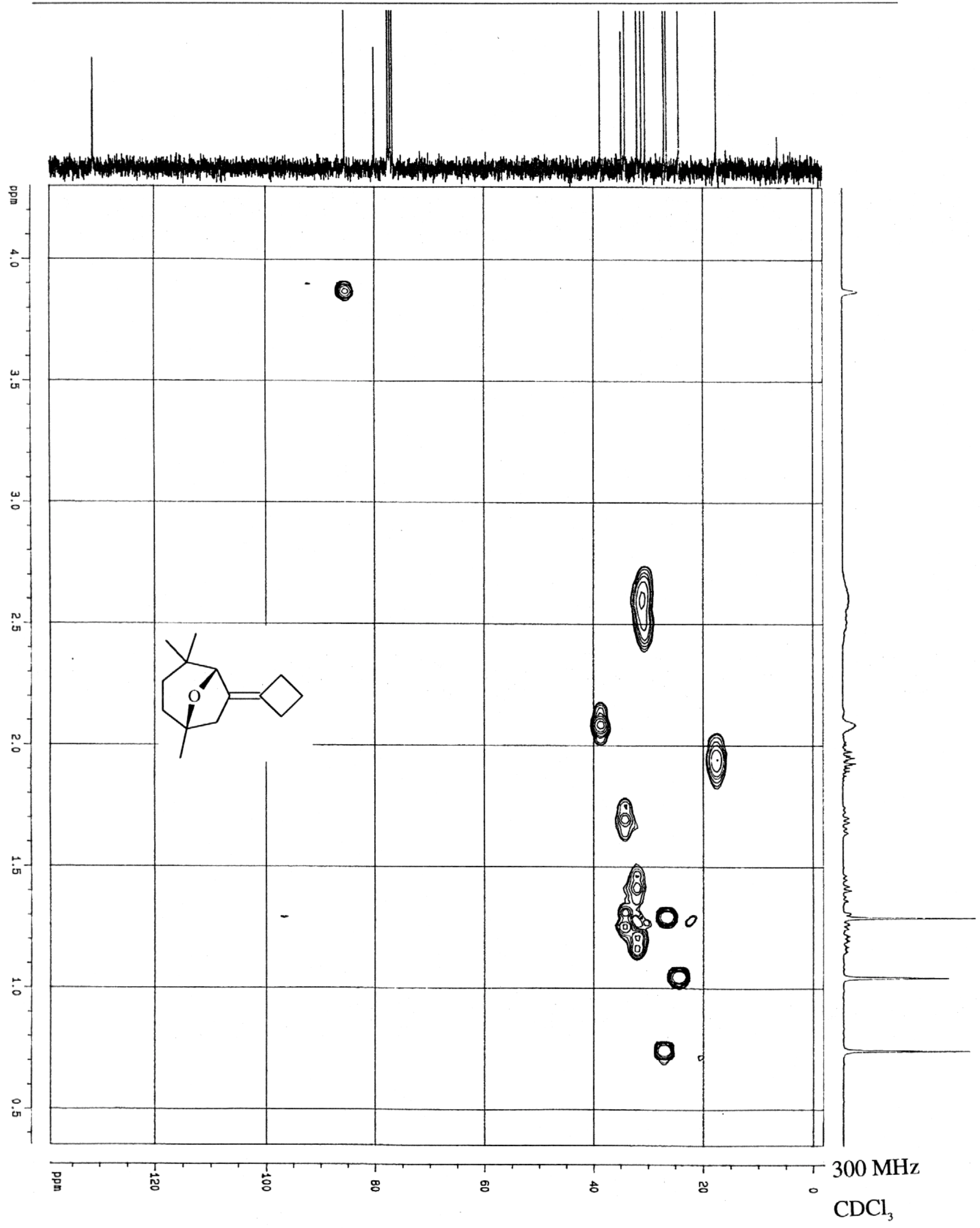

Abb. 117: HMQC-Spektrum von 6-Cyclobutyliden-1,4,4-trimethyl-8-oxa-bicyclo[3.2.1]octan (138) 

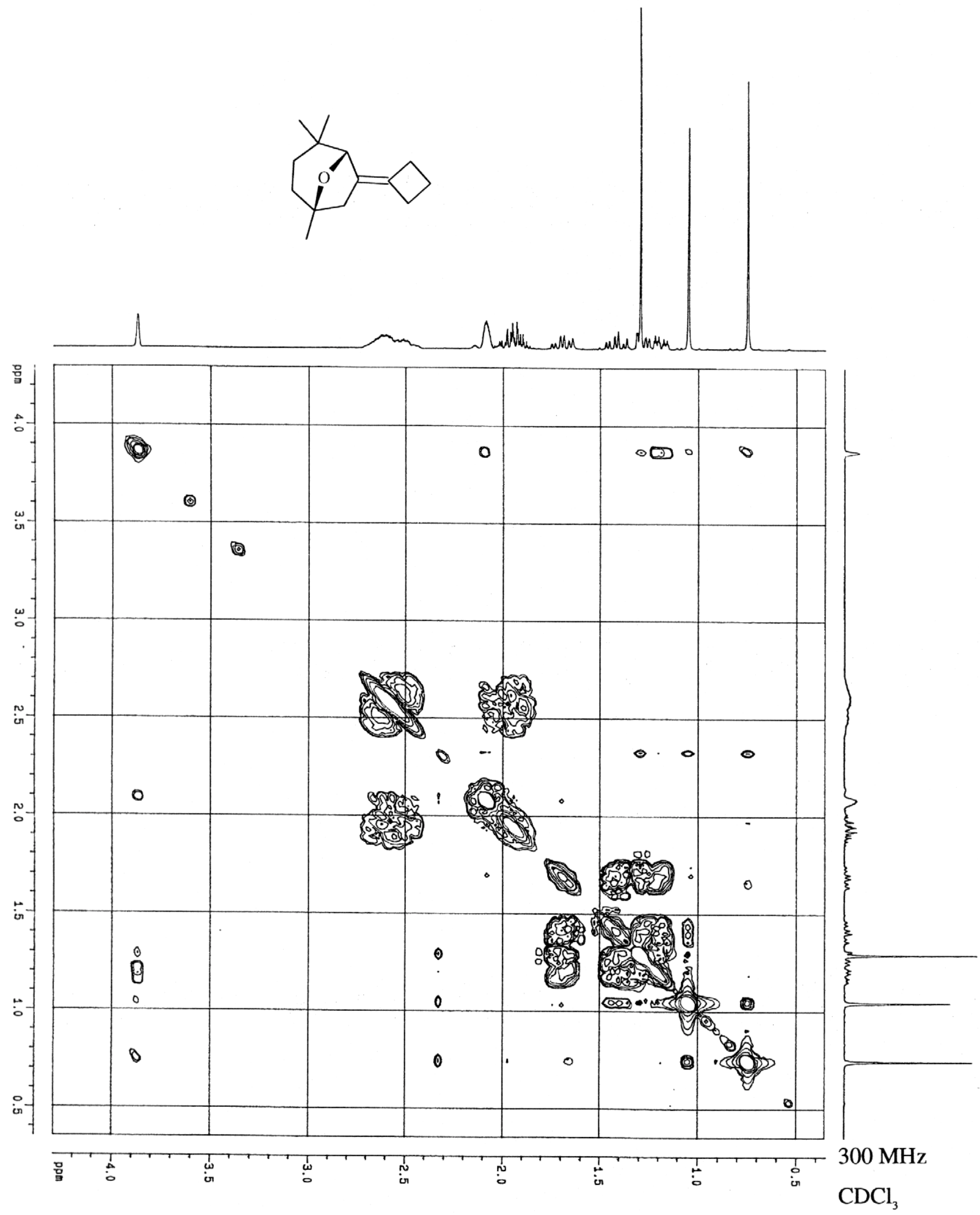

Abb. 118: COSY-Spektrum von 6-Cyclobutyliden-1,4,4-trimethyl-8-oxa-bicyclo[3.2.1]octan (138) 


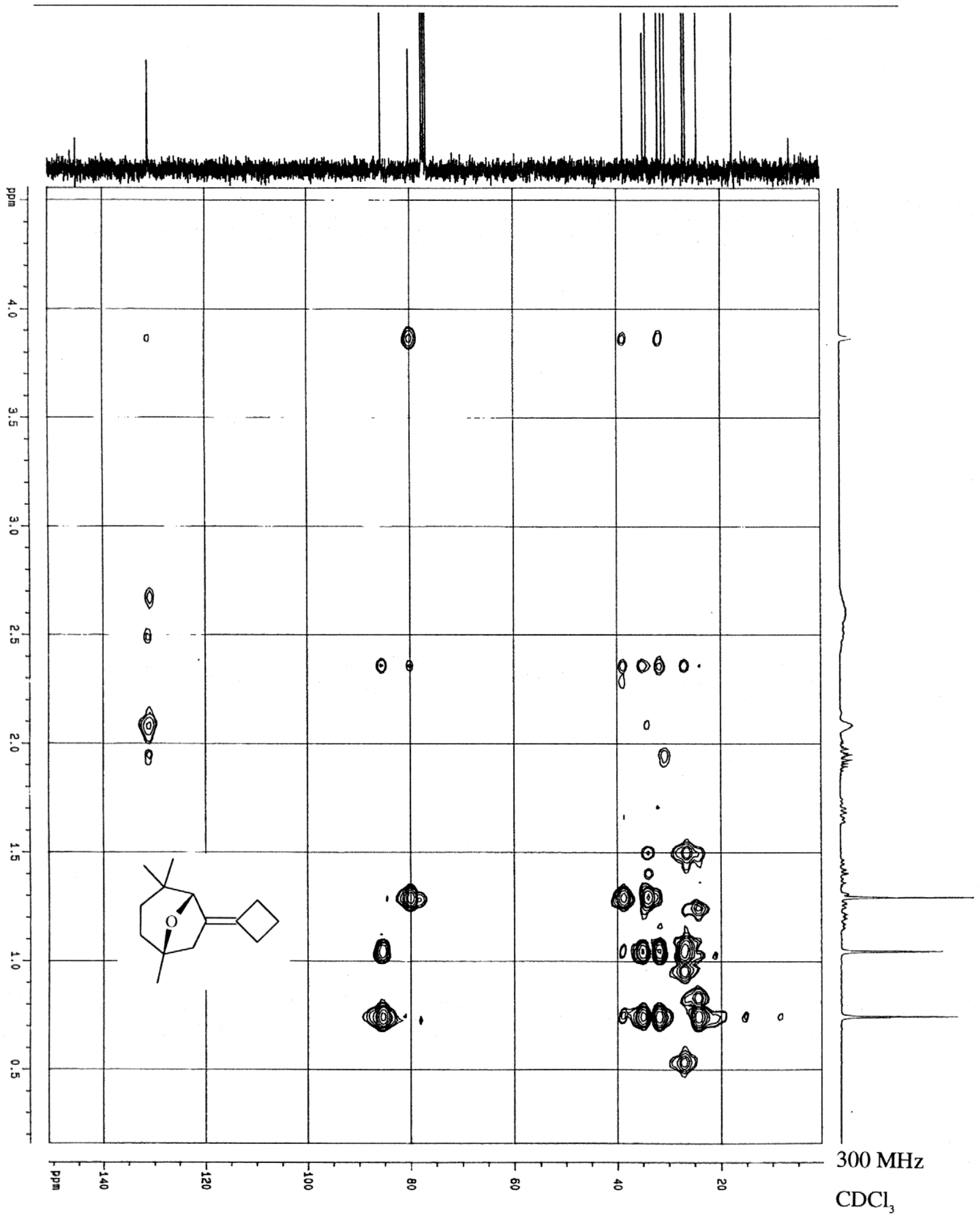

Abb. 119: HMBC-Spektrum von 6-Cyclobutyliden-1,4,4-trimethyl-8-oxa-bicyclo[3.2.1]octan (138) 


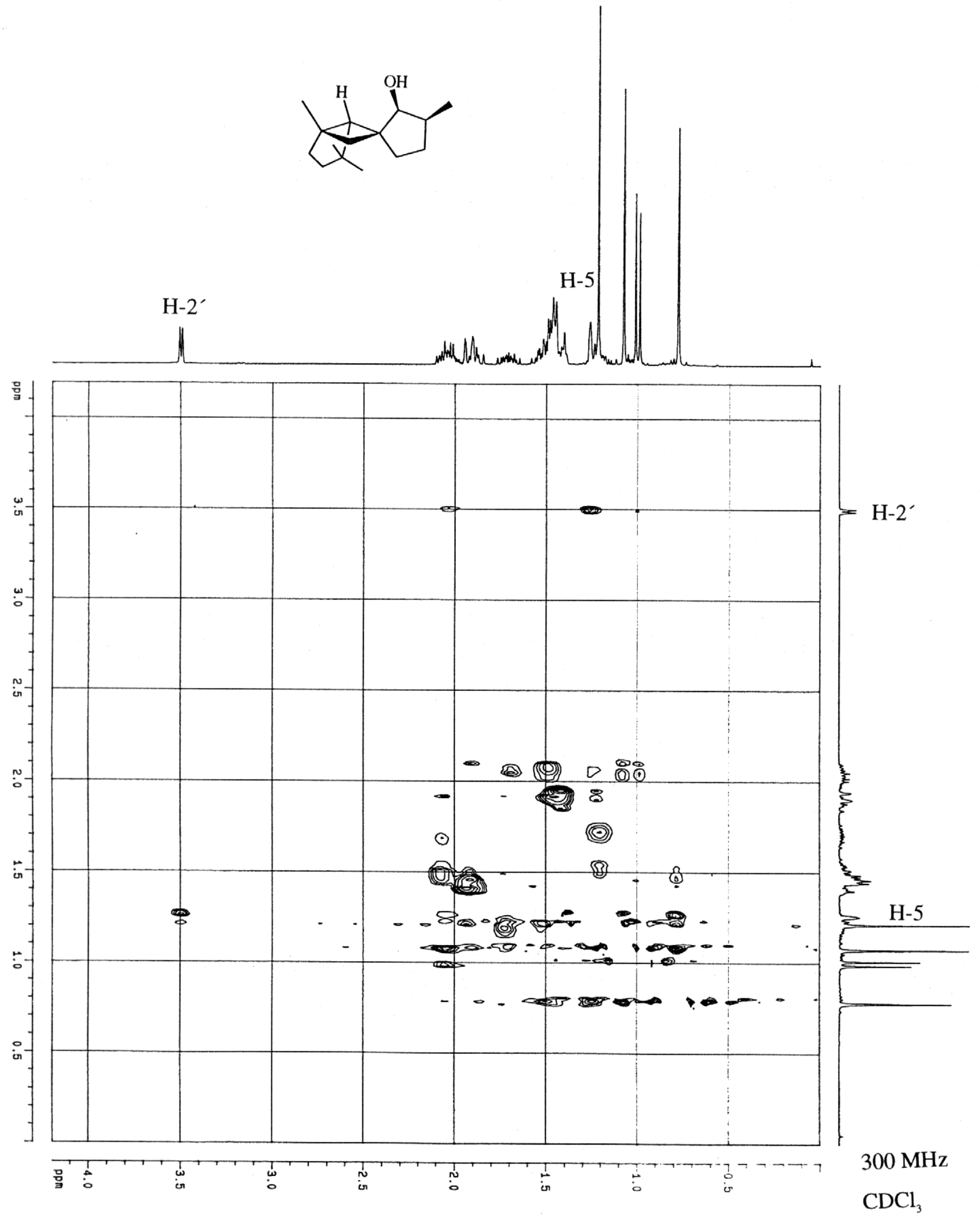

Abb. 120: NOESY-Spektrum von $\left(1 R^{*}, 5 R^{*}, 6 R^{*}, 2^{\prime} R^{*}, 3^{\prime} R^{*}\right)-1,4,4,3^{\prime}$-Tetramethylspiro$\{$ bicyclo[3.2.0]heptan-6,1'-cyclopentan-2'-ol\}(60) 


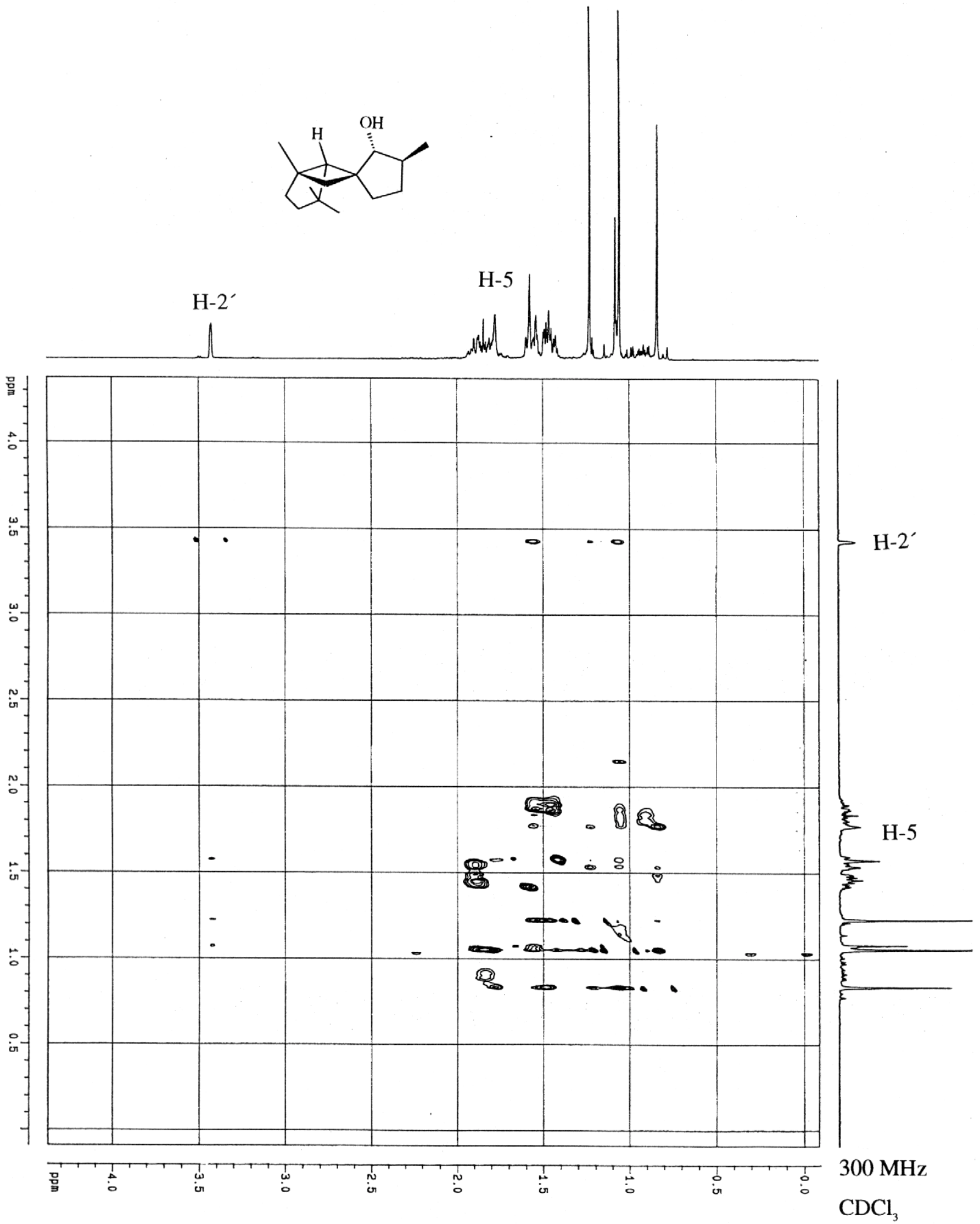

Abb. 121: NOESY-Spektrum von $\left(1 R^{*}, 5 R^{*}, 6 R^{*}, 2^{\prime} S^{*}, 3^{\prime} R^{*}\right)-1,4,4,3^{\prime}-$ Tetramethylspiro\{bicyclo[3.2.0]heptan-6,1'-cyclopentan-2'-ol\} (64) 

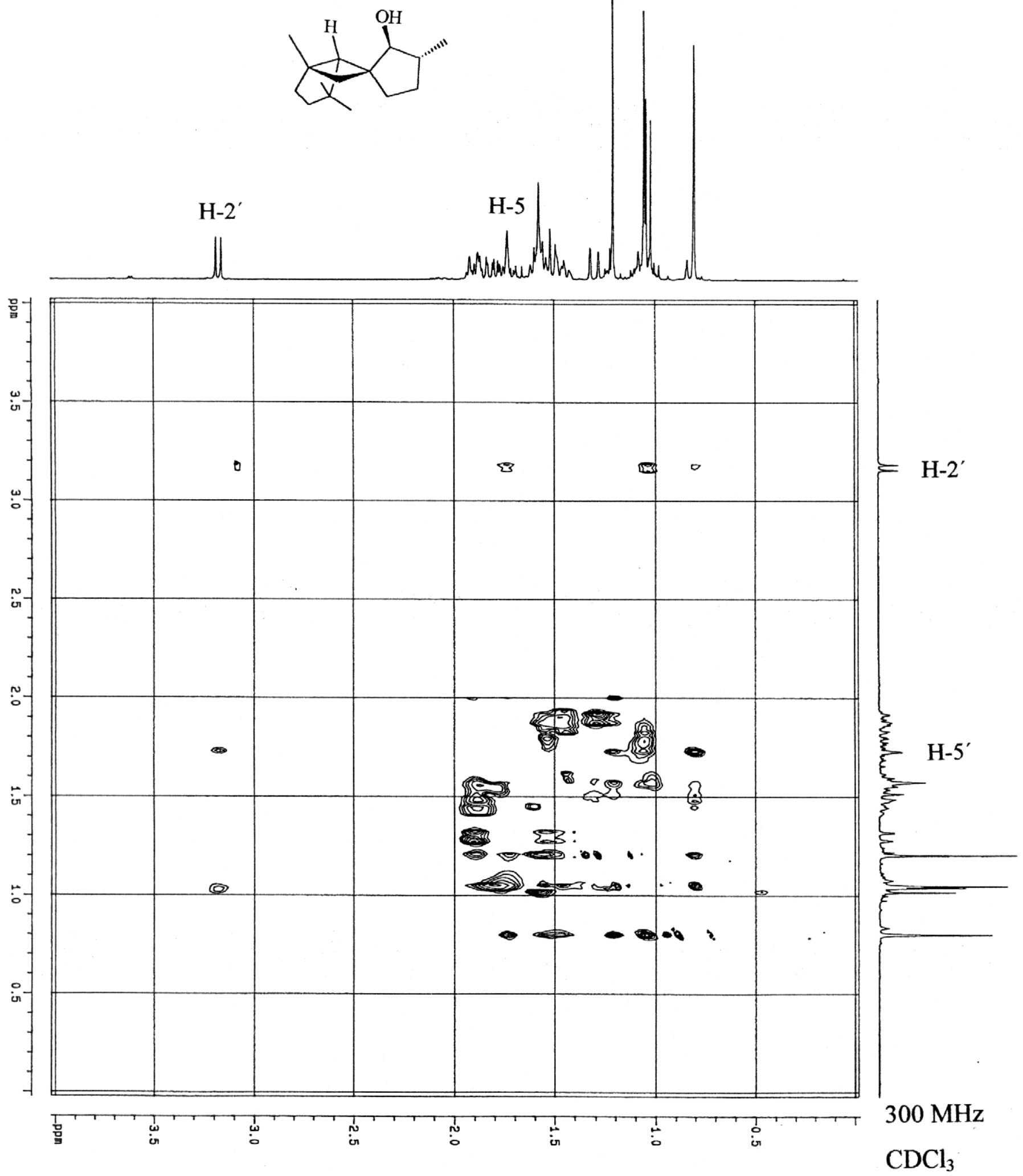

Abb. 122: NOESY-Spektrum von $\left(1 R^{*}, 5 R^{*}, 6 R^{*}, 2^{\prime} R^{*}, 3^{\prime} S^{*}\right)-1,4,4,3^{\prime}$-Tetramethylspiro\{bicyclo[3.2.0]heptan-6,1'-cyclopentan-2'-ol\} (66) 


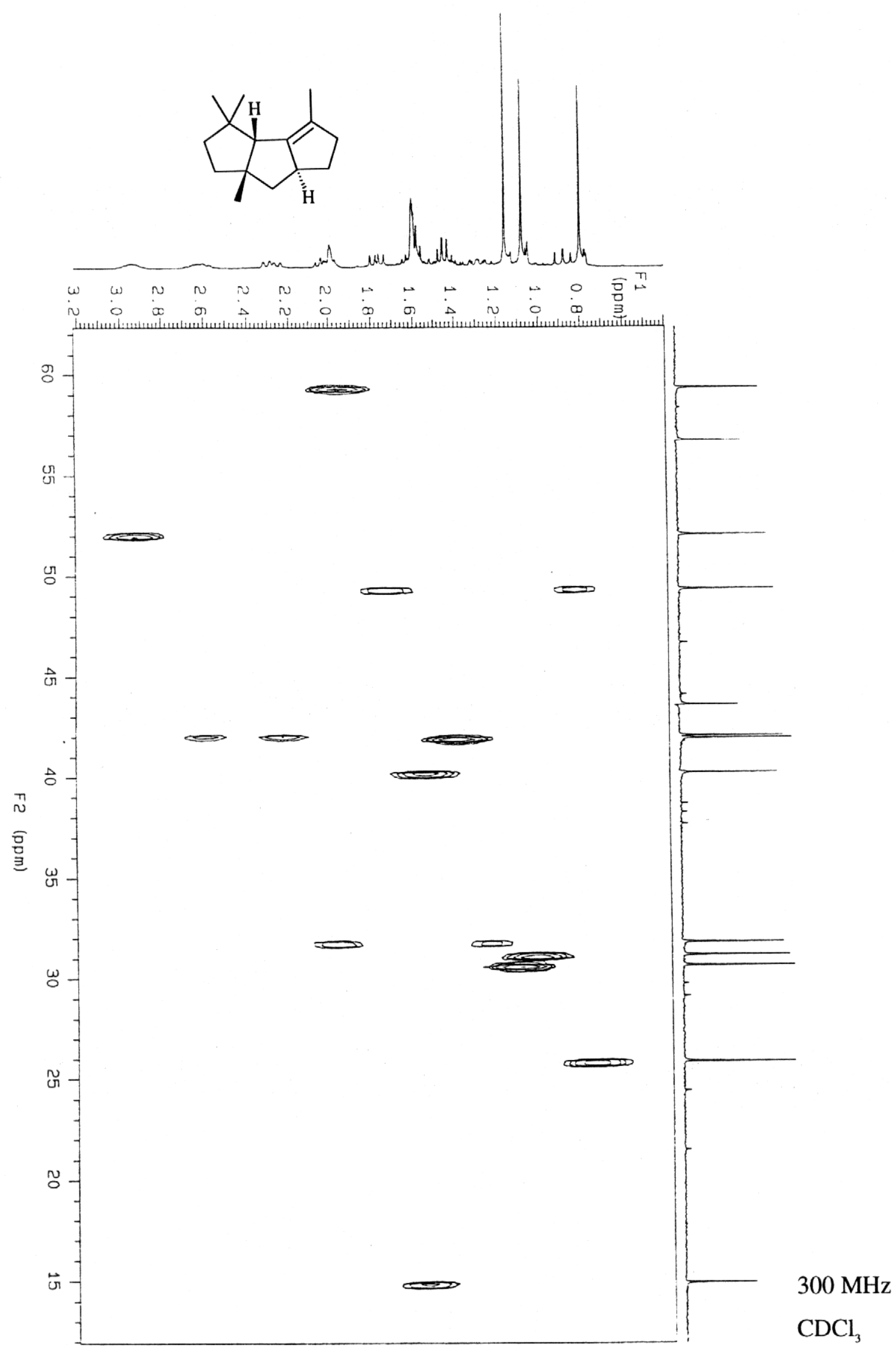

Abb. 123: HMQC-Spektrum von (3aR*,6aR*,7aR*)-3,3,4,7a-Tetramethyl2,3,3a,5,6,6a,7,7a-octahydro-1 $H$-cyclopenta[a]pentalen (68) 


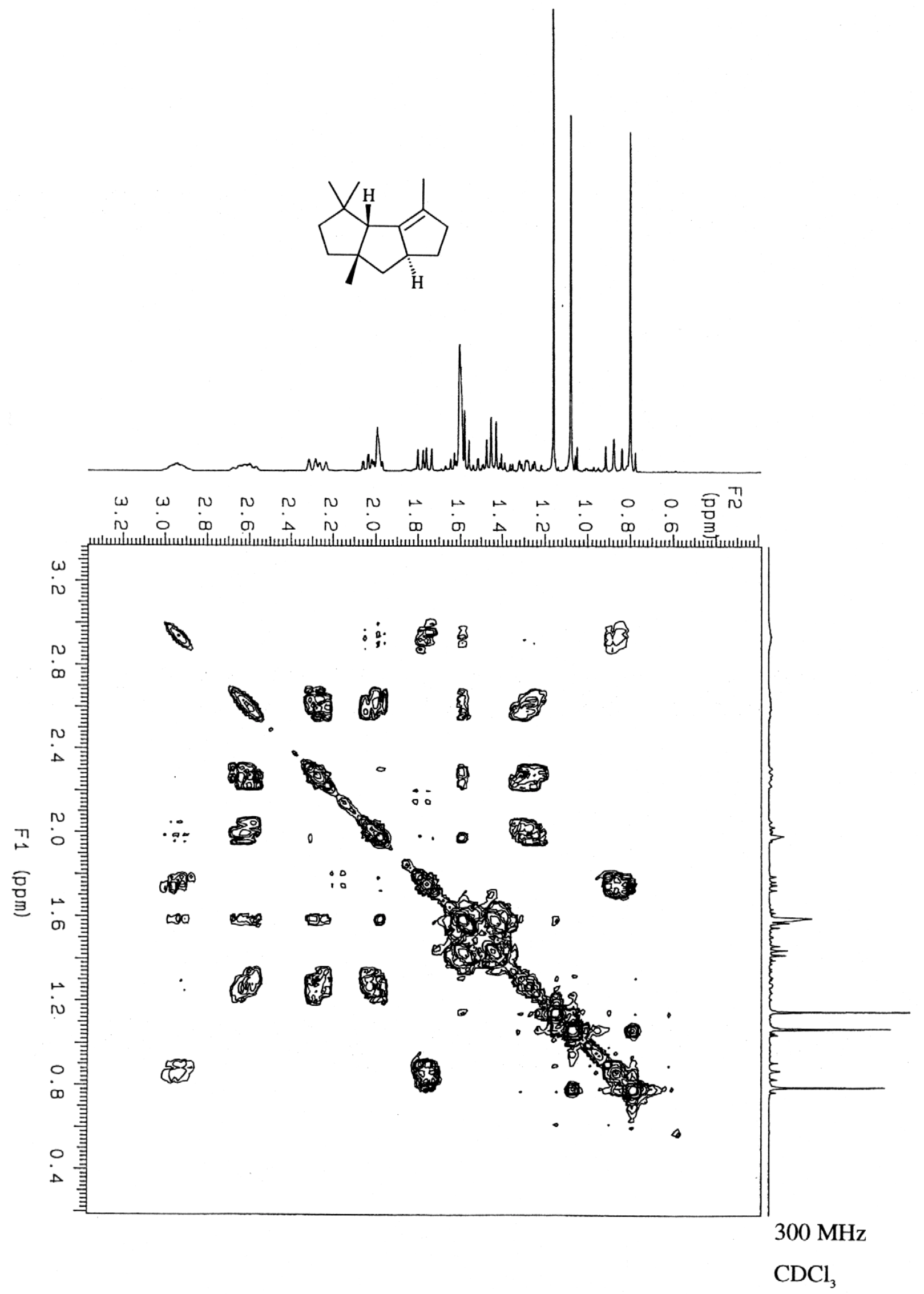

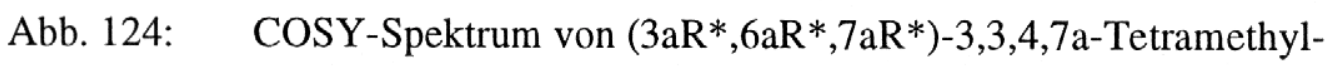
2,3,3a,5,6,6a,7,7a-octahydro- $1 H$-cyclopenta[a]pentalen $(\mathbf{6 8})$ 


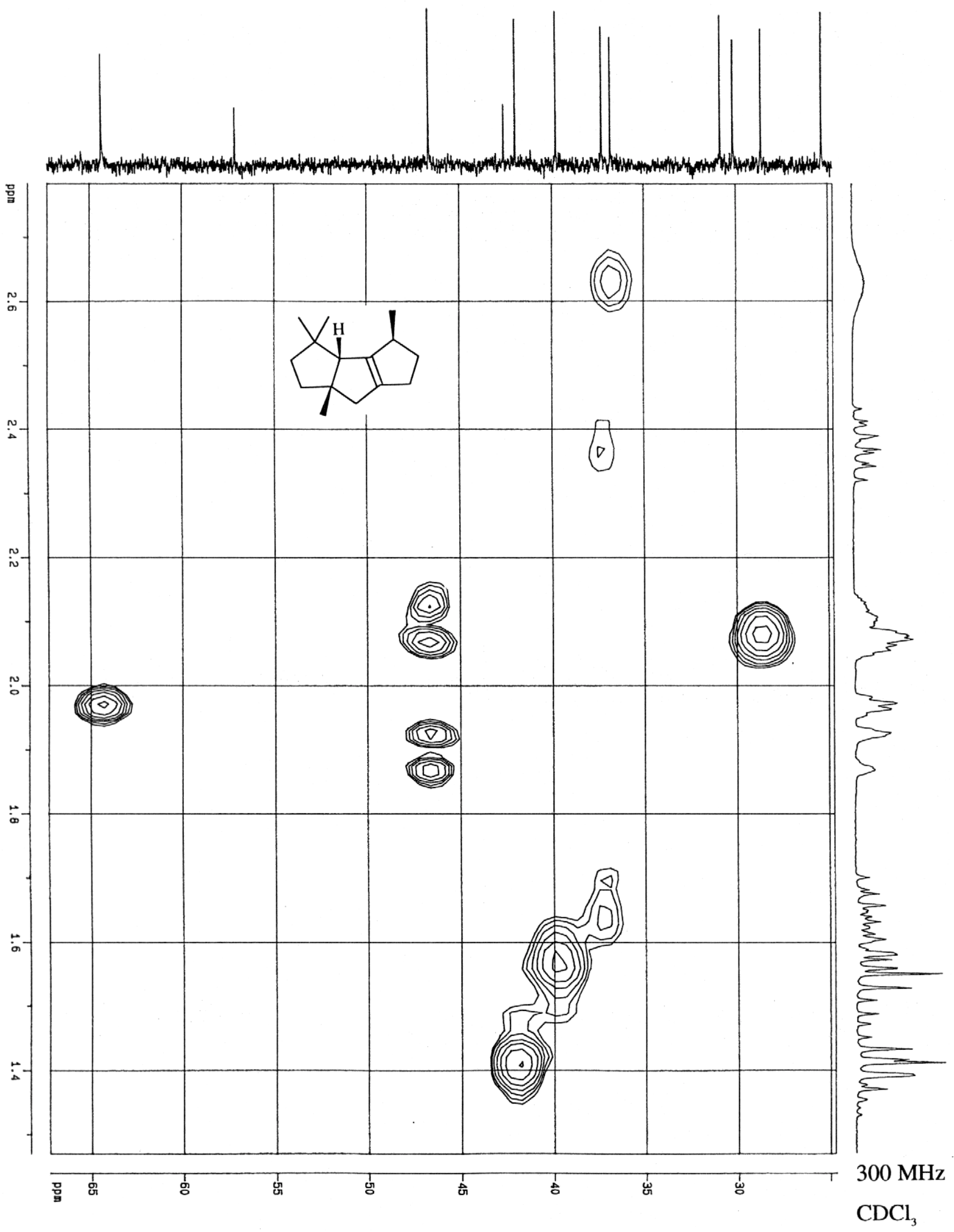

Abb. 125: HMQC-Spektrum von $\left(3 \mathrm{aR}^{*}, 4 \mathrm{R}^{*}, 7 \mathrm{aR} *\right)-3,3,4,7 \mathrm{a}$-Tetramethyl2,3,3a,4,5,6,7,7a-octahydro-1 $\mathrm{H}$-cyclopenta[a]pentalen (69) 


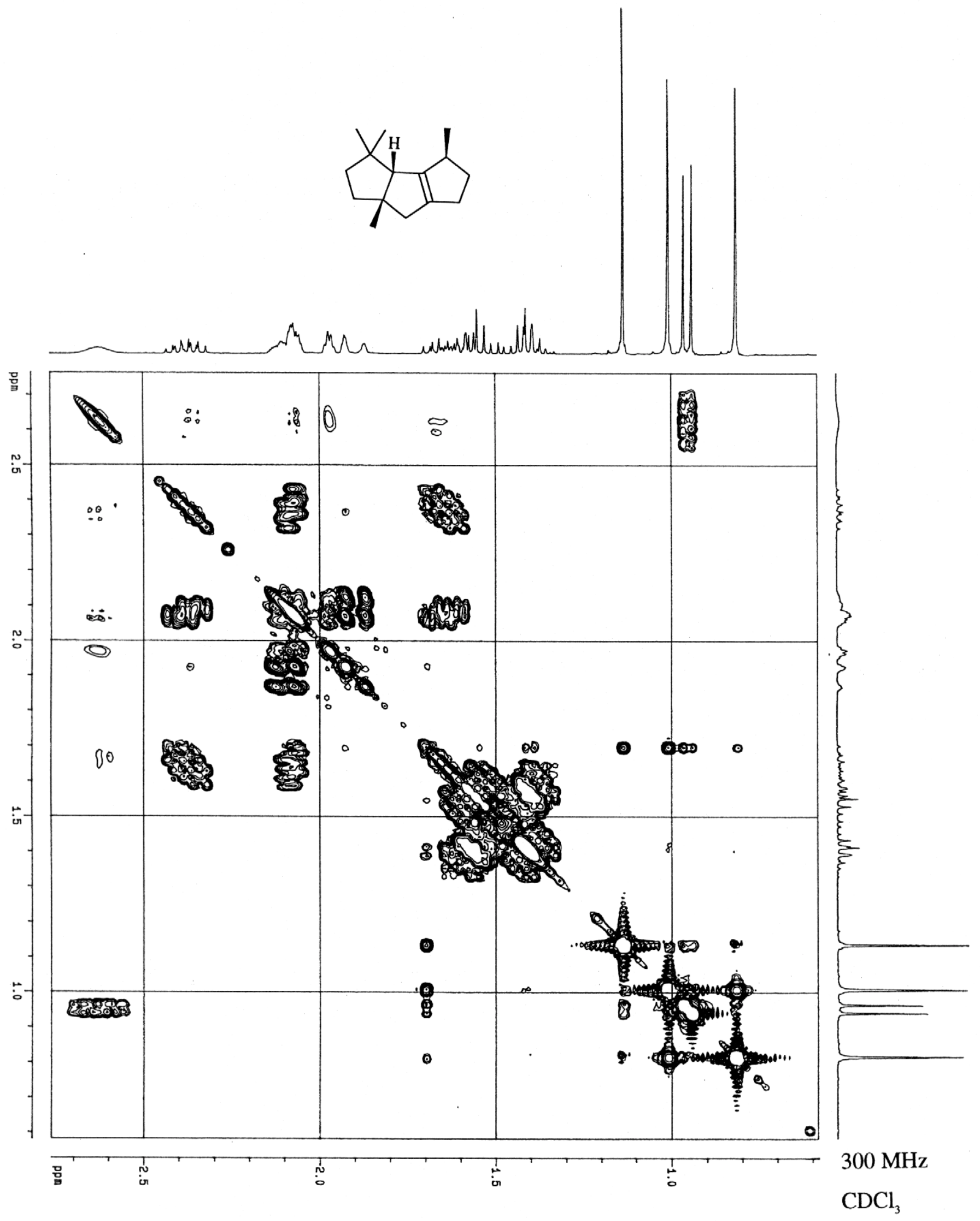

Abb. 126: COSY-Spektrum von $\left(3 \mathrm{aR}^{*}, 4 \mathrm{R}^{*}, 7 \mathrm{aR} *\right)-3,3,4,7 \mathrm{a}-$ Tetramethyl2,3,3a,4,5,6,7,7a-octahydro-1 $H$-cyclopenta[a]pentalen (69) 


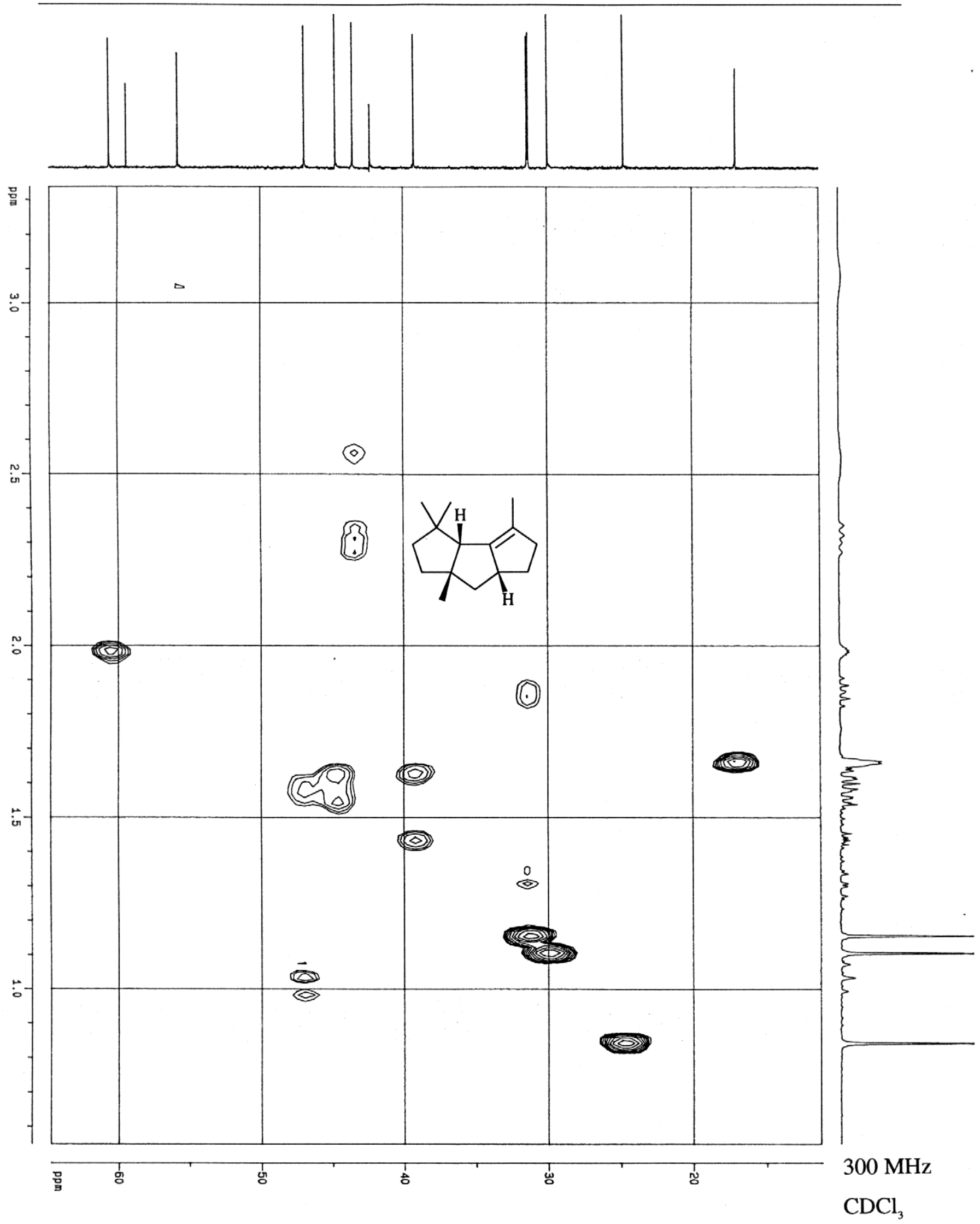

Abb. 127: $\mathrm{HMQC-Spektrum} \mathrm{von} \mathrm{(3aR*,6aS*,7aR*)-3,3,4,7a-Tetramethyl-}$ 2,3,3a,5,6,6a,7,7a-octahydro- $1 H$-cyclopenta[a]pentalen (70) 

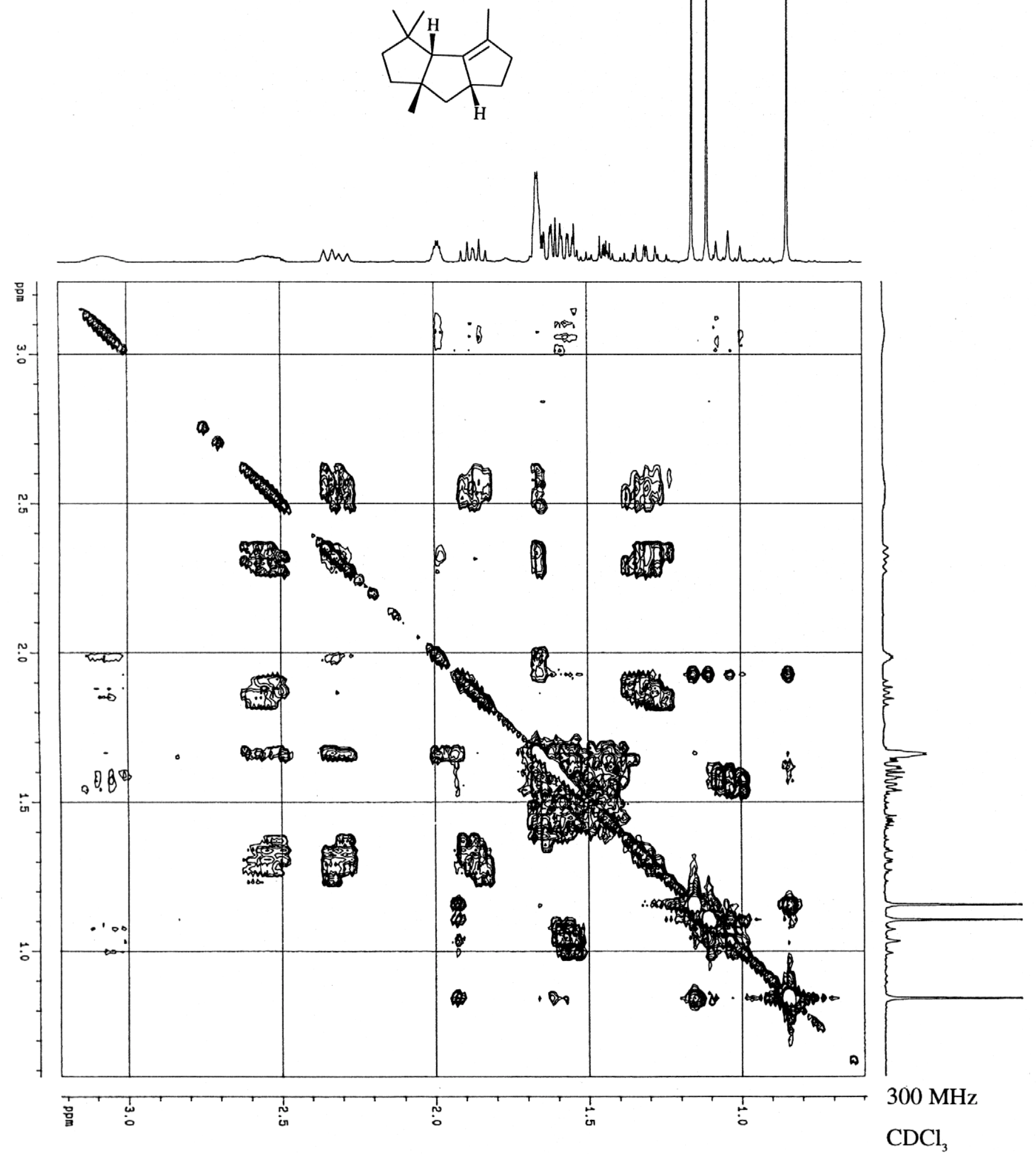

Abb. 128: COSY-Spektrum von $(3 \mathrm{aR} *, 6 \mathrm{aS} *, 7 \mathrm{aR} *)-3,3,4,7 \mathrm{a}-$ Tetramethyl2,3,3a,5,6,6a,7,7a-octahydro- $1 H$-cyclopenta[a]pentalen (70) 


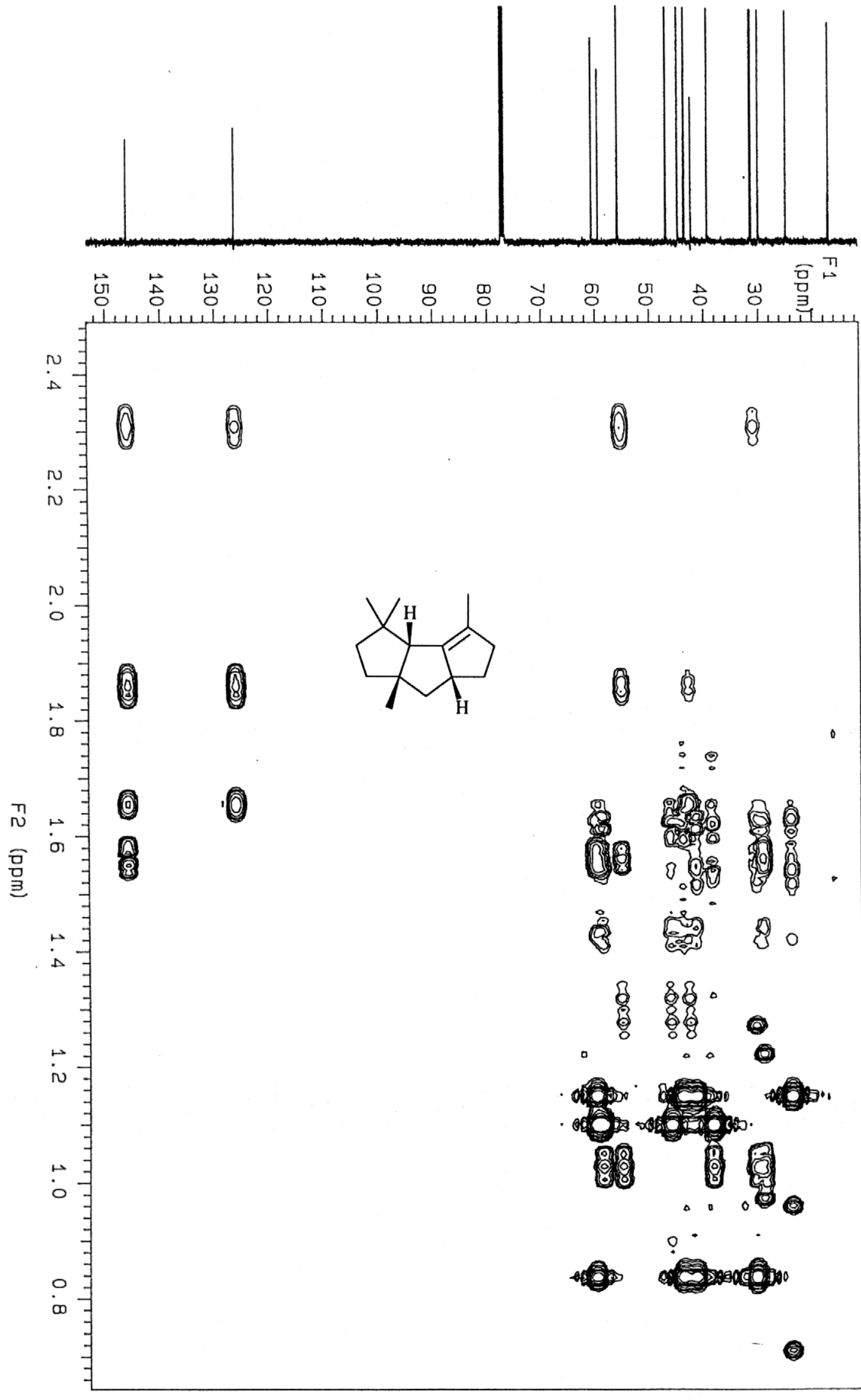

$500 \mathrm{MHz}$

Abb. 129: HMBC-Spektrum von (3aR*,6aS*,7aR*)-3,3,4,7a-Tetramethyl-

$\mathrm{CDCl}_{3}$ 2,3,3a,5,6,6a,7,7a-octahydro- $1 H$-cyclopenta[a]pentalen (70) 


\section{ABBILDUNGSVERZEICHNIS}

Seite

Abb. 1: $\quad$ Kristallstruktur von $\left(1 R^{*}, 5 R^{*}, 6 R^{*}, 2^{\prime} R^{*}, 3^{\prime} R^{*}\right)-1,4,4,3^{\prime}$-Tetra-

methylspiro $\left\{\right.$ bicyclo[3.2.0]heptan-6, $1^{\prime}$-cyclopentan-2'-ol $\}(\mathbf{6 0})$

Abb. 2: $\quad$ Kristallstruktur von $\left(1 R^{*}, 5 R^{*}, 6 R^{*}, 2^{\prime} R^{*}, 3^{\prime} S^{*}\right)-1,4,4,3^{\prime}$-Tetra-

methylspiro $\{$ bicyclo[3.2.0]heptan-6,1'-cyclopentan-2'-ol $\}$ (66)

Abb. 3:

IR-Spekrum von 1,2,2-Trimethyl-cyclobutylchlorid (97)

Abb. 4:

IR-Spektrum von $\left(1 R^{*}, 2 S^{*}\right)$-1,2-Dimethyl-cyclobutancarbonsäure (98a)

Abb. 5: $\quad$ IR-Spektrum von $\left(1 R^{*}, 2 R^{*}\right)$-1,2-Dimethyl-cyclobutancarbonsäure $(\mathbf{9 8 b})$

Abb. 6:

IR-Spektrum von 1,2,2-Trimethyl-cyclobutancarbonsäure (100)

Abb. 7:

IR-Spektrum von 2,2-Spiro-cyclobutyl-3,3-spiro-[(1R*,5R*,6R*)-

1',4',4'-trimethyl-bicyclo[3.2.0]heptan-6,6-yl]-oxiran (55)

Abb. 8:

IR-Spektrum von 2,2-Spiro-cyclobutyl-3,3-spiro-[(1 $\left.R^{*}, 5 R^{*}, 6 S^{*}\right)$ 1,4,4-trimethyl-bicyclo[3.2.0]heptan-6,6-yl]-oxiran (56)

Abb. 9:

IR-Spektrum von $\left(1 R^{*}, 5 R^{*}, 6 R^{*}\right)-1,4,4$-Trimethylspiro\{ bicyclo-

[3.2.0]heptan-6, $1^{\prime}$-cyclopentan-2'-on\} (57)

Abb. 10:

IR-Spektrum von 6-Cyclobutyliden-1,4,4-trimethyl-8-oxa-bicyclo[3.2.1]octan (138)

Abb. 11:

IR-Spektrum von $\left(1 R^{*}, 5 R^{*}, 6 R^{*}, 3 S^{*}\right)-1,4,4,3^{\prime}$-Tetramethylspiro-

\{bicyclo[3.2.0]heptan-6,1'-cyclopentan-2'-on\} (141) und

$\left(1 R^{*}, 5 R^{*}, 6 R^{*}, 3 R^{*}\right)-1,4,4,3^{\prime}-$ Tetramethylspiro $\{$ bicyclo[3.2.0]-

heptan-6,1'-cyclopentan-2'-on\} (142)

Abb. 12: $\quad$ IR-Spektrum von $\left(1 R^{*}, 5 R^{*}, 6 R^{*}\right)-1,4,4,3^{\prime}, 3^{\prime}$-Pentamethylspiro\{bicyclo[3.2.0]heptan-6,1'-cyclopentan-2'-on\} (143)

Abb. 13:

IR-Spektrum von $\left(1 R^{*}, 5 R^{*}, 6 R^{*}, 2^{\prime} R^{*}, 3^{\prime} R^{*}\right)-1,4,4,3^{\prime}$-Tetramethyl96 spiro $\{$ bicyclo[3.2.0]heptan-6,1'-cyclopentan-2'-ol $\}$ (60) 
Abb. 14: IR-Spektrum von $\left(1 R^{*}, 5 R^{*}, 6 R^{*}, 2^{\prime} S^{*}, 3^{\prime} R^{*}\right)-1,4,4,3^{\prime}$-Tetramethyl-

spiro $\{$ bicyclo[3.2.0]heptan-6,1'-cyclopentan-2'-ol $\}(64)$

Abb. 15: IR-Spektrum von $\left(1 R^{*}, 5 R^{*}, 6 R^{*}, 2^{\prime} R^{*}, 3^{\prime} S^{*}\right)-1,4,4,3^{\prime}-$ Tetramethyl-

spiro $\{$ bicyclo[3.2.0]heptan-6,1'-cyclopentan-2'-ol $\}$ (66)

Abb. 16: $\quad$ IR-Spektrum von $\left(1 R^{*}, 2 S^{*}\right)-2,1^{\prime}$-Dimethyl-bicyclobutyl-1-ol (104b)

Abb. 17:

IR-Spektrum von 2,2,1'-Trimethyl-bicyclobutyl-1-ol (105)

Abb. 18:

IR-Spektrum von $\left(1^{\prime} R^{*}, 2^{\prime} S^{*}\right)-2^{\prime}$-Methyl-bicyclobutyl-1-ol (106a)

Abb. 19:

IR-Spektrum von $\left(1^{\prime} R^{*}, 2^{\prime} R^{*}\right)-2^{\prime}$-Methyl-bicyclobutyl-1-ol (106b)

Abb. 20:

IR-Spektrum von $\left(1^{\prime} R^{*}, 2^{\prime} S^{*}\right)-1^{\prime}, 2^{\prime}$-Dimethyl-bicyclobutyl-1-ol (107a)

Abb. 21:

IR-Spektrum von $\left(1^{\prime} R^{*}, 2^{\prime} R^{*}\right)-1^{\prime}, 2^{\prime}$-Dimethyl-bicyclobutyl-1-ol (107b)

Abb. 22:

IR-Spektrum von $\left(1 S^{*}, 5 S^{*}, 6 S^{*}, 1^{\prime} S^{*}, 2^{\prime} S^{*}\right)-1,4,4-$ Trimethyl-6-

(2'-methyl-cyclobutyl)-bicyclo[3.2.0]heptan-6-ol (46) und

$\left(1 S^{*}, 5 S^{*}, 6 S^{*}, 1^{\prime} R^{*}, 2^{\prime} R^{*}\right)$ - 1,4,4-Trimethyl-6-(2'-methyl-cyclobutyl)-

bicyclo[3.2.0]heptan-6-ol (49)

Abb. 23: $\quad$ IR-Spektrum von $\left(1 S^{*}, 5 S^{*}, 6 S^{*}, 1^{\prime} S^{*}, 2^{\prime} R^{*}\right)-1,4,4-$ Trimethyl-6-

(2'-methyl-cyclobutyl)-bicyclo[3.2.0]heptan-6-ol (47)

Abb. 24: $\quad$ IR-Spektrum von $\left(1 S^{*}, 5 S^{*}, 6 S^{*}, 1^{\prime} R^{*}, 2^{\prime} S^{*}\right)-1,4,4-$ Trimethyl-6-

(2'-methyl-cyclobutyl)-bicyclo[3.2.0]heptan-6-ol (48)

Abb. 25: $\quad$ IR-Spektrum von $\left(3 \mathrm{a} R^{*}, 6 \mathrm{a} S^{*}\right)-3 \mathrm{a}, 6,6 \mathrm{a}-$ Trimethyl-1,2,3,3a,4,6ahexahydro-pentalen (118)

Abb. 26:

${ }^{1} \mathrm{H}-\mathrm{NMR}-\mathrm{Spektrum}$ von $\left(1 R^{*}, 2 S^{*}\right)$-2-Methyl-cyclobutancarbonsäure (81a)

Abb. 27: $\quad{ }^{1}$ H-NMR-Spektrum von $\left(1 R^{*}, 2 R^{*}\right)$-2-Methyl-cylobutancarbonsäure (81b)

Abb. 28:

${ }^{1}$ H-NMR-Spektrum von 1,2,2-Trimethyl-cyclobutylchlorid (97)

Abb. 29: säure (98a) 
Abb. 30: $\quad{ }^{1} \mathrm{H}-\mathrm{NMR}-\mathrm{Spektrum}$ von $\left(1 R^{*}, 2 R^{*}\right)$-1,2-Dimethyl-cyclobutancarbon-

104 säure (98b)

Abb. 31: $\quad{ }^{1}$ H-NMR-Spektrum von 1,2,2-Trimethyl-cyclobutancarbonsäure (100) 105

Abb. 32:

${ }^{1}$ H-NMR-Spektrum von 4,5-Dimethyl-hex-4-en-säure (102) 105

Abb. 33:

${ }^{1} \mathrm{H}-\mathrm{NMR}-$ Spektrum von 2,2-Spiro-cyclobutyl-3,3-spiro-

106 [(1R*,5R*,6R*)-1,4,4-trimethyl-bicyclo[3.2.0]heptan-6,6-yl]oxiran (55)

Abb. 34: $\quad{ }^{1}$ H-NMR-Spektrum von 2,2-Spiro-cyclobutyl-3,3-spiro106 [(1 $\left.R^{*}, 5 R^{*}, 6 S^{*}\right)-1,4,4$-trimethyl-bicyclo[3.2.0]heptan-6,6-yl]oxiran (56)

Abb. 35: $\quad{ }^{1} \mathrm{H}-\mathrm{NMR}-\mathrm{Spektrum}$ von $\left(1 R^{*}, 5 R^{*}, 6 R^{*}\right)-1,4,4$-Trimethylspiro107 \{bicyclo[3.2.0]heptan-6,1'-cyclopentan-2'-on\} (57)

Abb. 36: $\quad{ }^{1}$ H-NMR-Spektrum von 6-Cyclobutyliden-1,4,4-trimethyl-8-oxabicyclo[3.2.1]octan (138)

Abb. 37: ${ }^{1} \mathrm{H}-\mathrm{NMR}-\mathrm{Spektrum}$ von $\left(1 R^{*}, 5 R^{*}, 6 R^{*}, 3 S^{*}\right)-1,4,4,3^{\prime}$-Tetramethyl108 spiro\{bicyclo[3.2.0]heptan-6,1'-cyclopentan-2'-on\} (141) und $\left(1 R^{*}, 5 R^{*}, 6 R^{*}, 3 R^{*}\right)-1,4,4,3^{\prime}-$ Tetramethylspiro\{bicyclo[3.2.0]heptan-6,1'-cyclopentan-2'-on\} (142)

Abb. 38: ${ }^{1} \mathrm{H}-\mathrm{NMR}-\mathrm{Spektrum}$ von $\left(1 R^{*}, 5 R^{*}, 6 R^{*}\right)-1,4,4,3^{\prime}, 3^{\prime}$-Pentamethylspiro\{bicyclo[3.2.0]heptan-6,1'-cyclopentan-2'-on\} (143)

Abb. 39: ${ }^{1} \mathrm{H}-\mathrm{NMR}-\mathrm{Spektrum}$ von $\left(1 R^{*}, 5 R^{*}, 6 R^{*}, 2^{\prime} R^{*}, 3^{\prime} R^{*}\right)-1,4,4,3^{\prime}-$ Tetramethylspiro $\{$ bicyclo[3.2.0]heptan-6,1'-cyclopentan-2'-ol $\}(\mathbf{6 0})$

Abb. 40: ${ }^{1} \mathrm{H}-\mathrm{NMR}-\mathrm{Spektrum}$ von $\left(1 R^{*}, 5 R^{*}, 6 R^{*}, 2^{\prime} S^{*}, 3^{\prime} R^{*}\right)-1,4,4,3^{\prime}-$ Tetramethylspiro $\{$ bicyclo[3.2.0]heptan-6,1'-cyclopentan-2'-ol $\}$ (64)

Abb. 41: ${ }^{1} \mathrm{H}-\mathrm{NMR}-$ Spektrum von $\left(1 R^{*}, 5 R^{*}, 6 R^{*}, 2^{\prime} R^{*}, 3^{\prime} S^{*}\right)-1,4,4,3^{\prime}-$ Tetramethylspiro $\{$ bicyclo[3.2.0]heptan-6,1'-cyclopentan-2'-ol $\}$ (66)

Abb. 42: ${ }^{1}$ H-NMR-Spektrum von $\left(1 R^{*}, 2 S^{*}\right)-2,1^{\prime}$-Dimethyl-bicyclobutyl-1-ol (104b)

Abb. 43: ${ }^{1}$ H-NMR-Spektrum von 2,2,1'-Trimethyl-bicyclobutyl-1-ol (105) 
Abb. 44: $\quad{ }^{1}$ H-NMR-Spektrum von $\left(1^{\prime} R^{*}, 2^{\prime} S^{*}\right)-2^{\prime}$-Methyl-bicyclobutyl-1-ol (106a) 111

Abb. 45: $\quad{ }^{1}$ H-NMR-Spektrum von $\left(1^{\prime} R^{*}, 2^{\prime} R^{*}\right)-2^{\prime}$-Methyl-bicyclobutyl-1-ol (106b) 112

Abb. 46: $\quad{ }^{1}$ H-NMR-Spektrum von $\left(1^{\prime} R^{*}, 2^{\prime} S^{*}\right)-1^{\prime}, 2^{\prime}$-Dimethyl-bicyclo112 butyl-1-ol (107a)

Abb. 47: $\quad{ }^{1} \mathrm{H}-\mathrm{NMR}-\mathrm{Spektrum}$ von $\left(1^{\prime} R^{*}, 2^{\prime} R^{*}\right)-1^{\prime}, 2^{\prime}$-Dimethyl-bicyclo113 butyl-1-ol (107b)

Abb. 48: $\quad{ }^{1}$ H-NMR-Spektrum von $\left(1 S^{*}, 5 S^{*}, 6 S^{*}, 1^{\prime} S^{*}, 2^{\prime} S^{*}\right)-1,4,4-$ Trimethyl-

6-(2'-methyl-cyclobutyl)-bicyclo[3.2.0]heptan-6-ol (46) und $\left(1 S^{*}, 5 S^{*}, 6 S^{*}, 1^{\prime} R^{*}, 2^{\prime} R^{*}\right)-1,4,4-$ Trimethyl-6-(2'-methyl-cyclobutyl)bicyclo[3.2.0]heptan-6-ol (49)

Abb. 49: $\quad{ }^{1} \mathrm{H}-\mathrm{NMR}-\mathrm{Spektrum}$ von $\left(1 S^{*}, 5 S^{*}, 6 S^{*}, 1^{\prime} S^{*}, 2^{\prime} R^{*}\right)-1,4,4-$ Trimethyl6-(2'-methyl-cyclobutyl)-bicyclo[3.2.0]heptan-6-ol (47)

Abb. 50: ${ }^{1} \mathrm{H}-\mathrm{NMR}-$ Spektrum von $\left(1 S^{*}, 5 S^{*}, 6 S^{*}, 1^{\prime} R^{*}, 2^{\prime} S^{*}\right)-1,4,4-$ Trimethyl6-(2'-methyl-cyclobutyl)-bicyclo[3.2.0]heptan-6-ol (48)

Abb. 51: $\quad{ }^{1}$ H-NMR-Spektrum von (3a $\left.R^{*}, 6 a S^{*}\right)-3 \mathrm{a}, 6-$ Dimethyl-1,2,3,3a,4,6ahexahydro-pentalen (109)

Abb. 52: ${ }^{1} \mathrm{H}-\mathrm{NMR}-$ Spektrum von $\left(3 \mathrm{a} R^{*}, 6 \mathrm{a} S^{*}\right)-3 \mathrm{a}, 6,6 \mathrm{a}-$ Trimethyl-1,2,3,3a,4,6ahexahydro-pentalen (118)

Abb. 53: ${ }^{1} \mathrm{H}-\mathrm{NMR}$-Spektrum von 1,1,3a-Trimethyl-1,2,3,3a,4,5-hexahydropentalen (120)

Abb. 54: ${ }^{1}$ H-NMR-Spektrum von 2,6,6-Trimethyl-4-(2-methylcyclobutyl)cyclohepta-1,4-dien (122)

Abb. 55: $\quad{ }^{1}$ H-NMR-Spektrum von 3,6,6-Trimethyl-1-(2-methyl-cyclobutyl)cyclohepta-1,3-dien (123)

Abb. 56: ${ }^{1}$ H-NMR-Spektrum von 1,5,5-Trimethyl-3-(2-methyl-cyclobutyliden)cyclohept-1-en (124)

Abb. 57:

${ }^{1}$ H-NMR-Spektrum von 1,5,5-Trimethyl-3-(2-methyl-cyclobutyliden)- 118 cyclohept-1-en (125) 
Abb. 58: $\quad{ }^{1}$ H-NMR-Spektrum von 1,4,4-Trimethyl-6-(2-methyl-cyclobutyl)bicyclo[3.2.0]hept-6-en (130 und 131)

Abb. 59: $\quad{ }^{1}$ H-NMR-Spektrum von 1,4,4-Trimethyl-6-(2-methyl-cyclobutyliden)bicyclo[3.2.0]heptan $(\mathbf{1 3 2 / 1 3 3 / 1 3 4 / 1 3 5 )}$

Abb. 60: $\quad{ }^{1} \mathrm{H}-\mathrm{NMR}-\mathrm{Spektrum}$ von (3aR*,4R*,7aR*)-3,3,4,7a-Tetramethyl2,3,3a,4,5,6,7,7a-octahydro-1 $H$-cyclopenta[a]pentalen (67) und (3aR*,6aR*,7aR*)-3,3,4,7a-Tetramethyl-2,3,3a,5,6,6a,7,7a-octahydro- $1 H$-cyclopenta[a]pentalen $(\mathbf{6 8})$

Abb. 61: $\quad{ }^{1} \mathrm{H}-\mathrm{NMR}-\mathrm{Spektrum}$ von $\left(3 \mathrm{aR}^{*}, 6 \mathrm{aR}^{*}, 7 \mathrm{aR} *\right)-3,3,4,7 \mathrm{a}-$ Tetramethyl120 2,3,3a,5,6,6a,7,7a-octahydro-1H-cyclopenta[a]pentalen (68)

Abb. 62: $\quad{ }^{1} \mathrm{H}-\mathrm{NMR}-\mathrm{Spektrum}$ von $\left(3 \mathrm{aR}^{*}, 4 \mathrm{R}^{*}, 7 \mathrm{aR} *\right)-3,3,4,7 \mathrm{a}-$ Tetramethyl2,3,3a,4,5,6,7,7a-octahydro-1H-cyclopenta[a]pentalen (69)

Abb. 63: $\quad{ }^{1} \mathrm{H}-\mathrm{NMR}-\mathrm{Spektrum}$ von $(3 \mathrm{aR} *, 6 \mathrm{aS} *, 7 \mathrm{aR} *)-3,3,4,7 \mathrm{a}-$ Tetramethyl2,3,3a,5,6,6a,7,7a-octahydro- $1 H$-cyclopenta[a]pentalen (70)

Abb. 64: $\quad{ }^{13}$ C-NMR-Spektrum von $\left(1 R^{*}, 2 S^{*}\right)$-2-Methyl-cyclobutancarbonsäure (81a)

Abb. 65: $\quad{ }^{13} \mathrm{C}-\mathrm{NMR}-\mathrm{Spektrum}$ von $\left(1 R^{*}, 2 R^{*}\right)-2-$ Methyl-cylobutancarbonsäure (81b)

Abb. 66: ${ }^{13}$ C-NMR-Spektrum von 1,1-Dimethyl-2-methylencyclobutan (91)

Abb. 68:

${ }^{13}$ C-NMR-Spektrum von 1,2,2-Trimethyl-cyclobutylchlorid (97)

Abb. 69:

${ }^{13} \mathrm{C}-\mathrm{NMR}-$ Spektrum von $\left(1 R^{*}, 2 S^{*}\right)$-1,2-Dimethyl-cyclobutancarbonsäure (98a)

Abb. 70: ${ }^{13} \mathrm{C}-\mathrm{NMR}-\mathrm{Spektrum}$ von $\left(1 R^{*}, 2 R^{*}\right)$-1,2-Dimethyl-cyclobutancarbonsäure (98b)

Abb. 71: ${ }^{13} \mathrm{C}-\mathrm{NMR}-$ Spektrum von 1,2,2-Trimethyl-cyclobutancarbonsäure (100)

Abb. 72: ${ }^{13}$ C-NMR-Spektrum von 4,5-Dimethylhex-4-en-säure (102) 125

Abb. 73: $\left.6 R^{*}\right)$-1,4,4-trimethyl-bicyclo[3.2.0]heptan-6,6-yl]-oxiran (55) 
Abb. 74: $\quad{ }^{13}$ C-NMR-Spektrum von 2,2-Spiro-cyclobutyl-3,3-spiro-[( $1 R^{*}, 5 R^{*}$, 126 6S*)-1,4,4-trimethyl-bicyclo[3.2.0]heptan-6,6-yl]-oxiran (56)

Abb. 75: $\quad{ }^{13} \mathrm{C}-\mathrm{NMR}-\mathrm{Spektrum}$ von $\left(1 R^{*}, 5 R^{*}, 6 R^{*}\right)-1,4,4-$ Trimethylspiro\{bicyclo[3.2.0]heptan-6,1'-cyclopentan-2'-on $\}(57)$

Abb. 76: $\quad{ }^{13}$ C-NMR-Spektrum von 6-Cyclobutyliden-1,4,4-trimethyl-8-oxabicyclo[3.2.1] octan (138)

Abb. 77:

${ }^{13} \mathrm{C}-\mathrm{NMR}-$ Spektrum von $\left(1 R^{*}, 5 R^{*}, 6 R^{*}, 3 S^{*}\right)-1,4,4,3^{\prime}-$ Tetramethylspiro- 128 $\left\{\right.$ bicyclo[3.2.0]heptan-6, $1^{\prime}$-cyclopentan-2'-on $\}$ (141) und $\left(1 R^{*}, 5 R^{*}\right.$, $\left.6 R^{*}, 3 R^{*}\right)-1,4,4,3^{\prime}$-Tetramethylspiro\{bicyclo[3.2.0]heptan-6,1'-cyclopentan-2'-on\} (142)

Abb. 78:

${ }^{13}$ C-NMR-Spektrum von $\left(1 R^{*}, 5 R^{*}, 6 R^{*}\right)-1,4,4,3^{\prime}, 3^{\prime}$-Pentamethylspiro128 \{bicyclo[3.2.0]heptan-6,1'-cyclopentan-2'-on $\}$ (143)

Abb. 79:

${ }^{13} \mathrm{C}-\mathrm{NMR}-$ Spektrum von $\left(1 R^{*}, 5 R^{*}, 6 R^{*}, 2^{\prime} R^{*}, 3^{\prime} R^{*}\right)-1,4,4,3^{\prime}$-Tetramethylspiro $\{$ bicyclo[3.2.0]heptan-6,1'-cyclopentan-2'-ol $\}(\mathbf{6 0})$

Abb. 80:

${ }^{13} \mathrm{C}-\mathrm{NMR}-\mathrm{Spektrum}$ von $\left(1 R^{*}, 5 R^{*}, 6 R^{*}, 2^{\prime} S^{*}, 3^{\prime} R^{*}\right)-1,4,4,3^{\prime}$-Tetramethylspiro $\{$ bicyclo[3.2.0]heptan-6,1'-cyclopentan-2'-ol $\}$ (64)

Abb. 81:

${ }^{13} \mathrm{C}-\mathrm{NMR}-\mathrm{Spektrum}$ von $\left(1 R^{*}, 5 R^{*}, 6 R^{*}, 2^{\prime} R^{*}, 3^{\prime} S^{*}\right)-1,4,4,3^{\prime}$-Tetra130 methylspiro $\{$ bicyclo[3.2.0]heptan-6,1'-cyclopentan-2'-ol $\}$ (66)

Abb. 82:

${ }^{13} \mathrm{C}-\mathrm{NMR}-$ Spektrum von $\left(1 R^{*}, 2 S^{*}\right)-2,1^{\prime}$-Dimethyl-bicyclobutyl-1-ol (104b)

Abb. 83:

${ }^{13}$ C-NMR-Spektrum von 2,2,1'-Trimethyl-bicyclobutyl-1-ol (105)

Abb. 84:

${ }^{13} \mathrm{C}-\mathrm{NMR}-$ Spektrum von $\left(1^{\prime} R^{*}, 2^{\prime} S^{*}\right)-2^{\prime}$-Methyl-bicyclobutyl-1-ol (106a)

Abb. 85: ${ }^{13} \mathrm{C}-\mathrm{NMR}-$ Spektrum von $\left(1^{\prime} R^{*}, 2^{\prime} R^{*}\right)-2^{\prime}$-Methyl-bicyclobutyl-1-ol (106b)

Abb. 86: ${ }^{13} \mathrm{C}-\mathrm{NMR}-$ Spektrum von $\left(1^{\prime} R^{*}, 2^{\prime} S^{*}\right)-1^{\prime}, 2^{\prime}$-Dimethyl-bicyclobutyl-1-ol (107a)

Abb. 87: (107b) 
Abb. 88: $\quad{ }^{13}$ C-NMR-Spektrum von $\left(1 S^{*}, 5 S^{*}, 6 S^{*}, 1^{\prime} S^{*}, 2^{\prime} S^{*}\right)-1,4,4-$ Trimethyl-6-

(2'-methyl-cyclobutyl)-bicyclo[3.2.0]heptan-6-ol (46) und $\left(1 S^{*}, 5 S^{*}, 6 S^{*}, 1^{\prime} R^{*}, 2^{\prime} R^{*}\right)-1,4,4-$ Trimethyl-6-(2'-methyl-cyclobutyl)bicyclo[3.2.0]heptan-6-ol (49)

Abb. 89: $\quad{ }^{13}$ C-NMR-Spektrum von $\left(1 S^{*}, 5 S^{*}, 6 S^{*}, 1^{\prime} S^{*}, 2^{\prime} R^{*}\right)-1,4,4-$ Trimethyl-6(2'-methyl-cyclobutyl)-bicyclo[3.2.0]heptan-6-ol (47)

Abb. 90:

${ }^{13} \mathrm{C}-\mathrm{NMR}-\mathrm{Spektrum}$ von $\left(1 S^{*}, 5 S^{*}, 6 S^{*}, 1^{\prime} R^{*}, 2^{\prime} S^{*}\right)-1,4,4-$ Trimethyl-6-

(2'-methyl-cyclobutyl)-bicyclo[3.2.0]heptan-6-ol (48)

Abb. 91:

${ }^{13} \mathrm{C}-\mathrm{NMR}-$ Spektrum von $\left(3 \mathrm{a} R^{*}, 6 \mathrm{a} S^{*}\right)-3 \mathrm{a}, 6,6 \mathrm{a}-$ Trimethyl-1,2,3,3a,4,6a- 135 hexahydro-pentalen (118)

Abb. 92: $\quad{ }^{13}$ C-NMR-Spektrum von 1,1,3a-Trimethyl-1,2,3,3a,4,5-hexahydro135 pentalen (120)

Abb. 93

${ }^{13}$ C-NMR-Spektrum von 2,6,6-Trimethyl-4-(2-methylcyclobutyl)cyclohepta-1,4-dien (122)

Abb. 94:

${ }^{13}$ C-NMR-Spektrum von 3,6,6-Trimethyl-1-(2-methyl-cyclobutyl)136 cyclohepta-1,3-dien (123)

Abb. 95:

${ }^{13}$ C-NMR-Spektrum von 1,5,5-Trimethyl-3-(2-methyl-cyclobutyliden)- 137 cyclohept-1-en (124)

Abb. 96:

${ }^{13}$ C-NMR-Spektrum von 1,5,5-Trimethyl-3-(2-methyl-cyclobutyliden)- 137 cyclohept-1-en (125)

Abb. 97:

${ }^{13}$ C-NMR-Spektrum von 1,4,4-Trimethyl-6-(2-methyl-cyclobutyl)bicyclo[3.2.0]hept-6-en (130 und 131)

Abb. 98:

${ }^{13}$ C-NMR-Spektrum von 1,4,4-Trimethyl-6-(2-methyl-cyclobutyliden)- 138 bicyclo[3.2.0]heptan (132/133/134/135)

Abb. 99:

${ }^{13} \mathrm{C}-\mathrm{NMR}-$ Spektrum von $\left(3 \mathrm{aR}^{*}, 4 \mathrm{R}^{*}, 7 \mathrm{aR} *\right)-3,3,4,7 \mathrm{a}-$ Tetramethyl2,3,3a,4,5,6,7,7a-octahydro-1H-cyclopenta[a]pentalen (67) und (3aR*,6aR*,7aR*)-3,3,4,7a-Tetramethyl-2,3,3a,5,6,6a,7,7a-octahydro- $1 H$-cyclopenta[a]pentalen $(\mathbf{6 8})$

Abb. 100: ${ }^{13} \mathrm{C}-\mathrm{NMR}-$ Spektrum von $\left(3 \mathrm{aR}^{*}, 6 \mathrm{aR} *, 7 \mathrm{aR} *\right)-3,3,4,7 \mathrm{a}-$ Tetramethyl2,3,3a,5,6,6a,7,7a-octahydro-1H-cyclopenta[a]pentalen (68) 
Abb. 101: $\quad{ }^{13} \mathrm{C}-\mathrm{NMR}-$ Spektrum von $\left(3 \mathrm{aR}^{*}, 4 \mathrm{R}^{*}, 7 \mathrm{aR} *\right)-3,3,4,7 \mathrm{a}-$ Tetramethyl- $\quad 140$ 2,3,3a,4,5,6,7,7a-octahydro-1H-cyclopenta[a]pentalen (69)

Abb. 102: $\quad{ }^{13} \mathrm{C}-\mathrm{NMR}-\mathrm{Spektrum}$ von $\left(3 \mathrm{aR}^{*}, 6 \mathrm{aS}^{*}, 7 \mathrm{aR} *\right)-3,3,4,7 \mathrm{a}-$ Tetramethyl140 2,3,3a,5,6,6a,7,7a-octahydro-1H-cyclopenta[a]pentalen (70)

Abb. 103: $\quad$ HMQC-Spektrum von 1,1,3a-Trimethyl-1,2,3,3a,4,5-hexahydropentalen (120)

Abb. 104: COSY-Spektrum von 1,1,3a-Trimethyl-1,2,3,3a,4,5-hexahydropentalen (120)

Abb. 105: $\quad$ HMBC-Spektrum von 1,1,3a-Trimethyl-1,2,3,3a,4,5-hexahydropentalen (120)

Abb. 106: HMQC-Spektrum von 2,6,6-Trimethyl-4-(2-methylcyclobutyl)cyclohepta-1,4-dien (122)

Abb. 107: $\quad$ COSY-Spektrum von 2,6,6-Trimethyl-4-(2-methylcyclobutyl)cyclohepta-1,4-dien (122)

Abb. 108: HMQC-Spektrum von 3,6,6-Trimethyl-1-(2-methyl-cyclobutyl)146 cyclohepta-1,3-dien (123)

Abb. 109: $\quad$ COSY-Spektrum von 3,6,6-Trimethyl-1-(2-methyl-cyclobutyl)cyclohepta-1,3-dien (123)

Abb. 110: $\quad$ HMBC-Spektrum von 3,6,6-Trimethyl-1-(2-methyl-cyclobutyl)cyclohepta-1,3-dien (123)

Abb. 111: HMQC-Spektrum von 1,5,5-Trimethyl-3-(2-methyl-cyclobutyliden)cyclohept-1-en (124)

Abb. 112: COSY-Spektrum von 1,5,5-Trimethyl-3-(2-methyl-cyclobutyliden)cyclohept-1-en (124)

Abb. 113: HMQC-Spektrum von 1,5,5-Trimethyl-3-(2-methyl-cyclobutyliden)cyclohept-1-en (125)

Abb. 114: COSY-Spektrum von 1,5,5-Trimethyl-3-(2-methyl-cyclobutyliden)cyclohept-1-en (125)

Abb. 115: HMQC-Spektrum von 1,4,4-Trimethyl-6-(2-methyl-cyclobutyliden)bicyclo[3.2.0]heptan (132/133/134/135) 
Abb. 116: $\quad$ COSY-Spektrum von 1,4,4-Trimethyl-6-(2-methyl-cyclobutyliden)bicyclo[3.2.0]heptan $(\mathbf{1 3 2} / \mathbf{1 3 3} / \mathbf{1 3 4} / \mathbf{1 3 5})$

Abb. 117: $\quad$ HMQC-Spektrum von 6-Cyclobutyliden-1,4,4-trimethyl-8-oxabicyclo[3.2.1] octan (138)

Abb. 118: $\quad$ COSY-Spektrum von 6-Cyclobutyliden-1,4,4-trimethyl-8-oxabicyclo[3.2.1]octan (138)

Abb. 119: HMBC-Spektrum von 6-Cyclobutyliden-1,4,4-trimethyl-8-oxabicyclo[3.2.1] octan (138)

Abb. 120: $\quad$ NOESY-Spektrum von $\left(1 R^{*}, 5 R^{*}, 6 R^{*}, 2^{\prime} R^{*}, 3^{\prime} R^{*}\right)-1,4,4,3^{\prime}$-Tetramethylspiro $\{$ bicyclo[3.2.0]heptan-6,1'-cyclopentan-2'-ol $\}(\mathbf{6 0})$

Abb. 121: $\quad$ NOESY-Spektrum von $\left(1 R^{*}, 5 R^{*}, 6 R^{*}, 2^{\prime} S^{*}, 3^{\prime} R^{*}\right)-1,4,4,3^{\prime}$-Tetramethylspiro $\{$ bicyclo[3.2.0]heptan-6,1'-cyclopentan-2'-ol $\}$ (64)

Abb. 122: $\quad$ NOESY-Spektrum von $\left(1 R^{*}, 5 R^{*}, 6 R^{*}, 2^{\prime} R^{*}, 3^{\prime} S^{*}\right)-1,4,4,3^{\prime}-$ Tetramethylspiro $\{$ bicyclo[3.2.0]heptan-6,1'-cyclopentan-2'-ol $\}$ (66)

Abb. 123: HMQC-Spektrum von (3aR*,6aR*,7aR*)-3,3,4,7a-Tetramethyl2,3,3a,5,6,6a,7,7a-octahydro-1 $H$-cyclopenta[a]pentalen (68)

Abb. 124: $\quad$ COSY-Spektrum von $\left(3 \mathrm{aR}^{*}, 6 \mathrm{aR}^{*}, 7 \mathrm{aR} *\right)-3,3,4,7 \mathrm{a}-\mathrm{Tetramethyl-}$ 2,3,3a,5,6,6a,7,7a-octahydro-1 $H$-cyclopenta[a]pentalen $(\mathbf{6 8})$

Abb. 125: HMQC-Spektrum von $\left(3 \mathrm{aR}^{*}, 4 \mathrm{R}^{*}, 7 \mathrm{aR} *\right)-3,3,4,7 \mathrm{a}-$ Tetramethyl2,3,3a,4,5,6,7,7a-octahydro-1H-cyclopenta[a]pentalen (69)

Abb. 126: $\quad$ COSY-Spektrum von (3aR*,4R*,7aR*)-3,3,4,7a-Tetramethyl2,3,3a,4,5,6,7,7a-octahydro-1H-cyclopenta[a]pentalen (69)

Abb. 127: HMQC-Spektrum von (3aR*,6aS*,7aR*)-3,3,4,7a-Tetramethyl2,3,3a,5,6,6a,7,7a-octahydro- $1 H$-cyclopenta[a]pentalen (70)

Abb. 128: $\quad$ COSY-Spektrum von $\left(3 \mathrm{aR}^{*}, 6 \mathrm{aS} *, 7 \mathrm{aR} *\right)-3,3,4,7 \mathrm{a}$-Tetramethyl2,3,3a,5,6,6a,7,7a-octahydro-1H-cyclopenta[a]pentalen (70)

Abb. 129: $\quad$ HMBC-Spektrum von (3aR*,6aS*,7aR*)-3,3,4,7a-Tetramethyl- 


\section{LITERATURVERZEICHNIS}

1 Zur Umlagerung von Bicyclobutyliden und zwei dibenzoanellierten Derivaten siehe: Finkelshtein, E. S.; Strelchik, B. S.; Vdovin, V. M.; Nametkin, N. S. Dokl. Akad. Nauk SSSR 1975, 220, 131 - 134; Dokl. Chem. 1975, 220, 36 - 39. Barton, J. W.; Shepherd, M. K. J. Chem. Soc. Perkin Trans. I 1987, 1561 - 1565.

2 Wong, H. N. C. In Methods of Organic Chemistry (Houben-Weyl); de Meijere, A., Ed., Georg Thieme Verlag: Stuttgart 1997; Vol. E 17e, S.495 ff.

3 Fitjer, L.; Gerke, R.; Anger, T. Synthesis 1994, 893 - 894.

4 El-Hachach, N.; Fischbach, M.; Gerke, R.; Fitjer, L. Tetrahedron 1999, 55, 6119 6128.

$5 \quad$ Mandelt, K.; Fitjer, L. Synthesis 1998, 1523 - 1526.

6 Anger, T.; Graalmann, O.; Schröder, H.; Gerke, R.; Kaiser, U.; Fitjer, L.; Noltemeyer, M. Tetrahedron 1998, 54, 10713 - 10720.

7 Takeda, R.; Naoki, H.; Iwashita, T.; Mizukawa, K.; Hirose, Y.; Isida, T.; Inoue, M. Bull. Chem. Soc. Jpn. 1983, 56, 1125 - 1132.

8 Die Synthese eines Stereoisomers ist beschrieben: Solaja, B.; Huguet, J.; Karpf, M.; Dreiding, A. S. Tetrahedron 1987, 53, 4875 - 4886.

9 Weiser, J. Dissertation, Universität Göttingen 1994.

10 Weiser, J.; Holthausen, M. C.; Fitjer, L. J. Comput. Chem. 1997, 18, 1265 - 1281.

11 Meyers, A. I.; Bienz, S. J. Org. Chem. 1990, 55, 791 - 798 und dort angegebene Literatur.

12 Schwarzer, S. Dissertation, Universität Göttingen 1994. 
13 (a) Whitesell, J. K.; Matthews, R. S.; Helbling, H. J. Org. Chem. 1978, 43, 784 - 786.

(b) Paquette, L. A.; Leone-Bay, A. J. Am. Chem. Soc. 1983, 105, 7352 - 7358.

14 (a) Fitjer, L.; Kanschik, A.; Majewski, M. Tetrahedron 1994, 50, 10867 - 10878.

(b) Fitjer, L., Majewski, M.; Monzó-Oltra, H. Tetrahedron 1995, 51, 8835 - 8852.

15 Sprague, J. T.; Tai, J. C.; Yuh, Y.; Allinger, N. L. J. Comput. Chem. 1987, 8, 581 603.

16 Zusammenfassende Übersichten siehe:

(a) Kharasch, Reinmuth, O. Grignard Reactions of NonmetallicSubstances, PrenticeHall, New York, 1954.

(b) Wakefield B. J. In Comprehensive Organometallic Chemistry; Sir Wilkinson, G., Ed. Pergamon Press: Oxford 1982; Vol 7, Kap. 44.

(c) Lai Y.-H. Synthesis 1981, 585 - 604.

17 Falkenberg-Anderson, C.; Ranganayakulu, K.; Schmitz, L. R.; Sorensen, T. S. J. Am. Chem. Soc. 1984, 106, 178 - 182.

18 Hittich, R. Org. Magn. Reson. 1982, 18, 214 - 218.

19 Cason. J.; Allen, C. F. J. Org. Chem. 1949, 14, 1036 - 1038.

20 Blomquist, A. T.; Wolinsky, J. Org. Chem. 1956, 21, 1371 - 1373.

21 Conia, J.-M.; Gore, J. Bull. Soc. Chim. France 1963, 735 - 743.

22 Hill, E. L.; Chen, A. T.; Doughty, A. J. Am. Chem. Soc. 1975, 98, 167 - 170.

23 Török, B.; Molnár, Á. J. Chem. Soc. Perkin Trans. I 1993, 801 - 804.

24 Becker, K. B.; Geisel, M.; Grob, C. A.; Kuhnen, F. Synthesis 1973, 493 - 494.

25 Crowley, K. J. Tetrahedron 1965, 21, 1001 - 1014.

26 Shand jr. W.; Schomaker V.; Fischer, J. R. J. Am. Chem. Soc. 1944, 66, 636 - 637. 
27 Jacobs, T.L.; Johnson, R. N. J. Am. Chem. Soc. 1960, 82, 6397 - 6404. [ ${ }^{1}$ H-NMR $\left(\mathrm{CDCl}_{3}\right): 1.03(\mathrm{t}, \mathrm{J}=8 \mathrm{~Hz}, 3 \mathrm{H}), 1.54(\mathrm{~s}, 3 \mathrm{H}), 1.82\left(\mathrm{~m}_{\mathrm{c}}, 2 \mathrm{H}\right), 2.23\left(\mathrm{~m}_{\mathrm{c}}, 2 \mathrm{H}\right), 3.70\left(\mathrm{~m}_{\mathrm{c}}\right.$, 2H)]

28 Erickson, K. L. J. Org. Chem. 1971, 36, 1031 - 1036.

29 Trost, B. M.; Keeley, D. E.; Arndt, H. C.; Rigby, J. H.; Bogdanowicz, M. J. J. Am. Chem. Soc. 1977, 99, 3080 - 3087.

30 Trost, B. M.; Vladuchick, W.C. Synthesis 1978, 821.

31 Bestian, H.; Guenter, D. Angew. Chem. 1963, 75, 841 - 845.

32 Conia, J.-M.; Salaün, J. Bull. Soc. Chim. France 1964, 1957 - 1963.

33 Agosta, W. C.; Herron, D. K. J. Org. Chem. 1969, 34, 2782 - 2785.

34 Mieloszynski, J. L.; Andrieu, C. G.; Schneider, M.; Paquer, D. Recl. Trav. Chim. Pays-Bas 1985, 104, 9 - 15.

35 Schläger, M. Diplomarbeit, Universität Göttingen 1996.

36 Griesbaum, K.; Mach, H. Chem. Ber. 1982, 115, 3818 - 3829.

37 Pechhold, E.; Adams, D. G.; Fraenkel, G. J. Org. Chem. 1971, 36, 1368 - 1374.

38 Die verunreinigte Cyclobutancarbonsäure 100 wurde vorher schon durch Methylierung von 2,2-Dimethylcyclobutancarbonsäure erhalten. Spektroskopische Daten wurden nicht angegeben: Beckwith, A. L. J.; Moad, G. J. Chem. Perkin Trans. II 1980 , $1083-1092$.

39 Korte, F.; Christoph, H. Chem. Ber. 1961, 94, 1966 - 1976.

40 Gassmann, P. G.; Bottdorff, K. J. J. Am. Soc. 1987, 109, 7547 - 7548.

41 Allgemeine Übersichten siehe:

(a) Hill, A. E. Adv. Organomet. Chem. 1977, 16, 131 - 165. 
(b) Lindsell, W. E. In Comprehensive Organometallic Chemistry; Sir Wilkinson, G., Ed. Pergamon Press: Oxford 1982; Vol 1, Kap. 4, 155 - 253.

42 Imamoto, T.; Takiyama, N.; Nakamura, K.;Hatajima, T.; Kamiya, Y. J. Am. Chem. Soc. 1989, 111, 4392 - 4398.

43 (a) Eliel, E. L.; Pietrusiewicz, K. M. Org. Magn. Res. 1980, 13, 193 - 196.

(b) Hesse, M., Meier H., Zeeh, B. Spektroskopische Methoden in der organischen Chemie, Georg Thieme Verlag, Stuttgart 1987.

44 Whitesell, J. K.; Matthews, R. S. J. Org. Chem. 1977, 42, 3878 - 3882.

45 Brown, H. C.; Hammar, W. J. Tetrahedron 1978, 34, 3405 - 3411.

46 Gassmann, P. G.; Valcho, J. J.; Proehl, G. S.; Cooper, C. F. J. Am. Chem. Soc. 1980, $102,6519-6526$.

47 Billington, D. C.; Kerr, W: J. ; Pauson, P. L.; Farnocchi, C. J. Organomet. Chem. 1988, $356,213-219$.

48 Thiele, S.; Erker, G. Chem. Ber. 1997, 130, 201 - 207.

49 Whitesell, J. K.; Matthews, R. S.; Solomon, P. A. Tetrahedron Lett. 1976, 1549 - 1552.

50 Haufe, G.; Wolf, A.; Schulze, K. Tetrahedron 1986, 42, 4719 - 4728.

51 Mallien, M.; Haupt, E. T. K.; tom Dieck, H. Angew. Chem. 1988, 100, 1091 - 1092; Angew. Chem. Int. Ed. Engl. 1988, 27, 1062 - 1063.

52 Baldenius, K.-U.; tom Dieck, H. Angew. Chem. 1992, 104, 338 - 340; Angew. Chem. Int. Ed. Engl. 1992, 31, 305 - 307.

53 Olah, G. A., Synthesis 1986, 513 - 531.

54 Mann G., Muchall H. M. In Methods of Organic Chemistry (Houben-Weyl); de Meijere, A., Ed., Georg Thieme Verlag: Stuttgart 1997; Vol. E 17e, S.485 ff.

55 McDonald, R. N.; Reineke, C. E. J. Org. Chem. 1967, 32, 1878 - 1887.

56 Diaz, A. F.; Miller, R. D. J. Am. Chem. Soc. 1978, 66, 5905 - 5910. 
57 El-Hachach, N. Diplomarbeit, Universität Göttingen 1999.

58 Mandelt, K. Diplomarbeit, Universität Göttingen 1997.

59 Rei, M.-H. J. Org. Chem. 1978, 43, 2173 - 2178.

60 Pretsch, E.; Clerc, T.; Seibl, J.; Simon, W. Tabellen zur Strukturaufklärung organischer Verbindungen mit spektroskopischen Methoden, Springer Verlag, Berlin, Heidelberg, New York, 3.Auflage, 1990, I125.

61 Blacket, B. N.; Coxon, J. M.; Hartshorn, M. P.; Richards, K. E. J. Am. Chem. Soc. 1970, 92,2574 - 2575.

62 Allgemeine Übersichten siehe:

(a) Kirmse, W. Top. Curr. Chem., 1979, 80, 89 - 124.

(b) Saunders, M.; Chandrasekhar J.; Schleyer, P. v. R. Rearrangements of Carbocations. In Rearrangements in Ground and Excited States; de Mayo, P. Ed.; Academic Press: New York 1980; 1 - 53.

63 APT-Pulssequenz: Patt, S. L.; Shoolery, J. J. Magn. Res. 1982, 46, 535 - 539. 


\section{DANKSAGUNG}

Für die Messungen der NMR-Spektren danke ich Frau Andrea Godawa, Frau Carola Zolke, Herrn Ulrich Leonhardt und Herrn Reinhard Machinek.

Dank an Dr. Remberg und Frau Györgi Udvarnoki für die Aufnahme der Massenspektren.

Für die Bearbeitung der Elementaranalysen bedanke ich mich bei Herrn Hambloch.

Für die Bereitstellung von ausgewählten Chemikalien und allgegenwärtige Unterstützung danke ich Herrn Ralf Gerke.

Ich danke allen Mitgliedern der Abteilung Fitjer für ihr unkompliziertes Verhalten im Labor, ihre stete Hilfsbereitschaft und das angenehme Arbeitsklima. Imelda Meyer, Onno Graalmann und Nizar El-Hachach danke ich für die zahlreichen fördernden Diskussionen. Bei Imelda Meyer möchte ich mich für ihre angenehme Labornachbarschaft bedanken. Desweiteren bedanke ich mich bei Oliver Lauenstein für die schnellen GC-MS-Messungen.

Weiterhin gilt mein Dank für die Unterstützung ausserhalb des Labors allen meinen Freunden und Bekannten. An dieser Stelle seien insbesondere Praios, Helios, Neo, Turrium, Trevor, Nica, Xawin und Eorn genannt.

Meiner Schwester danke ich für die unermüdlichen Korrekturen und ihr starkes Interesse an meiner Arbeit.

Mein besonderer Dank gilt meinen Eltern, die mir dieses Studium überhaupt erst ermöglicht haben und für ihre Unterstützung während des gesamten Studiums.

Meinen Söhnen Maximilian und Lucas danke ich für die zahlreichen netten Stunden ausserhalb des normalen Arbeitstages.

Ganz besonders bedanke ich mich bei meiner Frau Judit für ihr Verständnis und ihre Hilfe während meines gesamten Studiums. 


\section{LEBENSLAUF}

Am 1.6.1969 wurde ich, Klaus Mandelt, in Göttingen als Sohn von Helga Mandelt, geborene Köhler, und Gerhard Mandelt geboren. Von 1975 - 1979 besuchte ich die Grundschule Bovenden, von 1979 - 1989 das Otto-Hahn-Gymnasium in Göttingen, wo ich 1989 auch die Allgemeine Hochschulreife erwarb. Von Juni 1989 - September 1990 absolvierte ich meinen Grundwehrdienst in Göttingen. Im Wintersemester 1990 nahm ich das Chemiestudium an der Georg-August-Universität in Göttingen auf. Im Februar 1995 bestand ich dort die Diplomvorprüfung im Fach Chemie. Im August 1995 heiratete ich die Arzthelferin Judit Buxadé Martí. Unter der Leitung von Prof. Dr. L. Fitjer begann ich im August 1996 am Organisch Chemischen Institut in Göttingen mit meiner Diplomarbeit über das Thema „1-Methylcyclobutylmagnesiumchlorid: Ein Reagenz zur Anellierung von Cyclopentenen“ und legte im Juni 1997 die Diplomprüfung im Fach Chemie ab. Seit Juli 1997 arbeite ich im gleichen Arbeitskreis an meiner Dissertation. Unser Sohn Maximilian wurde im April 1998 und unser Sohn Lucas im April 2000 geboren. 